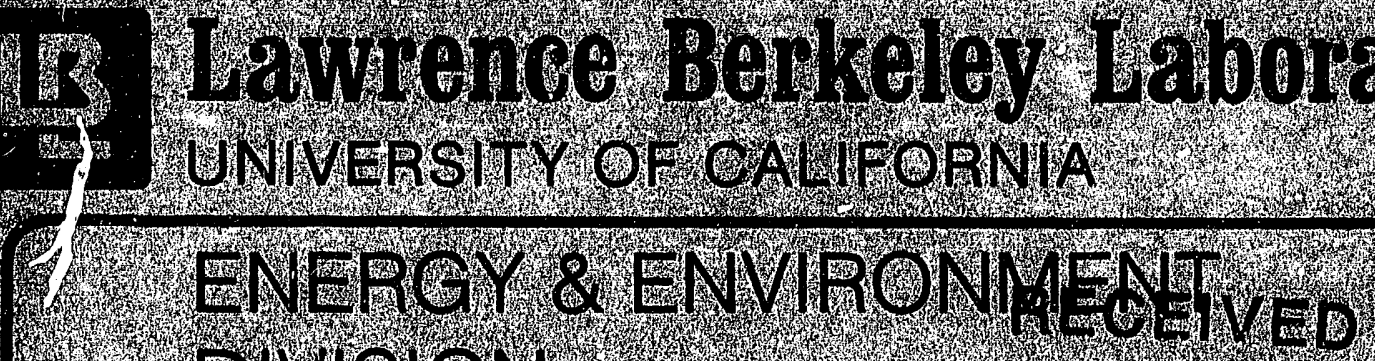 DUVISION

\author{
JuN 17.203 \\ OSTI
}

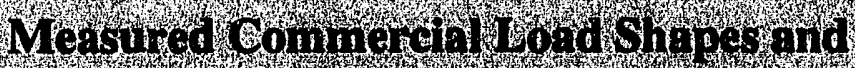

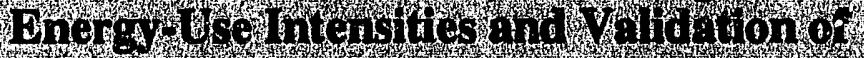

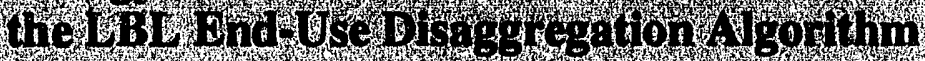

1. Akbari, L. Rainer, $\mathrm{K}$. Heinemeie,

January 1993

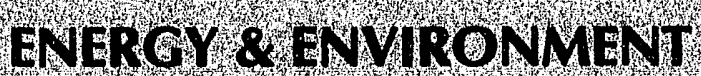
DNWSION 


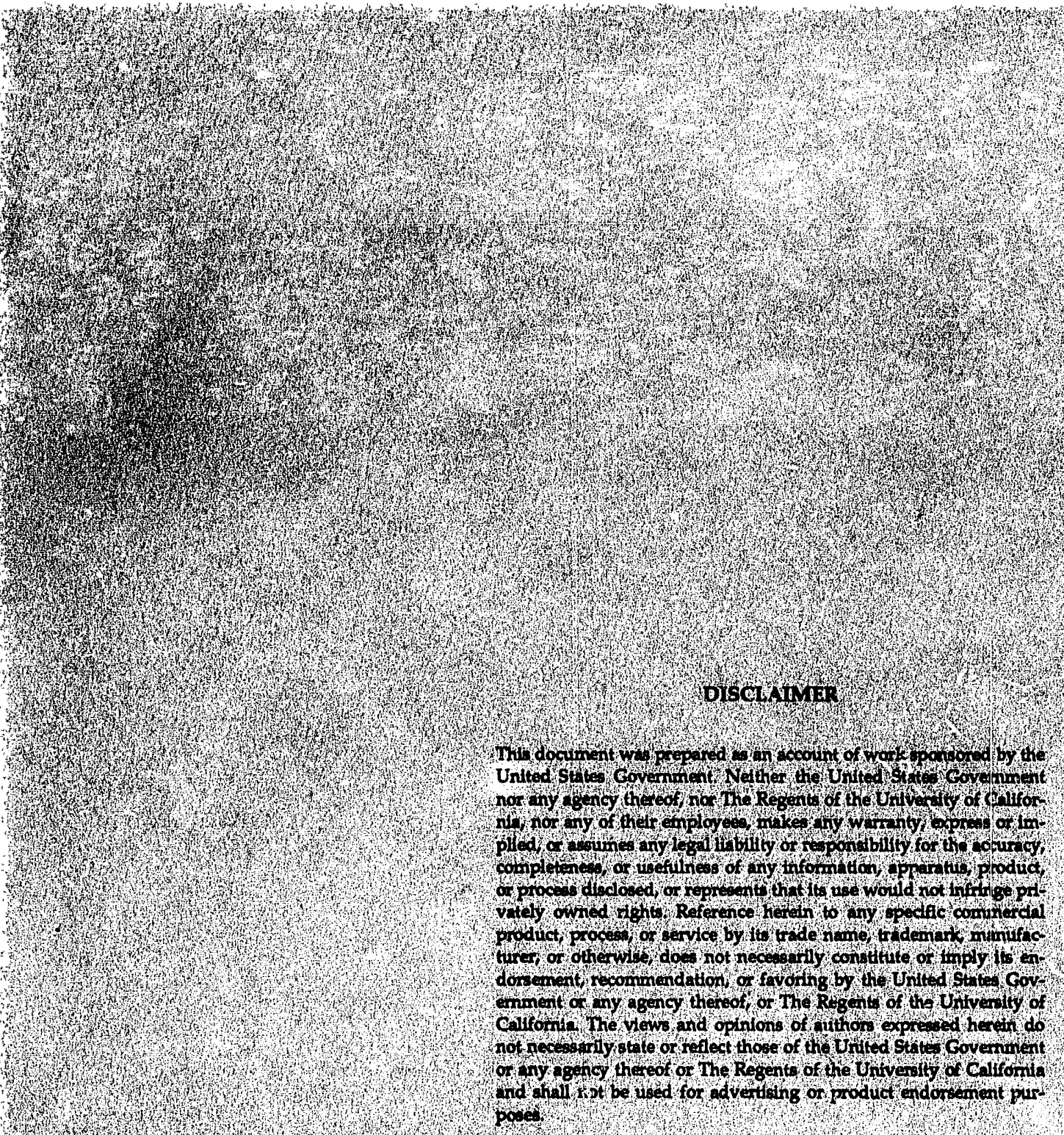

This report has been reproduced directy from the bet avilablecopy.

Avallable to DOE and BOE Contractor:

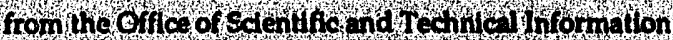

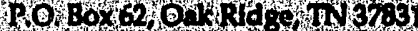

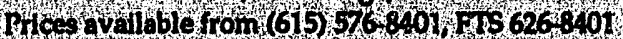

Avallable to the public fiom the

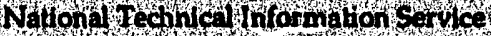

U. Doptum of Commero.

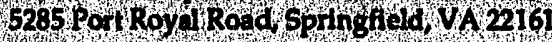

Whren ce Berkeley Labotatory is an equal opportunity enploy er. 
LBL-32193

UC-000

\title{
Measured Commercial Load Shapes and Energy-Use Intensities and Validation of the LBL End-Use Disaggregation Algorithm
}

\section{FINAL REPORT}

January 1993

\author{
A report prepared for the \\ Southern California Edison Company (SCE) \\ and \\ California Institute for Energy Efficiency (CIEE)
}

H. Akbari, L. Rainer, K. Heinemeier, J. Huang, and E. Franconi

Energy Analysis Program

Energy and Environment Division

Lawrence Berkeley Laboratory

Berkeley, CA 94720

This project was jointly funded by the California Institute for Energy Efficiency (CIEE) and the Southern California Edison Company (SCE) through the U.S. Department of Energy under Contract No. DEAC03-76SF00098. 


\section{Acknowledgement}

This project is jointly funded by the California Institute for Energy Efficiency (CIEE) and the Southern California Edison Company (SCE). We acknowledge the project support and thoughtful comments of Ishtiaq Chisti and Mark Martinez of SCE. 


\title{
Measured Commercial Load Shapes and Energy-Use Intensities and Validation of the LBL End-use Disaggregation Algorithm
}

\author{
H. Akbari, L. Rainer, K. Heinemeier, J. Huang, and E. Franconi
}

\begin{abstract}
The Southern California Edison Company (SCE) has conducted an extensive metering project in which electricity end use in 53 commercial buildings in Southern California has been measured. The building types monitored include offices, retail stores, groceries, restaurants, and warehouses. One year (June 1989 through May 1990) of the SCE measured hourly end-use data are reviewed in this report. Annual whole-building and end-use energy use intensities (EUIs) and monthly load shapes (LSs) have been calculated for the different building types based on the monitored data. This report compares the monitored buildings' EUIs and LSs to EUIs and LSs determined using whole-building load data and the End-Use Disaggregation Algorithm (EDA). Two sets of EDA determined EUIs and LSs are compared to the monitored data values. The data sets represent: 1) average buildings in the SCE service territory and 2) specific buildings that were monitored.

The EDA model was developed at Lawrence Berkeley Laboratory (LBL). It uses characteristics of the whole-building electric load and disaggregates it into major end-use components. The EDA based EUI and LS values presented in this study for average buildings have been determined in a previous study conducted by LBL and jointly sponsored by the California Energy Commission (CEC) and Southern California Edison. The average building types are the same as the monitored building types. The average buildings are based on the average characteristics of buildings found in SCE's service area. The data indicate that the EDA estimates and the measured average end use EUIs and LSs for all the building types reviewed, except the grocery, statistically compare well. The LBL LSs developed for non-standard days (weekends and holidays) show some questionable characteristics. These LSs were found to differ from those in the limited sample of end-use metered buildings.

The measured data from two of the end-use metered buildings (an office and a retail store) have been used to validate the LBL EDA model. The characteristics of the buildings and their measured whole-building loads were used with EDA to estimate their end use loads. In the retail store analysis, the EDA estimates of hourly end-use compare remarkably well with the monitored end-use data (average error of less than 5\% during daytime operation). For the office building, the model gives a consistent bias of about $30 \%$ in over estimating the HVAC electric load at the expense of under estimating the miscellaneous load. This can be attributed to the presence of inconsistencies between the office audit information and its measured end-use data. A three-fold difference between the auditor's estimate for miscellaneous energy use (EDA input) and the metered amount has been found. The validation, however, indicates great promise for the application of EDA to whole-building load data for obtaining reliable end-use data.
\end{abstract}




\section{Table of Contents}

Chapter I: Introduction and Purpose ............................................................................... 1

Chapter II: Analysis of Measured End-Use Data .................................................................... 5

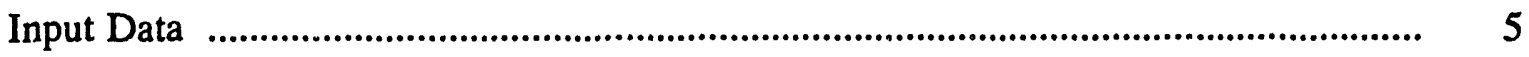

EUI Analysis and Comparisons with Forecasting Models ............................................ 9

Office buildings .................................................................................................. 10

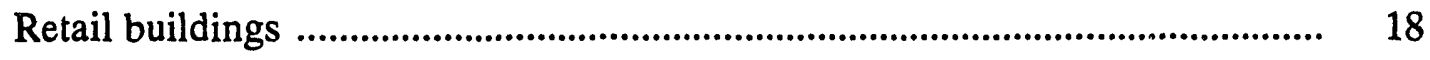

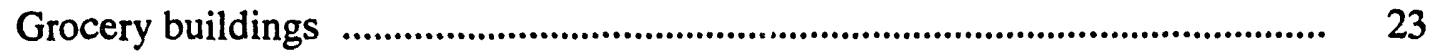

Restaurant buildings ........................................................................................ 30

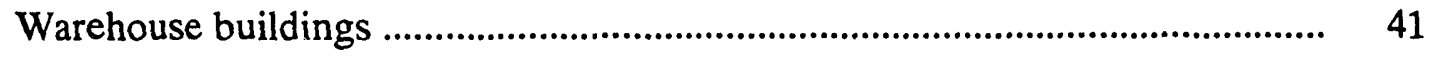

Statistical Comparison ........................................................................................ 46

Load-shape Analysis and Comparison with Forecasting Models ................................... 50

Large Office buildings .................................................................................. 50

Small Office buildings ................................................................................. 82

Large Retail buildings ......................................................................................... 82

Small Retail buildings ..................................................................................... 82

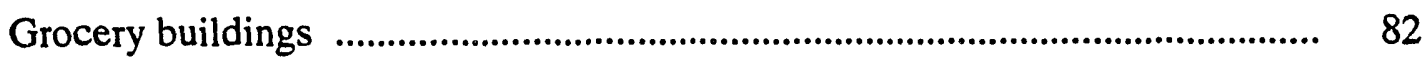

Restaurant buildings ........................................................................................... 83

Warehouse buildings .......................................................................................... 83

Summary ......................................................................................................... 84

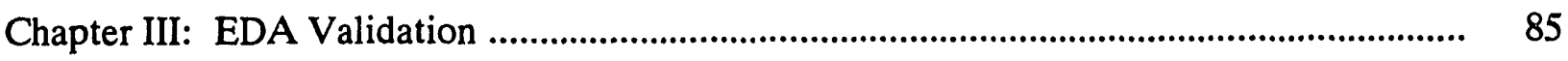

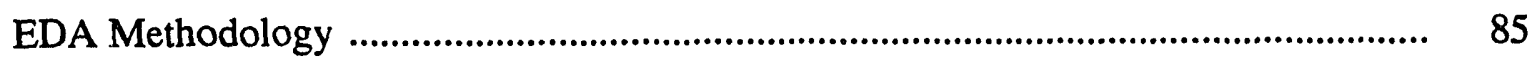

Validation .................................................................................................................. 86

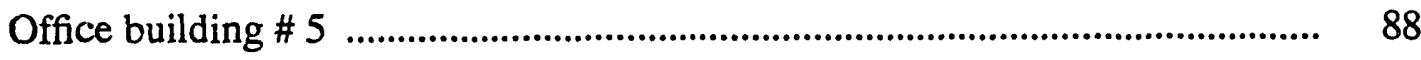

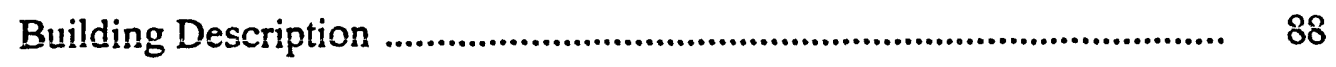


DOE-2 Simulation

Load/Temperature Regressions ........................................................... 94

EDA Output and Comparison with Monitored End Uses .......................... 99

Retail building \# 1 ............................................................................................ 111

Building Description .................................................................................. 111

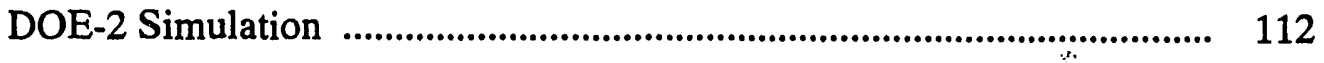

Load/Temperature Regressions ........................................................... 115

EDA Output and Comparison with Monitored End Uses .......................... 122

Chapter IV: Conclusions and Recommendations .............................................................. 133

Load-Temperature Regressions ............................................................................ 133

Components of the Non-Temperature-Dependent Load ............................................ 142

"Permissible" Hourly End-use Load Variations .............................................................. 143

Recommendations ............................................................................................ 149

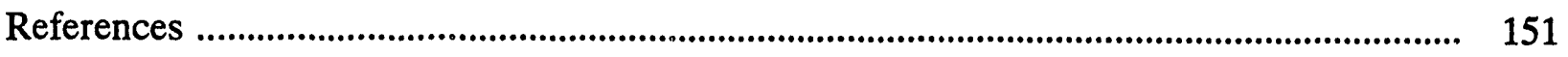

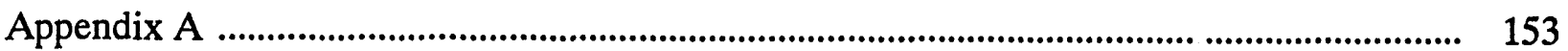




\section{Chapter I: Introduction and Purpose}

Reliable end-use electric load shape data are essential for accurate load forecasting. Metering projects to obtain the needed data are very expensive and, consequently, difficult to justify. Analytical techniques to obtain these data are promising alternatives to metering because they are far less costly. Nevertheless, validation of these techniques must rely ultimately on end-use metered data.

In 1988, the Southern California Edison Company (SCE) and the California Energy Commission (CEC) jointly sponsored a unique project at the Lawrence Berkeley Laboratory (LBL). The project was to develop an analytical technique to estimate typical commercial sector load shapes (LSs) and end-use energy use intensities (EUIs) using whole-building load research data, individual building audit data, and mail survey data from many buildings (Akbari et al. 1989). The building types and end uses addressed in the SCE/CEC project are summarized in Table I.1. These categories are the same as those used in SCE's energy forecasting model. For the project, LBL developed a disaggregation inethod and determined EUIs and LSs for each building type in three SCE climate zones. The climate zones are represented by Los Angeles, Burbank, and Norton Air Force Base weather.

The development of a disaggregation method is significant because such an analytical technique promises to be far less expensive than end-use metering for obtaining end-use LSs and EUIs. The LBL project was unique because it produced a new method for performing this type of analysis. The method is based on a combination of engineering analysis, building performance computer simulations and reconciliation with measured whole-building electric load data (Akbari et al. 1988). The project was also unique because it represented a convergence of SCE and CEC energy demand forecasting research efforts with the end result of a common set of data for use in future forecasts.

An important missing piece of the project was the absence of high quality end-use metered data to validate the technique. Although validation was envisioned in the scope of the initial effort, the needed data were not yet available. Now that the SCE metering projects are mature, we can use existing data to refine and validate the LSs and EUIs developed by LBL. 


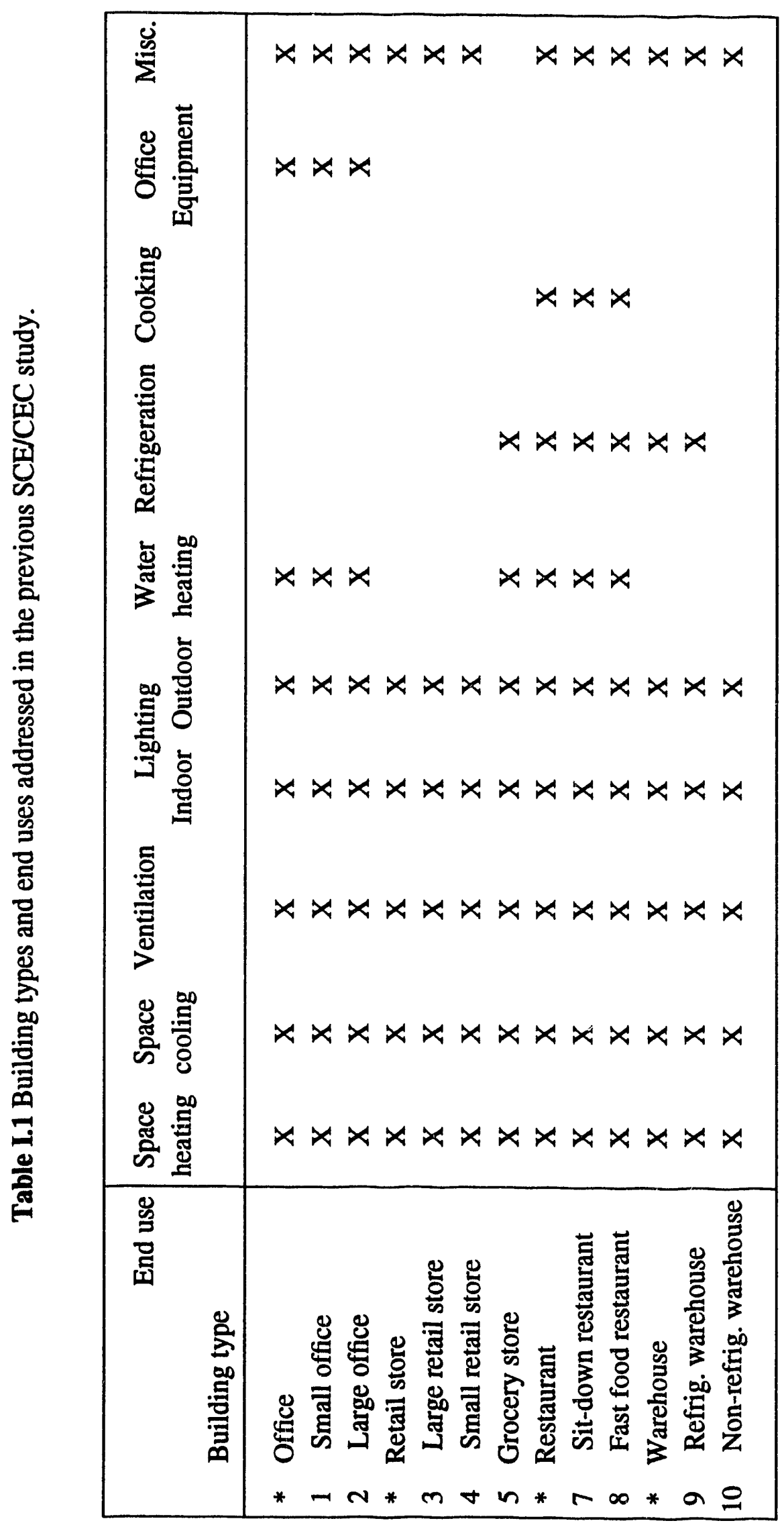


A comparison of data collected in the SCE end-use metering project (Table 1.2) and enduse data required for the forecasting model (Table I.1) indicates that some further disaggregation of the monitored data is required to make them useful for the model. For instance, the metered end-use data for HVAC should be further disaggregated to Heating, Ventilation, and AirConditioning loads. One of the purposes of this project is to compare the metered end-use data with our previous estimates of EUIs and LSs resulting from the SCE/CEC-sponsored project and, accordingly, refine the EUIs and LSs.

In the first part of this report, we compare the EUIs and LS for the 53 SCE buildings to EUIs and LS predicted by the disaggregation algorithm for the average building population. The average EUIs and LS were developed in the 1989 LBL study. We discuss the metered end-use data obtained from SCE and review them for completeness. We briefly review the characteristics of the buildings that have been submetered and compare their characteristics to those which represent the population of commercial buildings in the SCE service territory. The objective of this comparison is to assess the feasibility for making generalized findings from the limited SCE submetered building sample and relating these findings to the building population. We discuss using the end-use metered data to assess the estimates of EUIs and LSs resulting from the previous LBL project. The assessment was initially planned to be carried out for the building categories of office, retail, and grocery. But in addition, we have been able to analyze data for restaurant and warehouse buildings.

In the second part of this report, we apply the EDA to two individual buildings in the SCE metering sample, an office building and a retail building. We compare the measured EUIs and LS and the EDA-developed EUIs and LS for each building. The comparison allows for the refinement and validation of the EDA methodology.

The report concludes with a sımmary of the project's major findings. The possible uses for the end-use data in further validation and refinement of EDA are discussed. 


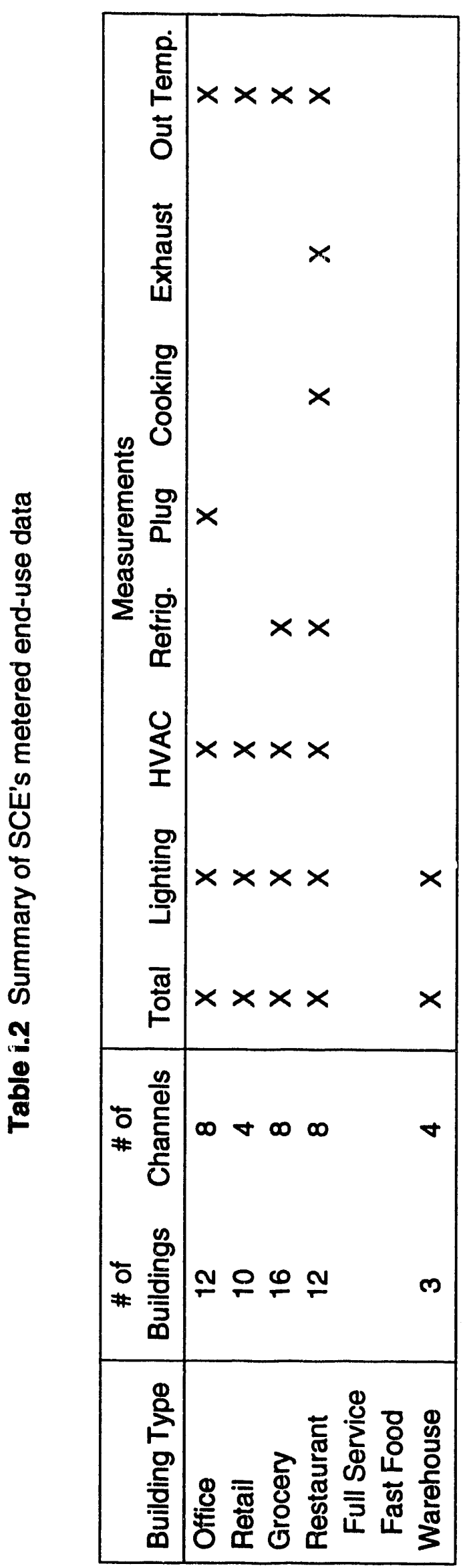




\section{Chapter II: Analysis of Measured End-Use Data}

\section{Input Data}

The SCE end-use monitoring project has collected short-interval (15-minutes to hourly) data for up to six end uses for five building types in 53 buildings. The building types monitored include offices, retail stores, grocery stores, restaurants, and warehouses. Only electricity consumption data are collected. The end uses monitored include total building electricity use, HVAC, lighting, refrigeration, cooking, plug load, and exhaust fan. In addition to electricity use data, outdoor temperature was also collected for some buildings. Table 1.2 provides a summary of monitored building types, number of buildings, end uses, and the number of monitored channels per building. A detailed review of these data is reported in SCE (1989 and 1990).

To limit metering costs, the SCE project has been selective in monitoring end uses. A minimum of two end uses, total building and lighting energy use, has been collected for all building types. Total HVAC energy have been collected for all building types but the warehouses. Plug load and refrigeration load have been collected for offices and groceries, respectively. For restaurants, refrigeration, cooking, and exhaust fan end uses have also been monitored.

The buildings selected for the SCE end-use metering projects were a subset of the 375 buildings that had detailed on-site survey data collected for them during 1985-1986 (CEC 1986). The data were used in the $1989 \mathrm{LBL}$ project for defining average building characteristics in the SCE service territory. We received the on-site survey data for the building set in electronic form, the files were formatted for use with Statistical Analysis Software (SAS). In the electronic data set, information regarding the building identification (i.e. address) was removed before the data were transferred to LBL. As part of the end-use metering project, SCE collected updated building and system information for the 53 buildings. Hard copies of the updated data along with the hourly end-use data were provided to LBL for use in this study.

We received one year of metered data (June 1, 1989 through May 31, 1990) for most of the building types. In addition to the monitored end-use data, we received a year of outside dry bulb temperature data, revised and updated information on the conditioned and unconditioned floor areas for each building, and the suggested floor area weighting factors. The weighing factors are used for estimating the population EUIs and LSs from the sample buildings' EUIs and LSs. The SCE weighting factors are derived from the fraction of floor area, by building type, that are 
represented by the monitored buildings in each of the SCE's four planning regions.

Since our analysis required data starting on January 1 , we wrapped the metered values and created one complete calendar year of data such that January 1 to May 31 of 1990 appears at the beginning of the data stream, followed by June 1 to December 31 of 1989 . For buildings in which monitoring started later than June 1,1989 , the wrapping of data caused discontinuities to appear in the plots. However, it did not affect our analysis.

For quick visual check of the data, we have generated three dimensional plots of the hourly data. A sample of these 3-D plots for an office building (Office \# 5) with HVAC, lighting, receptacles, and total building metered electricity use is shown in Figure II.1. Similar plots have been generated for each of the metered buildings and are presented in Appendix $A$.

Note that the HVAC electricity use for Office \#5 is primarily driven by schedules. Besides daily operational schedules of the HVAC system, two levels of HVAC use corresponding to June through November 1989 and December 1989 to May 1990 periods are observed. In fact, the observed HVAC energy use, during January and February 1990, seems to be different from the other two periods. The same variation is also observed in the plug energy use, with apparently a few weeks of significant variations from 'normal' schedules during the months of March and April 1990. The lighting energy use apparently follows a uniform daily schedule during the year.

As we will discuss in the following sections, the total building electricity use is key input data to the EDA algorithm. The disaggregation of total electricity use data into end-use consumption is based on the two principle hypotheses of EDA, namely:

1. the total building electric load carries the true signature of all the major building end uses and

2. the temperature dependence of the total building electricity use is caused solely by HVAC energy use.

Metered end-use data provide a means for validating these hypotheses. Using the SCE submetered data however, resulted in two immediaie problems. First, not all building end uses are monitored; the total building electricity use is usually larger than the summation of all the end uses. ${ }^{1}$ Second, the definition of the metered end uses are not consistent. For instance, the plug

\footnotetext{
1 As discussed in the appendix, two buildings have total building energy use less than the summation of all the monitored end uses.
} 

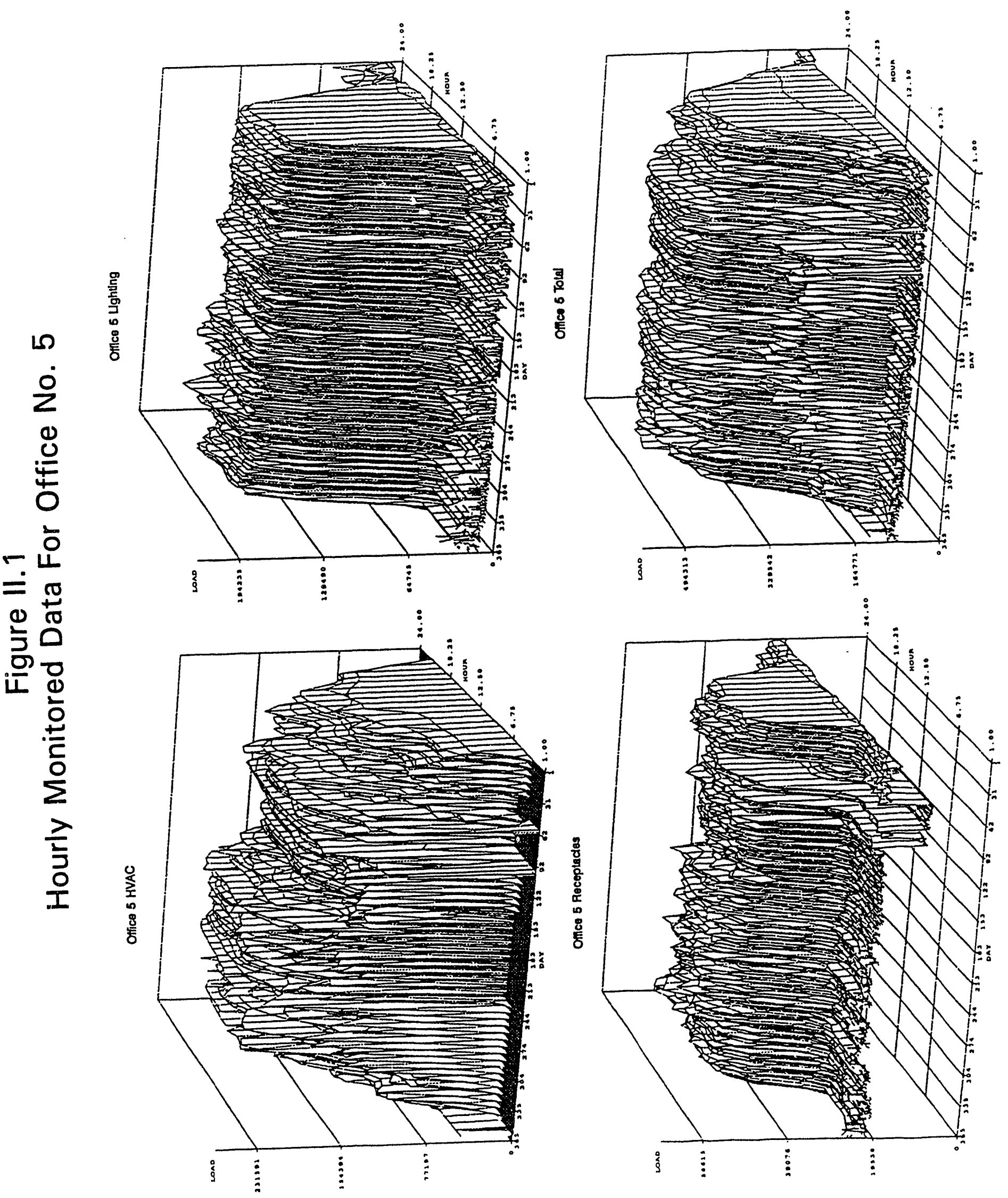
energy use may include task lighting or weather dependent end uses such as electric space heaters or fans.

Despite the above-mentioned problems, it is still possible to observe the characteristics of the HVAC load in the whole-building hourly electricity use. HVAC electric loads are characterized by operating schedules and climate conditions. For the two buildings examined, the periods of daily schedule changes that are observed for both the whole-building and the HVAC electricity use are; June through November 1989, December 1989 through February 1990, and March through May 1990. We will discuss and present the temperature dependency of the HVAC and whole-building load in a subsequent section. 


\section{EUI Analysis and Comparison with Forecasting Models}

EUIs were developed for all monitored end uses using total building load data from the sample of monitored buildings. In determining the annual EUIs, we summed the energy use for all the hours that were monitored. In those cases where a year of data was not available, the estimated EUIs were prorated based on the ratio of 8760 to the hours of available data. For example, the sum of the energy use for all valid, metered hours of Office \#6 (this building has 8,575 hours of valid data) is multiplied by the ratio of 8,760 over 8,575 in order to estimate the building's EUIs. Such a simple proration, in general, will yield an acceptable amount of accuracy for EUIs, provided that the number of hours of missing data is only a small fraction (less than 25\%) of all the data. The prorated EUIs are then used to develop weighted and unweighted average EUIs for each building type.

Hourly data are not valid and not included in the analysis if

- the data are labeled missing (indicated by ' ' in the SAS file),

- the data are labeled questionably (indicated by negative numbers in the SAS file),

- or the data collection for a building started later than June 1, 1989 (shown as missing data).

In the following sections, we present a summary table of EUIs including the estimated average EUIs for each building type. We compare the end use intensity values with each other and with the EUIs estimated in the 1989 LBL study. 


\section{Office buildings}

There are 12 office buildings in the data sample with total floor areas ranging from 2,400 to $160,000 \mathrm{ft}^{2}$ and total electric EUls ranging from 3.4 to $59 \mathrm{kWh} / \mathrm{ft}^{2}$ year. We partitioned the offices into two categories; small and large. SCE has also partioned their commercial customers into small and large building categories. We used the same criteria as SCE for partitioning. The size partitions are based on 15 minute-interval whole-building Load Research Data (LRD) collected by SCE; all those offices with annual electricity use of over $400,000 \mathrm{kWh} /$ year are considered large and those with annual electricity use less than $400,000 \mathrm{kWh} /$ year are considered small. With this partitioning criteria, offices $1,4,5$, and 12 are in the large office group and offices 3 and 6 through 10 are in the small office group.

Upon examination of the electricity use data, we found that office \#2 is actually a warehouse (EUI=3.4 kWh / $\mathrm{ft}^{2}$ year) and office \#11 has an activity which uses a large of amount energy (EUI $=59 \mathrm{kWh} / \mathrm{ft}^{2}$, such as a printing shop) that should be dealt with separately. For these reasons, in calculating the average EUIs, we did not use data from these two buildings. Note that the total building EUI, excluding office \#2 and \#11, ranges from 7.7 to $26.8 \mathrm{~W} / \mathrm{ft}^{2}$ year. Table II.1 presents the summary data for all offices. The metered data summary includes the hours of valid and bad data, the estimated EUIs based on the conditioned and total building floor area, and the weighted and unweighted EUIs.

Figure II.2 compares the total electricity use for the twelve offices with the office total energy use developed using EDA by LBL in the 1989 study. The comparison is favorable for small office buildings but for large office buildings the LBL data is about $20 \%$ larger than the average of the monitored large offices. Knowing that the LBL values have been developed from SCE's building-class billing data and 1985 on-site building survey data, the difference between the average EUIs can probably be attributed to the small sample size of the monitored offices.

Figure II.3 compares the measured HVAC EUIs for large and small offices with the LBL EUI values. The LBL study provides data for three climate regions. However, the limited sample size of the monitored building does not allow a comparison at a regional climate zone level. Hence, we took the EUIs developed for the Burbank climate zone from the LBL study as an average of the entire SCE service area for all of our comparisons of HVAC EUIs. 
Table II.1

End Use Intensities for Office Buildings

\begin{tabular}{|c|c|c|c|c|c|c|c|c|}
\hline \multirow{2}{*}{$\begin{array}{c}\text { Bldg } \\
\text { Type } \\
\text { OFF LGE } \\
\text { OFF LGE } \\
\text { OFF LGE } \\
\text { OFF LGE }\end{array}$} & \multirow{2}{*}{$\begin{array}{c}\begin{array}{c}\text { Bidg } \\
\text { Num }\end{array} \\
1 \\
1 \\
1 \\
1\end{array}$} & End Use & \multicolumn{2}{|c|}{$\begin{array}{l}\text { Observations } \\
\text { Valid Bad }\end{array}$} & $\begin{array}{l}\text { Total } \\
\text { Floor } \\
\text { Area }\end{array}$ & $\begin{array}{c}\text { Conditioned } \\
\text { Floor } \\
\text { Area }\end{array}$ & \multicolumn{2}{|c|}{$\begin{array}{l}\text { End Use Intensities } \\
\left(\mathrm{kWh} / \mathrm{tt}^{2} \cdot \mathrm{yr}\right)\end{array}$} \\
\hline & & $\begin{array}{l}\text { HVAC } \\
\text { Lighting } \\
\text { Receptacle } \\
\text { Total }\end{array}$ & $\begin{array}{l}8760 \\
8760 \\
8760 \\
8760\end{array}$ & $\begin{array}{l}0 \\
0 \\
0 \\
0\end{array}$ & $\begin{array}{l}19800 \\
19800 \\
19800 \\
19800\end{array}$ & $\begin{array}{l}19800 \\
19800 \\
1.9800 \\
19800\end{array}$ & $\begin{array}{r}9.130 \\
12.064 \\
0.549 \\
26.798\end{array}$ & $\begin{array}{r}9.130 \\
12.064 \\
0.549 \\
26.798\end{array}$ \\
\hline $\begin{array}{l}\text { OFF" } \\
\text { OFF" } \\
\text { OFF" } \\
\text { OFF" }\end{array}$ & $\begin{array}{l}2 \\
2 \\
2 \\
2\end{array}$ & \begin{tabular}{l|} 
HVAC \\
Lighting \\
Receptacle \\
Total
\end{tabular} & $\begin{array}{l}8760 \\
8760 \\
8760 \\
8760\end{array}$ & $\begin{array}{l}0 \\
0 \\
0 \\
0\end{array}$ & $\begin{array}{l}58632 \\
58632 \\
58632 \\
58632 \\
\end{array}$ & $\begin{array}{l}9462 \\
9462 \\
9462 \\
9462\end{array}$ & $\begin{array}{l}0.392 \\
2.299 \\
0.378 \\
3.395\end{array}$ & $\begin{array}{r}2.433 \\
14.248 \\
2.344 \\
21.040\end{array}$ \\
\hline $\begin{array}{l}\text { OFF SML } \\
\text { OFF SML } \\
\text { OFF SML } \\
\text { OFF SML }\end{array}$ & $\begin{array}{l}3 \\
3 \\
3 \\
3\end{array}$ & $\begin{array}{l}\text { HVAC } \\
\text { Lighting } \\
\text { Receptacle } \\
\text { Total }\end{array}$ & $\begin{array}{l}8759 \\
8760 \\
8760 \\
8759\end{array}$ & $\begin{array}{l}1 \\
0 \\
0 \\
1\end{array}$ & $\begin{array}{l}28880 \\
28880 \\
28880 \\
28880\end{array}$ & $\begin{array}{l}28880 \\
28880 \\
28880 \\
28880\end{array}$ & \begin{tabular}{|r|}
3.243 \\
2.338 \\
3.919 \\
11.338 \\
\end{tabular} & \begin{tabular}{r|}
3.243 \\
2.338 \\
3.919 \\
11.338 \\
\end{tabular} \\
\hline $\begin{array}{l}\text { OFF LGE } \\
\text { OFF LGE } \\
\text { OFF LGE } \\
\text { OFF LGE }\end{array}$ & $\begin{array}{l}4 \\
4 \\
4 \\
4 \\
\end{array}$ & $\begin{array}{l}\text { HVAC } \\
\text { Lighting } \\
\text { Receptacle } \\
\text { Total } \\
\end{array}$ & \begin{tabular}{|l|}
7738 \\
8549 \\
8549 \\
8548 \\
\end{tabular} & $\begin{array}{r}1022 \\
211 \\
211 \\
212 \\
\end{array}$ & $\begin{array}{l}51500 \\
51500 \\
51500 \\
51500 \\
\end{array}$ & $\begin{array}{l}51500 \\
51500 \\
51500 \\
51500 \\
\end{array}$ & \begin{tabular}{|r|}
2.513 \\
3.430 \\
2.405 \\
17.274 \\
\end{tabular} & $\begin{array}{r}2.513 \\
3.430 \\
2.405 \\
17.274 \\
\end{array}$ \\
\hline $\begin{array}{l}\text { OFF LGE } \\
\text { OFF LGE } \\
\text { OFF LGE } \\
\text { OFF LGE }\end{array}$ & $\begin{array}{l}5 \\
5 \\
5 \\
5\end{array}$ & \begin{tabular}{l|} 
HVAC \\
Lighting \\
Receptacle \\
Total \\
\end{tabular} & $\begin{array}{l}8757 \\
8757 \\
8757 \\
8757\end{array}$ & $\begin{array}{l}3 \\
3 \\
3 \\
3 \\
\end{array}$ & \begin{tabular}{|l|}
117600 \\
117600 \\
117600 \\
117600 \\
\end{tabular} & $\begin{array}{l}101136 \\
101136 \\
101136 \\
101136 \\
\end{array}$ & \begin{tabular}{|r|}
5.961 \\
4.419 \\
2.149 \\
17.127 \\
\end{tabular} & $\begin{array}{r}6.932 \\
5.139 \\
2.499 \\
19.916 \\
\end{array}$ \\
\hline $\begin{array}{l}\text { OFF SML } \\
\text { OFF SML } \\
\text { OFF SML } \\
\text { OFF SML }\end{array}$ & $\begin{array}{l}6 \\
6 \\
6 \\
6\end{array}$ & $\begin{array}{l}\text { HVAC } \\
\text { Lighting } \\
\text { Receptacle } \\
\text { Total }\end{array}$ & $\begin{array}{l}8575 \\
8575 \\
8575 \\
8575\end{array}$ & $\begin{array}{l}185 \\
185 \\
185 \\
185\end{array}$ & $\begin{array}{l}46125 \\
46125 \\
46125 \\
46125\end{array}$ & $\begin{array}{l}37420 \\
37420 \\
37420 \\
37420\end{array}$ & $\begin{array}{l}4.007 \\
2.964 \\
1.219 \\
7.714\end{array}$ & $\begin{array}{l}4.939 \\
3.654 \\
1.503 \\
9.509\end{array}$ \\
\hline $\begin{array}{l}\text { OFF SML } \\
\text { OFF SML } \\
\text { OFF SML } \\
\text { OFF SML }\end{array}$ & $\begin{array}{l}7 \\
7 \\
7 \\
7\end{array}$ & $\begin{array}{l}\text { HVAC } \\
\text { Lighting } \\
\text { Receptacie } \\
\text { Total }\end{array}$ & $\begin{array}{l}7751 \\
7751 \\
7751 \\
7751\end{array}$ & $\begin{array}{l}1009 \\
1009 \\
1009 \\
1009\end{array}$ & $\begin{array}{l}21300 \\
21300 \\
21300 \\
21300\end{array}$ & $\begin{array}{l}21300 \\
21300 \\
21300 \\
21300\end{array}$ & \begin{tabular}{|r|}
8.646 \\
8.968 \\
3.202 \\
21.846 \\
\end{tabular} & $\begin{array}{r}8.646 \\
8.968 \\
3.202 \\
21.846\end{array}$ \\
\hline $\begin{array}{l}\text { OFF SML } \\
\text { OFF SML } \\
\text { OFF SML } \\
\text { OFF SML }\end{array}$ & $\begin{array}{l}8 \\
8 \\
8 \\
8 \\
\end{array}$ & $\begin{array}{l}\text { HVAC } \\
\text { Lighting } \\
\text { Receptacle } \\
\text { Total } \\
\end{array}$ & $\begin{array}{l}6694 \\
7617 \\
7617 \\
7617 \\
\end{array}$ & $\begin{array}{r}1442 \\
543 \\
519 \\
519 \\
\end{array}$ & $\begin{array}{l}32800 \\
32800 \\
32800 \\
32800\end{array}$ & $\begin{array}{l}32800 \\
32800 \\
32800 \\
32800\end{array}$ & $\begin{array}{r}2.079 \\
5.260 \\
2.907 \\
14.185\end{array}$ & $\begin{array}{r}2.079 \\
5.260 \\
2.907 \\
14.185 \\
\end{array}$ \\
\hline $\begin{array}{l}\text { OFF SML } \\
\text { OFF SML } \\
\text { OFF SML } \\
\text { OFF SML }\end{array}$ & $\begin{array}{l}9 \\
9 \\
9 \\
9 \\
\end{array}$ & $\begin{array}{l}\text { HVAC } \\
\text { Lighting } \\
\text { Receptacle } \\
\text { Total } \\
\end{array}$ & $\begin{array}{l}7920 \\
7920 \\
7920 \\
7920\end{array}$ & $\begin{array}{l}216 \\
240 \\
216 \\
216\end{array}$ & $\begin{array}{l}19150 \\
19150 \\
19150 \\
19150\end{array}$ & $\begin{array}{l}16852 \\
16852 \\
16852 \\
16852 \\
\end{array}$ & $\begin{array}{r}5.259 \\
6.145 \\
0.540 \\
21.673\end{array}$ & $\begin{array}{r}5.976 \\
6.983 \\
0.614 \\
24.629\end{array}$ \\
\hline $\begin{array}{l}\text { OFF SML } \\
\text { OFF SML } \\
\text { OFF SML }\end{array}$ & $\begin{array}{l}10 \\
10 \\
10\end{array}$ & $\begin{array}{l}\text { HVAC } \\
\text { Lighting } \\
\text { Total }\end{array}$ & $\begin{array}{l}7536 \\
7536 \\
7535\end{array}$ & $\begin{array}{l}264 \\
264 \\
265\end{array}$ & $\begin{array}{l}2396 \\
2396 \\
2396\end{array}$ & $\begin{array}{l}2396 \\
2396 \\
2396\end{array}$ & $\begin{array}{r}4.466 \\
7.900 \\
15.073\end{array}$ & $\begin{array}{r}4.466 \\
7.900 \\
15.073\end{array}$ \\
\hline
\end{tabular}


Table II. 1 (continued)

End Use Intensities for Office Buildings

\begin{tabular}{|c|c|c|c|c|c|c|c|c|}
\hline \multirow{2}{*}{\begin{tabular}{|l} 
Bldg \\
Type \\
OFF* \\
OFF" \\
OFF* \\
OFF"
\end{tabular}} & $\begin{array}{l}\text { Bldg } \\
\text { Num }\end{array}$ & End Use & \multicolumn{2}{|c|}{$\begin{array}{l}\text { Observations } \\
\text { Valid Bad }\end{array}$} & $\begin{array}{l}\text { Total } \\
\text { Floor } \\
\text { Area }\end{array}$ & $\begin{array}{l}\text { Conditioned } \\
\text { Floor } \\
\text { Area }\end{array}$ & \multicolumn{2}{|c|}{$\begin{array}{l}\text { End Use Intensities } \\
\left(k W h / t^{2} \cdot y r\right)\end{array}$} \\
\hline & $\begin{array}{l}11 \\
11 \\
11 \\
11\end{array}$ & $\begin{array}{l}\text { HVAC } \\
\text { Lighting } \\
\text { Receptacle } \\
\text { Total }\end{array}$ & $\begin{array}{l}6781 \\
6740 \\
7560 \\
7560\end{array}$ & $\begin{array}{r}1019 \\
1060 \\
240 \\
240\end{array}$ & $\begin{array}{l}15900 \\
15900 \\
15900 \\
15900\end{array}$ & $\begin{array}{l}15900 \\
15900 \\
15900 \\
15900\end{array}$ & $\begin{array}{r}18.903 \\
10.743 \\
3.116 \\
59.462 \\
\end{array}$ & $\begin{array}{r}18.903 \\
10.743 \\
3.116 \\
59.462\end{array}$ \\
\hline $\begin{array}{l}\text { OFF LGE } \\
\text { OFF LGE } \\
\text { OFF LGE } \\
\text { OFF LGE }\end{array}$ & $\begin{array}{l}12 \\
12 \\
12 \\
12\end{array}$ & \begin{tabular}{l|} 
HVAC \\
Lighting \\
Receptacle \\
Total
\end{tabular} & $\begin{array}{l}5784 \\
5784 \\
5544 \\
5543\end{array}$ & $\begin{array}{r}0 \\
0 \\
264 \\
241\end{array}$ & $\begin{array}{l}81914 \\
81914 \\
81914 \\
81914 \\
\end{array}$ & $\begin{array}{l}50787 \\
50787 \\
50787 \\
50787 \\
\end{array}$ & \begin{tabular}{|r|}
5.316 \\
2.922 \\
0.971 \\
11.332 \\
\end{tabular} & \begin{tabular}{r|}
8.573 \\
4.712 \\
1.566 \\
18.277 \\
\end{tabular} \\
\hline $\begin{array}{l}\text { Weighted } \\
\text { OFF SML } \\
\text { OFF SML } \\
\text { OFF SML } \\
\text { OFF SML. }\end{array}$ & Averag & $\begin{array}{l}\text { ges : } \\
\text { HVAC } \\
\text { Lighting } \\
\text { Receptacle } \\
\text { Total }\end{array}$ & & & & & $\begin{array}{r}6.002 \\
5.515 \\
1.310 \\
17.085 \\
\end{array}$ & $\begin{array}{r}7.554 \\
6.408 \\
1.630 \\
20.549 \\
\end{array}$ \\
\hline $\begin{array}{l}\text { OFF LGE } \\
\text { OFF LGE } \\
\text { OFF LGE } \\
\text { OFF LGE }\end{array}$ & & \begin{tabular}{l|} 
HVAC \\
Lighting \\
Receptacle \\
Total \\
\end{tabular} & & & & & \begin{tabular}{|r|}
5.818 \\
5.924 \\
2.280 \\
15.518 \\
\end{tabular} & $\begin{array}{r}6.186 \\
6.220 \\
2.382 \\
16.346 \\
\end{array}$ \\
\hline $\begin{array}{l}\text { UnWeight } \\
\text { OFF SML } \\
\text { OFF SML } \\
\text { OFF SML } \\
\text { OFF SML }\end{array}$ & ed Ave & $\begin{array}{l}\text { Rrages: } \\
\text { HVAC } \\
\text { Lighting } \\
\text { Receptacle } \\
\text { Total }\end{array}$ & & & & & \begin{tabular}{|r|}
5.730 \\
5.709 \\
1.518 \\
18.133 \\
\end{tabular} & $\begin{array}{r}6.787 \\
6.336 \\
1.755 \\
20.566 \\
\end{array}$ \\
\hline $\begin{array}{l}\text { OFF LGE } \\
\text { OFF LGE } \\
\text { OFF LGE } \\
\text { OFF LGE }\end{array}$ & & $\begin{array}{l}\text { HVAC } \\
\text { Lighting } \\
\text { Receptacle } \\
\text { Total }\end{array}$ & & & & & \begin{tabular}{|r|}
4.617 \\
5.596 \\
1.964 \\
15.305
\end{tabular} & $\begin{array}{r}4.892 \\
5.851 \\
2.024 \\
16.097\end{array}$ \\
\hline
\end{tabular}

- Buildings 2 and 11 were anomalous and not used in calculating average EUI's. 


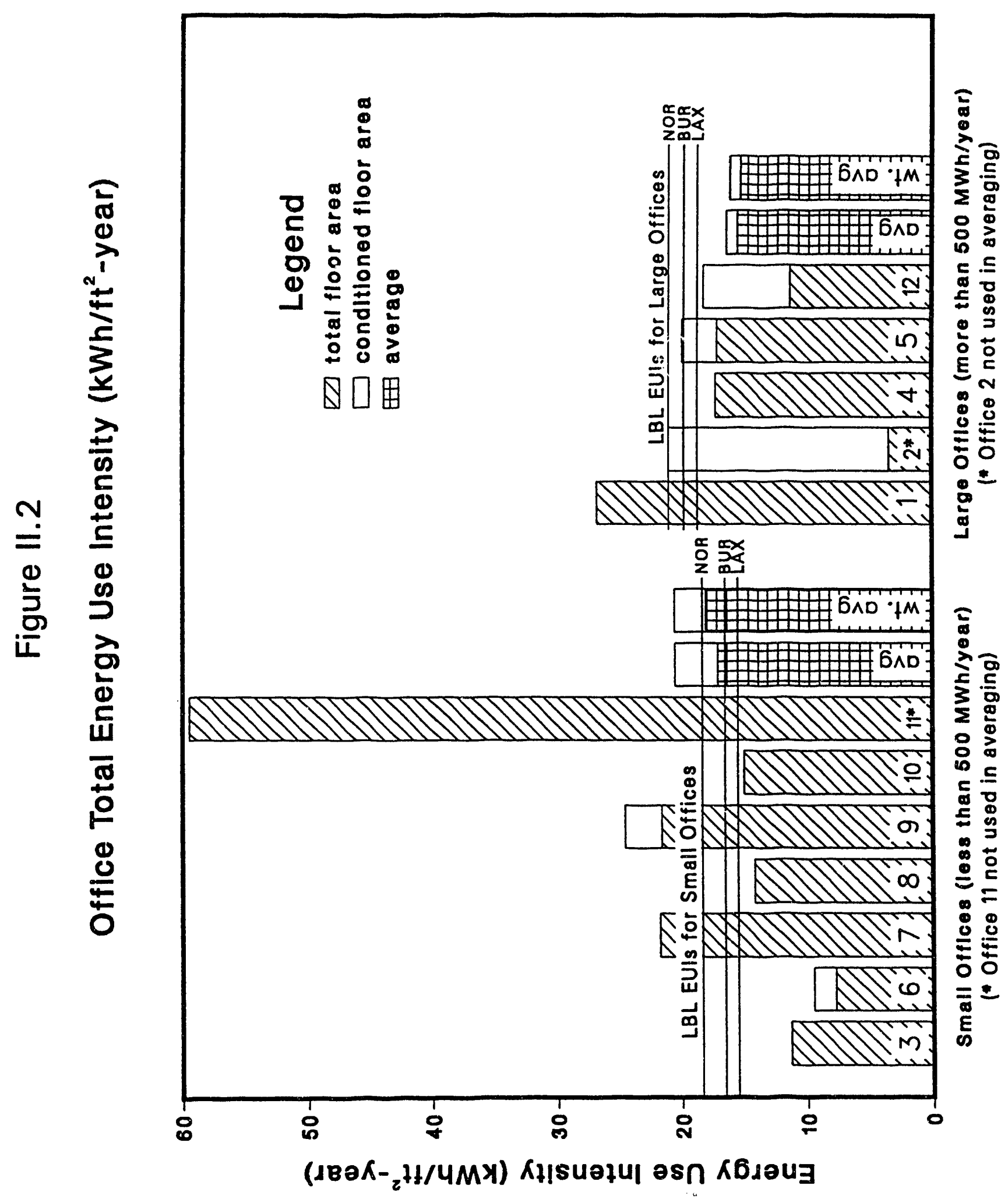




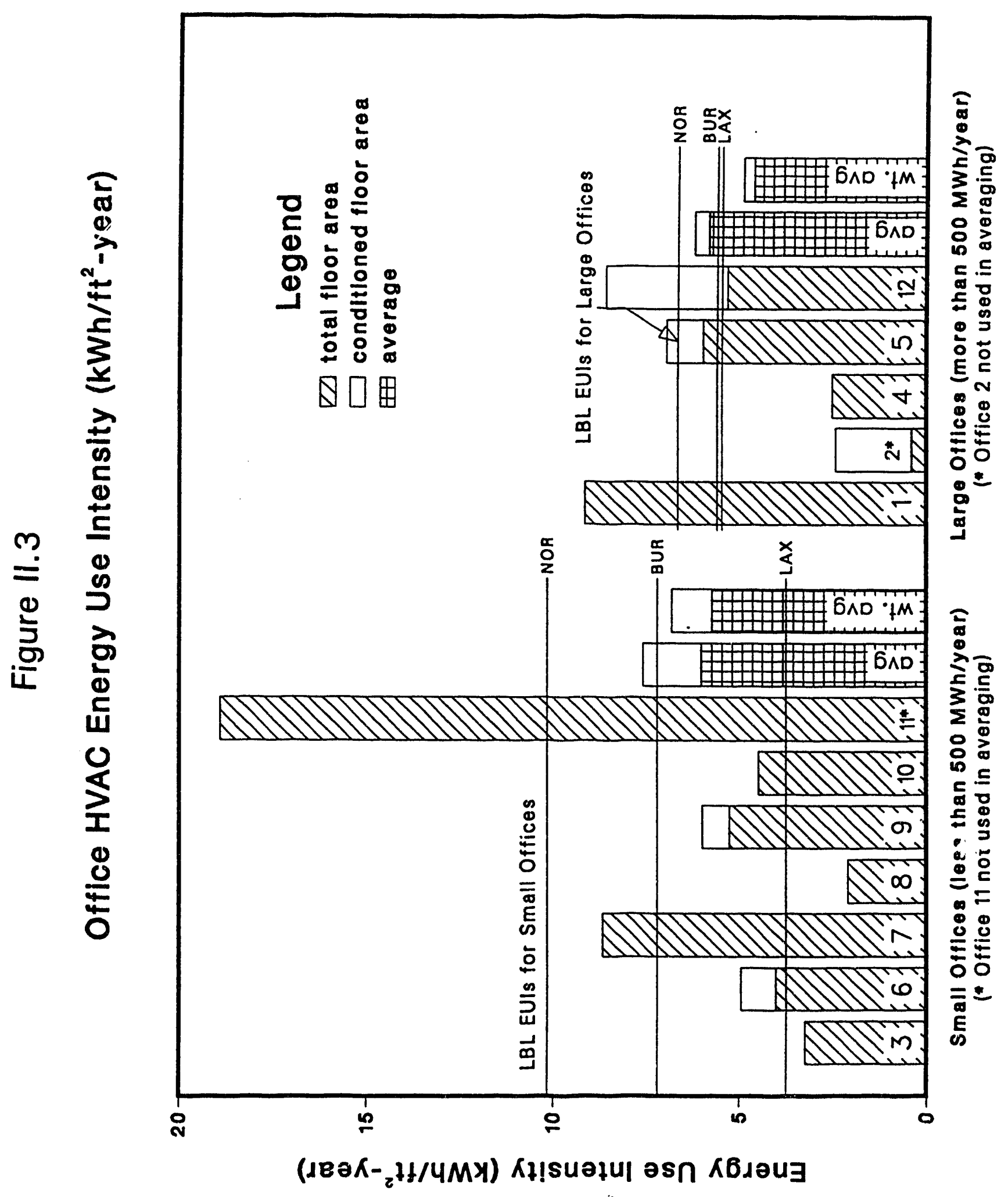


The HVAC EUIs developed for the small office building compare well with the monitored data. The comparison for the large office buildings is not as close, yet still acceptable.

Figure II.4 shows the lighting EUIs for both small and large office buildings. The agreement between the measured and the LBL values for small offices is good, but the EUIs for large offices are significantly different. Further analysis of the data showed that while the characteristic office building in the LBL study has significant nighttime lighting, the nighttime lighting is very small for the monitored buildings. Also, the LBL data include the nighttime outdoor lighting in the lighting end-use value. We cannot tell whether the monitored buildings included outdoor lighting in the lighting channel.

Figure II.5 compares the 'plug' end-use data of the monitored buildings with the 'miscellaneous' end-use data estimated in the LBL study. The differences between these data are due to the different definition of these end uses. Note that the LBL EUIs for miscellaneous end uses do not include lighting, HVAC, refrigeration, cooking and water heating loads; but for the monitored buildings, the plug loads may include these end uses. 


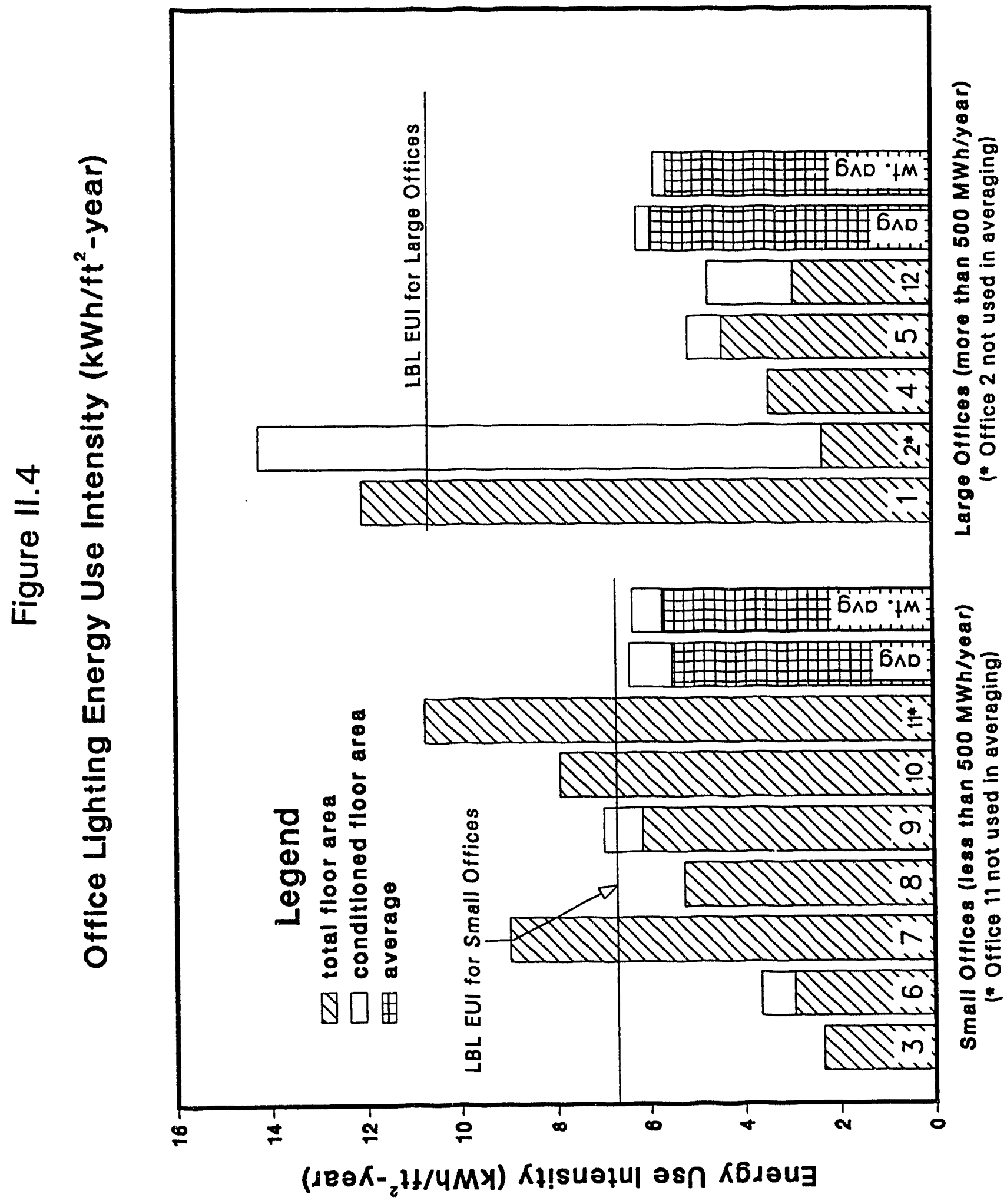




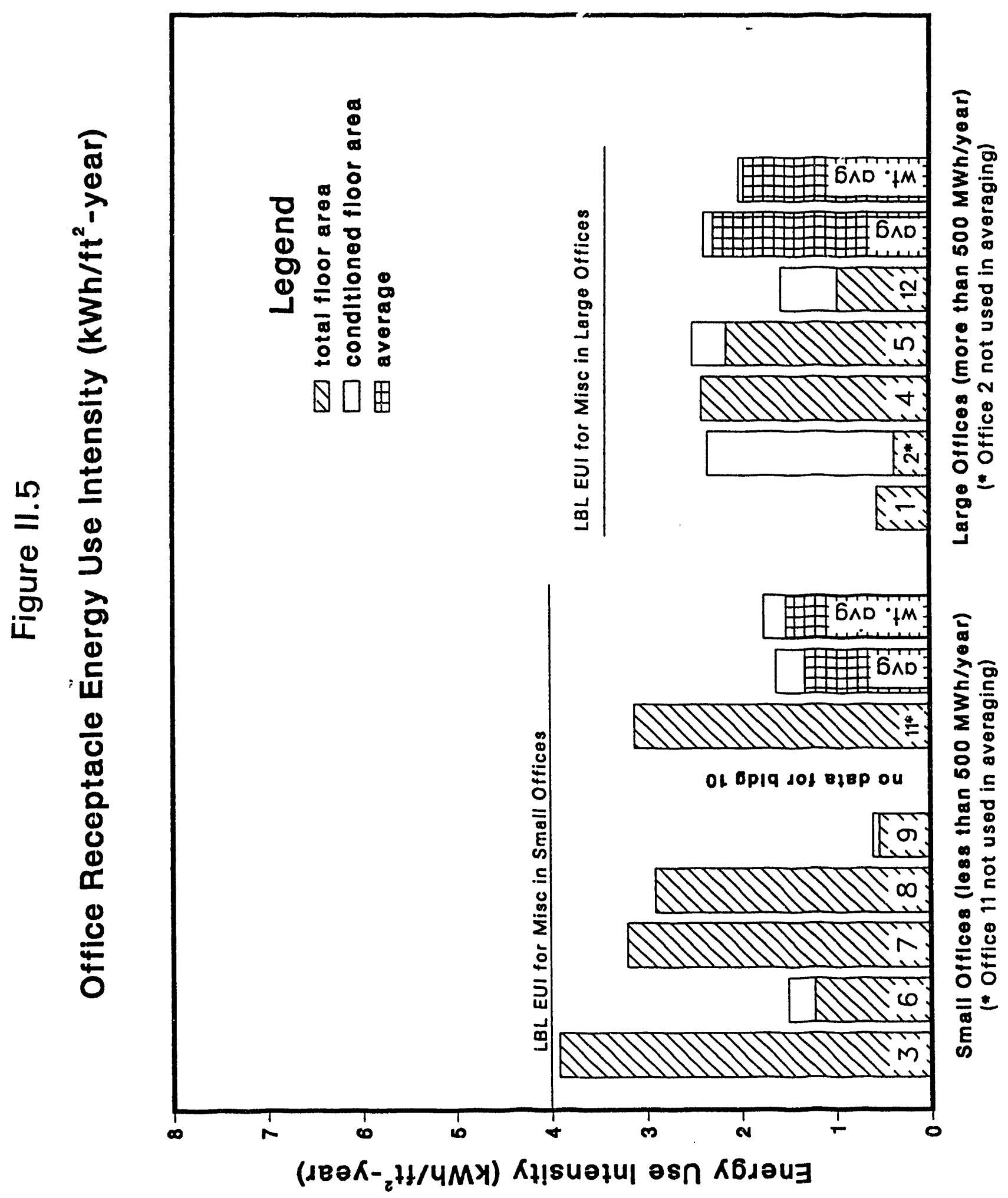




\section{Retail buildings}

There are 10 retail buildings in the submetered building sample with total floor areas ranging from 14,500 to $66,800 \mathrm{ft}^{2}$ and with total electric EUIs ranging from 10.4 to 34.2 $\mathrm{kWh} / \mathrm{ft}^{2}$ year. As in the office category, we partitioned the buildings in two categories of small and large retails, using the same criteria that SCE uses in their partioning. All the retail buildings with annual electricity use of over $400,000 \mathrm{kWh} /$ year are considered large and those with annual electricity use less than $400,000 \mathrm{kWh} / \mathrm{year}$ are considered small. With this partitioning criteria, buildings $1,3,4$, and 9 are in the small retail group and buildings 2,5 through 8 , and 10 are in the large retail group.

Table I.2 presents the summary data for all retail buildings including the number of missing or 'bad' data, estimated EUIs based on the conditioned and total building floor area, and weighted and unweighted end-use EUIs.

Figure II.6 compares the total building EUIs for large and small retail buildings with the LBL estimates for retail buildings. For both sizes of retail buildings, the LBL whole-building EUI is about 15 to 20 percent lower than the monitored buildings. Knowing that the LBL values are based on SCE's building-class sales data and 1985 on-site building survey data, the difference between the average total building EUIs can be probably traced to the small sample size of the monitored retail buildings.

Figure II.7 compares the HVAC EUIs for large and small retail. The LBL study predicted EUIs for three climate regions. However, the limited sample size of the monitored building does not allow a comparison at a regional climate zone level. Hence, we took LBL values developed for the Burbank climate region as an average of the entire SCE service area for all of our comparisons of HVAC EUIs.

The LBL estimate for HVAC EUI for small retail buildings is about $20 \%$ lower than average value of the the monitored data. The comparison of the large retail building HVAC EUIs is very close.

Figure II.8 shows the lighting EUIs for both small and large retail buildings. The agreement for small retail is good, but the LBL EUIs for large retail are about $20 \%$ lower than the monitored data. 
Table 11.2

End Use Intensities for Retail Buildings

\begin{tabular}{|c|c|c|c|c|c|c|c|c|}
\hline \multirow{2}{*}{$\begin{array}{l}\text { Bldg } \\
\text { Type }\end{array}$} & \multirow[b]{2}{*}{$\begin{array}{l}\text { Bldg } \\
\text { Num }\end{array}$} & \multirow[b]{2}{*}{ End Use } & \multirow{2}{*}{\multicolumn{2}{|c|}{ Observations }} & \multirow{2}{*}{$\begin{array}{l}\text { Total } \\
\text { Floor } \\
\text { Area }\end{array}$} & \multirow{2}{*}{$\begin{array}{l}\text { Conditioned } \\
\text { Floor } \\
\text { Area }\end{array}$} & \multicolumn{2}{|c|}{$\begin{array}{l}\text { End Use Intensities } \\
\left(\mathrm{kWh} / \mathrm{tt}^{2} \cdot \mathrm{yr}\right)\end{array}$} \\
\hline & & & & & & & $\begin{array}{l}\text { per tot } \\
\text { flr area }\end{array}$ & $\begin{array}{l}\text { per cond. } \\
\text { flr area }\end{array}$ \\
\hline $\begin{array}{l}\text { RTL SML } \\
\text { RTL SML } \\
\text { RTL SML }\end{array}$ & $\begin{array}{l}1 \\
1 \\
1\end{array}$ & $\begin{array}{l}\text { HVAC } \\
\text { Lighting } \\
\text { Total }\end{array}$ & $\begin{array}{l}8608 \\
8608 \\
8608\end{array}$ & $\begin{array}{l}152 \\
152 \\
152\end{array}$ & $\begin{array}{l}23396 \\
23396 \\
23396\end{array}$ & $\begin{array}{l}23396 \\
23396 \\
23396\end{array}$ & \begin{tabular}{|r|}
4.415 \\
8.319 \\
13.049 \\
\end{tabular} & \begin{tabular}{|r|}
4.415 \\
8.319 \\
13.049
\end{tabular} \\
\hline $\begin{array}{l}\text { RTL LGE } \\
\text { RTL LGE } \\
\text { RTL LGE }\end{array}$ & $\begin{array}{l}2 \\
2 \\
2\end{array}$ & $\begin{array}{l}\text { HVAC } \\
\text { Lighting } \\
\text { Total }\end{array}$ & $\begin{array}{l}8760 \\
8760 \\
8760\end{array}$ & $\begin{array}{l}0 \\
0 \\
0\end{array}$ & $\begin{array}{l}25500 \\
25500 \\
25500\end{array}$ & $\begin{array}{l}25500 \\
25500 \\
25500\end{array}$ & \begin{tabular}{|r|}
8.188 \\
24.208 \\
34.262
\end{tabular} & $\begin{array}{r}8.188 \\
24.208 \\
34.262\end{array}$ \\
\hline $\begin{array}{l}\text { RTL SML } \\
\text { RTL SML } \\
\text { RTL SML }\end{array}$ & $\begin{array}{l}3 \\
3 \\
3\end{array}$ & $\begin{array}{l}\text { HVAC } \\
\text { Lighting } \\
\text { Total }\end{array}$ & $\begin{array}{l}6683 \\
8377 \\
8382\end{array}$ & $\begin{array}{r}18 \overline{37} \\
143 \\
138\end{array}$ & $\begin{array}{l}26900 \\
26900 \\
26900\end{array}$ & $\begin{array}{l}26900 \\
26900 \\
26900\end{array}$ & $\begin{array}{r}3.801 \\
4.596 \\
10.405 \\
\end{array}$ & $\begin{array}{r}3.801 \\
4.596 \\
10.405\end{array}$ \\
\hline $\begin{array}{l}\text { RTL SML } \\
\text { RTL SML } \\
\text { RTL SML }\end{array}$ & $\begin{array}{l}4 \\
4 \\
4\end{array}$ & $\begin{array}{l}\text { HVAC } \\
\text { Lighting } \\
\text { Total }\end{array}$ & $\begin{array}{l}8625 \\
8625 \\
8625\end{array}$ & $\begin{array}{l}135 \\
135 \\
135\end{array}$ & $\begin{array}{l}19162 \\
19162 \\
19162 \\
\end{array}$ & $\begin{array}{l}19162 \\
19162 \\
19162\end{array}$ & $\begin{array}{r}7.536 \\
8.235 \\
16.285 \\
\end{array}$ & $\begin{array}{r}7.536 \\
8.235 \\
16.285\end{array}$ \\
\hline $\begin{array}{l}\text { RTL LGE } \\
\text { RTL LGE } \\
\text { RTL LGE }\end{array}$ & $\begin{array}{l}5 \\
5 \\
5\end{array}$ & $\begin{array}{l}\text { HVAC } \\
\text { Lighting } \\
\text { Total }\end{array}$ & $\begin{array}{l}8709 \\
7128 \\
8709 \\
\end{array}$ & $\begin{array}{r}51 \\
1632 \\
51 \\
\end{array}$ & \begin{tabular}{|l|}
20640 \\
20640 \\
20640 \\
\end{tabular} & $\begin{array}{l}19402 \\
19402 \\
19402 \\
\end{array}$ & \begin{tabular}{|r|}
9.704 \\
14.493 \\
24.888 \\
\end{tabular} & $\begin{array}{l}10.324 \\
15.417 \\
26.476 \\
\end{array}$ \\
\hline $\begin{array}{l}\text { RTL LGE } \\
\text { RTL LGE } \\
\text { RTL LGE }\end{array}$ & $\begin{array}{l}6 \\
6 \\
6\end{array}$ & $\begin{array}{l}\text { HVAC } \\
\text { Lighting } \\
\text { Total }\end{array}$ & $\begin{array}{l}8760 \\
8760 \\
8760 \\
\end{array}$ & $\begin{array}{l}0 \\
0 \\
0\end{array}$ & $\begin{array}{l}66800 \\
66800 \\
66800 \\
\end{array}$ & $\begin{array}{l}66800 \\
66800 \\
66800 \\
\end{array}$ & $\begin{array}{r}5.664 \\
8.594 \\
20.989 \\
\end{array}$ & $\begin{array}{r}5.664 \\
8.594 \\
20.989 \\
\end{array}$ \\
\hline $\begin{array}{l}\text { RTL LGE } \\
\text { RTL LGE } \\
\text { RTL LGE }\end{array}$ & $\begin{array}{l}7 \\
7 \\
7\end{array}$ & $\begin{array}{l}\text { HVAC } \\
\text { Lighting } \\
\text { Total }\end{array}$ & $\begin{array}{l}8759 \\
8759 \\
8759\end{array}$ & $\begin{array}{l}1 \\
1 \\
1\end{array}$ & $\begin{array}{l}37500 \\
37500 \\
37500\end{array}$ & $\begin{array}{l}37500 \\
37500 \\
37500\end{array}$ & $\begin{array}{r}2.841 \\
4.455 \\
10.694 \\
\end{array}$ & $\begin{array}{r}2.841 \\
4.455 \\
10.694\end{array}$ \\
\hline $\begin{array}{l}\text { RTL LGE } \\
\text { RTL LGE } \\
\text { RTL LGE }\end{array}$ & $\begin{array}{l}8 \\
8 \\
8\end{array}$ & $\begin{array}{l}\text { HVAC } \\
\text { Lighting } \\
\text { Total }\end{array}$ & $\begin{array}{l}8537 \\
8760 \\
8760\end{array}$ & $\begin{array}{r}223 \\
0 \\
0\end{array}$ & $\begin{array}{l}29350 \\
29350 \\
29350\end{array}$ & $\begin{array}{l}29350 \\
29350 \\
29350\end{array}$ & \begin{tabular}{|r|}
7.010 \\
13.851 \\
22.632 \\
\end{tabular} & $\begin{array}{r}7.010 \\
13.851 \\
22.632\end{array}$ \\
\hline $\begin{array}{l}\text { RTL SML } \\
\text { RTL SML } \\
\text { RTL SML }\end{array}$ & $\begin{array}{l}9 \\
9 \\
9\end{array}$ & $\begin{array}{l}\text { HVAC } \\
\text { Lighting } \\
\text { Total }\end{array}$ & $\begin{array}{l}8757 \\
8757 \\
8757\end{array}$ & $\begin{array}{l}3 \\
3 \\
3\end{array}$ & $\begin{array}{l}14500 \\
14500 \\
14500\end{array}$ & $\begin{array}{l}14500 \\
14500 \\
14500\end{array}$ & \begin{tabular}{|r|}
11.305 \\
5.565 \\
17.197 \\
\end{tabular} & $\begin{array}{r}11.305 \\
5.565 \\
17.197\end{array}$ \\
\hline $\begin{array}{l}\text { RTL LGE } \\
\text { RTL LGE } \\
\text { RTL LGE }\end{array}$ & $\begin{array}{l}10 \\
10 \\
10\end{array}$ & $\begin{array}{l}\text { HVAC } \\
\text { Lighting } \\
\text { Total }\end{array}$ & \begin{tabular}{|l|}
7536 \\
7536 \\
7536 \\
\end{tabular} & $\begin{array}{l}600 \\
600 \\
600 \\
\end{array}$ & $\begin{array}{l}30000 \\
30000 \\
30000\end{array}$ & $\begin{array}{l}30000 \\
30000 \\
30000 \\
\end{array}$ & $\begin{array}{r}6.970 \\
11.086 \\
20.432 \\
\end{array}$ & $\begin{array}{r}6.970 \\
11.086 \\
20.432 \\
\end{array}$ \\
\hline $\begin{array}{l}\text { Weighted } \\
\text { RTL SML } \\
\text { RTL SML } \\
\text { RTL SML }\end{array}$ & verag & $\begin{array}{l}\text { es: } \\
\text { HVAC } \\
\text { Lighting } \\
\text { Total }\end{array}$ & & & & & $\begin{array}{r}6.871 \\
6.688 \\
14.334\end{array}$ & $\begin{array}{r}6.871 \\
6.688 \\
14.334\end{array}$ \\
\hline $\begin{array}{l}\text { RTL LGE } \\
\text { RTL LGE } \\
\text { RTL LGE }\end{array}$ & & $\begin{array}{l}\text { HVAC } \\
\text { Lighting } \\
\text { Total }\end{array}$ & & & & & $\begin{array}{r}6.489 \\
12.571 \\
22.288 \\
\end{array}$ & $\begin{array}{r}6.629 \\
12.780 \\
22.647 \\
\end{array}$ \\
\hline $\begin{array}{l}\text { UnWeighte } \\
\text { RTL SML } \\
\text { RTL SML } \\
\text { RTL SML }\end{array}$ & Ave & $\begin{array}{l}\text { rages: } \\
\text { HVAC } \\
\text { Lighting } \\
\text { Total }\end{array}$ & & & & & $\begin{array}{r}6.764 \\
6.679 \\
14.234\end{array}$ & $\begin{array}{r}6.764 \\
6.679 \\
14.234\end{array}$ \\
\hline $\begin{array}{l}\text { RTL LGE } \\
\text { RTL LGE } \\
\text { RTL LGE }\end{array}$ & & $\begin{array}{l}\text { HVAC } \\
\text { Lighting } \\
\text { Total } \\
\end{array}$ & & & & & \begin{tabular}{|r|}
6.730 \\
12.781 \\
22.316 \\
\end{tabular} & $\begin{array}{r}6.833 \\
12.935 \\
22.581 \\
\end{array}$ \\
\hline
\end{tabular}




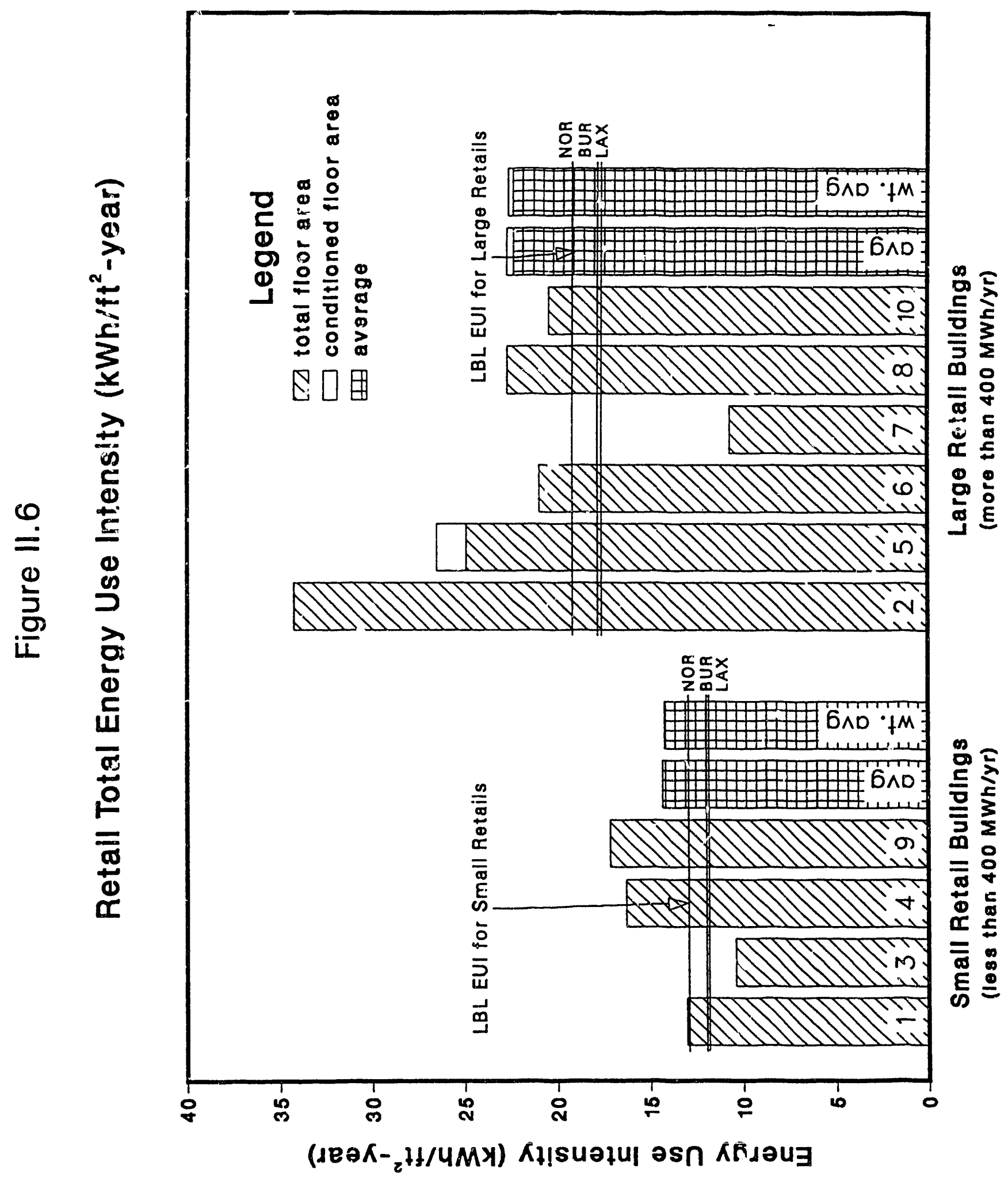




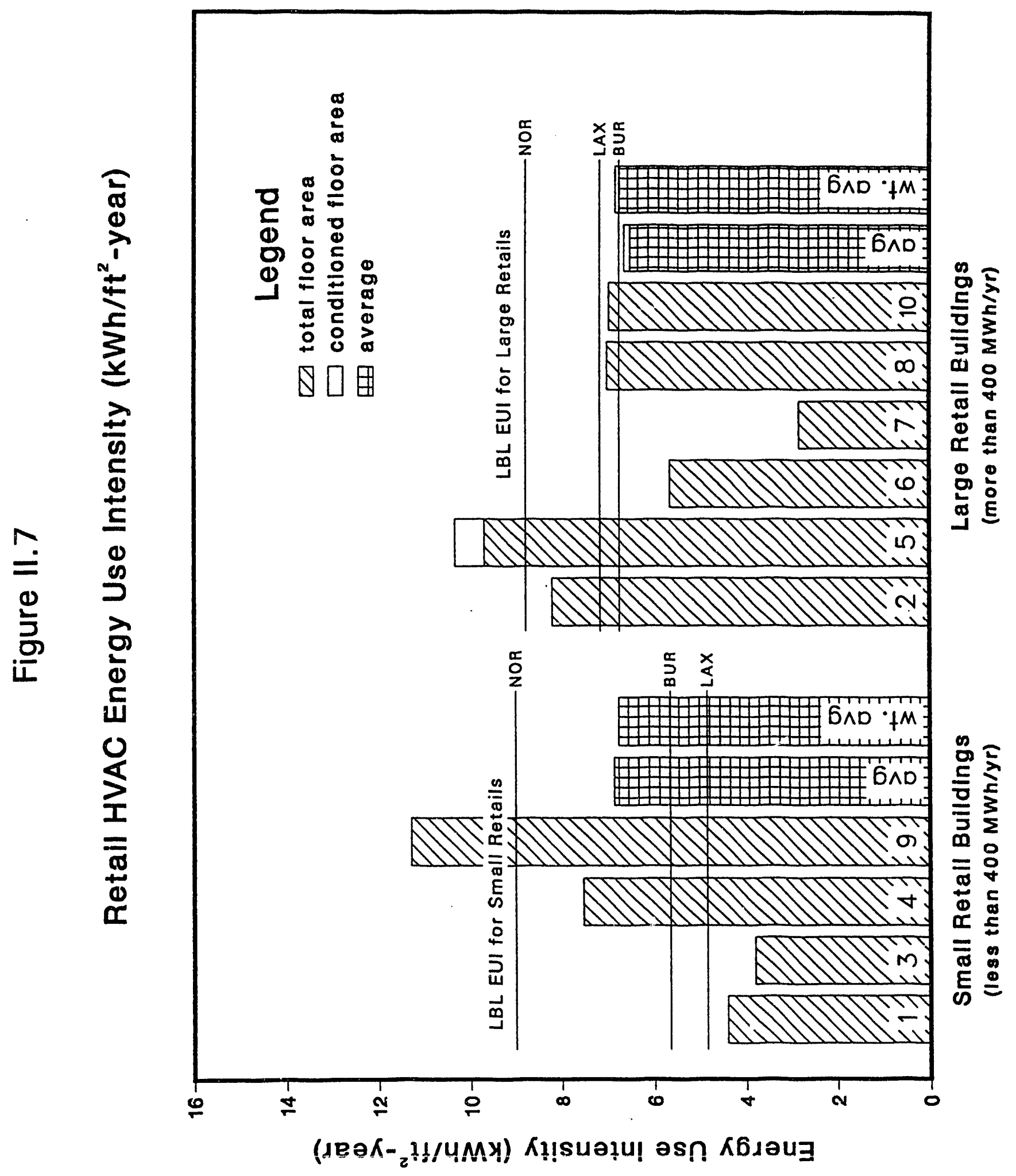




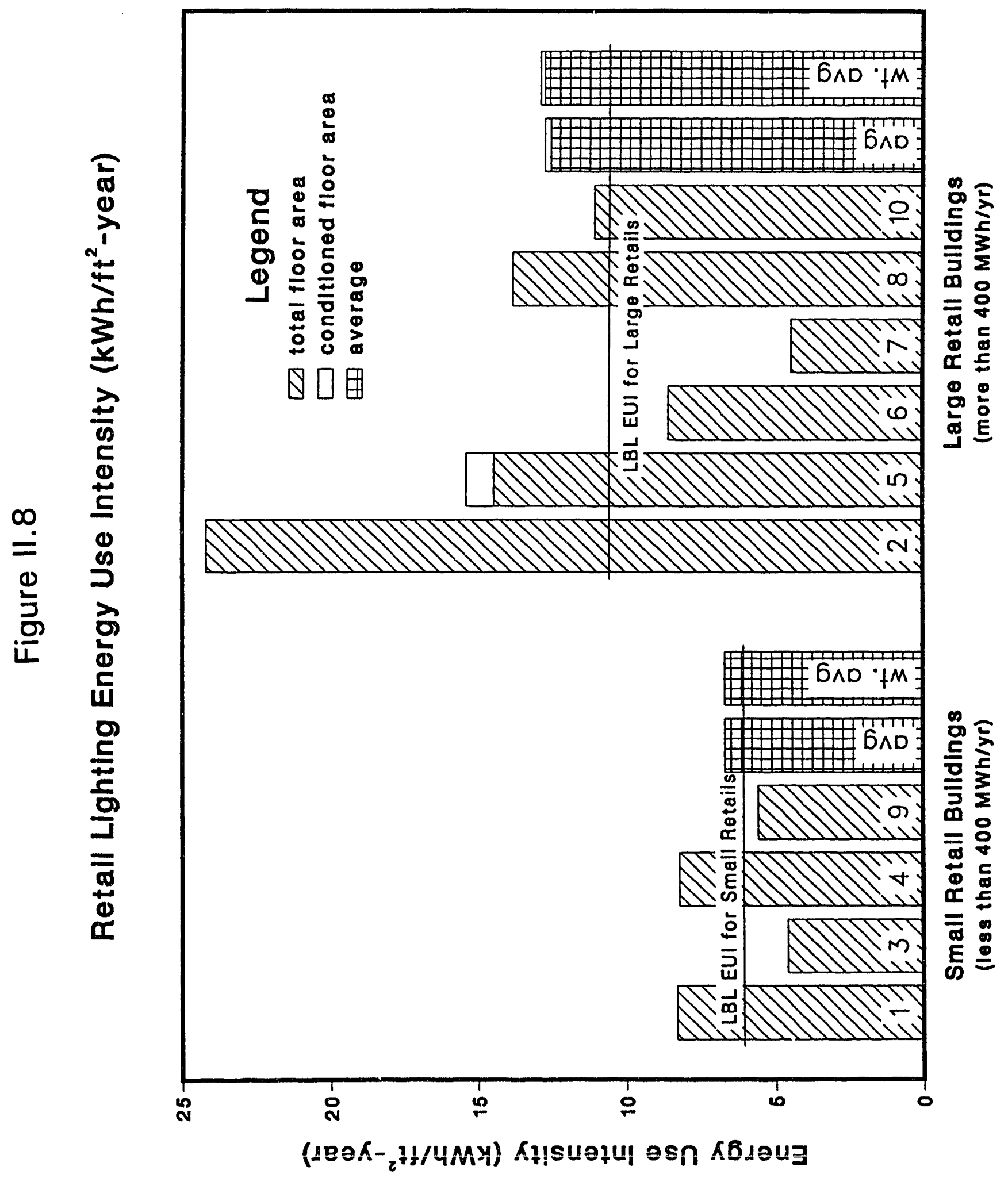


Grocery buildings

There are 14 grocery buildings in our sample with floor areas ranging from 12,000 to over $61,000 \mathrm{ft}^{2}$ and with total electric EUIs ranging from 36 to $89 \mathrm{kWh} / \mathrm{ft}^{2}$ year. Although SCE does not partition groceries by energy use, these groceries are large, having supermarket characteristics. Table II.3 presents the summary data for the groceries. The summary includes the amount of valid and invalid hourly data, the EUIs based on the conditioned and total building floor area, and the weighted and unweighted end-use EUIs.

Figure II.9 compares the total building EUIs for grocery buildings with the LBL estimates. The SCE monitored groceries are supermarkets, whereas those used in the previous LBL study are mostly smaller "mom and pop" neighborhood grocery stores that differ significantly in both operation and equipment. Since most large supermarkets are open 24-hours a day, it is expected that the LBL estimates, based on 16 to 18 hours of operation, be on the average about $30 \%$ lower than the monitored buildings. Figure II.9, indeed, agrees with this hypothesis.

Figure II.10 compares the HVAC EUIs for grocery buildings. Although the LBL study provided data for three climate regions, we use the Burbank data as an average value representing the entire SCE service area for this comparison. The LBL HVAC EUIs for grocery buildings is about 20 to $30 \%$ lower than the EUIs based on the monitored HVAC data and the total building floor area and the conditioned floor area. However, if adjustments are made for the different hours of operation between the on-site survey sample (data used by LBL) and that of the monitored buildings, this difference will be reduced significantly.

Figure II.11 compares the lighting EUIs for grocery buildings. The LBL lighting EUIs for grocery buildings is about 10 to $20 \%$ lower than the monitored data. The difference can be traced to the different hours of operation and the different lighting systems in large and small groceries.

Figure II.12 shows the refrigeration EUIs for grocery buildings. The agreement between the LBL study and the monitored data is fairly good. This is consistent with our other findings since the refrigeration EUIs of groceries are insensitive to operating hours. 
Table II. 3

End Use Intensities for Grocery Buildings

\begin{tabular}{|c|c|c|c|c|c|c|c|c|}
\hline \multirow{2}{*}{$\begin{array}{l}\text { Bldg } \\
\text { Type } \\
\text { GRY } \\
\text { GRY } \\
\text { GRY } \\
\text { GRY }\end{array}$} & $\begin{array}{l}\text { Bldg } \\
\text { Num }\end{array}$ & End Use & \multicolumn{2}{|c|}{$\begin{array}{l}\text { Observations } \\
\text { Valid Bad }\end{array}$} & $\begin{array}{l}\text { Total } \\
\text { Floor } \\
\text { Area }\end{array}$ & $\begin{array}{l}\text { Conditioned } \\
\text { Floor } \\
\text { Area }\end{array}$ & \multicolumn{2}{|c|}{$\begin{array}{l}\text { End Use Intensities } \\
\left(k W h / t^{2} \cdot y r\right)\end{array}$} \\
\hline & $\begin{array}{l}1 \\
1 \\
1 \\
1\end{array}$ & $\begin{array}{l}\text { HVAC } \\
\text { Lighting } \\
\text { Refrig } \\
\text { Total }\end{array}$ & $\begin{array}{l}7342 \\
7342 \\
7342 \\
7342\end{array}$ & $\begin{array}{l}1418 \\
1418 \\
1418 \\
1418\end{array}$ & $\begin{array}{l}26440 \\
26440 \\
26440 \\
26440\end{array}$ & $\begin{array}{l}22738 \\
22738 \\
22738 \\
22738\end{array}$ & $\begin{array}{r}2.920 \\
19.553 \\
26.312 \\
57.619\end{array}$ & $\begin{array}{r}3.394 \\
22.737 \\
30.595 \\
66.991\end{array}$ \\
\hline $\begin{array}{l}\text { GRY } \\
\text { GRY } \\
\text { GRY } \\
\text { GRY }\end{array}$ & $\begin{array}{l}2 \\
2 \\
2 \\
2\end{array}$ & $\begin{array}{l}\text { HVAC } \\
\text { Lighting } \\
\text { Refrig } \\
\text { Total }\end{array}$ & $\begin{array}{l}8450 \\
8104 \\
8489 \\
6559\end{array}$ & $\begin{array}{r}310 \\
656 \\
271 \\
2201\end{array}$ & $\begin{array}{l}23200 \\
23200 \\
23200 \\
23200\end{array}$ & $\begin{array}{l}20416 \\
20416 \\
20416 \\
20416\end{array}$ & $\begin{array}{r}1.773 \\
20.912 \\
23.814 \\
74.043\end{array}$ & $\begin{array}{r}2.015 \\
23.763 \\
27.061 \\
84.139\end{array}$ \\
\hline $\begin{array}{l}\text { GRY } \\
\text { GRY } \\
\text { GRY } \\
\text { GRY }\end{array}$ & $\begin{array}{l}3 \\
3 \\
3 \\
3\end{array}$ & $\begin{array}{l}\text { HVAC } \\
\text { Lighting } \\
\text { Refrig } \\
\text { Total }\end{array}$ & $\begin{array}{l}8473 \\
8474 \\
8493 \\
8134\end{array}$ & $\begin{array}{l}287 \\
286 \\
267 \\
626\end{array}$ & $\begin{array}{l}15923 \\
15923 \\
15923 \\
15923\end{array}$ & $\begin{array}{l}12420 \\
12420 \\
12420 \\
12420\end{array}$ & $\begin{array}{r}1.313 \\
15.705 \\
32.651 \\
81.050\end{array}$ & $\begin{array}{r}1.683 \\
20.135 \\
41.860 \\
103.910\end{array}$ \\
\hline $\begin{array}{l}\text { GRY } \\
\text { GRY } \\
\text { GRY } \\
\text { GRY }\end{array}$ & $\begin{array}{l}4 \\
4 \\
4 \\
4\end{array}$ & $\begin{array}{l}\text { HVAC } \\
\text { Lighting } \\
\text { Refrig } \\
\text { Total }\end{array}$ & $\begin{array}{l}8760 \\
8760 \\
8760 \\
8760\end{array}$ & $\begin{array}{l}0 \\
0 \\
0 \\
0\end{array}$ & $\begin{array}{l}30427 \\
30427 \\
30427 \\
30427 \\
\end{array}$ & $\begin{array}{l}24950 \\
24950 \\
24950 \\
24950\end{array}$ & $\begin{array}{r}3.477 \\
12.825 \\
26.210 \\
52.080\end{array}$ & $\begin{array}{r}4.240 \\
15.640 \\
31.963 \\
63.513 \\
\end{array}$ \\
\hline $\begin{array}{l}\text { GRY } \\
\text { GRY } \\
\text { GRY } \\
\text { GRY }\end{array}$ & $\begin{array}{l}5 \\
5 \\
5 \\
5\end{array}$ & $\begin{array}{l}\text { HVAC } \\
\text { Lighting } \\
\text { Refrig } \\
\text { Total }\end{array}$ & $\begin{array}{l}8744 \\
8744 \\
8635 \\
8635\end{array}$ & $\begin{array}{r}16 \\
16 \\
125 \\
125 \\
\end{array}$ & $\begin{array}{l}25565 \\
25565 \\
25565 \\
25565 \\
\end{array}$ & $\begin{array}{l}19429 \\
19429 \\
19429 \\
19429 \\
\end{array}$ & \begin{tabular}{r|}
2.628 \\
12.476 \\
18.240 \\
40.329 \\
\end{tabular} & $\begin{array}{r}3.458 \\
16.417 \\
24.000 \\
53.066 \\
\end{array}$ \\
\hline $\begin{array}{l}\text { GRY } \\
\text { GRY } \\
\text { GRY } \\
\text { GRY }\end{array}$ & $\begin{array}{l}6 \\
6 \\
6 \\
6\end{array}$ & $\begin{array}{l}\text { HVAC } \\
\text { Lighting } \\
\text { Refrig } \\
\text { Total }\end{array}$ & $\begin{array}{l}8189 \\
8743 \\
8743 \\
8742\end{array}$ & $\begin{array}{r}571 \\
17 \\
17 \\
18\end{array}$ & $\begin{array}{l}55000 \\
55000 \\
55000 \\
55000\end{array}$ & $\begin{array}{l}53350 \\
53350 \\
53350 \\
53350\end{array}$ & $\begin{array}{r}2.021 \\
7.116 \\
10.171 \\
27.856 \\
\end{array}$ & $\begin{array}{r}2.083 \\
7.336 \\
10.486 \\
28.717 \\
\end{array}$ \\
\hline $\begin{array}{l}\text { GRY } \\
\text { GRY } \\
\text { GRY } \\
\text { GRY }\end{array}$ & $\begin{array}{l}7 \\
7 \\
7 \\
7\end{array}$ & $\begin{array}{l}\text { HVAC } \\
\text { Lighting } \\
\text { Refrig } \\
\text { Total }\end{array}$ & $\begin{array}{l}8742 \\
8742 \\
8742 \\
8741 \\
\end{array}$ & $\begin{array}{l}18 \\
18 \\
18 \\
19\end{array}$ & $\begin{array}{l}22632 \\
22632 \\
22632 \\
22632 \\
\end{array}$ & $\begin{array}{l}19916 \\
19916 \\
19916 \\
19916 \\
\end{array}$ & $\begin{array}{r}5.572 \\
20.912 \\
31.934 \\
89.160 \\
\end{array}$ & $\begin{array}{r}6.332 \\
23.763 \\
36.289 \\
101.319\end{array}$ \\
\hline $\begin{array}{l}\text { GRY } \\
\text { GRY } \\
\text { GRY } \\
\text { GRY }\end{array}$ & $\begin{array}{l}8 \\
8 \\
8 \\
8\end{array}$ & $\begin{array}{l}\text { HVAC } \\
\text { Lighting } \\
\text { Refrig } \\
\text { Total }\end{array}$ & $\begin{array}{l}8759 \\
8760 \\
8759 \\
8759 \\
\end{array}$ & $\begin{array}{l}1 \\
0 \\
1 \\
1\end{array}$ & $\begin{array}{l}23400 \\
23400 \\
23400 \\
23400 \\
\end{array}$ & $\begin{array}{l}18486 \\
18486 \\
18486 \\
18486 \\
\end{array}$ & \begin{tabular}{|r|}
2.484 \\
16.425 \\
35.398 \\
60.290 \\
\end{tabular} & $\begin{array}{r}3.145 \\
20.791 \\
44.807 \\
76.317 \\
\end{array}$ \\
\hline $\begin{array}{l}\text { GRY } \\
\text { GRY } \\
\text { GRY } \\
\text { GRY }\end{array}$ & $\begin{array}{l}9 \\
9 \\
9 \\
9\end{array}$ & $\begin{array}{l}\text { HVAC } \\
\text { Lighting } \\
\text { Refrig } \\
\text { Total }\end{array}$ & $\begin{array}{l}8759 \\
8759 \\
8543 \\
8759\end{array}$ & $\begin{array}{r}1 \\
1 \\
217 \\
1\end{array}$ & $\begin{array}{l}24400 \\
24400 \\
24400 \\
24400\end{array}$ & $\begin{array}{l}20008 \\
20008 \\
20008 \\
20008\end{array}$ & $\begin{array}{r}2.559 \\
16.693 \\
27.034 \\
60.603 \\
\end{array}$ & $\begin{array}{r}3.120 \\
20.357 \\
32.969 \\
73.906 \\
\end{array}$ \\
\hline $\begin{array}{l}\text { GRY } \\
\text { GRY } \\
\text { GRY } \\
\text { GRY }\end{array}$ & $\begin{array}{l}10 \\
10 \\
10 \\
10\end{array}$ & $\begin{array}{l}\text { HVAC } \\
\text { Lighting } \\
\text { Refrig } \\
\text { Total }\end{array}$ & $\begin{array}{l}8735 \\
8760 \\
8760 \\
8759\end{array}$ & $\begin{array}{r}25 \\
0 \\
0 \\
1\end{array}$ & $\begin{array}{l}20720 \\
20720 \\
20720 \\
20720\end{array}$ & $\begin{array}{l}14090 \\
14090 \\
14090 \\
14090\end{array}$ & \begin{tabular}{|r|}
3.198 \\
15.575 \\
23.402 \\
70.818 \\
\end{tabular} & $\begin{array}{r}4.702 \\
22.904 \\
34.414 \\
104.140\end{array}$ \\
\hline $\begin{array}{l}\text { GRY } \\
\text { GRY } \\
\text { GRY } \\
\text { GRY }\end{array}$ & $\begin{array}{l}11 \\
11 \\
11 \\
11\end{array}$ & $\begin{array}{l}\text { HVAC } \\
\text { Lighting } \\
\text { Refrig } \\
\text { Total }\end{array}$ & $\begin{array}{l}7679 \\
7679 \\
7679 \\
7679\end{array}$ & $\begin{array}{l}457 \\
457 \\
457 \\
481\end{array}$ & $\begin{array}{l}21400 \\
21400 \\
21400 \\
21400\end{array}$ & $\begin{array}{l}16050 \\
16050 \\
16050 \\
16050\end{array}$ & \begin{tabular}{|r|}
1.082 \\
7.517 \\
17.065 \\
35.928 \\
\end{tabular} & $\begin{array}{r}1.443 \\
10.023 \\
22.754 \\
47.904\end{array}$ \\
\hline
\end{tabular}


Table II.3 (continued)

End Use Intensities for Grocery Buildings

\begin{tabular}{|c|c|c|c|c|c|c|c|c|}
\hline \multirow{2}{*}{$\begin{array}{l}\text { Bldg } \\
\text { Type } \\
\text { GRY } \\
\text { GRY } \\
\text { GRY } \\
\text { GRY }\end{array}$} & \multirow{2}{*}{$\begin{array}{c}\text { Bldg } \\
\text { Num } \\
12 \\
12 \\
12 \\
12\end{array}$} & \multirow{2}{*}{$\begin{array}{l}\text { End Use } \\
\text { HVAC } \\
\text { Lighting } \\
\text { Refrig } \\
\text { Total }\end{array}$} & \multicolumn{2}{|c|}{$\begin{array}{l}\text { Observations } \\
\text { Valid Bad }\end{array}$} & \multirow{2}{*}{$\begin{array}{c}\text { Total } \\
\text { Floor } \\
\text { Area } \\
12000 \\
12000 \\
12000 \\
12000 \\
\end{array}$} & \multirow{2}{*}{$\begin{array}{c}\begin{array}{c}\text { Conditioned } \\
\text { Floor } \\
\text { Area }\end{array} \\
9000 \\
9000 \\
9000 \\
9000\end{array}$} & \multicolumn{2}{|c|}{$\begin{array}{l}\text { End Use Intensities } \\
\left.\text { (kWh/ } / \mathrm{t}^{2} \cdot \mathrm{yr}\right) \\
\text { per tot per cond. } \\
\text { fir area fir area }\end{array}$} \\
\hline & & & $\begin{array}{l}7768 \\
7907 \\
7908 \\
7907 \\
\end{array}$ & $\begin{array}{l}368 \\
229 \\
252 \\
229 \\
\end{array}$ & & & \begin{tabular}{r|}
1.620 \\
3.432 \\
23.674 \\
41.845 \\
\end{tabular} & $\begin{array}{r}2.159 \\
4.576 \\
31.565 \\
55.793\end{array}$ \\
\hline $\begin{array}{l}\text { GRY } \\
\text { GRY } \\
\text { GRY } \\
\text { GRY }\end{array}$ & $\begin{array}{l}13 \\
13 \\
13 \\
13 \\
\end{array}$ & $\begin{array}{l}\text { HVAC } \\
\text { Lighting } \\
\text { Refrig } \\
\text { Total } \\
\end{array}$ & \begin{tabular}{|l|}
5757 \\
5758 \\
5169 \\
5171 \\
\end{tabular} & $\begin{array}{r}27 \\
26 \\
639 \\
613 \\
\end{array}$ & $\begin{array}{l}61616 \\
61616 \\
61616 \\
61616 \\
\end{array}$ & $\begin{array}{l}43747 \\
43747 \\
43747 \\
43747 \\
\end{array}$ & $\begin{array}{r}5.714 \\
18.999 \\
12.671 \\
55.808 \\
\end{array}$ & $\begin{array}{r}8.048 \\
26.759 \\
17.847 \\
78.604 \\
\end{array}$ \\
\hline $\begin{array}{l}\text { GRY } \\
\text { GRY } \\
\text { GRY } \\
\text { GRY }\end{array}$ & $\begin{array}{l}14 \\
14 \\
14 \\
14\end{array}$ & $\begin{array}{l}\text { HVAC } \\
\text { Lighting } \\
\text { Refrig } \\
\text { Total }\end{array}$ & $\begin{array}{l}5760 \\
5760 \\
5760 \\
5760 \\
\end{array}$ & $\begin{array}{l}24 \\
24 \\
48 \\
24\end{array}$ & $\begin{array}{l}51072 \\
51072 \\
51072 \\
51072 \\
\end{array}$ & $\begin{array}{l}51072 \\
51072 \\
51072 \\
51072 \\
\end{array}$ & \begin{tabular}{|r|}
3.399 \\
23.978 \\
22.508 \\
64.581 \\
\end{tabular} & $\begin{array}{r}3.399 \\
23.978 \\
22.508 \\
64.581\end{array}$ \\
\hline \multicolumn{3}{|c|}{$\begin{array}{ll}\text { Weighted } & \text { Averages : } \\
\text { GRY } & \text { HVAC } \\
\text { GRY } & \text { Lighting } \\
\text { GRY } & \text { Refrig } \\
\text { GRY } & \text { Total }\end{array}$} & & & & & $\begin{array}{r}2.936 \\
15.762 \\
25.629 \\
62.884 \\
\end{array}$ & $\begin{array}{r}3.586 \\
19.262 \\
31.598 \\
77.381\end{array}$ \\
\hline $\begin{array}{l}\text { Unwe } \\
\text { GRY } \\
\text { GRY } \\
\text { GRY } \\
\text { GRY }\end{array}$ & & $\begin{array}{l}\text { Herages: } \\
\text { HVAC } \\
\text { Lighting } \\
\text { Refrig } \\
\text { Total }\end{array}$ & & & & & $\begin{array}{r}2.840 \\
15.151 \\
23.649 \\
58.000\end{array}$ & $\begin{array}{r}3.516 \\
18.513 \\
29.223 \\
71.636\end{array}$ \\
\hline
\end{tabular}




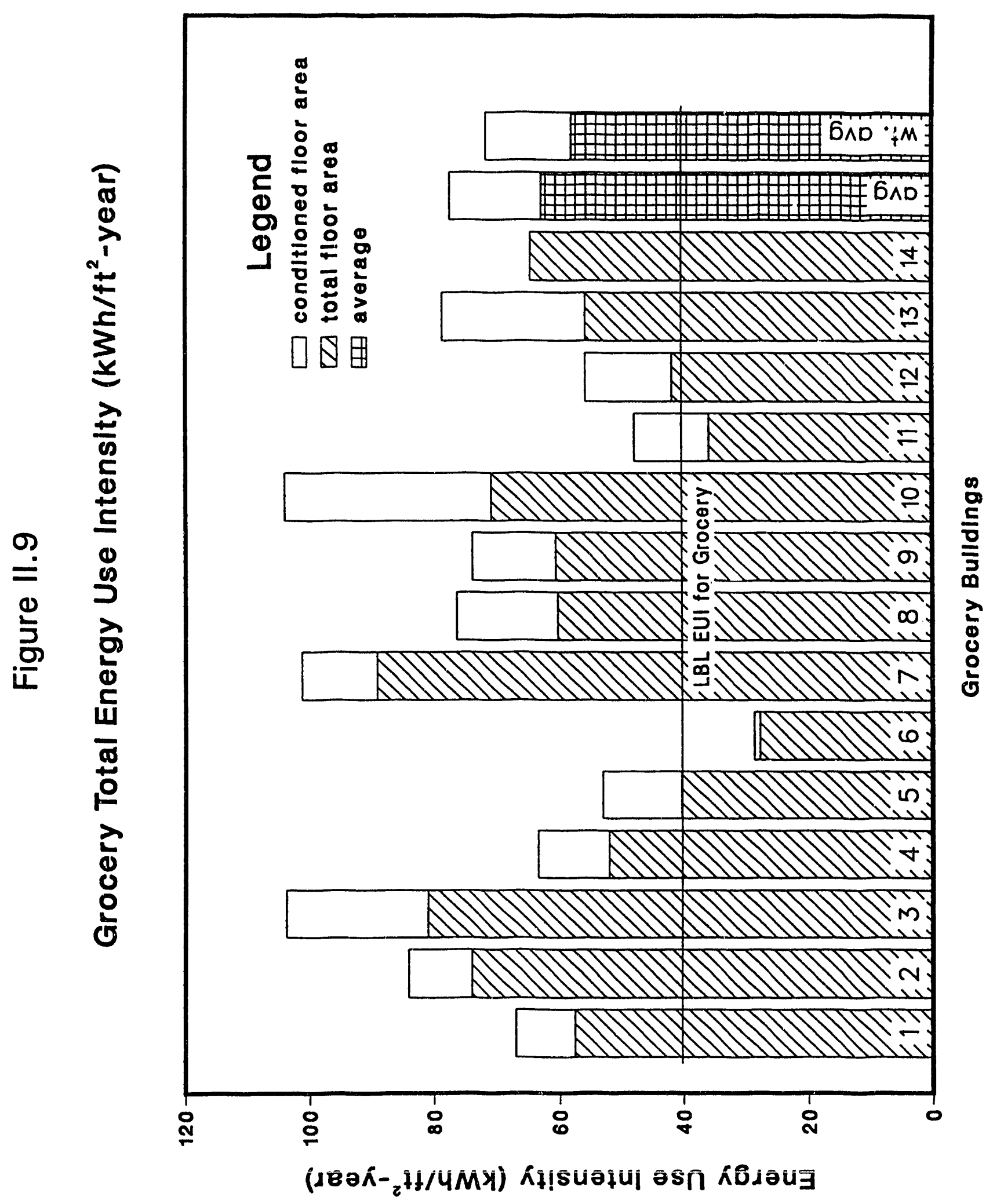




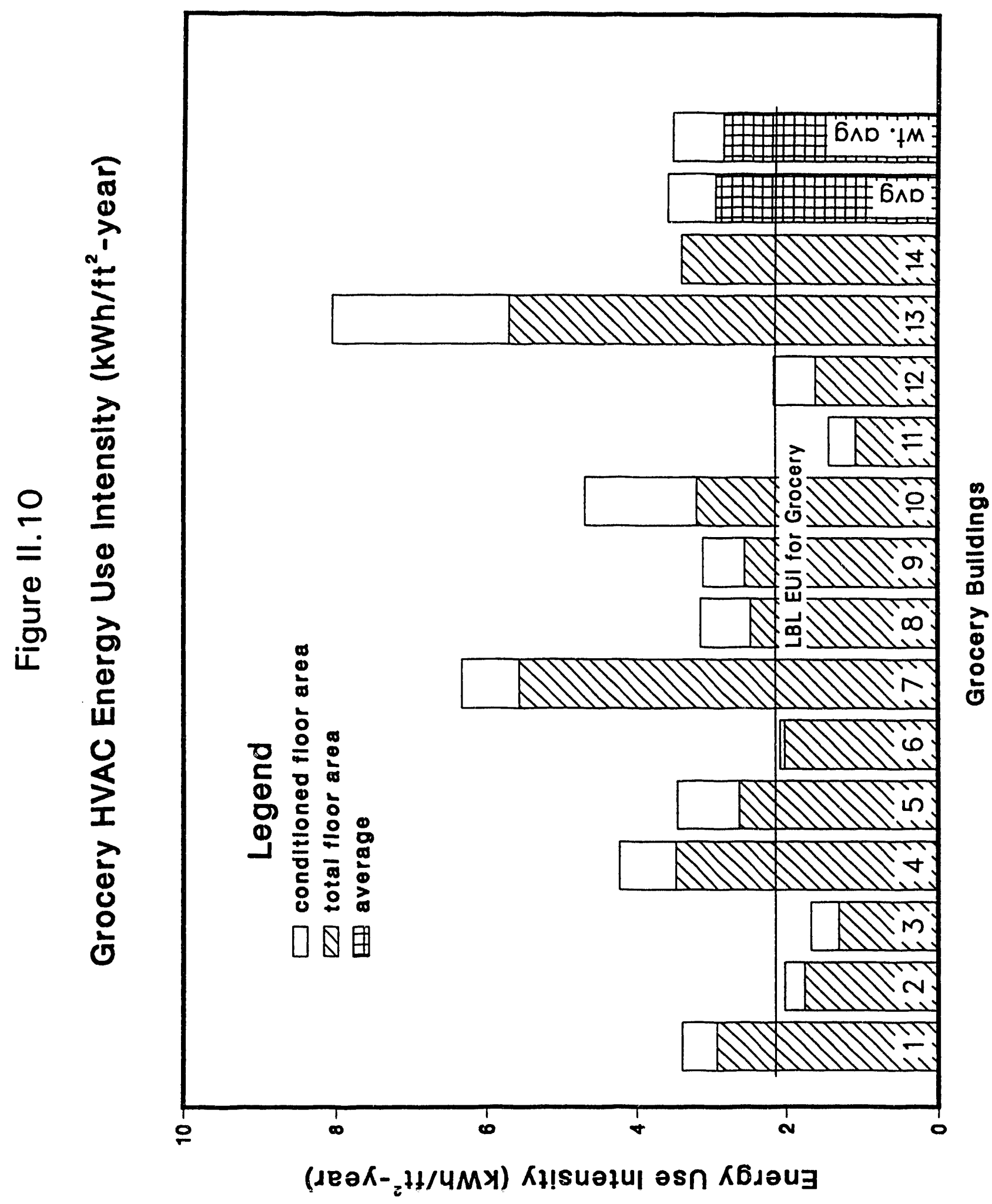




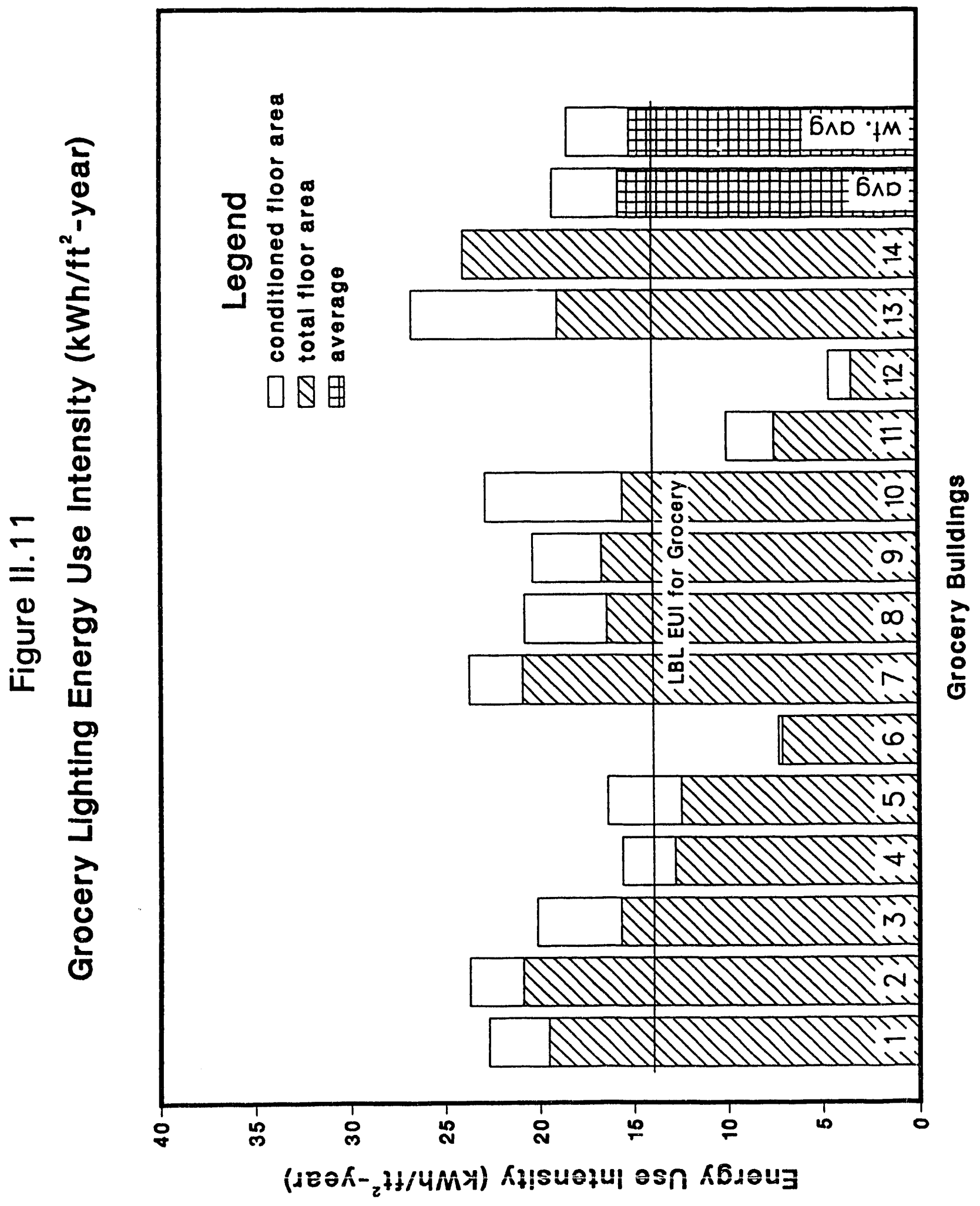




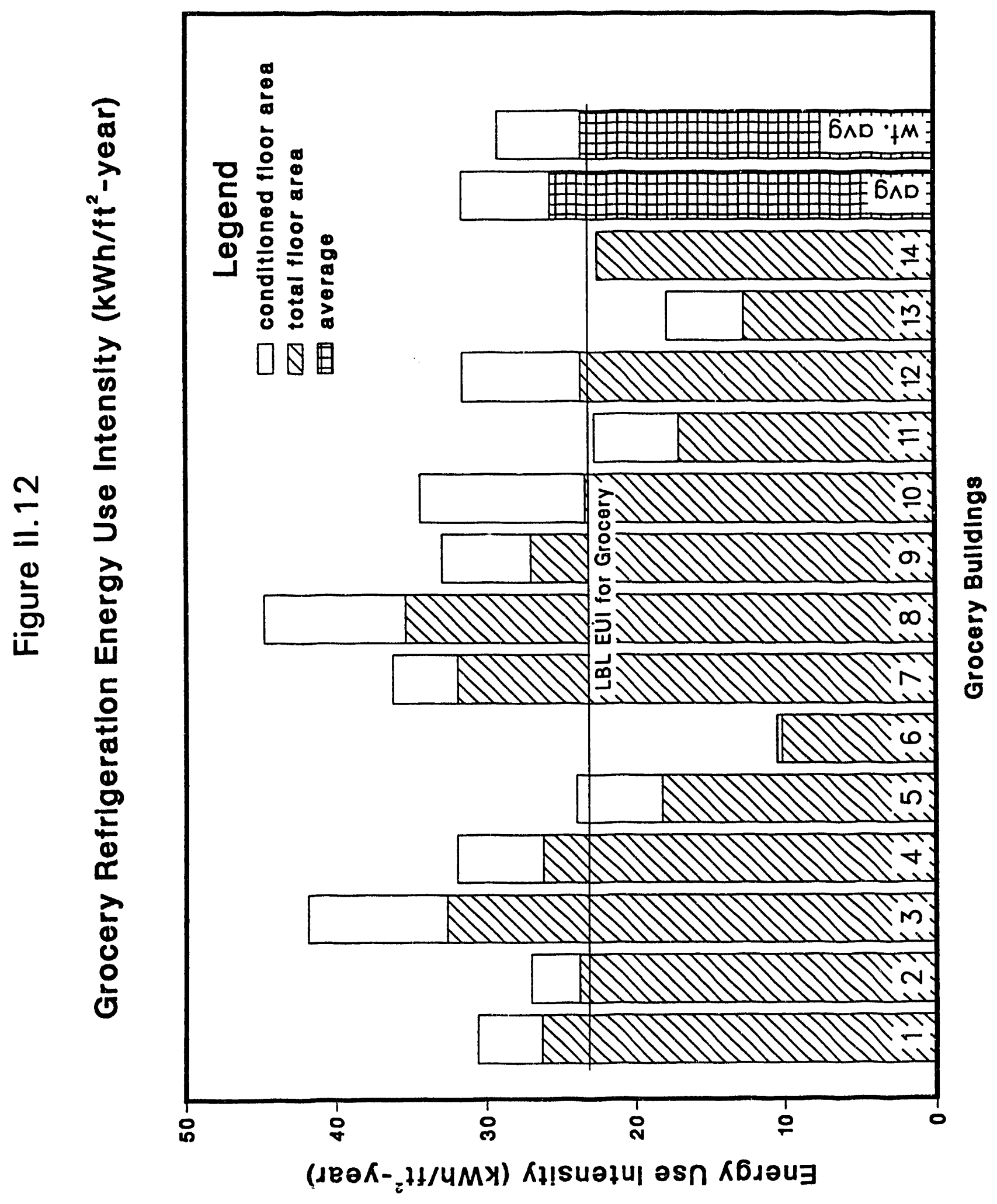




\section{Restaurant buildings}

Of the 14 restaurants monitored in the SCE service area, 10 are sit-down restaurants and 4 are fast food. The fast food restaurants monitored are comparable in size and in whole-building electric EUIs. The floor areas are in a narrow range of 3,650 to $5,780 \mathrm{ft}^{2}$ and the total electric EUIs range from 63 to $132 \mathrm{kWh} / \mathrm{ft}^{2}$ year. The variation in size of the 10 sit-down restaurants is slightly larger. Their fioor areas range from 7,250 to $23,5000 \mathrm{ft}^{2}$ and the whole-building EUIs range from 24 to $58 \mathrm{kWh} / \mathrm{ft}^{2}$ year.

Tables II.4 and II.5 present the summary data for the 14 sit-down and fast-food restaurants. Included in the summary are the number of hours of valid and bad data, estimated EUIs based on the conditioned and total building floor area, and weighted and unweighted end-use EUIs.

Figure II.13 compares the total building EUls for fast food and sit-down restaurants with the LBL estimates. The LBL study predicted EUIs and LSs for the combined group of fast food and sit-down restaurants. Although, the on-site survey data distinguishes between the fast food and sit-down restaurants, the load research data combines both restaurant types together. Hence, the LBL study only provides performance data for the combined group. As is to be expected, the fast-food restaurants are more energy intensive than the sit-down restaurants. The LBL total building electric EUI estimate for the combined group is between the monitored values for the fast-food and sit-down restaurants.

Figure II.14 compares the HVAC EUIs for fast-food and sit-down restaurants in the monitored building set. The LBL average EUI for restaurants is also included. Although the LBL study developed EUIs for three climate regions, the limited sample size of the monitored restaurants does not allow a comparison at a regional climate zone level. Hence, we take Burbank data from the LBL study as an average for the restaurants in the SCE service area for all of our comparisons of HVAC EUIs. Again, the LBL HVAC EUI is between the EUIs for the fast-food and sit-down restaurants. An interesting observation from the monitored data is that the HVAC systems for the fast food restaurants are about four times as energy intensive as the sit-down restaurants.

Figure II.15 shows the lighting EUIs for both fast-food and sit-down restaurants. Again the LBL EUI is between that of the fast food and sit-down restaurants and the lighting EUI for the fast food restaurants, on the average, is about twice that of the sit-down restaurants. 
Table II.4

End Use Intensities for Sit-down Restaurant Buildings

\begin{tabular}{|c|c|c|c|c|c|c|c|c|}
\hline \multirow{2}{*}{\begin{tabular}{|c} 
Bldg \\
Type
\end{tabular}} & $\begin{array}{l}\text { Bldg } \\
\text { Num }\end{array}$ & End Use & \multicolumn{2}{|c|}{$\begin{array}{l}\text { Observations } \\
\text { Valid Bad }\end{array}$} & $\begin{array}{l}\text { Total } \\
\text { Floor } \\
\text { Area } \\
\end{array}$ & $\begin{array}{c}\text { Conditioned } \\
\text { Floor } \\
\text { Area }\end{array}$ & \multicolumn{2}{|c|}{$\begin{array}{l}\text { End Use Intensities } \\
\left(\mathrm{kWW} / \mathrm{ft}^{2} \cdot \mathrm{yr}\right)\end{array}$} \\
\hline & $\begin{array}{l}1 \\
1 \\
1 \\
1 \\
1 \\
1\end{array}$ & $\begin{array}{l}\text { HVAC } \\
\text { Lighting } \\
\text { Cooking } \\
\text { Exhaust-fan } \\
\text { Refrig } \\
\text { Total } \\
\end{array}$ & $\begin{array}{l}8136 \\
8136 \\
7557 \\
8708 \\
8700 \\
8699 \\
\end{array}$ & $\begin{array}{r}624 \\
624 \\
1203 \\
52 \\
60 \\
61 \\
\end{array}$ & $\begin{array}{l}7550 \\
7550 \\
7550 \\
7550 \\
7550 \\
7550\end{array}$ & $\begin{array}{l}7324 \\
7324 \\
7324 \\
7324 \\
7324 \\
7324\end{array}$ & \begin{tabular}{|r|}
15.862 \\
8.751 \\
0.433 \\
2.460 \\
4.396 \\
56.859 \\
\end{tabular} & $\begin{array}{r}16.351 \\
9.021 \\
0.447 \\
2.536 \\
4.531 \\
58.613\end{array}$ \\
\hline \begin{tabular}{|l|}
$R S T$ SD \\
RST SD \\
RST SD \\
RST SD \\
RST SD \\
RST SD \\
\end{tabular} & $\begin{array}{l}2 \\
2 \\
2 \\
2 \\
2 \\
2 \\
\end{array}$ & \begin{tabular}{l|} 
HVAC \\
Lighting \\
Cooking \\
Exhaust-fan \\
Refrig \\
Total \\
\end{tabular} & \begin{tabular}{|l|}
8760 \\
8759 \\
7926 \\
8134 \\
8134 \\
8759 \\
\end{tabular} & $\begin{array}{r}0 \\
1 \\
834 \\
626 \\
626 \\
1 \\
\end{array}$ & \begin{tabular}{|l|}
11122 \\
11122 \\
11122 \\
11122 \\
11122 \\
11122 \\
\end{tabular} & $\begin{array}{l}11122 \\
11122 \\
11122 \\
11122 \\
11122 \\
11122 \\
\end{array}$ & \begin{tabular}{|r|}
3.368 \\
4.963 \\
4.026 \\
1.489 \\
4.411 \\
33.504 \\
\end{tabular} & \begin{tabular}{r|}
3.368 \\
4.963 \\
4.026 \\
1.489 \\
4.411 \\
33.504 \\
\end{tabular} \\
\hline \begin{tabular}{|l} 
RST SD \\
RST SD \\
RST SD \\
RST SD \\
RST SD \\
RST SD \\
\end{tabular} & $\begin{array}{l}3 \\
3 \\
3 \\
3 \\
3 \\
3 \\
\end{array}$ & \begin{tabular}{l|} 
HVAC \\
Lighting \\
Cooking \\
Exhaust-fan \\
Refrig \\
Total \\
\end{tabular} & $\begin{array}{l}8708 \\
3708 \\
8006 \\
7761 \\
7797 \\
8048\end{array}$ & $\begin{array}{r}52 \\
52 \\
754 \\
999 \\
963 \\
712 \\
\end{array}$ & \begin{tabular}{|l|}
11020 \\
11020 \\
11020 \\
11020 \\
11020 \\
11020 \\
\end{tabular} & $\begin{array}{l}11020 \\
11020 \\
11020 \\
11020 \\
11020 \\
11020 \\
\end{array}$ & \begin{tabular}{|r|}
7.731 \\
12.801 \\
7.858 \\
0.457 \\
1.471 \\
46.158 \\
\end{tabular} & $\begin{array}{r}7.731 \\
12.801 \\
7.858 \\
0.457 \\
1.471 \\
46.158 \\
\end{array}$ \\
\hline \begin{tabular}{|l} 
RST SD \\
RST SD \\
RST SD \\
RST SD \\
RST SD \\
RST SD \\
\end{tabular} & $\begin{array}{l}4 \\
4 \\
4 \\
4 \\
4 \\
4\end{array}$ & \begin{tabular}{l|} 
HVAC \\
Lighting \\
Cooking \\
Exhaust-fan \\
Refrig \\
Total \\
\end{tabular} & $\begin{array}{l}8690 \\
8690 \\
8759 \\
8759 \\
8759 \\
8759 \\
\end{array}$ & $\begin{array}{r}70 \\
70 \\
1 \\
1 \\
1 \\
1 \\
\end{array}$ & $\begin{array}{l}15430 \\
15430 \\
15430 \\
15430 \\
15430 \\
15430 \\
\end{array}$ & $\begin{array}{l}15430 \\
15430 \\
15430 \\
15430 \\
15430 \\
15430 \\
\end{array}$ & \begin{tabular}{|r|}
5.332 \\
6.686 \\
2.121 \\
2.843 \\
8.365 \\
33.721 \\
\end{tabular} & $\begin{array}{r}5.332 \\
6.686 \\
2.121 \\
2.843 \\
8.365 \\
33.721 \\
\end{array}$ \\
\hline $\begin{array}{l}\text { RST SD } \\
\text { RST SD } \\
\text { RST SD } \\
\text { RST SD } \\
\text { RST SD } \\
\text { RST SD } \\
\end{array}$ & $\begin{array}{l}5 \\
5 \\
5 \\
5 \\
5 \\
5 \\
\end{array}$ & $\begin{array}{l}\text { HVAC } \\
\text { Lighting } \\
\text { Cooking } \\
\text { Exhaust-fan } \\
\text { Refrig } \\
\text { Total } \\
\end{array}$ & \begin{tabular}{|l|}
8756 \\
8758 \\
8758 \\
8758 \\
8758 \\
8758 \\
\end{tabular} & $\begin{array}{l}4 \\
2 \\
2 \\
2 \\
2 \\
2 \\
\end{array}$ & $\begin{array}{l}8834 \\
8834 \\
8834 \\
8834 \\
8834 \\
8834 \\
\end{array}$ & $\begin{array}{l}8569 \\
8569 \\
8569 \\
8569 \\
8569 \\
8569 \\
\end{array}$ & \begin{tabular}{|r|}
8.536 \\
12.117 \\
16.894 \\
2.559 \\
8.624 \\
58.416 \\
\end{tabular} & $\begin{array}{r}8.280 \\
11.753 \\
16.387 \\
2.482 \\
8.365 \\
56.664 \\
\end{array}$ \\
\hline \begin{tabular}{|l|} 
RST SD \\
RST SD \\
RST SD \\
RST SD \\
RST SD \\
RST SD \\
\end{tabular} & $\begin{array}{l}8 \\
8 \\
8 \\
8 \\
8 \\
8 \\
\end{array}$ & $\begin{array}{l}\text { HVAC } \\
\text { Lighting } \\
\text { Cooking } \\
\text { Exhaust-fan } \\
\text { Refrig } \\
\text { Total } \\
\end{array}$ & $\begin{array}{l}8654 \\
6073 \\
8653 \\
8640 \\
8635 \\
8653 \\
\end{array}$ & $\begin{array}{r}106 \\
2687 \\
107 \\
120 \\
125 \\
107 \\
\end{array}$ & $\begin{array}{l}8300 \\
8300 \\
8300 \\
8300 \\
8300 \\
8300 \\
\end{array}$ & $\begin{array}{l}8051 \\
8051 \\
8051 \\
8051 \\
8051 \\
8051 \\
\end{array}$ & \begin{tabular}{|r|}
8.811 \\
3.328 \\
12.577 \\
0.627 \\
2.936 \\
41.578 \\
\end{tabular} & $\begin{array}{r}9.084 \\
3.431 \\
12.966 \\
0.646 \\
3.027 \\
42.864 \\
\end{array}$ \\
\hline $\begin{array}{l}\text { RST SD } \\
\text { RST SD } \\
\text { RST SD } \\
\text { RST SD } \\
\text { RST SD } \\
\text { RST SD } \\
\end{array}$ & $\begin{array}{l}9 \\
9 \\
9 \\
9 \\
9 \\
9\end{array}$ & $\begin{array}{l}\text { HVAC } \\
\text { Lighting } \\
\text { Cooking } \\
\text { Exhaust-fan } \\
\text { Refrig } \\
\text { Total } \\
\end{array}$ & $\begin{array}{l}6072 \\
6332 \\
6280 \\
6281 \\
6332 \\
6279 \\
\end{array}$ & $\begin{array}{l}2688 \\
2428 \\
2480 \\
2167 \\
2428 \\
2481 \\
\end{array}$ & $\begin{array}{l}6308 \\
6308 \\
6308 \\
6308 \\
6308 \\
6308 \\
\end{array}$ & $\begin{array}{l}5993 \\
5993 \\
5993 \\
5993 \\
5993 \\
5993 \\
\end{array}$ & $\begin{array}{r}10.480 \\
8.724 \\
10.116 \\
1.853 \\
5.257 \\
47.936 \\
\end{array}$ & $\begin{array}{r}11.031 \\
9.182 \\
10.648 \\
1.950 \\
5.533 \\
50.456 \\
\end{array}$ \\
\hline
\end{tabular}


Table 11.4 (continued)

End Use Intensities for Sit-down Restaurant Buildings

\begin{tabular}{|c|c|c|c|c|c|c|c|c|}
\hline $\begin{array}{l}\text { Bldg } \\
\text { Type }\end{array}$ & $\begin{array}{l}\text { Bldg } \\
\text { Num }\end{array}$ & End Use & \multicolumn{2}{|c|}{$\begin{array}{l}\text { Observations } \\
\text { Valid Bad }\end{array}$} & $\begin{array}{l}\text { Total } \\
\text { Floor } \\
\text { Area }\end{array}$ & $\begin{array}{l}\text { Conditioned } \\
\text { Floor } \\
\text { Area }\end{array}$ & \multicolumn{2}{|c|}{$\begin{array}{c}\text { End Use Intensities } \\
\text { (kWh/ft' } y r)\end{array}$} \\
\hline $\begin{array}{l}\text { RST SD } \\
\text { RST SD } \\
\text { RST SD } \\
\text { RST SD } \\
\text { RST SD } \\
\text { RST SD }\end{array}$ & $\begin{array}{l}10 \\
10 \\
10 \\
10 \\
10 \\
10\end{array}$ & $\begin{array}{l}\text { HVAC } \\
\text { Lighting } \\
\text { Cooking } \\
\text { Exhaust-fan } \\
\text { Refrig } \\
\text { Total }\end{array}$ & $\begin{array}{l}6853 \\
6859 \\
7339 \\
7339 \\
7339 \\
8237\end{array}$ & $\begin{array}{r}1907 \\
1901 \\
1421 \\
1421 \\
1421 \\
523\end{array}$ & $\begin{array}{l}9083 \\
9083 \\
9083 \\
5083 \\
5083 \\
9083\end{array}$ & $\begin{array}{l}9083 \\
9083 \\
9083 \\
9083 \\
9083 \\
9083\end{array}$ & $\begin{array}{r}9.177 \\
9.358 \\
5.622 \\
1.710 \\
6.913 \\
53.792 \\
\end{array}$ & $\begin{array}{r}9.177 \\
9.358 \\
5.622 \\
1.710 \\
6.913 \\
53.792\end{array}$ \\
\hline $\begin{array}{l}\text { RST SD } \\
\text { RST SD } \\
\text { RST SD } \\
\text { RST SD } \\
\text { RST SD } \\
\text { RST SD }\end{array}$ & $\begin{array}{l}12 \\
12 \\
12 \\
12 \\
12 \\
12\end{array}$ & $\begin{array}{l}\text { HVAC } \\
\text { Lighting } \\
\text { Cooking } \\
\text { Exhaust-fan } \\
\text { Refrig } \\
\text { Total }\end{array}$ & $\begin{array}{l}8707 \\
8707 \\
8577 \\
8576 \\
8577 \\
8577\end{array}$ & $\begin{array}{r}53 \\
53 \\
183 \\
184 \\
183 \\
183\end{array}$ & $\begin{array}{l}7254 \\
7254 \\
7254 \\
7254 \\
7254 \\
7254\end{array}$ & $\begin{array}{l}7181 \\
7181 \\
7181 \\
7181 \\
7181 \\
7181\end{array}$ & $\begin{array}{r}6.092 \\
2.714 \\
7.477 \\
0.750 \\
7.251 \\
26.974 \\
\end{array}$ & $\begin{array}{r}6.154 \\
2.742 \\
7.553 \\
0.758 \\
7.325 \\
27.248\end{array}$ \\
\hline \begin{tabular}{|l} 
RST SD \\
RST SD \\
RST SD \\
RST SD \\
RST SD \\
RST SD \\
\end{tabular} & $\begin{array}{l}14 \\
14 \\
14 \\
14 \\
14 \\
14\end{array}$ & $\begin{array}{l}\text { HVAC } \\
\text { Lighting } \\
\text { Cooking } \\
\text { Exhaust-tan } \\
\text { Refrig } \\
\text { Total } \\
\end{array}$ & \begin{tabular}{|l|}
7776 \\
7776 \\
7734 \\
7733 \\
7734 \\
7734 \\
\end{tabular} & $\begin{array}{l}312 \\
312 \\
354 \\
379 \\
354 \\
354 \\
\end{array}$ & $\begin{array}{l}23519 \\
23519 \\
23519 \\
23519 \\
23519 \\
23519 \\
\end{array}$ & $\begin{array}{l}22813 \\
22813 \\
22813 \\
22813 \\
22813 \\
22813 \\
\end{array}$ & \begin{tabular}{|r|}
5.893 \\
6.127 \\
2.315 \\
0.566 \\
2.431 \\
23.850 \\
\end{tabular} & $\begin{array}{r}6.075 \\
6.316 \\
2.385 \\
0.584 \\
2.506 \\
24.588 \\
\end{array}$ \\
\hline $\begin{array}{l}\text { Weighte } \\
\text { RST SD } \\
\text { RST SD } \\
\text { RST SD } \\
\text { RST SD } \\
\text { RST SO } \\
\text { RST SD }\end{array}$ & & $\begin{array}{l}\text { ages: } \\
\text { HVAC } \\
\text { Lighting } \\
\text { Cooking } \\
\text { Exhaust-fan } \\
\text { Refrig } \\
\text { Total }\end{array}$ & & & & & \begin{tabular}{|r|}
7.889 \\
7.261 \\
7.499 \\
1.421 \\
5.121 \\
40.727
\end{tabular} & $\begin{array}{r}8.057 \\
7.375 \\
7.658 \\
1.445 \\
5.199 \\
41.474\end{array}$ \\
\hline $\begin{array}{l}\text { Unweigh } \\
\text { RST SD } \\
\text { RST SD } \\
\text { RST SD } \\
\text { RST SD } \\
\text { RST SD } \\
\text { RST SD }\end{array}$ & ted Av & $\begin{array}{l}\text { erages: } \\
\text { HVAC } \\
\text { Lighting } \\
\text { Cooking } \\
\text { Exhaust-fan } \\
\text { Refrig } \\
\text { Total }\end{array}$ & & & & & \begin{tabular}{|r|}
8.128 \\
7.557 \\
6.944 \\
1.531 \\
5.205 \\
42.279
\end{tabular} & $\begin{array}{r}8.284 \\
7.662 \\
7.052 \\
1.553 \\
5.271 \\
42.936\end{array}$ \\
\hline
\end{tabular}


Table 11.5

End Use Intensities for Fast Food Restaurant Buildings

\begin{tabular}{|c|c|c|c|c|c|c|c|c|}
\hline \multirow{2}{*}{$\begin{array}{l}\text { BIdg } \\
\text { Type } \\
\text { RST FF } \\
\text { RST FF } \\
\text { RST FF } \\
\text { RST FF } \\
\text { RST FF } \\
\text { RST FF }\end{array}$} & \multirow{2}{*}{ 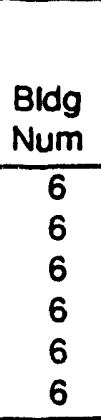 } & \multirow[b]{2}{*}{\begin{tabular}{l|} 
End Use \\
HVAC \\
Lighting \\
Cooking \\
Exhaust-fan \\
Refrig \\
Total
\end{tabular}} & \multicolumn{2}{|c|}{$\begin{array}{l}\text { Observations } \\
\text { Valid Bad }\end{array}$} & \multirow{2}{*}{\begin{tabular}{|l} 
Total \\
Floor \\
Area \\
3650 \\
3650 \\
3650 \\
3650 \\
3650 \\
3650 \\
\end{tabular}} & $\begin{array}{c}\text { Conditioned } \\
\text { Floor } \\
\text { Area } \\
\end{array}$ & \multicolumn{2}{|c|}{$\begin{array}{l}\text { End Use Intensities } \\
\left.\text { (kWhut }{ }^{2} \cdot y r\right) \\
\text { per tot per cond. } \\
\text { fir area fir area }\end{array}$} \\
\hline & & & $\begin{array}{l}8753 \\
8752 \\
7103 \\
7103 \\
8755 \\
7103 \\
\end{array}$ & $\begin{array}{r}7 \\
8 \\
1657 \\
1657 \\
5 \\
1657 \\
\end{array}$ & & $\begin{array}{l}3504 \\
3504 \\
3504 \\
3504 \\
3504 \\
3504 \\
\end{array}$ & \begin{tabular}{|r|}
34.582 \\
19.813 \\
34.768 \\
3.387 \\
14.404 \\
131.911 \\
\end{tabular} & $\begin{array}{r}36.023 \\
20.638 \\
36.217 \\
3.528 \\
15.004 \\
137.408\end{array}$ \\
\hline $\begin{array}{l}\text { RST FF } \\
\text { RST FF } \\
\text { RST FF } \\
\text { RST FF } \\
\text { RST FF } \\
\text { RST FF } \\
\end{array}$ & $\begin{array}{l}7 \\
7 \\
7 \\
7 \\
7 \\
7 \\
\end{array}$ & \begin{tabular}{l|} 
HVAC \\
Lighting \\
Cooking \\
Exhaust-fan \\
Reirig \\
Total \\
\end{tabular} & $\begin{array}{l}8759 \\
8758 \\
8760 \\
8760 \\
8760 \\
8760 \\
\end{array}$ & $\begin{array}{l}1 \\
2 \\
0 \\
0 \\
0 \\
0 \\
\end{array}$ & \begin{tabular}{|l|}
5780 \\
5780 \\
5780 \\
5780 \\
5780 \\
5780 \\
\end{tabular} & \begin{tabular}{l|}
3468 \\
3468 \\
3468 \\
3468 \\
3468 \\
3468 \\
\end{tabular} & $\begin{array}{r}22.189 \\
10.499 \\
12.131 \\
2.916 \\
6.693 \\
63.668 \\
\end{array}$ & $\begin{array}{r}36.981 \\
17.499 \\
20.219 \\
4.860 \\
11.156 \\
106.113 \\
\end{array}$ \\
\hline $\begin{array}{l}\text { RST FF } \\
\text { RST FF } \\
\text { RST FF } \\
\text { RST FF } \\
\text { RST FF } \\
\text { RST FF } \\
\end{array}$ & $\begin{array}{l}11 \\
11 \\
11 \\
11 \\
11 \\
11 \\
\end{array}$ & \begin{tabular}{l|} 
HVAC \\
Lighting \\
Cooking \\
Exhaust-fan \\
Retrig \\
Total \\
\end{tabular} & \begin{tabular}{|l|}
8755 \\
8755 \\
8659 \\
8755 \\
8755 \\
8755 \\
\end{tabular} & $\begin{array}{r}5 \\
5 \\
101 \\
5 \\
5 \\
5 \\
\end{array}$ & $\begin{array}{l}4025 \\
4025 \\
4025 \\
4025 \\
4025 \\
4025 \\
\end{array}$ & $\begin{array}{l}3824 \\
3824 \\
3824 \\
3824 \\
3824 \\
3824 \\
\end{array}$ & \begin{tabular}{|r|}
36.157 \\
27.120 \\
34.903 \\
4.421 \\
12.614 \\
115.102 \\
\end{tabular} & $\begin{array}{r}38.057 \\
28.545 \\
36.738 \\
4.653 \\
13.277 \\
121.152 \\
\end{array}$ \\
\hline $\begin{array}{l}\text { RST FF } \\
\text { RST FF } \\
\text { RST FF } \\
\text { RST FF } \\
\text { RST FF } \\
\text { RST FF } \\
\end{array}$ & $\begin{array}{l}13 \\
13 \\
13 \\
13 \\
13 \\
13\end{array}$ & \begin{tabular}{l|} 
HVAC \\
Lighting \\
Cooking \\
Exhaust-fan \\
Refrig \\
Total \\
\end{tabular} & \begin{tabular}{|l|}
7293 \\
7374 \\
6680 \\
7913 \\
7817 \\
7913 \\
\end{tabular} & $\begin{array}{r}1131 \\
1050 \\
1744 \\
511 \\
607 \\
511 \\
\end{array}$ & $\begin{array}{l}4800 \\
4800 \\
4800 \\
4800 \\
4800 \\
4800 \\
\end{array}$ & $\begin{array}{l}2208 \\
2208 \\
2208 \\
2208 \\
2208 \\
2208 \\
\end{array}$ & \begin{tabular}{r|}
36.960 \\
19.527 \\
21.345 \\
4.712 \\
8.413 \\
98.136 \\
\end{tabular} & $\begin{array}{r}80.348 \\
42.451 \\
46.403 \\
10.243 \\
18.289 \\
213.340 \\
\end{array}$ \\
\hline $\begin{array}{l}\text { Weightec } \\
\text { RST FF } \\
\text { RST FF } \\
\text { RST FF } \\
\text { RST FF } \\
\text { RST FF } \\
\text { RST FF } \\
\end{array}$ & & \begin{tabular}{l|} 
ages: \\
HVAC \\
Lighting \\
Cooking \\
Exhaust-fan \\
Relrig \\
Total \\
\end{tabular} & & & & & $\begin{array}{r}32.749 \\
19.232 \\
24.870 \\
3.962 \\
10.118 \\
100.117 \\
\end{array}$ & $\begin{array}{r}51.434 \\
29.020 \\
35.843 \\
6.348 \\
14.742 \\
151.048 \\
\end{array}$ \\
\hline $\begin{array}{l}\text { Unweigh } \\
\text { RST FF } \\
\text { RST FF } \\
\text { RST FF } \\
\text { RST FF } \\
\text { RST FF } \\
\text { RST FF }\end{array}$ & $\overline{d A V}$ & $\begin{array}{l}\text { erages: } \\
\text { HVAC } \\
\text { Lighting } \\
\text { Cooking } \\
\text { Exhaust-fan } \\
\text { Refrig } \\
\text { Total }\end{array}$ & & & & & $\begin{array}{r}32.472 \\
19.240 \\
25.787 \\
3.859 \\
10.531 \\
102.204 \\
\end{array}$ & $\begin{array}{r}47.852 \\
27.283 \\
34.894 \\
5.821 \\
14.432 \\
144.503 \\
\end{array}$ \\
\hline
\end{tabular}




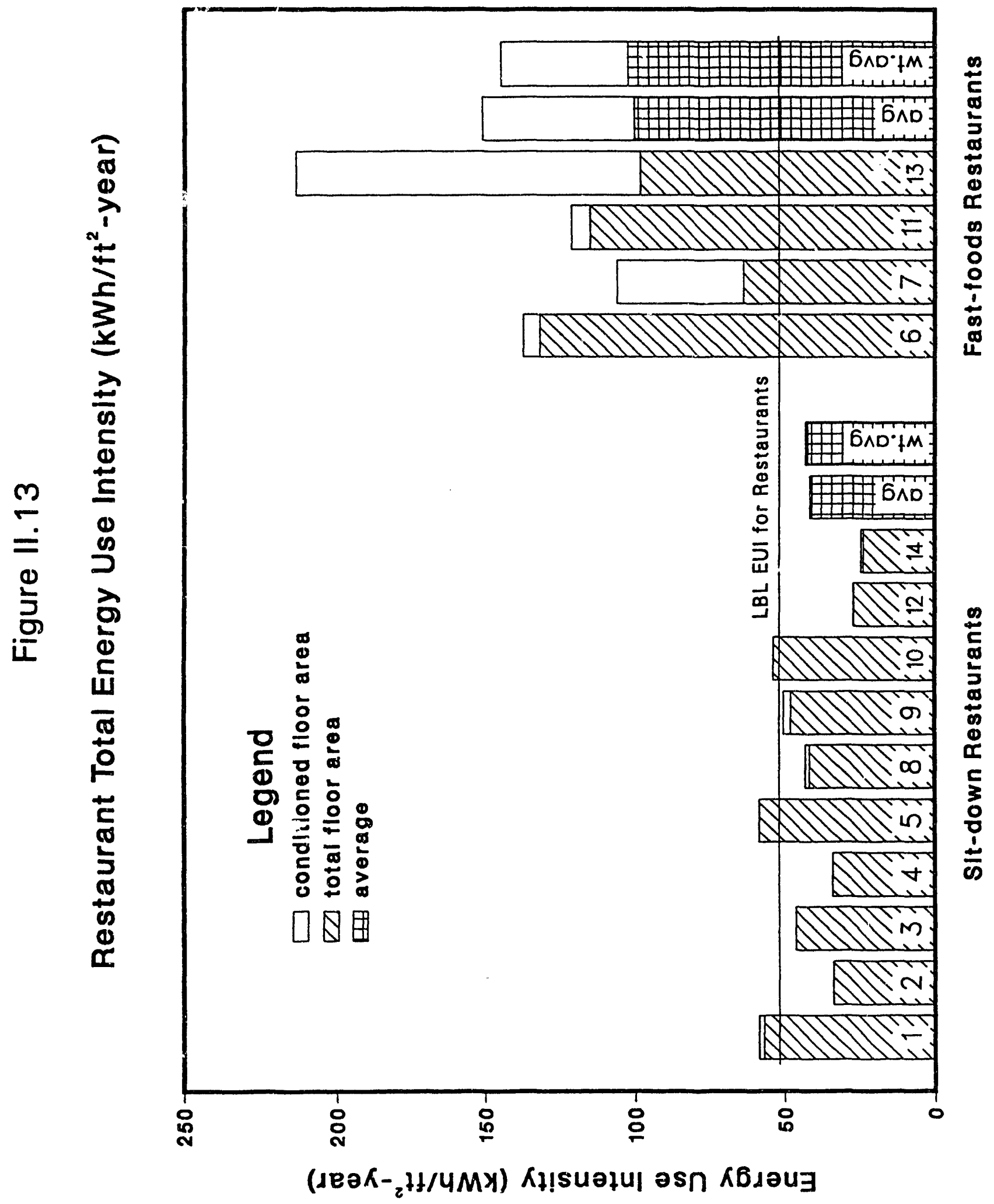




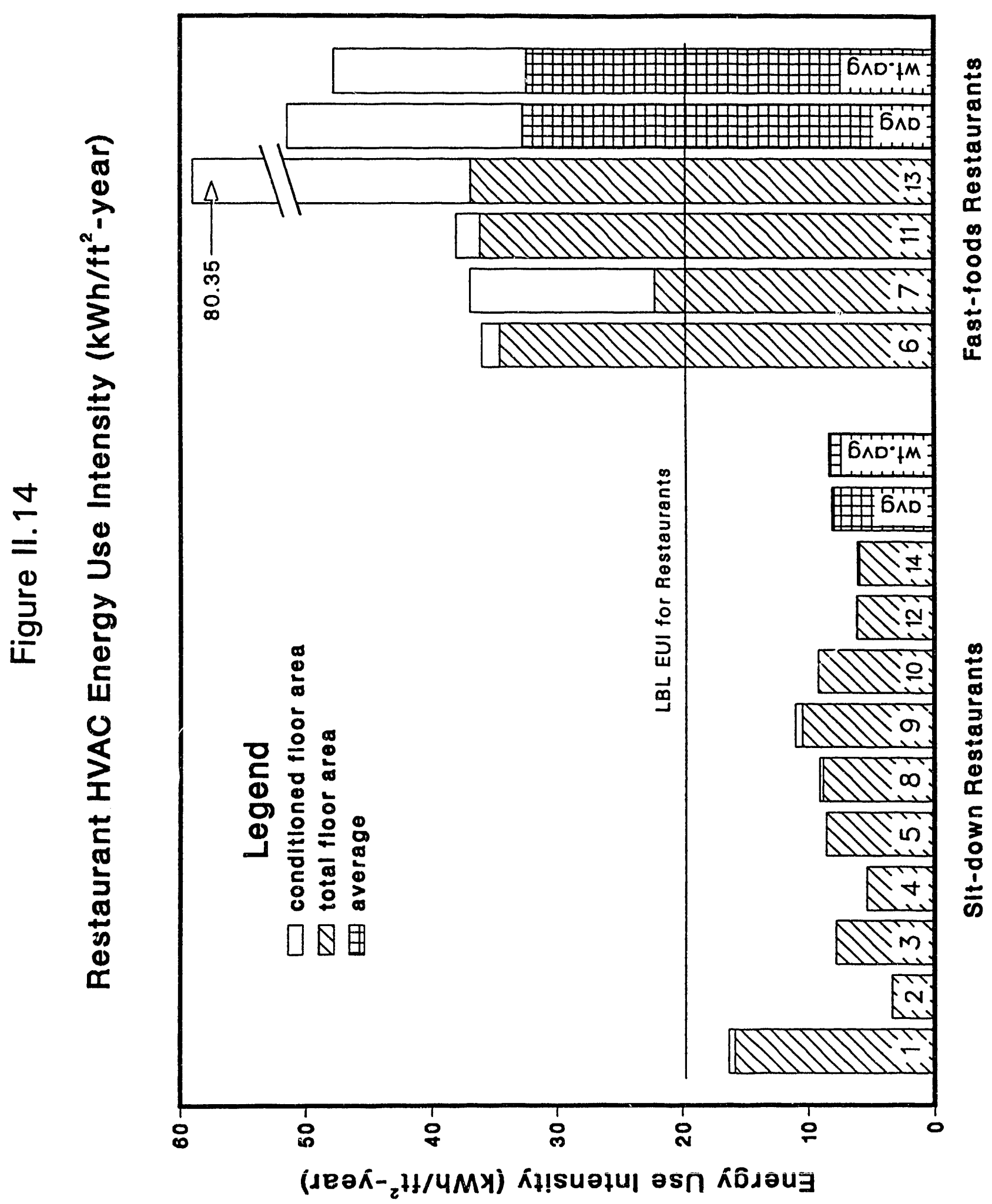




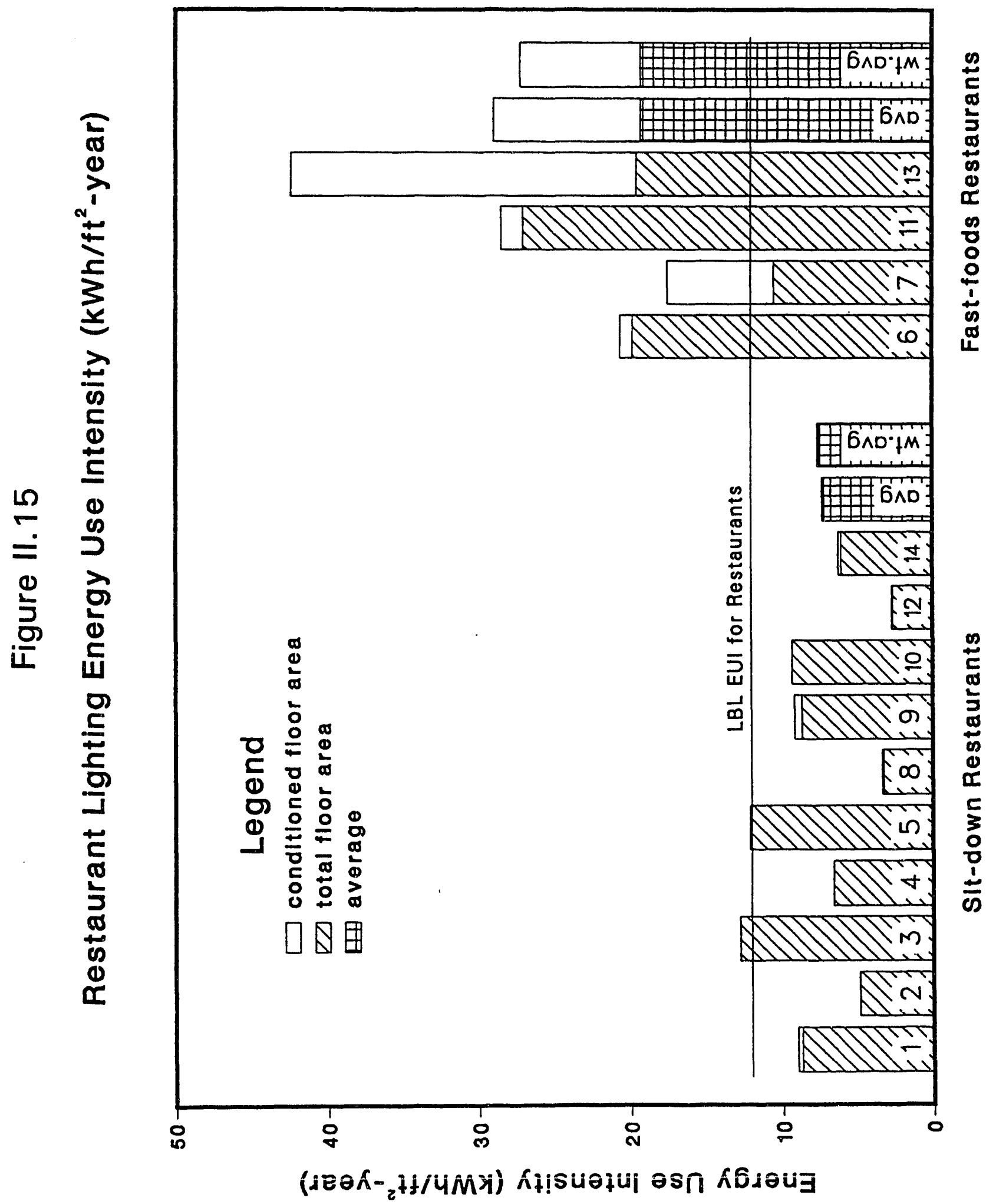


Figure II.16 compares the electric cooking EUIs of the monitored buildings with the LBL data. The LBL electric cooking EUIs are consistently smaller than monitored fast food and sitdown restaurants. The basic difference arises from the LBL study assumption of mostly gas cooking in restaurants.

Figures II.17 and II.18 show the refrigeration and exhaust fan EUIs and compare them with the LBL data. The LBL refrigeration EUI is higher than both the fast food and sit-down restaurants. LBL has used the on-site survey data to estimate the refrigeration EUI for restaurants. The LBL 'miscellaneous' EUI is comparable to the monitored restaurants' exhaust fan EUIs. 


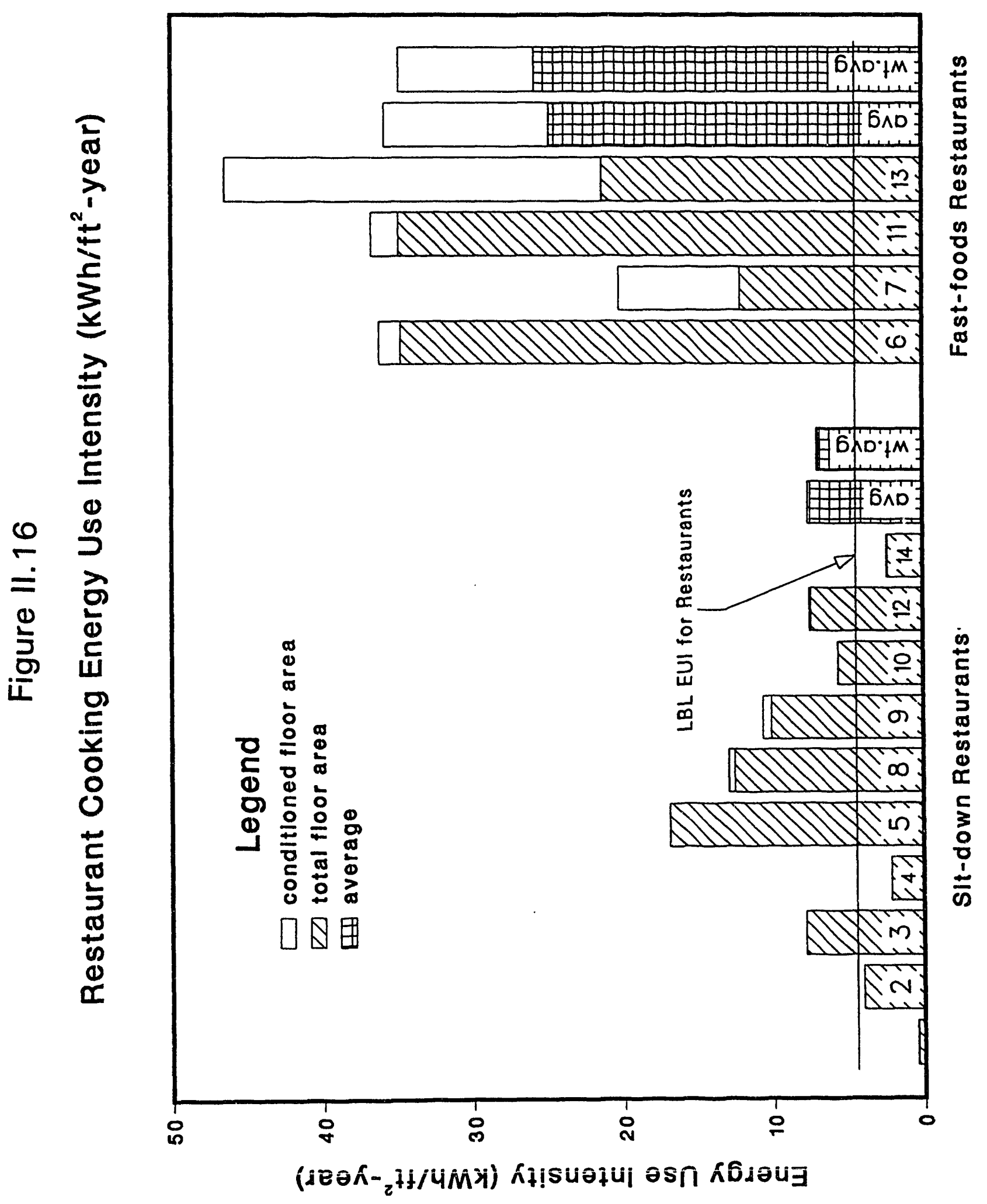




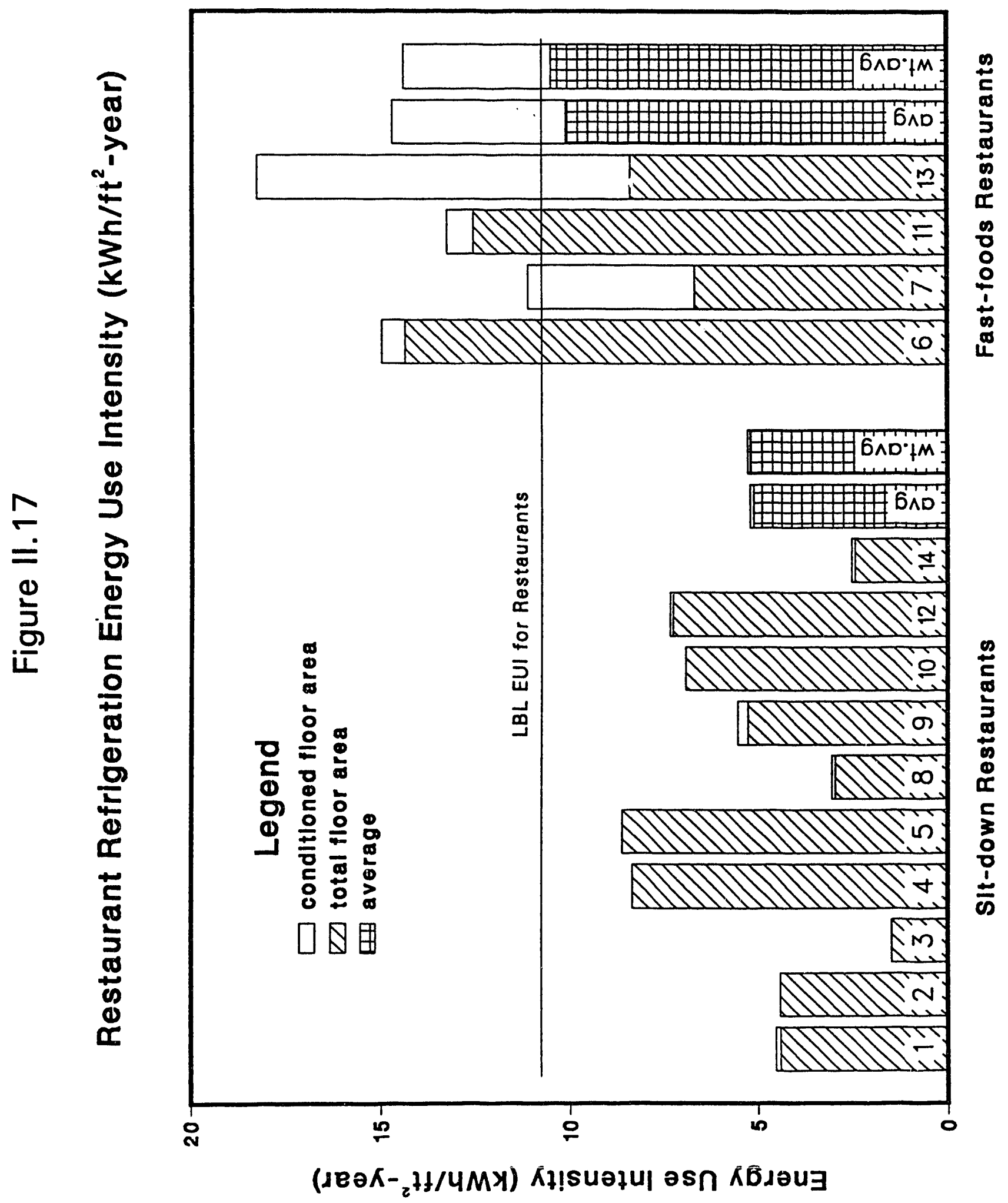




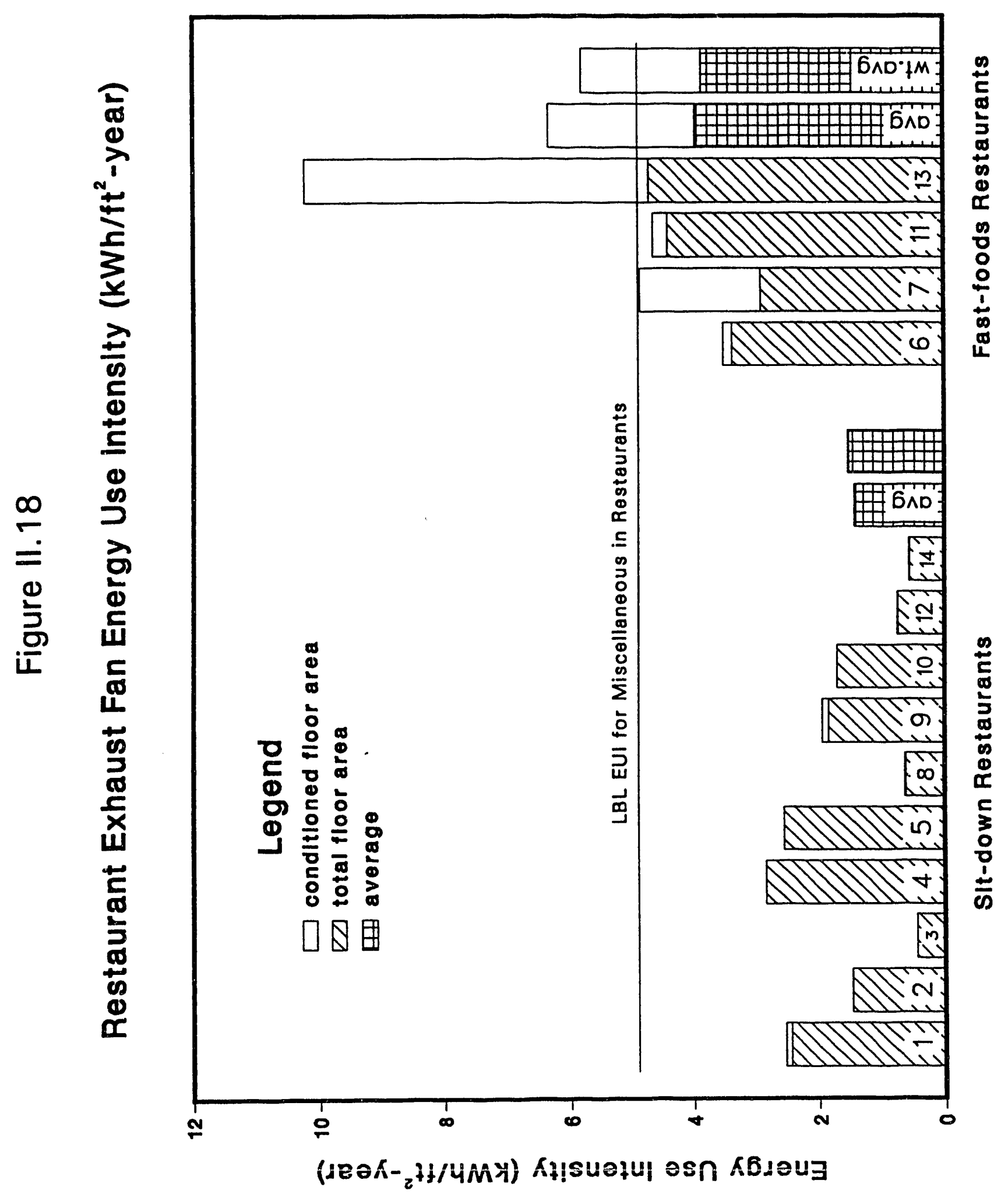




\section{Warehouse buildings}

Only three non-refrigerated warehouses have been monitored. Their floor areas range from 41,000 to $300,000 \mathrm{ft}^{2}$ and their total electric EUIs range from 2.5 to $8.9 \mathrm{kWh} / \mathrm{ft}^{2}$ year. Table II.6 presents the summary data for the three warehouses including the amount of valid and missing data, the estimated EUIs based on the conditioned and total building floor area, and the weighted and unweighted end-use EUIs.

Since, the sample of monitored buildings is very limited, not much can be concluded about the EUI comparison for the warehouses. Figures II.19, II.20, and II.21 show the total building EUIs, HVAC EUIs, and lighting EUIs, respectively, for all monitored warehouses. In the figures, the values are compared with the LBL estimates. Although the sample size is very limited, there is a good comparison between the monitored and LBL whole-building electric EUIs. 
Table 11.6

End Use Intensities for Warehouse Buildings

\begin{tabular}{|c|c|c|c|c|c|c|c|c|}
\hline \multirow{3}{*}{\begin{tabular}{|l} 
Bldg \\
Type \\
WHS \\
WHS
\end{tabular}} & \multirow{3}{*}{$\frac{\begin{array}{c}\text { Bldg } \\
\text { Num }\end{array}}{1}$} & \multirow{3}{*}{$\begin{array}{l}\text { End Use } \\
\text { Lighting } \\
\text { Total }\end{array}$} & \multirow{2}{*}{\multicolumn{2}{|c|}{$\begin{array}{l}\text { Observations } \\
\text { Valid Bad }\end{array}$}} & \multirow{3}{*}{$\begin{array}{c}\text { Total } \\
\text { Floor } \\
\text { Area } \\
300000 \\
300000\end{array}$} & \multirow{2}{*}{$\begin{array}{l}\text { Conditioned } \\
\text { Floor } \\
\text { Area }\end{array}$} & \multicolumn{2}{|c|}{$\begin{array}{l}\text { End Use Intensities } \\
\left(\mathrm{kWh} / \mathrm{t}^{2} \cdot \mathrm{yr}\right)\end{array}$} \\
\hline & & & & & & & $\begin{array}{l}\text { per tot } \\
\text { fir area }\end{array}$ & $\begin{array}{l}\text { per cond. } \\
\text { fir area }\end{array}$ \\
\hline & & & $\begin{array}{l}8375 \\
8375\end{array}$ & $\begin{array}{l}385 \\
385\end{array}$ & & $\begin{array}{l}39000 \\
39000\end{array}$ & $\begin{array}{l}2.818 \\
5.080\end{array}$ & $\begin{array}{l}21.676 \\
39.074\end{array}$ \\
\hline $\begin{array}{l}\text { WHS } \\
\text { WHS } \\
\text { WHS } \\
\text { WHS }\end{array}$ & $\begin{array}{l}2 \\
2 \\
2 \\
2\end{array}$ & $\begin{array}{l}\text { HVAC } \\
\text { Lighting } \\
\text { Equipment } \\
\text { Total }\end{array}$ & $\begin{array}{l}8759 \\
8759 \\
8758 \\
8709 \\
\end{array}$ & $\begin{array}{l}1 \\
1 \\
2 \\
3 \\
\end{array}$ & $\begin{array}{l}41400 \\
41400 \\
41400 \\
41400\end{array}$ & $\begin{array}{l}8280 \\
8280 \\
8280 \\
8280 \\
\end{array}$ & $\begin{array}{l}0.696 \\
0.864 \\
6.766 \\
8.950 \\
\end{array}$ & $\begin{array}{r}3.481 \\
4.318 \\
33.829 \\
44.748 \\
\end{array}$ \\
\hline $\begin{array}{l}\text { WHS } \\
\text { WHS }\end{array}$ & $\begin{array}{l}3 \\
3\end{array}$ & $\begin{array}{l}\text { Lighting } \\
\text { Total }\end{array}$ & $\begin{array}{l}7175 \\
7175\end{array}$ & $\begin{array}{l}1273 \\
1249\end{array}$ & $\begin{array}{l}110000 \\
110000\end{array}$ & $\begin{array}{l}28600 \\
28600\end{array}$ & \begin{tabular}{|l|}
1.473 \\
2.537
\end{tabular} & $\begin{array}{l}5.664 \\
9.758\end{array}$ \\
\hline $\begin{array}{l}\text { Weigh } \\
\text { WHS } \\
\text { WHS } \\
\text { WHS } \\
\text { WHS }\end{array}$ & $\operatorname{ted} A v$ & $\begin{array}{l}\text { Erages: } \\
\text { HVAC } \\
\text { Lighting } \\
\text { Equipment } \\
\text { Total }\end{array}$ & & & & & $\begin{array}{l}0.696 \\
1.718 \\
6.766 \\
5.522 \\
\end{array}$ & $\begin{array}{r}3.481 \\
10.553 \\
33.829 \\
31.193 \\
\end{array}$ \\
\hline $\begin{array}{l}\text { Unwe } \\
\text { WHS } \\
\text { WHS } \\
\text { WHS } \\
\text { WHS }\end{array}$ & & $\begin{array}{l}\text { Averages : } \\
\text { HVAC } \\
\text { Lighting } \\
\text { Equipment } \\
\text { Total }\end{array}$ & & & & & $\begin{array}{l}0.696 \\
1.718 \\
6.766 \\
5.522 \\
\end{array}$ & $\begin{array}{r}3.481 \\
10.553 \\
33.829 \\
31.193 \\
\end{array}$ \\
\hline
\end{tabular}




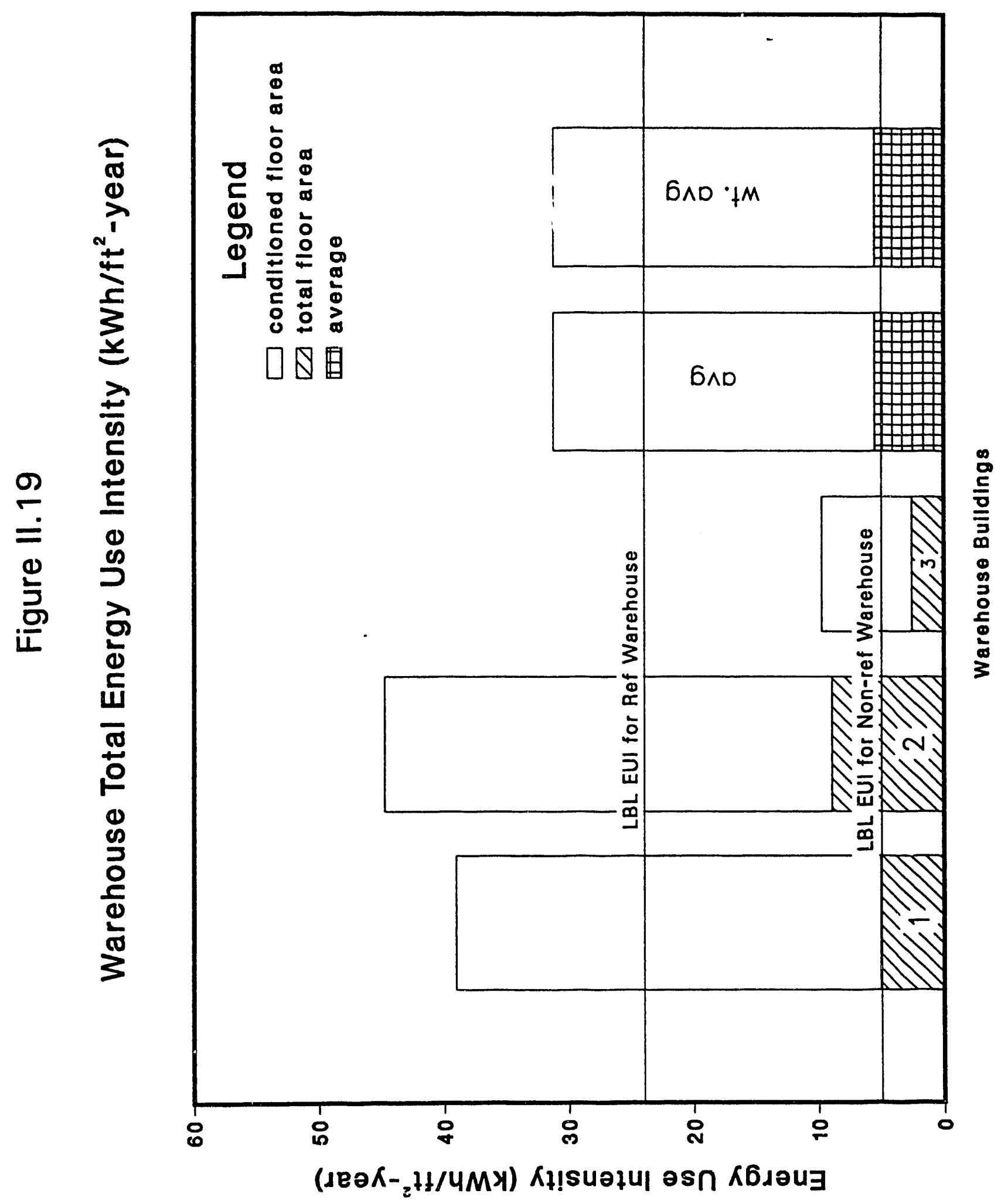




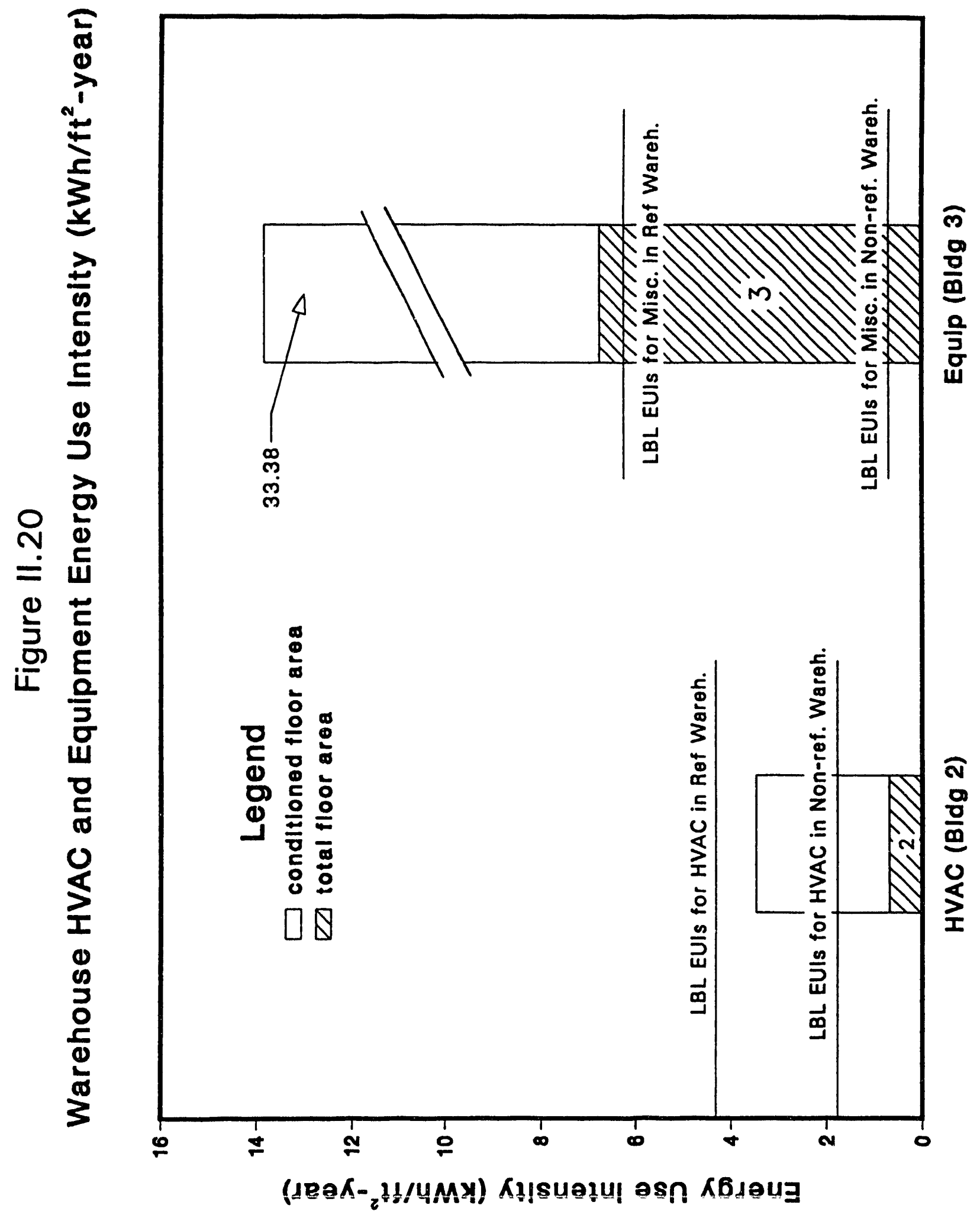




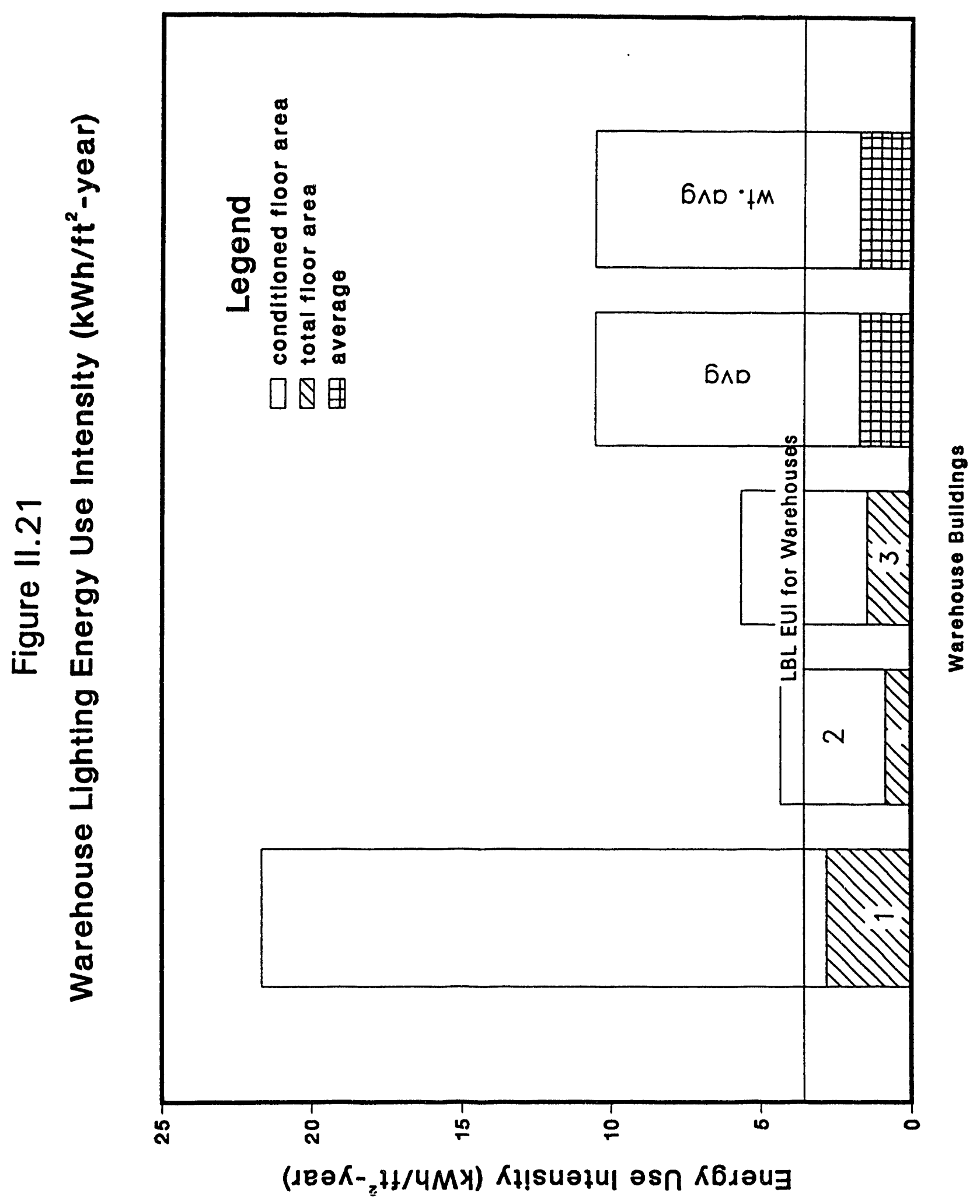




\section{Statistical Comparison}

To further compare the EUIs from the monitored buildings to those of the LBL estimates, we performed two sets of statistical tests: the t-test (student test) and the u-test, a non-parametric test. The t-test is statistical check used when the population distribution is approximately normal but the standard deviation is unknown. A non-parametric test is used when the distribution of the population is not known.

Table II.7 shows the result of the statistical comparison for all of the buildings. The hypothesis being tested is that the monitored building's mean EUI is not significantly different, within a 95\% confidence limit, from the population mean (it is assumed that the LBL data presents the population of commercial buildings in SCE service area). The first column of Table II.7 shows the range of EUIs for the monitored building sample; column 2 is the standard deviation of the sample; column 3 is the sample mean; column 4 is the population mean (LBL EUIs); column 5 is the ' $t$ '-value for normal distribution, column 7 is the ' $u$ '-value for the nonparametric test. Columns 6 and 8 indicate the statistical significance of the sample set based on the criteria stated above using the two types of tests. The $t$ and $u$ values are found using the following equations:

$$
\begin{gathered}
\mathrm{t}=(\text { sample mean-population mean }) /(\text { sample std. dev } / \sqrt{n}) \\
\mathrm{u}=(\text { sample mean-population mean }) / \text { sample range }
\end{gathered}
$$

For a more detailed discussion of the statistical tests, refer to a standard statistics textbook, such as Lipson and Narendra (1973).

Judging from the results of the non-parametric test one can conclude that, except for groceries and restaurants, there is no significant difference between the EUIs estimated from the monitored buildings and the LBL EUIs. For groceries, the lighting and refrigeration EUIs are not statistically different. For restaurants, the HVAC, lighting, and tctal EUIs are also not statistically different from each other. However, the HVAC EUIs and total building EUI for the grocery buildings are significantly different. The results of the statistical comparisons using the $t-$ test is somewhat different from the non-parametric test, as shown in Table II.7 . For those instances in which the two tests reached the same conclusion, there is a higher confidence in the conclusion. Likewise, the results can be viewed with lower confidence if both tests don't accept or reject the hypothesis. 
Table 11.7

Monitored Building Mean EUI Compared to Population Mean

\begin{tabular}{|c|c|c|c|c|c|c|c|c|}
\hline $\begin{array}{l}\text { Building Type (N) } \\
\text { End Use } \\
\end{array}$ & Range & $\begin{array}{l}\text { Standard } \\
\text { Deviation } \\
\end{array}$ & $\begin{array}{c}\text { Sample } \\
\text { Mean }\end{array}$ & $\begin{array}{c}\text { Poplulation } \\
\text { Mean }\end{array}$ & $t$ & $\begin{array}{l}\text { Hypotheses } \\
\text { Accepted }\end{array}$ & u & $\begin{array}{l}\text { Hypotheses } \\
\text { Accepted }\end{array}$ \\
\hline \multicolumn{9}{|l|}{ Large Office (4) } \\
\hline HVAC & 6.62 & 1.22 & 7.56 & 5.90 & 2.721 & yes & 0.251 & yes \\
\hline LIGHT & 9.14 & 2.17 & 5.55 & 10.66 & -4.707 & no & -0.559 & yes \\
\hline MISC & 8.84 & 1.66 & 5.89 & 3.21 & 3.224 & no & 0.304 & yes \\
\hline TOTAL & 15.47 & 3.50 & 17.14 & 19.88 & -1.562 & yes & -0.177 & yes \\
\hline \multicolumn{9}{|l|}{ Small Office (6) } \\
\hline HVAC & 6.57 & 1.00 & 6.19 & 7.02 & -2.033 & yes & -0.127 & yes \\
\hline LIGHT & 6.63 & 1.26 & 5.92 & 6.71 & -1.535 & yes & -0.119 & yes \\
\hline MISC & 9.53 & 1.22 & 3.78 & 3.59 & 0.373 & yes & 0.019 & yes \\
\hline TOTAL & 14.14 & 2.88 & 15.52 & 16.82 & -1.109 & yes & -0.092 & yes \\
\hline \multicolumn{9}{|l|}{ Large Retail (6) } \\
\hline HVAC & 7.48 & 1.24 & 6.71 & 7.56 & -1.668 & yes & -0.113 & yes \\
\hline LIGHT & 20.74 & 3.38 & 12.89 & 10.58 & 1.674 & yes & 0.111 & yes \\
\hline OTHER & 6.24 & 1.04 & 3.30 & 1.67 & 3.862 & no & 0.2062 & yes \\
\hline TOTAL & 24.93 & 3.89 & 22.77 & 18.22 & 2.861 & no & 0.182 & yes \\
\hline \multicolumn{9}{|l|}{ Small Retail (4) } \\
\hline HVAC & 7.89 & 1.85 & 7.61 & 6.50 & 1.202 & yes & 0.141 & yes \\
\hline LIGHT & 3.76 & 0.95 & 6.74 & 6.07 & 1.425 & yes & 0.179 & yes \\
\hline OTHER & 1.72 & 0.41 & 0.79 & 1.94 & -5.591 & no & -0.666 & yes \\
\hline TOTAL & 7.20 & 1.64 & 14.60 & 12.25 & 2.857 & yes & 0.326 & yes \\
\hline \multicolumn{9}{|l|}{ Grocery (14) } \\
\hline HVAC & 6.61 & 0.47 & 3.59 & 2.14 & 11.520 & no & 0.219 & no \\
\hline LIGHT & 20.55 & 1.37 & 15.76 & 13.97 & 4.894 & no & 0.087 & yes \\
\hline REFRIG & 25.23 & 2.12 & 25.63 & 23.17 & 4.328 & no & 0.097 & yes \\
\hline OTHER & 25.40 & 2.88 & 18.56 & 2.04 & 21.454 & no & 0.650 & no \\
\hline TOTAL & 61.30 & 5.21 & 62.88 & 40.27 & 16.229 & no & 0.369 & no \\
\hline \multicolumn{9}{|l|}{ Restaurant (14) } \\
\hline HVAC & 64.93 & 5.12 & i\$.23 & 19.74 & -0.375 & yes & -0.008 & yes \\
\hline LIGHT & 24.41 & 1.84 & 10.85 & 12.03 & -2.398 & no & -0.048 & yes \\
\hline REFRIG & 12.93 & 0.92 & 6.62 & 10.78 & -16.924 & no & -0.322 & no \\
\hline COOKING & 34.47 & 2.65 & 12.71 & 4.46 & 11.658 & no & 0.239 & no \\
\hline EXHAUST & 4.25 & 0.38 & 2.18 & 0.00 & 21.513 & no & 0.514 & no \\
\hline OTHER & 25.07 & 1.72 & 10.83 & 4.92 & 12.844 & no & 0.236 & no \\
\hline TOTAL & 108.06 & 8.22 & 58.54 & 51.91 & 3.019 & no & 0.061 & yes \\
\hline \multicolumn{9}{|l|}{ Warehouse (4) } \\
\hline HVAC & 1.05 & 0.37 & 2.95 & 1.78 & 4.476 & yes & 1.119 & yes \\
\hline LIGHT & 1.96 & 0.44 & 1.86 & 3.55 & -7.764 & no & -0.861 & no \\
\hline MISC & 6.68 & 1.55 & 2.85 & 1.14 & 2.216 & yes & 0.257 & yes \\
\hline TOTAL & 6.41 & 1.42 & 4.99 & 5.02 & -0.039 & yes & -0.004 & yes \\
\hline
\end{tabular}


We also looked at the question of the statistical differences of the end-use EUIs in terms of the percentage of the whole-building electric EUI. The results are shown in Table II.8. For the non parametric test, the comparison is somewhat less favorable than the results of the previous comparison. The percentage of lighting EJJIs for the large office and grocery, HVAC EUI for large retail, and the refrigeration EUI for the grocery are significantly different from that of the corresponding LBL percentages.

In summary, in the absence of a statistically representative data, one can conclude that the monitored data are not statistically different from a good portion of the LBL derived end-use EUIs. 
Table 11.8

End Use EUls as Percentage of Whole Building Load

\begin{tabular}{|c|c|c|c|c|c|c|c|c|}
\hline $\begin{array}{l}\text { Building Type (N) } \\
\text { End Use }\end{array}$ & Range & $\begin{array}{l}\text { Standard } \\
\text { Deviation }\end{array}$ & $\begin{array}{c}\text { Sample } \\
\text { Mean }\end{array}$ & $\begin{array}{l}\text { Poplulation } \\
\text { Mean }\end{array}$ & $t$ & $\begin{array}{l}\text { Hypocheses } \\
\text { Accepted }\end{array}$ & u & $\begin{array}{l}\text { Hypotheses } \\
\text { Accepted }\end{array}$ \\
\hline \multicolumn{9}{|l|}{ Large Orfice (4) } \\
\hline HVAC & 0.611 & 0.131 & 0.499 & 0.297 & 3.082 & yes & 0.331 & yes \\
\hline LIGHT & 0.251 & 0.052 & 0.297 & 0.536 & -9.259 & no & -0.950 & no \\
\hline MISC & 0.444 & 0.083 & 0.347 & 0.161 & 4.457 & no & 0.418 & yes \\
\hline \multicolumn{9}{|l|}{ Small Office (6) } \\
\hline HVAC & 0.494 & 0.071 & 0.440 & 0.417 & 0.785 & yes & 0.046 & yes \\
\hline LIGHT & 0.318 & 0.028 & 0.377 & 0.399 & -1.956 & yes & -0.070 & yes \\
\hline MISC & 0.412 & 0.066 & 0.227 & 0.213 & 0.487 & yes & 0.032 & yes \\
\hline \multicolumn{9}{|l|}{ Large Retail (6) } \\
\hline HVAC & 0.176 & 0.030 & 0.295 & 0.415 & -9.804 & no & -0.678 & no \\
\hline LIGHT & 0.295 & 0.054 & 0.526 & 0.581 & -2.499 & yes & -0.186 & yes \\
\hline OTHER & 0.294 & 0.062 & 0.185 & 0.092 & 3.694 & no & 0.316 & yes \\
\hline \multicolumn{9}{|l|}{ Small Retail (4) } \\
\hline HVAC & 0.298 & 0.072 & 0.498 & 0.531 & -0.900 & yes & -0.109 & yes \\
\hline LIGHT & 0.317 & 0.066 & 0.471 & 0.496 & -0.745 & yes & -0.077 & yes \\
\hline OTHER & 0.176 & 0.042 & 0.066 & 0.158 & -4.384 & no & -0.523 & yes \\
\hline \multicolumn{9}{|l|}{ Grocery (14) } \\
\hline HVAC & 0.124 & 0.007 & 0.059 & 0.053 & 3.491 & no & 0.050 & yes \\
\hline LIGHT & 0.289 & 0.014 & 0.253 & 0.347 & -24.439 & no & -0.324 & no \\
\hline REFRIG & 0.360 & 0.026 & 0.416 & 0.575 & -23.169 & no & -0.443 & no \\
\hline OTHER & 0.305 & 0.028 & 0.282 & 0.051 & 30.413 & no & 0.759 & no \\
\hline \multicolumn{9}{|l|}{ Restaurant (14) } \\
\hline HVAC & 0.593 & 0.042 & 0.279 & 0.380 & -9.087 & no & -0.171 & no \\
\hline LIGHT & 0.197 & 0.015 & 0.179 & 0.232 & -12.881 & no & -0.267 & no \\
\hline REFRIG & 0.237 & 0.019 & 0.127 & 0.208 & -15.422 & no & -0.339 & no \\
\hline COOKING & 0.296 & 0.022 & 0.201 & 0.086 & 19.401 & no & 0.391 & no \\
\hline EXHAUST & 0.074 & 0.005 & 0.038 & 0.000 & 25.813 & no & 0.505 & no \\
\hline OTHER & 0.456 & 0.032 & 0.219 & 0.095 & 14.494 & no & 0.272 & no \\
\hline \multicolumn{9}{|l|}{ Warehouse (4) } \\
\hline HVAC & 0.326 & 0.115 & 0.552 & 0.355 & 2.420 & yes & 0.605 & yes \\
\hline LIGHT & 0.580 & 0.130 & 0.477 & 0.707 & -3.560 & no & -0.397 & yes \\
\hline MISC & 0.617 & 0.128 & 0.474 & 0.227 & 3.847 & no & 0.401 & yes \\
\hline
\end{tabular}




\section{Load-shape Analysis and Comparison with Forecasting Models}

We used the same procedure discussed in the previous sections to develop average monthly load shapes by end use for each building type and to compare them with the LBL results. The load shapes are grouped by weekdays and weekend days. The results are shown in Figures II.22a,b through 28a,b. All ' $a$ ' figures correspond to the SCE monitored data and ' $b$ ' figures correspond to load-shape data from the LBL study. The following is a key to the list of figures.

\begin{tabular}{|c|c|c|}
\hline Figure & Code & Building Type \\
\hline ----------- & ----- & 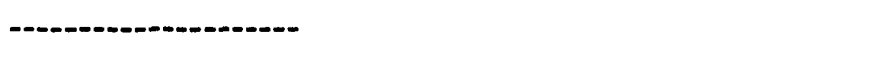 \\
\hline Fig. II.22a & OFFLG & large office measured data \\
\hline Fig. II.22b & OFFLG & large office EDA results \\
\hline Fig. II.23a & OFFSM & small office measured data \\
\hline Fig. II.23b & OFFSM & small office EDA results \\
\hline Fig. II.24a & RTLLG & large retail measured data \\
\hline Fig. II.24b & RTLLG & large retail EDA results \\
\hline Fig. 11.25a & RTLSM & small retail measured data \\
\hline Fig. II.25b & RTLSM & small retail EDA results \\
\hline Fig. II.26a & GRY & grocery (or food store) measured data \\
\hline Fig. II.26b & GRY & food store EDA results \\
\hline Fig. II.27a.1 & RSTSD & sit-down restaurant measured data \\
\hline Fig. II.27a.2 & RSTFF & fast-food restaurant measured data \\
\hline Fig. II.27a & RST & combined restaurant ( $63 \%$ sit-down, $37 \%$ fast food) \\
\hline Fig. II.27b & RST & restaurant EDA results \\
\hline Fig. II.28a & WHS & warehouse measured data \\
\hline Fig. II.28b & WHS & warehouse EDA results \\
\hline
\end{tabular}

Since the 1989 LBL study focused on developing load shapes for a standard weekday, we will only concentrate on the comparison of the LSs for the standard weekday.

\section{Large Office (Figures II.22a\&b)}

The average monthly whole-building electric load shapes developed in the LBL study and those produced from the monitored data compare well qualitatively. Significant differences 
Figure 11.22a

\section{Large Office Monitored Data}

Solid lines for winter months, dashed lines for summer months

OFFLG HVAC on Weekdays

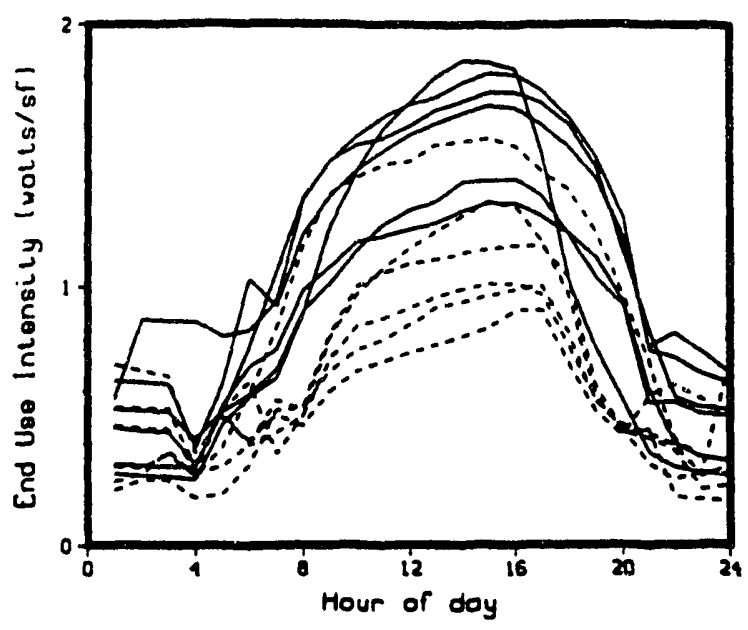

OFFLG Lighting on Heokdoys

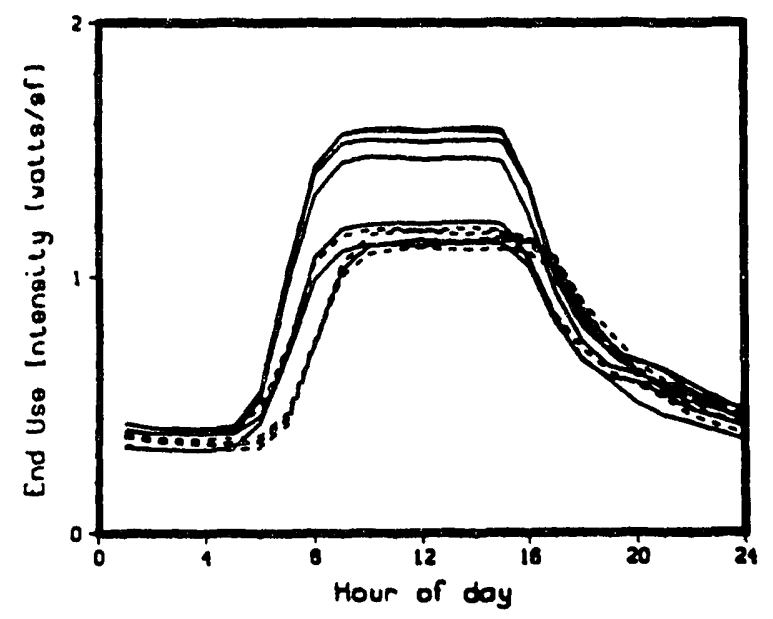

OFFLG Receptacle on Heakdays

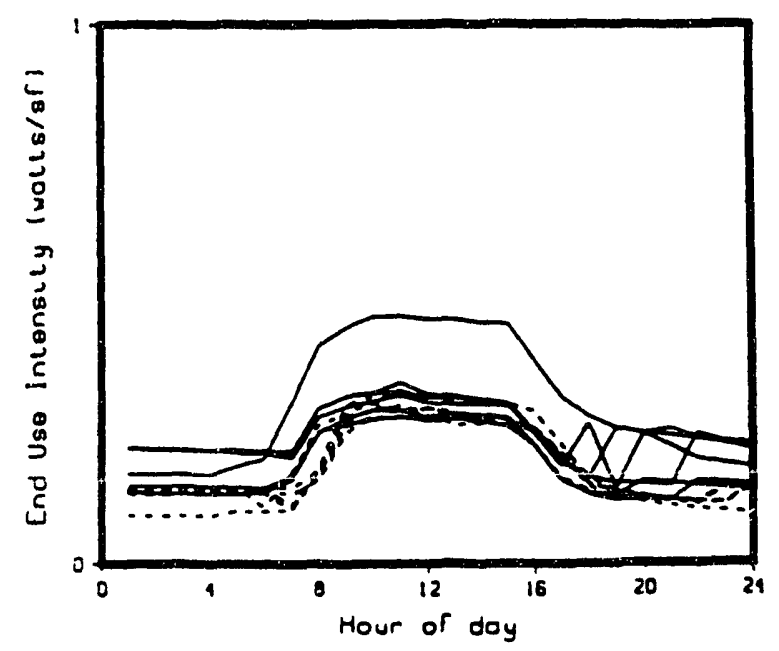

OFFLG HVAC on Weokends

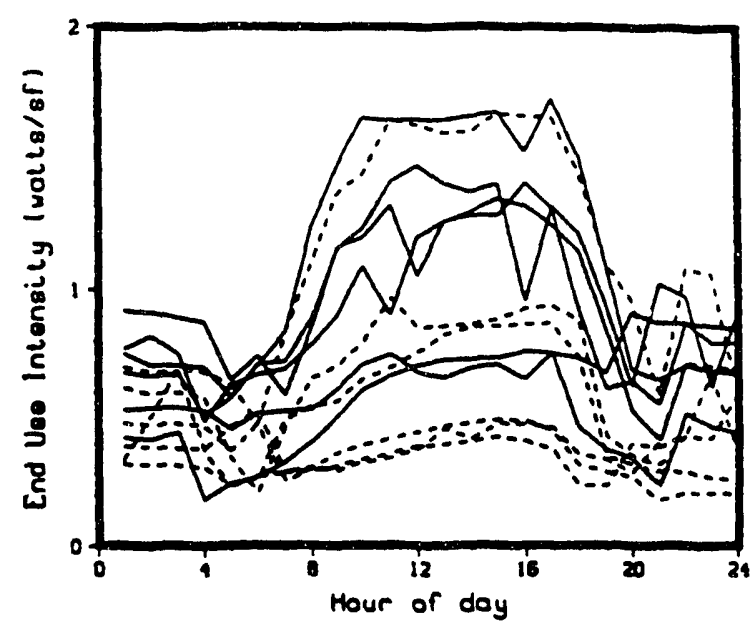

OFFLG Lighting on Wookends

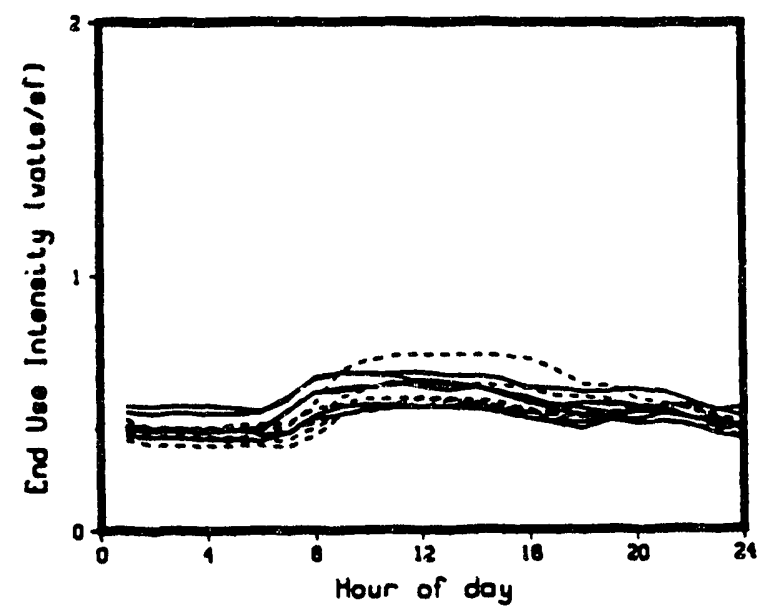

OFFLG Receptocle on Weekends

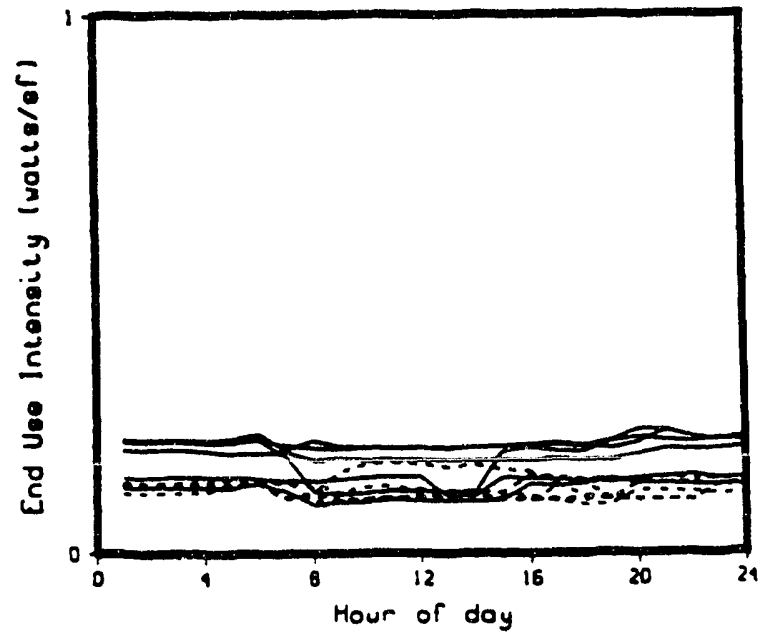


Figure II.22a (continued)

\section{Large Office Monitored Data}

Solid lines for winter months, dashed lines for summer months

OFFLG Totol on Weekdoys

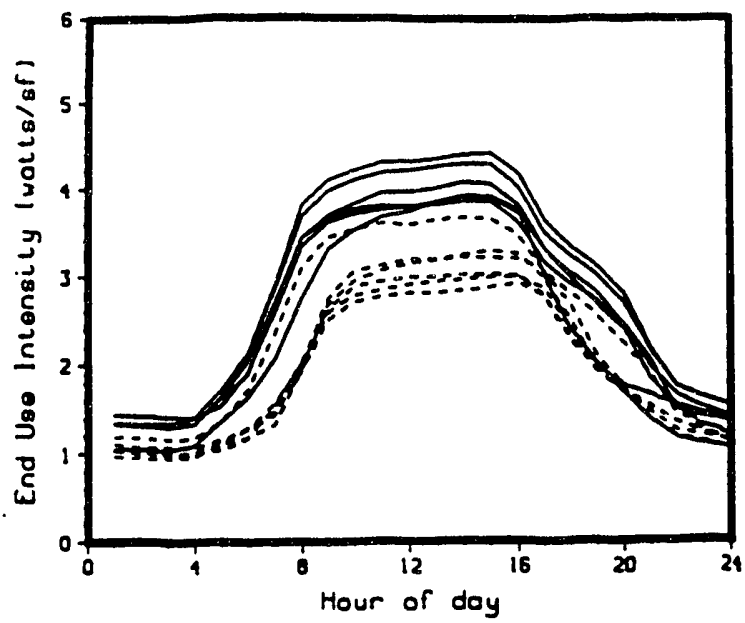

OFFLG Total on Werkends

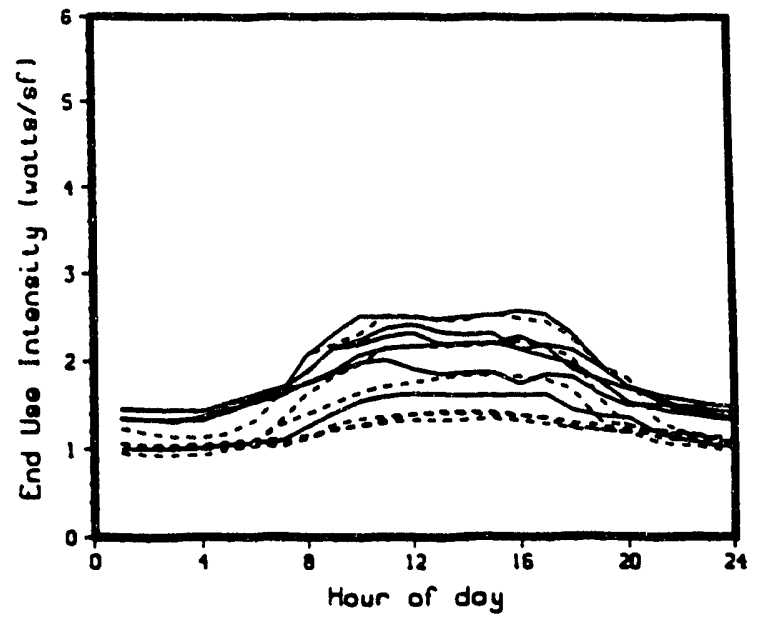




\section{Figure II.22b \\ Large Office EDA Results}

Solid lines for winter months, dashed lines for summer months

OFFLG EOA HVAC on Weekdoys

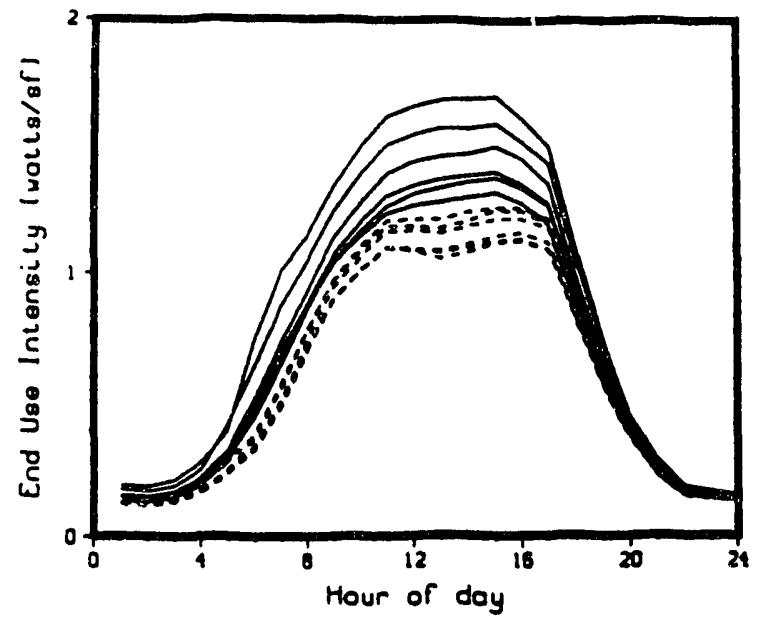

OFFLG EDA Lighting on Weokdays

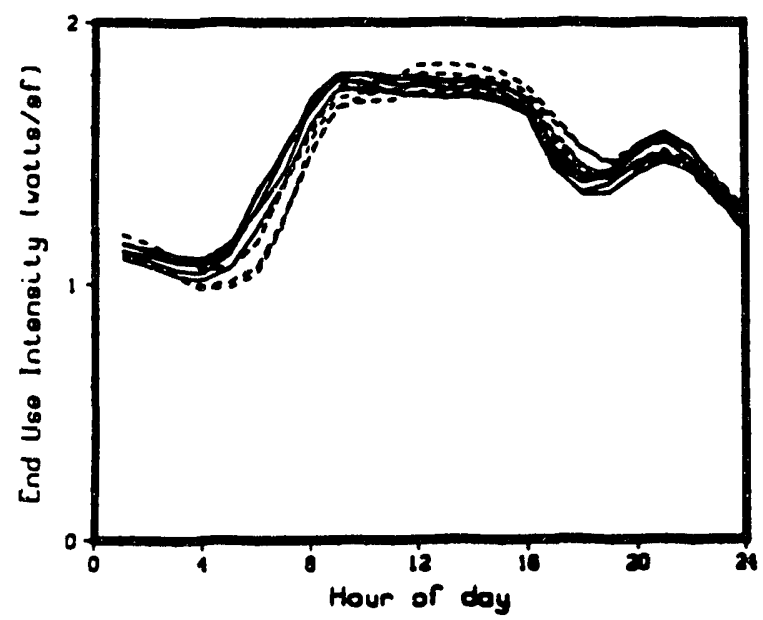

OFFLG EDA Mise on Hookdoys

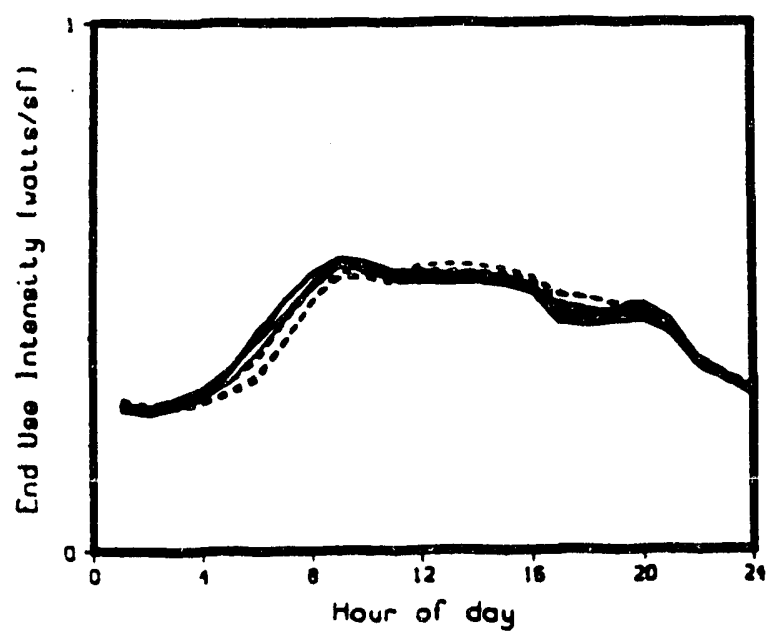

OFFLG EDA HVAC on Weokends

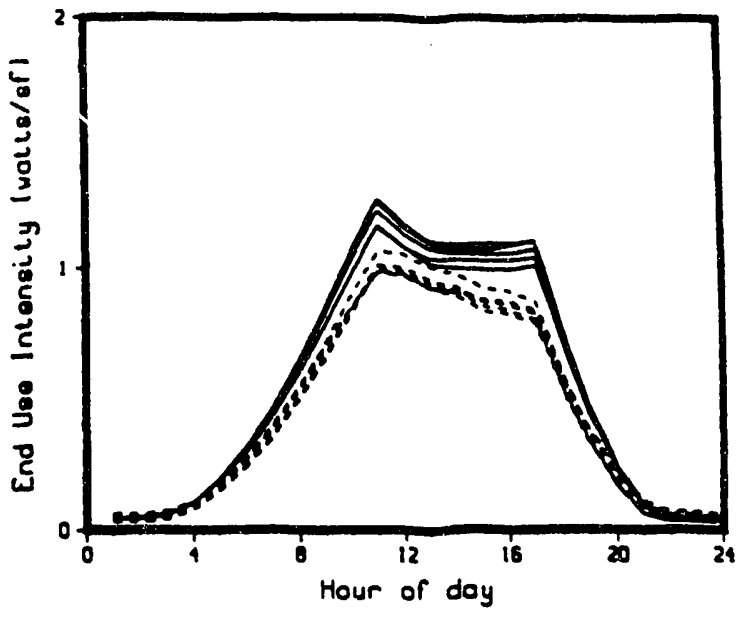

OFFLG EDA Lighting on Heokends

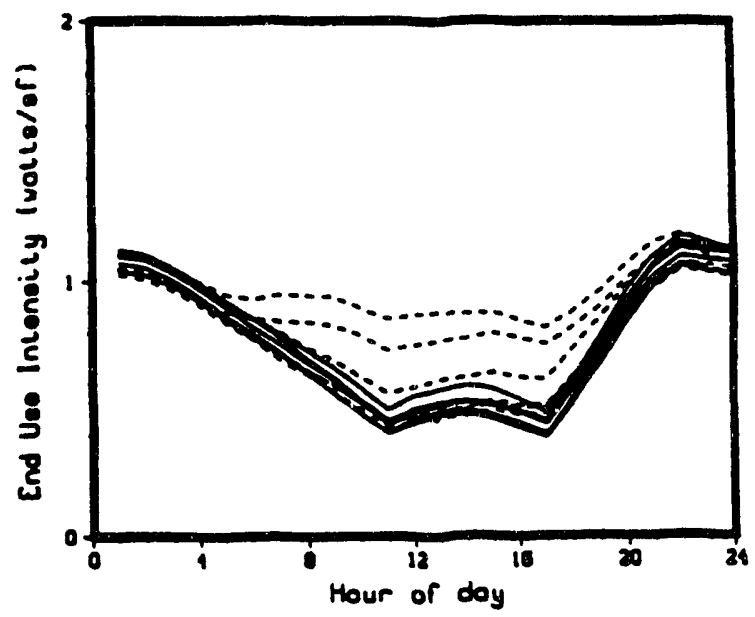

OFFLG EOA Mise on Weakends

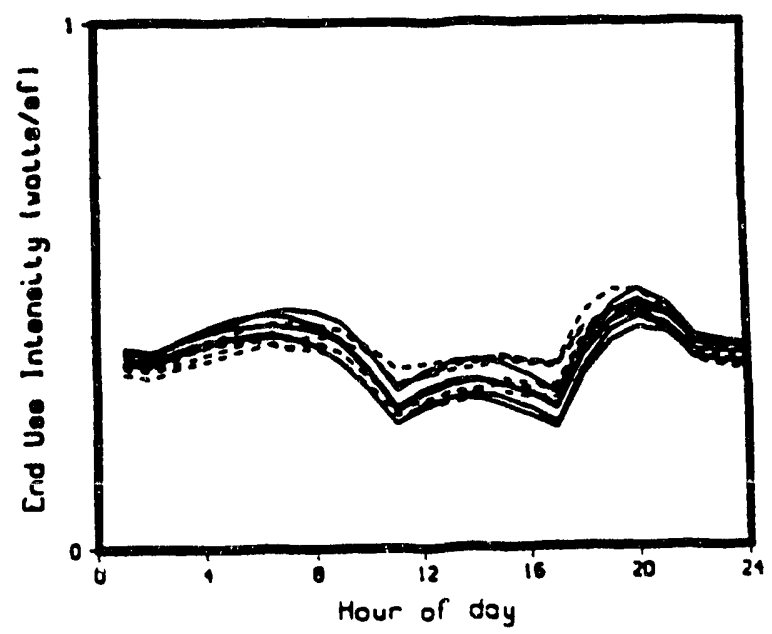


Figure II.22b (continued)

\section{Large Office EDA Results}

Solid lines for winter months, dashed lines for summer months

OFFLG EDA Totol on Weekdays

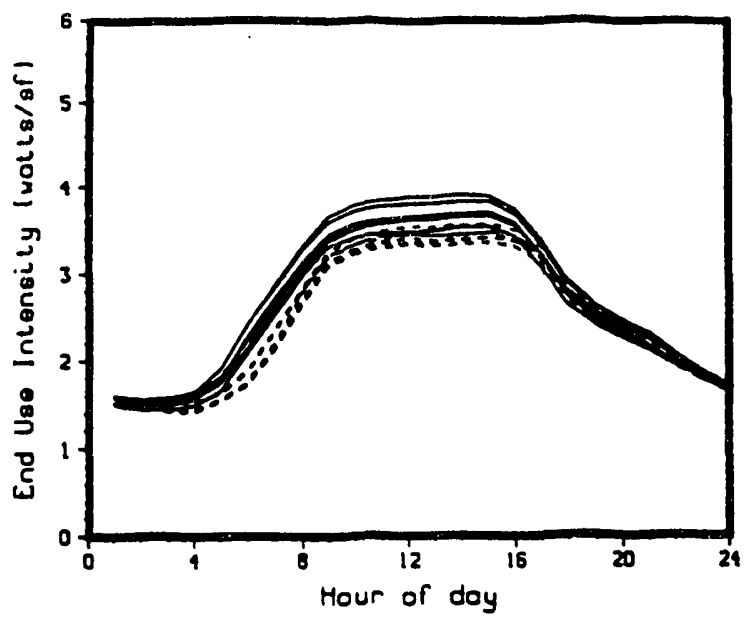

OFFLG EOA Tocol on Weekends

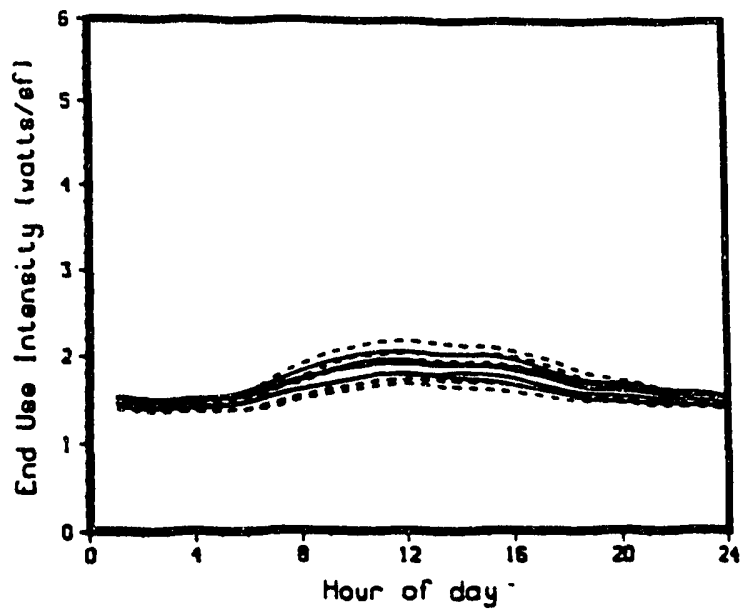




\section{Figure II.23a \\ Small Office Monitored Data}

Solid lines for winter months, dashed lines for summer months

OFFSM HVAC on Weokdays

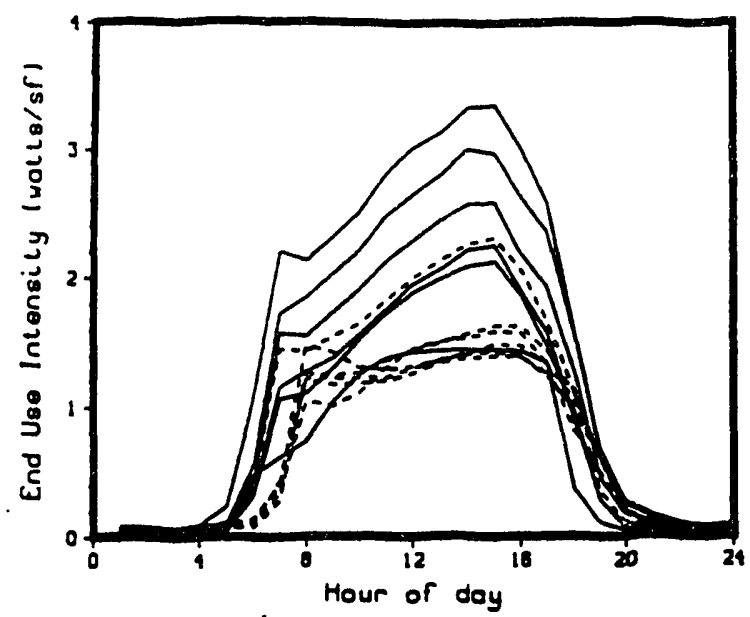

OFFSM Lighting on Heokdoys

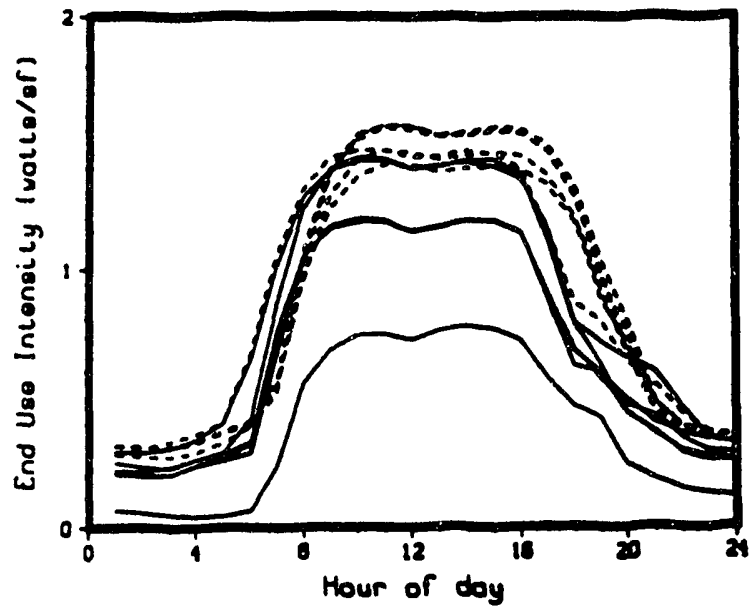

OFFSM Receptocle on Heckdoye

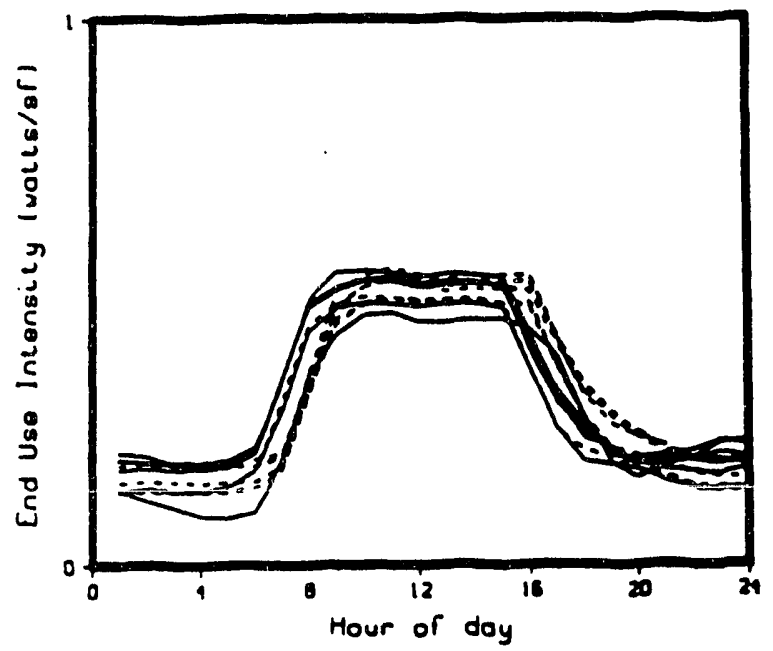

OFFSM HVAC on Weekends

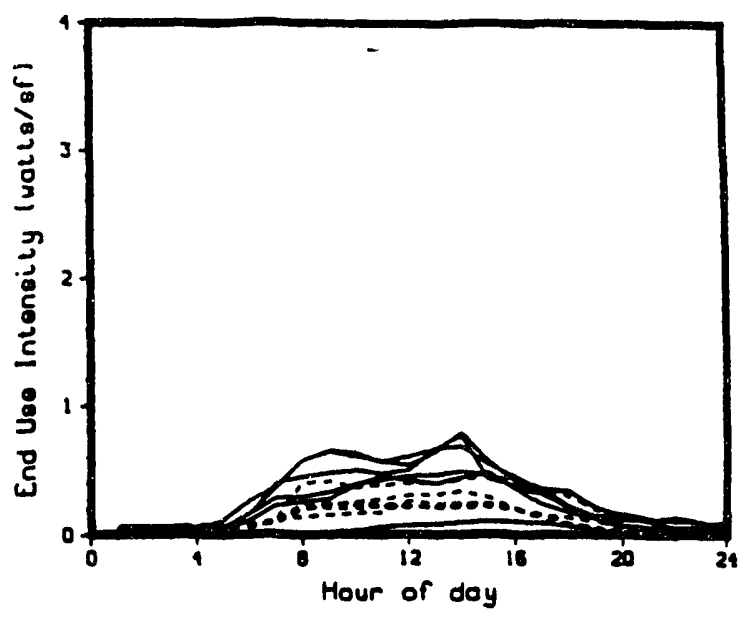

OFFSM Lighting on Weokende

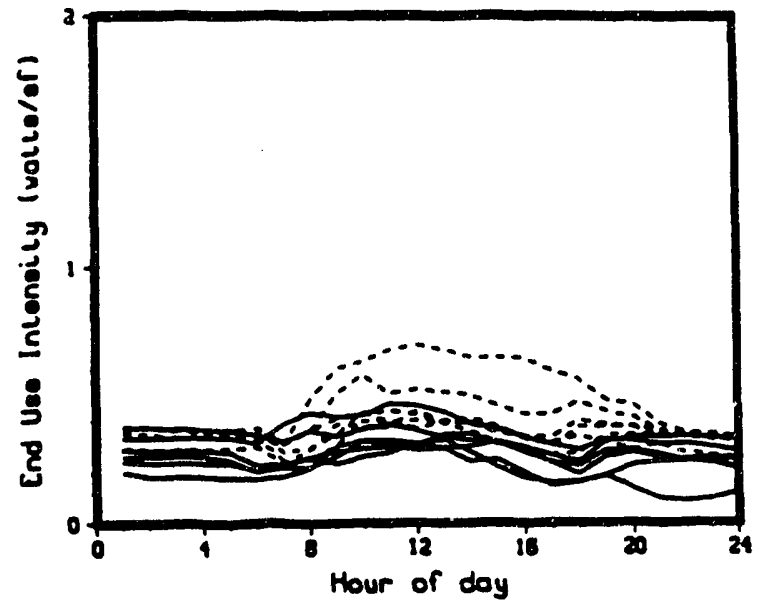

OFFSM Receptocle on Weokends

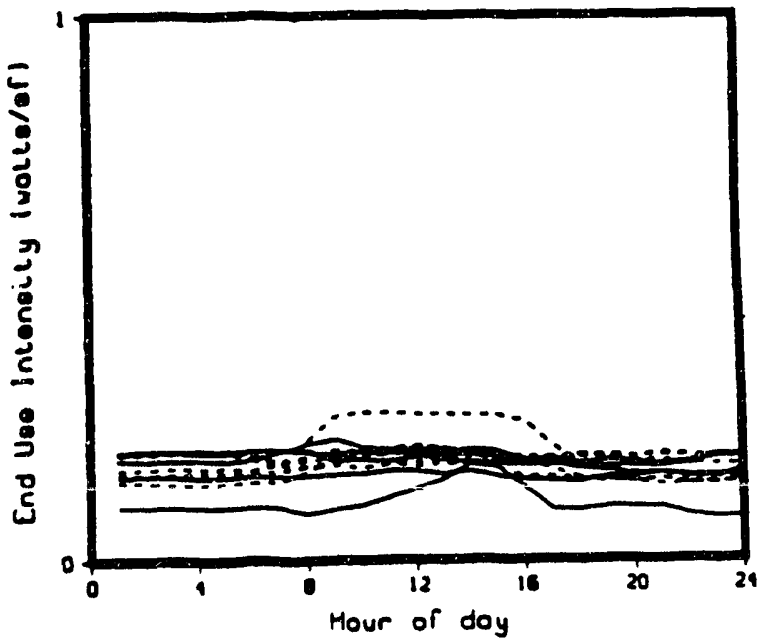


Figure II.23a (continued)

Small Office Monitored Data

Solid lines for winter months, dashed lines for summer months

OFFSM Totol on Weokdoys

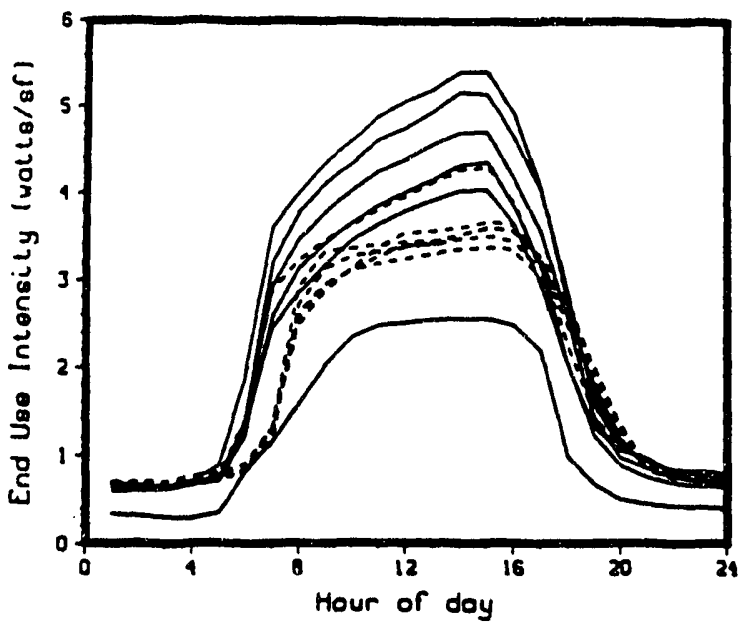

OFFSM Tolol on Werkends

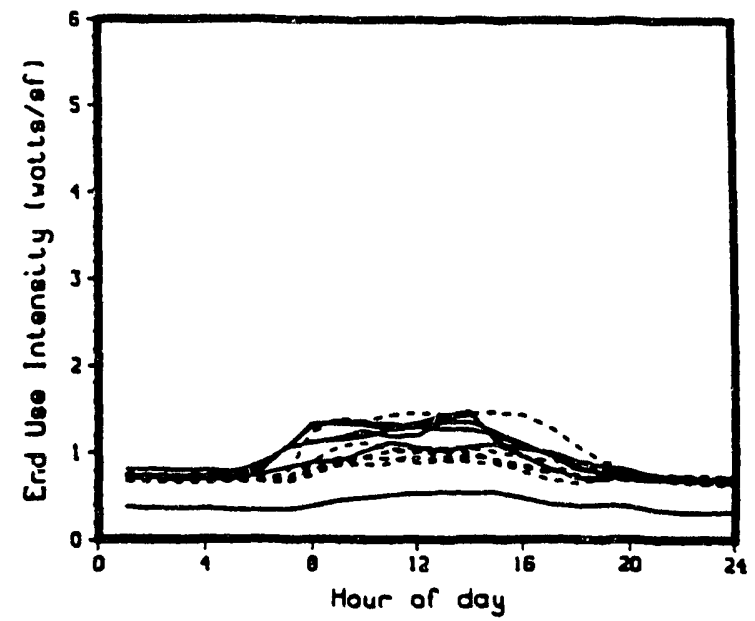




\section{Figure II.23b \\ Small Office EDA Results}

Solid lines for winter months, dashed lines for summer months

OFFSM EOA HVAC on Weokdoys

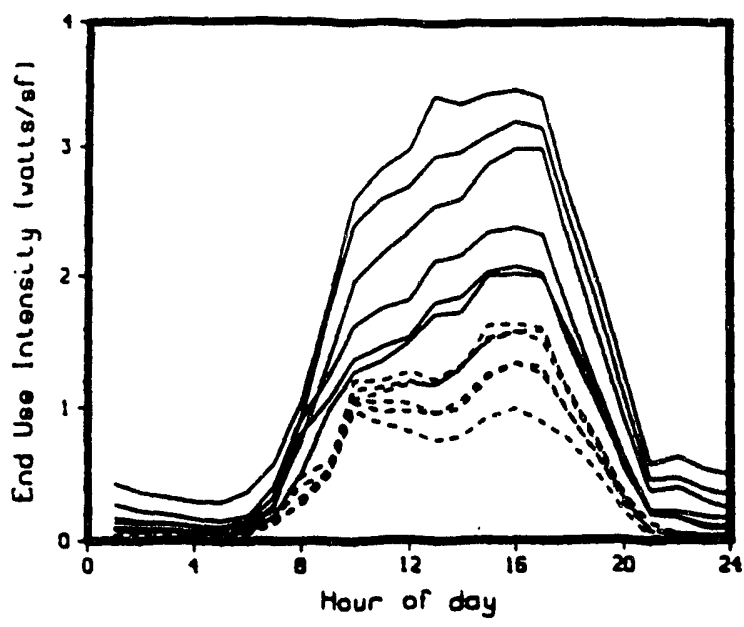

OFFSM EDA Lighting on Woskdoys

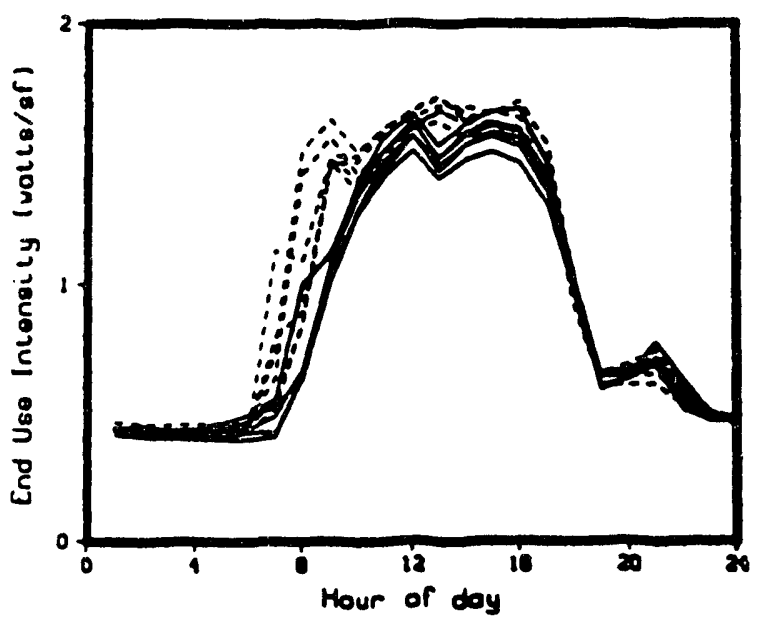

OFFSM EDA Mise on Weehdoys

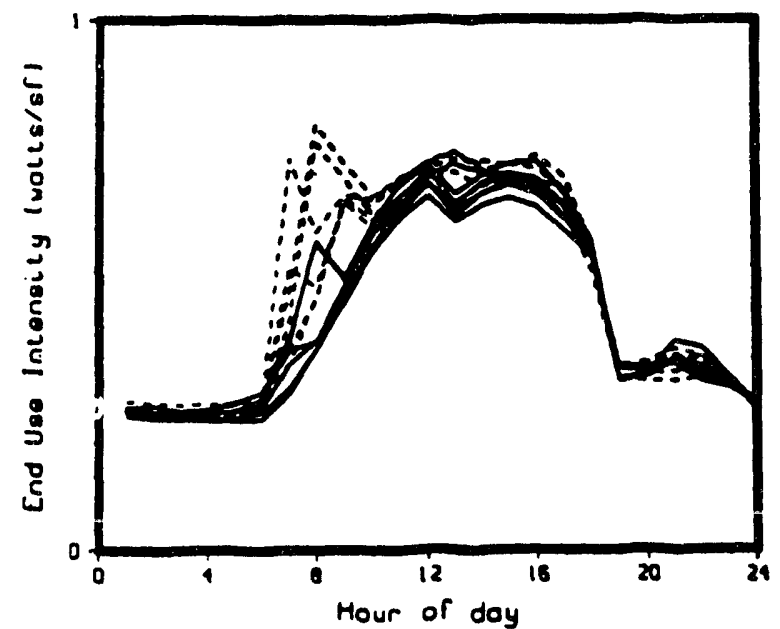

OFFSM EDA HVAC on Weokends

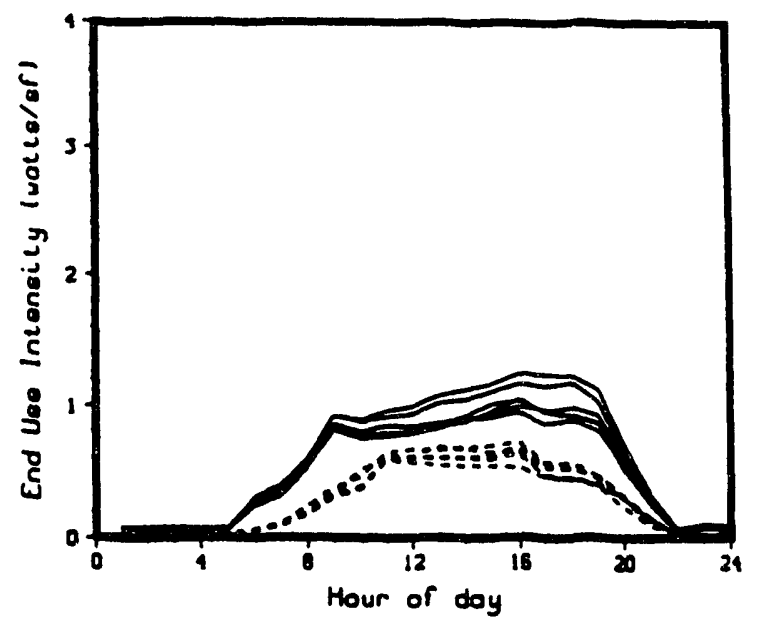

OFFSM EOA Lighting on Weokends

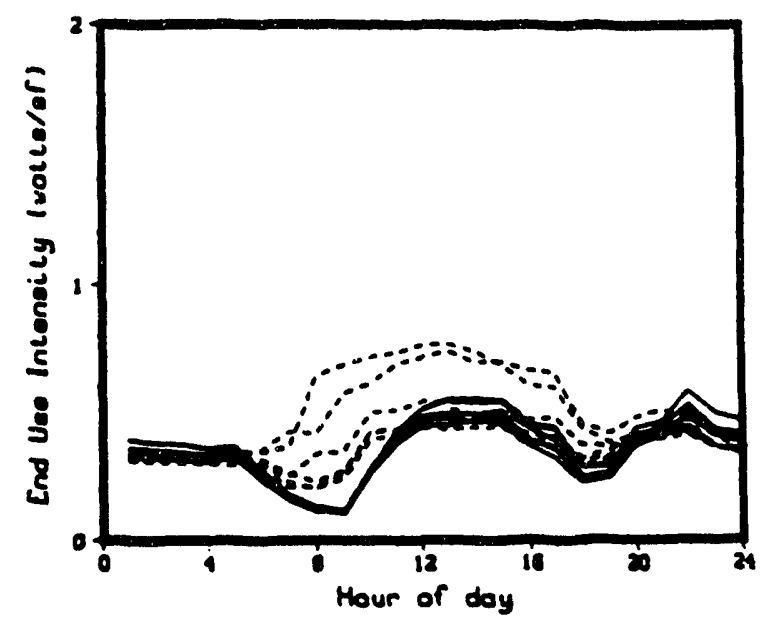

OFFSM EDA Mise on Hoekende

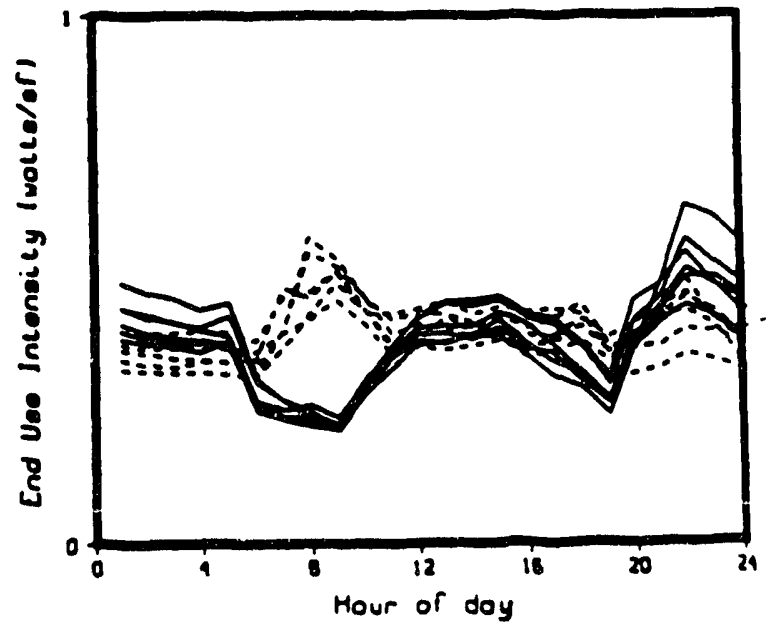


Figure II.23b (continued) Small Office EDA Results

Solid lines for winter months, dashed lines for summer months

OFFSM EOA TOLOL on Werkdoys

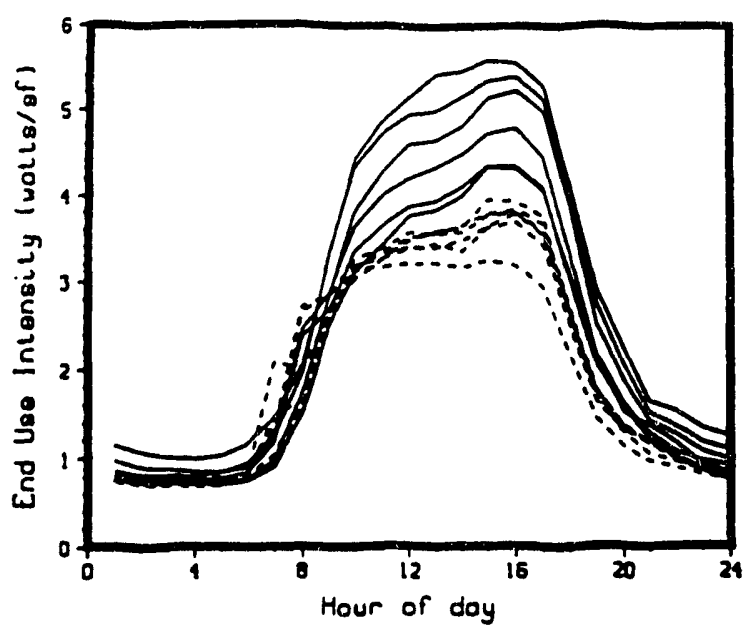

OFFSM EOA TotoL on Weekends

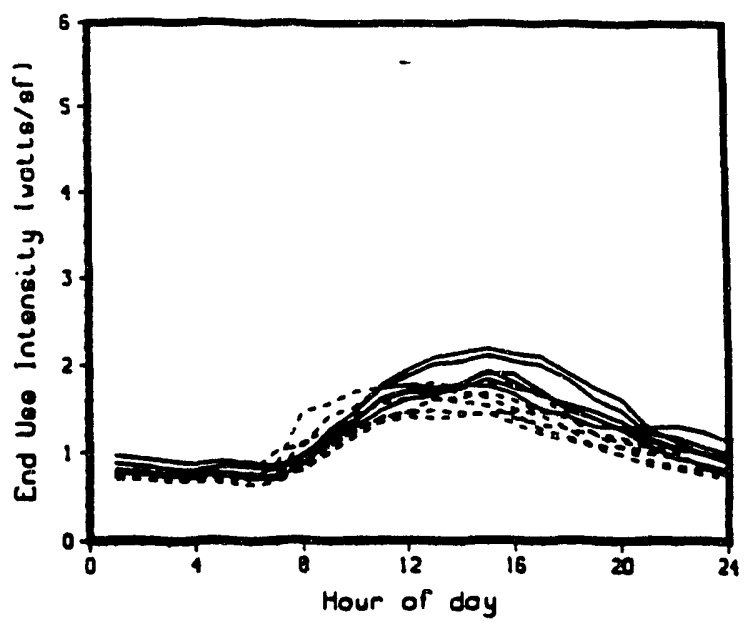


Figure II.24a

\section{Large Retail Monitored Data}

Solid lines for winter months, dashed lines for summer months

RTLLG HVAC on Weokdoys

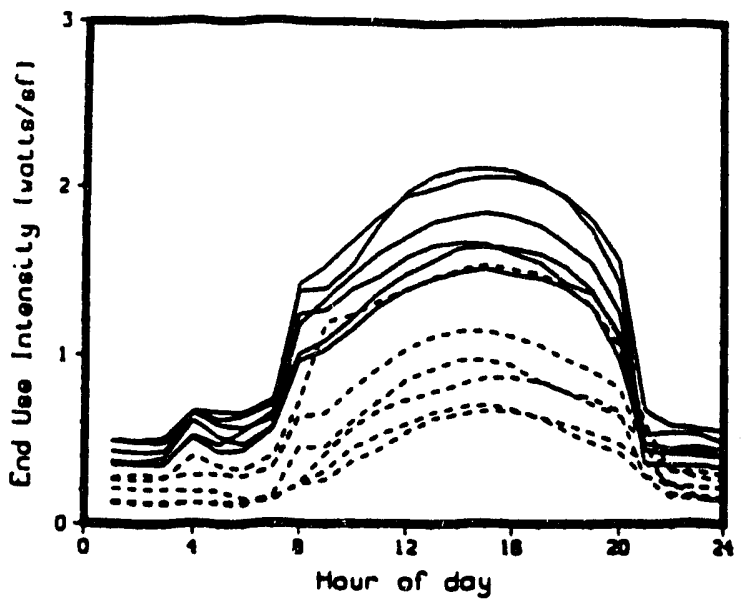

RILLG Lighting on Wookdoys

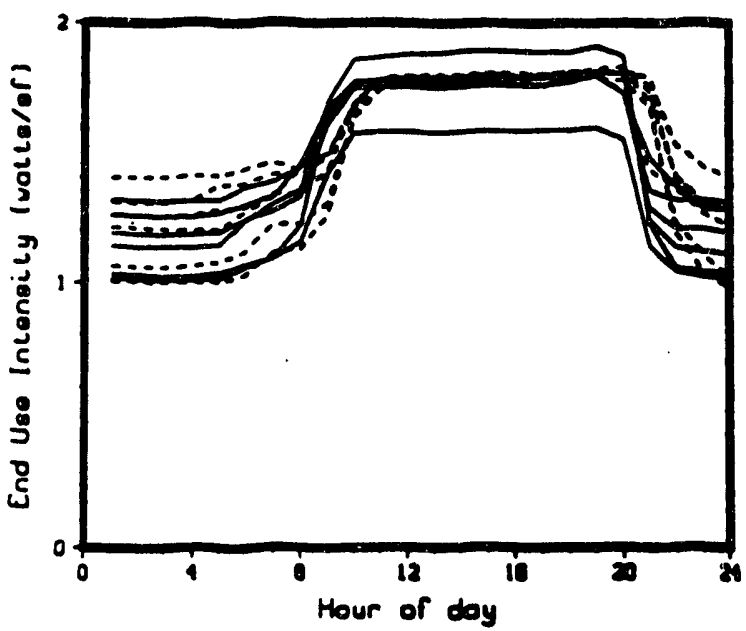

RILLG Totol on Heckdoye

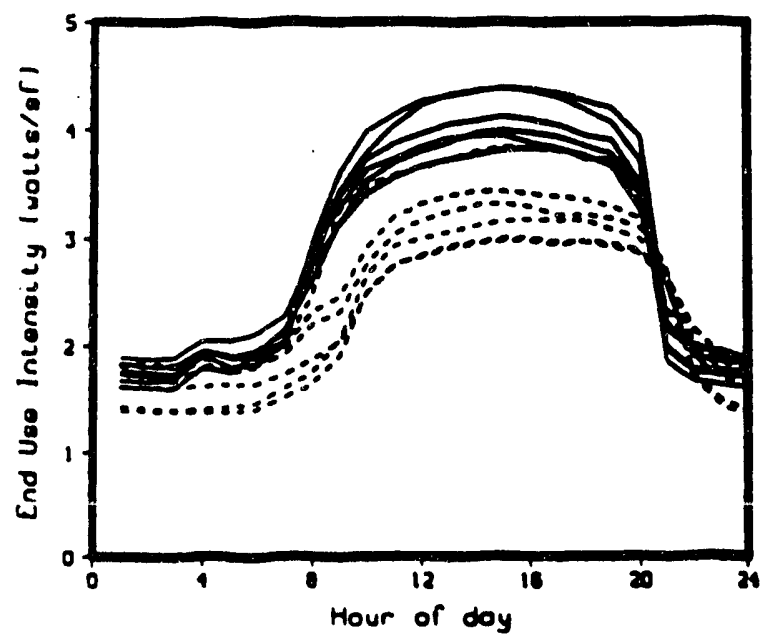

RTLLG HVAC on Weokends

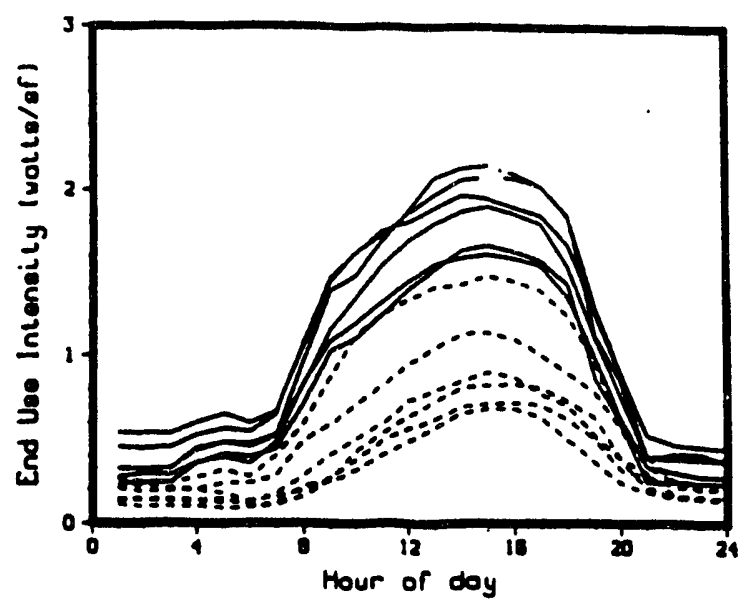

RTLLG Lighting on Weokends

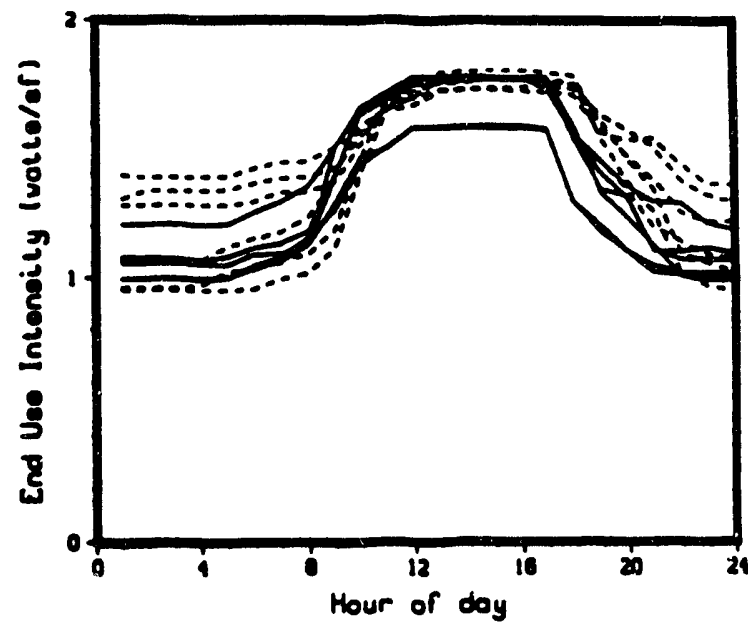

RTLLG Tolol on Heokends

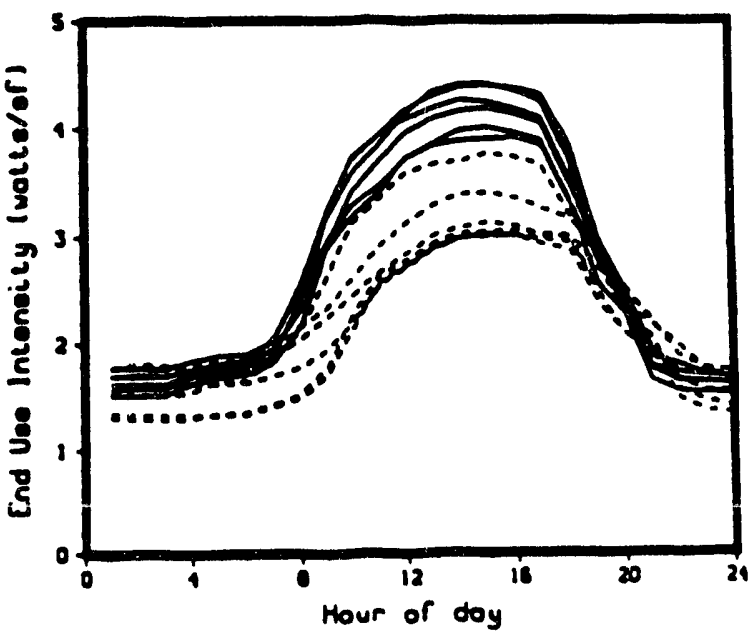




\section{Figure II.24b \\ Large Retail EDA Results}

Solid lines for winter months, dashed lines for summer months

RILLG EDA HVAC on Weekdoys

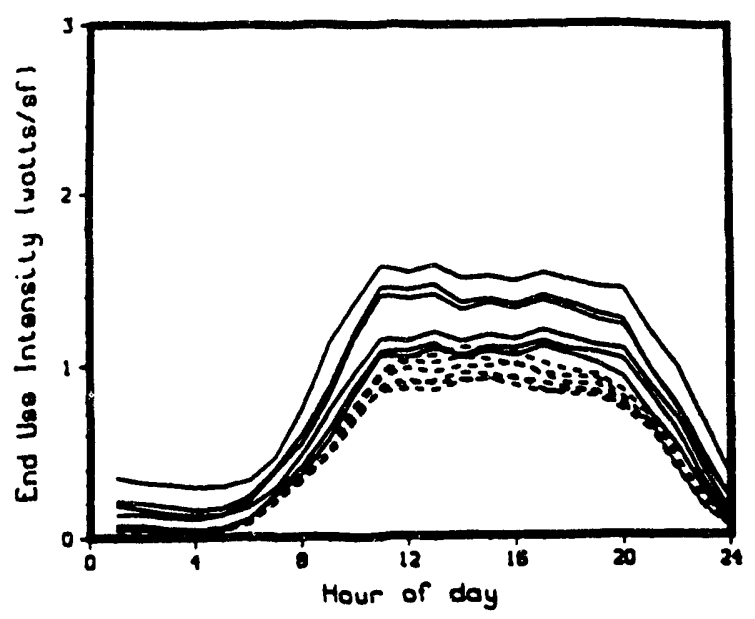

RTLLG EOR Lighting on Wookdoys

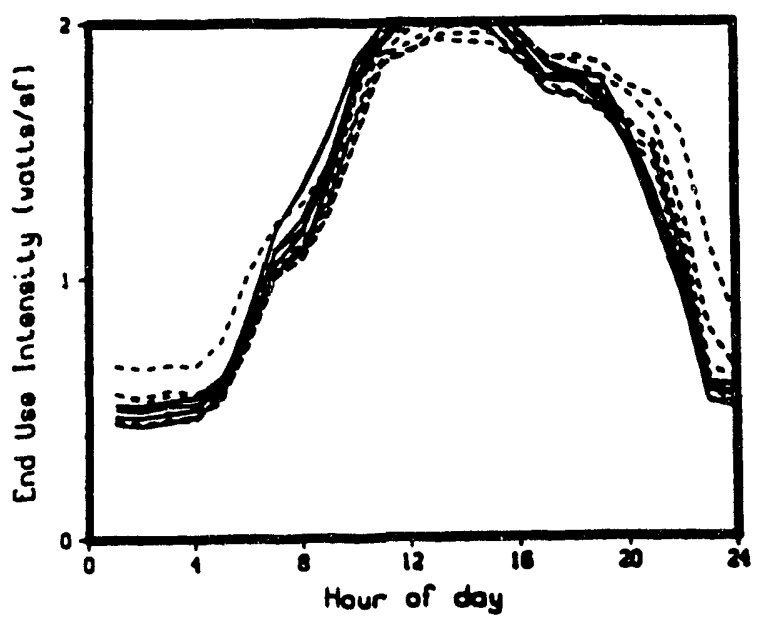

RILLG EOA Misec on Woekdoys

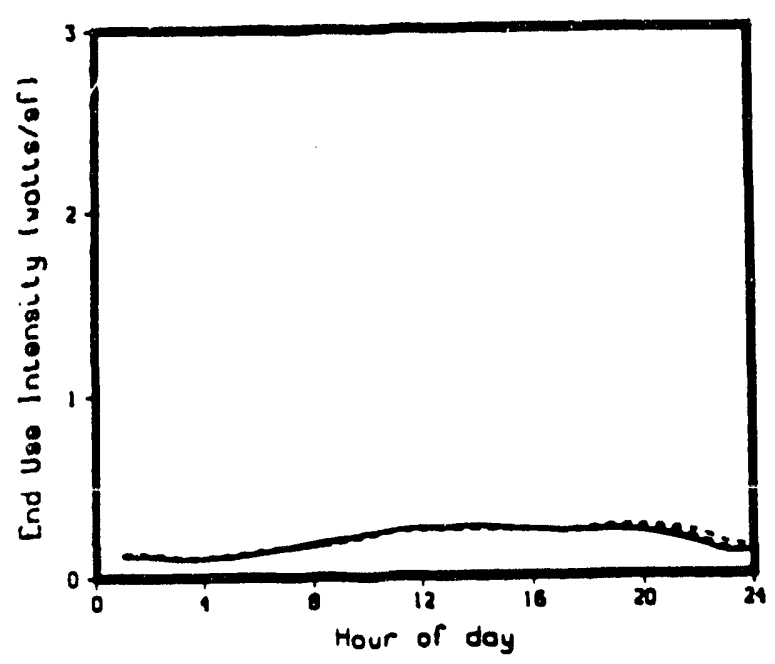

RTLLG EDA HVAC on Weokends

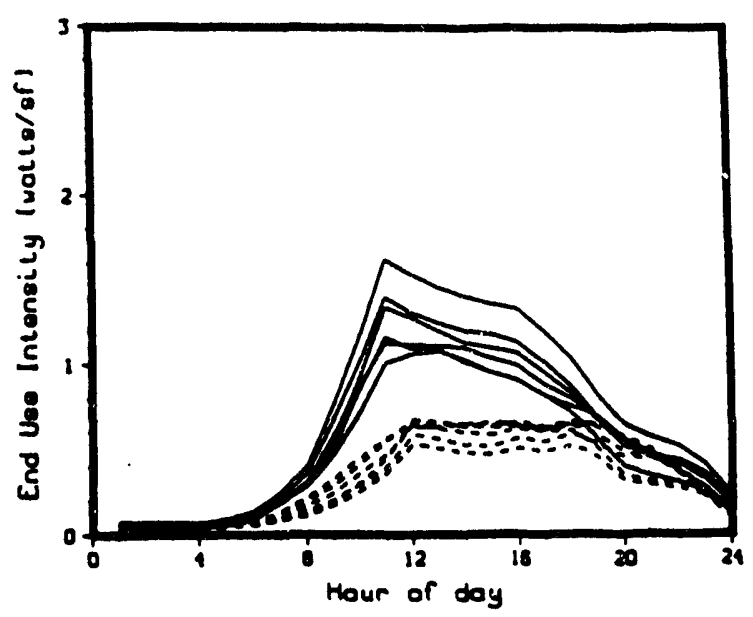

RILLG EDR Lighting on Weokenda

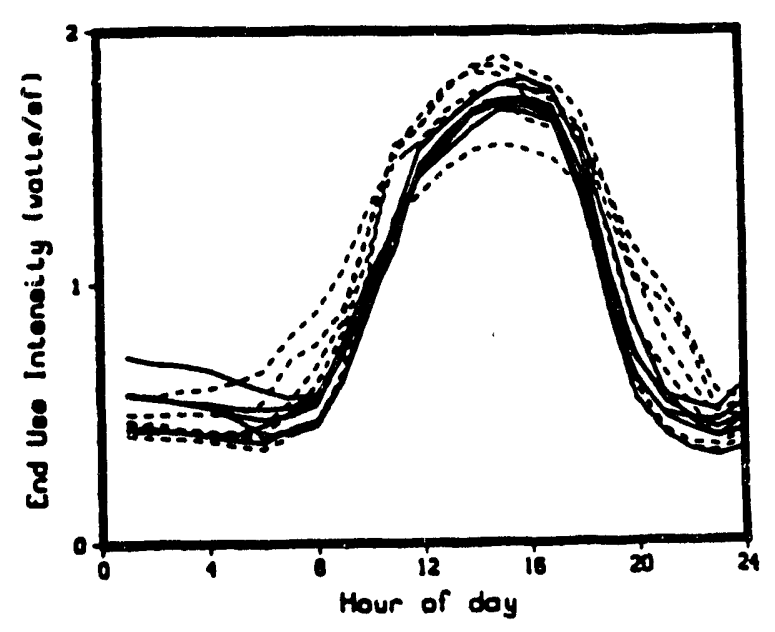

RILLG EDP Mise on Weokends

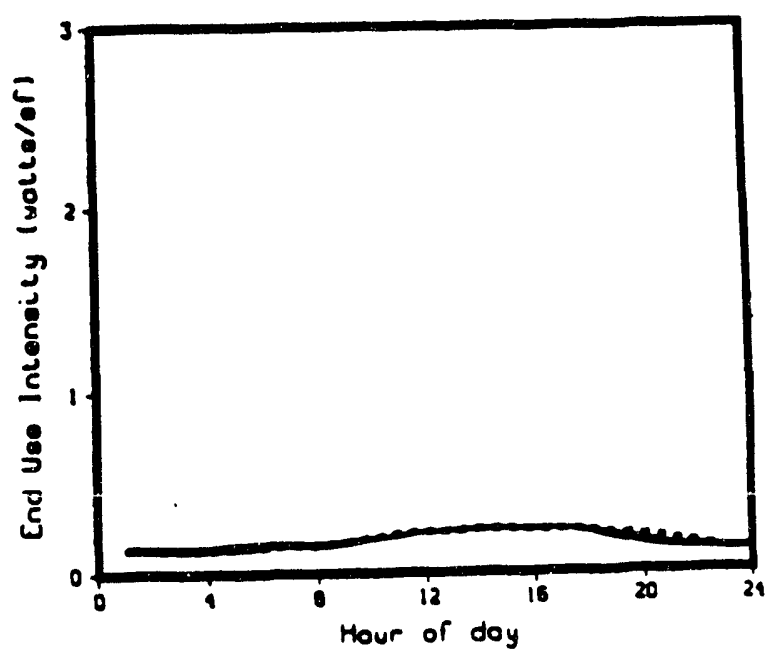


Figure II.24b (continued)

\section{Large Retail EDA Results}

Solid lines for winter months, dashed lines for summer months

RTLLG EDA Tolol on Weakdoys

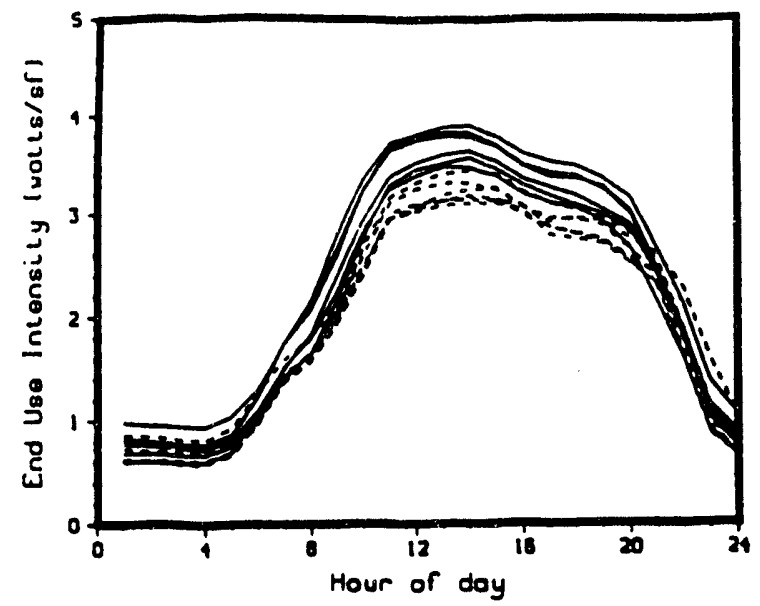

RTLLG ERA Tolol on Weakends

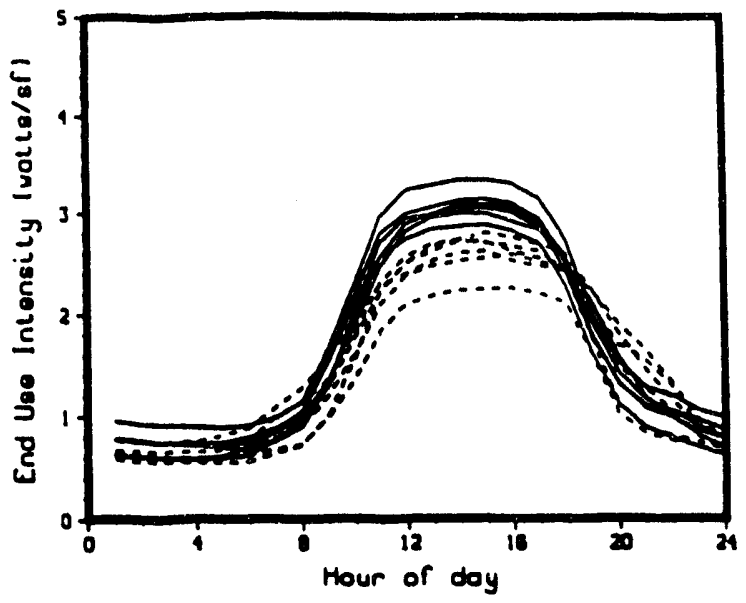




\section{Figure II.25a \\ Small Retail Monitored Data}

Solid lines for winter months, dashed lines for summer months

RTLSM HVAC on Weekdays

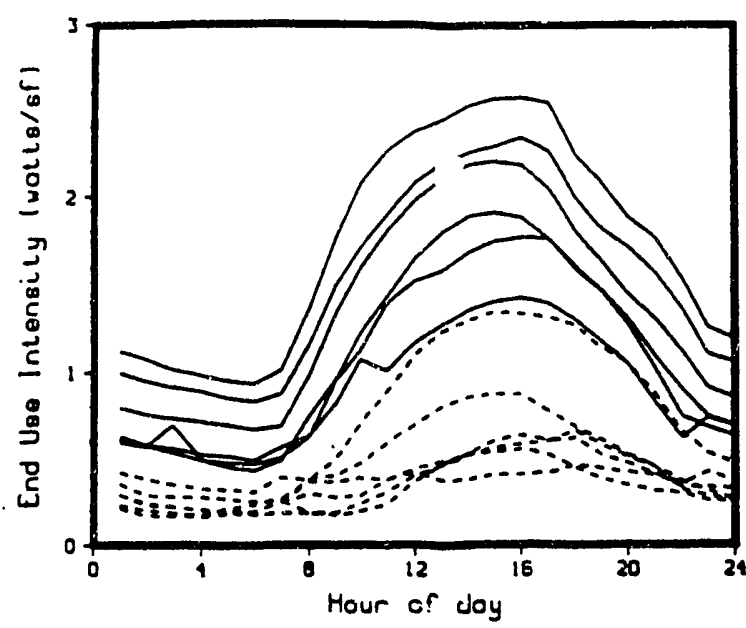

RILSM Light.ing on Weokdoys

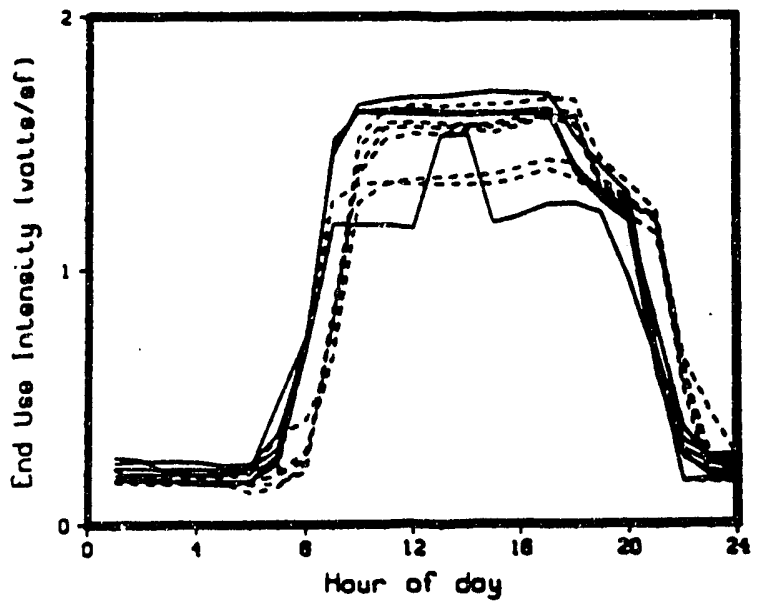

RTLSM Totol on Weokdoys

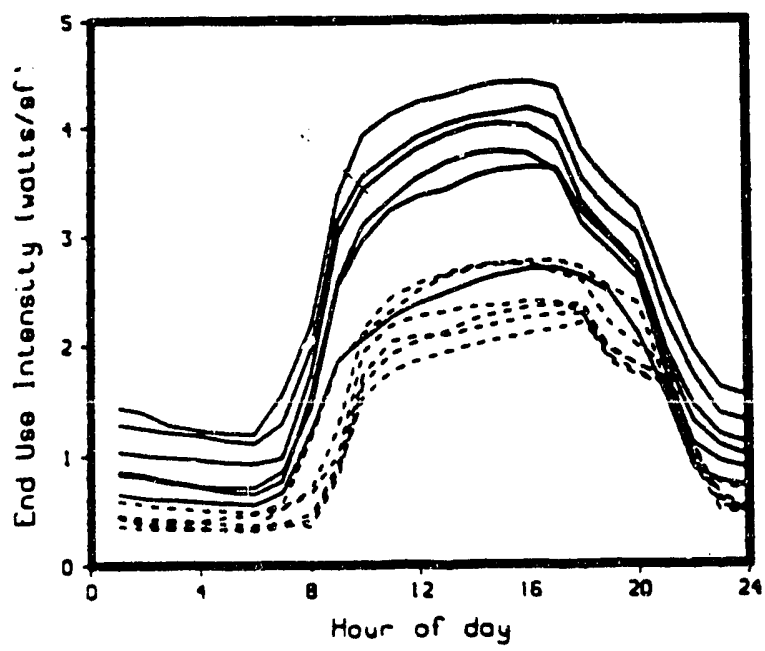

RILSM HVAC on Weokends

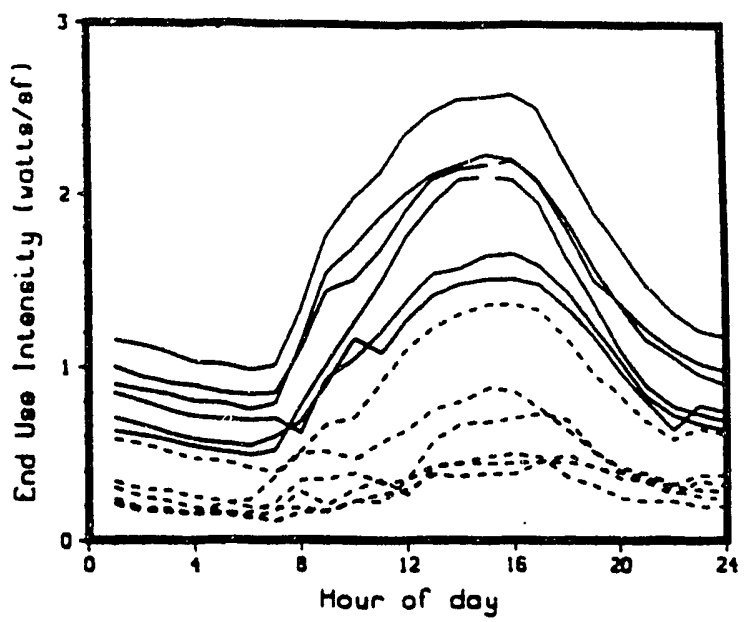

RTLSM Lighting on Weokends

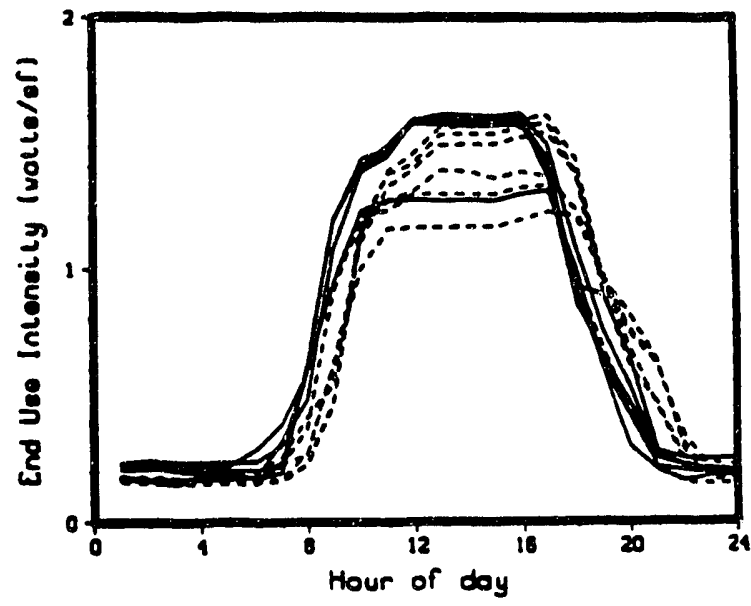

RTLSM Total on Heakends

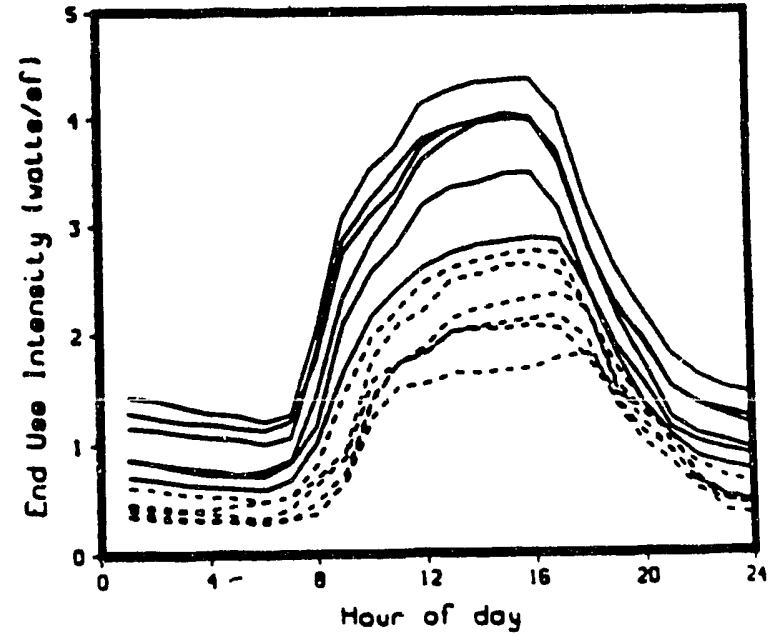




\section{Figure II.25b \\ Small Retail EDA Results}

Solid lines for winter months, dashed lines for summer months

RTLSM EDA HVAC on Weokdoys

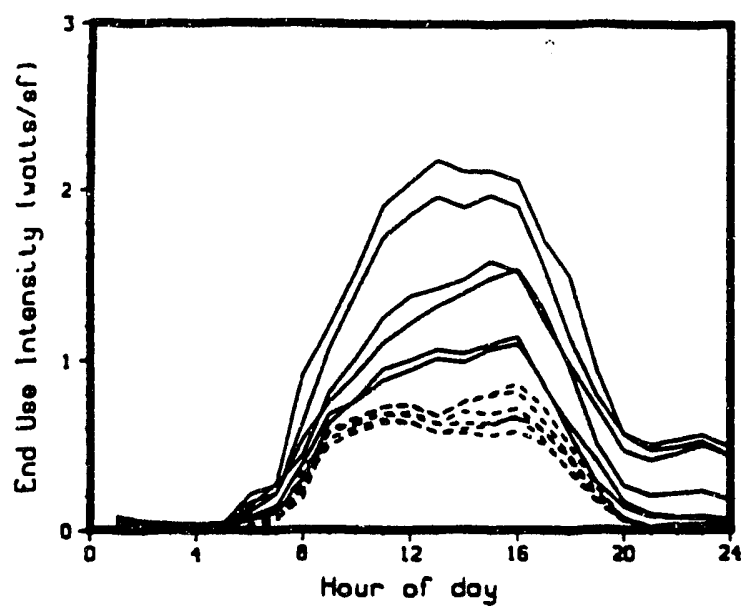

RTLSM EOA Lighting on Woekdoys

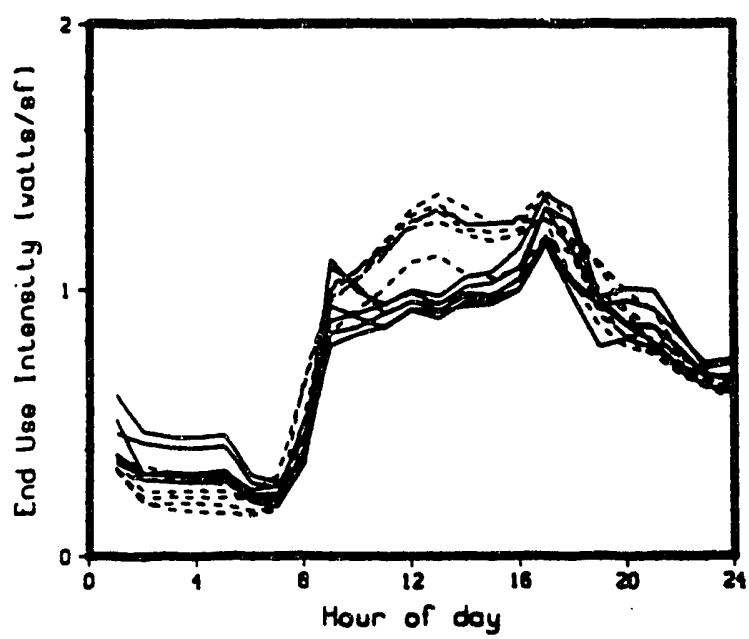

RILSM EDA Misc on Heekdoys

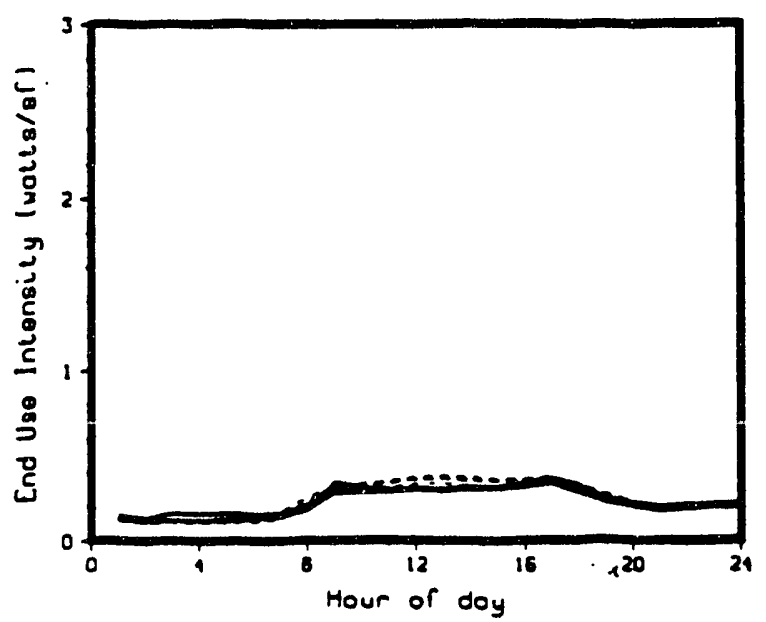

RTLSM EDA HVAC on Weokends

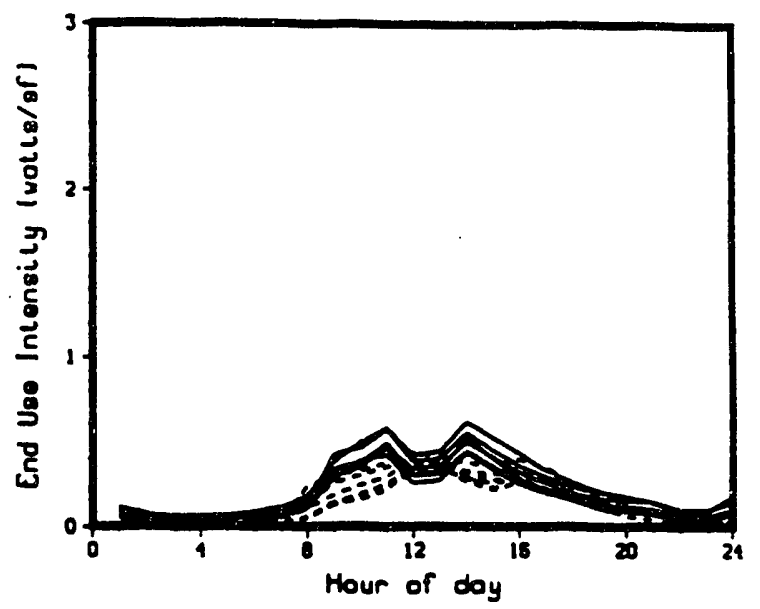

RTLSM EOA Lighting on Weokende

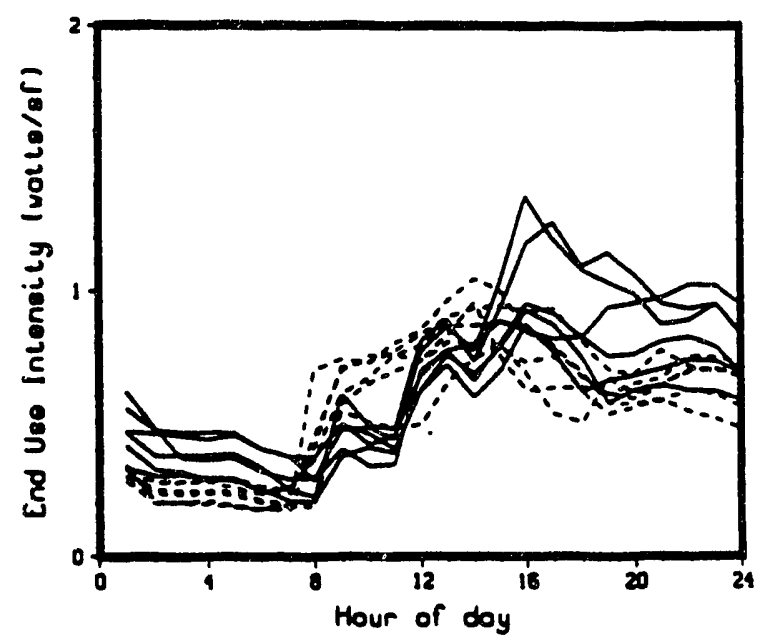

RILSM EOA Misc on Weokends

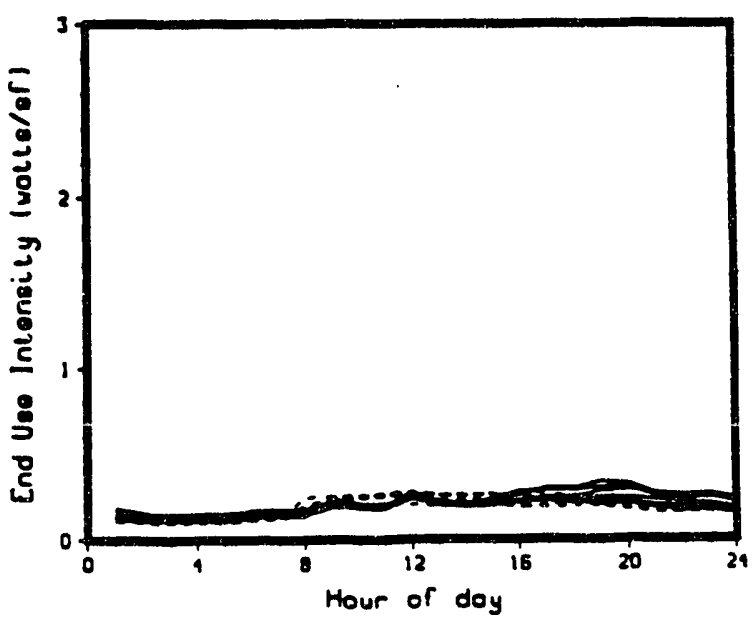


Figure II.25b (continued)

Small Retail EDA Results

Solid lines for winter months, dashed lines for summer months

RTLSM EDA Tolal on Weakdoys

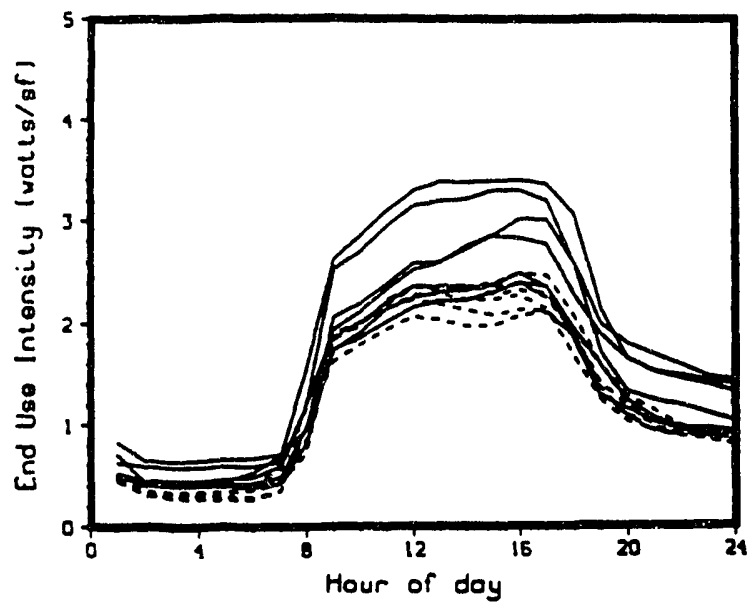

RTLSM EOA Total on Weekends

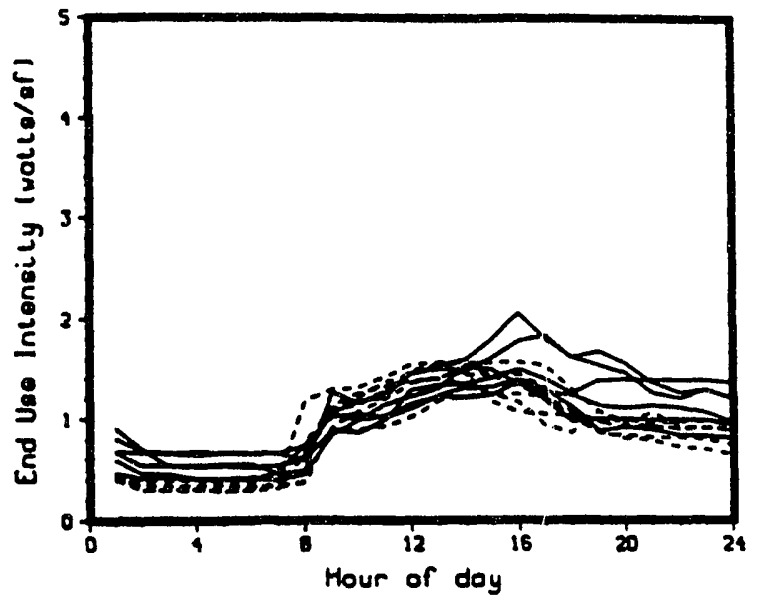




\section{Figure II.26a \\ Grocery Monitored Data}

Solid lines for winter months, dashed lines for summer months

GRY HVAC on Weokdays

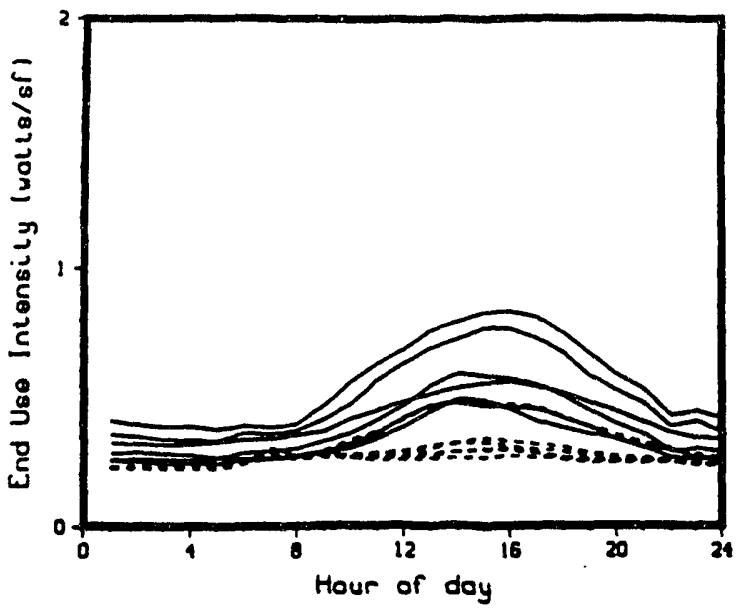

GRY Lighting on Heekdoys

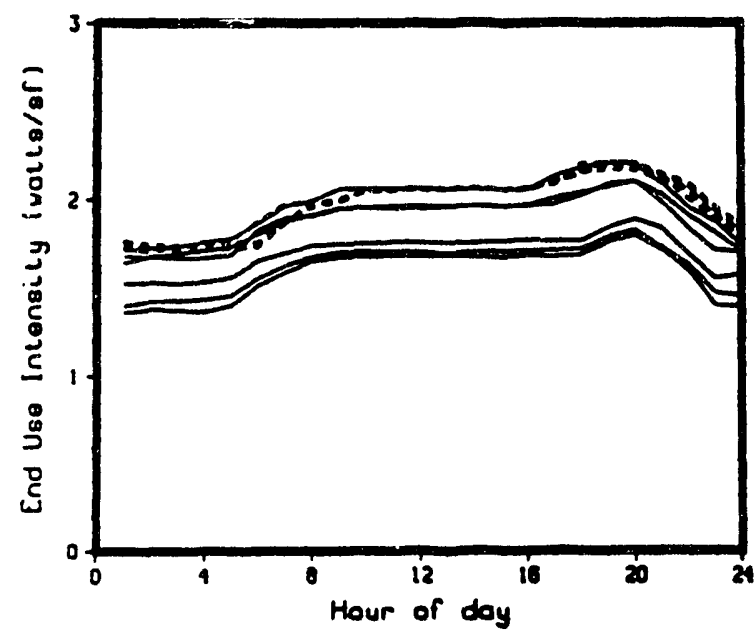

GRY Refrig on Heakdoys

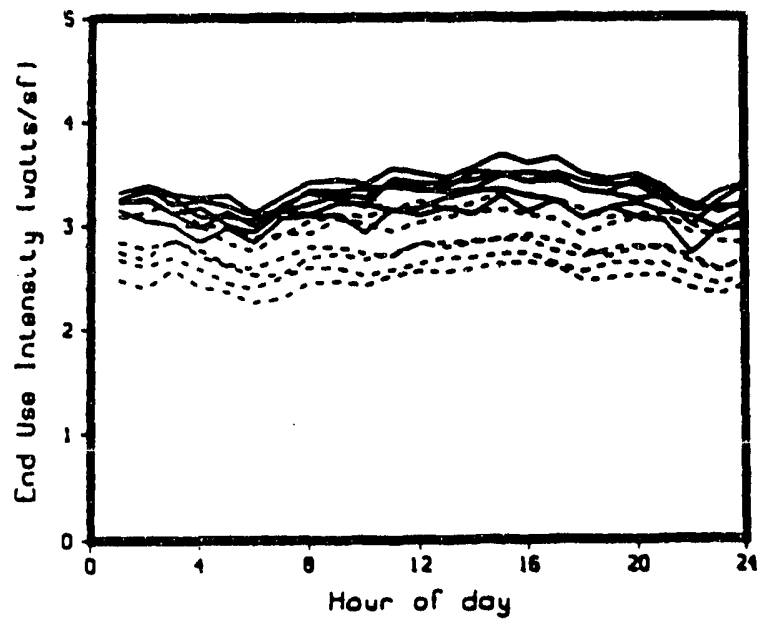

GRY HVAC on Weakends

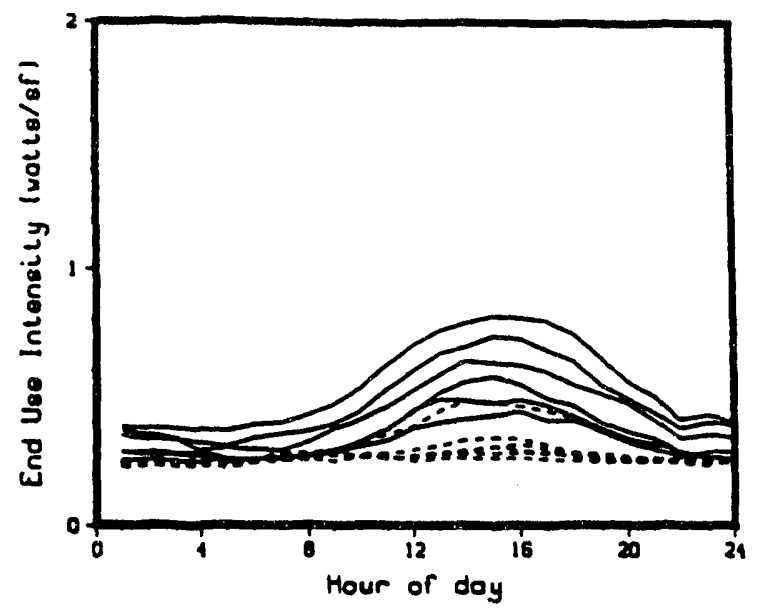

GRY Lighting on Wookends

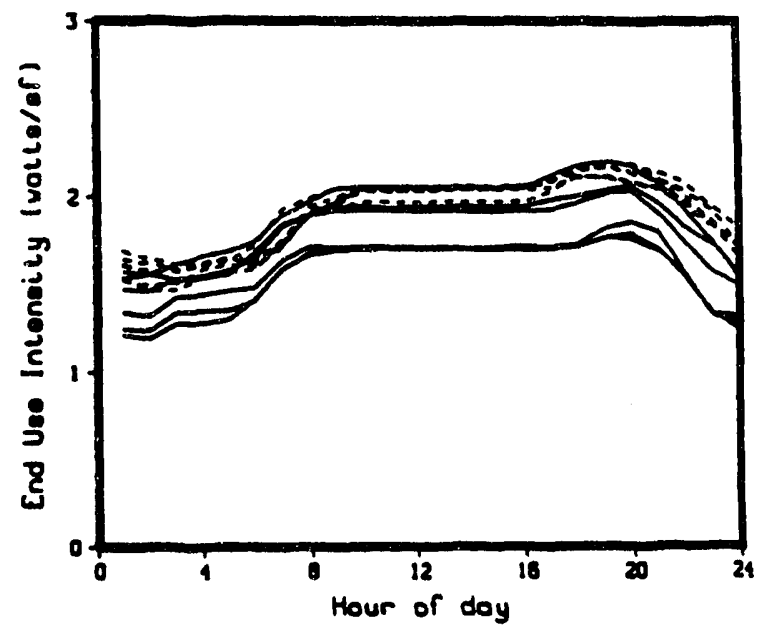

CRY Refrig on Weakends

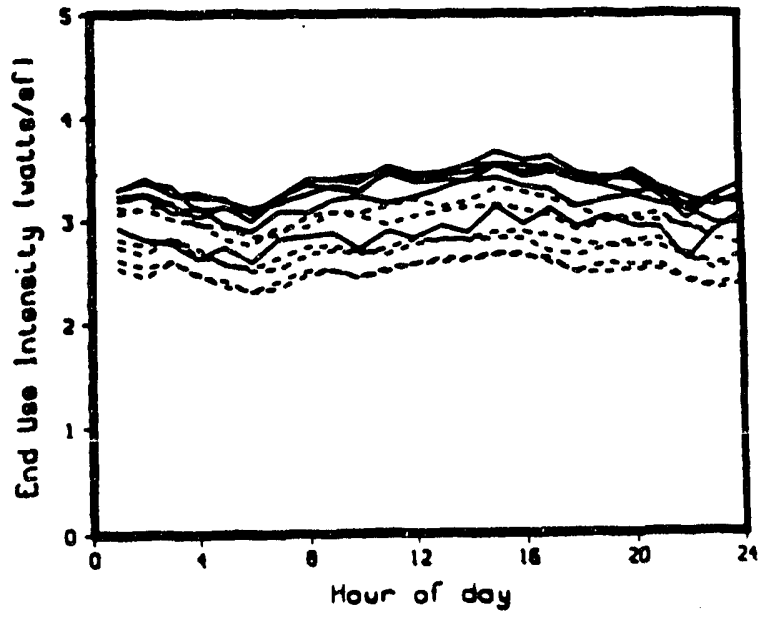


Figure II.26a (continued)

\section{Grocery Monitored Data}

Solid lines for winter months, dashed lines for summer months

GRY Total on Weekdays

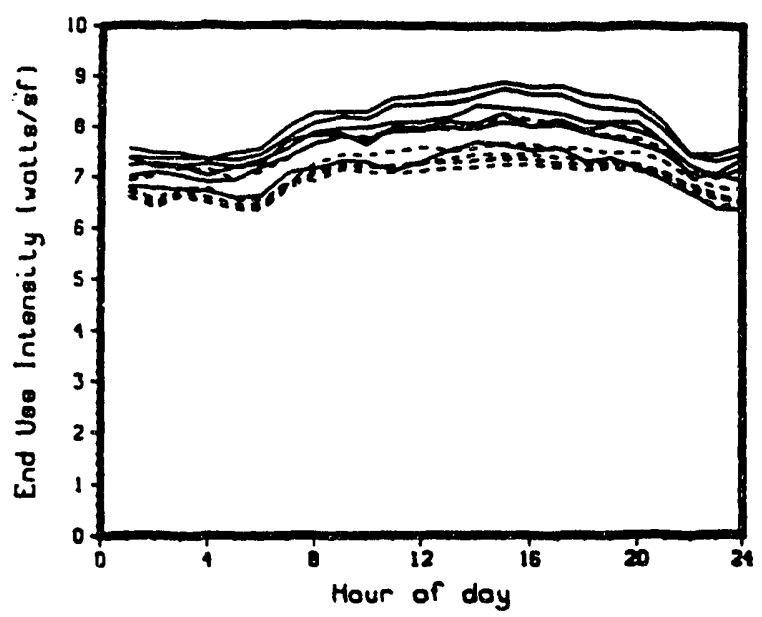

GRY Tolal on Heekends

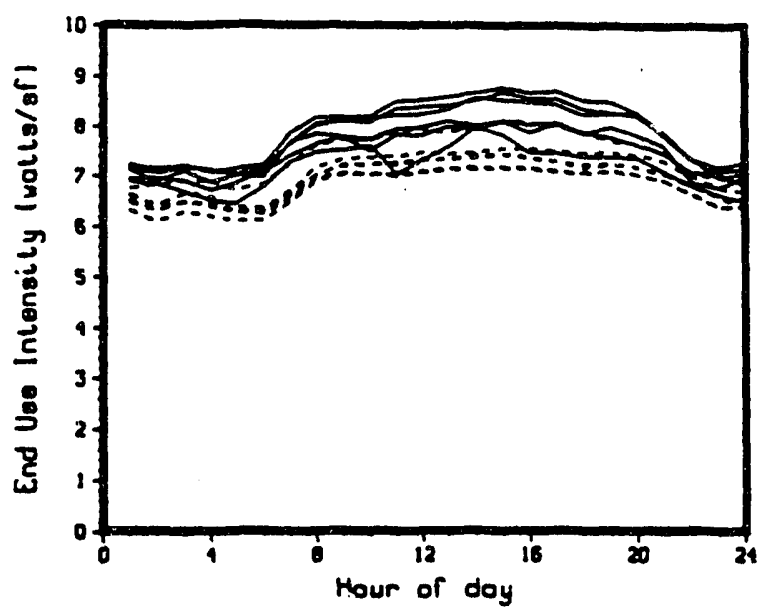




\section{Figure II.26b \\ Grocery EDA Results}

Solid lines for winter months, dashed lines for summer months

GRY EDA HVAC on Weokdoys

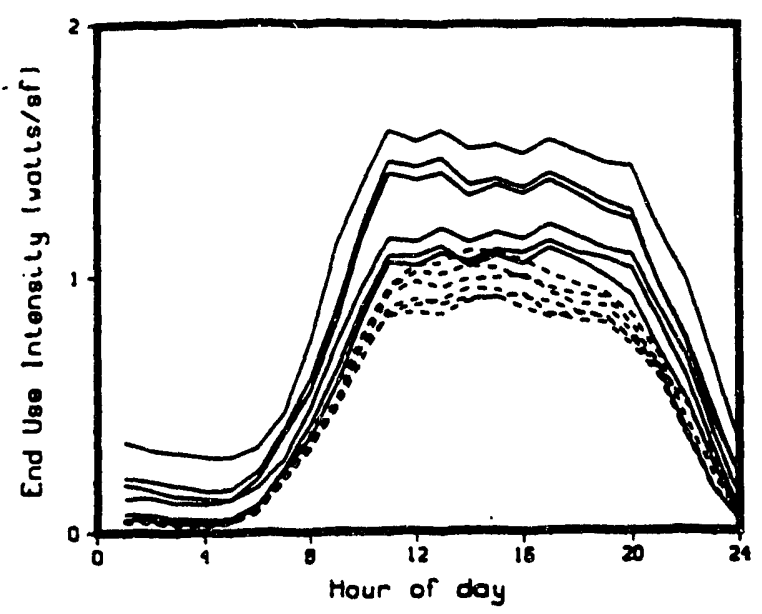

GRY EDR Lighting on Heokdoys

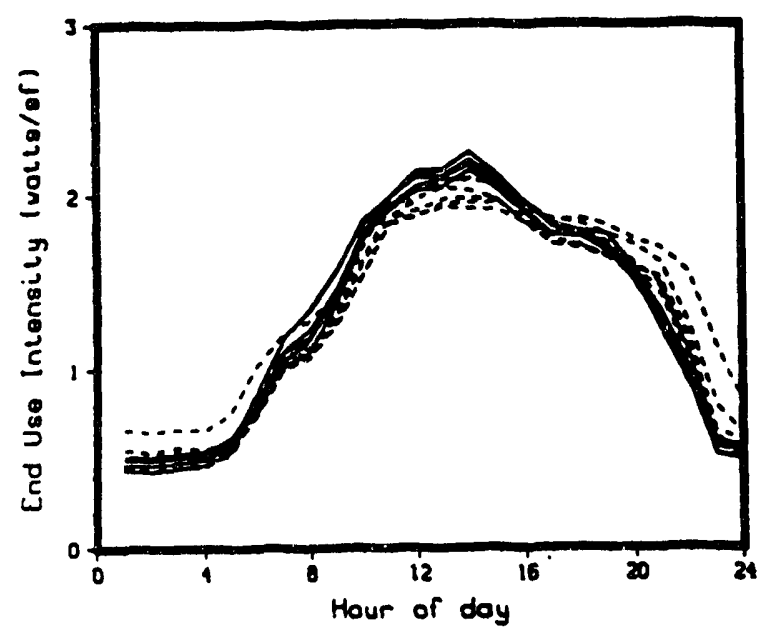

GRY EDR Misc on Heekdoys

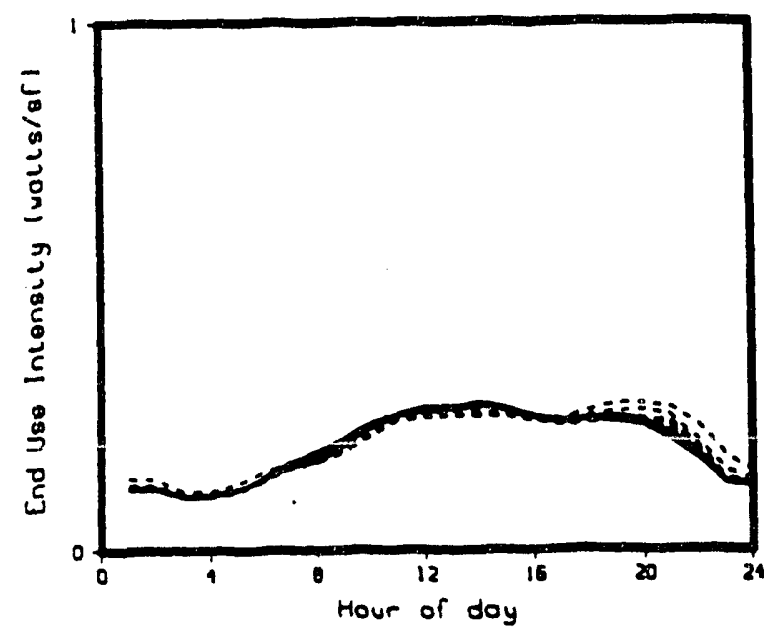

GRY EDR HVAC on Woekends

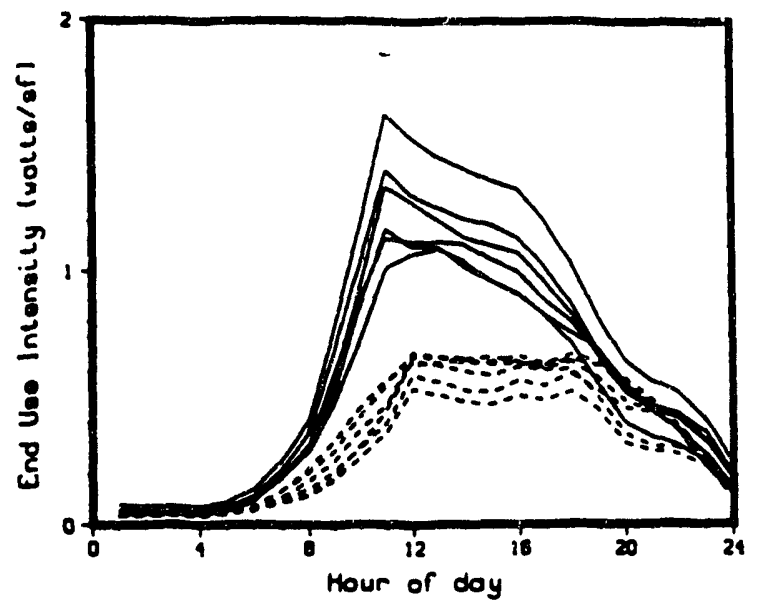

GRY EDA Lighting on Heckends

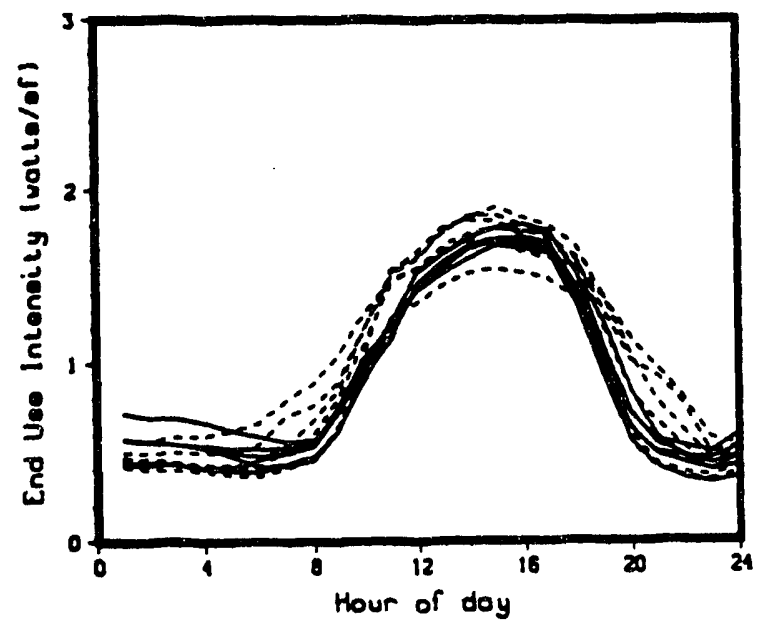

GRY EOR Misc on Weokends

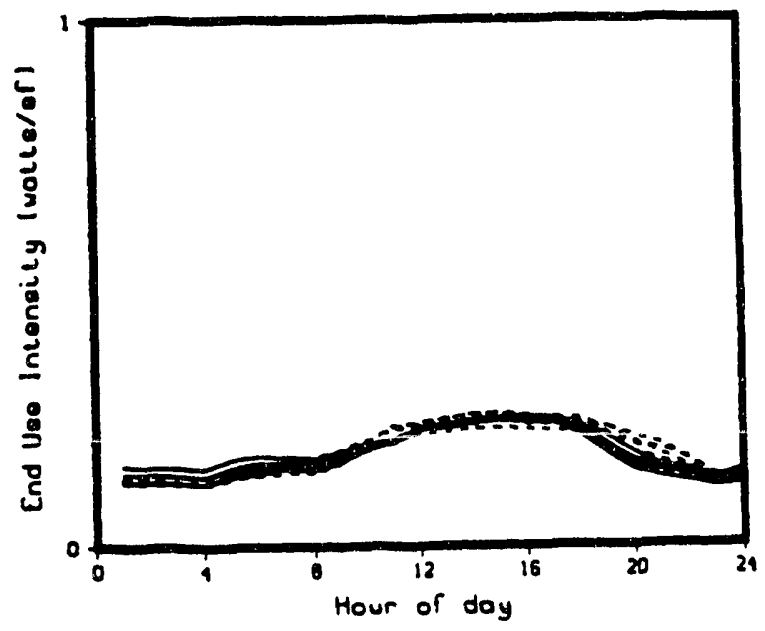


Figure II.26b (continued)

\section{Grocery EDA Results}

Solid lines for winter months, dashed lines for summer months

GRY EDA Tolal on Weekdoys

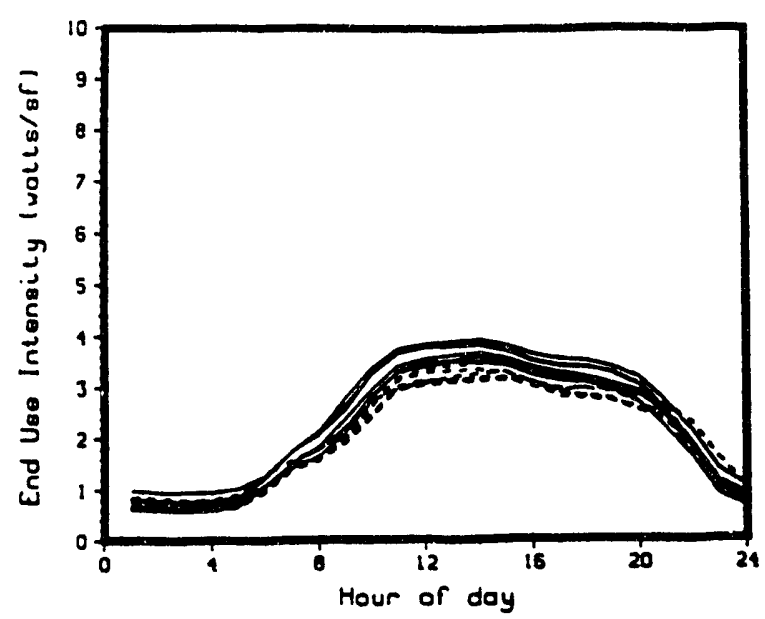

GRY EDR Total on Weokends

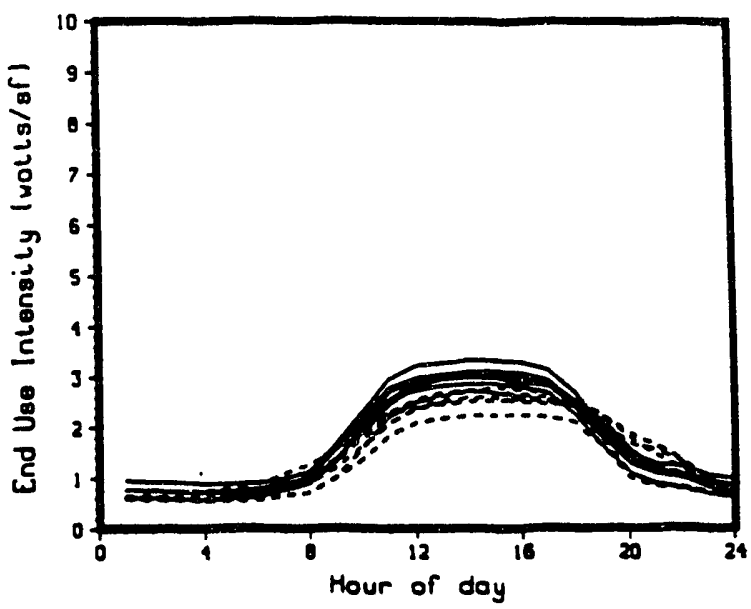


Figure II.27a.1

Sit-Down Restaurant Monitored Data

Solid lines for winter months, dashed lines for summer months

RSTSD HVAC on Weokdoys

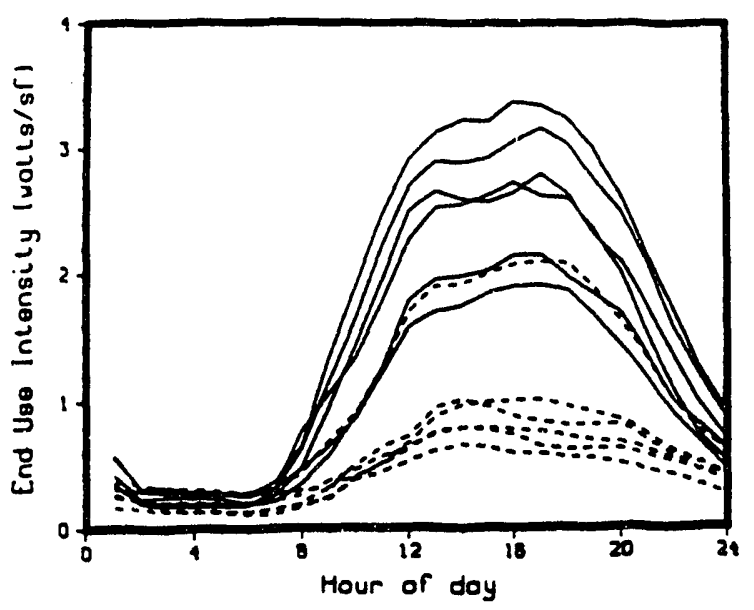

RSTSD Lighting on Weokdays

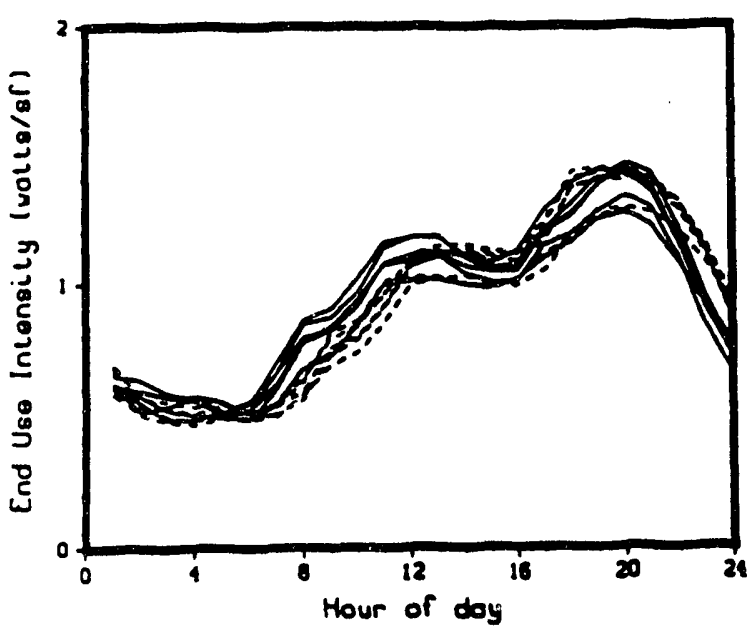

RSTSD Cooking on Hookdoys

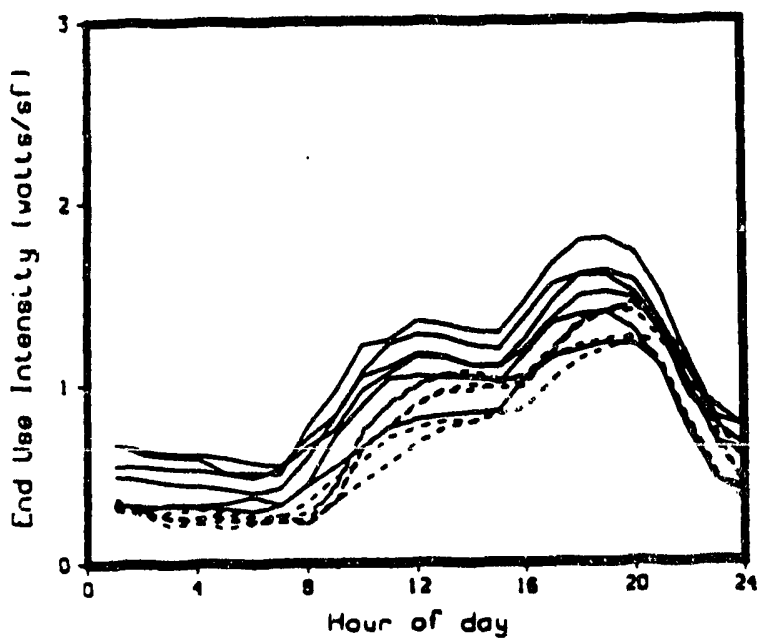

RSTSD HVAC on Wookends

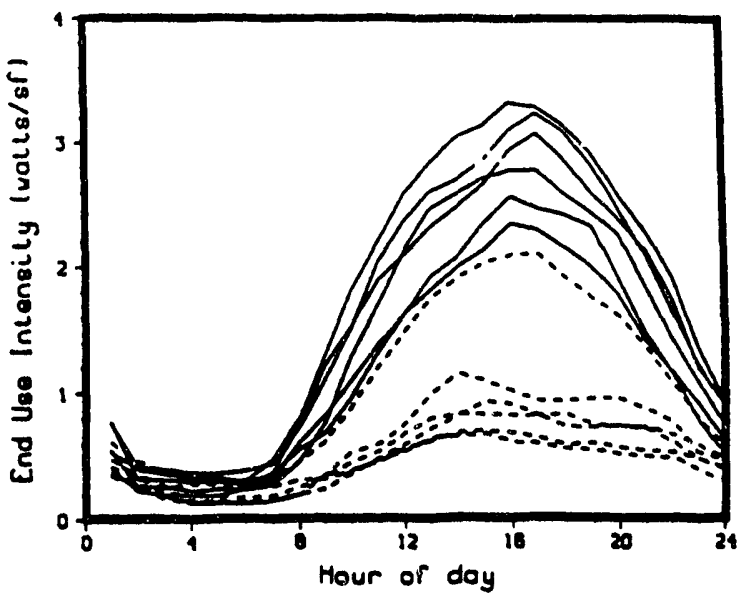

RSTSD Lighting on Weekends

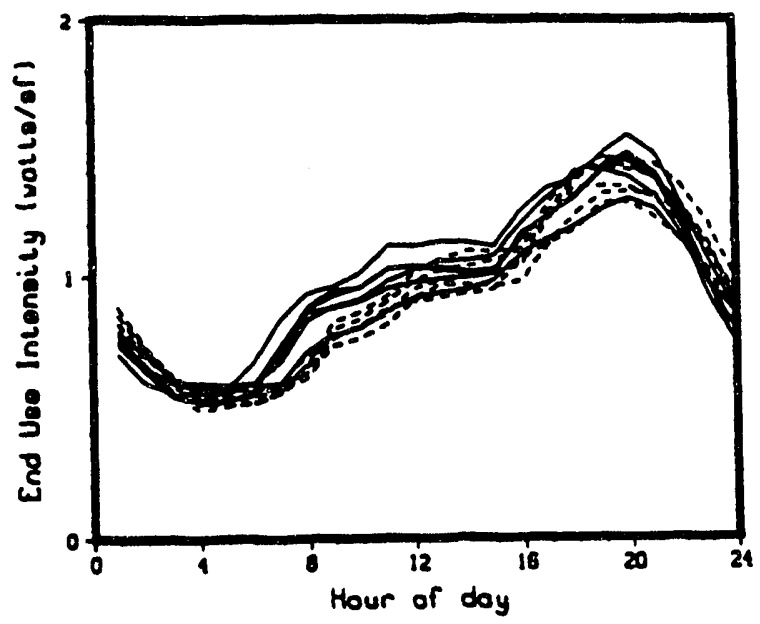

RSTSD Cooking on Weokends

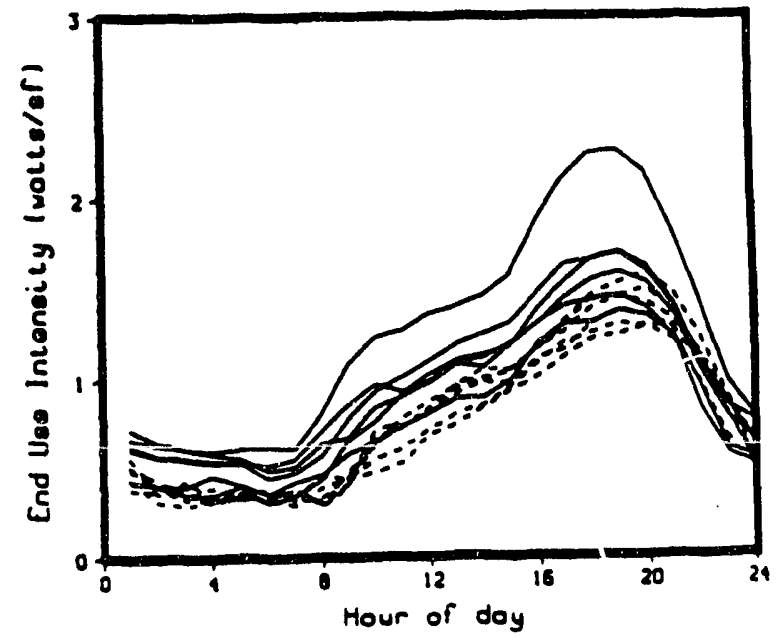


Figure II.27a.1 (continued)

Sit-Down Restaurant Monitored Data

Solid lines for winter months, dashed lines for summer months

RSTSO Exhaust-fan on Weokdays

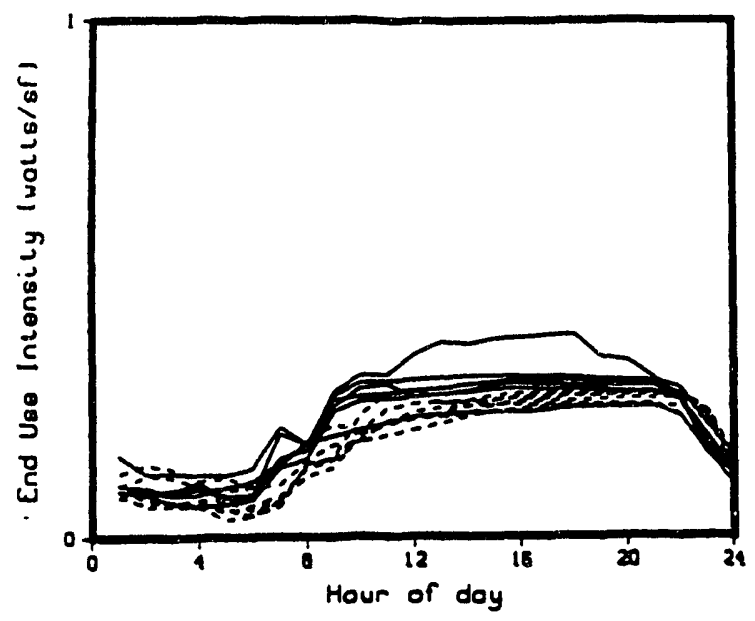

RSTSO Refrig on Weekdoys

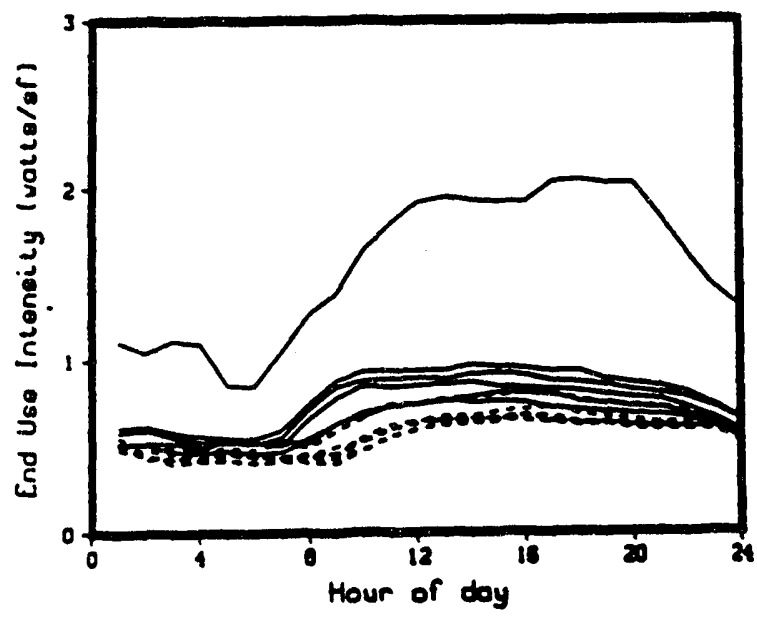

RSTSD Totol on Hookdoye

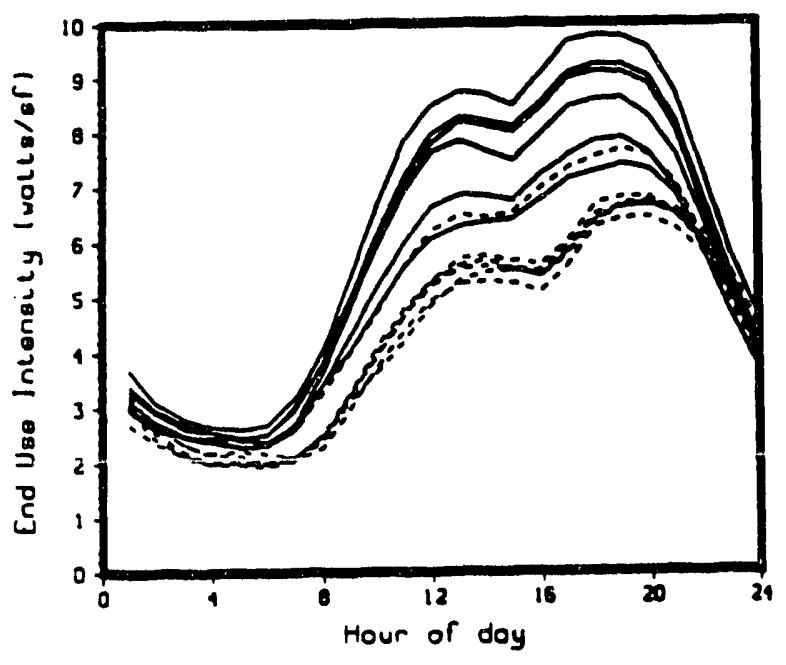

RSTSD Exhoust-fon on Weokends

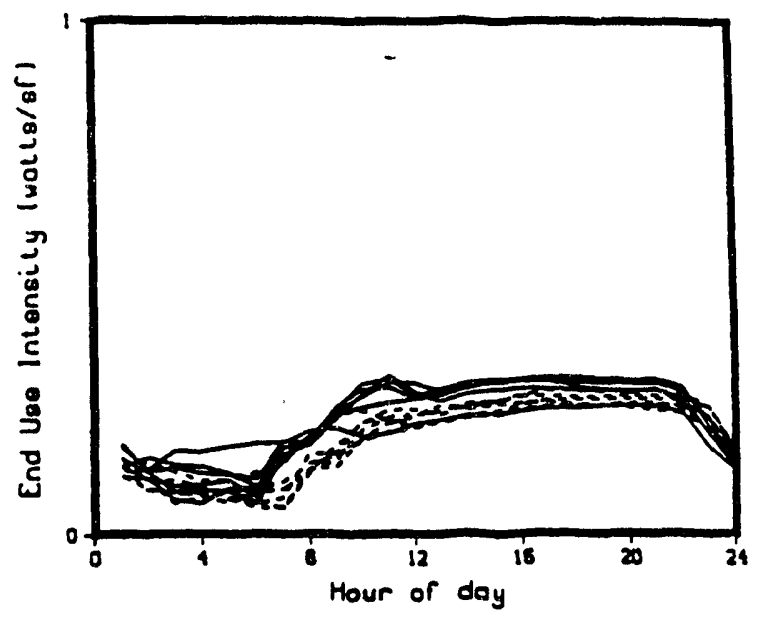

RSTSO Refrig on Weokends

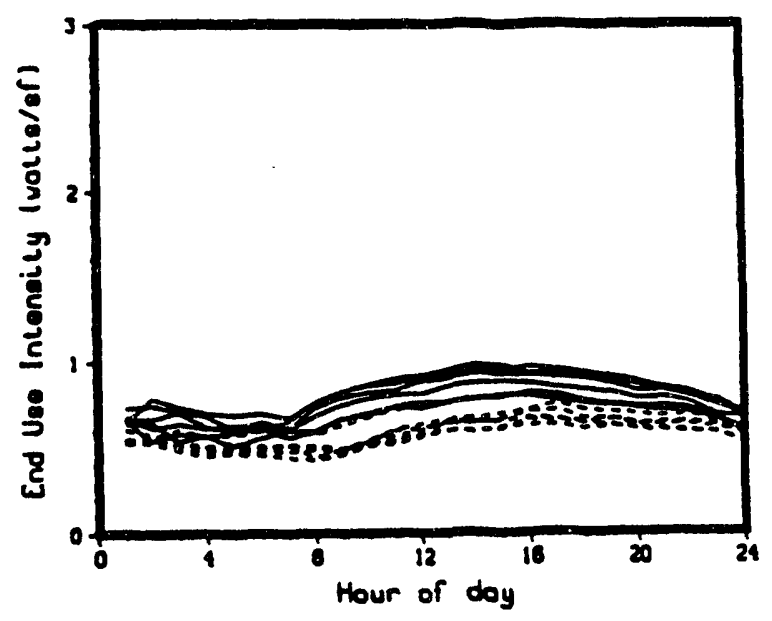

RSTSO Total on Heokends

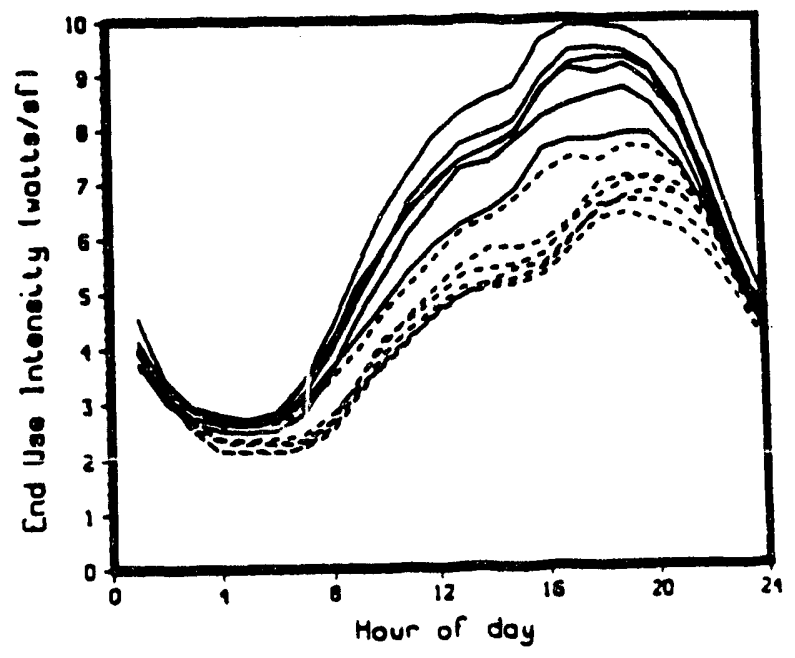


Figure II.27a.2

Fast-food Restaurant Monitored Data Solid lines for winter months, dashed lines for summer months

RSTFF HVAC on Weokdoys

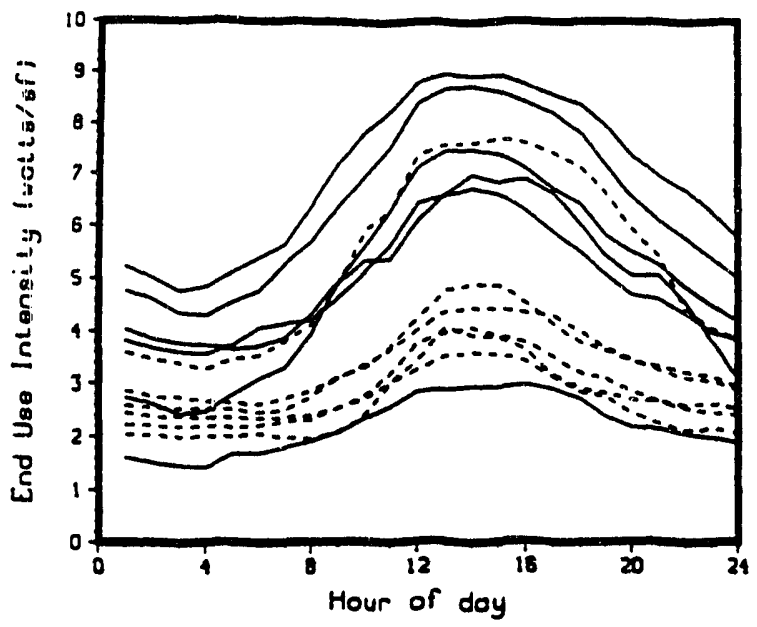

RSTFF Lighting on Heokdoys

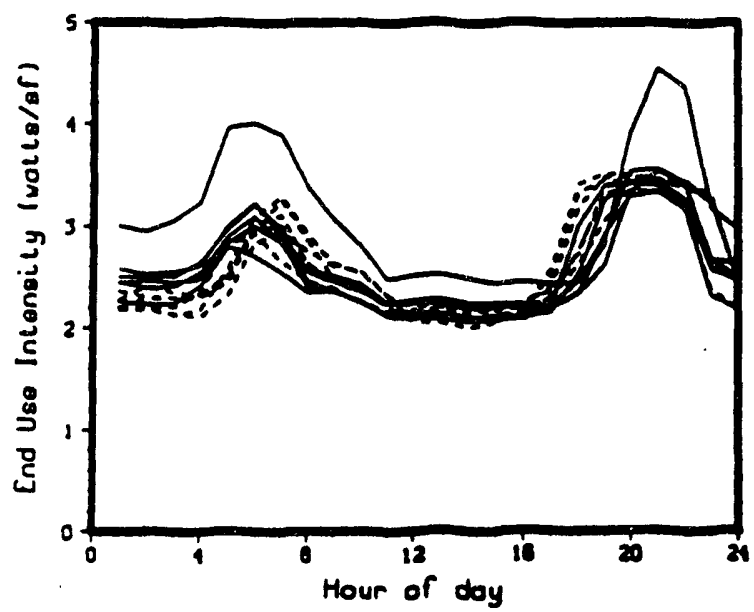

RSTFF Cooking on Wookdoys

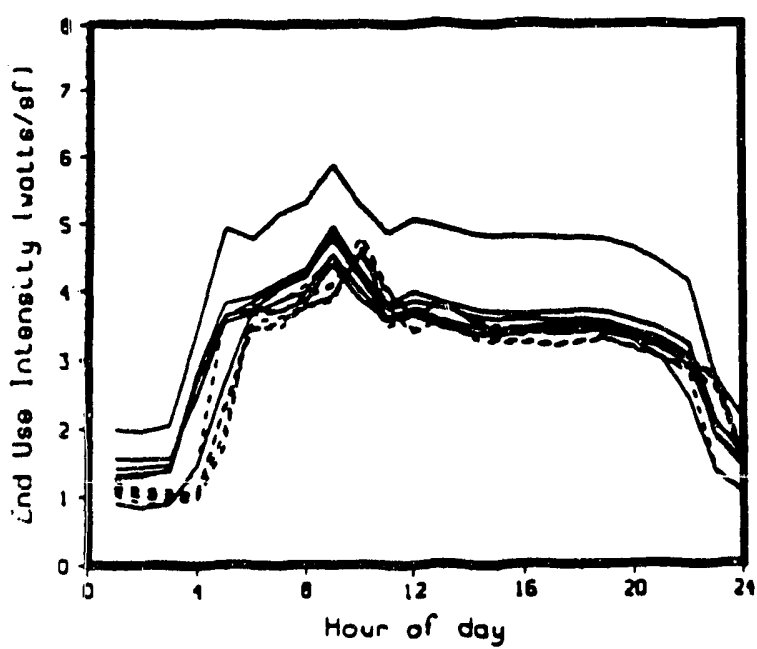

RSTFF HVAC on Weekends

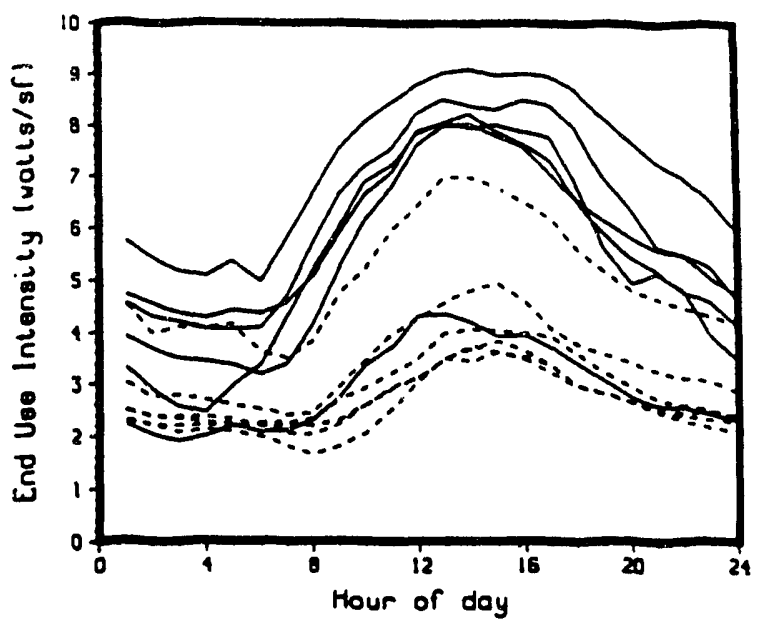

RSTFF Lighting on Weokends

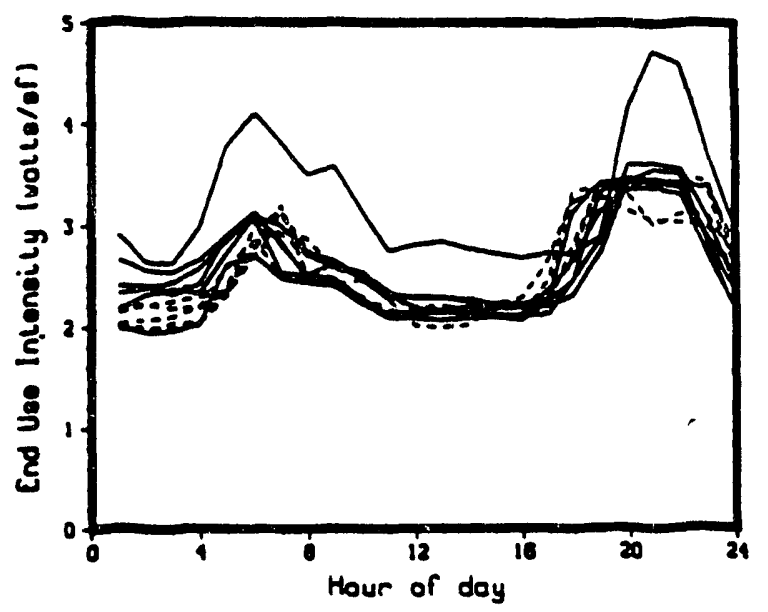

RSTFF Cooking on Weekends

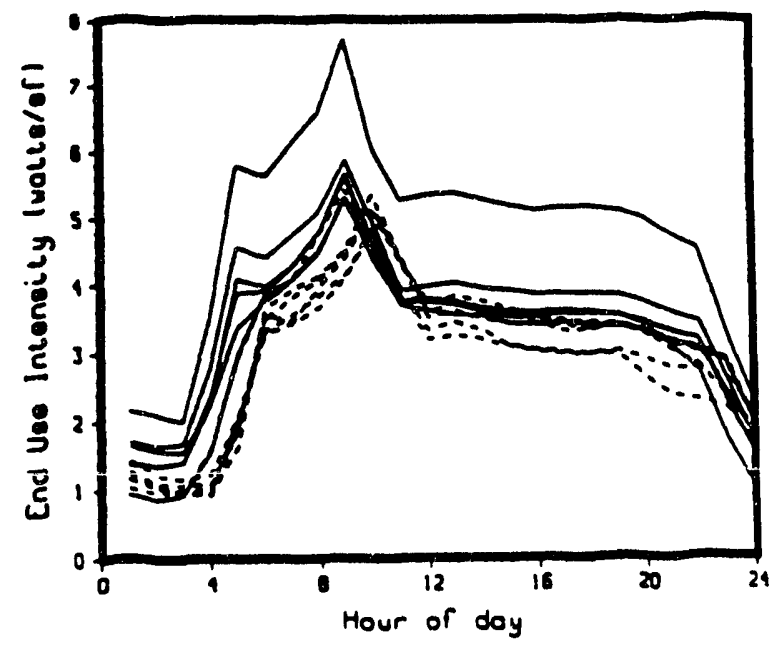


Figure II.27a.2 (continued)

Fast-food Restaurant Monitored Data

Solid lines for winter months, dashed lines for summer months

RSTFF Exhoust-fan on Weokdoys

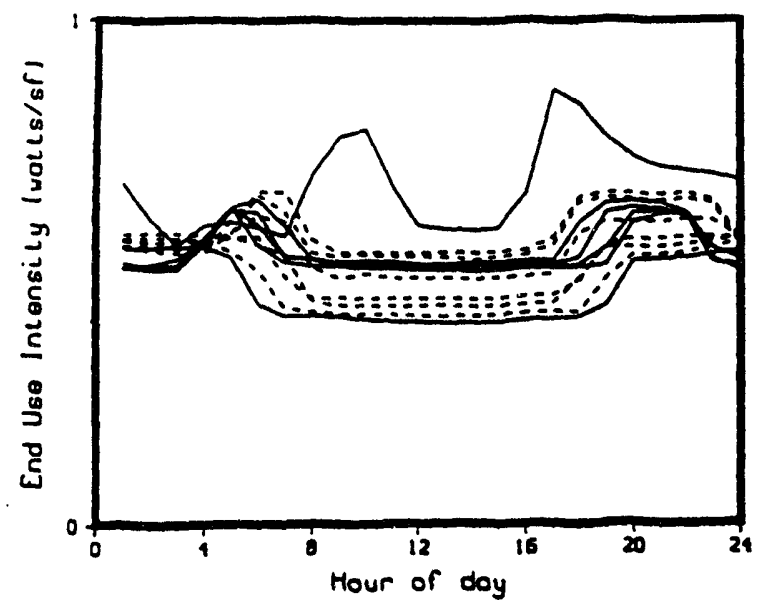

RSIfF Rofrig on Woekdoys

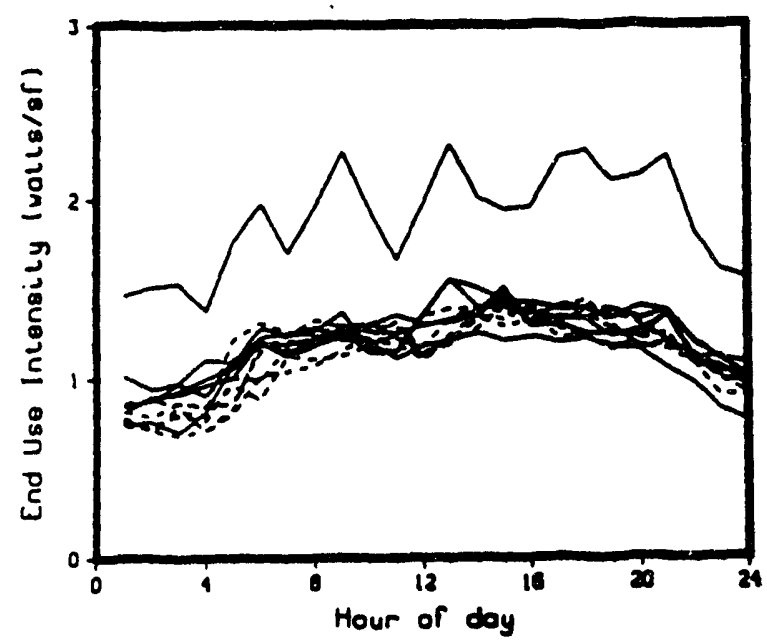

RSTFF Tolal on Heckdoys

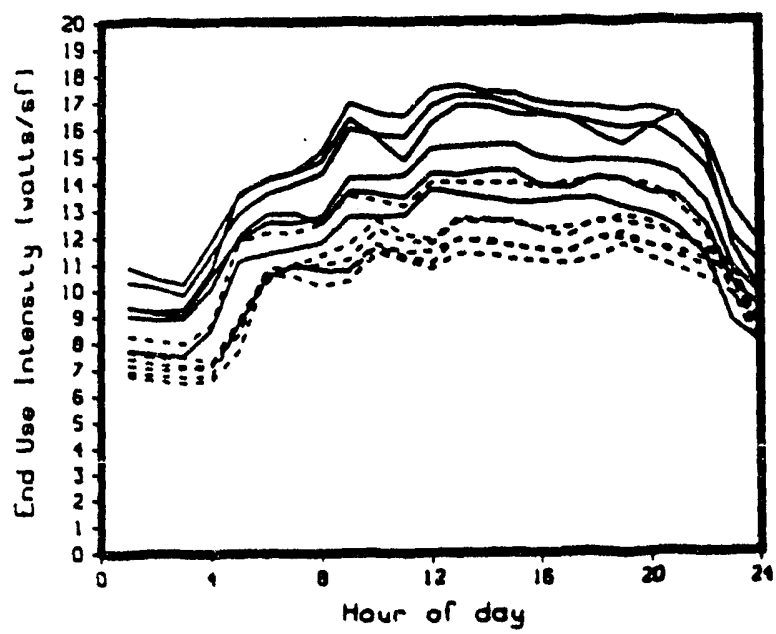

RSTFF Exhoust-f an on Weokends

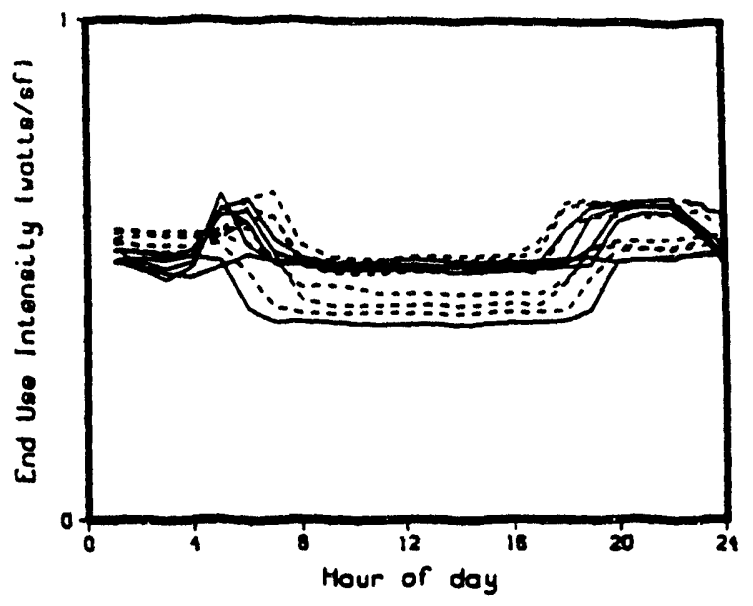

RSTFF Refrig on Woekends

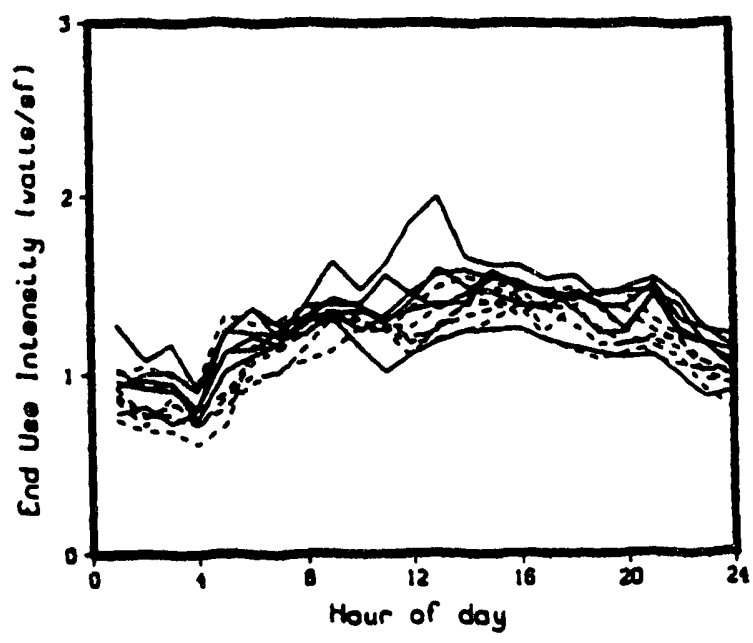

RSTFF Totol on Weekends

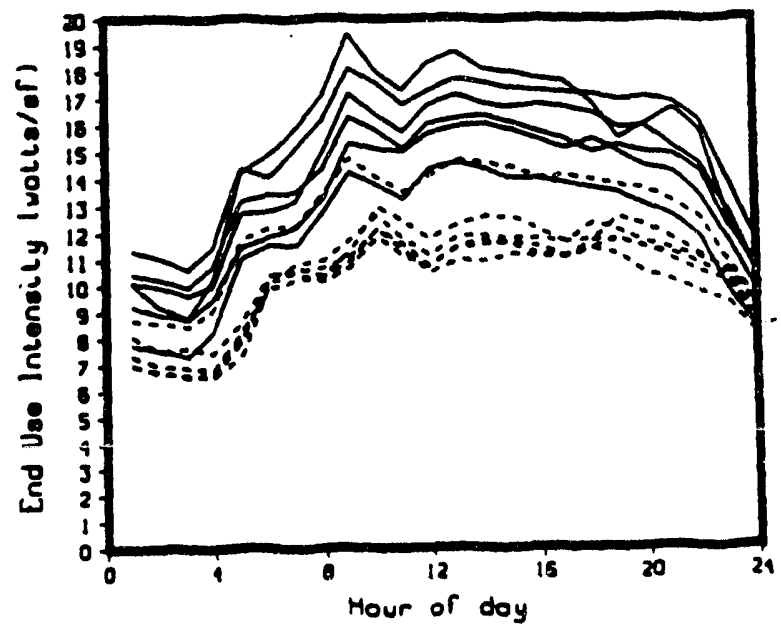


Figure II.27a

Combined Restaurant Monitored Data

Solid lines for winter months, dashed lines for summer months

RST HVAC on Wookdoys

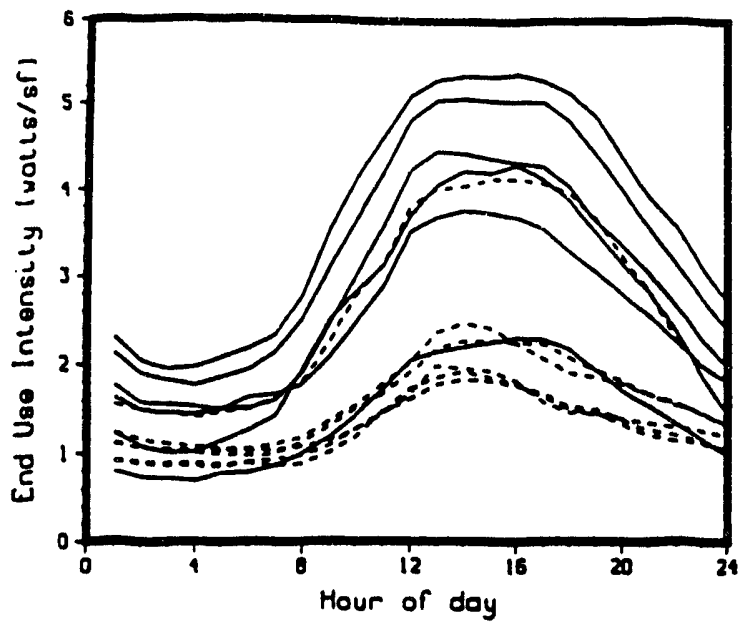

RST Lighting on Weekdays

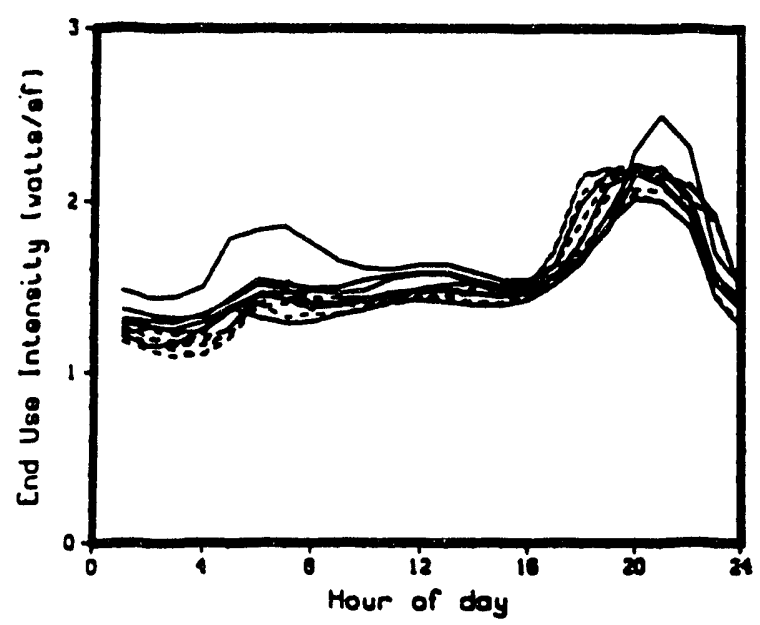

RST Cooking on Heekdoys

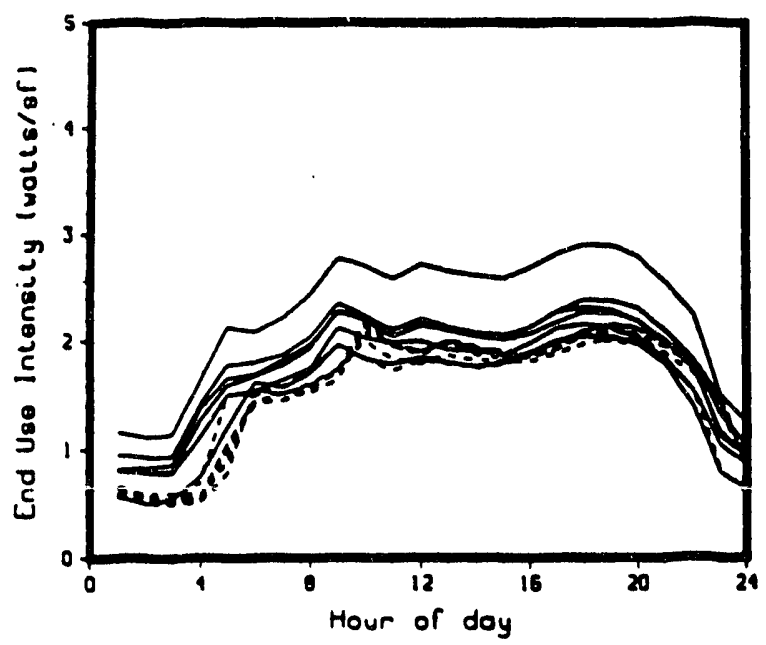

RST HVAC on Woekends

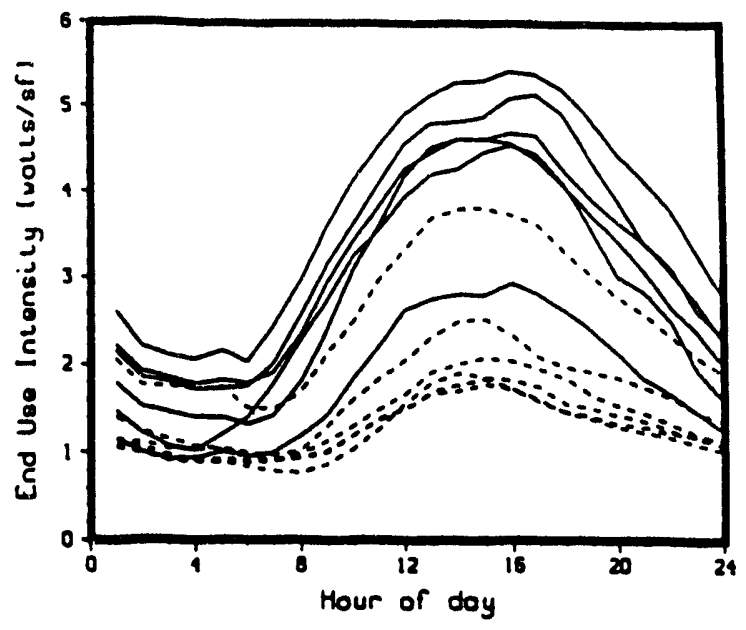

RST Lighting on Weokends

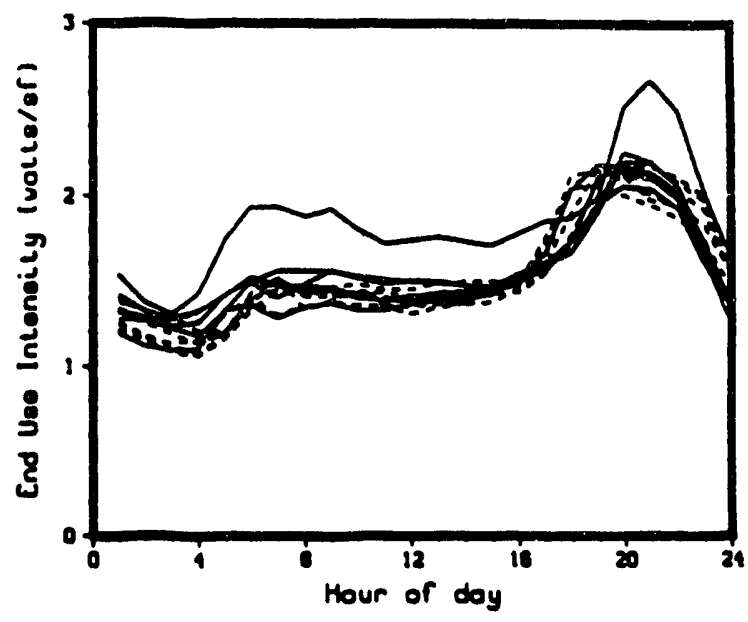

RST Cooking on Weokends

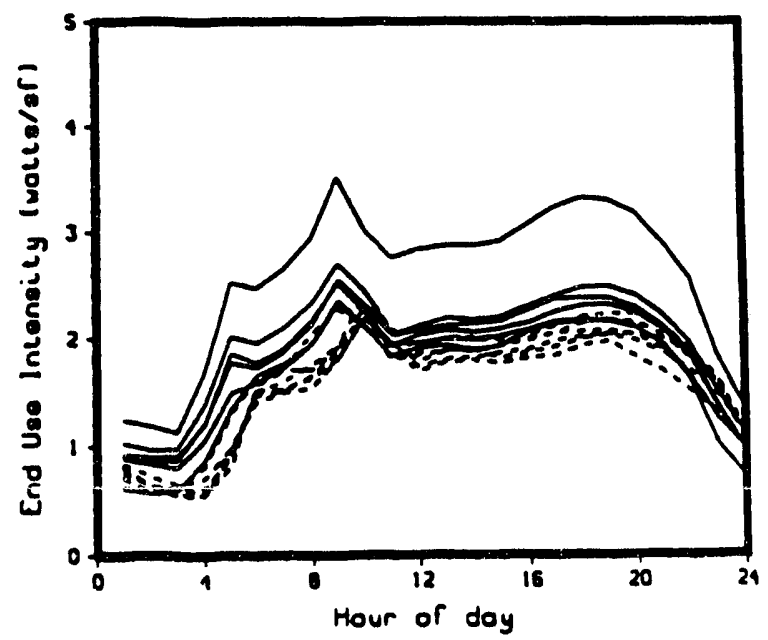




\section{Figure II.27a (continued) \\ Combined Restaurant Monitored Data}

Solid lines for winter months, dashed lines for summer months

RST Exhoust-fan on Wookdays

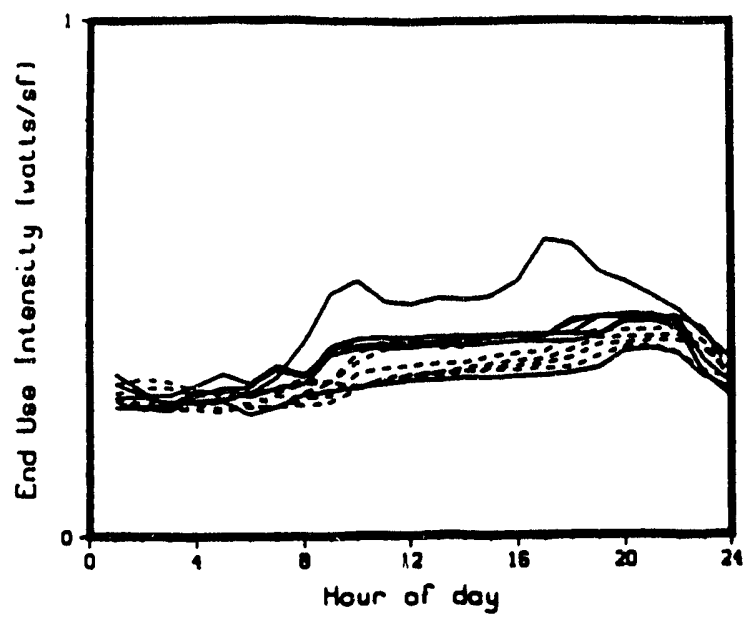

RST Refrig on Weokdoys

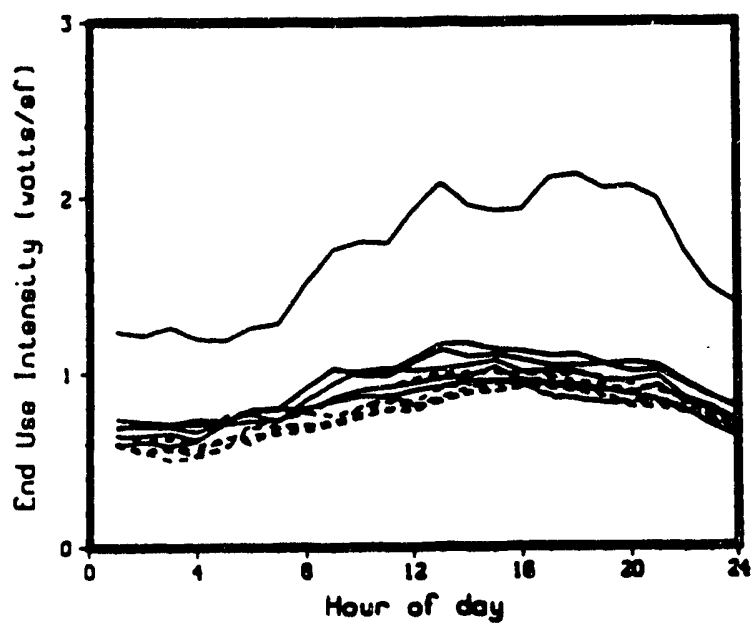

RST Totol on Hechiogye

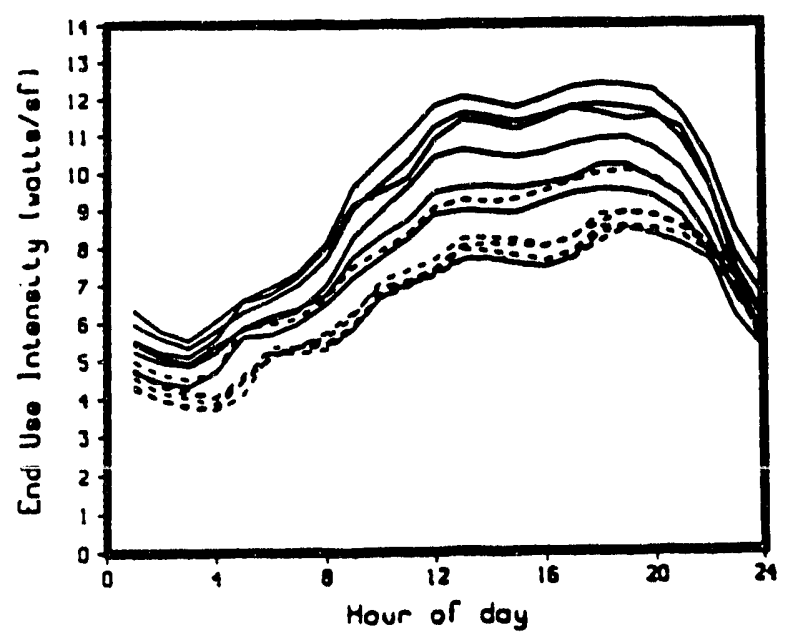

RST Exhoust-fon on Weakends

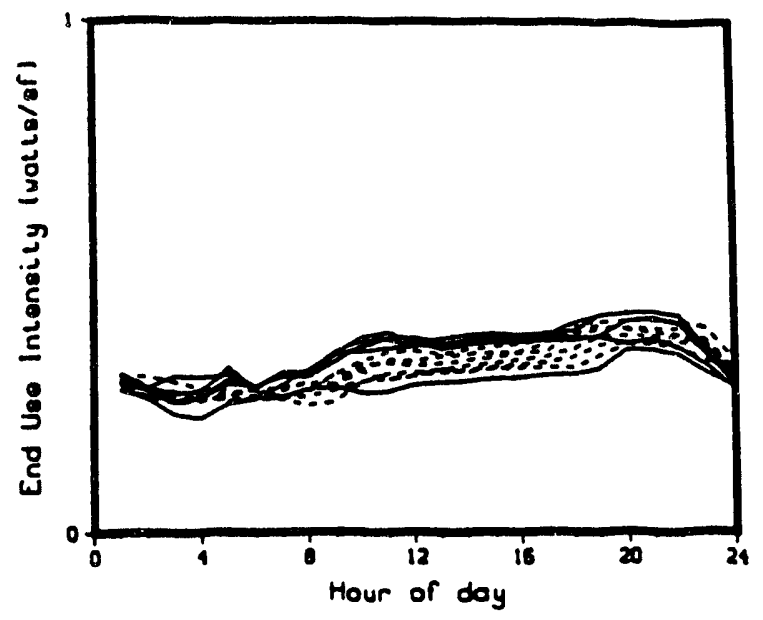

RST Refrig on Weokends

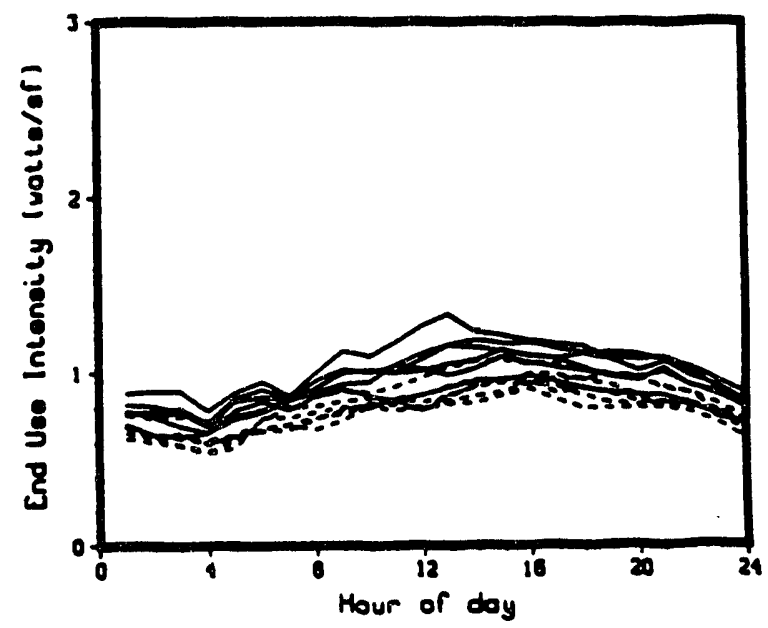

RST Total on Heokends

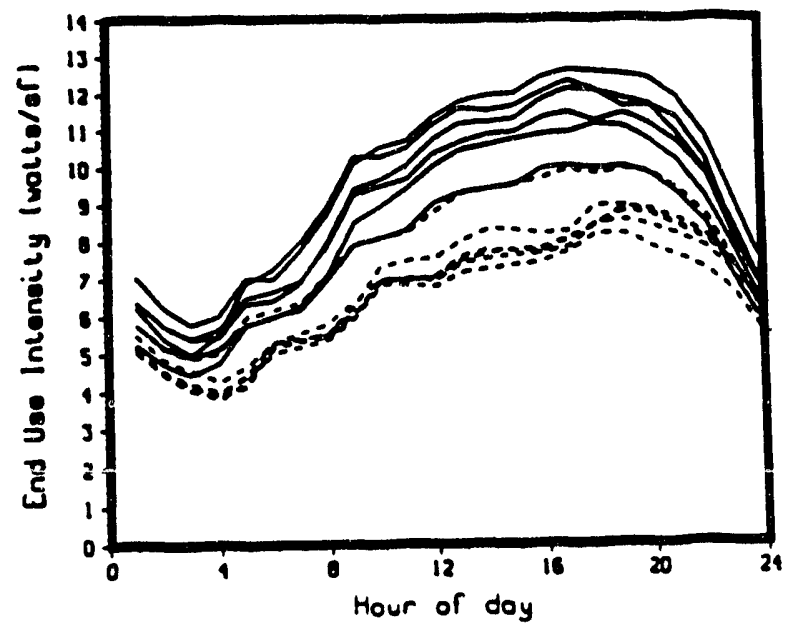




\section{Figure II.27b \\ Restaurant EDA Results}

Solid lines for winter months, dashed lines for summer months

RST EDA Refrig on Wookdoys

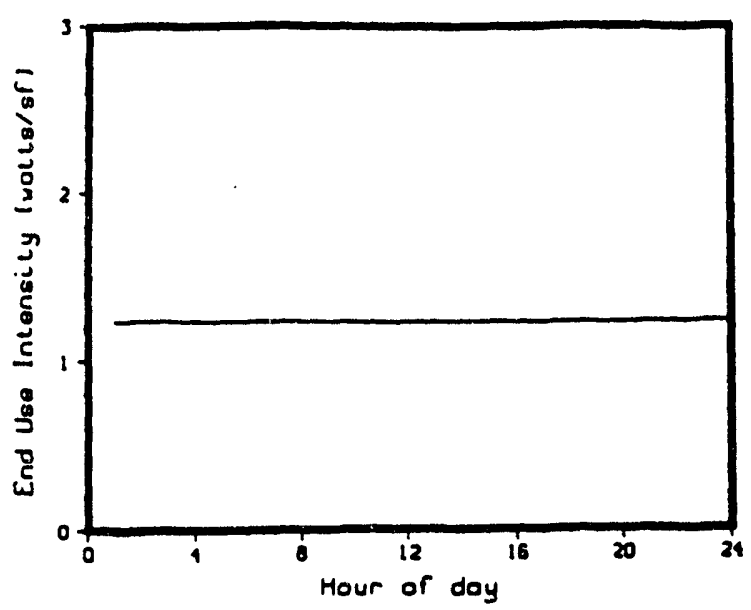

RST EDR Equip on Weokdays

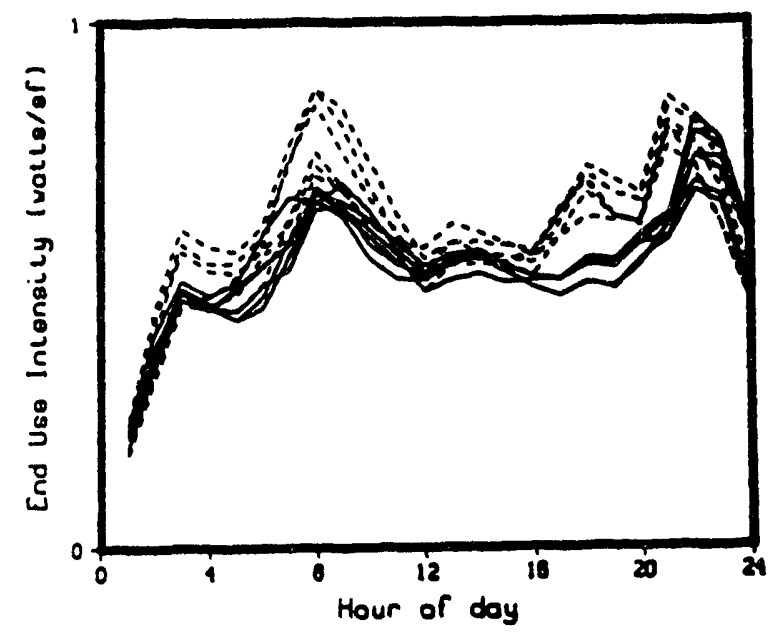

RST EDA Hot Water on Heakdoys

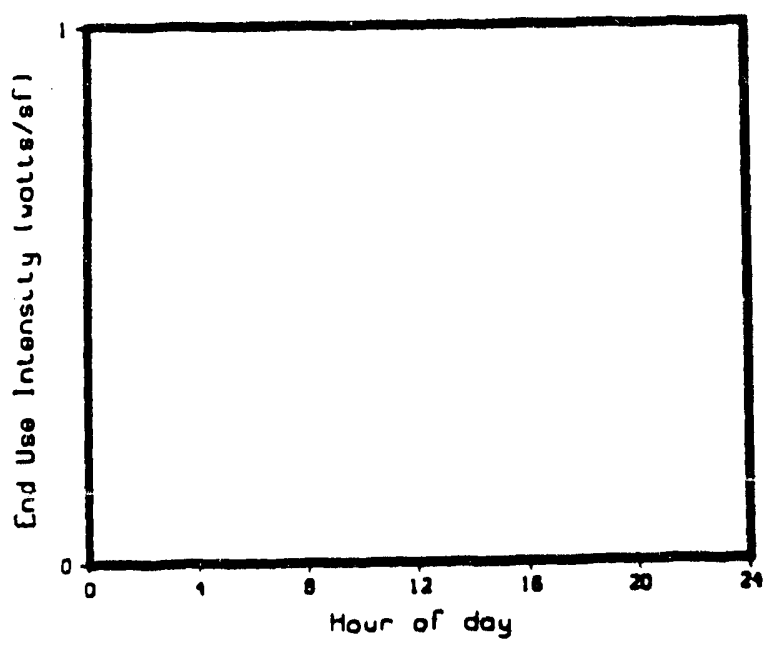

RST EOA Refrig on Weokends

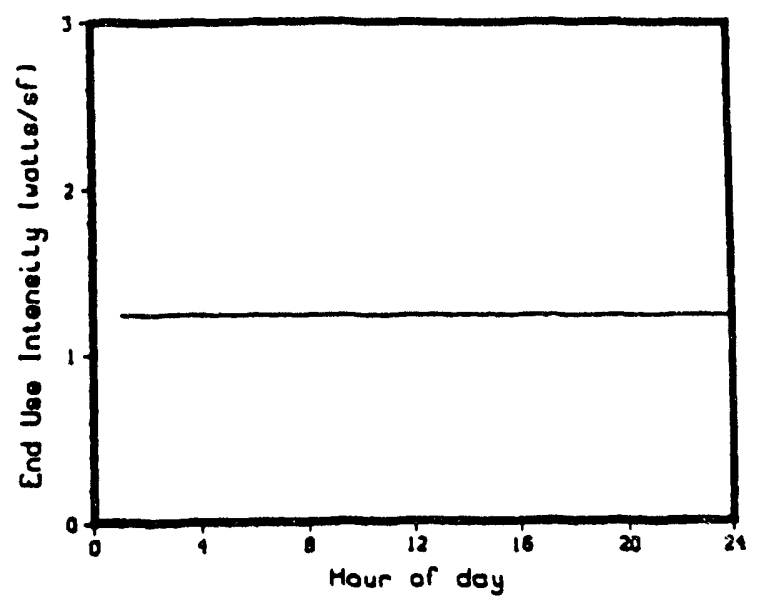

RST EDA Equip on Weokends

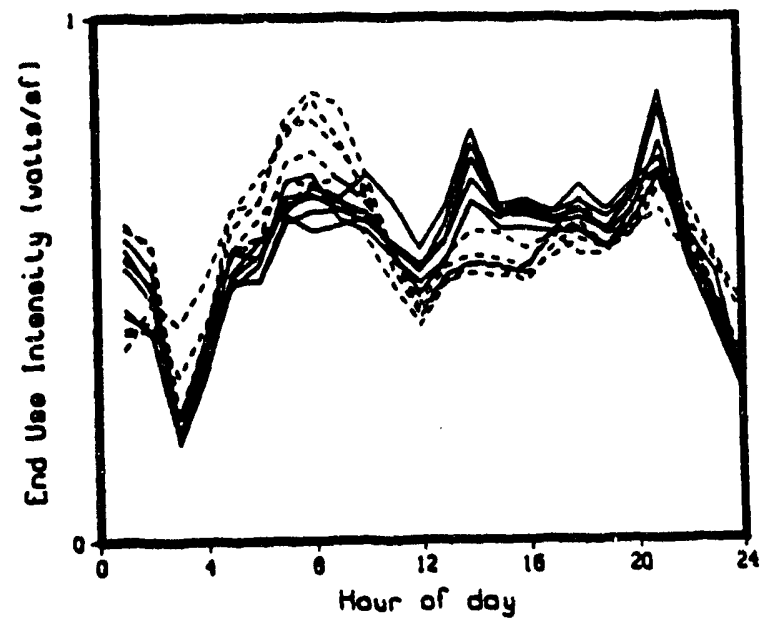

RST EDA Hot Woter on Weakends

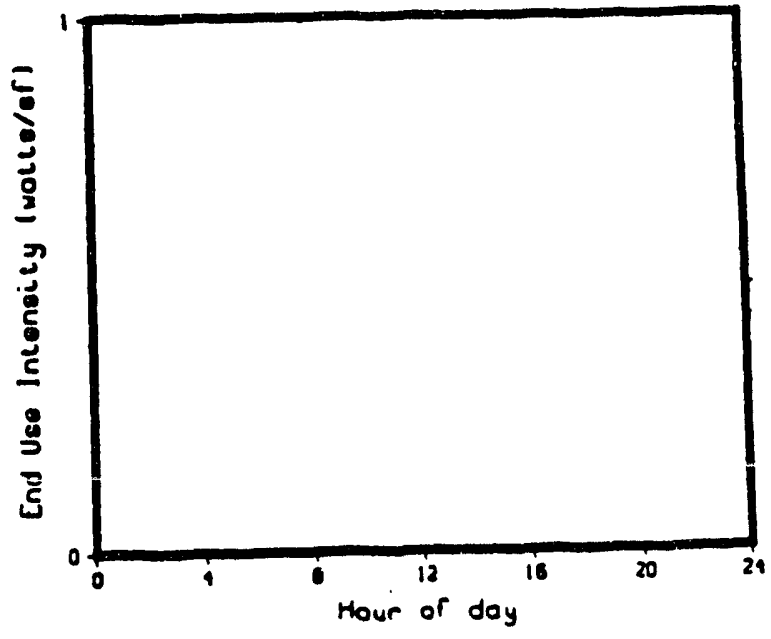


Figure II.27b (continued)

\section{Restaurant EDA Results}

Solid lines for winter months, dashed lines for summer months

RST EDA HVAC on Woekdays

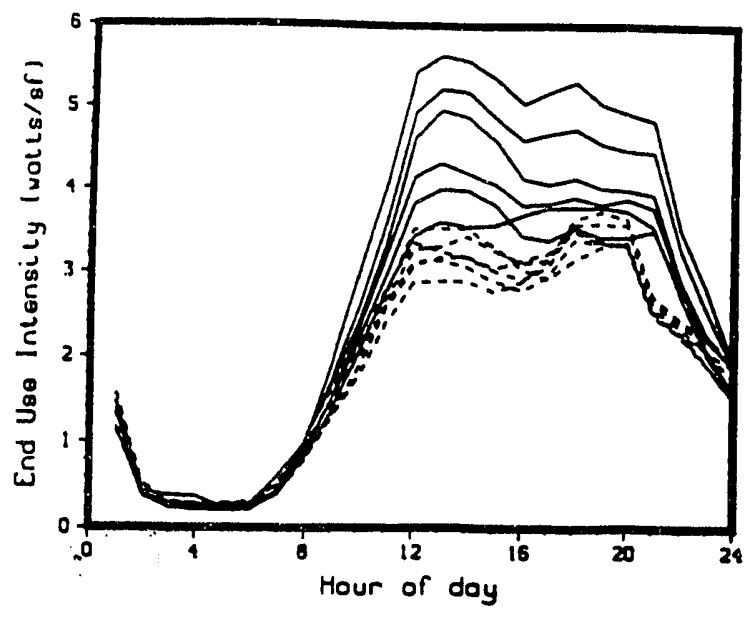

RST EDA Lighting on Woekdoys

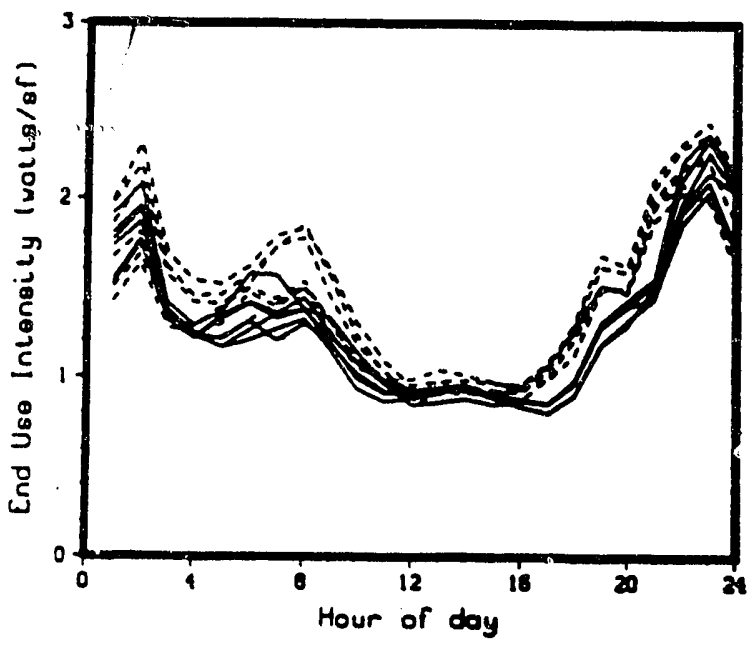

RST EDA Cooking on Hoekdoys

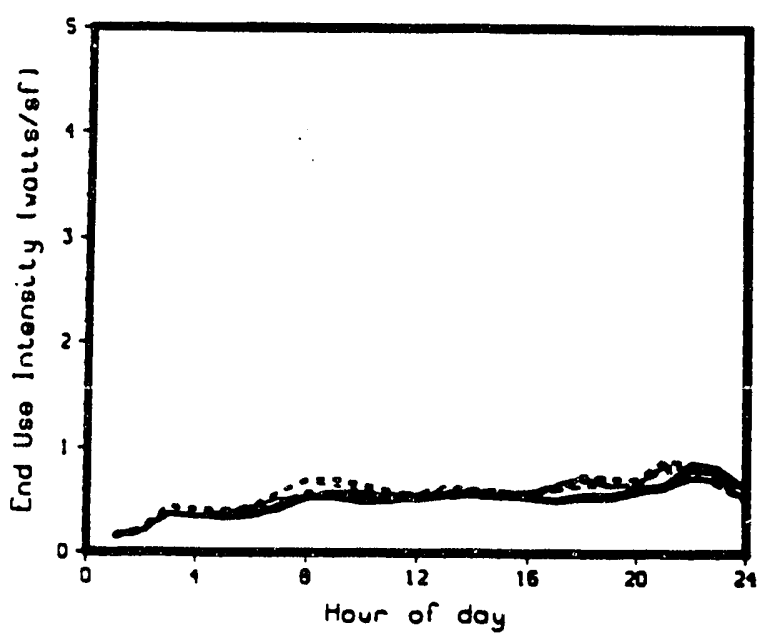

RST EDA HVAC on Weokends

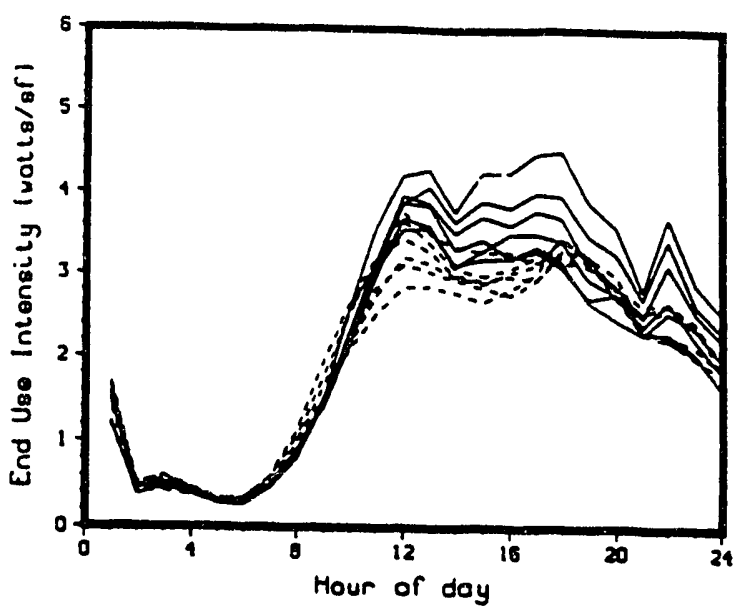

RST EDA Lighting on Weekends

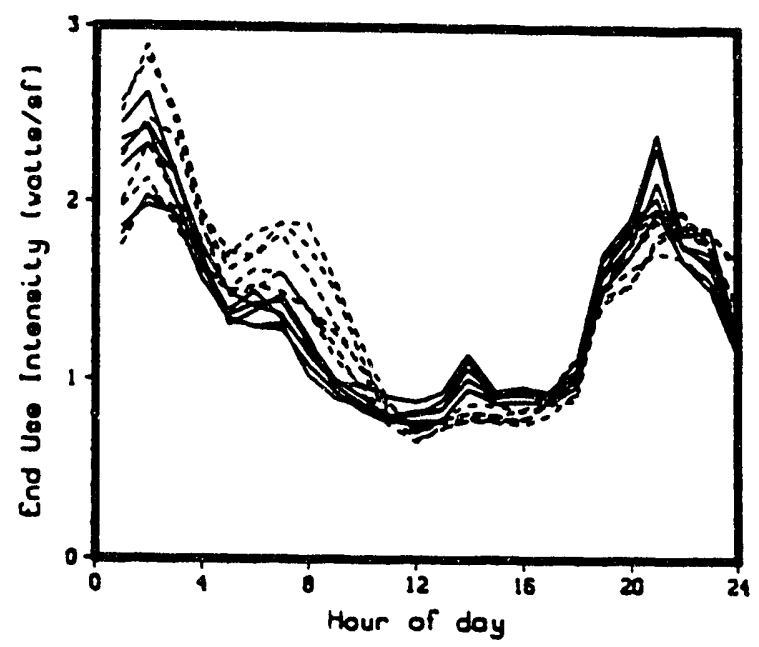

R'j! EDA Cooking on Woekends

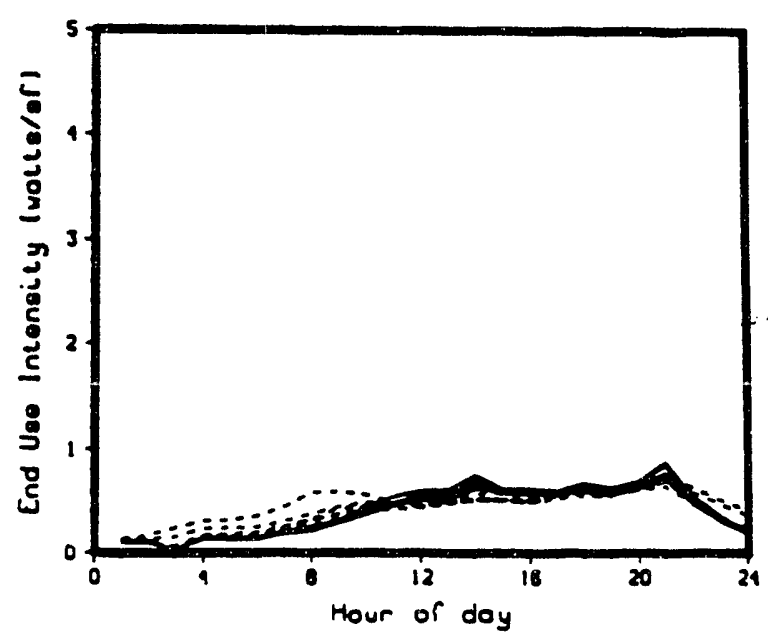


Figure II.27b (continued)

Restaurant EDA Results

Solid lines for winter months, dashed lines for summer months

RST EDA Total on Weokdays

RST EDA Total on Weekends
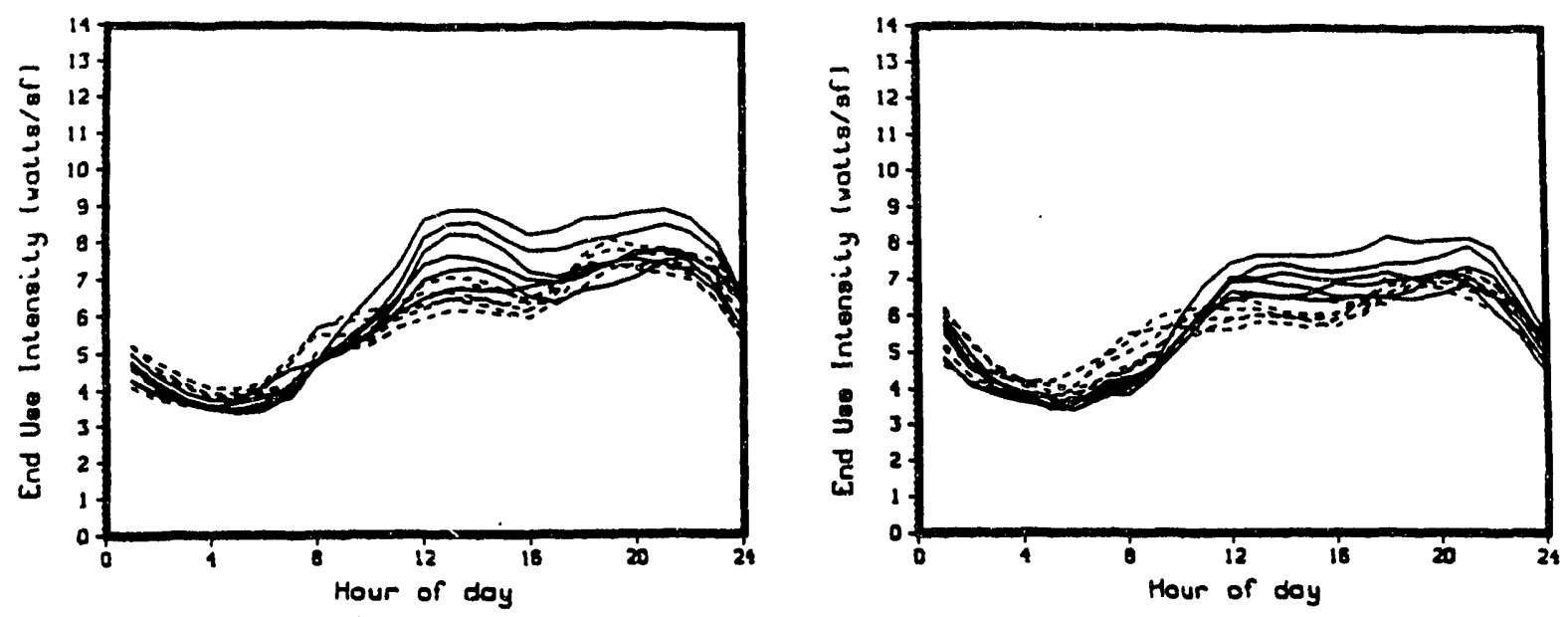


\section{Figure II.28a \\ Warehouse Monitored Data}

Solid lines for winter months, dashed lines for summer months

WHS HVAC on Weekdays

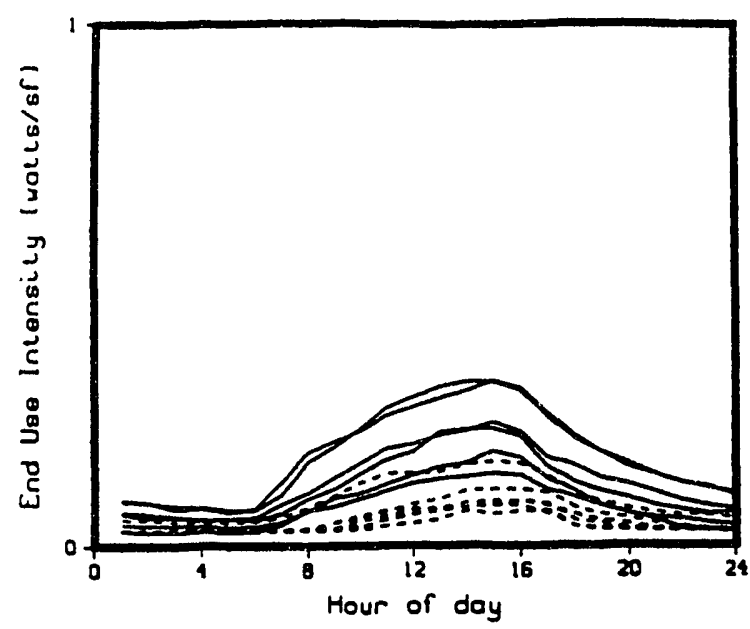

WHS Lighting on Weekdays

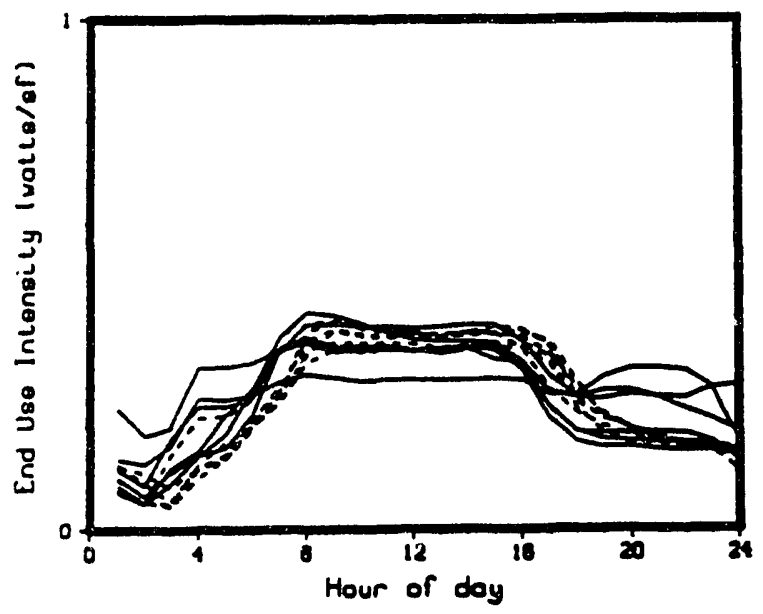

WHS Equipment on Heekdoys

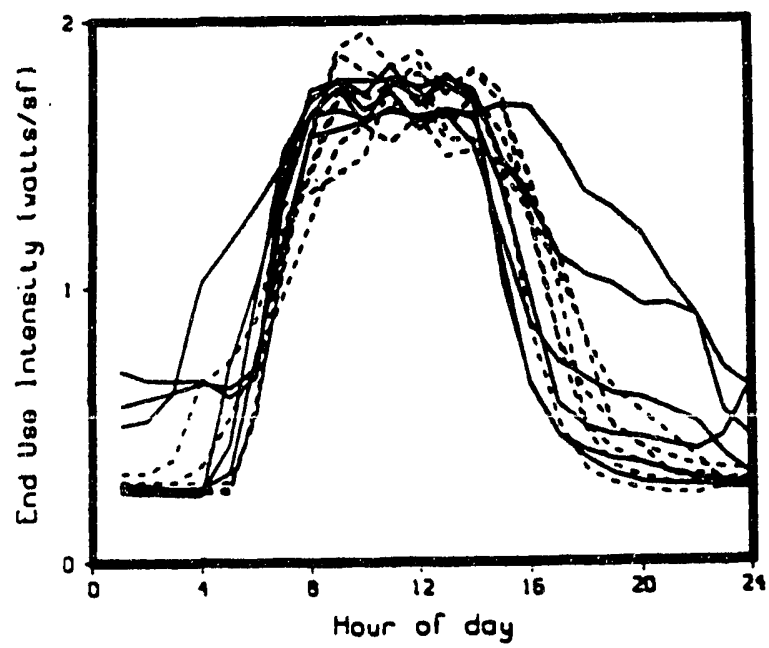

WHS HVAC on Weekends

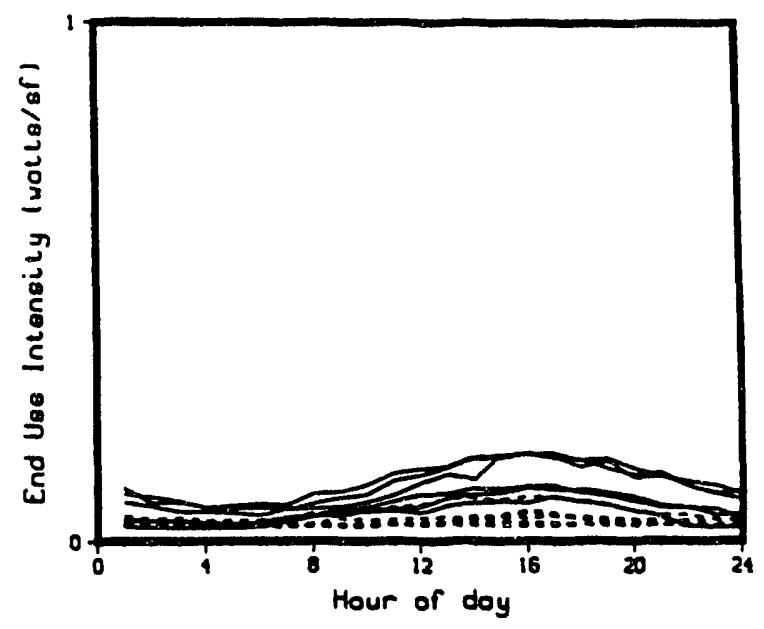

WHS Lighting on Weekends

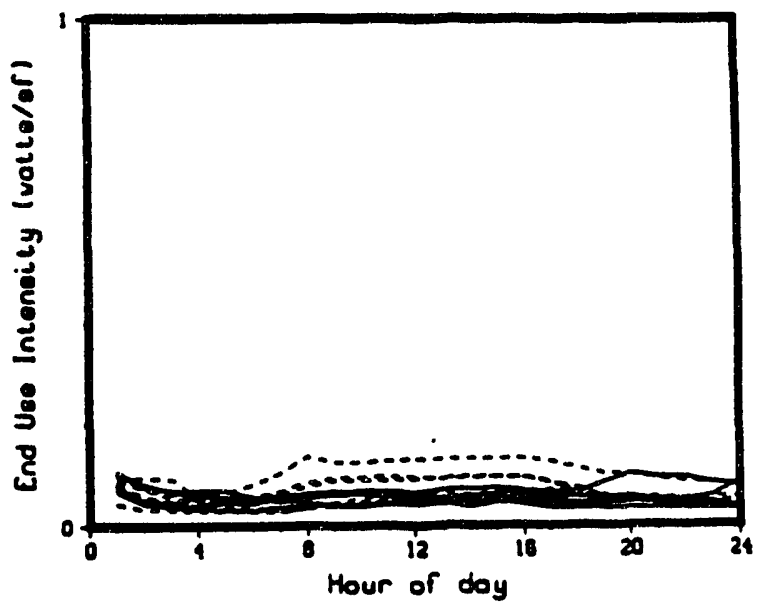

WHS Equipenent on Weokends

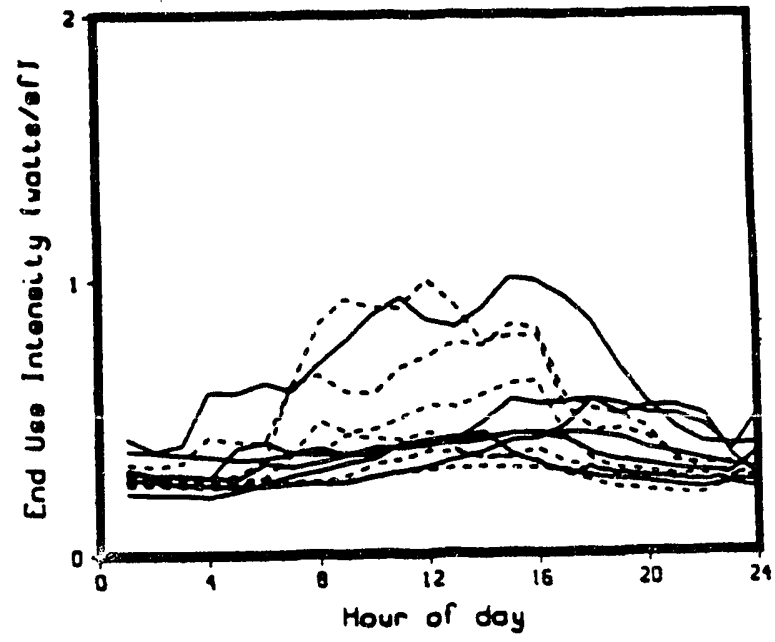


Figure II.28a (continued)

Warehouse Monitored Data

Solid lines for winter months, dashed lines for summer months

WHS Totol on Weokdoys

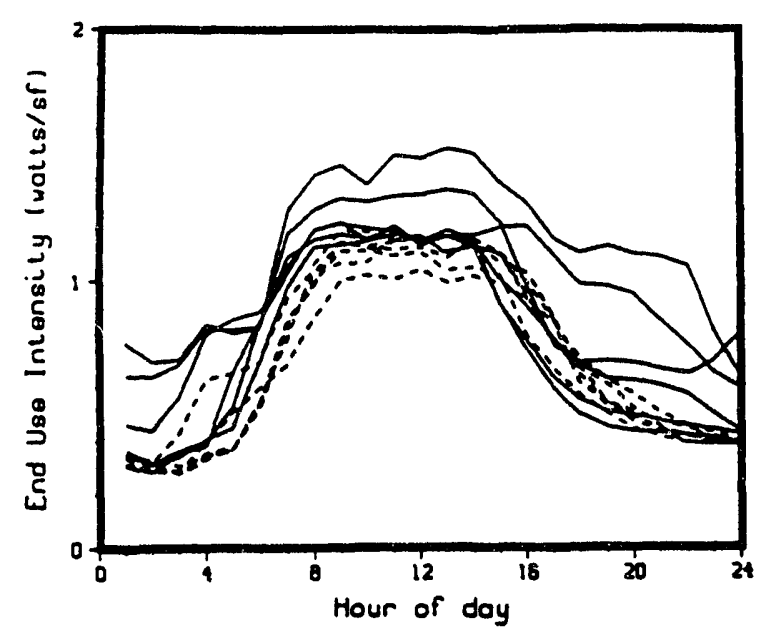

WHS Total on Weekends

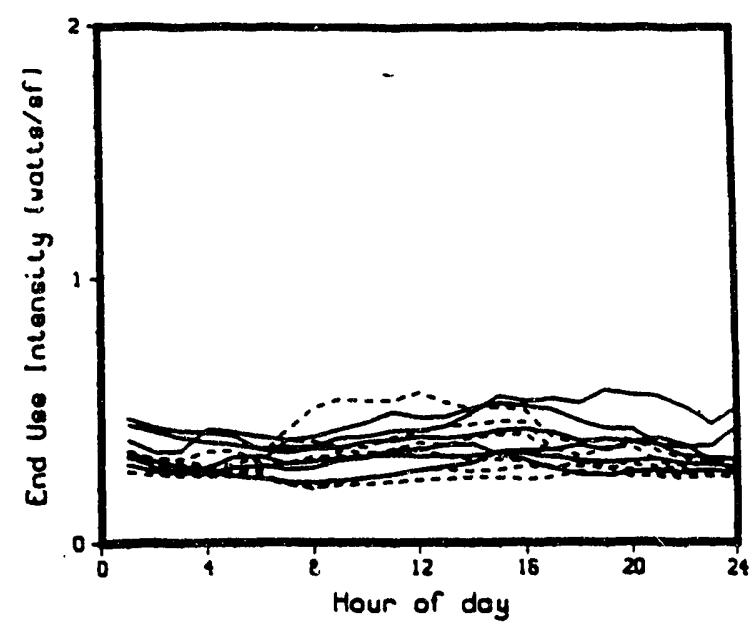


Figure II.28b

\section{Warehouse EDA Results}

Solid lines for winter months, dashed lines for summer months

WHS EDA HVAC on Weokdoys

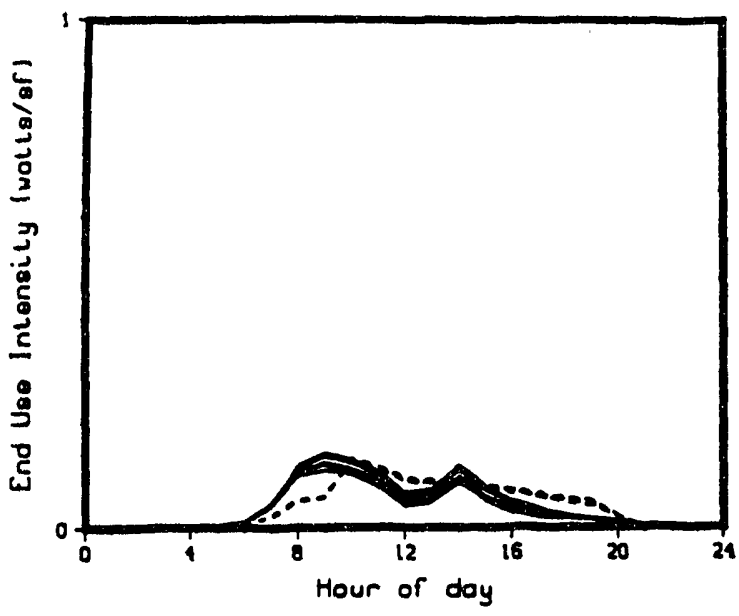

WHS EOR Lighting on Weokdoys

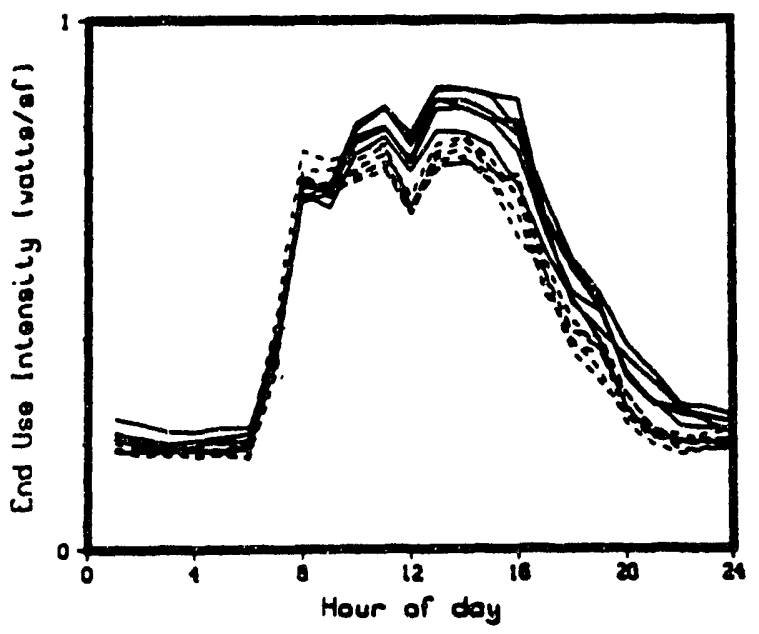

WHS EOR Misse on Heokdoys

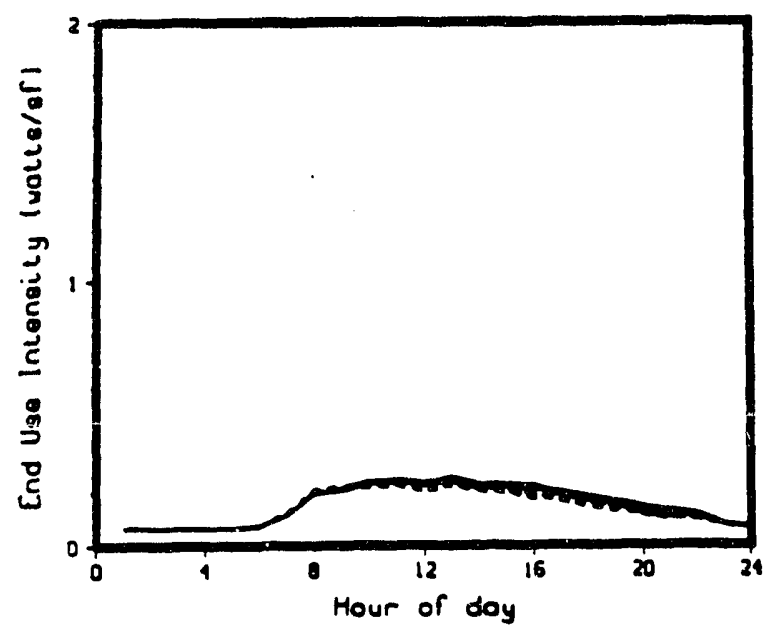

WHS EDA HVAC on Weekends

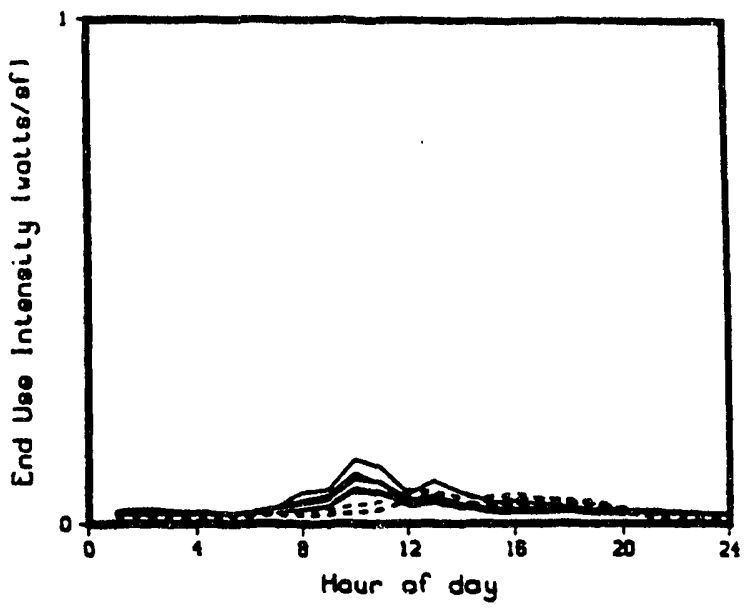

WHS EDA Lighting on Weekends

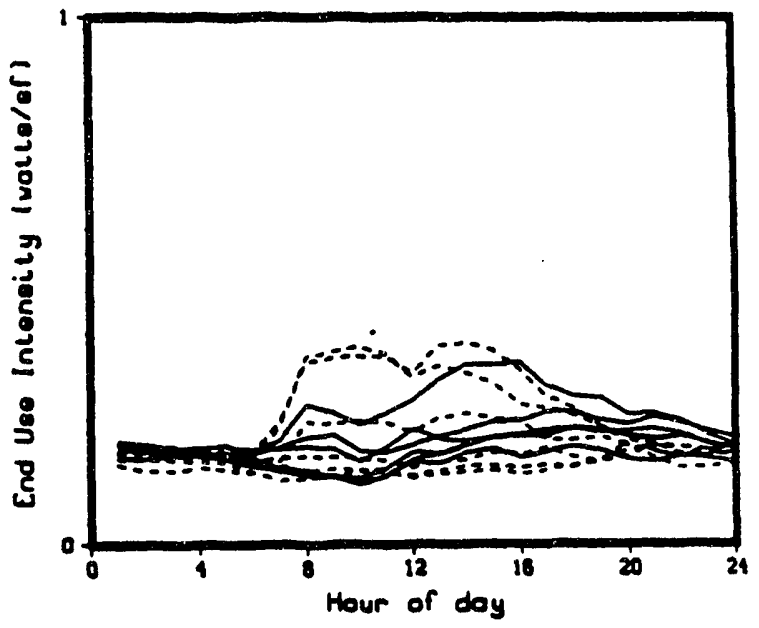

WHS EOA Mise on Haokends

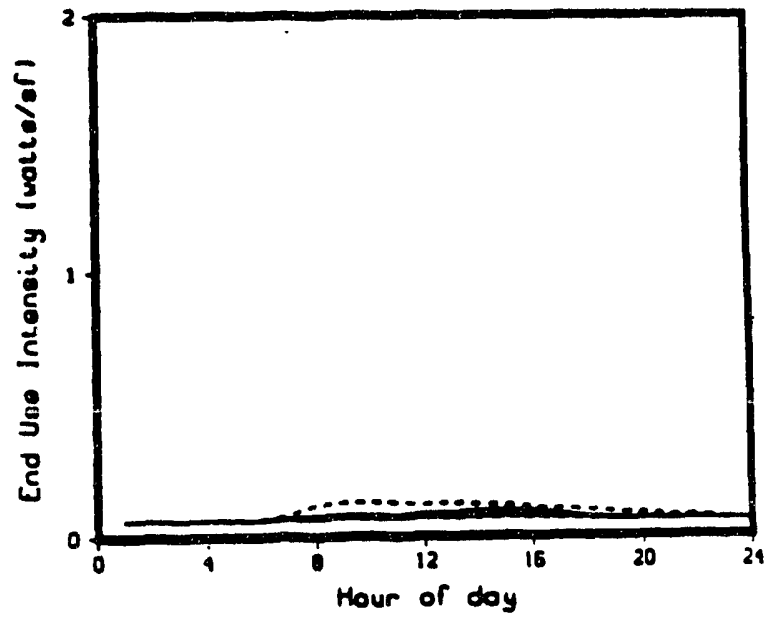


Figure II.28b (continued)

Warehouse EDA Results

Solid lines for winter months, dashed lines for summer months

WHS EDA Total on Weekdays

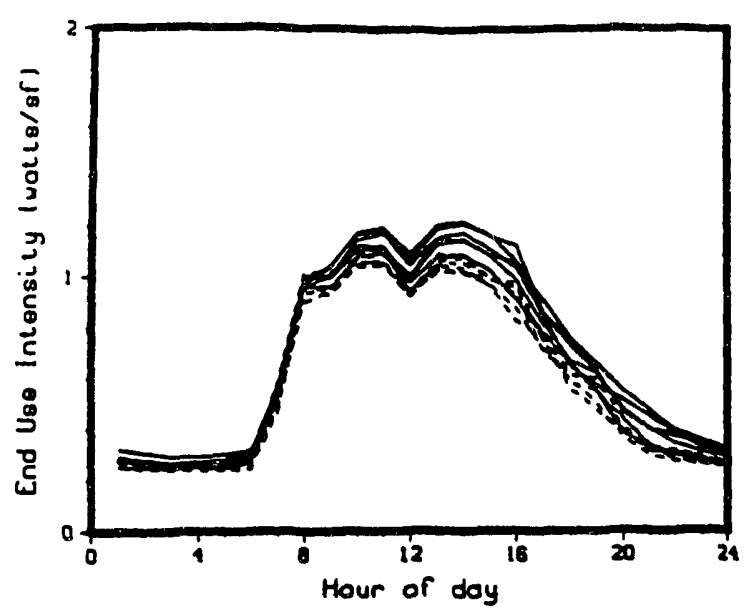

WHS EDA Total on Weekends

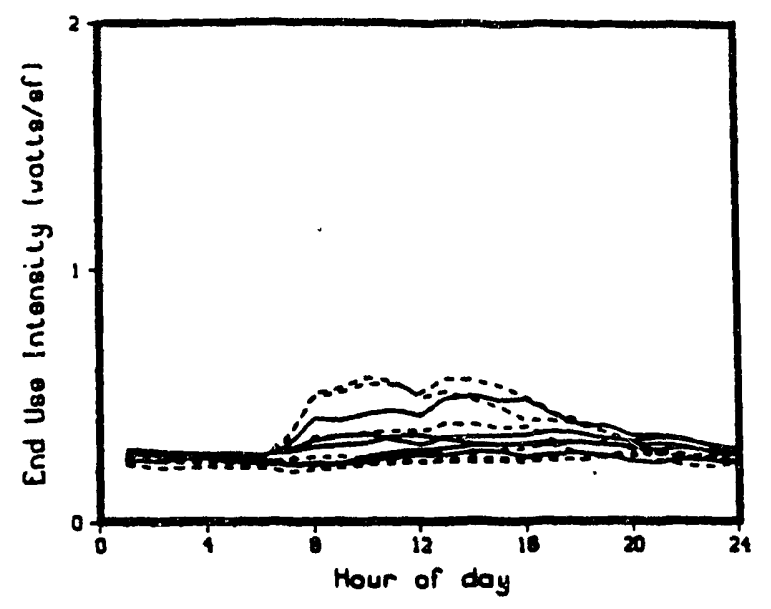

81 
between the monthly load shapes in the monitored data graphs, can be attributed to some months having different building data sets. Some months are based on data from more buildings than other months. Since the monitoring did not start for all the buildings at the same time, more data were available for the later months than the earlier ones.

The major differences between the monitored and LBL LSs are in the maximum daytime intensity (monitored: $4.2 \mathrm{~W} / \mathrm{ft}^{2}$, LBL: $3.7 \mathrm{~W} / \mathrm{ft}^{2}$ ) and evening shoulder hours (monitored: shorter hours of evening usage, LBL: longer hours of evening usage). It should be noted that the LBL whole-building LSs are averages based on the SCE load research data.

The HVAC and plug (miscellaneous) LSs for weekdays compare well, but the lighting LSs of the LBL study show a secondary peak during the evening hours. Thus the lighting end use accounts for the total electric load-shape differences in the evening hours that occur in the load research data and the monitored data.

Small Office (Figures II.23a\&b)

The comments on large office buildings also applies to the small office load shapes.

\section{Large Retail (Figures I1.24a\&b)}

Average monthly whole-building electric load shapes from the LBL study and the monitored data are significantly different, particularly during the nighttime hours. This difference directly contributes to significant differences in load shapes for all end uses, especially HVAC and lighting.

Small Retail (Figures II.25a\&b)

Average monthly whole-building electric load shapes from the LBL study and the monitored data are somewhat different during the nighttime hours. This difference directly contributes to significant differences in all end uses, especially HVAC and lighting LSs. The HVAC LSs for the monitored data shows a higher level of activity during the night. Also, the lighting LSs of the monitored data do not show a secondary peak during the evening shoulder hours.

Grocery (Figures II.26a\&b)

Average monthly whole-building electric load shapes from the LBL study and the monitored data are significantly different particularly during the nighttine hours. The main reasciil for this difference is the difference between the types of buildings in the LBL study and SCE monitoring program. Recall that the grocery stores in the LBL work are fairly small, averaging about $6,000 \mathrm{ft}^{2}$ in floor area and operating mainly during the day, while the monitcred buildings 
are larger and appear to operate 24-hours a day. Furthermore, the peak, whole-building electric use intensity determined in the LBL study is about $4 \mathrm{kWh} / \mathrm{ft}^{2}$ year versus $9 \mathrm{kWh} / \mathrm{ft}^{2}$ year for the monitored buildings. These differences contribute directly to the significant differences in all end uses, especially HVAC and lighting LSs.

Restaurant (Figures II.27a.1, II.27a.2, II.27a\&b)

The SCE monitoring project has provided data for both sit-down and fast-food restaurants. But the LBL study, which uses SCE LRD based data, only provides LSs for the combined group of restaurants. From the monitored data, we have developed LSs for both sit-down (Figure 27a.1) and fast-food (Figure 27a.2) restaurants and combined them together (the mixture of restaurants are two-thirds sit-down and one-third fast food to be consistent with the LBL study). (Figure 27a).

A qualitative comparison between the whole-building load shapes reveals the same load shape for these two sources. But the load shapes for the end uses are, in general, different. The HVAC load shapes are different in the early morning hours and in the number of peaks which occur during the day. The lighting LSs (including outdoor lighting) of the LBL study show two peaks during morning and evening hours, but the monitored data show flat profiles during the day with sharp nighttime peaks. We are not sure whether the monitored lighting includes outdoor lighting.

The monitored data LSs shows much more electric cooking than the LBL LSs. This, however, is a saturation effect and can be dealt with by separately modeling the EUIs and LSs for restaurant electric cooking. The refrigeration LS of LBL study is flat but the monitored data show small peaks during the day.

\section{Warehouse (Figures II.28a\&b)}

There are only three buildings in the sample of the monitored data. The average LSs for these buildings are plotted and compared with the EDA result. In general, the comparison hetween the whole-building LSs and HVAC LSs are fairly good. But the equipment and lighting LSs are significantly different in their shape and maximum intensities. For instance, the average maximum equipment intensity for the monitored buildings is about $1.7 \mathrm{~W} / \mathrm{ft}^{2}$, while the corresponding figure for LBL study is only $0.3 \mathrm{~W} / \mathrm{ft}^{2}$. 


\section{Summary}

In summary, for standard weekdays and for most building types, the whole-building and HVAC load shapes developed in the LBL study and those calculated from the monitored data compare well. There are some differences in the lighting LSs that are caused mainly by the different operating schedules of the buildings in the two data sets. The weekend load shapes developed in the LBL study show some physically suspect characteristics and have not been given additional consideration at this time. The budget limitations of this study did not permit their reexamination. Since weekend load shapes are of secondary importance for forecasting models, we have concentrated on the development of the weekday loads. 


\section{Chapter III: EDA Validation}

\section{EDA Methodology}

The End-Use Disaggregation Algorithm (EDA) developed at LBL is an integrated method for the estimation of EUIs and LSs, which relies explicitly on measured whole-building hourly load to reconcile preliminary engineering estimates. The end-use disaggregation is a two step process. First, we develop preliminary end-use EUIs and LSs for the building of interest using the integrated on-site survey data, the non-HVAC EUI/LS and DOE-2 Input Generator (NELDIG), and the DOE-2 building energy analysis program. NELDIG performs two functions: 1) it estimates preliminary LSs and annual EUIs for non-HVAC end uses and 2) it prepares building input data for simulations. For a group of buildings, NELDIG prepares prototypical building characteristics by averaging building characteristics of sample buildings. The building is then simulated, using DOE-2, to obtain preliminary EUIs and LSs for the HVAC end uses.

Second, using the initial building loads by end use from the first step and the measured whole-building hourly loads, we apply the End-use Disaggregation Algorithm (EDA) to obtain adjusted, reconciled end-use load profiles for the building. The corresponding EUIs are simply the integration of the hourly profiles for the entire year.

EDA is a deterministic model that primarily utilizes the statistical characteristics of the measured, hourly, whole-building load and its inferred dependence on temperature. Simulation is only used to supply information that is not evident from the load/temperature relationship. In the EDA, the sum of the end uses is constrained, at hourly intervals, to be equal to the measured whole-building load. This constraint provides a reality check that is not always possible with pure simulation. In addition, the load/temperature relationship helps to characterize the HVAC end use, providing an additional constraint on the remaining end uses and preventing some of the errors possible with simple proration. Finally, EDA also attempts to deal with the fluctuations of hourly loads by incorporating observed statistical variation.

The primary component of the EDA is regression of hourly loads with climatic variables. If the weather dependency of the building load changes with season, we use two season-specific (summer and winter) sets of weather regression coefficients. The weather regression equations are used to separate the load predicted by the regression into a temperature-dependent part and a temperature-independent part. We assume the temperature-dependent load is attributable to HVAC equipment. The temperature-independent load is the sum of loads such as lighting, 
ventilation, and miscellaneous equipment, as well as temperature-independent cooling at base weather conditions. Because the regression will provide no information about how to break down the temperature-independent load, we simply prorate it according to the loads predicted by simulation. The actual load at a particular hour on a particular day will probably not lie on the best-fit regression line, so the difference between the actual load and the regressed load is split between the two parts of the load.

A flow chart of the EDA and its data requirements are shown schematically in Figure III.1. For each building, the inputs to the EDA are:

- the actual hourly whole-building load during a given period of time;

- the actual measured outside weather conditions during this same period of time;

- $\quad$ statistics from the regression of load with the selected weather variables, calculated separately for summer and winter and

- the results of simulating the building at the base weather condition.

Using these data, EDA disaggregates the whole-building hourly load into end uses. The output of the EDA is hourly load profile estimated for all end uses described in the initial conditions. The hourly end-use load profiles can be used to develop end-use load shapes by type of day, month, season, or for the entire year. A detailed description of the EDA and a comparison of its performance versus pure simulation is reported in Akbari et al. (1988).

EDA can be used for analysis of measured hourly data for both prototypical and individual buildings. EDA has been applied to SCE data and average end-use data have been calculated for each of the SCE planning regions (Akbari et al 1989,1990). For the validation purposes in this analysis, we apply the EDA to two buildings from SCE's end-use metering project. We develop hourly end-use load profiles for each of the selected buildings and compare the results with the metered end-use data.

\section{Validation}

The buildings we have selected for the validation are an office and a retail store. These buildings were selected because of their continuous data sets (little or no missing data), complete on-site surveys, and reasonable load shapes (no apparent changes in occupancy or operation).

In the remainder of this chapter, we discuss the process of validation for the two selected buildings. For each building, we first present a brief description of the building followed by a 
Figure III.1

Integrated Commercial LS and EUI Estimation Methodology

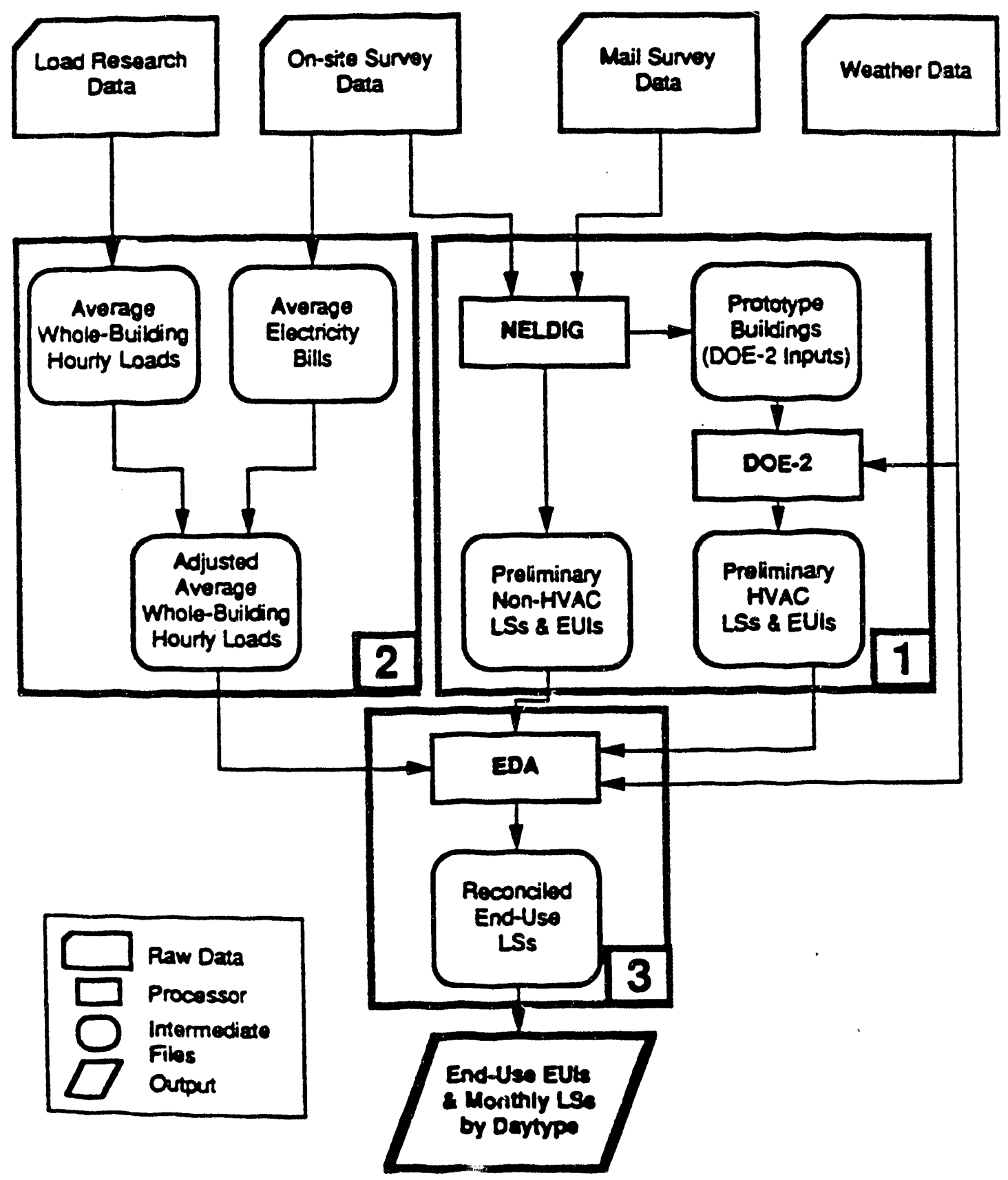


discussion of the DOE-2 simulation input and results. Then, we discuss the hourly correlations of the whole-building load with respect to weather parameters, particularly drybulb temperature and relative humidity. The audit information, DOE-2 results, and the temperature correlations are used in the EDA to develop end-use load profiles. Finally, the EDA output is compared with the monitored data with particular emphasis on the strengths and weaknesses of the model.

\section{Office building \# 5}

\section{Building Description}

This $117,600 \mathrm{ft}^{2}$ building is a six story multi-tenant office built in 1971 in the coastal region of SCE's service area (SCE 1990, 1989). About $86 \%$ of the building floor area is conditioned office space and $14 \%$ basement parking. Natural gas is used for heating and hot water. All other systems are electric.

The building is occupied by 435 people and operated Monday through Friday from 7 a.m. to 6 p.m. All the interior lights are fluorescent with installed intensity of $1.9 \mathrm{~W} / \mathrm{ft}^{2}$ (based on conditioned floor area, $1.6 \mathrm{~W} / \mathrm{ft}^{2}$ based on gross floor area). The lights are controlled by local switches and have typical operating hours of 7 a.m. to 6 p.m. The outdoor lighting is fluorescent and has an installed intensity of about $0.04 \mathrm{~W} / \mathrm{ft}^{2}$ (based on the gross total building floor area), operated from 6 p.m. to 6 a.m., Monday through Friday, and controlled by the energy management system. The installed intensity of other building loads (personal computers, typewriters, copiers, water coolers, air compressor, elevators, and water pumps), as estimated by the auditor, is about $0.65 \mathrm{~W} / \mathrm{ft}^{2}$ (based on the total building floor area).

The building has three multi-zone HVAC systems. Heating is provided by forced air furnaces with total capacity of $1.6 \mathrm{MBtu} / \mathrm{h}$ and cooling by two hermetic reciprocating compressors with total capacity of 190 tons. Total electricity load for HVAC systems is $250 \mathrm{~kW}$. The heating and cooling set point is $74^{\circ} \mathrm{F}$ from 7 a.m. to 6 p.m., Monday through Friday, operated and controlled manually.

DOE-2 Simulation

We used information provided to us by the hardcopy of the 1989 audit and the 1985 on-site survey data to simulate the energy use of the building with DOE-2. The audit data provide information on the operation, schedules, equipment type, and equipment energy use for the building. The audit does not provide information on the architectural and construction characteristics of the building. 
We simulated the building, using a square floor plan in 6 stories with a total conditioned floor area of $101,000 \mathrm{ft}^{2}$ and total gross floor area of $117,000 \mathrm{ft}^{2}$. The building was simulated with a forced air gas furnace, two central reciprocating chillers with cooling towers, and three multizone air handling units. The total heating capacity was $1.6 \mathrm{MBtu} / \mathrm{h}$ and the total cooling capacity was 190 tons. Each air handling unit (AHU) served two floors of the building; each floor was simulated with two zones: a perimeter and a core zone. The internal load of the building was calculated using the methodology discussed in Akbari et al. (1989). The HVAC schedule of the building was modified, using the characteristics of the whole-building load.

We did not have the complete actual weather data to simulate HVAC energy use for 1989. 1990. Instead, we used the following alternative. The building was simulated using the Los Angeles WYEC (Weather Year for Energy Calculations) weather tape and the resulting hourly HVAC energy use was regressed against the WYEC drybulb temperature. The resulting correlations were then used in conjunction with actual 1989-1990 hourly drybulb temperatures to estimate the hourly cooling energy use for the period of June 1, 1989 to May 31, 1990.

The simulated annual HVAC, indoor lighting, and plug loads are 8.36, 4.62, and 0.99 $\mathrm{kWh} / \mathrm{ft}^{2}$, respectively. The simulated whole-building energy use only accounts for $14.0 \mathrm{kWh} / \mathrm{ft}^{2}$ compared to $17.1 \mathrm{kWh} / \mathrm{ft}^{2}$ as measured for this office building. Also, the shape of the simulated whole-building load is significantly different from that of the measured load, both during the peak day and during an average day. Simulation indicated that, in contrast to measured data, building electricity use peaks during the early morning hours, mainly due to the high start up cooling demands. EDA will adopt the characteristics of the measured whole-building load in the reconciliation process (shown in Figure III.2).

It is also interesting to note that the hourly plot of the simulated HVAC electricity use versus drybulb temperature does not indicate a base temperature (i.e. a temperature below which there is no temperature dependency) (See Figure III.3). This is consistent with the observed characteristics of the measured data which are discussed in the next section. The regression results of the simulated hourly HVAC load against the WYEC drybulb temperatures are presented in Table III.1. Using these correlations and the measured drybulb temperatures, we calculated the new estimate of the simulated HVAC electricity use for the actual year of 1989 . 1990. For a typical summer day, Figure III.4 compares the new estimate of simulated HVAC electricity use for the 1989-1990 temperature data with the original DOE-2 results based on simulations with WYEC weather data. This process reduced the simulated annual HVAC 


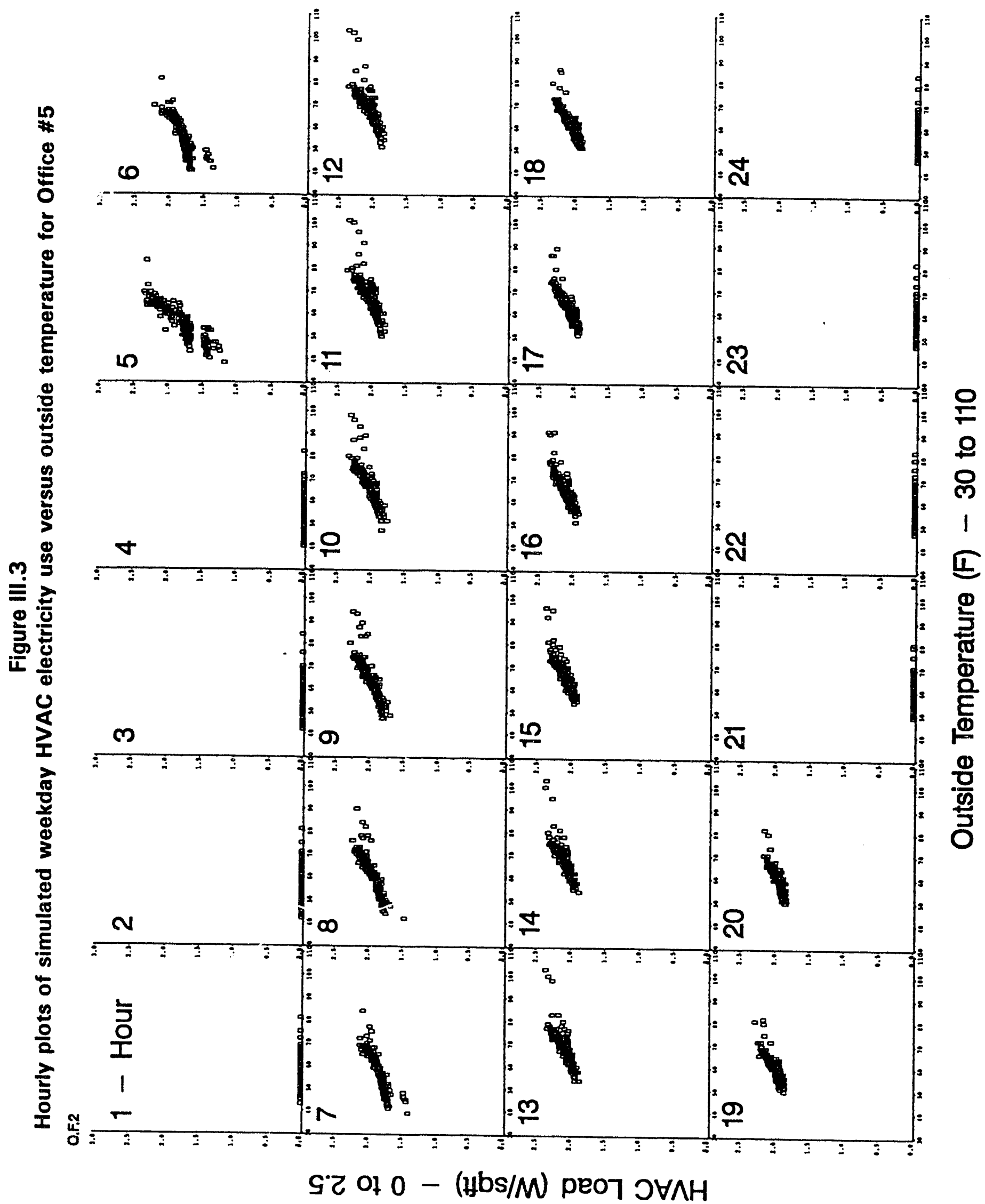




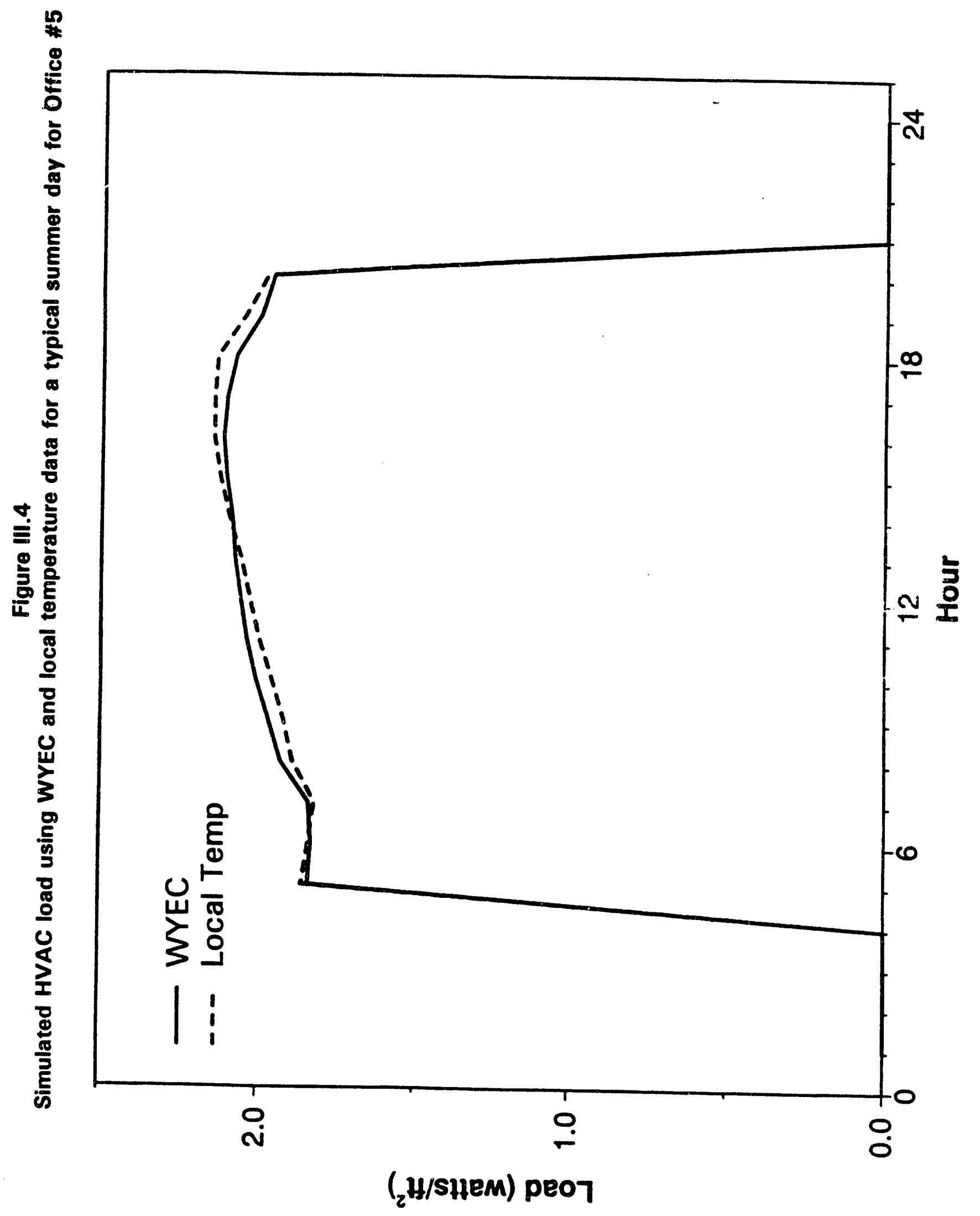


energy use from 8.36 to $7.99 \mathrm{kWh} / \mathrm{ft}^{2}$.

Load/Temperature Regressions

Figures III.5-III.7 show the yearly, summer, and winter plots of hourly whole-building load against the ambient drybulb temperatures. The summer and winter period are defined as May 1 to October 31 and November 1 to April 30, respectively. These figures indicate a few interesting points that warrant further discussion. The hours that the building is shut down, judged by the lowest level of energy use of $0.7 \mathrm{~W} / \mathrm{ft}^{2}$, are between 11 p.m. to $3 \mathrm{a} . \mathrm{m}$. This indirectly contradicts the building audit which indicate the hours of operation are from 7 a.m. to 6 p.m. In fact, judging from the whole-building hourly load, the normal hours of operation of the building are between 9 a.m. and 5 p.m. Hours 4 a.m. to 8 a.m. and 6 p.m. to 10 p.m. are morning and evening shoulder hours, respectively.

It is also interesting to note that neither of the plots indicate a definite and identifiable base temperature for any hours of the day, except the period between 11 p.m. to 3 a.m. As we will discuss later, this has introduced some difficulties in identifying a clear base cooling load for the building. Of the $0.7 \mathrm{~W} / \mathrm{ft}^{2}$ nighttime load, only about $0.04 \mathrm{~W} / \mathrm{ft}^{2}$ is for outdoor lighting (based on on-site survey data). The rest is for nighttime indoor lighting (including emergency lighting) and other miscellaneous equipment (identified or not identified in the audit).

We regressed the whole-building hourly load in summer and winter against drybulb temperature. Table III.2 shows the summary of regression statistics. The table indicates that for both summer and winter significant correlations for most hours of the day exist. Significant correlations exist for summer from 10 a.m. to 7 p.m. and 10 p.m. to 3 a.m., however a closer review of the table indicates that the nighttime correlations are statistical artifacts and indeed the correlation coefficients are fairly weak during the nighttime hours. In the winter, significant correlations exist from 5 a.m. to 9 p.m.

The $\mathrm{R}^{2}$ values for both the summer and winter regressions are generally poor, in the range of 0.14 to 0.23 for the summer and 0.13 to 0.41 for winter. One reason that the winter time $\mathrm{R}^{2}$ are better than the summer time $R^{2}$ is the wider range of temperature variation during the winter. Other probable reasons are further discussed in Chapter IV in the review of the characteristics of the measured end-use data. The poor statistics of the whole-building load indicate that temperature variations can only describe a small portion of the variation in the whole-building load. These poor statistics may be the result of the manual operation of the HVAC system, indicating a poor temperature control of the HVAC system. 


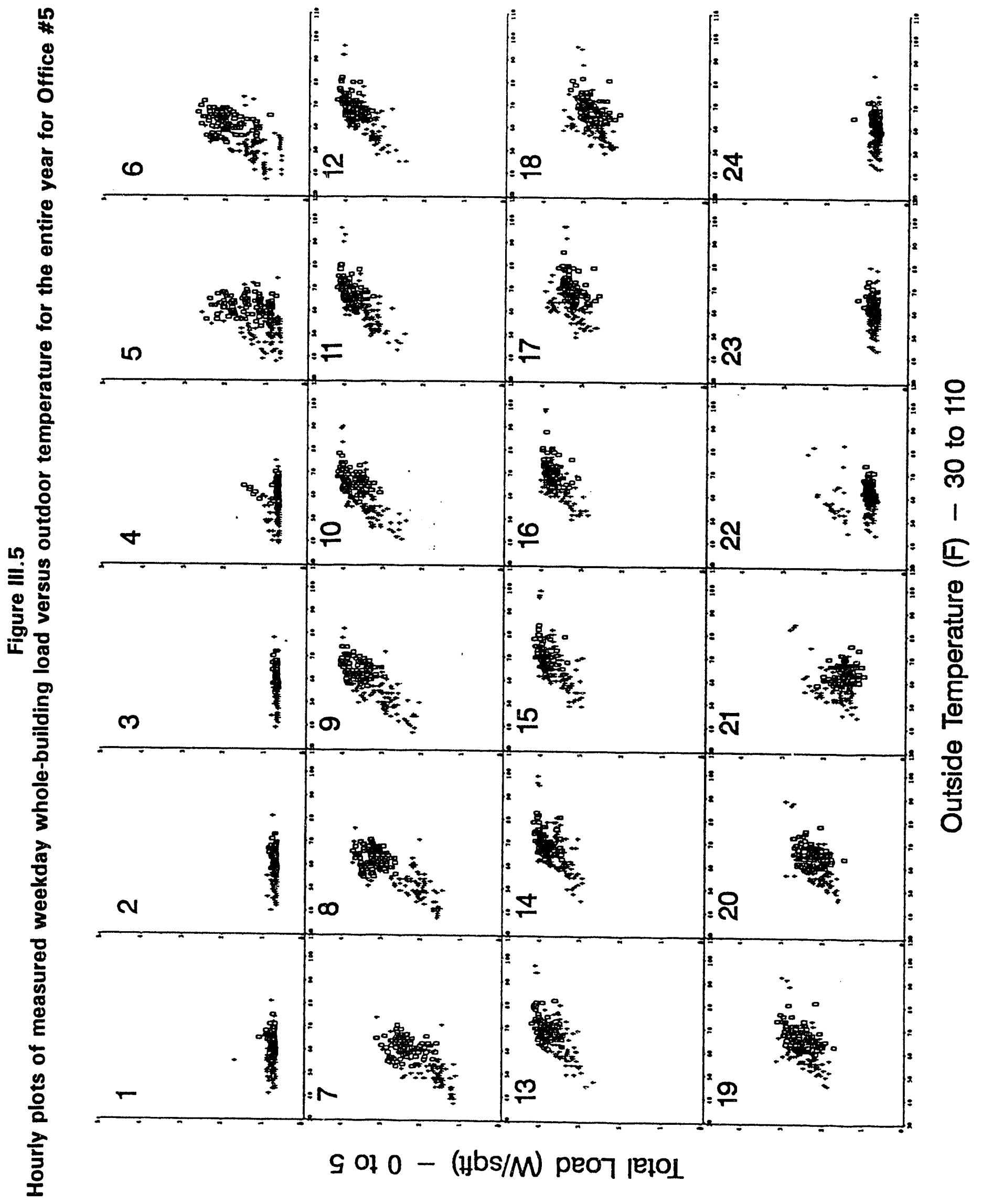




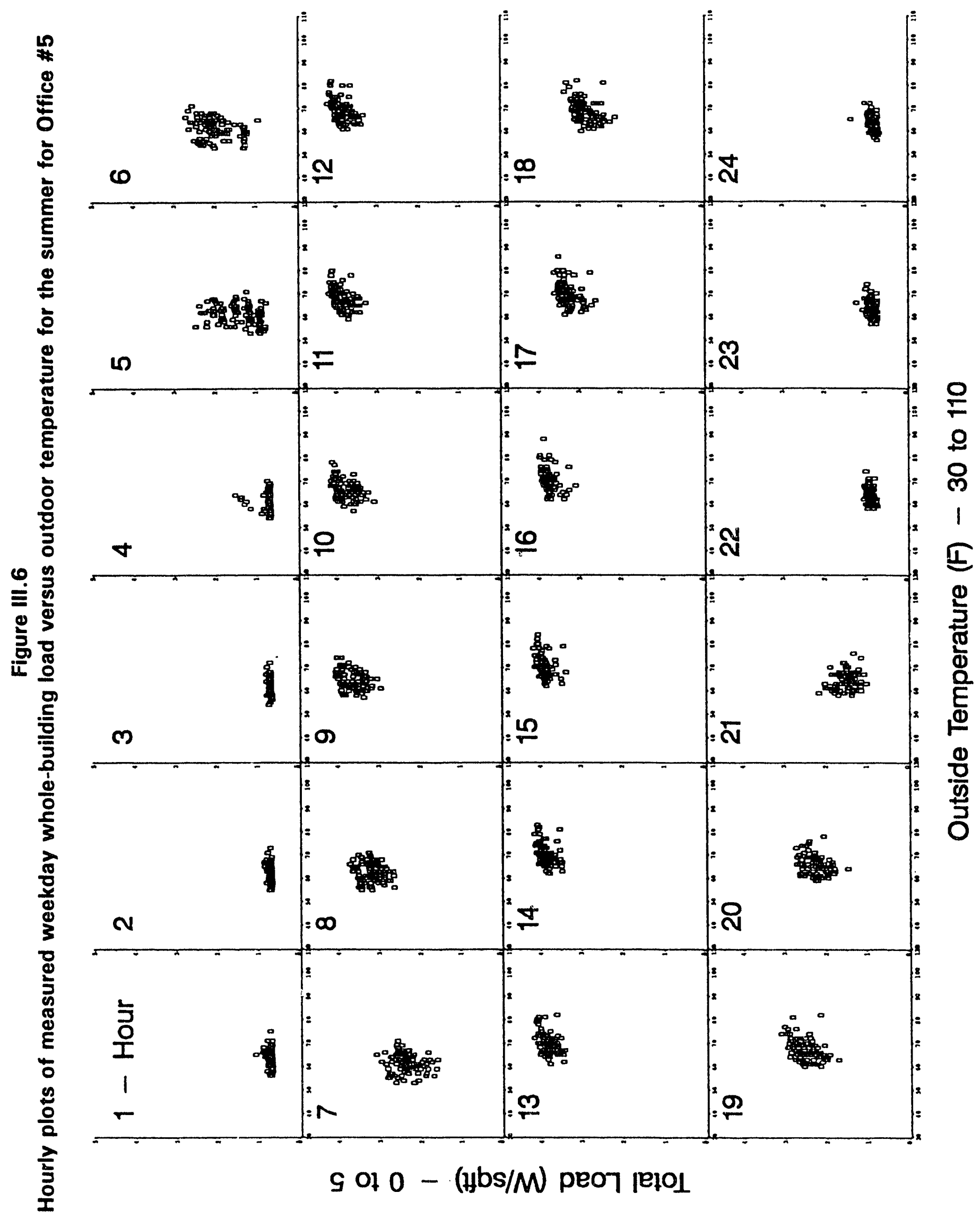




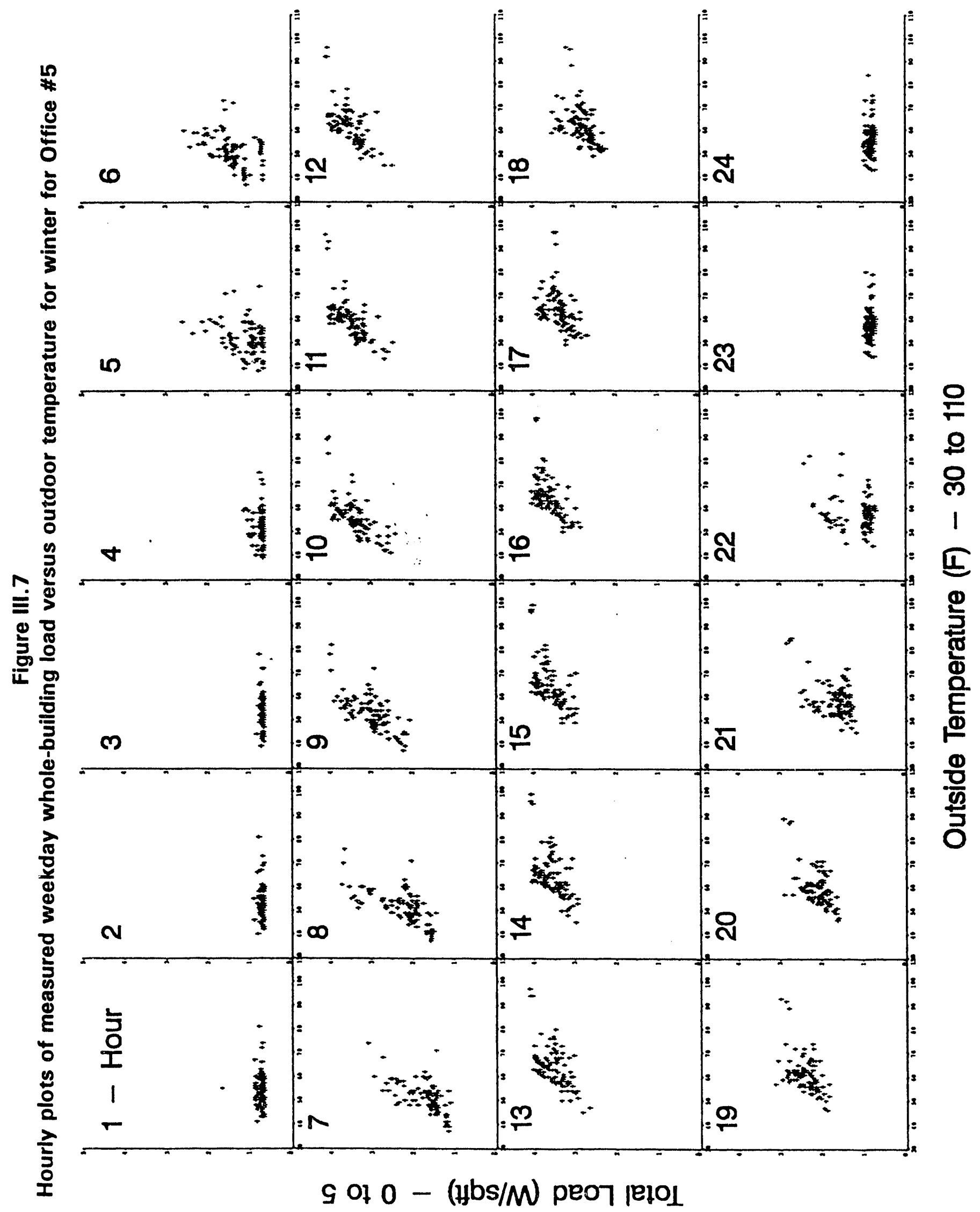




\begin{tabular}{|c|c|c|}
\hline \multirow{5}{*}{ 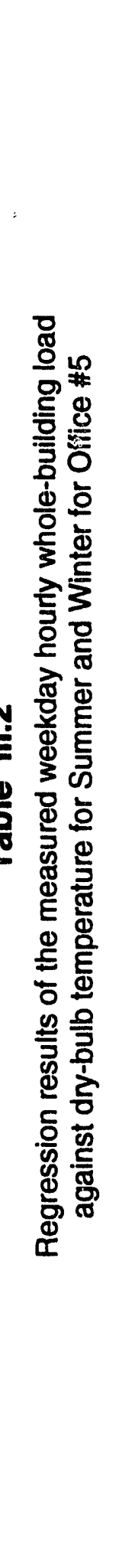 } & 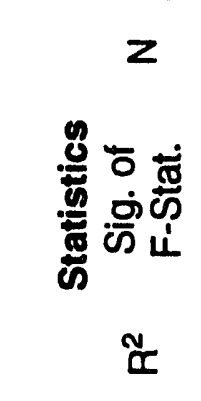 & 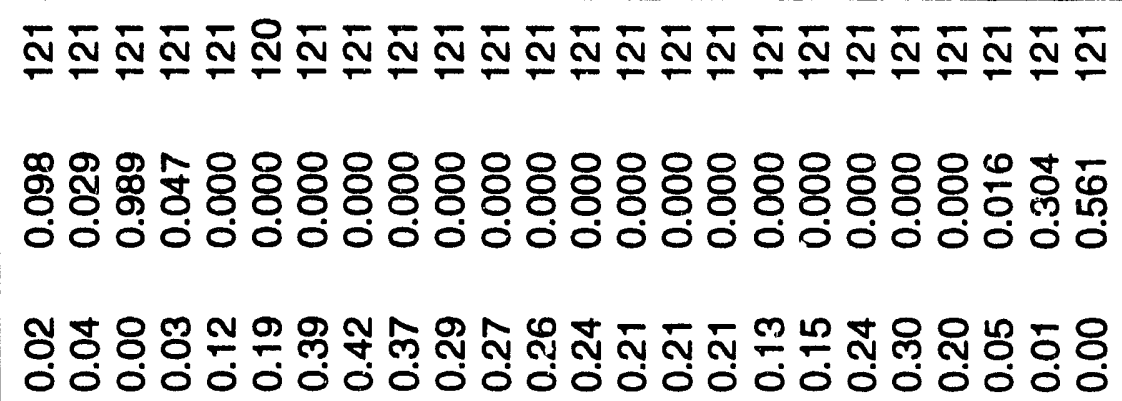 \\
\hline & 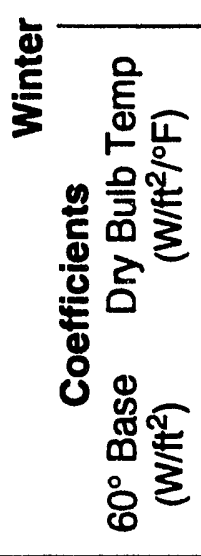 & 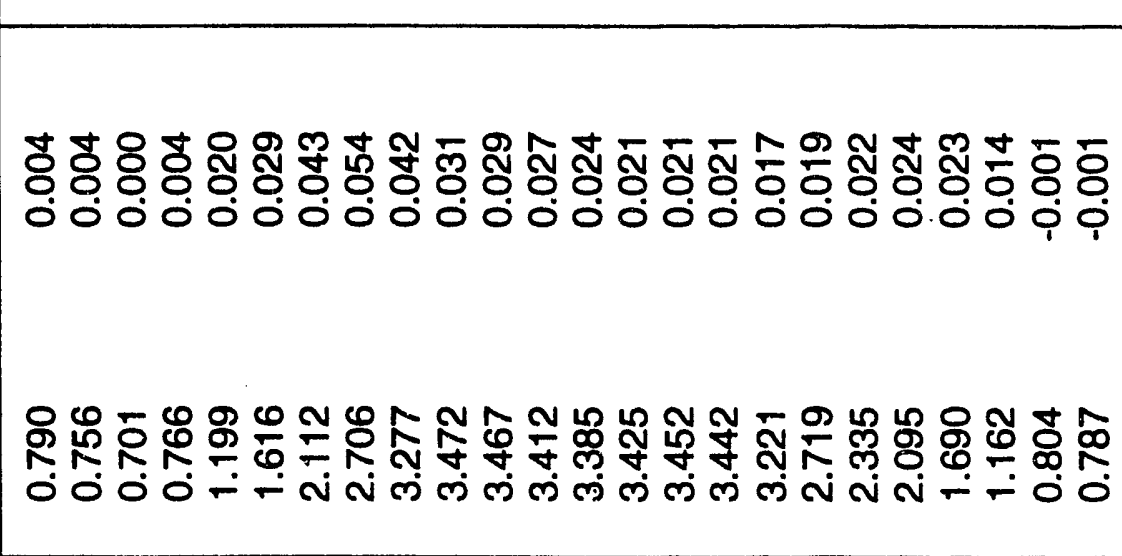 \\
\hline & 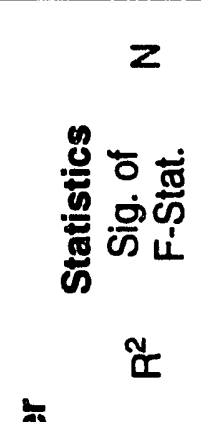 & 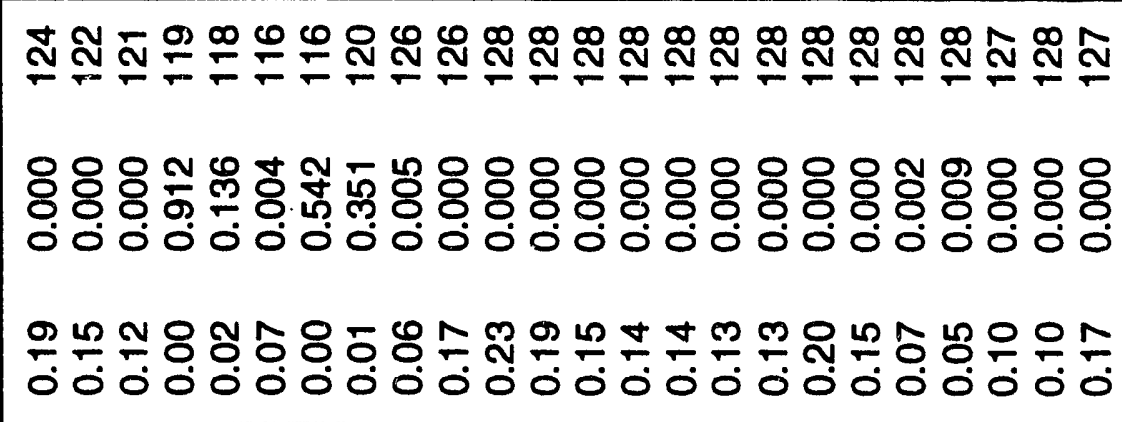 \\
\hline & 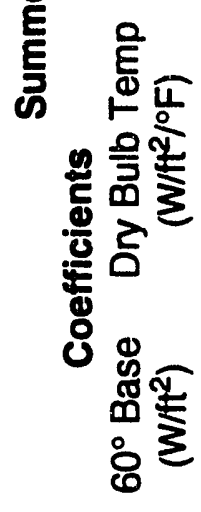 & 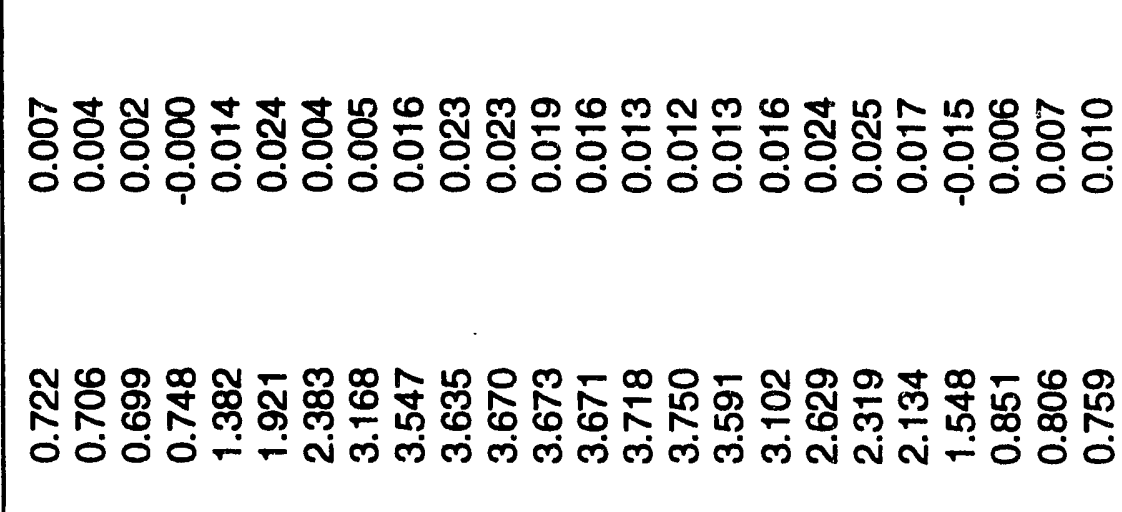 \\
\hline & 호ํ & 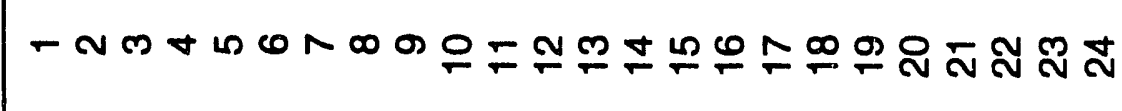 \\
\hline
\end{tabular}


In order to improve the statistics, we regressed the whole-building load against both drybulb temperature and relative humidity. The addition of relative humidity to the summer and winter regressions did not significantly improve the $\mathrm{R}^{2}$ (Table III.3). Regressions of the load against relative humidity alone indicated no correlation between these variables (Table III.4). Hence, for the remainder of this analysis, we only considered the variation of the whole-building load against the drybulb temperature (Table III.2).

To further investigate methods of improving the statistics of the load temperature correlations, we regressed the load against drybulb temperature using data for the entire year; see Table III.5. The $\mathbf{R}^{2}$ of the regressions improved significantly, particularly during the morning hours. Of special attention is the robustness of the base load at $60^{\circ} \mathrm{F}$ which for most part of the normal hours of the day is about $3.5 \mathrm{~W} / \mathrm{ft}^{2}$ (10 a.m. to 4 p.m.). We also regressed the total daily electricity use against the average daily drybulb and relative humidity. The results indicated that daily whole-building load is sensitive to drybulb temperature and not sensitive to relative humidity (Table III.6). The statistics for the daily correlations are much better than the hourly ones: The average daily electricity use at $60^{\circ} \mathrm{F}$ ambient temperature is about $54 \mathrm{Wh} / \mathrm{ft}^{2}$ and the daily electricity use increases by about $0.55 \mathrm{Wh} / \mathrm{ft}^{2}$ per degree $\mathrm{F}$. We will discuss the daily regressions in further detail in Chapter IV.

\section{EDA Output and Comparison with Monitored End Uses}

We performed a series of successive EDA runs to estimate the EUIs and LSs for the office building. The results of the runs as well as the summary of the monitored data and the DOE-2 simulations are summarized in Table III.7.

For comparing the EDA run results with measured data, we used the same end-use categories that are represented in the submetered data. The monitored data include channels for the HVAC end uses, indoor lighting, plug loads, and whole-building electricity use. Note that of the total $17.12 \mathrm{kWh} / \mathrm{ft}^{2}$ annual electricity use, the end-use monitored data account for 13.50 $\mathrm{kWh} / \mathrm{ft}^{2}$, leaving some $3.62 \mathrm{kWh} / \mathrm{ft}^{2}$ unaccounted for. For this reason, we combined the plug load and unaccounted for loads into the single class of miscellaneous load and used the miscellaneous load in the comparison. The first row of data in Table III.7 summarizes the measured end-use EUIs.

The second row of data indicates the annual electricity use of interior lighting and miscellaneous end uses as obtained from analysis of on-site survey information, and HVAC electricity use (using WYEC weather tapes) simulated using DOE-2. Although, at this stage we have not 


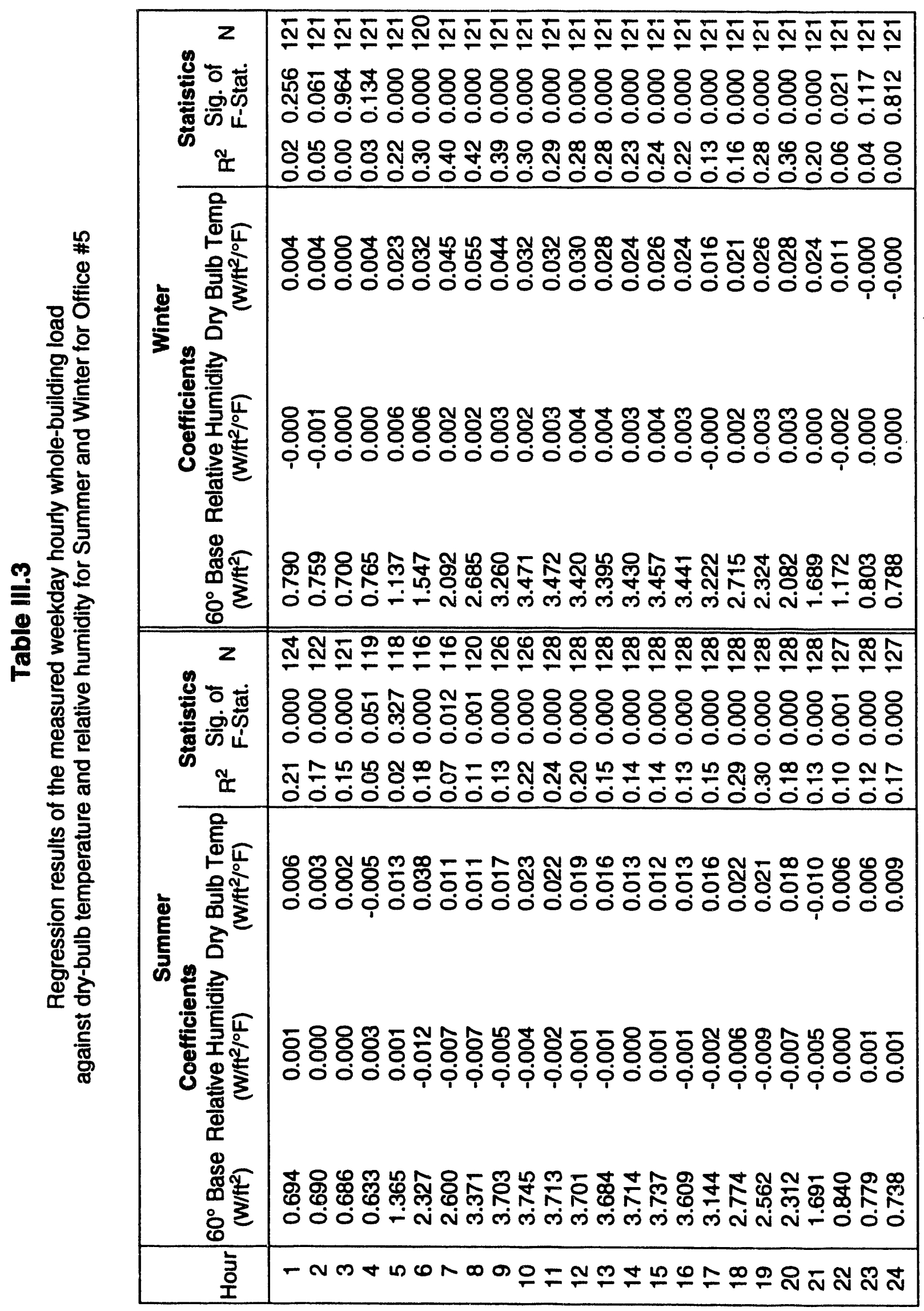




\begin{tabular}{|c|c|c|}
\hline \multirow{5}{*}{ 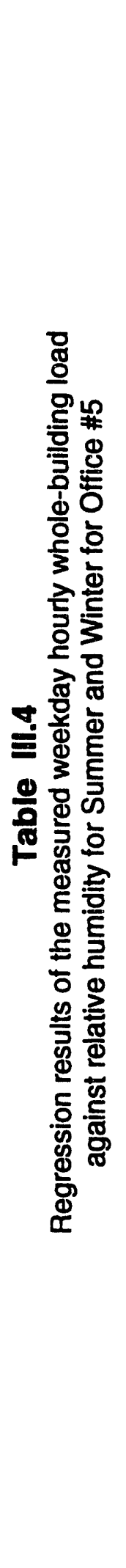 } & 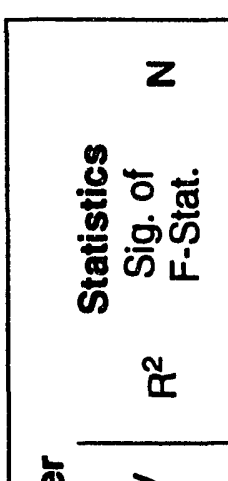 & 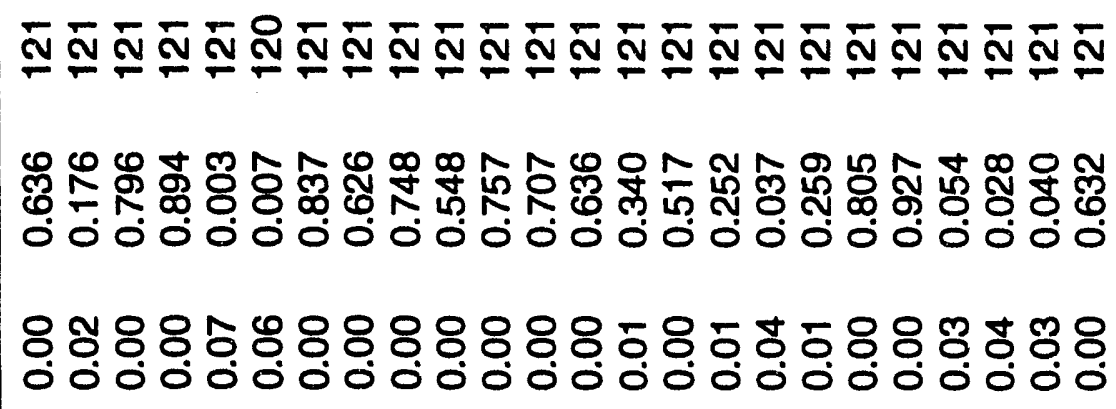 \\
\hline & 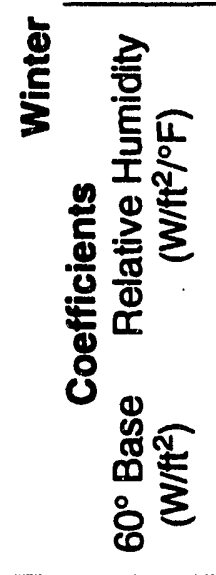 & 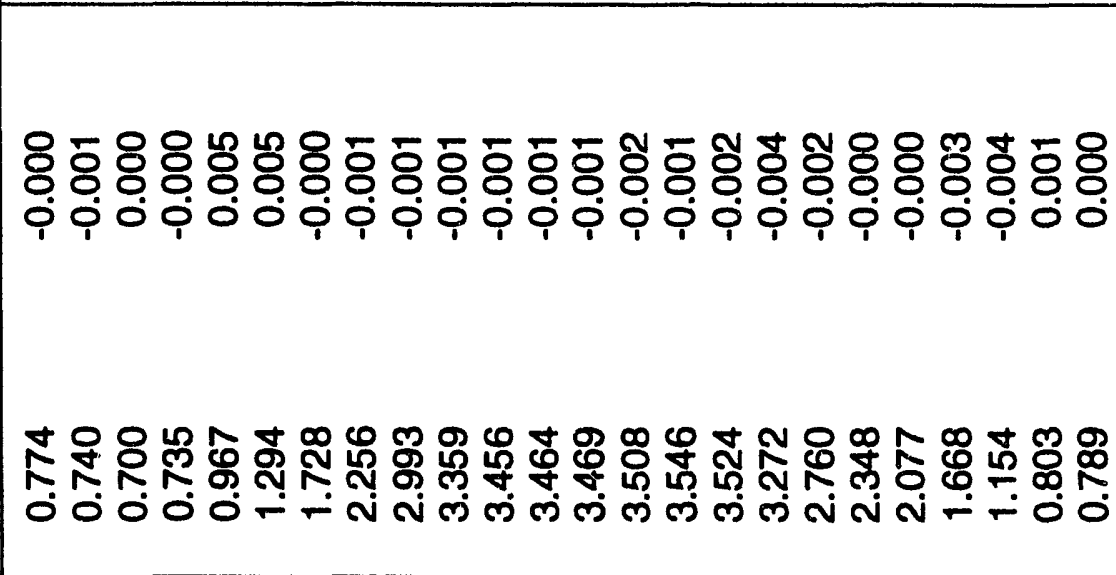 \\
\hline & 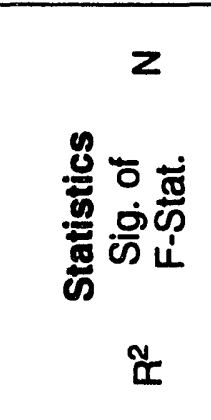 & 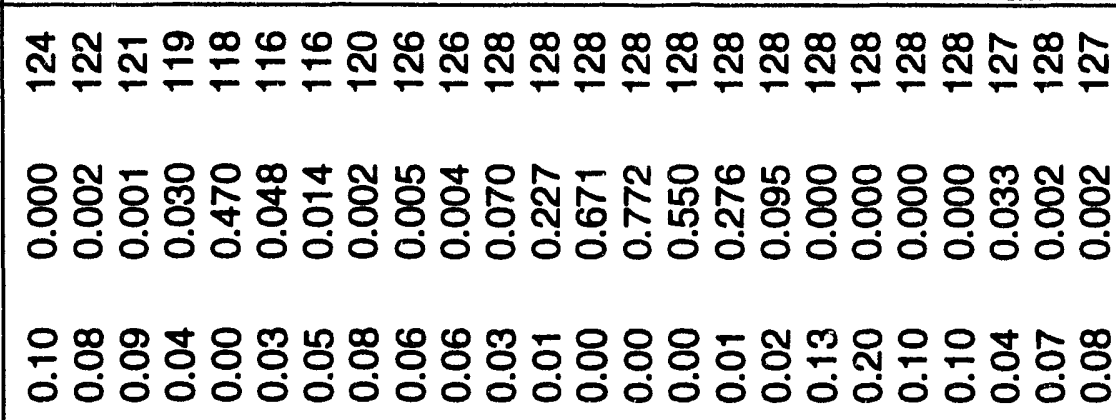 \\
\hline & 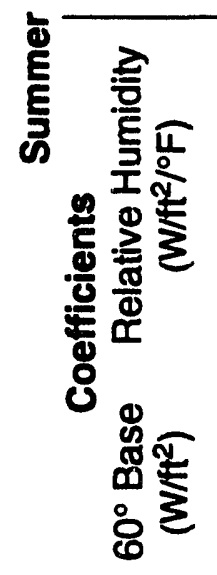 & 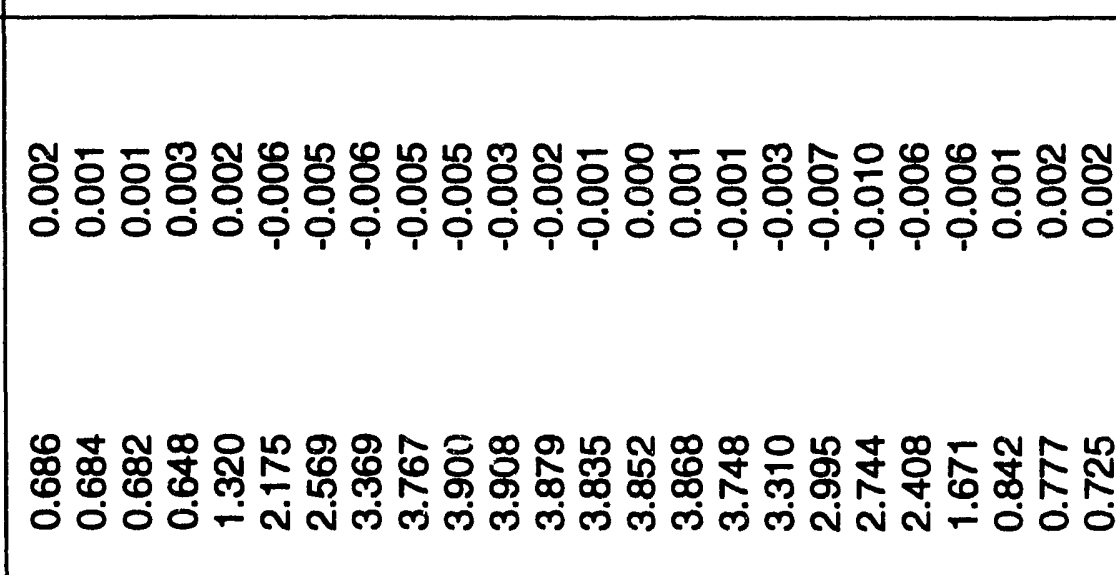 \\
\hline & 혼 & 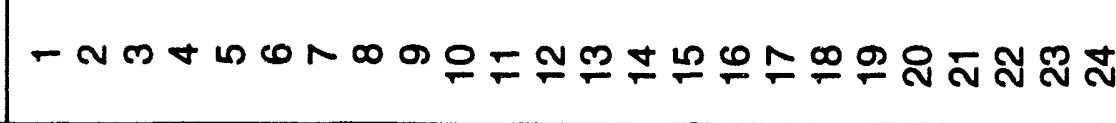 \\
\hline
\end{tabular}


Table III.5

Regression results of the measured weekday hourly whole-building load against dry-bulb temperature for the entire year for Office \#5

\begin{tabular}{|c|c|c|c|c|c|}
\hline \multirow[b]{2}{*}{ Hour } & \multicolumn{2}{|c|}{ Coefficients } & \multicolumn{3}{|c|}{ Statistics } \\
\hline & $\begin{array}{l}60^{\circ} \text { Base } \\
\left(\mathrm{W} / \mathrm{ft}^{2}\right)\end{array}$ & $\begin{array}{l}\text { Dry Bulb Temp } \\
\left(\mathrm{W} / \mathrm{ft}^{2} /{ }^{\circ} \mathrm{F}\right)\end{array}$ & $\mathbf{R}^{2}$ & $\begin{array}{l}\text { Sig. of } \\
\text { F-Stat. }\end{array}$ & $\mathbf{N}$ \\
\hline 1 & 0.758 & 0.002 & 0.01 & 0.163 & 246 \\
\hline 2 & 0.726 & 0.002 & 0.01 & 0.064 & 244 \\
\hline 3 & 0.703 & 0.000 & 0.01 & 0.098 & 243 \\
\hline 4 & 0.747 & 0.002 & 0.01 & 0.105 & 241 \\
\hline 5 & 1.306 & 0.026 & 0.20 & 0.000 & 240 \\
\hline 6 & 1.805 & 0.041 & 0.38 & 0.000 & 237 \\
\hline 7 & 2.225 & 0.047 & 0.51 & 0.000 & 238 \\
\hline 8 & 2.898 & 0.062 & 0.60 & 0.000 & 242 \\
\hline 9 & 3.362 & 0.046 & 0.53 & 0.000 & 248 \\
\hline 10 & 3.527 & 0.034 & 0.43 & 0.000 & 248 \\
\hline 11 & 3.529 & 0.033 & 0.41 & 0.000 & 250 \\
\hline 12 & 3.483 & 0.031 & 0.37 & 0.000 & 250 \\
\hline 13 & 3.462 & 0.028 & 0.33 & 0.000 & 250 \\
\hline 14 & 3.503 & 0.025 & 0.30 & 0.000 & 250 \\
\hline 15 & 3.529 & 0.025 & 0.29 & 0.000 & 250 \\
\hline 16 & 3.473 & 0.021 & 0.25 & 0.000 & 250 \\
\hline 17 & 3.183 & 0.013 & 0.10 & 0.000 & 250 \\
\hline 18 & 2.700 & 0.019 & 0.18 & 0.000 & 250 \\
\hline 19 & 2.335 & 0.023 & 0.25 & 0.000 & 250 \\
\hline 20 & 2.099 & 0.023 & 0.26 & 0.000 & 250 \\
\hline 21 & 1.558 & 0.004 & 0.01 & 0.158 & 250 \\
\hline 22 & 1.000 & -0.001 & 0.00 & 0.666 & 249 \\
\hline 23 & 0.820 & 0.002 & 0.02 & 0.018 & 250 \\
\hline 24 & 0.791 & 0.001 & 0.01 & 0.108 & 249 \\
\hline
\end{tabular}


Table III.6

Regression results of the measured weekday daily whole-building load against dry-bulb temperture for Office \#5

\begin{tabular}{|l|ccc|ccc|}
\hline Season & $\begin{array}{c}30^{\circ} \text { Base } \\
\left(\mathrm{W} / \mathrm{ft}^{2}\right)\end{array}$ & $\begin{array}{c}\text { Coefficients } \\
\text { Relative Humidity } \\
\left(\mathrm{W} / \mathrm{ft}^{2} /{ }^{\circ} \mathrm{F}\right)\end{array}$ & $\begin{array}{c}\text { Dry Bulb Temp } \\
\left(\mathrm{W} / \mathrm{ft}^{2} /{ }^{\circ} \mathrm{F}\right)\end{array}$ & $\mathrm{R}^{2}$ & $\begin{array}{c}\text { Statistics } \\
\text { Sig. of } \\
\text { F-Stat. }\end{array}$ & $\mathrm{N}$ \\
\hline Summer & 55.455 & - & 0.320 & 0.16 & 0.000 & 110 \\
Winter & 53.080 & - & 0.557 & 0.38 & 0.000 & 121 \\
Year $^{1}$ & 53.634 & - & 0.573 & 0.48 & 0.000 & 232 \\
Year $^{1}$ & 53.892 & - & 0.550 & 0.58 & 0.000 & 230 \\
Summer & 58.649 & -0.062 & - & 0.05 & 0.014 & 110 \\
Winter & 51.565 & -0.017 & - & 0.00 & 0.568 & 121 \\
Summer & 57.791 & -0.113 & 0.436 & 0.32 & 0.000 & 110 \\
Winter & 52.941 & 0.054 & 0.606 & 0.40 & 0.000 & 121 \\
Year $^{1}$ & 53.325 & 0.021 & 0.562 & 0.48 & 0.000 & 232 \\
Year ${ }^{2}$ & 53.636 & 0.018 & 0.541 & 0.59 & 0.000 & 230 \\
\hline
\end{tabular}

1. Excluding two outliers. 


\section{Table III.7}

Comparison of the EDA estimated EUIs with measured EUIs

for Office \#5

\begin{tabular}{|c|c|c|c|c|c|}
\hline \multirow[b]{2}{*}{ Run } & \multicolumn{4}{|c|}{ EUI $\left(\mathrm{kWh} / \mathrm{ft}^{2} / \mathbf{y r}\right)$} & \multirow[b]{2}{*}{ Total } \\
\hline & HVAC & $\begin{array}{c}\text { Indoor } \\
\text { Lighting }\end{array}$ & Plugs & $\begin{array}{l}\text { Miscellaneous } \\
\text { (Including Plugs) }\end{array}$ & \\
\hline Monitored & 6.93 & 4.42 & 2.15 & 5.78 & 17.12 \\
\hline DOE-2 estimate with WYEC weather & 8.36 & 4.62 & & 0.99 & 13.97 \\
\hline DOE-2 with 1989 weather & 7.99 & 4.62 & & 0.99 & 13.60 \\
\hline EDA with cooling based on regression of total load & 7.73 & 5.63 & & 3.73 & 17.09 \\
\hline EDA with cooling based on DOE-2 estimates & 5.69 & 6.87 & & 4.38 & 16.94 \\
\hline
\end{tabular}


used the monitored data in the EDA analysis, note that there is a significant difference of miscellaneous electricity use between the monitored data and on-site survey information. In the third row of the table we have estimated the 1989 HVAC electricity use from the DOE-2 simulation results of the second row. The hourly information on the third row is used as the initial condition for EDA. Figure III.8 shows the simulated DOE-2 end-use load shape for the building peak day. In these simulations, based on the analysis of the measured whole-building load, we have extended the hours of operation of HVAC systems from 7 a.m. to 6 p.m. to 5 a.m. to 8 p.m. Note that the simulated peak cooling load occur at 5 a.m. to cool down the building from the last night accumulated heat.

The results of applying EDA to the DOE-2 simulated initial conditions are summarized in rows 4 and 5. In the EDA process we have used the annual regressions of whole-building hourly loads. Row 4 provides end-use EUIs with all end uses at all hours having equal weight. Comparing the results with the monitored data indicate that the lighting and HVAC EUIs are overestimated by about $27 \%$ and $12 \%$, respectively. The miscellaneous EUI is underestimated by $5 \%$. The majority of the discrepancy for the lighting and miscellaneous EUIs initiate from a illdefined, initial condition miscellaneous end use. Recall that EDA relies entirely on the initial condition information in apportioning loads between non-HVAC (i.e., lighting and miscellaneous) end uses. In an attempt to improve the EUIs for lighting and miscellaneous, we repeated the EDA calculation using varying weight for different end uses (row 5). We used higher weights for lighting based on the assumption that the on-site survey information are probably more accurate for the interior lighting than miscellaneous end uses. The weighting only slightly modified the resulting EUIs and LSs. We also performed another EDA run, using the DOE-2 simulation results as HVAC initial conditions (row 5). The result was an underestimation of HVAC and miscellaneous EUIs by $18 \%$ and $24 \%$ respectfully. The lighting EUI was over estimated by $55 \%$.

In order to gain insight into the differences between the EDA results and the monitored data, in Figures III.9 and III.10, we compare the average hourly energy use load shapes of the monitored data with those estimated by EDA. Figure III.9 corresponds to EDA simulations with cooling based on regression of total hoad and Figure III.10 is for EDA simulations with cooling based on DOE-2 estimates. The differences between the EDA estimates and measured data are plotted in Figures III.11 and III.12. 


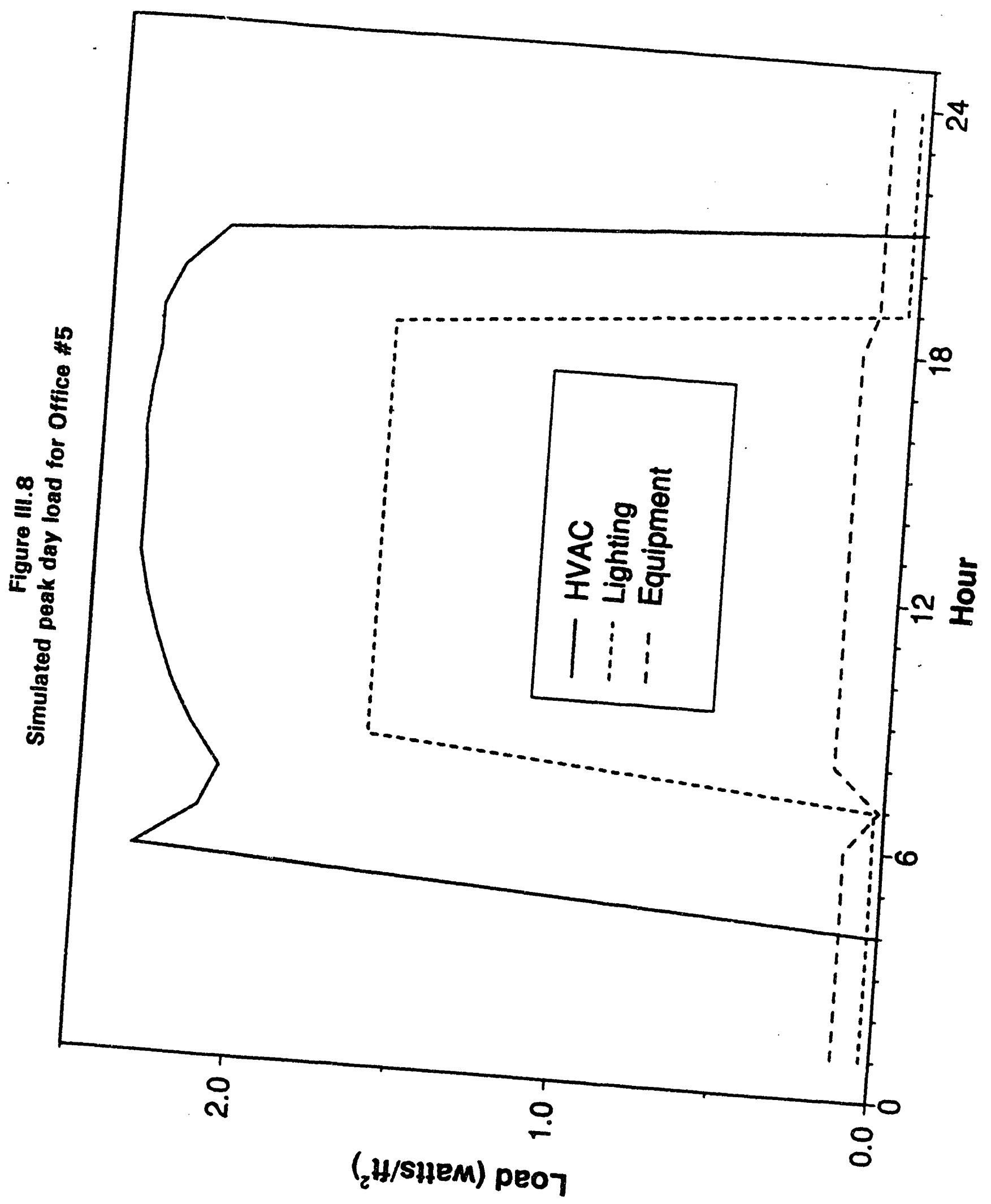




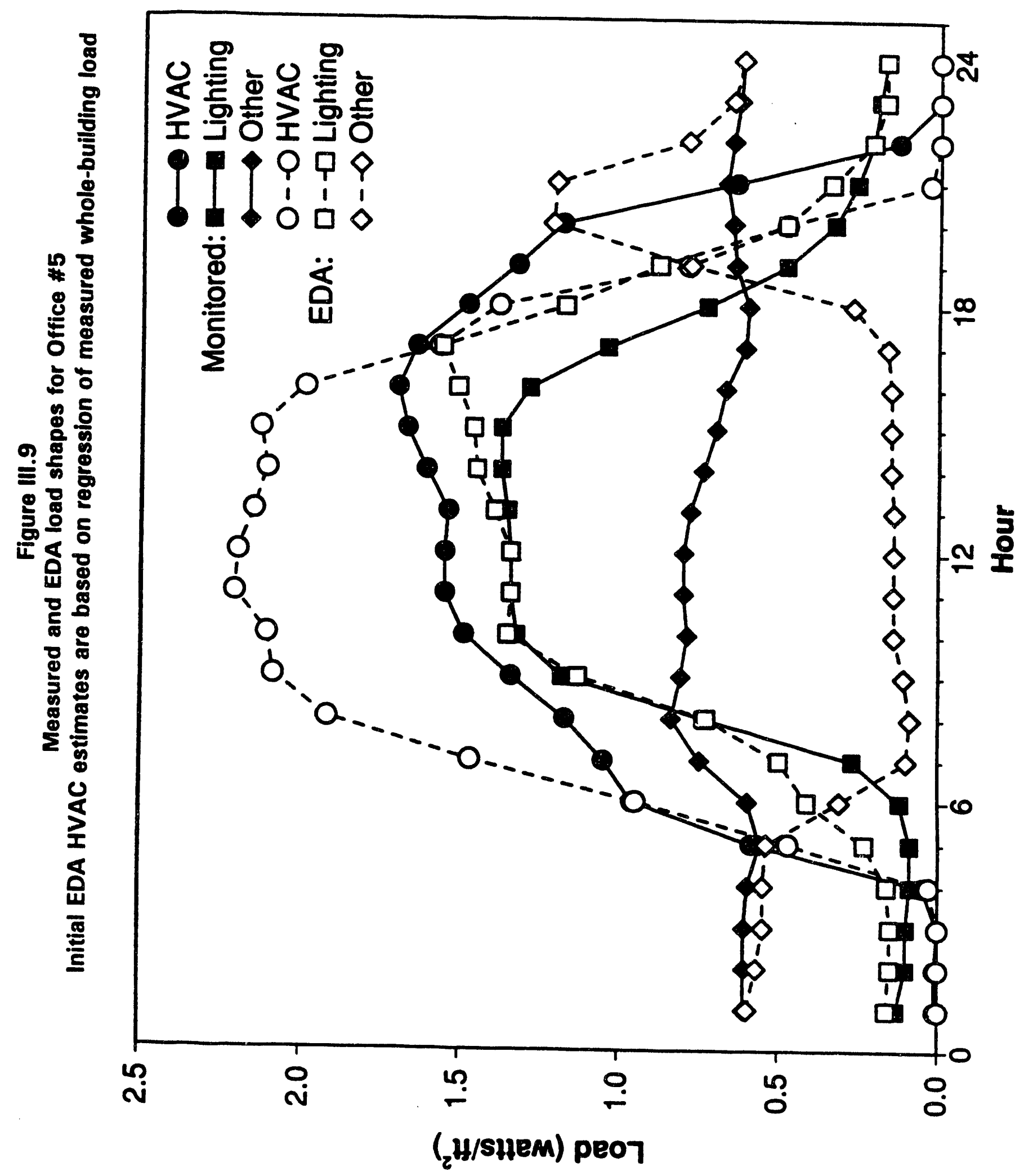




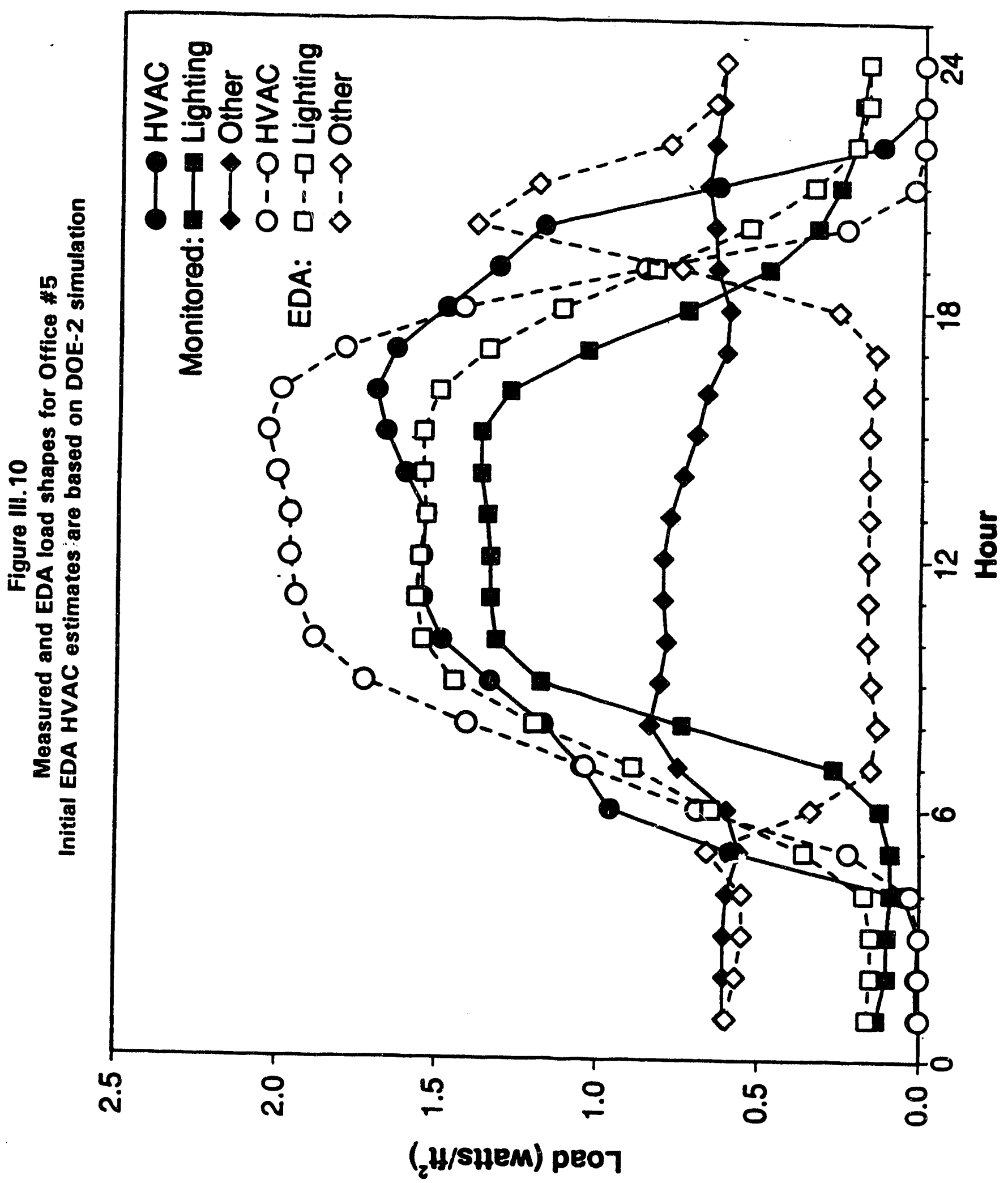




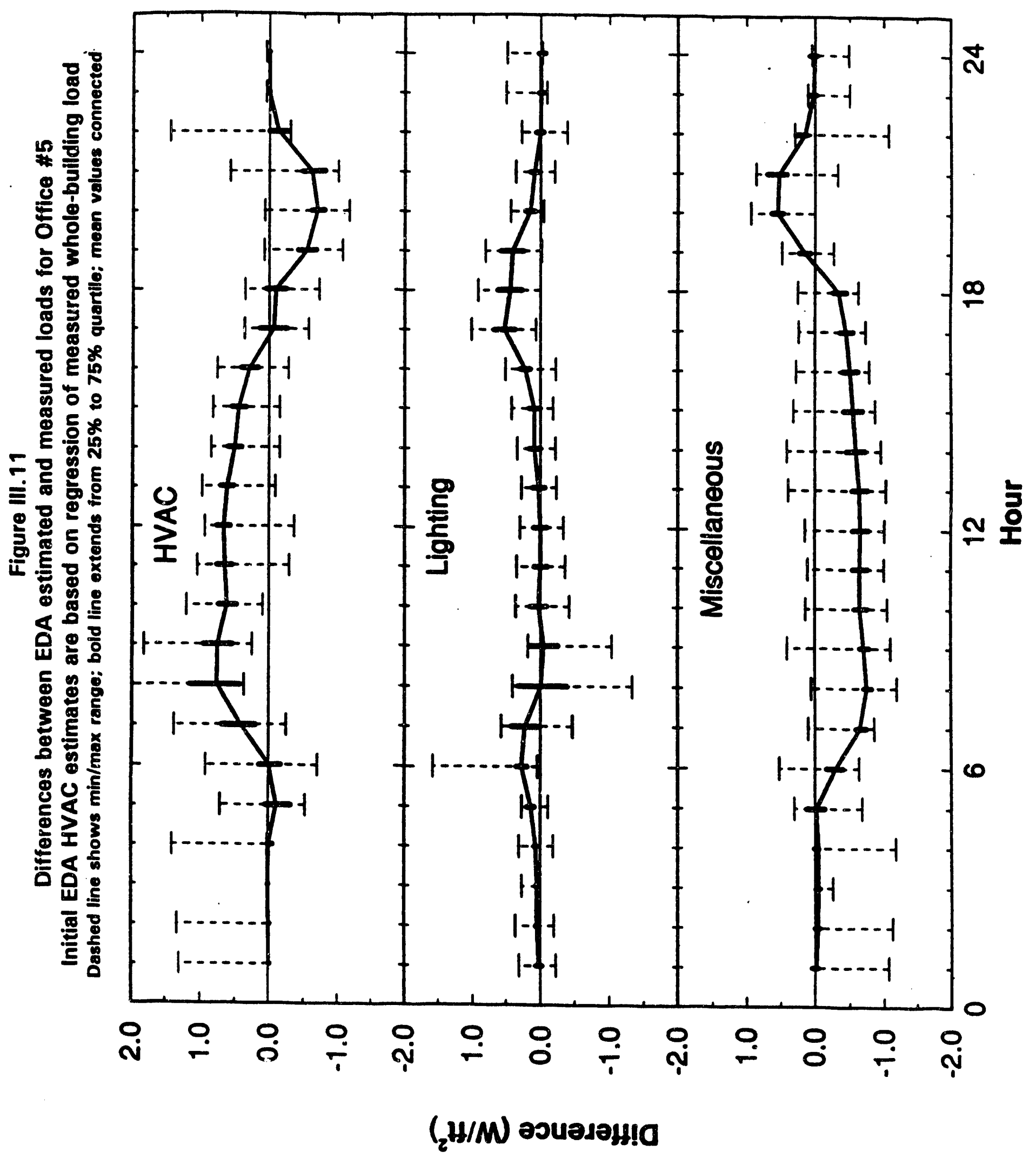




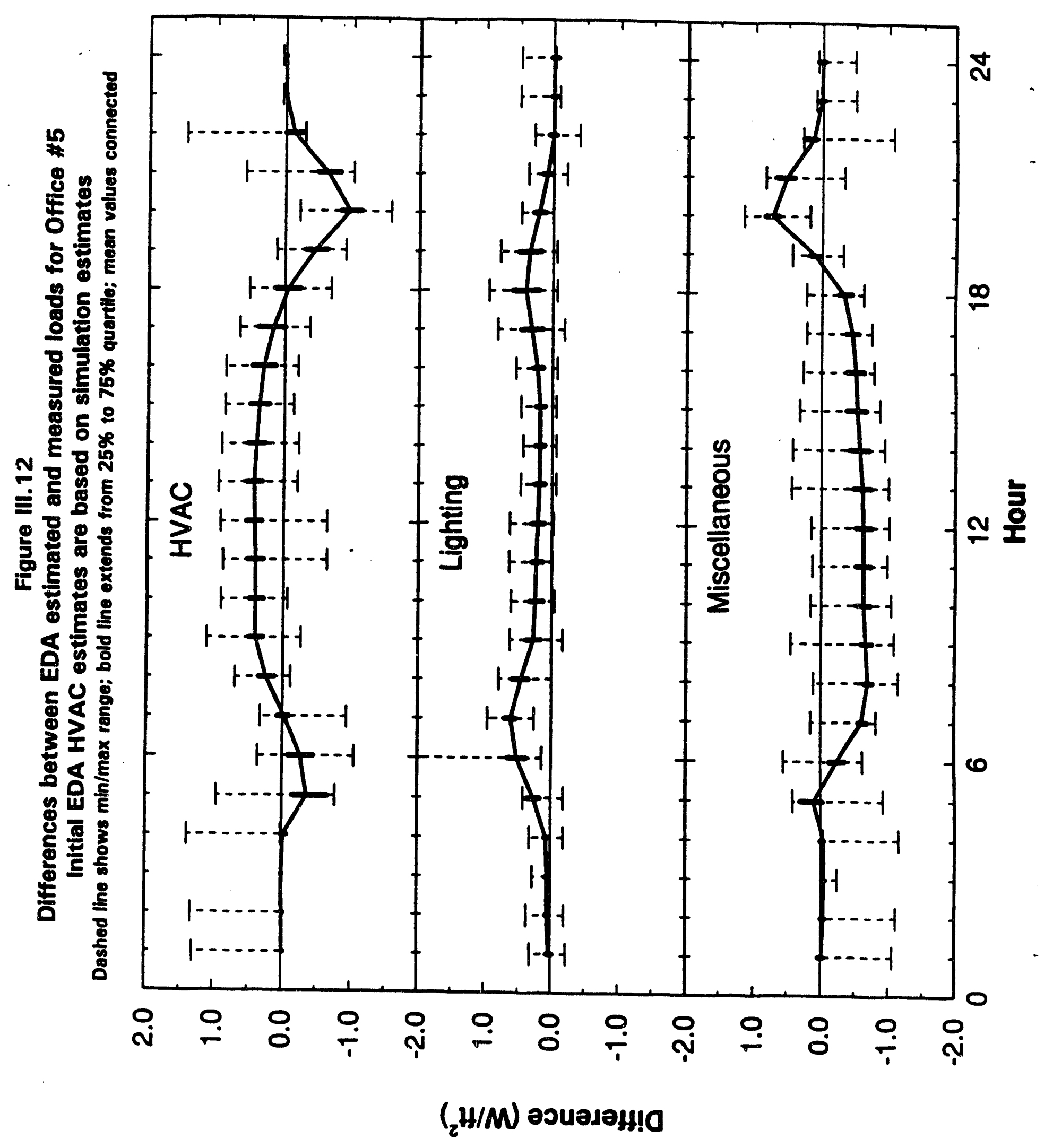


In inspecting these figures, two points become evident. First, during the night, estimates of electricity use by EDA for all end uses agree very well with those of the measured data. This is no surprise, since the initial conditions for air-conditioning electricity use during the night is zero. When the HVAC energy equals zero, EDA allocates the difference between the measured and estimated whole-building load to the end uses, namely miscellaneous and indoor lighting. During the day, when the air conditioning is an end use, most of the differences between the measured whole-building load and the simulated initial conditions is allocated to the lighting and air conditioning. In fact, there is a consistent positive bias in EDA's estimates of air conditioning at the expense of miscellaneous end use. The EDA-estimated miscellaneous end-use intensity drops from the nighttime levels of approximately $0.6 \mathrm{~W} / \mathrm{ft}^{2}$ to daytime levels of approximately $0.1 \mathrm{~W} / \mathrm{ft}^{2}$.

Clearly, the EDA-estimated end-use load shape for the miscellaneous end use is in contradiction with the audit information (see Figure III.8). The audit estimates for the miscellaneous end use electricity consumption is about $0.2 \mathrm{~W} / \mathrm{ft}^{2}$, during the day, and $0.15 \mathrm{~W} / \mathrm{ft}^{2}$ during the night. There are two ways to adjust this inconsistency in miscellaneous load shape: either by reducing the nighttime level miscellaneous load (resulting in an increase in nighttime lighting and HVAC usage) or by increasing the daytime level (resulting in a decrease in daytime lighting and HVAC usage). Without comparison with the measured data, it is hard to decide what to do. The measured data however indicate that the nighttime miscellaneous electricity use is probably correct and the daytime use should be increased. If we assume that the nighttime miscellaneous electricity use is indeed a lower estimate of the hourly electricity use for this end use, we can develop a new set of initial conditions for EDA simulations and repeat the calculation.

\section{Retail building \# 1}

Building Description

This $23,396 \mathrm{ft}^{2}$ building is a one story one-tenant retail store built in 1960 and located in Tustin (inland valley south of Los Angeles) (SCE 1990,1989). The entire store is conditioned (both heated and cooled); about $89 \%$ of the building is used for retail space and $11 \%$ as conditioned storage area. All building systems are electric.

The maximum occupancy of the building is 6 staff and 10 customers. The store is operated Monday through Saturday from 9:00 a.m. to 9:30 p.m. and Sunday from 10:00 a.m. to 7:00 p.m. Most interior lights ( $94 \%$ by installed capacity) are fluorescent but there are some incandescent lights as well $(6 \%)$. The total installed lighting intensity is $2.09 \mathrm{~W} / \mathrm{ft}^{2}$, operated by central 
switches with the same hours of operation as the store. The outdoor lighting is fluorescent and mercury vapor with an intensity of about $0.14 \mathrm{~W} / \mathrm{ft}^{2}$ operated from 6 p.m. to 6 a.m., every day. The audit of the building also indicates that the outdoor lighting is controlled by photocells. For our simulations, we rely on the fixed hours of outdoor lighting operation. The installed intensity of other building loads (refrigerator, drinking fountain, instantaneous domestic hot water heaters, cash register, microwave oven, coffee pot, etc.) is estimated at $0.37 \mathrm{~W} / \mathrm{ft}^{2}$.

The building has six single-zone heat-pump units. The total heating and cooling capacities are $816 \mathrm{kBtu} / \mathrm{h}$ and 65 tons, respectively. The total electricity load for all HVAC equipment is about $122 \mathrm{~kW}$. All heat-pump units have economizers. The heating and cooling set point is $66^{\circ} \mathrm{F}$ and $72^{\circ} \mathrm{F}$, respectively. The systems are operated during business hours and controlled by time clocks.

\section{DOE-2 Simulation}

We used information provided to us by the hardcopy of the audit (SCE 1989) and the 1985 on-site survey data to simulate the energy use of the building with DOE-2. Like the office building, the audit data provided information on the operation, schedule, equipment type, and equipment energy use for the building. The audit did not provide information on the architectural and construction characteristics of the building.

We simulated the building, with a square floor plan and a total conditioned floor area of $23,396 \mathrm{ft}^{2}$. The building was simulated with six single-zone heat-pump units. The internal load of the building was calculated using the methodology discussed in Akbari et al. (1989).

The DOE-2 simulations were performed using the Los Angeles WYEC weather tape and the resulting hourly HVAC energy use was regressed against the WYEC drybulb temperature. The resulting correlations were then used in conjunction with actual 1989-1990 hourly drybulb temperatures to estimate the hourly cooling energy use for the period of June 1, 1989 to May 31 , 1990.

The simulated annual HVAC, lighting, and other miscellaneous loads are 2.76, 6.91, and $0.28 \mathrm{kWh} / \mathrm{ft}^{2}$, respectively. Note that the simulated whole-building energy use of $9.95 \mathrm{kWh} / \mathrm{ft}^{2}$ is within $10 \%$ of the $9.06 \mathrm{kWh} / \mathrm{ft}^{2}$ measured energy use for this office building. EDA will adopt the characteristics of the measured whole-building load in the reconciliation process (See Figure III.13).

Contrary to the office building, the hourly plot of the simulated HVAC electricity use does indicate a base load (See Figure III.14). This is consistent with the observed characteristics of 


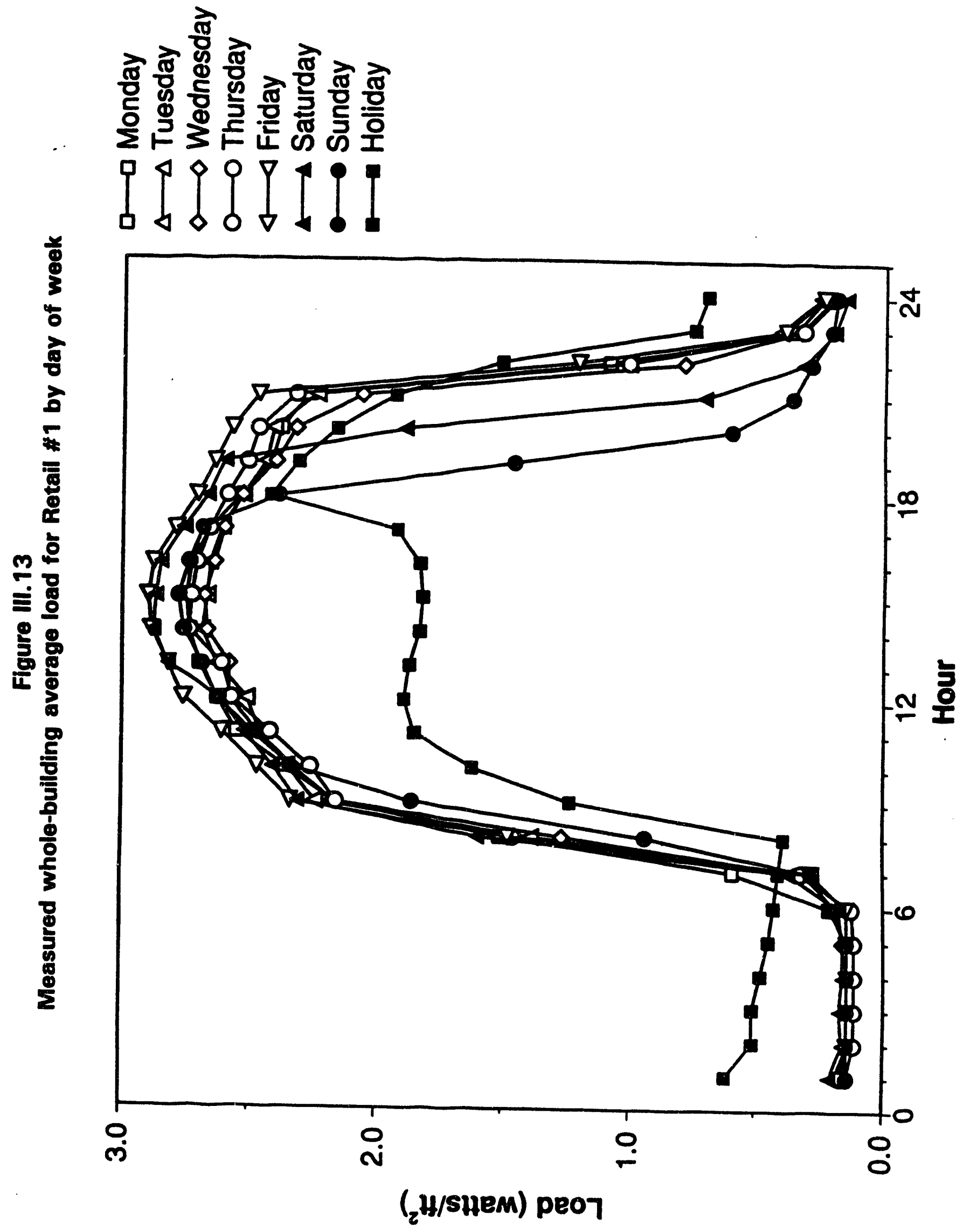




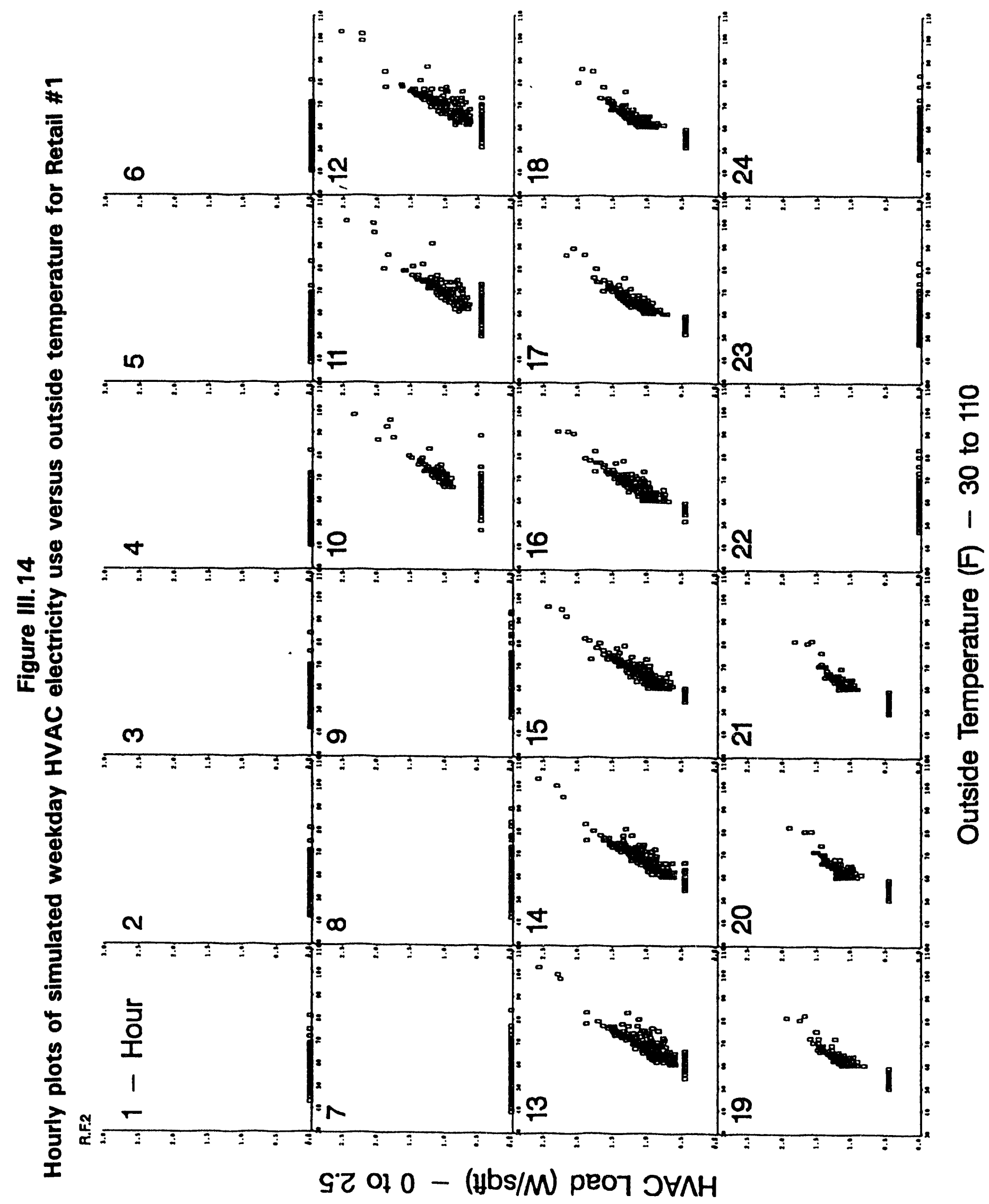


the measured data as are discussed in the next section. The regression results of the simulated hourly HVAC load against the WYEC drybulb temperatures are presented in Table III.8. Using these correlations and the measured drybulb temperatures, we calculated new estimates of the HVAC electricity end use. For a typical summer day, Figure III.15 compares the new estimate of simulated HVAC electricity use for the 1989-1990 temperature data with the original DOE-2 results, using WYEC weather data. This process modified the simulated annual HVAC energy use from 2.76 to $3.67 \mathrm{kWh} / \mathrm{ft}^{2}$.

Load/Temperature Regressions

Figures III.16-III.18 show the yearly, summer, and winter plots of hourly whole-building load against the ambient drybulb temperatures. The summer and winter peric $d$ are defined as May 1 to October 31 and November 1 to April 30, respectively. These figures indicate a few interesting points that warrant further discussions. Judging from the observed load data, the normal hours of operation are 9 a.m. to 9 p.m. (consistent with the audit information). There are some occasional activities during the early morning hours of 7 and $8 \mathrm{a} . \mathrm{m}$. and late evening hours of 10 and 11 p.m. The building load during the nighttime hours (12 p.m. to 6 a.m.) is almost flat at $0.2 \mathrm{~W} / \mathrm{ft}^{2}$.

The summer data does not show a clear base load, but both the winter data and the annual data indicate a flat base load for temperature conditions approximately below $65^{\circ} \mathrm{F}$. The base load varies by hour of day; for normal operating conditions it is between 2.0 to $2.2 \mathrm{~W} / \mathrm{ft}^{2}$. The audit of the building does not indicate any electricity use during the night. But the measured data indicate electricity use of about $0.2 \mathrm{~W} / \mathrm{ft}^{2}$ which is probably for the nighttime emergency indoor lighting and other miscellaneous equipment (identified or not identified in the audit).

We regressed the whole-building hourly load against drybulb temperature. Table III.9 shows the summary of regression statistics of the whole-building load versus drybulb temperature, for the entire year (summer and winter included). The table indicates that significant correlations for most hours of the day ( 8 a.m. to 10 p.m.) exist. The base load electricity use at $60^{\circ} \mathrm{F}$ varies between 1.9 to $2.2 \mathrm{~W} / \mathrm{ft}^{2}$, and the regression coefficients for drybulb temperature mostly are between 0.04 to $0.05 \mathrm{~W} / \mathrm{ft}^{2} / \mathrm{F}$. During hour 9 a.m., the drybulb temperature sensitivity of the hourly load is higher by $30 \%$; this may be only a statistical fluke.

The regression $\mathrm{R}^{2}$ values for all hours are in general better than those for the office building and are in the range of 0.3 to 0.7 . To try to improve the statistics, we regressed the whole- 
Table III.8

Regression results of the simulated weekday hourly HVAC load against dry-bulb temperature for Retail \#1

\begin{tabular}{|c|c|c|c|c|c|}
\hline \multirow[b]{2}{*}{ Hour } & \multicolumn{2}{|c|}{ Coefficients } & \multicolumn{3}{|c|}{ Statistics } \\
\hline & $\begin{array}{l}60^{\circ} \text { Base } \\
\left(\mathrm{w} / \mathrm{ft}^{2}\right)\end{array}$ & $\begin{array}{c}\text { Dry Bulb Temp } \\
\left(\mathrm{W} / \mathrm{ft}^{2} /{ }^{\circ} \mathrm{F}\right)\end{array}$ & $\mathbf{R}^{2}$ & $\begin{array}{l}\text { Sig. of } \\
\text { F-Stat. }\end{array}$ & $\mathbf{N}$ \\
\hline 1 & 0.000 & 0.000 & 0.00 & 0.000 & 96 \\
\hline 2 & 0.000 & 0.000 & 0.00 & 0.000 & 94 \\
\hline 3 & 0.000 & 0.000 & 0.00 & 0.000 & 90 \\
\hline 4 & 0.000 & 0.000 & 0.00 & 0.000 & 85 \\
\hline 5 & 0.000 & 0.000 & 0.00 & 0.000 & 84 \\
\hline 6 & 0.000 & 0.000 & 0.00 & 0.000 & 90 \\
\hline 7 & 0.000 & 0.000 & 0.00 & 0.000 & 102 \\
\hline 8 & 0.000 & 0.000 & 0.00 & 0.000 & 123 \\
\hline 9 & 0.000 & 0.000 & 0.00 & 0.000 & 156 \\
\hline 10 & -0.107 & 0.049 & 0.68 & 0.000 & 189 \\
\hline 11 & -0.008 & 0.048 & 0.71 & 0.000 & 210 \\
\hline 12 & 0.067 & 0.047 & 0.72 & 0.000 & 218 \\
\hline 13 & 0.150 & 0.046 & 0.77 & 0.000 & 224 \\
\hline 14 & 0.246 & 0.046 & 0.82 & 0.000 & 224 \\
\hline 15 & 0.341 & 0.045 & 0.84 & 0.000 & 223 \\
\hline 16 & 0.430 & 0.044 & 0.84 & 0.000 & 205 \\
\hline 17 & 0.487 & 0.045 & 0.85 & 0.000 & 172 \\
\hline 18 & 0.557 & 0.042 & 0.78 & 0.000 & 150 \\
\hline 19 & 0.581 & 0.040 & 0.75 & 0.000 & 139 \\
\hline 20 & 0.591 & 0.036 & 0.72 & 0.000 & 131 \\
\hline 21 & 0.596 & 0.033 & 0.76 & 0.000 & 123 \\
\hline 22 & 0.000 & 0.000 & 0.00 & 0.000 & 112 \\
\hline 23 & 0.000 & 0.000 & 0.00 & 0.000 & 110 \\
\hline 24 & 0.000 & 0.000 & 0.00 & 0.000 & 105 \\
\hline
\end{tabular}


Table III.9

Regression results of the measured weekday hourly whole-building load against dry-bulb temperature for the entire year for Retail \#1

\begin{tabular}{|c|cc|ccc|}
\hline & \multicolumn{3}{|c|}{ Coefficients } & \multicolumn{3}{c|}{ Statistics } \\
Hour & $\begin{array}{c}60^{\circ} \text { Base } \\
\left(\mathrm{W}^{\prime} \mathrm{ft}^{2} \text { ) }\right.\end{array}$ & $\begin{array}{c}\text { Dry Bulb Temp } \\
\left.\text { (W/ft }{ }^{2} /{ }^{\circ} \mathrm{F}\right)\end{array}$ & $\mathrm{R}^{2}$ & $\begin{array}{c}\text { Sig. of } \\
\text { F-Stat. }\end{array}$ & $\mathrm{N}$ \\
\hline 1 & 0.193 & 0.003 & 0.01 & 0.315 & 109 \\
2 & 0.169 & 0.004 & 0.03 & 0.104 & 99 \\
3 & 0.182 & 0.001 & 0.00 & 0.727 & 90 \\
4 & 0.161 & 0.004 & 0.03 & 0.107 & 80 \\
5 & 0.184 & 0.001 & 0.00 & 0.723 & 78 \\
6 & 0.243 & -0.007 & 0.01 & 0.319 & 75 \\
7 & 0.492 & -0.003 & 0.00 & 0.851 & 75 \\
8 & 1.590 & 0.054 & 0.19 & 0.000 & 86 \\
9 & 2.180 & 0.069 & 0.40 & 0.000 & 121 \\
10 & 2.147 & 0.046 & 0.31 & 0.000 & 149 \\
11 & 2.185 & 0.039 & 0.33 & 0.000 & 190 \\
12 & 2.126 & 0.042 & 0.37 & 0.000 & 201 \\
13 & 2.018 & 0.045 & 0.44 & 0.000 & 211 \\
14 & 1.974 & 0.046 & 0.48 & 0.000 & 221 \\
15 & 1.922 & 0.046 & 0.46 & 0.000 & 223 \\
16 & 1.908 & 0.047 & 0.47 & 0.000 & 224 \\
17 & 1.936 & 0.046 & 0.49 & 0.000 & 223 \\
18 & 2.006 & 0.051 & 0.76 & 0.000 & 214 \\
19 & 2.043 & 0.050 & 0.63 & 0.000 & 201 \\
20 & 2.114 & 0.050 & 0.58 & 0.000 & 186 \\
21 & 1.983 & 0.062 & 0.53 & 0.000 & 170 \\
22 & 0.803 & 0.036 & 0.08 & 0.000 & 157 \\
23 & 0.279 & 0.004 & 0.01 & 0.314 & 142 \\
24 & 0.224 & 0.002 & 0.00 & 0.551 & 123 \\
\hline
\end{tabular}




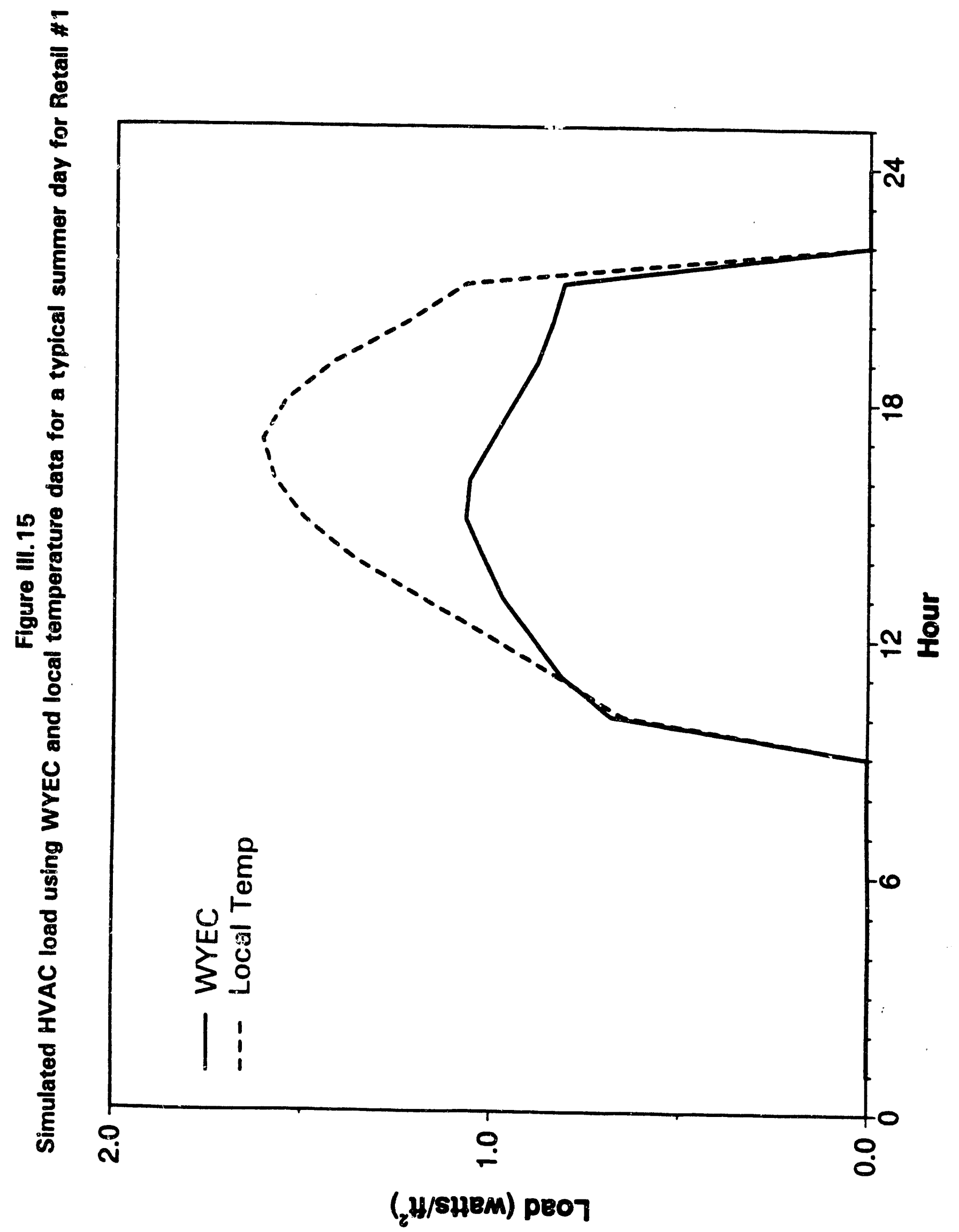




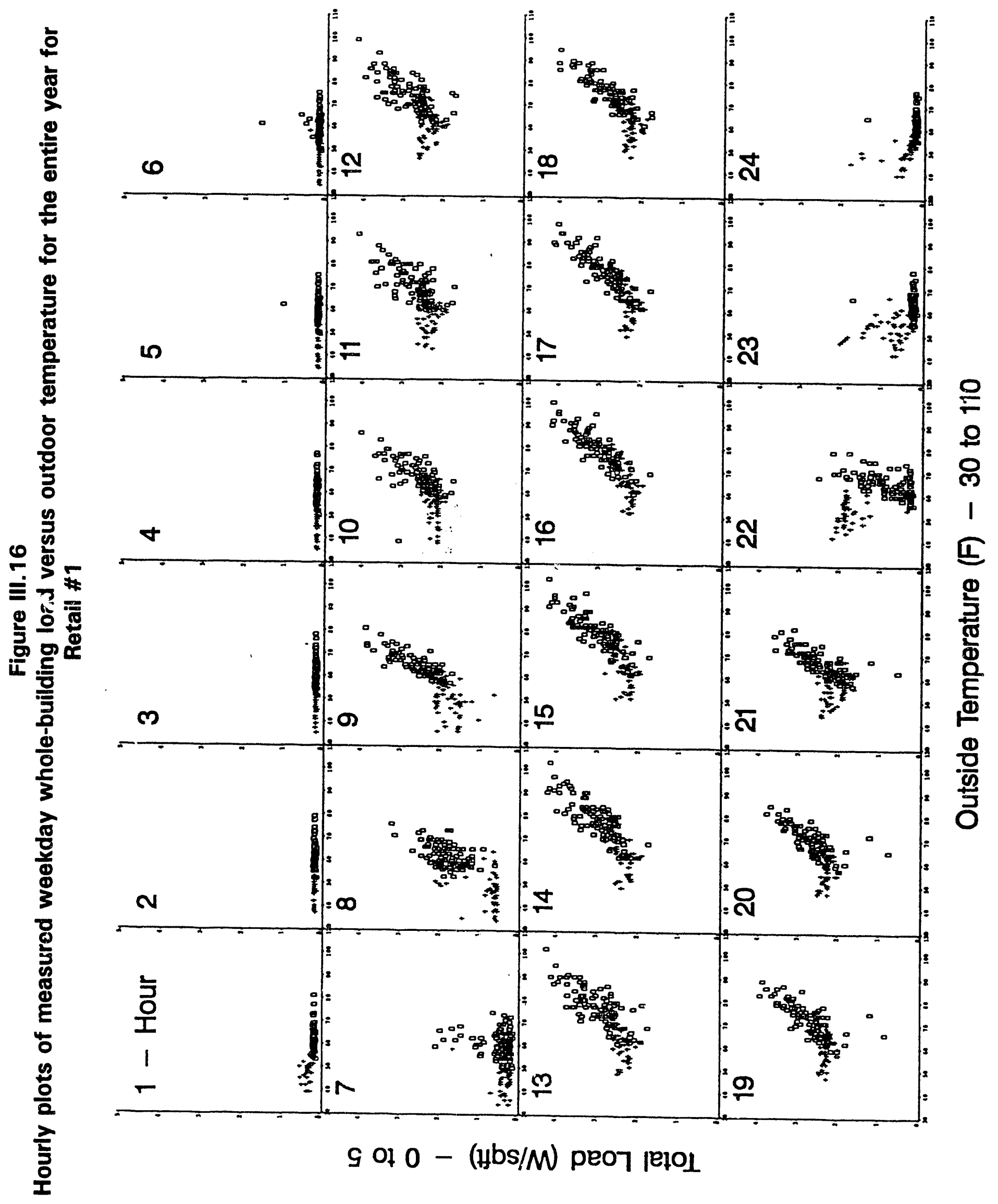




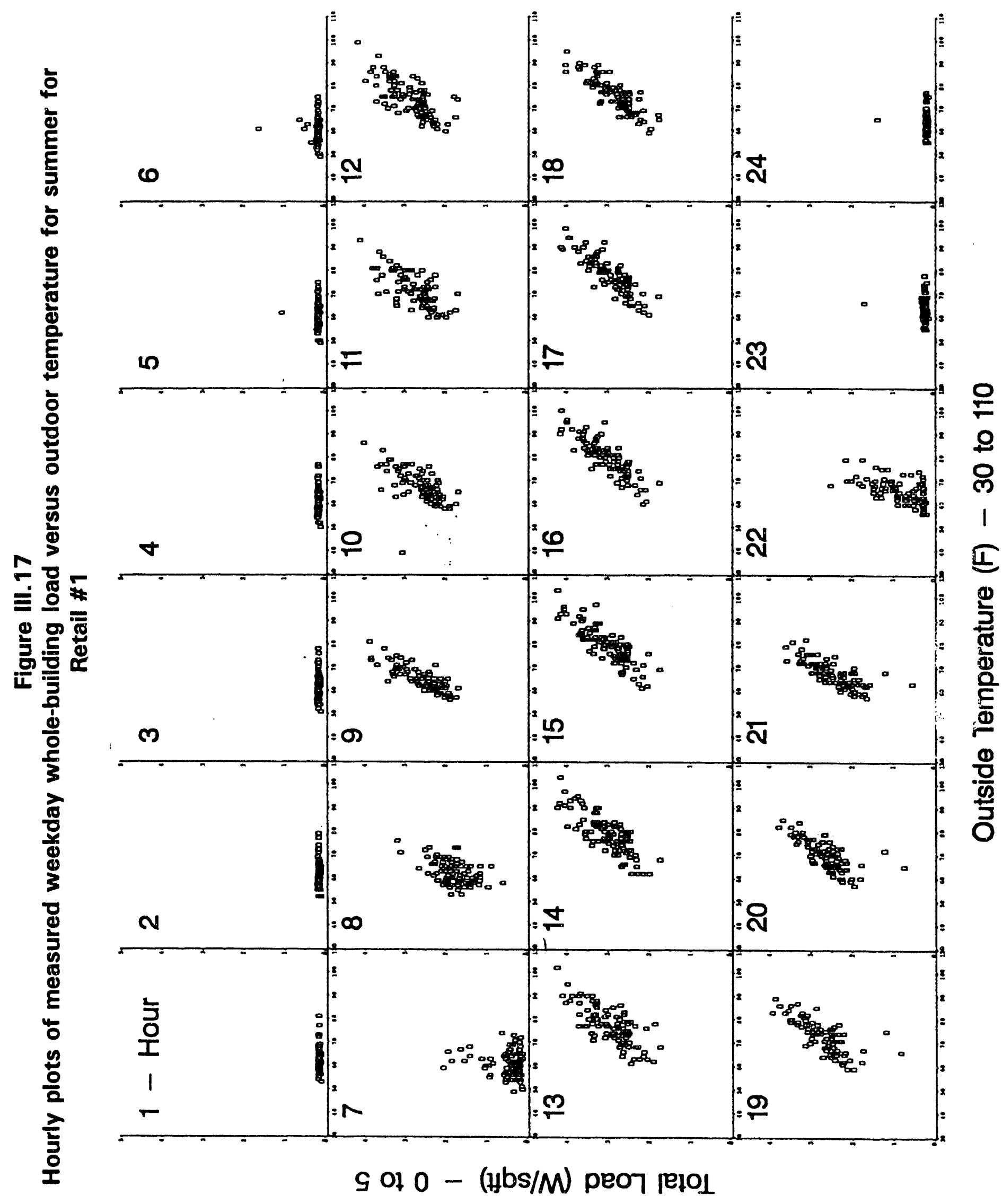




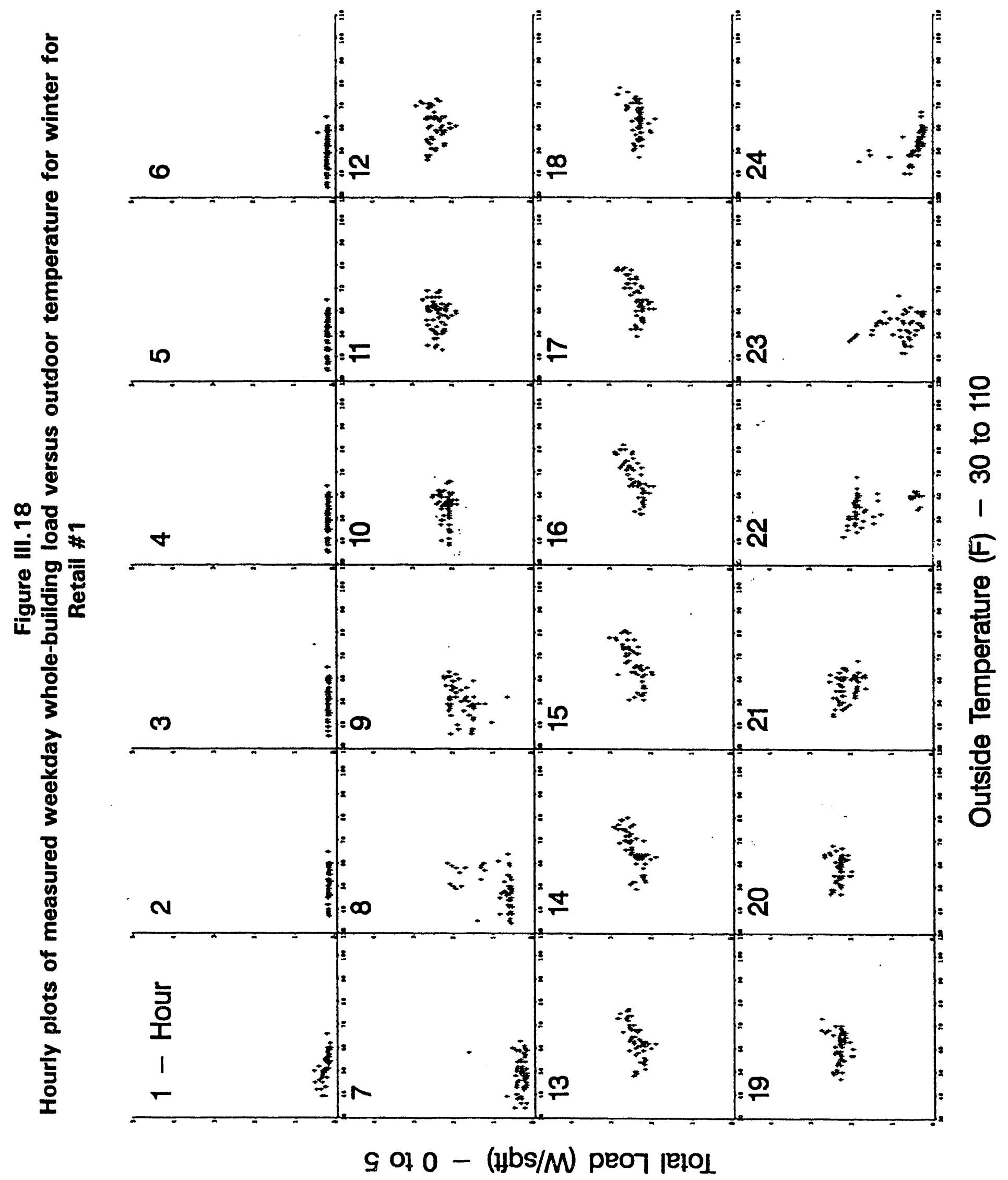


building load simultaneously against both urybulb temperature and relative humidity. The addition of the relative humidity did not significantly improve the $\mathrm{R}^{2}$ (see Table III.10). Regressions of the load against relative humidity alone indicated no correlation between these variables either (Table III.11). Hence, for the remainder of this analysis, we only considered the variation of the whole-building load against the drybulb temperature.

A closer inspection of the hourly data indicated that the operation of the building between days 19 to 96 (January 19 to April 7) is significantly different from the rest of the year. For this period, the whole-building hourly load is fairly constant and does not vary with outside temperature, even on hot days. Clearly, on these days the HVAC systems had not operated. Once, these data were filtered out of the regressions, the $R^{2}$ significantly improved (see Table III.12a). Regression of the hourly loads for the days 19 to 96 , except for a few hours in the evening (6 p.m. to 9 p.m.), indeed showed no correlation to the drybulb temperature (see Table III.12b). For the EDA analysis, we used regression results from Table III.12a.

We also regressed the total daily electricity use against the average daily drybulb and relative humidity. The results indicated that daily whole-building load is sensitive to drybulb temperature and not sensitive to relative humidity (Table III.13). The statistics for Day 19 through 96 are excluded from the data set. The daily correlations are much better than the hourly ones. The average daily electricity use at $60^{\circ} \mathrm{F}$ ambient temperature is about $33 \mathrm{~W} / \mathrm{ft}^{2}$ and the daily electricity use increases by about $1 \mathrm{~W} / \mathrm{ft}^{2} \mathrm{~F}$. We will discuss the daily regressions in further detail in Chapter IV.

EDA Output and Comparison with Monitored End Uses

We performed a series of successive EDA simulations to estimate the energy use intensities and LSs for this retail store. The results of the EDA runs as well as the summary of the monitored data and the DOE-2 simulations are summarized in Table III.14.

To establish a common reference for comparing these data we rely on the level of end-use disaggregation provided by the monitored data (row 1). The monitored data include channels for the HVAC and indoor lighting end uses and whole-building electricity use. Note that of the total $9.06 \mathrm{kWh} / \mathrm{ft}^{2}$ annual electricity use, the monitored end uses account for $8.85 \mathrm{kWh} / \mathrm{ft}^{2}$ leaving $0.21 \mathrm{kWh} / \mathrm{ft}^{2}$ unaccounted for. We assumed the unaccounted for load may be classified as miscellaneous load.

The second row of data indicates the annual electricity use for the interior lighting and the miscellaneous end uses as obtained from the analysis of on-site survey information and HVAC 
Table III.10

Regression results of the measured weekday hourly whole-building load against dry-bulb temperature and relative humidity for the entire year for Retail \#1

\begin{tabular}{|c|ccc|ccr|}
\hline Hour & $\begin{array}{c}60^{\circ} \text { Base } \\
\left(\mathrm{W} / \mathrm{ft}^{2}\right)\end{array}$ & $\begin{array}{c}\text { Coefficients } \\
\text { Relative Humidity } \\
\left(\mathrm{W} / \mathrm{ft}^{2} /{ }^{\circ} \mathrm{F}\right)\end{array}$ & $\begin{array}{c}\text { Dry Bulb Temp } \\
\left(\mathrm{W} / \mathrm{ft}^{2} /{ }^{\circ} \mathrm{F}\right)\end{array}$ & $\mathrm{R}^{2}$ & $\begin{array}{c}\text { Statistics } \\
\text { Sig. of } \\
\text { F-Stat. }\end{array}$ & $\mathrm{N}$ \\
\hline 1 & 0.302 & -0.004 & -0.003 & 0.20 & 0.000 & 109 \\
2 & 0.231 & -0.002 & 0.000 & 0.13 & 0.002 & 99 \\
3 & 0.252 & -0.003 & -0.002 & 0.21 & 0.000 & 90 \\
7 & 0.224 & -0.002 & 0.001 & 0.19 & 0.000 & 80 \\
5 & 0.270 & -0.003 & -0.002 & 0.13 & 0.005 & 78 \\
6 & 0.375 & -0.005 & -0.011 & 0.14 & 0.004 & 75 \\
7 & 0.587 & -0.004 & -0.005 & 0.01 & 0.616 & 75 \\
8 & 1.451 & 0.007 & 0.057 & 0.23 & 0.000 & 86 \\
9 & 1.809 & 0.017 & 0.089 & 0.58 & 0.000 & 121 \\
10 & 1.846 & 0.015 & 0.065 & 0.51 & 0.000 & 149 \\
11 & 2.068 & 0.010 & 0.048 & 0.43 & 0.000 & 190 \\
12 & 2.028 & 0.013 & 0.051 & 0.49 & 0.000 & 201 \\
13 & 1.975 & 0.014 & 0.055 & 0.56 & 0.000 & 211 \\
14 & 1.987 & 0.015 & 0.054 & 0.59 & 0.000 & 221 \\
15 & 1.952 & 0.014 & 0.053 & 0.56 & 0.000 & 223 \\
16 & 1.922 & 0.014 & 0.054 & 0.56 & 0.000 & 224 \\
17 & 1.927 & 0.012 & 0.053 & 0.59 & 0.000 & 223 \\
18 & 1.975 & 0.006 & 0.056 & 0.79 & 0.000 & 214 \\
19 & 1.972 & 0.007 & 0.058 & 0.68 & 0.000 & 201 \\
20 & 1.997 & 0.009 & 0.060 & 0.66 & 0.000 & 186 \\
21 & 1.836 & 0.009 & 0.073 & 0.59 & 0.000 & 170 \\
22 & 0.943 & -0.007 & 0.027 & 0.10 & 0.000 & 157 \\
23 & 0.430 & -0.008 & -0.004 & 0.23 & 0.000 & 142 \\
24 & 0.358 & -0.006 & -0.005 & 0.20 & 0.000 & 123 \\
\hline
\end{tabular}


Table III.11

Regression results of the measured weekday hourly whole-building load against relative humidity for the entire year for Retail \#1

\begin{tabular}{|c|cc|ccr|}
\hline & \multicolumn{3}{|c|}{ Coefficients } & \multicolumn{3}{c|}{ Statistics } \\
Hour & $\begin{array}{c}60^{\circ} \text { Base } \\
\left(\text { W/ft }^{2}\right)\end{array}$ & $\begin{array}{c}\text { Relative Humidity } \\
\left(\text { W/ft }{ }^{2} /{ }^{\circ} \text { ) }\right.\end{array}$ & $\mathrm{R}^{2}$ & $\begin{array}{c}\text { Sig. of } \\
\text { F-Stat. }\end{array}$ & $\mathrm{N}$ \\
\hline 1 & 0.284 & -0.004 & 0.20 & 0.000 & 109 \\
2 & 0.234 & -0.002 & 0.13 & 0.000 & 99 \\
3 & 0.239 & -0.003 & 0.20 & 0.000 & 90 \\
4 & 0.228 & -0.002 & 0.19 & 0.000 & 80 \\
5 & 0.258 & -0.003 & 0.13 & 0.001 & 78 \\
6 & 0.312 & -0.004 & 0.10 & 0.005 & 75 \\
7 & 0.561 & -0.004 & 0.01 & 0.351 & 75 \\
8 & 1.774 & 0.004 & 0.01 & 0.271 & 86 \\
9 & 2.485 & 0.006 & 0.03 & 0.075 & 121 \\
10 & 2.438 & 0.005 & 0.02 & 0.071 & 149 \\
11 & 2.533 & 0.002 & 0.01 & 0.251 & 190 \\
12 & 2.634 & 0.003 & 0.01 & 0.223 & 201 \\
13 & 2.687 & 0.003 & 0.01 & 0.301 & 211 \\
14 & 2.726 & 0.003 & 0.01 & 0.222 & 221 \\
15 & 2.728 & 0.004 & 0.01 & 0.178 & 223 \\
16 & 2.691 & 0.003 & 0.00 & 0.342 & 224 \\
17 & 2.633 & 0.002 & 0.00 & 0.418 & 223 \\
18 & 2.658 & -0.006 & 0.04 & 0.005 & 214 \\
19 & 2.598 & -0.004 & 0.02 & 0.047 & 201 \\
20 & 2.558 & -0.002 & 0.01 & 0.243 & 186 \\
21 & 2.430 & -0.002 & 0.01 & 0.284 & 170 \\
22 & 1.139 & -0.011 & 0.07 & 0.001 & 157 \\
23 & 0.405 & -0.007 & 0.22 & 0.000 & 142 \\
24 & 0.325 & -0.005 & 0.19 & 0.000 & 123 \\
\hline & & & & & \\
\hline
\end{tabular}


Table III.12a

Regression results of the measured weekday hourly whole-building load against dry-bulb temperature excluding days $19-96$ for Retail \#1

\begin{tabular}{|c|cc|ccc|}
\hline & \multicolumn{3}{|c|}{ Coefficients } & \multicolumn{3}{c|}{$\begin{array}{c}\text { Statistics } \\
\text { Hour }\end{array}$} & $\begin{array}{c}\text { Sig. of } \\
\text { (W/ft }{ }^{\circ} \text { ) }\end{array}$ & $\begin{array}{c}\text { Dry Bulb Temp } \\
\left.\text { (W/ft }{ }^{2} / \mathrm{F}\right)\end{array}$ & $\mathrm{R}^{2}$ & \multicolumn{3}{c|}{ F-Stat. } & $\mathrm{N}$ \\
\hline 1 & 0.166 & 0.000 & 0.00 & 0.797 & 99 \\
2 & 0.166 & 0.000 & 0.00 & 0.775 & 94 \\
3 & 0.167 & 0.000 & 0.00 & 0.935 & 85 \\
4 & 0.166 & -0.000 & 0.00 & 0.946 & 78 \\
5 & 0.186 & -0.002 & 0.00 & 0.584 & 76 \\
6 & 0.232 & -0.007 & 0.02 & 0.286 & 73 \\
7 & 0.473 & -0.003 & 0.00 & 0.848 & 73 \\
8 & 1.594 & 0.056 & 0.22 & 0.000 & 83 \\
9 & 2.160 & 0.083 & 0.62 & 0.000 & 115 \\
10 & 2.180 & 0.054 & 0.52 & 0.000 & 134 \\
11 & 2.208 & 0.047 & 0.55 & 0.000 & 162 \\
12 & 2.167 & 0.047 & 0.59 & 0.000 & 170 \\
13 & 2.130 & 0.047 & 0.65 & 0.000 & 175 \\
14 & 2.096 & 0.047 & 0.71 & 0.000 & 180 \\
15 & 2.024 & 0.049 & 0.73 & 0.000 & 180 \\
16 & 1.990 & 0.051 & 0.76 & 0.000 & 180 \\
17 & 1.974 & 0.052 & 0.77 & 0.000 & 179 \\
18 & 1.983 & 0.053 & 0.73 & 0.000 & 173 \\
19 & 2.033 & 0.053 & 0.59 & 0.000 & 165 \\
20 & 2.123 & 0.052 & 0.55 & 0.000 & 157 \\
21 & 1.954 & 0.070 & 0.55 & 0.000 & 147 \\
22 & 0.628 & 0.051 & 0.16 & 0.000 & 138 \\
23 & 0.261 & -0.002 & 0.00 & 0.571 & 127 \\
24 & 0.186 & 0.000 & 0.00 & 0.965 & 109 \\
\hline & & & & & \\
\hline
\end{tabular}


Table III.12b

Regression results of the measured weekday hourly whole-building load against dry-bulb temperature for days $19-96$ for Retail \#1

\begin{tabular}{|c|cc|ccc|}
\hline & \multicolumn{3}{|c|}{ Coefficients } & \multicolumn{3}{c|}{ Statistics } \\
Hour & $\begin{array}{c}60^{\circ} \text { Base } \\
\left(\text { W/ft } \mathrm{f}^{2}\right)\end{array}$ & $\begin{array}{c}\text { Dry Bulb Temp } \\
\left(\mathbf{W} / \mathrm{ft}^{2} / \mathrm{F} \text { F }\right)\end{array}$ & $\mathrm{R}^{2}$ & $\begin{array}{c}\text { Sig. of } \\
\text { F-Stat. }\end{array}$ & $\mathrm{N}$ \\
\hline 1 & 0.448 & 0.046 & 0.77 & 0.002 & 8 \\
2 & 0.342 & 0.046 & 0.62 & 0.212 & 3 \\
3 & 0.370 & 0.045 & 0.44 & 0.337 & 3 \\
4 & 0.000 & 0.000 & 0.00 & 0.000 & 0 \\
5 & 0.000 & 0.000 & 0.00 & 0.000 & 0 \\
6 & 0.000 & 0.000 & 0.00 & 0.000 & 0 \\
7 & 0.000 & 0.000 & 0.00 & 0.000 & 0 \\
8 & 1.891 & -0.124 & 1.00 & 0.000 & 1 \\
9 & 1.667 & -0.007 & 0.47 & 0.201 & 4 \\
10 & 1.673 & -0.000 & 0.00 & 0.997 & 13 \\
11 & 2.045 & -0.017 & 0.19 & 0.025 & 25 \\
12 & 2.054 & -0.012 & 0.08 & 0.135 & 28 \\
13 & 1.890 & -0.003 & 0.01 & 0.561 & 33 \\
14 & 1.846 & 0.000 & 0.00 & 0.963 & 38 \\
15 & 1.849 & 0.001 & 0.00 & 0.866 & 40 \\
16 & 1.840 & 0.001 & 0.00 & 0.817 & 41 \\
17 & 1.857 & 0.006 & 0.05 & 0.149 & 41 \\
18 & 2.049 & 0.047 & 0.85 & 0.000 & 38 \\
19 & 2.047 & 0.043 & 0.83 & 0.000 & 33 \\
20 & 2.027 & 0.043 & 0.78 & 0.000 & 26 \\
21 & 1.969 & 0.045 & 0.65 & 0.000 & 20 \\
22 & 1.930 & -0.021 & 0.09 & 0.245 & 16 \\
23 & 0.488 & 0.049 & 0.80 & 0.000 & 13 \\
24 & 0.517 & 0.036 & 0.79 & 0.000 & 12 \\
\hline & & & & & \\
\hline
\end{tabular}


Table III.13

Regression results of the measured weekday daily whole-building load against dry-bulb temperature and relative humidity for Retail \#1

\begin{tabular}{|c|c|c|c|c|c|c|}
\hline Season & $\begin{array}{c}60^{\circ} \text { Base } \\
\left(w / f t^{2}\right)\end{array}$ & $\begin{array}{l}\text { Coefficients } \\
\text { Relative Humidity } \\
\left(\mathrm{W} / \mathrm{ft}^{2} /{ }^{\circ} \mathrm{F}\right)\end{array}$ & $\begin{array}{l}\text { Dry Bulb Temp } \\
\left(\mathrm{W} / \mathrm{t}^{2} /{ }^{\circ} \mathrm{F}\right)\end{array}$ & $R^{2}$ & $\begin{array}{l}\text { Statistics } \\
\text { Sig. of } \\
\text { F-Stat. }\end{array}$ & $\mathbf{N}$ \\
\hline Summer & 31.773 & - & 1.110 & 0.77 & 0.000 & 115 \\
\hline Winter, & 33.355 & - & 0.441 & 0.43 & 0.000 & 47 \\
\hline Winter $^{1}$ & 34.544 & - & 0.518 & 0.44 & 0.000 & 28 \\
\hline Year, & 32.491 & - & 0.956 & 0.69 & 0.000 & 162 \\
\hline Year $^{1}$ & 32.708 & - & 1.014 & 0.75 & 0.000 & 143 \\
\hline Summer & 42.652 & -0.144 & - & 0.06 & 0.010 & 115 \\
\hline Winter & 35.248 & -0.075 & - & 0.14 & 0.010 & 47 \\
\hline Winter $^{1}$ & 36.280 & -0.079 & - & 0.27 & 0.004 & 28 \\
\hline Year, & 39.573 & -0.027 & - & 0.00 & 0.449 & 162 \\
\hline Year $^{1}$ & 40.846 & -0.082 & - & 0.03 & 0.040 & 143 \\
\hline Summer & 29.594 & 0.115 & 1.217 & 0.80 & 0.000 & 115 \\
\hline Winter & 33.463 & -0.017 & 0.414 & 0.43 & 0.000 & 47 \\
\hline Winter ${ }^{1}$ & 35.021 & -0.053 & 0.434 & 0.55 & 0.000 & 28 \\
\hline Year & 31.465 & 0.079 & 1.012 & 0.72 & 0.000 & 162 \\
\hline Year $^{1}$ & 32.298 & 0.028 & 1.032 & 0.76 & 0.000 & 143 \\
\hline
\end{tabular}

1. Day 19 through 96 are excluded from the data set. 
Table III.14

Comparison of the EDA estimated EUIs with measured EUIs for Retail \#1

\begin{tabular}{|c|c|c|c|c|}
\hline \multirow{2}{*}{ Run } & \multicolumn{4}{|c|}{ EUI (kWh/nt/yr) } \\
\hline & HVAC & Lighting & Miscellaneous & Total \\
\hline Monitored & 3.20 & 5.65 & 0.21 & 9.06 \\
\hline Doe-2 estimate & 2.76 & 6.91 & 0.28 & 9.95 \\
\hline Doe-2 with 1989 weather & 3.67 & 6.91 & 0.28 & 10.86 \\
\hline EDA with cooling based on regression of total load & 2.94 & 5.87 & 0.25 & 9.06 \\
\hline EDA with cooling based on Doe-2 estimates & 2.93 & 5.90 & 0.22 & 9.05 \\
\hline
\end{tabular}


electricity use simulated using DOE-2 (using WYEC weather tapes). Although at this stage we have not used the monitored data for further EDA analysis, note that the audit overestimates the lighting EUI by $22 \%$ and underestimates the HVAC EUI by $14 \%$. Once the HVAC EUI are calculated for the actual 1989 weather, the simulation overestimates the HVAC EUI by $15 \%$ (see row 3 of Table III.14). The hourly information on the third row is used as the initial condition for EDA. Figure III.19 shows the simulated DOE-2 end-use load shape for the building peak day. In these simulations, based on the analysis of the measured whole-building load, we adjusted the hours of operation of the HVAC system.

The results of the EDA application, using the DOE-2 simulated initial conditions, are summarized in rows 4 and 5 . In the EDA process we used the annual regressions of whole-building hourly loads. Row 4 provides end-use EUIs with all end uses at all hours having an equal weight. Comparing the results with the monitored data indicate that the lighting EUI is overestimated only by $4 \%$ and the HVAC EUI is underestimated by $8 \%$. In an attempt to improve the EUIs even further, we repeated the EDA calculation using varying weight for different end uses (row 5). We used higher weights for lighting based on the assumption that the on-site survey information are probably more accurate for the interior lighting than miscellaneous end uses. The weighting only slightly modified the resulting EUIs and LSs.

In order to gain insight into the differences between the EDA results and the monitored data, Figure III.20 compares the average daily energy use load shapes of the monitored data with those estimated by EDA. This figure corresponds to EDA simulations with cooling based on regression of total load, Row 4 of Table III.14. The differences between the EDA estimates and measured data are plotted in Figure III.21.

The results are very promising. In inspecting these figures it is clear that during both nighttime and daytime hours, EDA's estimates of electricity use for all end uses agree very well with those of the measured data. This is no surprise, since our initial conditions for all end uses were within $20 \%$ of measured data. The maximum average differences between the hourly measured load and EDA estimates are 0.2, 0.15, $0.02 \mathrm{~W} / \mathrm{ft}^{2}$, for HVAC, lighting and miscellaneous end uses, respectively. Maximum differences between the measured end-use data and the EDA estimates occur when there is a mismatch between the actual and the assumed schedules.

For the retail store, the EDA estimates of hourly end-use data compare remarkably well with those of the monitored data. This indicates great promise for applying the EDA analysis to whole-building load for obtaining reliable end-use data. 


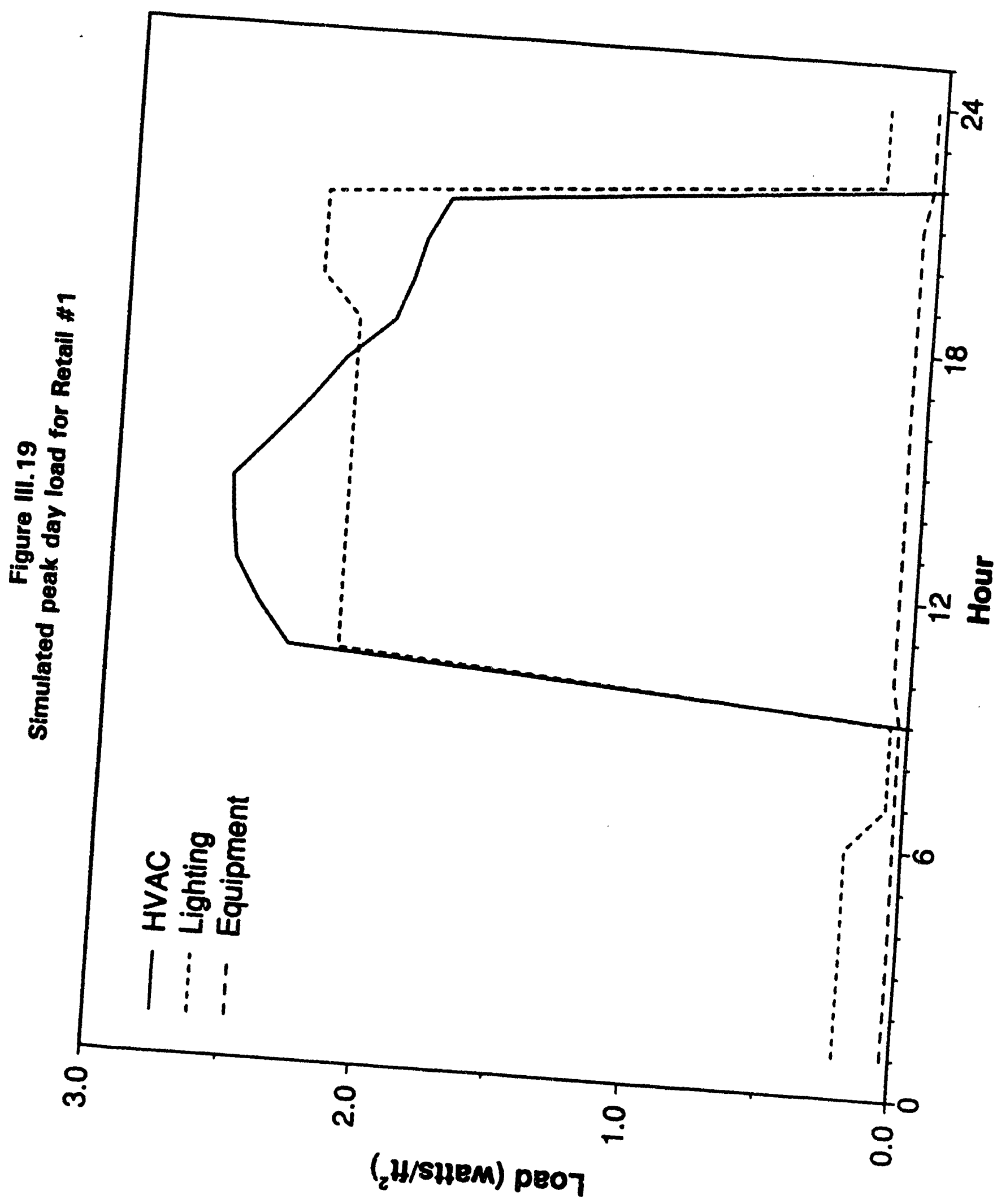




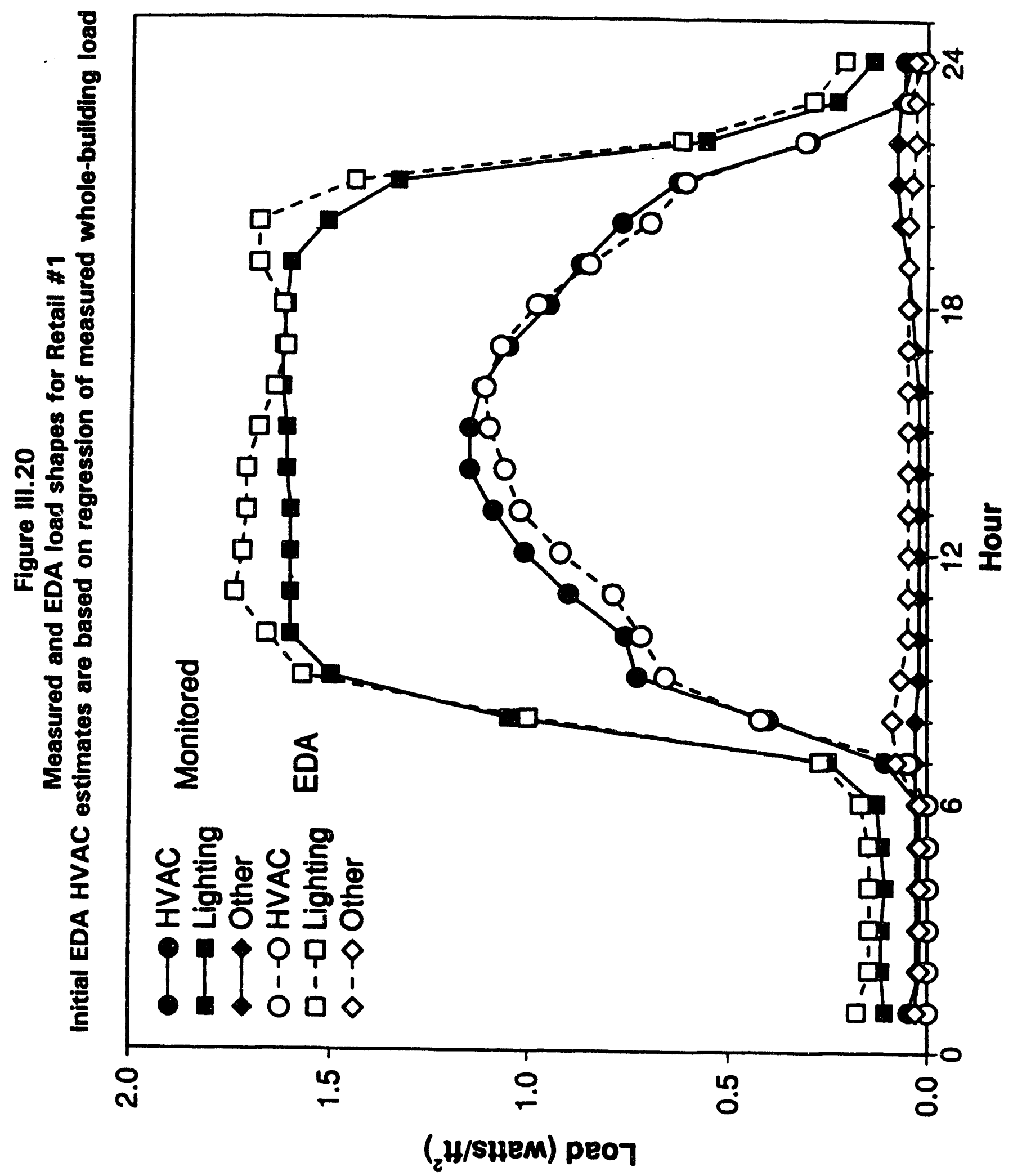




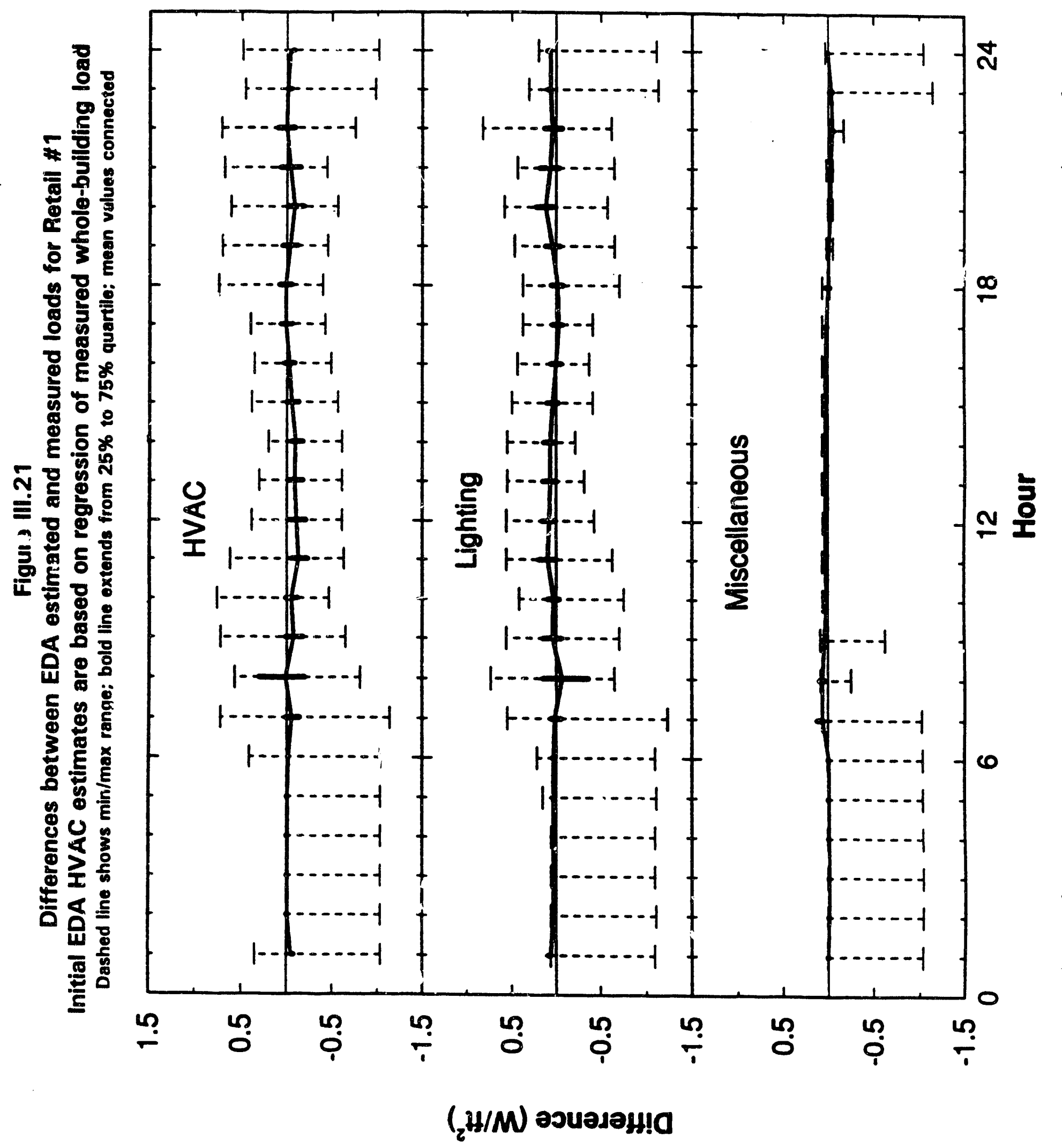




\section{Chapter IV: Conclusion and Recommendation}

In Chapter III, we compared the hourly end-use loads estimated by LBL/EDA model with the corresponding measured data. Before we make an attempt to recommend methods for improving the performance of the EDA, we will first review its strengths and weaknesses. Recall that EDA has three major components; analysis of hourly load-temperature regressions, estimation of the components of the non-temperature dependent load, and the review of the hourly end-use loads for "permissible" hourly end-use load variation. In this chapter, we will discuss the EDA assumptions for each of these components and verify them using measured data. We conclude the chapter with recommendations for improving EDA.

\section{Load-Temperature Regressions}

The principle EDA assumption is that all of the weather (in this report temperature) dependency of the whole-building load is due to the HVAC system. In other words, among all the end uses in a commercial building, only the HVAC load is sensitive to the outdoor temperature. Although, this assumption seems plausible in a large number of commercial buildings, particularly in offices, retail stores, schools, and colleges, there are other building types characterized by non-HVAC end uses such as refrigeration whose loads are also weather dependent. Restaurants, supermarkets, and refrigerated warehouses are examples of commercial buildings where HVAC is not the only weather-dependent load.

For the buildings we have studied in this report, the HVAC, lighting, and plug loads constitute the majority of the whole-building load and the contribution of the refrigeration load (which may be limited to a few residential refrigerators in each building) is fairly minimal. So for those cases where no significant refrigeration occurs, we can check our first hypothesis with the available end-use data.

Recall that the regressions of the hourly whole-building load against the outdoor temperature provide tw'o pieces of information; the weather-dependency of the hourly load (estimated by the regression coefficients) and the significance of the estimated correlations. The weatherdependency of the hourly whole-building load provides an estimate of the weather-dependency of the HVAC loads. The significance of the correlations identifies the operating schedules of the HVAC systems: if there is no significant correlation, the HVAC system is assumed to be off. 
Is the temperature dependency of the whole-building load the same as that of the HVAC load?

Tables IV.1a, IV.1b, and IV.2 summarize the hourly statistics of the drybulb temperature regression for the office and retail store. The summary statistics for the office building are presented separately for summer and winter periods, which have been defined by the HVAC seasonality. Table IV.1a shows low $\mathrm{R}^{2}$ values and thus fairly weak temperature correlation for the whole-building load when compared to the values for the HVAC load. This indicates that there is less scatter in the HVAC regression than in the whole-building regression. Also, the drybulb temperature regression coefficients for the whole-building load are significantly smaller than those for the HVAC load, indicating that the HVAC load is more temperature dependent.

For the office in the winter and for the retail store, the $R^{2}$ values and the drybulb temperature regression coefficients for the whole-building load compare very well with those for the HVAC load (see Tables IV.1b and IV.2). For the office building in winter, the temperature regression coefficients for the loads compare well, but the whole-building load correlations are weaker than those for the HVAC load. As shown for the retail store in Table IV.2, the $\mathrm{R}^{2}$ and regression coefficients for hours with significant correlations are very close, generally within a few percentage points of each other. Also, it is reassuring to note that the regressions do no indicate any temperature-dependency for the lighting and plug loads.

A closer look at the measured end-use data for the office building indicates that about $10 \%$ of the daytime whole-building load has not been sub-metered. This load is indicated by the "Other" category in Tables IV.1a and IV.1b. The Other load is calculated from the wholebuilding load by subtracting the HVAC, Lighting, and Plug loads. For the summer period, the hourly Other load has negative regression coefficients, indicating a decrease in the Other load as drybulb temperature increases. The unmonitored Other load may be caused by the electricity used in the air distribution reheat boxes. The Other load does not show significant correlation during the winter. Once the HVAC load is adjusted by including the Other load, the hourly regression coefficients compare very well with those of the whole-building load. If the weather-dependency of the Other load is, indeed, accurate and it accounts for some unmeasured part of the HVAC load, the HVAC load should be corrected for such unmeasured load. In those cases where some part of the HVAC load is not monitored, one may argue that the weather dependency of the HVAC load determined from the regression of the whole-building load represents the true characteristics of the HVAC load. 


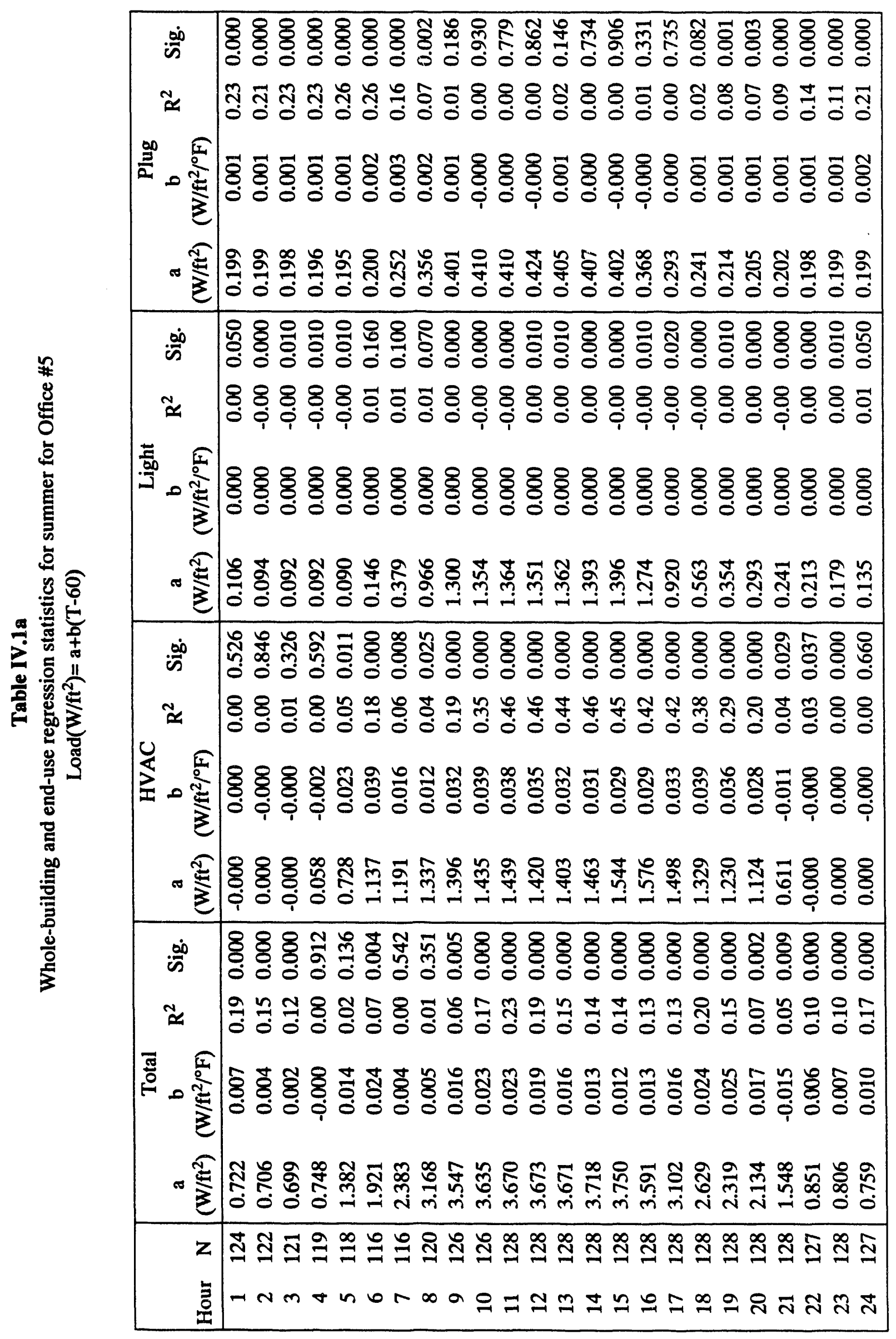




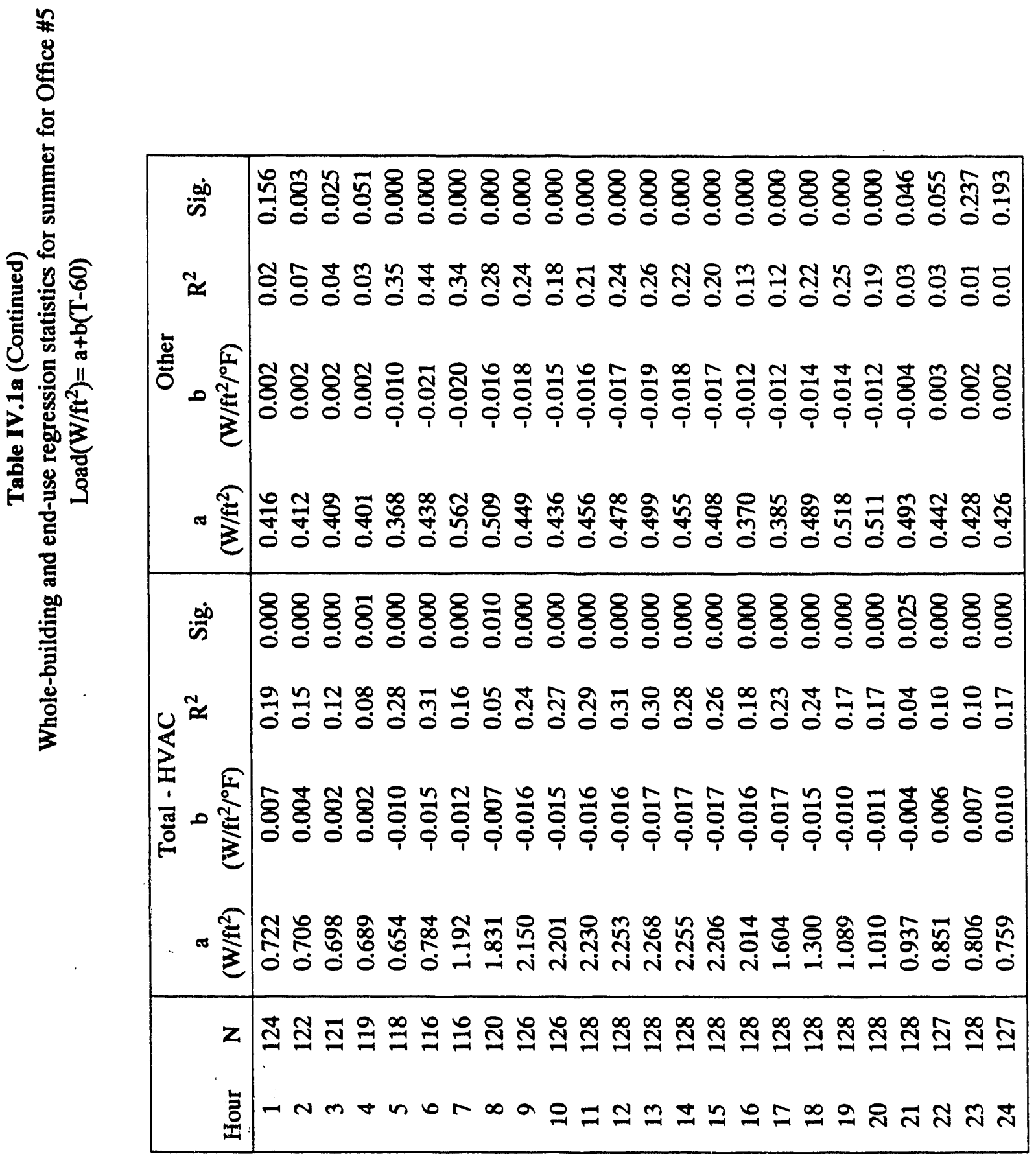




\begin{tabular}{|c|}
\hline 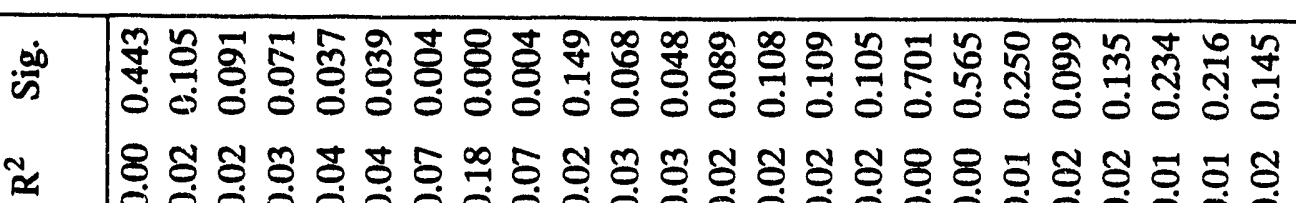 \\
\hline 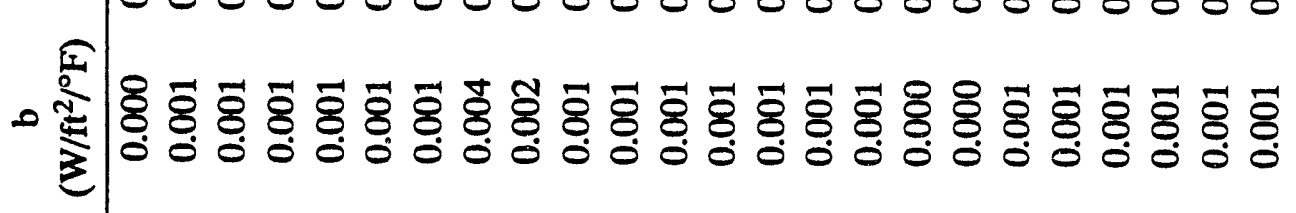 \\
\hline 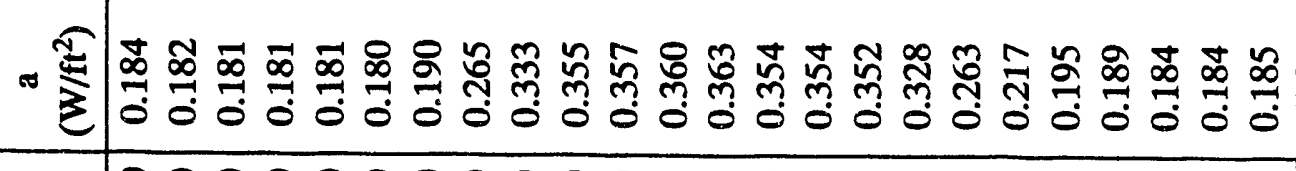 \\
\hline 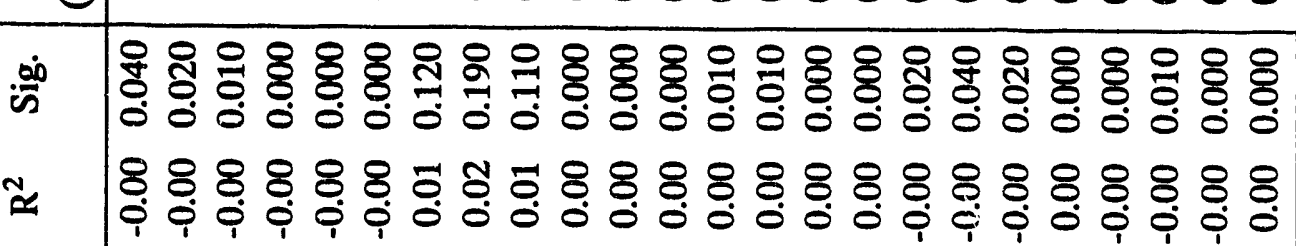 \\
\hline - \\
\hline 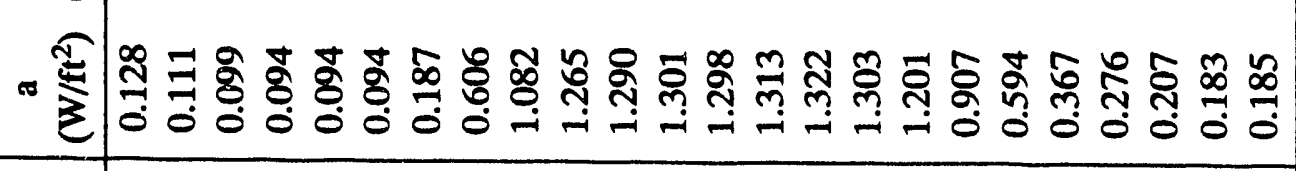 \\
\hline 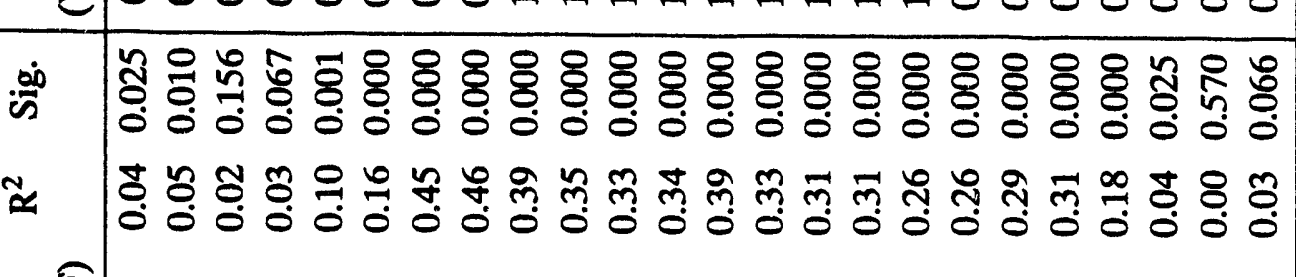 \\
\hline 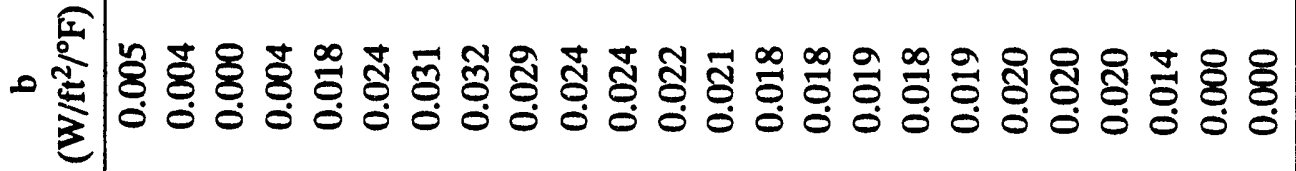 \\
\hline 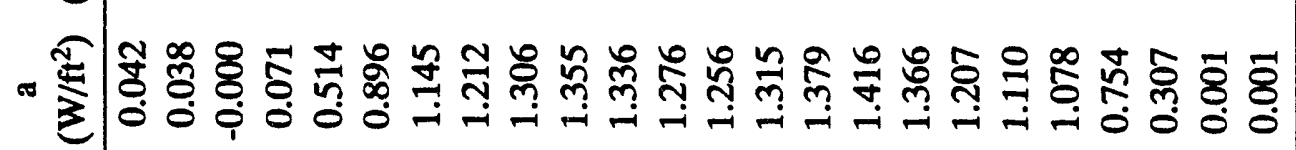 \\
\hline 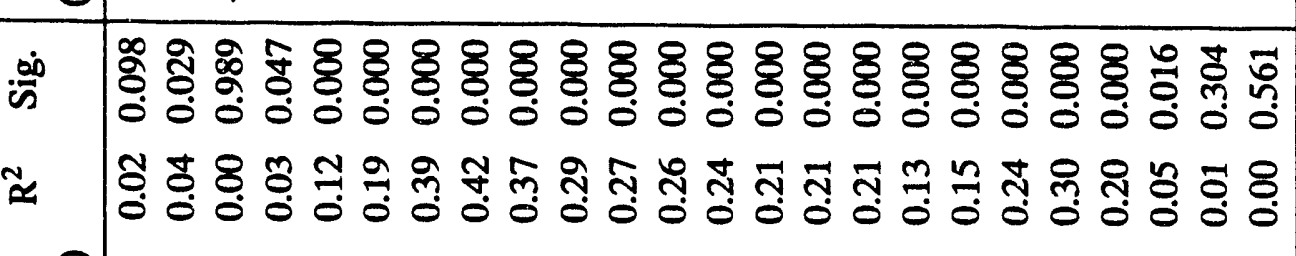 \\
\hline - \\
\hline 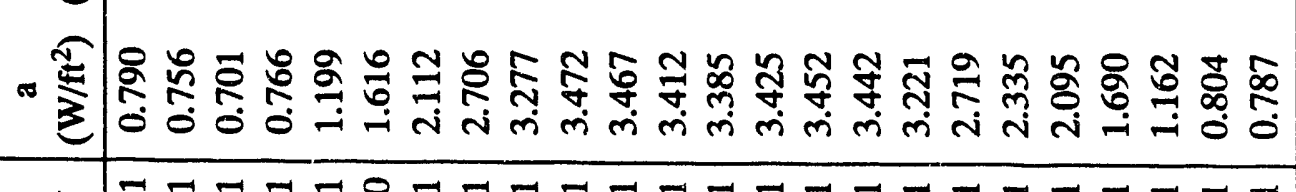 \\
\hline 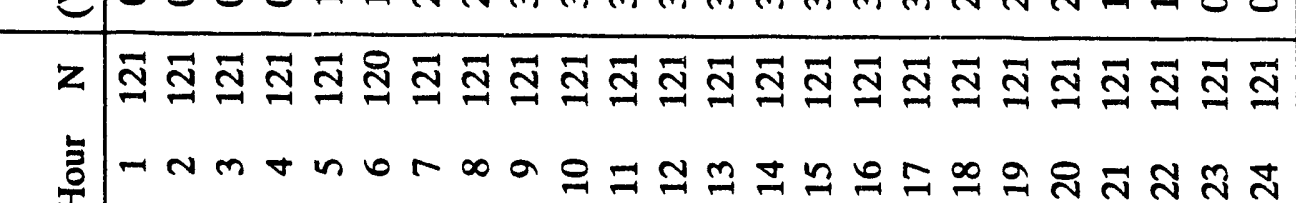 \\
\hline
\end{tabular}




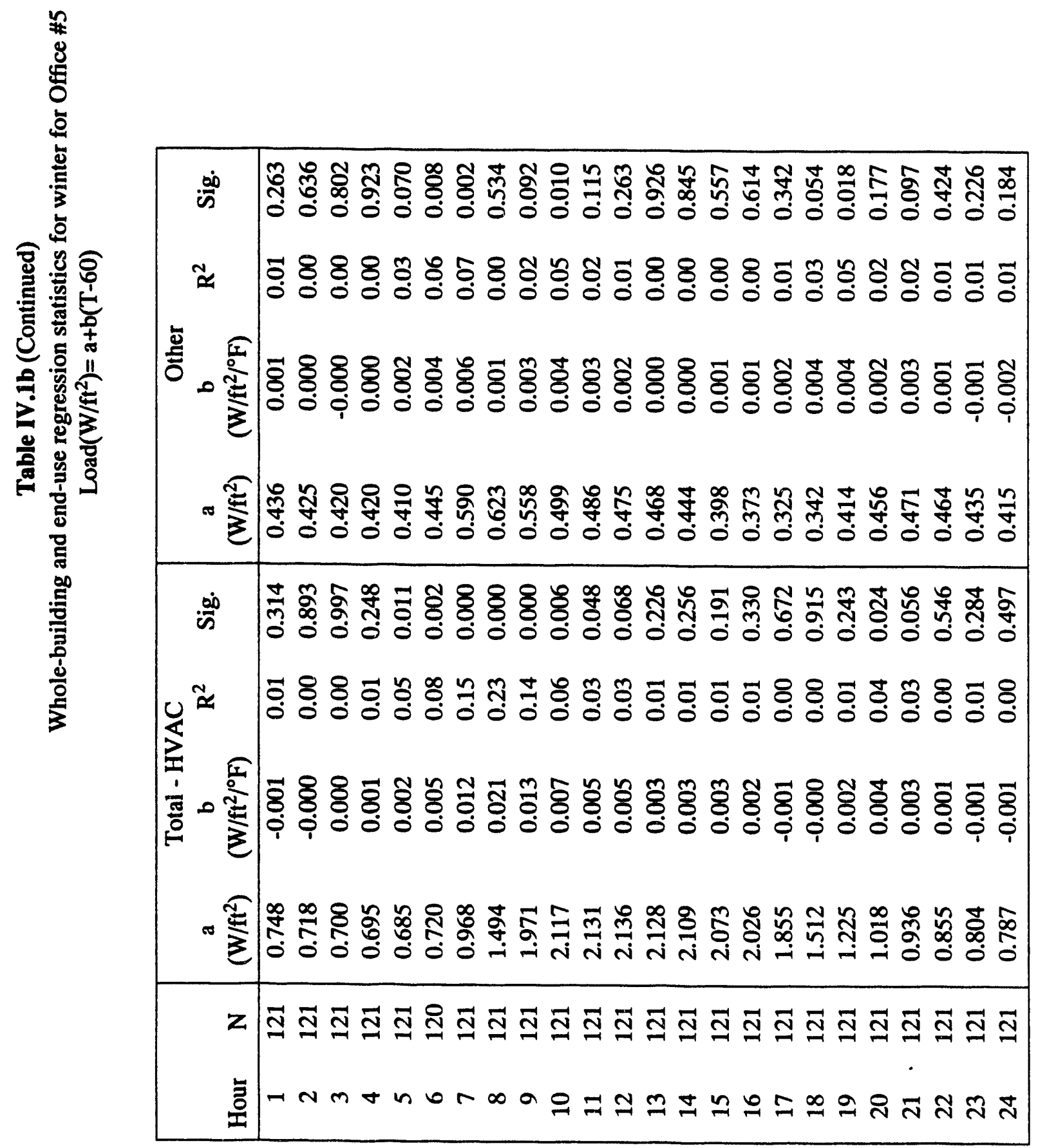




\begin{tabular}{|c|}
\hline \\
\hline 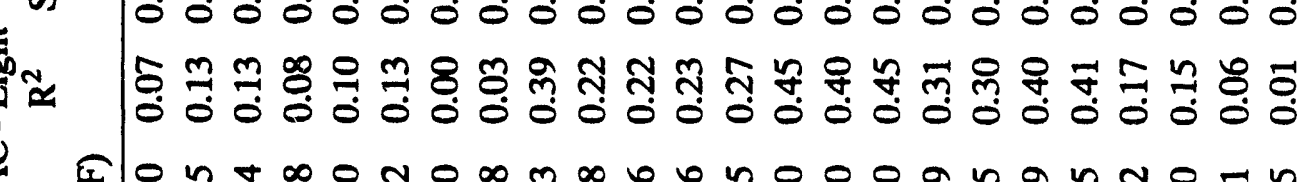 \\
\hline 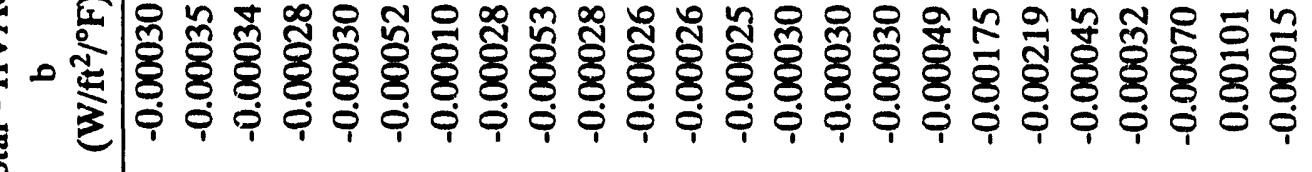 \\
\hline - \\
\hline 紊 \\
\hline ح \\
\hline 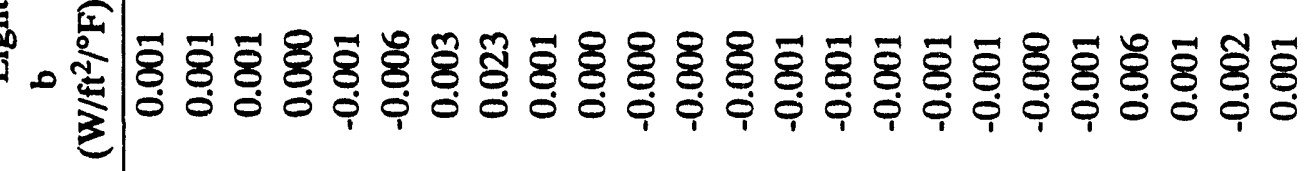 \\
\hline 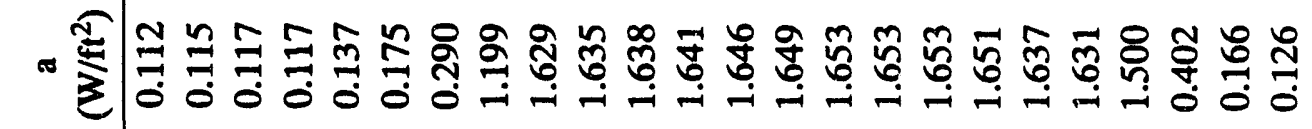 \\
\hline 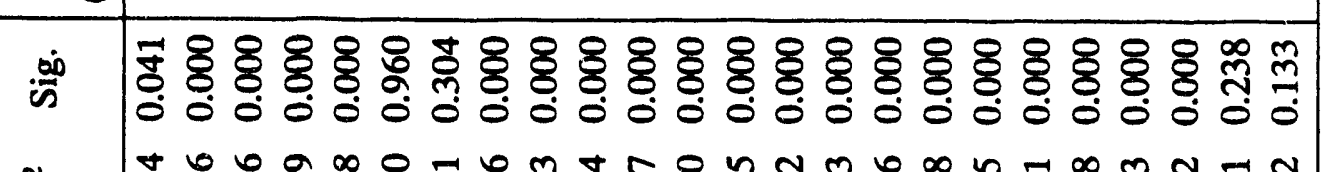 \\
\hline 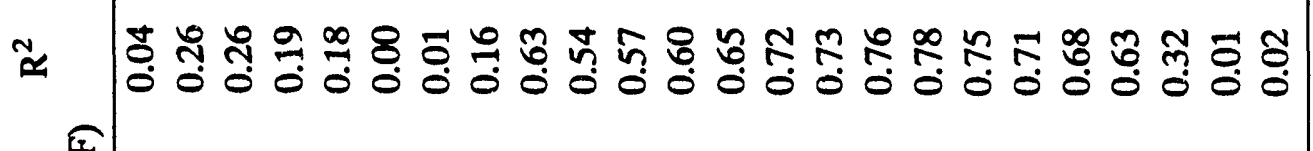 \\
\hline 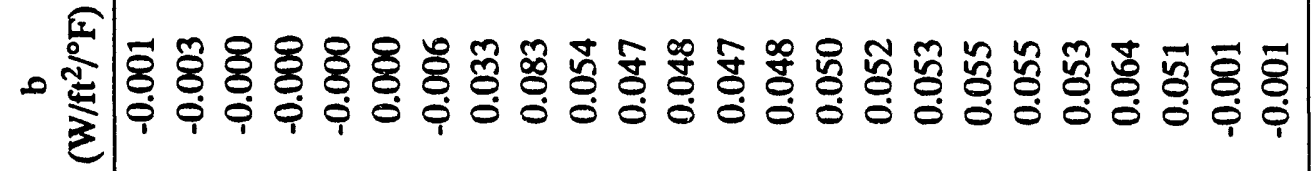 \\
\hline 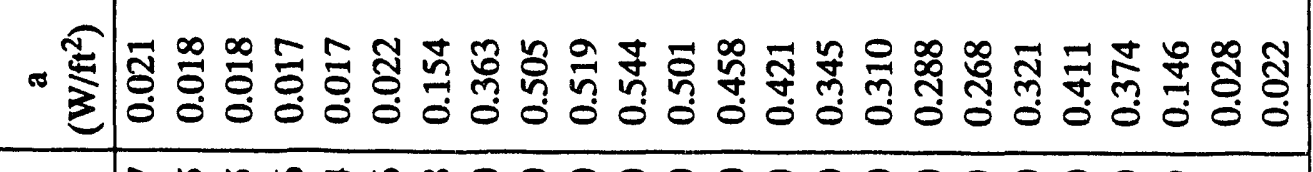 \\
\hline 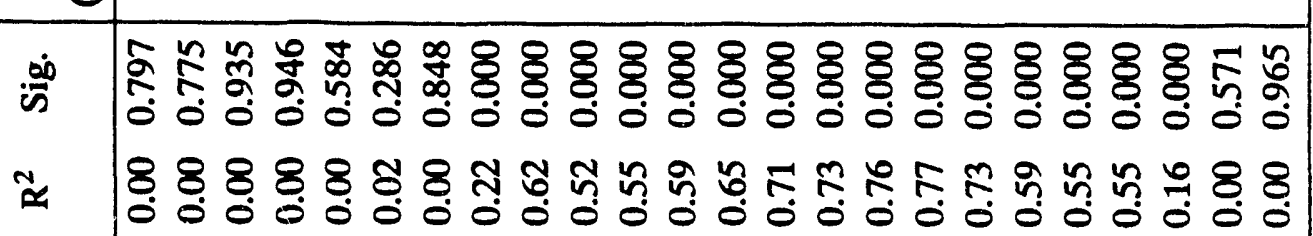 \\
\hline 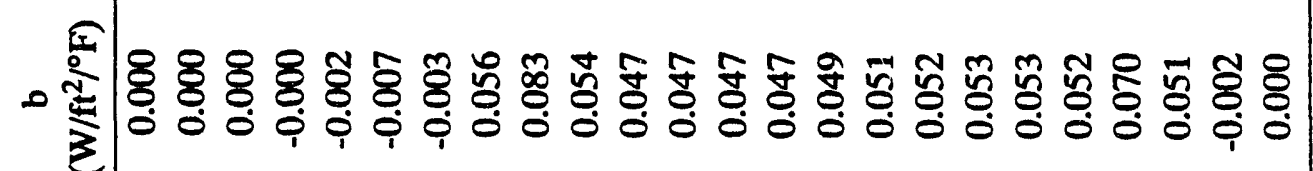 \\
\hline 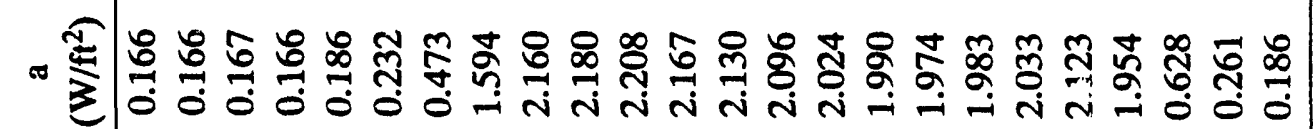 \\
\hline 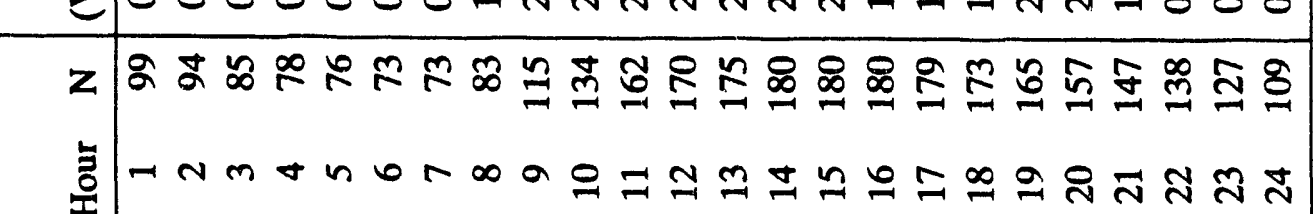 \\
\hline
\end{tabular}


We do not understand why there is not any apparent weather dependency for the Other end use during the winter. Also, it is not clear why the Other end use is showing higher nighttime intensities during the winter. We are not yet aware of end uses, other than HVAC, with a strong temperature correlation. Future studies determining the weather dependency of all end uses, using measured and synthetic data, must be conducted to better answer these questions and validate these assumptions.

Assuming that the Other load is an unmeasured HVAC load, our hypothesis has not been contradicted. Thus, given the uncertainties in the measured data, the analysis of the measured end-use data for these two buildings generally indicate that the temperature-dependency of the whole-building load is the same as that of the HVAC load.

Is the significance of the whole-building-load temperature correlation a valid indicator for the operating schedules of the HVAC systems?

The regression of the hourly whole-building load against the dry-bulb temperature, in addition to the regression coefficients, provides information about the significance of the correlation. The significance of a correlation is given as the probability that the obtained correlation is the result of statistical errors. A significance of 0.0001 indicates that a probability of $99.99 \%$ exists that the correlation is valid.

One of the uncertainties in the analysis of building energy use is usually the schedules of operation of the building and its systems. Scheduling information is usually obtained from the on-site survey of the building. Yet the analysis of the measured building data usually indicates a significant difference between actual and surveyed schedules.

For example, the whole-building load and the HVAC load for the office building show significant correlations with outdoor temperature during the hours 9 to 20 (the hours where correlation coefficients are clearly non-zero). The HVAC loads during the shoulder hours of 6 , 7,8 , and 21 do not show significant temperature correlations for either load. All other end uses do not show any significant temperature dependency except the Other end use. It shows significant correlations between the hours of 5 to 20 .

For the office during the winter, the hours of the significant temperature correlations for whole-building load and HVAC load are the same. The retail store data also show that the hours of significant correlations for the whole-building load and the HVAC load are the same.

The regression of the hourly whole-building load against outdoor temperature provides a fairly accurate tool to estimate, on the average, the temperature-dependency of the HVAC load. 
It also provides an indicator for modifying the audit estimates for the operating hours of the HVAC systems. During the shoulder hours, outdoor temperature alone will not provide an accurate estimate of the hours of HVAC operation. For these shoulder hours, other indicators should be developed.

Can we improve the load-temperature regressions statistics?

Although the regressions of the hourly whole-building load provide strong statistical correlations, the $R^{2}$ values are in general low. For the office building the $R^{2} s$ vary from 0.12 to 0.42 ; the lower $\mathbf{R}^{2} \mathrm{~s}$ usually correspond to shoulder hours. There are in general two ways to improve the load-temperature statistics; by performing regression analyses on a selected period of the year (winter vs summer vs yearly regressions) and by performing egression analyses on data for larger time intervals (using daily or weekly data).

In general, the $R^{2}$ values for the entire year are better than those for summer and for winter. Two factors explain the better yearly regression statistics; more data points and a larger, seasonal temperature range. In some cases, however, the changes in the seasonal operations of a building may result in different correlations between HVAC energy use and outdoor temperature. In such cases, one needs to review both seasonal and annual statistics of the data before deciding on the type of correlations to be used for EDA simulations.

Integrating hourly data into daily data will substantially improve the regression statistics. Table III.6 and III.13 (of Chapter III) show the statistics of the daily, whole-building load for standard day operations regressed against average daily drybulb and wetbulb temperature. As expected, the annual $R^{2}$ improved significantly. The $R^{2}$ for the office building during the summer did not improve as much as the others. This is mainly due to the smaller range of average daily temperature which occur during the summer and the fact that the air conditioner operation exhibited little temperature dependence since it was operating near full capacity during the summer.

Hence, one way to improve the EDA prediction may be to estimate the weather dependency of the whole-building load at the daily level. With the daily load defined, an algorithm can be developed, based on hourly temperatures and the operating schedules of the HVAC system, to distribute the daily load into hourly loads. The details of such an algorithm have yet to be designed. 


\section{Components of the Non-Temperature-Dependent Load}

Once the temperature-dependent part of the load is separated from the whole-building load, the remaining components include non-temperature-dependent HVAC, lighting, equipment, miscellaneous, refrigeration, and possibly other loads. We rely on three sources of data to disaggregate the non-temperature-dependent base load into its components:

1. audit estimates of the building and equipment energy intensity, characteristics, and operation;

2. DOE-2 simulation of the building; and

3. simplified engineering analysis.

In the following section, we discuss the various methods for base-load disaggregation in further detail.

How can we estimate the air-conditioning load at a base temperature from the analysis of the whole-building load?

We cannot accurately estimate the baseload, air-conditioning electricity use from the analysis of the whole-building load alone. In order to analyze the base load, we need to understand the contribution of each HVAC system component to the HVAC electricity use. During cooling, the two major components contributing to the HVAC energy use are the compressor and the ventilation fan. Assuming that we have separated the weather-dependent part of the wholebuilding load, we need to find correlations between the base-temperature HVAC load and the other loads in the building.

DOE-2 simulations of the building and its systems provide an estimate of the HVAC electricity use at a base temperature. Simulations require extensive information on the building and its HVAC system. Instead of DOE-2 simulations, one may also rely on simplified default values for the air-conditioning and ventilation electricity use. The fraction of the energy used by the HVAC system is primarily a function of HVAC type and its operation schedules. In order to develop default tables for estimating the HVAC contribution at the base temperature, one would need to review and analyze significant quantities of measured end-use data and condense the results of the analysis into simplified default values. The same rules also apply to estimating the electricity use for other end uses such as lighting and equipment. Currently, this information is supplied by the audit. 
Tables IV.3a, IV.3b, and IV.4 show the hourly electricity use by end use at the base $60^{\circ} \mathrm{F}$ temperature as a fraction of whole-building electricity use for the office and the retail building. For the office building during the hours 9 to 16 , the HVAC electricity use is $38 \%$ to $44 \%$ of the whole-building electricity use at the base temperature $60^{\circ} \mathrm{F}$. In the retail store, the HVAC contribution to the whole-building electricity use at base temperature is $20 \%$ to $25 \%$ (hours 9 to 14 ). These tables indicate that the whole-building-load temperature regressions alone cannot provide a good estimate of the air-conditioning load at the base temperature. One must rely on other sources (DOE-2 simulations or hourly rules-of-thumbs) for estimating the air-conditioning electricity use at the base temperature.

Can we estimate the non-HVAC end uses from the analysis of whole-building data?

In a majority of building types, chillers and air-handling systems are usually operated together during cooling. Hence, their presence can be identified from the regressions of the whole-building load. If the whole-building energy use data showed some hours with no significant correlation to temperature (usually nighttime hours), it can be assumed that the energy use for those hours are for non-HVAC end uses.

\section{"Permissible" Hourly End-use Load Variations}

One key factor in estimating end-use load shapes is the hour to hour variation of the end use load during normal operation of the building and systems. Even though abrupt hourly variations of the end-use loads are possible during the normal operation of the building, we expect these variations to be fairly smooth and, hence constraint our estimates to be smooth. In order to test this hypothesis, we performed statistical analyses for the normalized whole-building load and normalizet end-use loads. The hourly loads for each day were normalized by dividing them by the maximum hourly value for that day. The variation of the mean hourly end-use values over the day were compared as well as the standard deviation of the values for a particular hour of day over the analysis period. The results are presented in Tables IV.5 and IV.6.

For the retail store, the mean lighting electricity use between the hours 9 to 20 varies from 0.98 to 0.99 of the maximum daily use. This corresponds to about $1 \%$ variation in the hourly lighting load. The standard deviation of the normalized hourly load for hours 9 to 18 is about 4\%; for hours 19 and 20, it is $10 \%$. The mean HVAC electricity use varies between 0.64 to 0.93 during hours 9 to 20. The maximum hourly variation of the load is about $15 \%$ (changes between hours 10 to 11 and 19 to 20). The standard deviations for HVAC energy use are much larger 
Table IV.3a

Summary statisitics by end use for summer (May 1 to October 31 ) for Office \#5 $\operatorname{Total}\left(W / \mathrm{ft}^{2}\right)=\mathrm{a}+\mathrm{b}(\mathrm{T}-60)$; End-use $\left(\mathrm{W} / \mathrm{ft}^{2}\right)=\mathrm{a}\left(\mathrm{a}^{\prime}\right)+\mathrm{b}(\mathrm{T}-60)$

\begin{tabular}{|c|c|c|c|c|c|c|c|c|c|}
\hline \multirow[b]{2}{*}{ Hour } & \multirow[b]{2}{*}{$\mathbf{N}$} & \multicolumn{2}{|c|}{ Total } & \multicolumn{2}{|c|}{ HVAC } & \multicolumn{2}{|c|}{ Light } & \multicolumn{2}{|c|}{ Plug } \\
\hline & & $\begin{array}{c}a \\
\left(W / \mathrm{ft}^{2}\right)\end{array}$ & $\begin{array}{c}b \\
\left(W / \mathrm{ft}^{2} /{ }^{\circ} \mathrm{F}\right)\end{array}$ & $a^{\prime}$ & $\begin{array}{c}\mathrm{b} \\
\left(\mathrm{W} / \mathrm{ft}^{2} /{ }^{\circ} \mathrm{F}\right)\end{array}$ & $a^{\prime}$ & $\begin{array}{c}b \\
\left(W / \mathrm{ft}^{2} /{ }^{\circ} \mathrm{F}\right)\end{array}$ & $a^{\prime}$ & $\begin{array}{c}\mathrm{b} \\
\left(\mathrm{W} / \mathrm{ft}^{2} /{ }^{\circ} \mathrm{F}\right)\end{array}$ \\
\hline 1 & 124 & 0.722 & 0.007 & -0.000 & 0.000 & 0.147 & 0.000 & 0.276 & 0.001 \\
\hline 2 & 122 & 0.706 & 0.004 & 0.000 & -0.000 & 0.133 & 0.000 & 0.282 & 0.001 \\
\hline 3 & 121 & 0.699 & 0.002 & -0.000 & -0.000 & 0.132 & 0.000 & 0.283 & 0.001 \\
\hline 4 & 119 & 0.748 & -0.000 & 0.078 & -0.002 & 0.123 & 0.000 & 0.262 & 0.001 \\
\hline 5 & 118 & 1.382 & 0.014 & 0.527 & 0.023 & 0.065 & 0.000 & 0.141 & 0.001 \\
\hline 6 & 116 & 1.921 & 0.024 & 0.592 & 0.039 & 0.076 & 0.000 & 0.104 & 0.002 \\
\hline 7 & 116 & 2.383 & 0.004 & 0.500 & 0.016 & 0.159 & 0.000 & 0.106 & 0.003 \\
\hline 8 & 120 & 3.168 & 0.005 & 0.422 & 0.012 & 0.305 & 0.000 & 0.112 & 0.002 \\
\hline 9 & 126 & 3.547 & 0.016 & 0.394 & 0.032 & 0.367 & 0.000 & 0.113 & 0.001 \\
\hline 10 & 126 & 3.635 & 0.023 & 0.395 & 0.039 & 0.372 & 0.000 & 0.113 & -0.000 \\
\hline 11 & 128 & 3.670 & 0.023 & 0.392 & 0.038 & 0.372 & 0.000 & 0.112 & 0.000 \\
\hline 12 & 128 & 3.673 & 0.019 & 0.387 & 0.035 & 0.368 & 0.000 & 0.115 & -0.000 \\
\hline 13 & 128 & 3.671 & 0.016 & 0.382 & 0.032 & 0.371 & 0.000 & 0.110 & 0.001 \\
\hline 14 & 128 & 3.718 & 0.013 & 0.393 & 0.031 & 0.375 & 0.000 & 0.109 & 0.000 \\
\hline 15 & 128 & 3.750 & 0.012 & 0.412 & 0.029 & 0.372 & 0.000 & 0.107 & -0.000 \\
\hline 16 & 128 & 3.591 & 0.013 & 0.439 & 0.029 & 0.355 & 0.000 & 0.102 & -0.000 \\
\hline 17 & 128 & 3.102 & 0.016 & 0.483 & 0.033 & 0.297 & 0.000 & 0.094 & 0.000 \\
\hline 18 & 128 & 2.629 & 0.024 & 0.506 & 0.039 & 0.214 & 0.000 & 0.092 & 0.001 \\
\hline 19 & 128 & 2.319 & 0.025 & 0.530 & 0.036 & 0.153 & 0.000 & 0.092 & 0.001 \\
\hline 20 & 128 & 2.134 & 0.017 & 0.527 & 0.028 & 0.137 & 0.000 & 0.096 & 0.001 \\
\hline 21 & 128 & 1.548 & -0.015 & 0.395 & -0.011 & 0.156 & 0.000 & 0.130 & 0.001 \\
\hline 22 & 127 & 0.851 & 0.006 & -0.000 & -0.000 & 0.250 & 0.000 & 0.233 & 0.001 \\
\hline 23 & 128 & 0.806 & 0.007 & 0.000 & 0.000 & 0.222 & 0.000 & 0.247 & 0.001 \\
\hline 24 & 127 & 0.759 & 0.010 & 0.000 & -0.000 & 0.178 & 0.000 & 0.262 & 0.002 \\
\hline
\end{tabular}


Table IV.3b

Summary statisitics by end use for winter (Nov 1 to April 30) for Office \#5 $\operatorname{Total}\left(W / \mathrm{ft}^{2}\right)=\mathrm{a}+\mathrm{b}(\mathrm{T}-60) ;$ End-use $\left(W / \mathrm{ft}^{2}\right)=\mathrm{a}\left(\mathrm{a}^{2}\right)+\mathrm{b}(\mathrm{T}-60)$

\begin{tabular}{|c|c|c|c|c|c|c|c|c|c|}
\hline \multirow[b]{2}{*}{ Hour } & \multirow[b]{2}{*}{$\mathbf{N}$} & \multicolumn{2}{|c|}{ Total } & \multicolumn{2}{|c|}{ HVAC } & \multicolumn{2}{|c|}{ Light } & \multicolumn{2}{|c|}{ Plug } \\
\hline & & $\begin{array}{c}a \\
\left(W / f^{2}{ }^{2}\right)\end{array}$ & $\begin{array}{c}\mathrm{b} \\
\left(\mathrm{W} / \mathrm{ft}^{2} /{ }^{\circ} \mathrm{F}\right)\end{array}$ & $a^{\prime}$ & $\begin{array}{c}\mathrm{b} \\
\left(\mathrm{W} / \mathrm{ft}^{2} /{ }^{\circ} \mathrm{F}\right)\end{array}$ & $a^{\prime}$ & $\begin{array}{c}\mathrm{b} \\
\left(\mathrm{W} / \mathrm{ft}^{2} /{ }^{\circ} \mathrm{F}\right)\end{array}$ & $a^{\prime}$ & $\begin{array}{c}\mathrm{b} \\
\left(\mathrm{W} / \mathrm{ft}^{2} /{ }^{\circ} \mathrm{F}\right)\end{array}$ \\
\hline 1 & 121 & 0.790 & 0.004 & 0.053 & 0.005 & 0.162 & 0.000 & 0.233 & 0.000 \\
\hline 2 & 121 & 0.756 & 0.004 & 0.050 & 0.004 & 0.147 & 0.000 & 0.241 & 0.001 \\
\hline 3 & 121 & 0.701 & 0.000 & -0.000 & 0.000 & 0.141 & 0.000 & 0.258 & 0.001 \\
\hline 4 & 121 & 0.766 & 0.004 & 0.093 & 0.004 & 0.123 & 0.000 & 0.236 & 0.001 \\
\hline 5 & 121 & 1.199 & 0.020 & 0.429 & 0.018 & 0.078 & 0.000 & 0.151 & 0.001 \\
\hline 6 & 120 & 1.616 & 0.029 & 0.554 & 0.024 & 0.058 & 0.000 & 0.111 & 0.001 \\
\hline 7 & 121 & 2.112 & 0.043 & 0.542 & 0.031 & 0.089 & 0.000 & 0.090 & 0.001 \\
\hline 8 & 121 & 2.706 & 0.054 & 0.448 & 0.032 & 0.224 & 0.000 & 0.098 & 0.004 \\
\hline 9 & 121 & 3.277 & 0.042 & 0.399 & 0.029 & 0.330 & 0.000 & 0.102 & 0.002 \\
\hline 10 & 121 & 3.472 & 0.031 & 0.390 & 0.024 & 0.364 & 0.000 & 0.102 & 0.001 \\
\hline 11 & 121 & 3.467 & 0.029 & 0.385 & 0.024 & 0.372 & 0.000 & 0.103 & 0.001 \\
\hline 12 & 121 & 3.412 & 0.027 & 0.374 & 0.022 & 0.381 & 0.000 & 0.106 & 0.001 \\
\hline 13 & 121 & 3.385 & 0.024 & 0.371 & 0.021 & 0.383 & 0.000 & 0.107 & 0.001 \\
\hline 14 & 121 & 3.425 & 0.021 & 0.384 & 0.018 & 0.383 & 0.000 & 0.103 & 0.001 \\
\hline 15 & 121 & 3.452 & 0.021 & 0.399 & 0.018 & 0.383 & 0.000 & 0.103 & 0.001 \\
\hline 16 & 121 & 3.442 & 0.021 & 0.411 & 0.019 & 0.379 & 0.000 & 0.102 & 0.001 \\
\hline 17 & 121 & 3.221 & 0.017 & 0.424 & 0.018 & 0.373 & 0.000 & 0.102 & 0.000 \\
\hline 18 & 121 & 2.719 & 0.019 & 0.444 & 0.019 & 0.334 & 0.000 & 0.097 & 0.000 \\
\hline 19 & 121 & 2.335 & 0.022 & 0.475 & 0.020 & 0.254 & 0.000 & 0.093 & 0.001 \\
\hline 20 & 121 & 2.095 & 0.024 & 0.515 & 0.020 & 0.175 & 0.000 & 0.093 & 0.001 \\
\hline 21 & 121 & 1.690 & 0.023 & 0.446 & 0.020 & 0.163 & 0.000 & 0.112 & 0.001 \\
\hline 22 & 121 & 1.162 & 0.014 & 0.264 & 0.014 & 0.178 & 0.000 & 0.158 & 0.001 \\
\hline 23 & 121 & 0.804 & -0.001 & 0.001 & 0.000 & 0.228 & 0.000 & 0.229 & 0.001 \\
\hline 24 & 121 & 0.787 & -0.001 & 0.001 & 0.000 & 0.235 & 0.000 & 0.235 & 0.001 \\
\hline
\end{tabular}




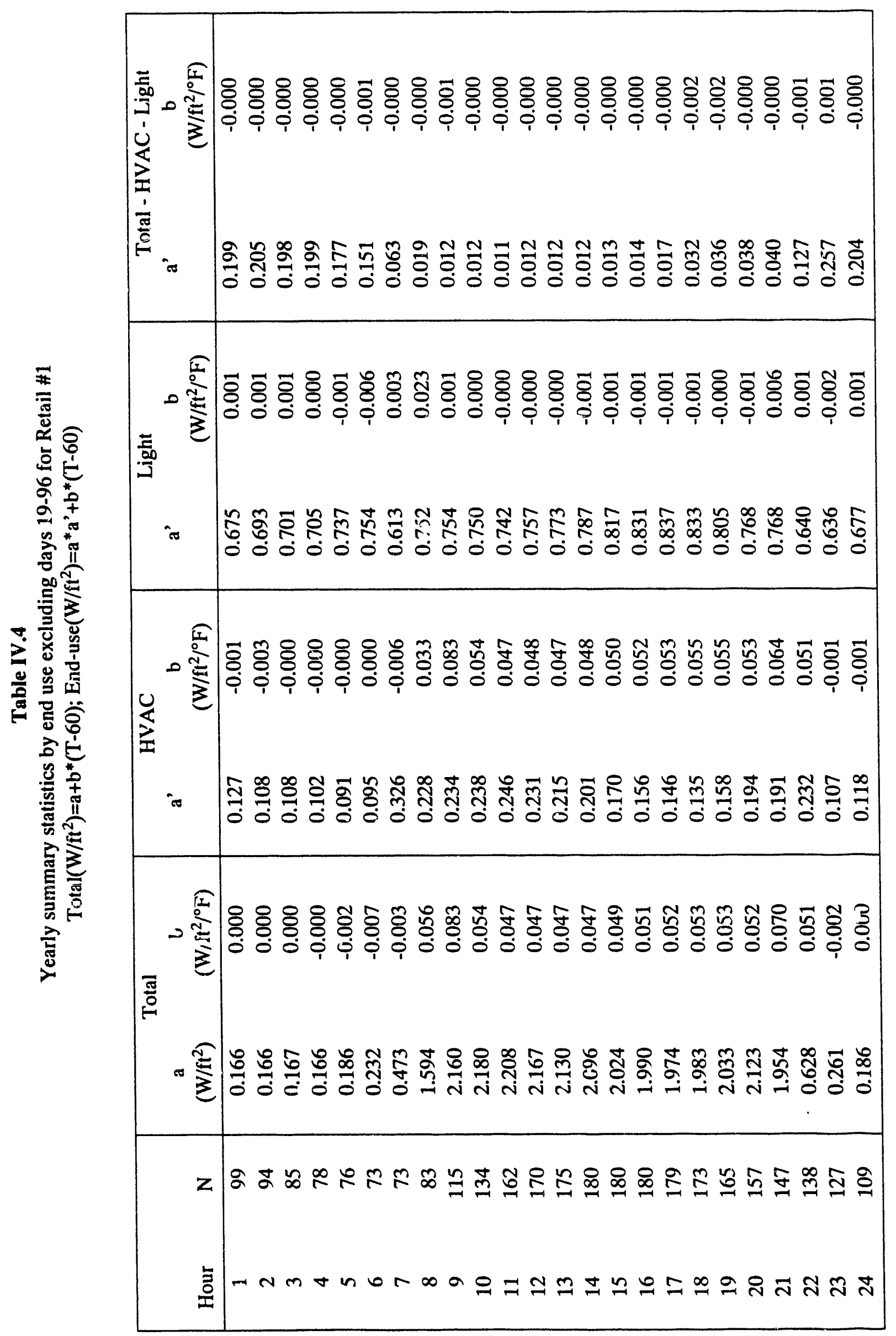




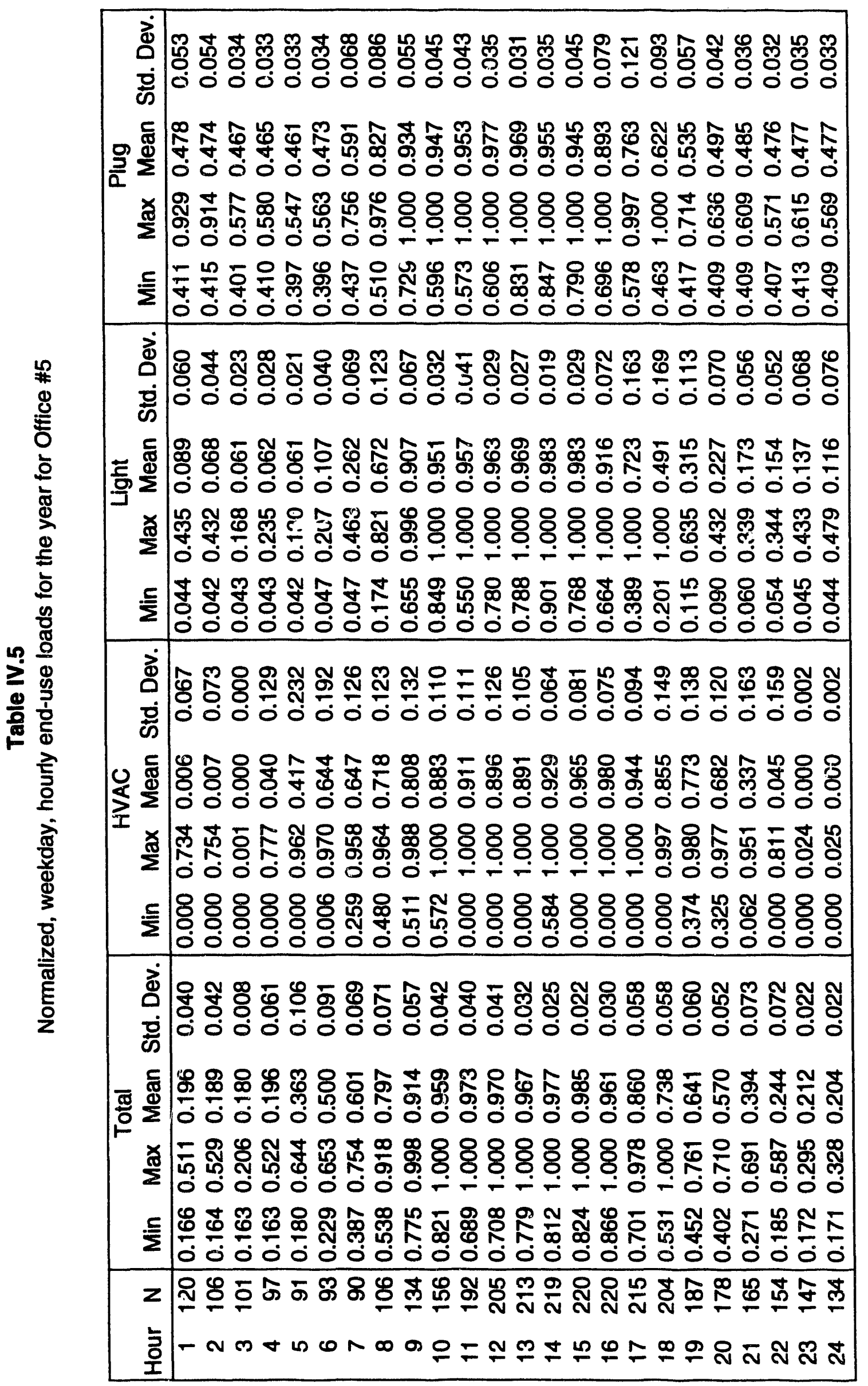




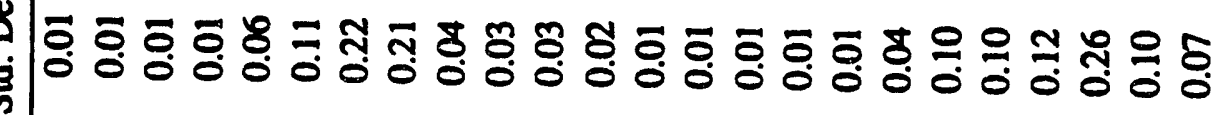

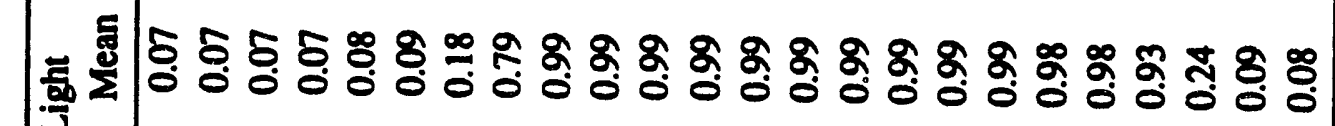
ב

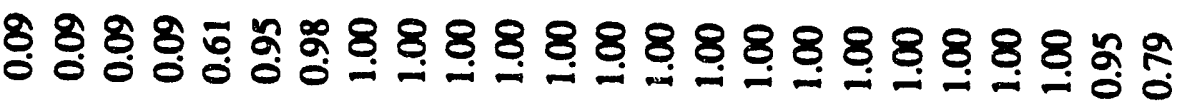

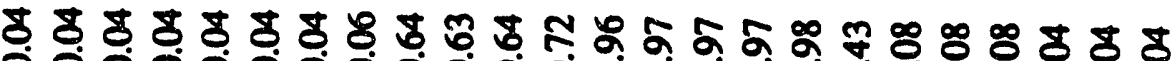

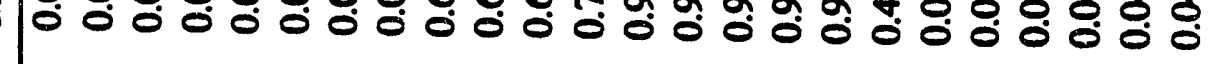

安

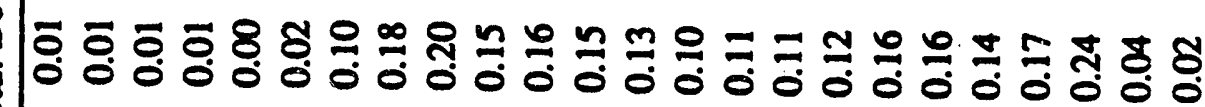

के

ч

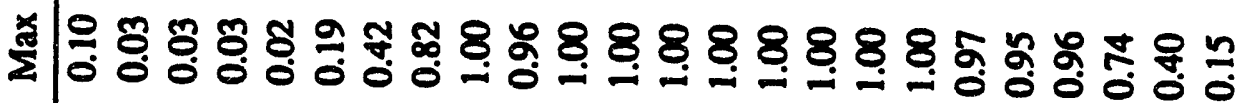

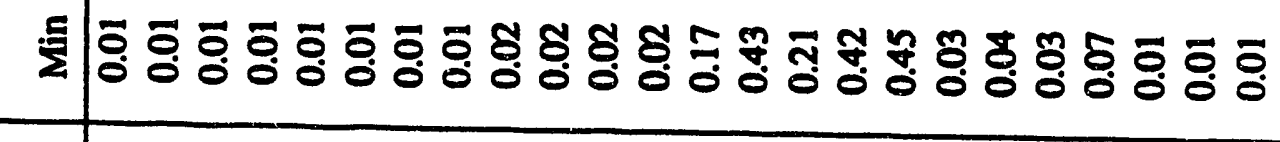

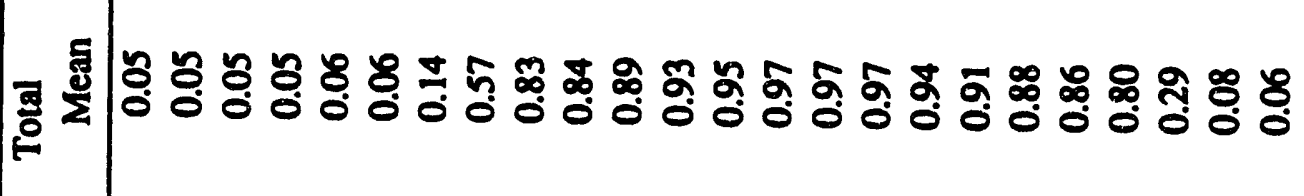

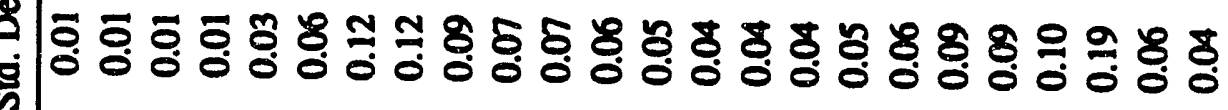
药 ₹

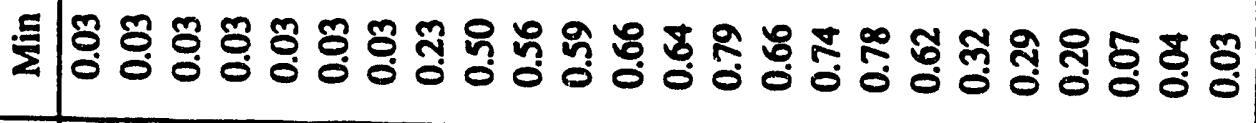

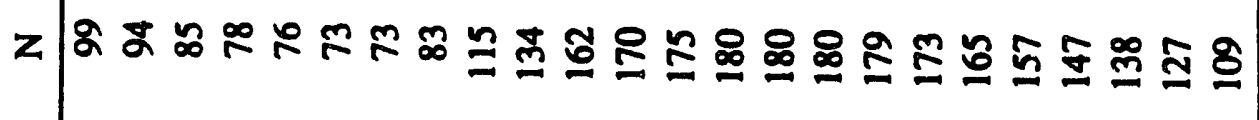

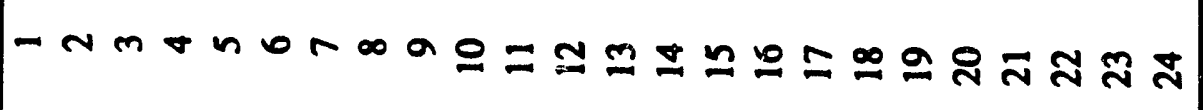


than the other end uses, typically 10 to $15 \%$ during daytime and up to $25 \%$ during the early morning and early evening hours. The mean whole-building load for hours 9 to 20 varies between 0.83 to 0.97 with typical standard deviations from $4 \%$ to $9 \%$.

For the office building, the mean lighting load between the hours 9 to 16 varies from 0.92 to 0.98 (about an $8 \%$ variation) with standard deviations ranging from 2 to $7 \%$. The normalized plug load for the same hours varies from 0.89 to 0.98 (about $11 \%$ variation) with slightly larger standard deviations. The mean values for the HVAC system has about the same variation as the lighting and plug loads but with standard deviations in the range of $7 \%$ to $13 \%$. Also, the office whole-building load for the same hours, is fairly flat; varying only from 0.91 to 0.98 with fairly small standard deviations.

This analysis of the two buildings indicates that most variations in the whole-building hourly load are typically caused by HVAC systems. Furthermore, the operations of the lighting, plug, and other end uses, on the average, seem to be fairly smooth.

We use this information in EDA to smooth out any abnormal variations in the estimated hourly, non-HVAC, end-use loads. These variations mainly occur during the shoulder hours, when there is a mismatch between the auditor's estimate of the operation of the building and that indicated by the measured whole-building load.

\section{Recommendation}

With the above background information, we see three general directions for improving the performance of EDA. The first improvement concerns integrating the hourly and daily regressions. Using the daily regressions enables us to obtain more robust estimates of the temperature-dependent HVAC electricity use by the day. Then with the help of the hourly regressions, we can distribute the daily electricity use to hours of the day. We have yet to design such an algorithm.

A second area for improving the performance of EDA concerns the initial estimates of the non-temperature dependent end uses (both HVAC and non-HVAC end uses). Currently, we rely on simulations based on audit information for estimating the non-temperature dependent end uses. Our analysis has indicated that both simulations and auditor's estimates can be grossly different from what measured data suggest. Utilities have collected end-use data that can be analyzed to provide information on the contribution of non-temperature dependent end uses to the whole-building load. To improve our estimates of the non-temperature-dependent end uses, we recommend analysis of all available measured end-use data and condensing the results of the 
analysis into look-up tables that can be used as initial estimates in EDA applications.

Finally, EDA, in its present form, cannot be applied to buildings that have extensive refrigeration, load-shaping technologies (thermal energy storage system and daylighting), coincident electric cooling and heating loads, erratic load shapes, or unreliable schedules. Although extending EDA to some of these applications may be fairly straightforward, completing such a task should be considered in future EDA development. 


\section{References}

Akbari, H.; Eto, J.; Turiel, I.; Heinemeier, K.; Lebot, B.; Nordman, B.; and Rainer, L. (1989). "Integrated Estimation of Commercial Sector End-Use Load Shapes and Energy Use Intensities," Lawrence Berkeley Laboratory Report, January.

Akbari, H.; Heinemeier, K.; Le Coniac, P.; and Flora, D. (1988). “An Algorithm to Disaggregate Commercial Whole-Building Electric Hourly Load into End Uses," Proceeding of ACEEE 1988 Summer Study on Energy Efficiency in Buildings, Vol 10, pp13-26, Asilomar CA, August.

Lipson, C.; Narendra, S. 1973. Statistical Design and Analysis of Engineering Experiments, McGraw Hill, New York.

SCE. 1990. "End use metered data for commercial buildings," A report prepared by ADM Associates for the Southern California Edison Company, 1988-1989 Annual Report (March).

SCE. 1989. "End use metered data for commercial buildings," A report prepared by ADM Associates for the Southern California Edison Company, Interim Reports Volumes I and II (April). 


\section{Appendix A}

\section{3-D Plots of Metered Data}

For a quick visual check of the SCE monitored data for the 53 commercial buildings, we have generated three dimensional plots of the hourly data. The total building metered electricity and the monitored end uses are shown. Since monitoring started in June of 1989 and ended in May of 1990, there may appear to be discontinuities in the data since we have wrapped the values to create one complete calender year. 

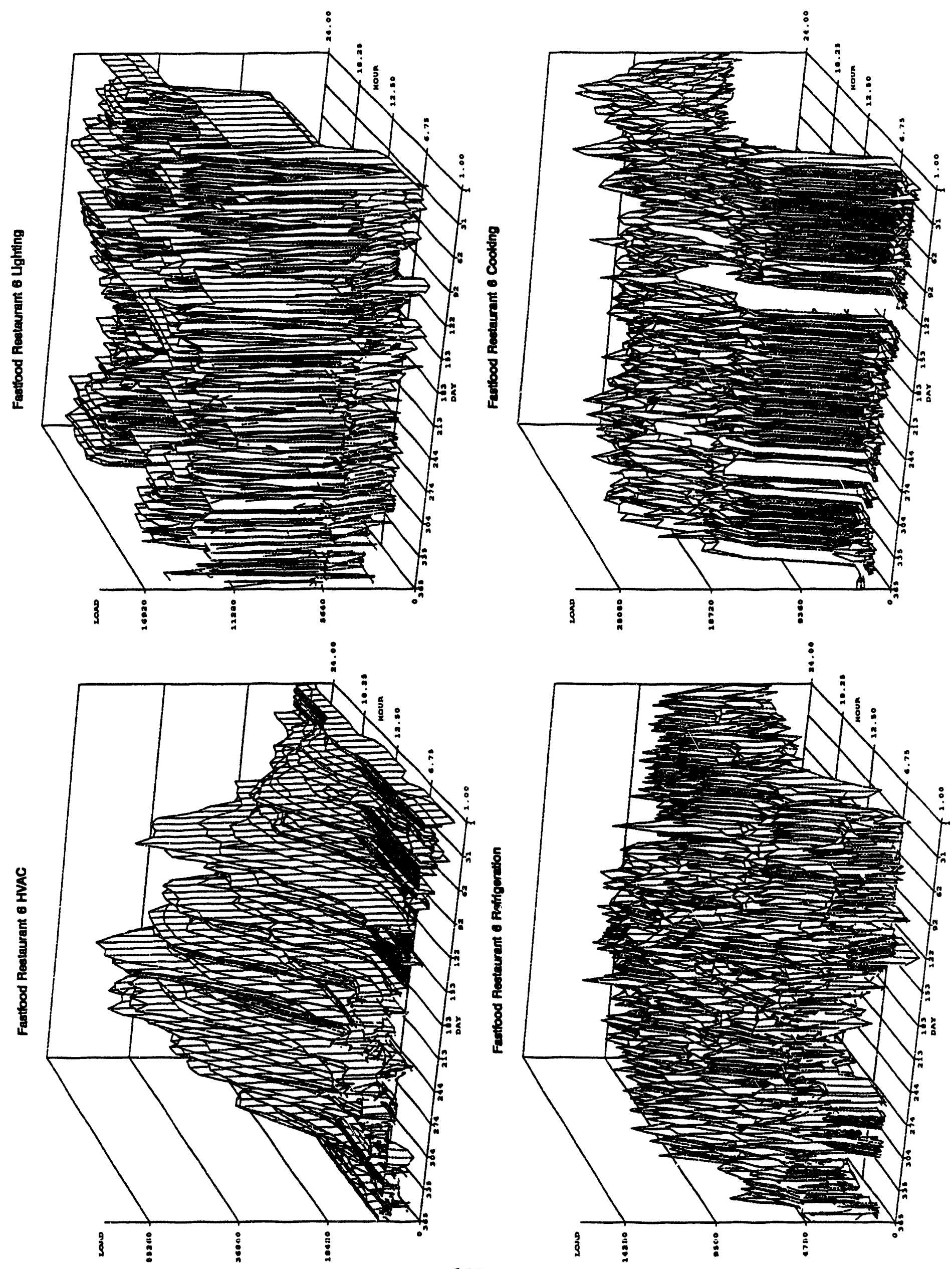

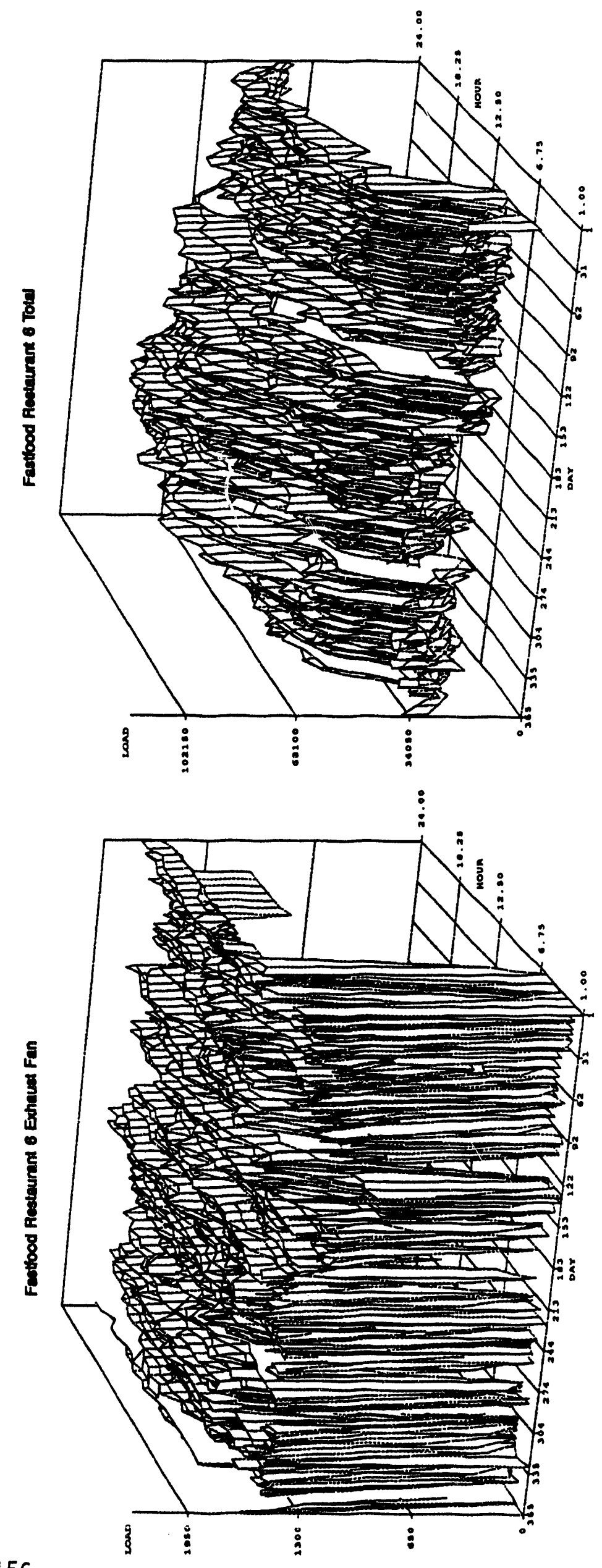

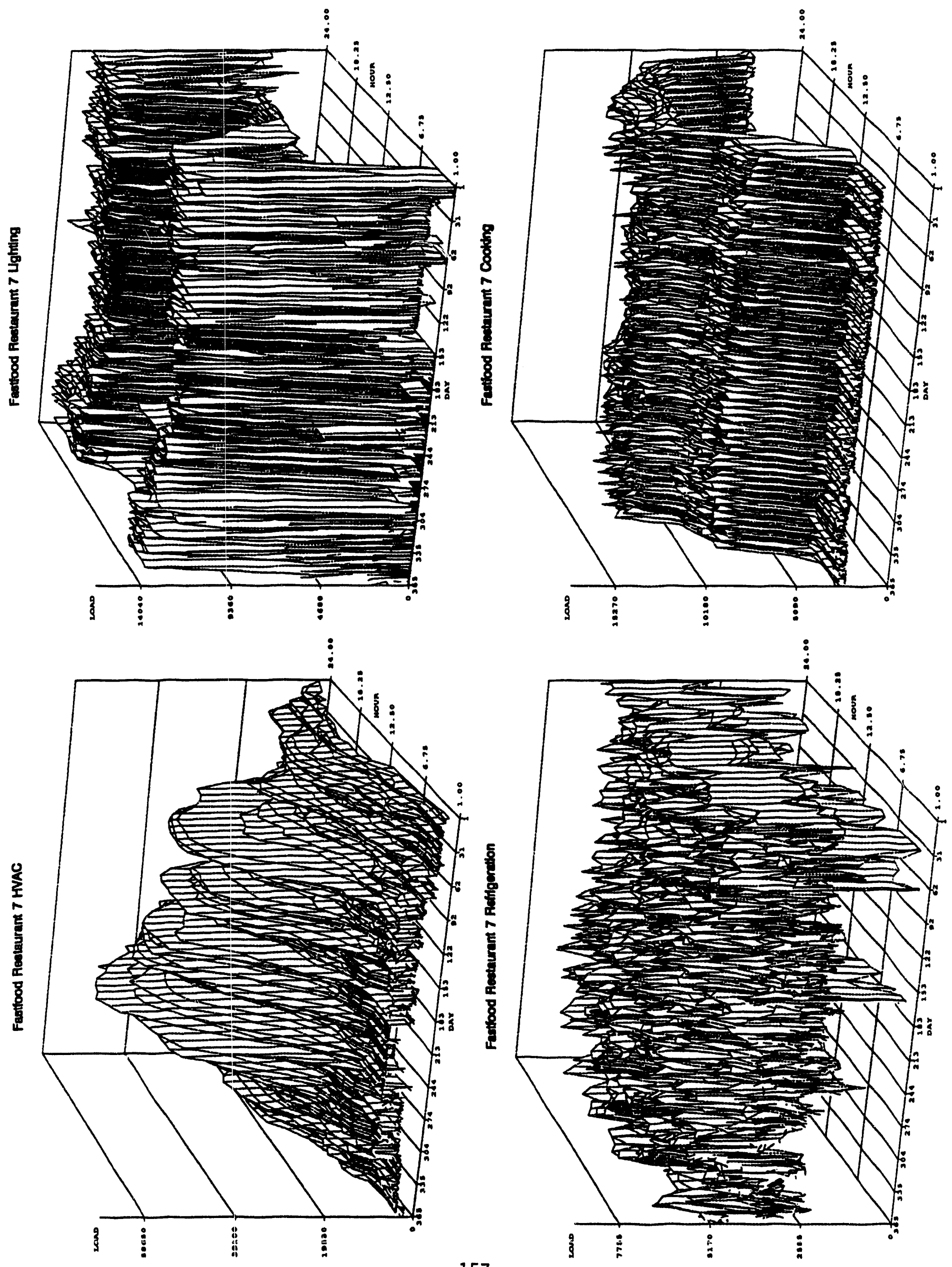


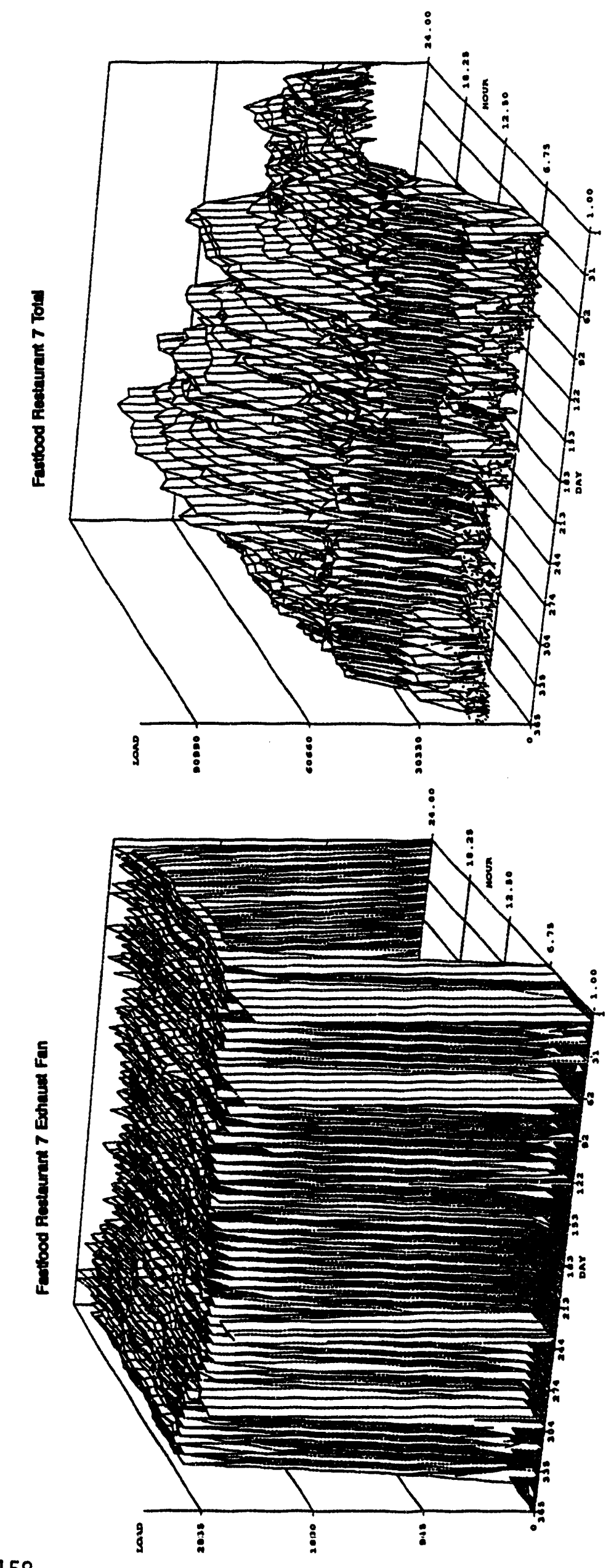



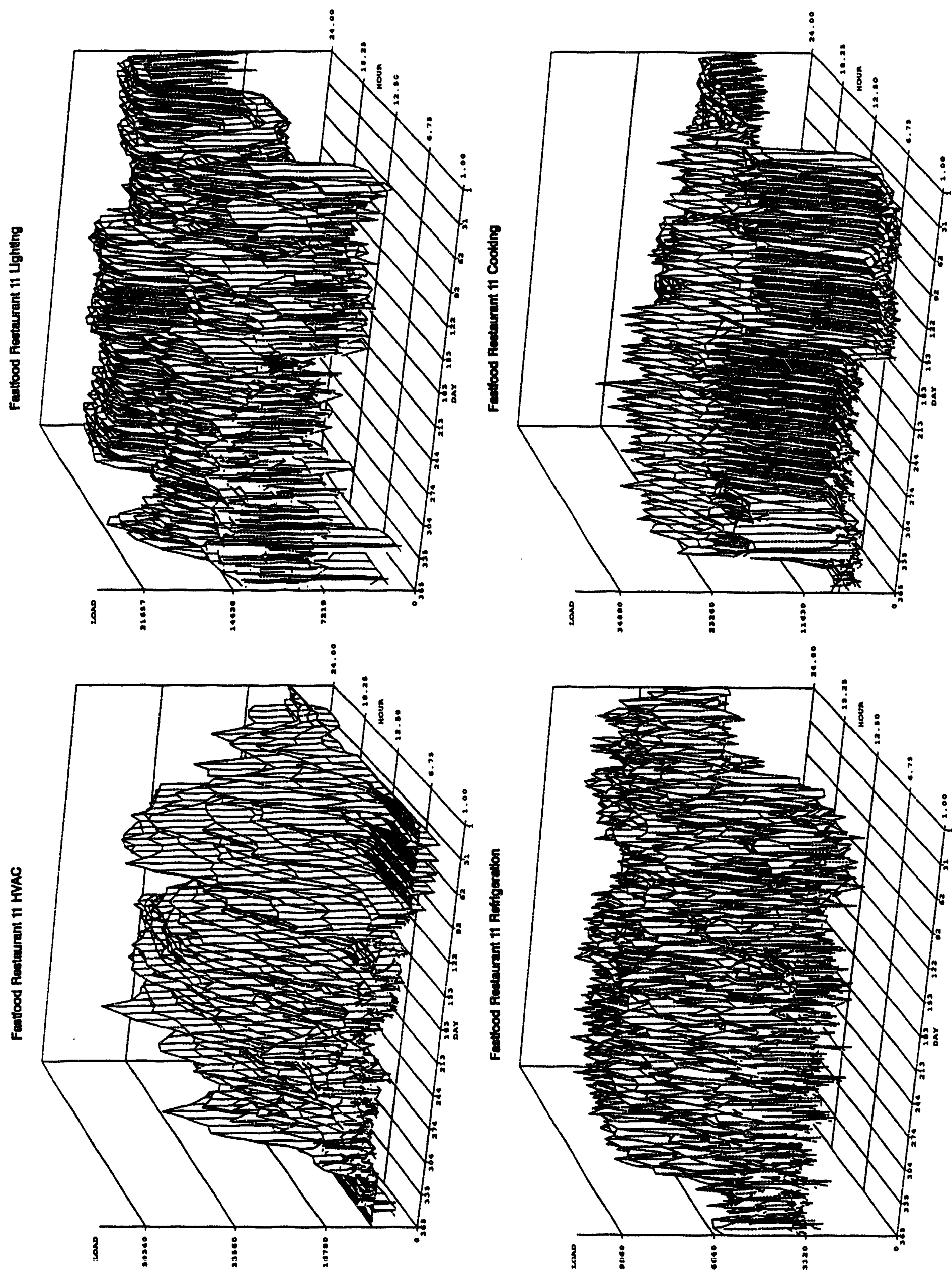

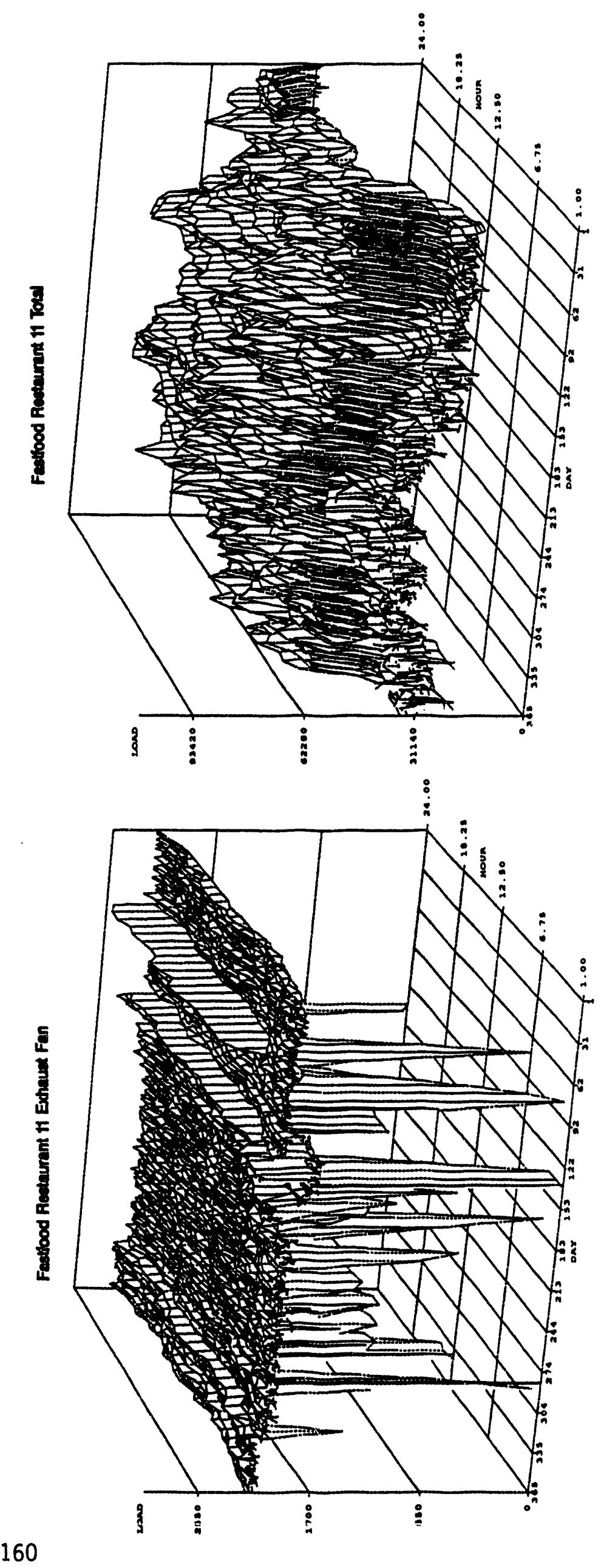

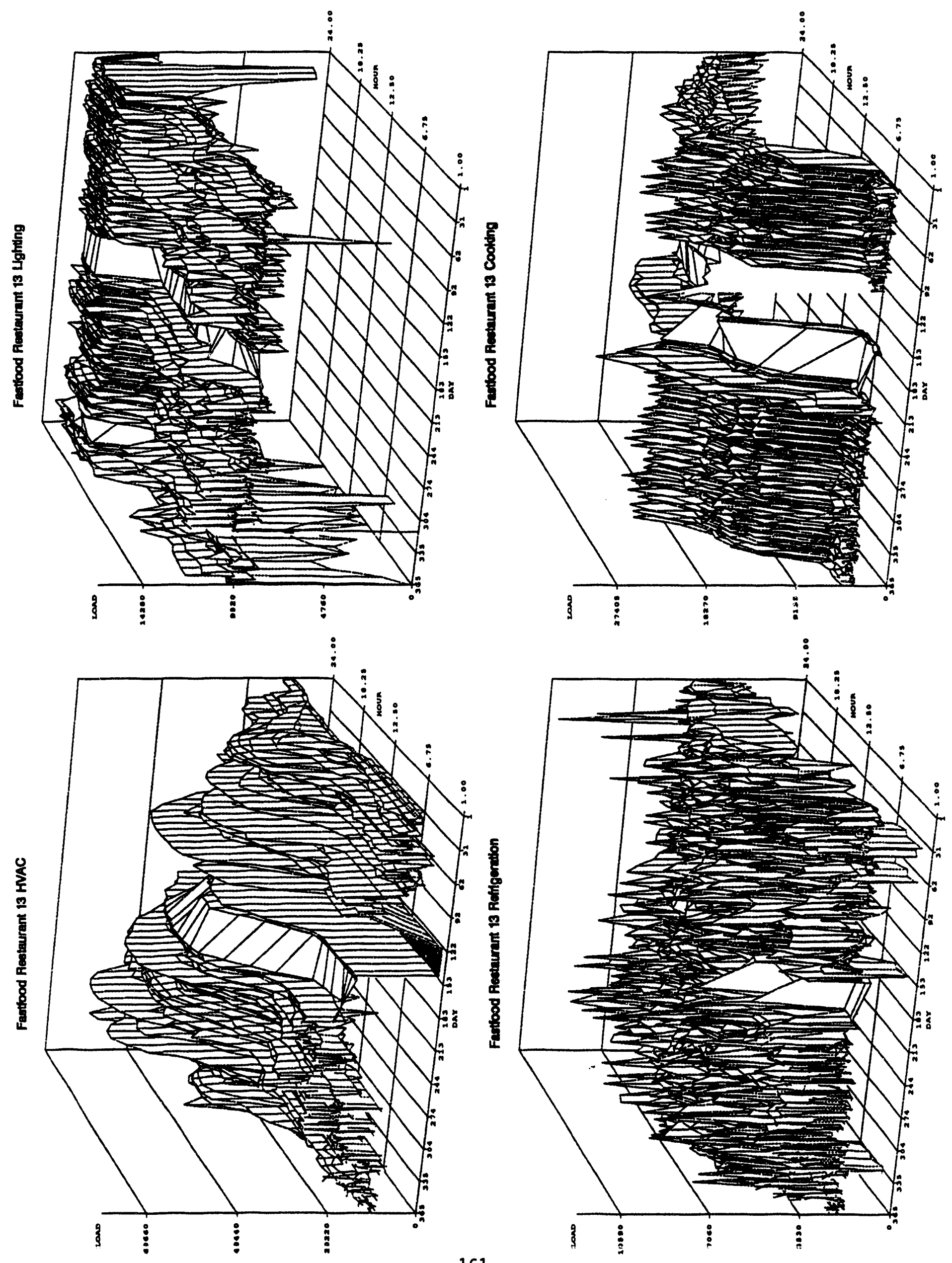

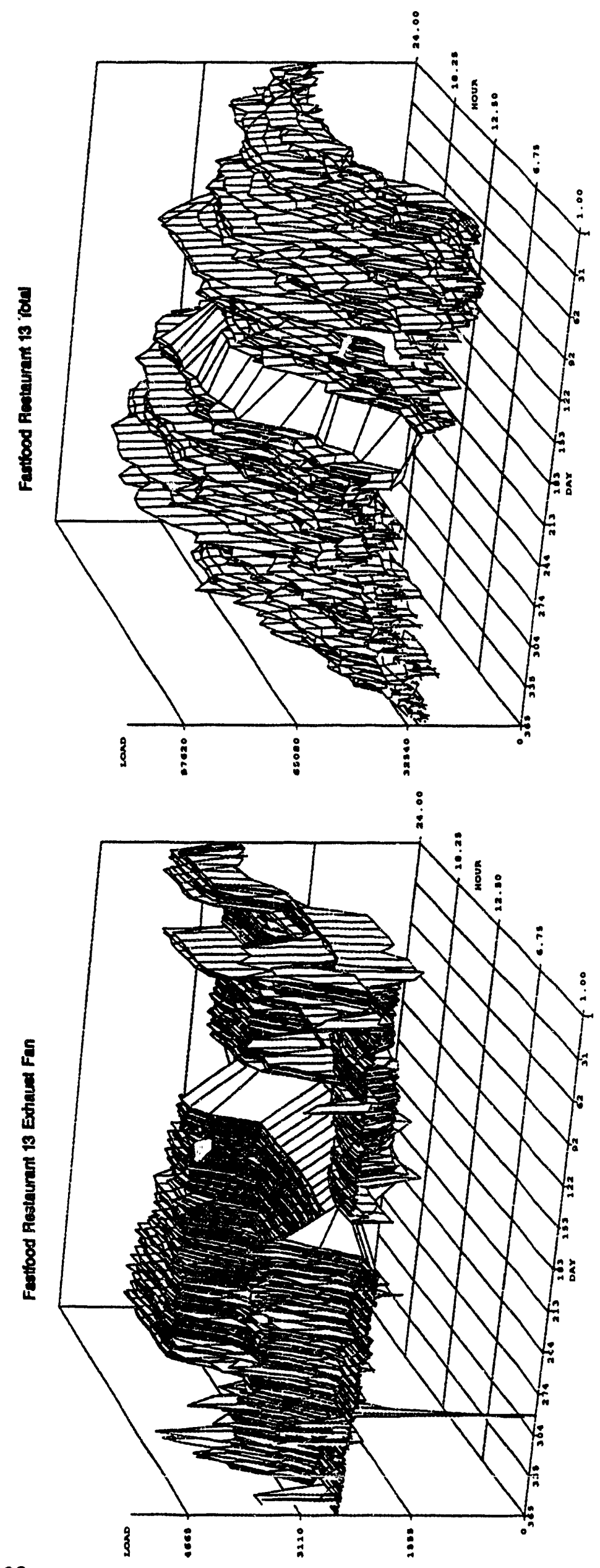

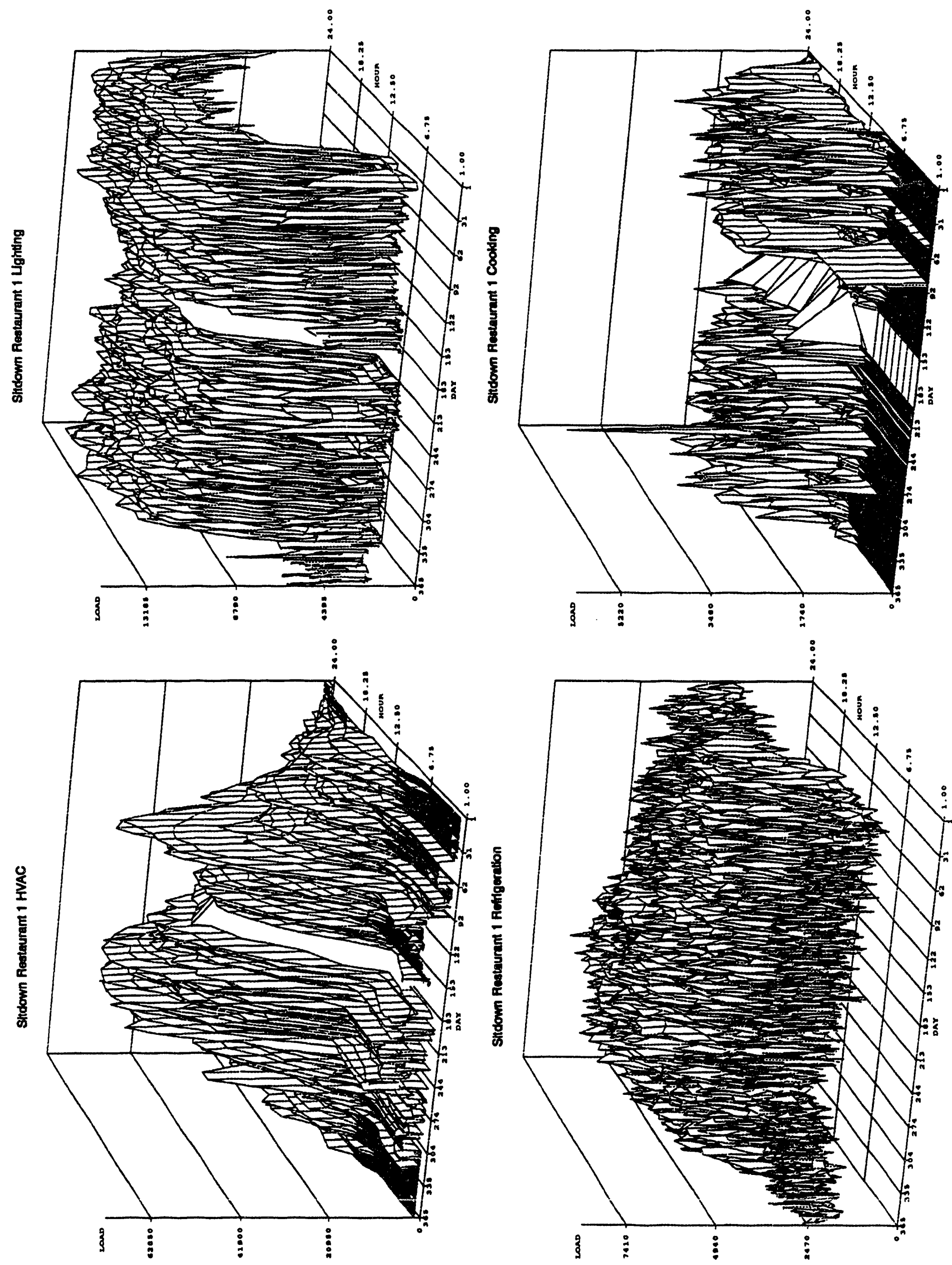

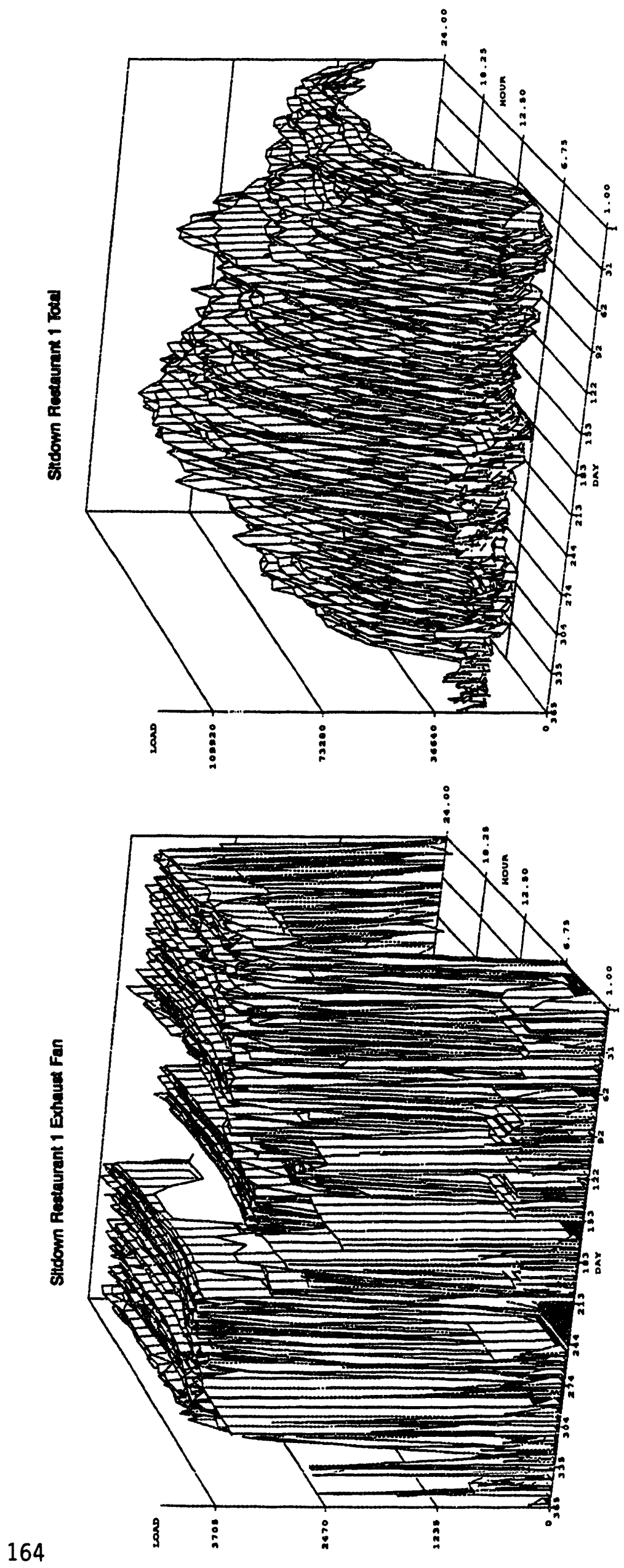

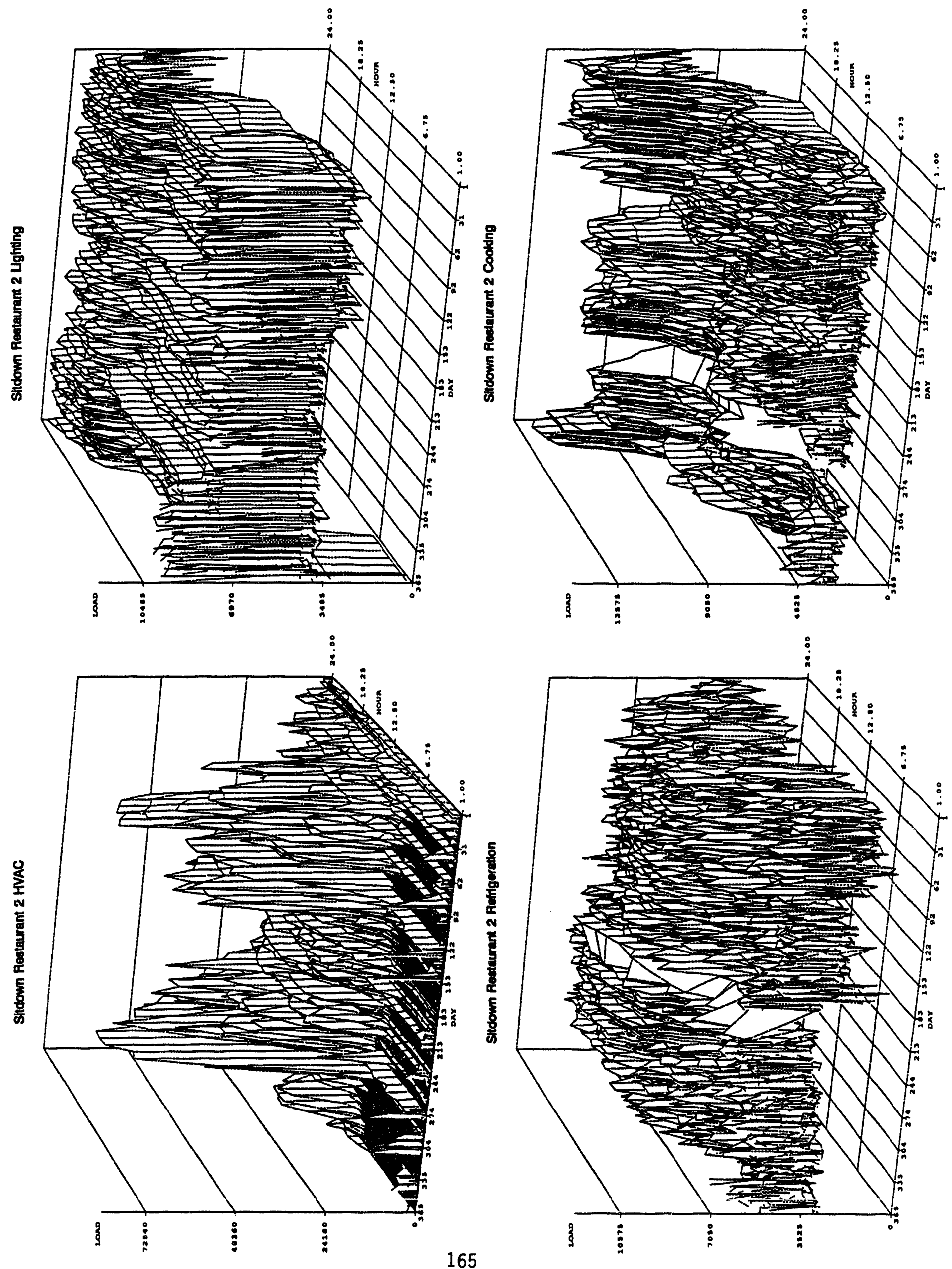

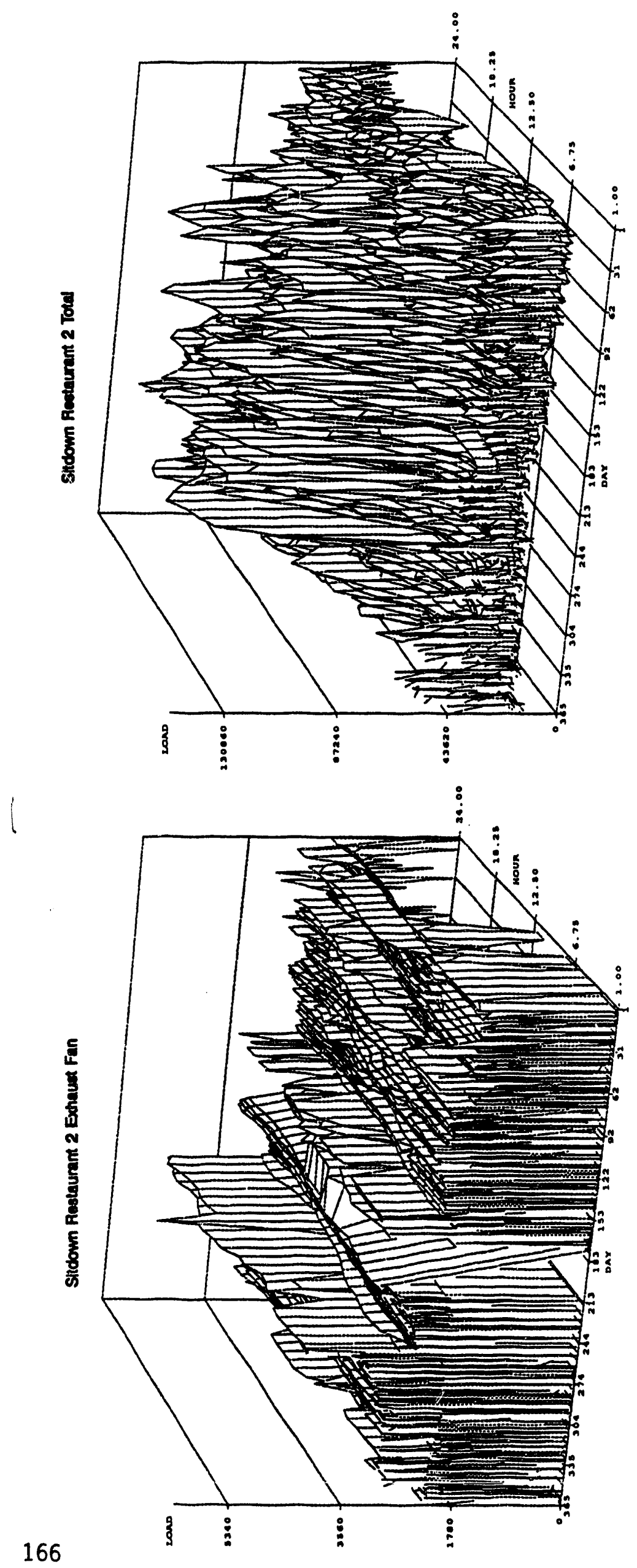

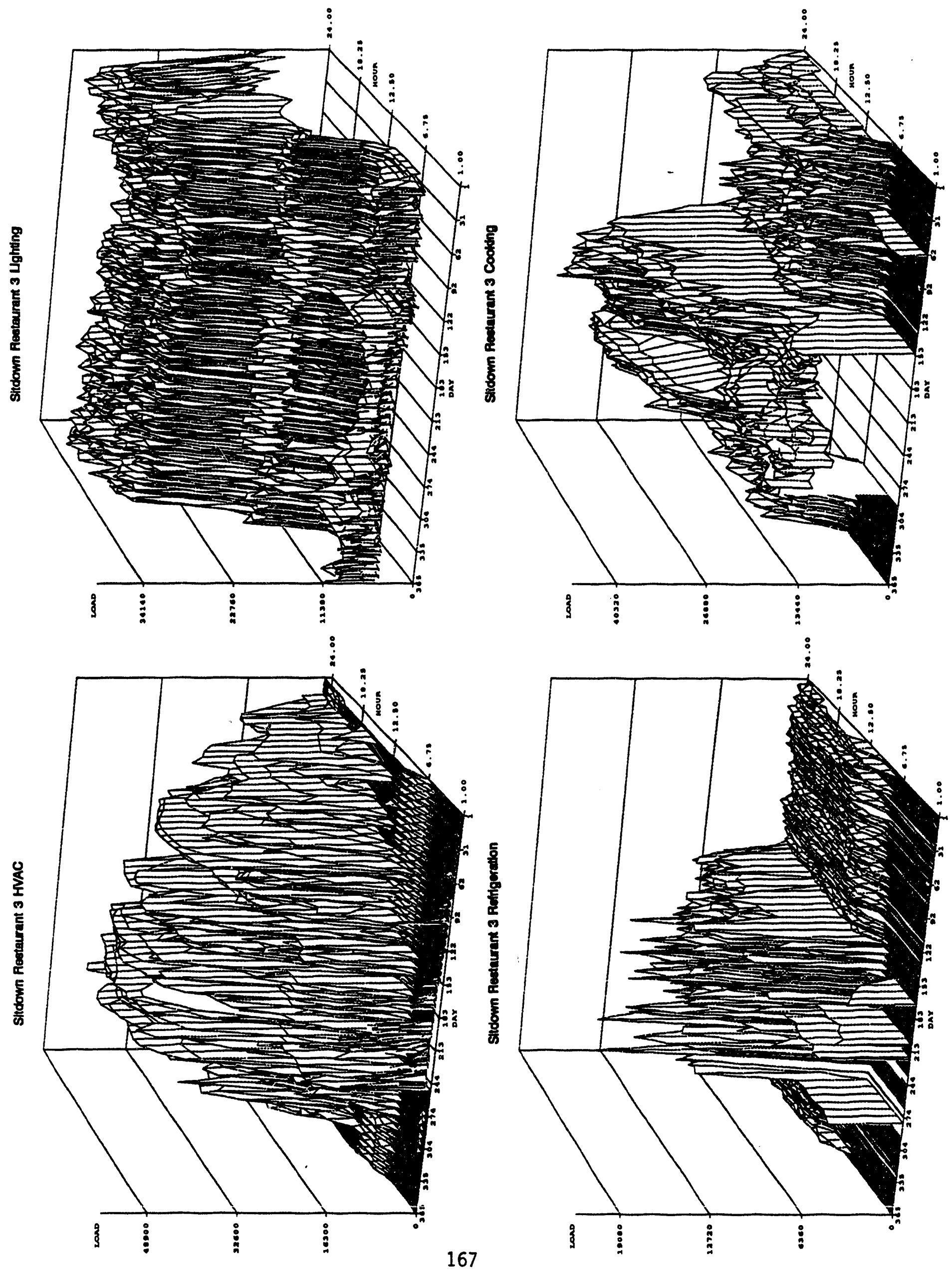

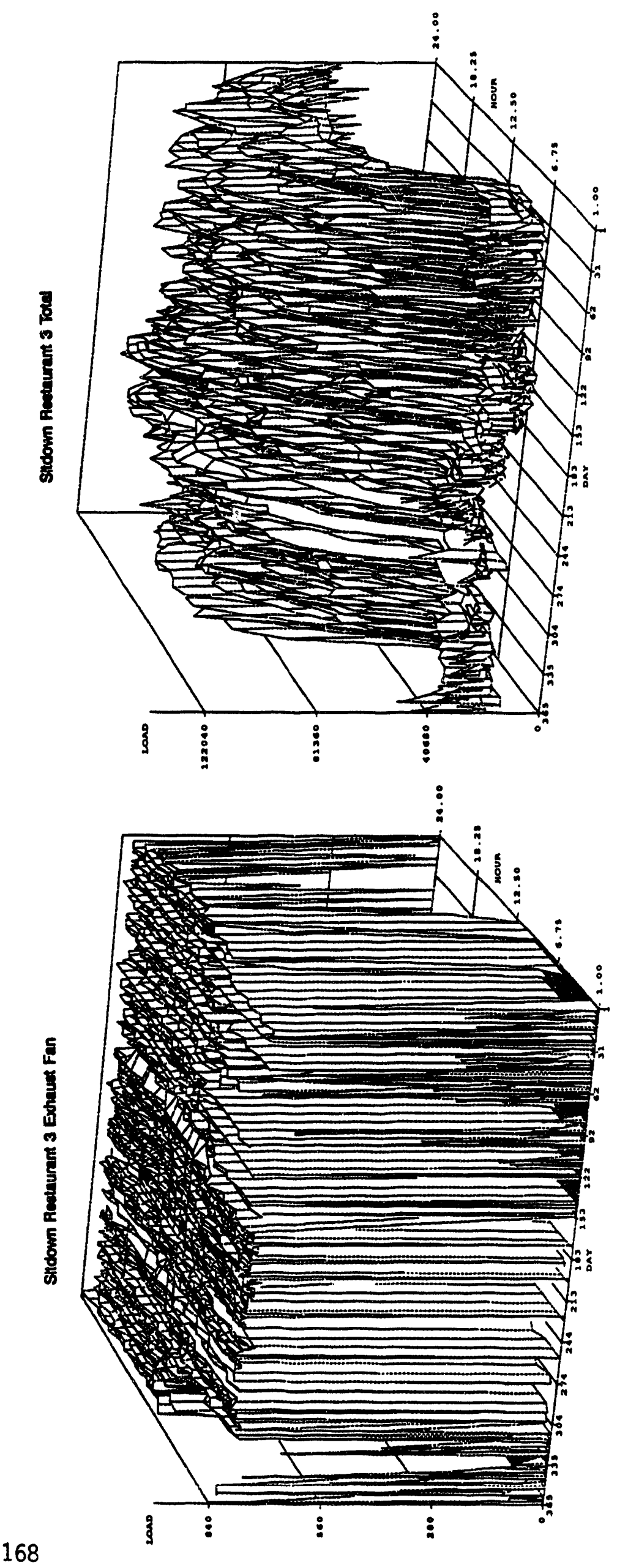

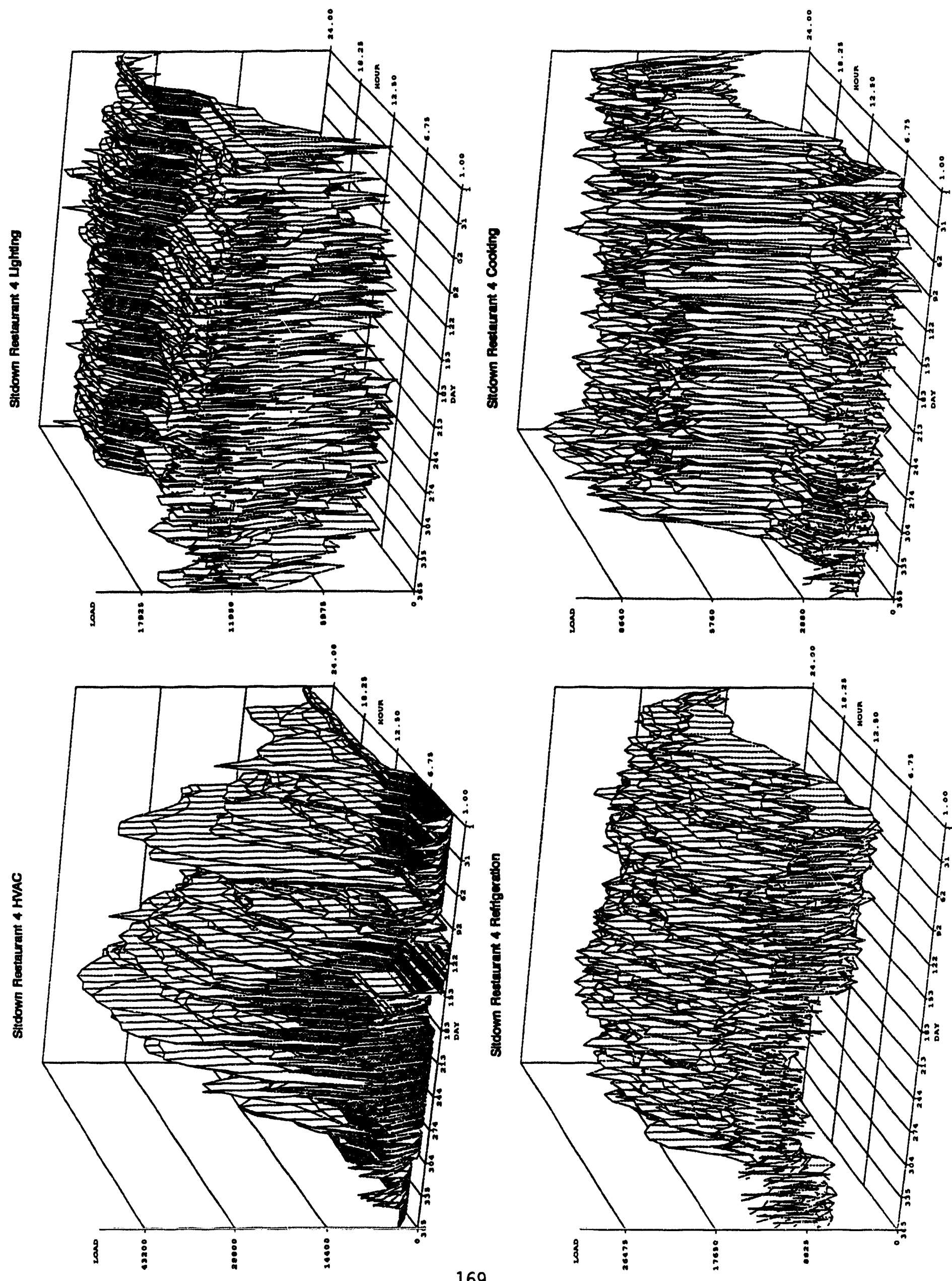


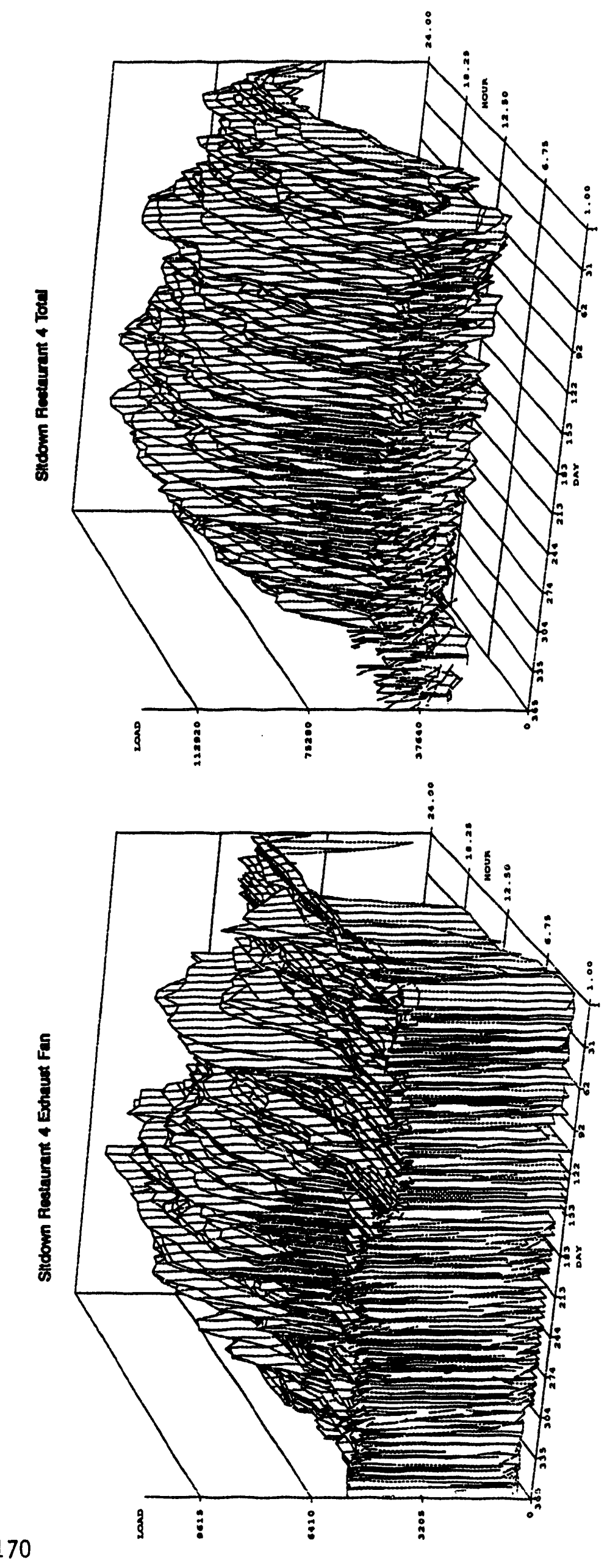



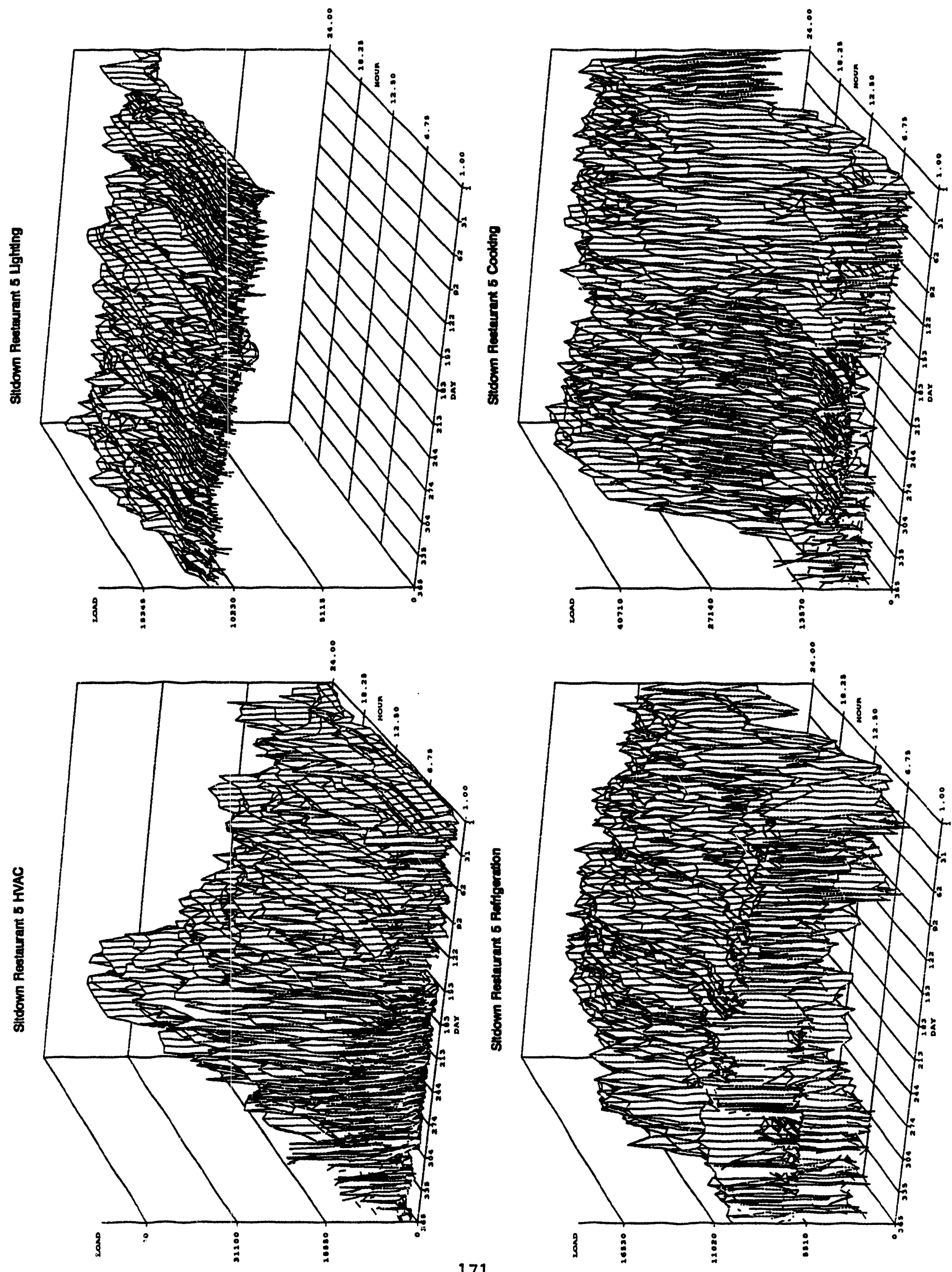


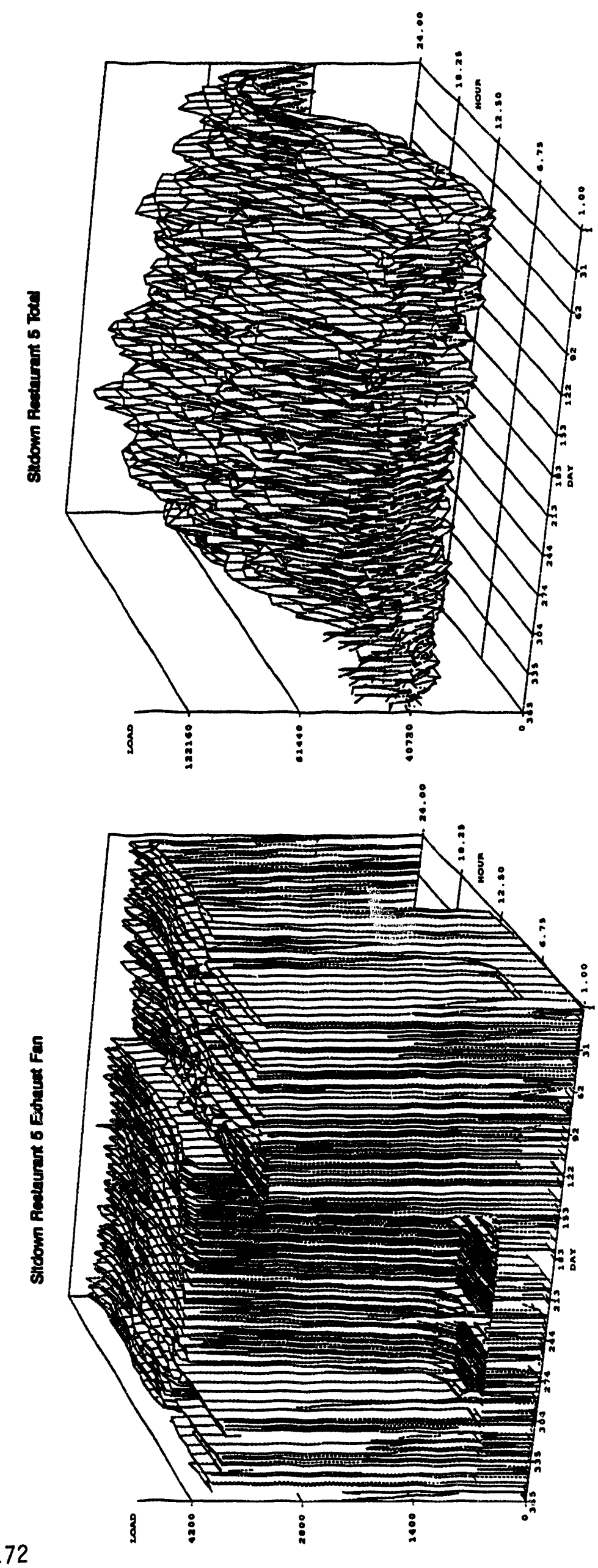



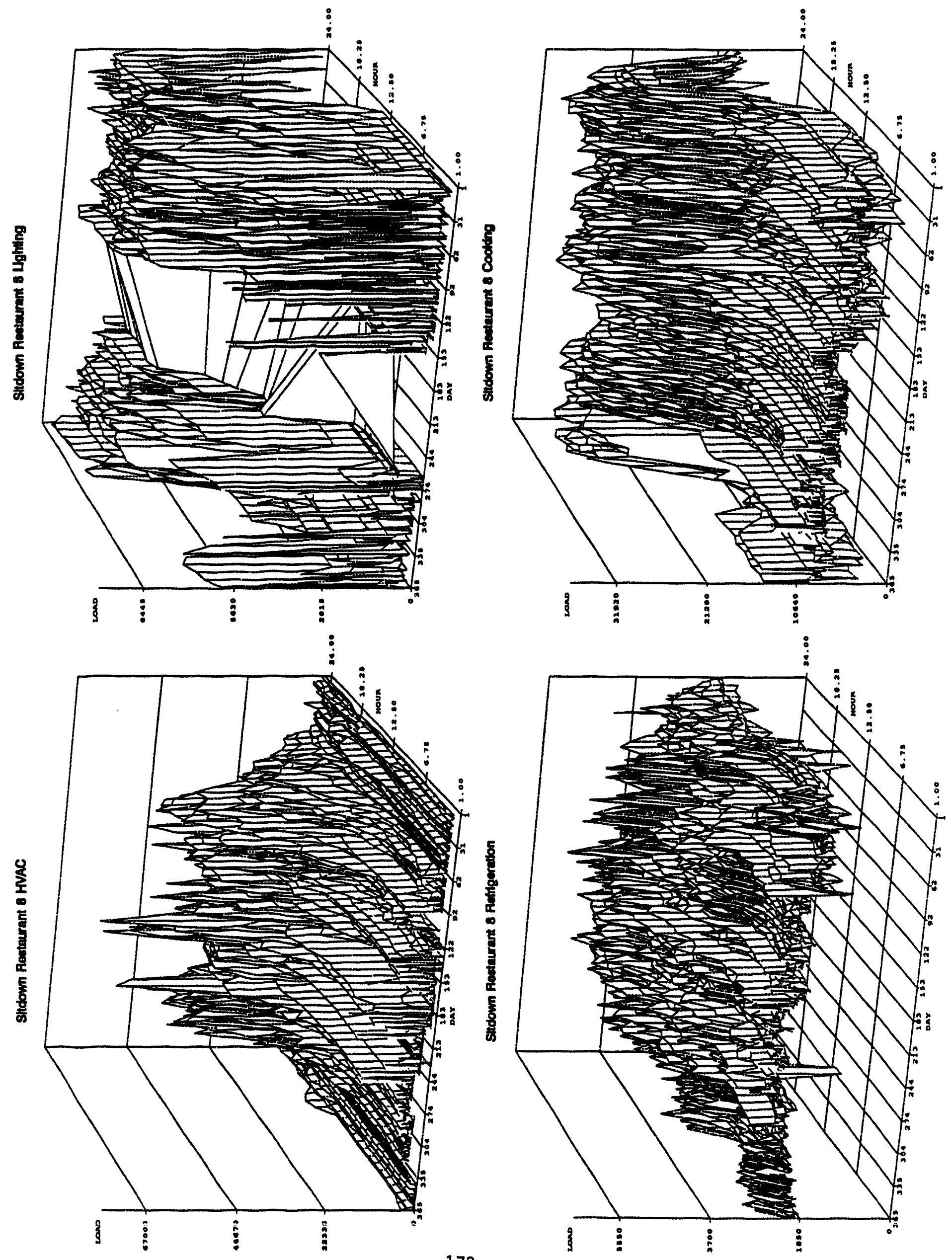

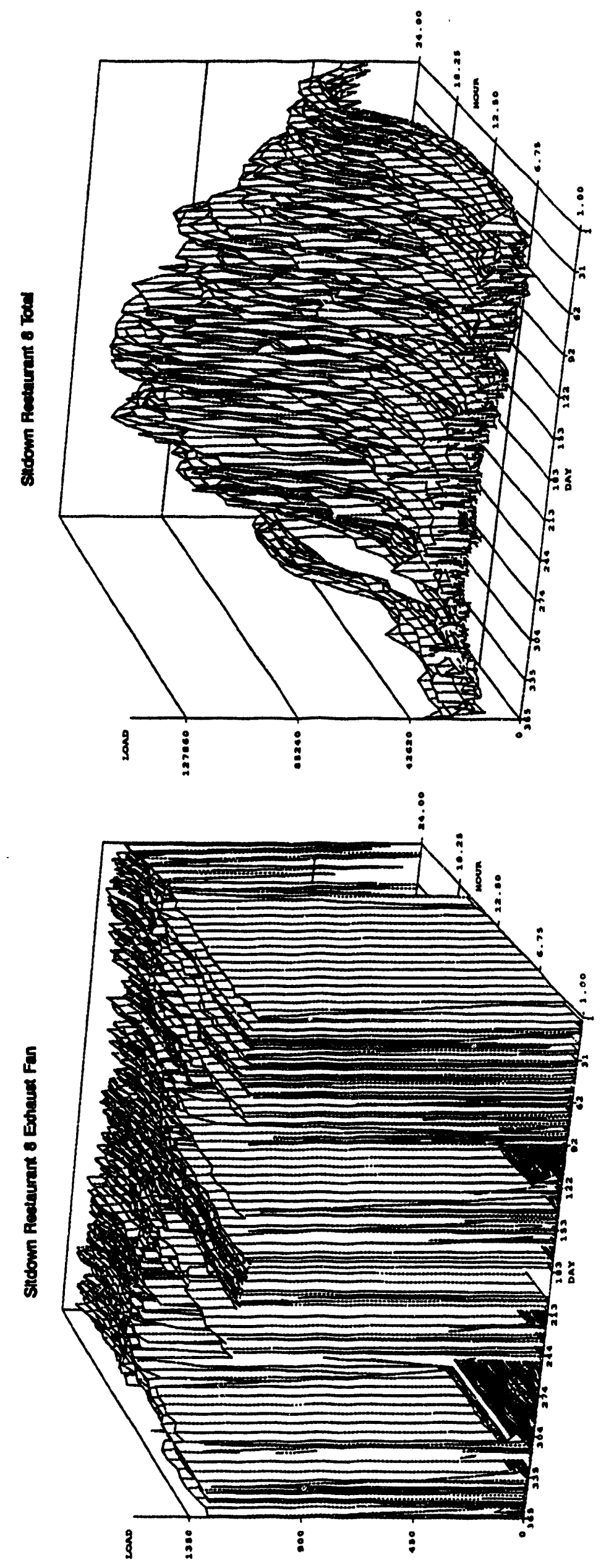

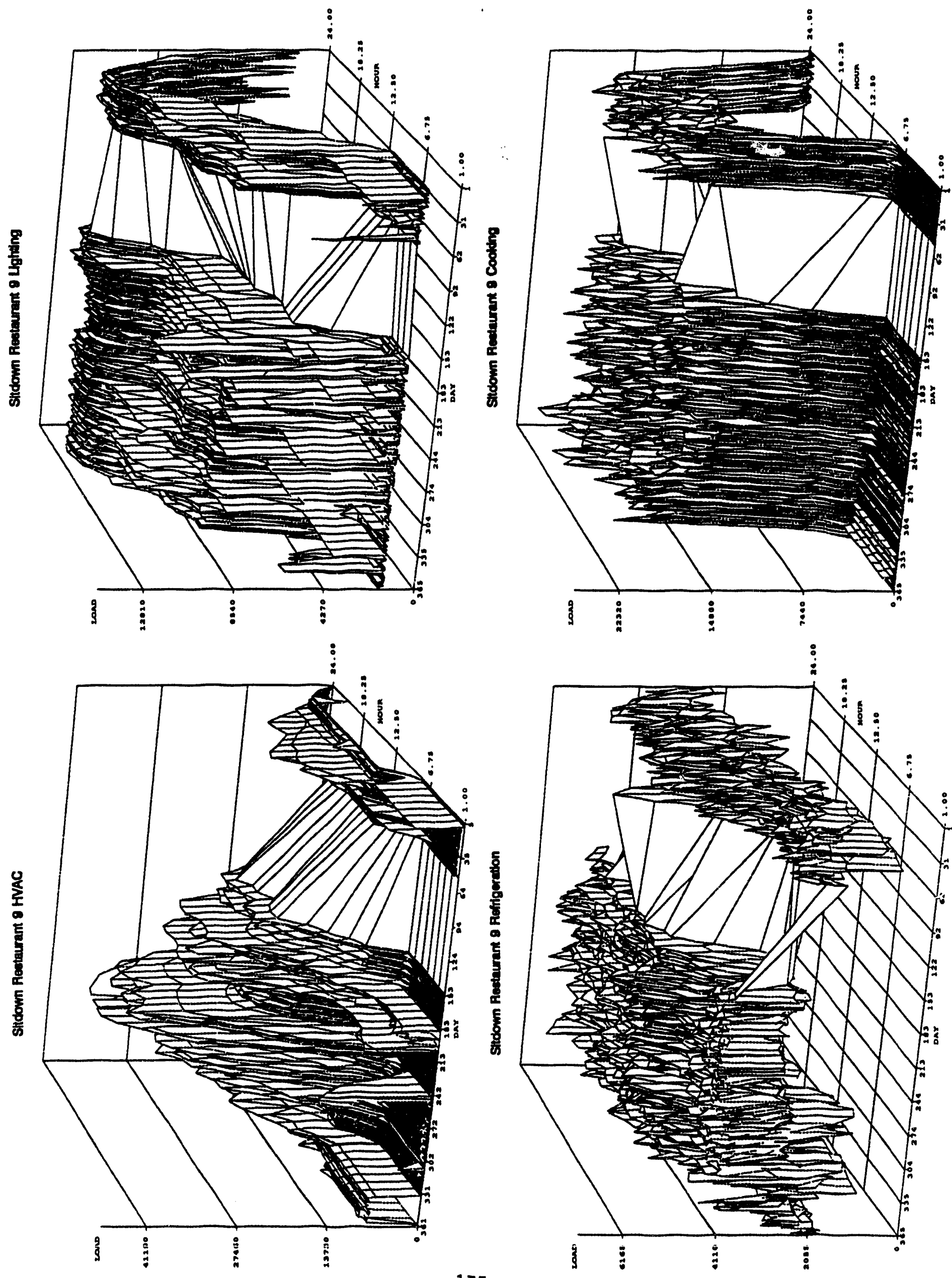


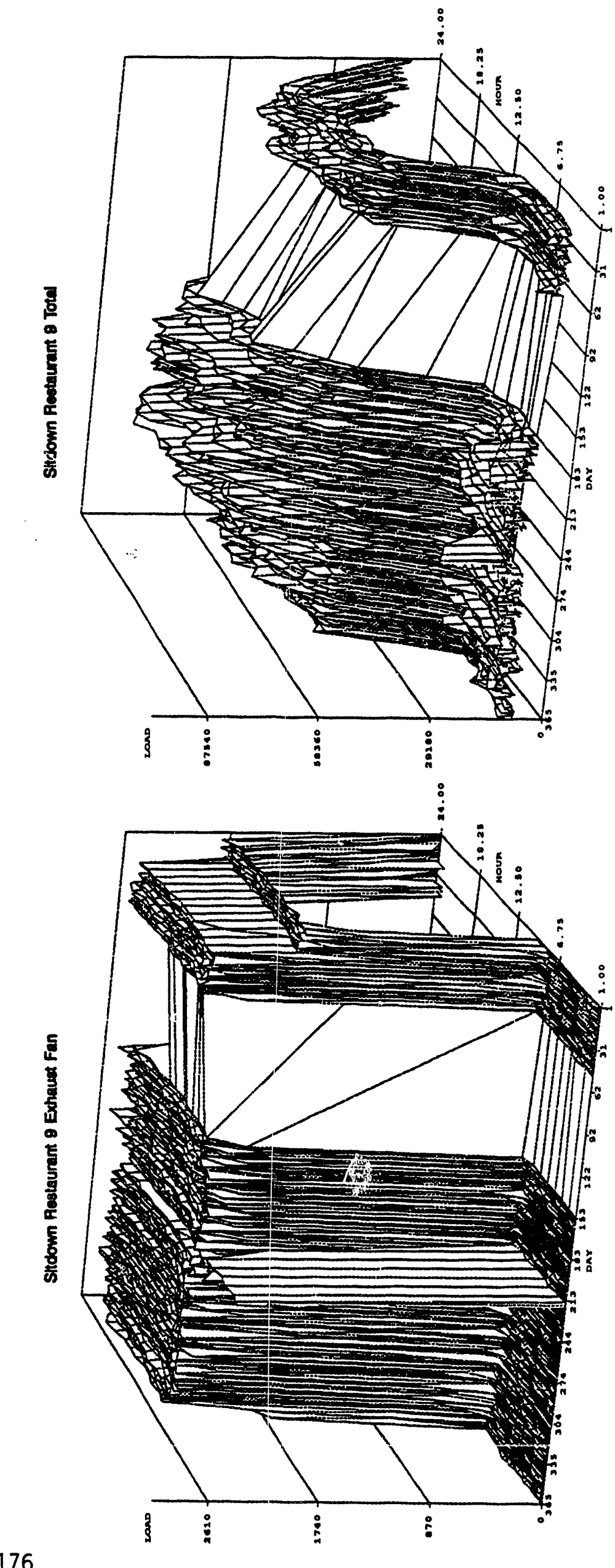



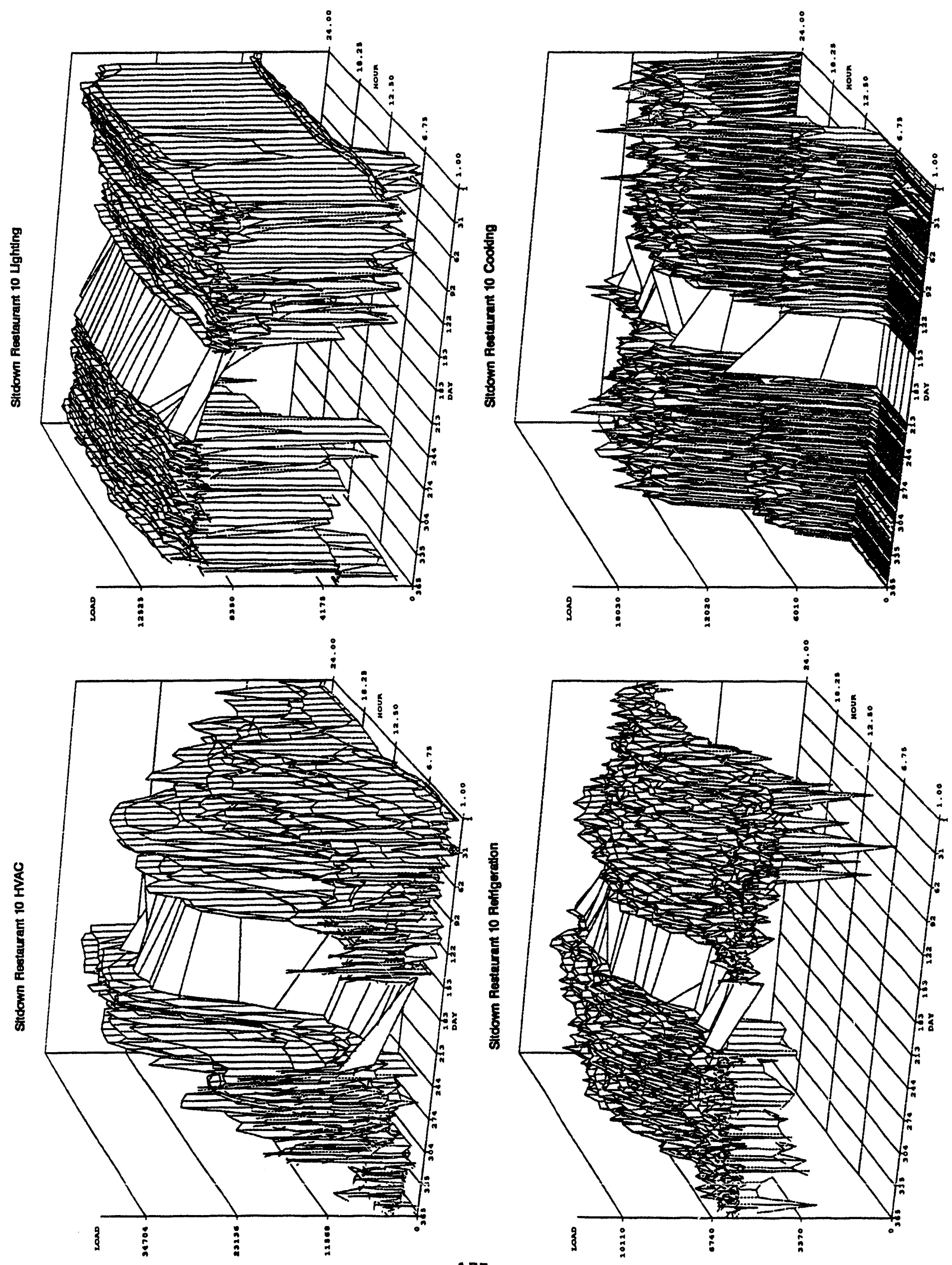


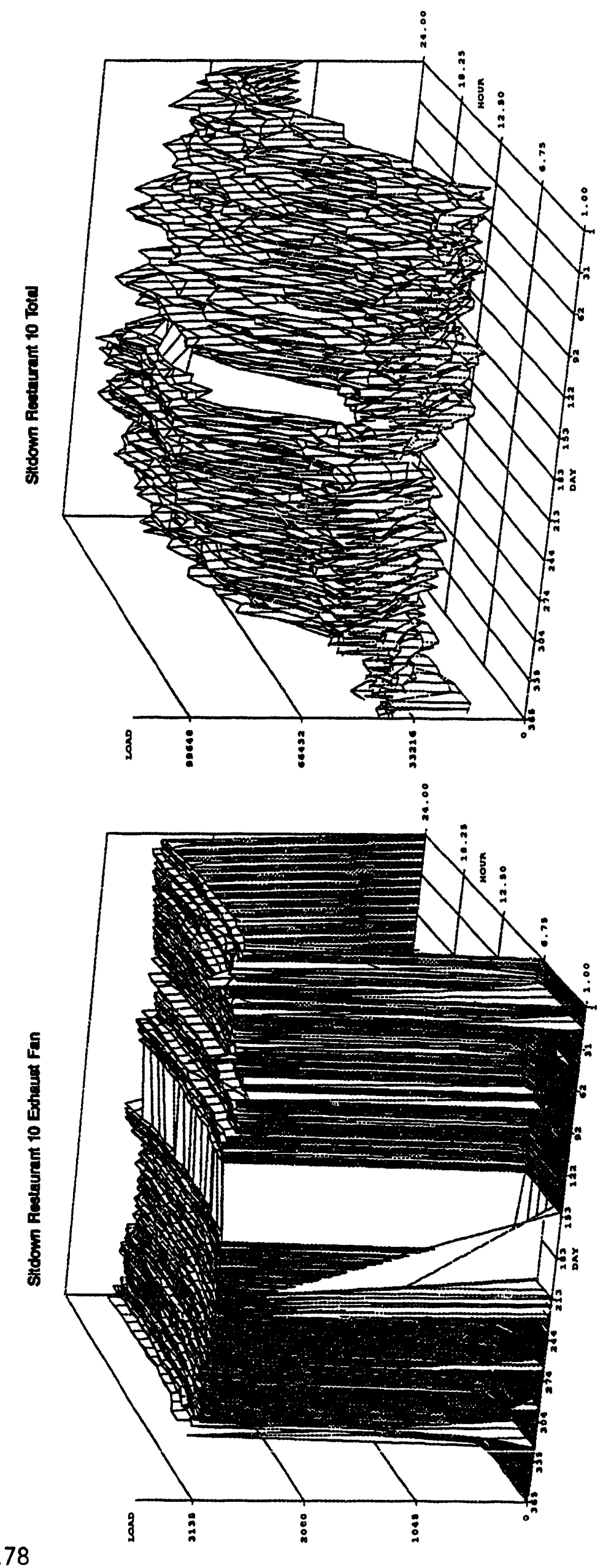



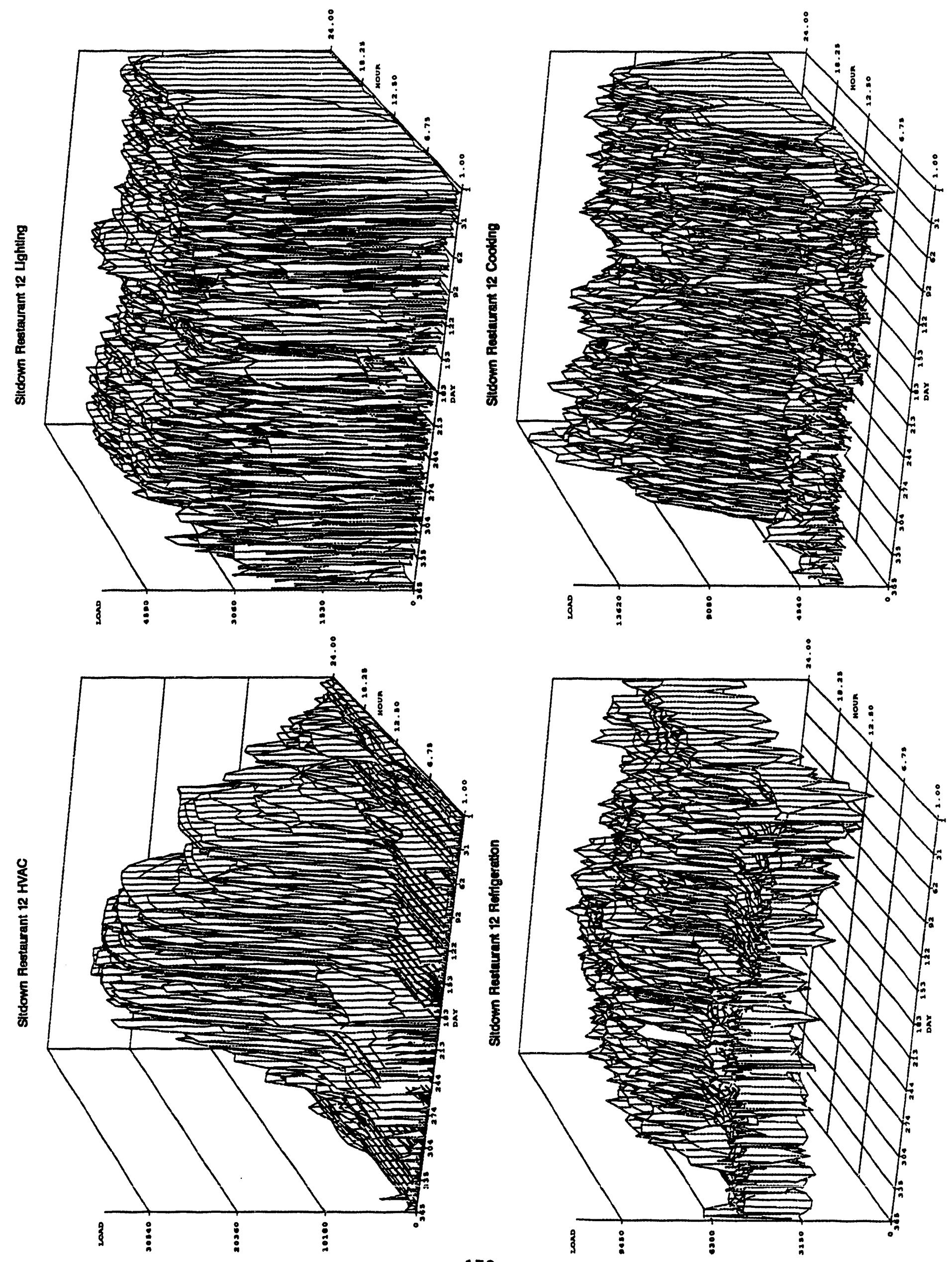


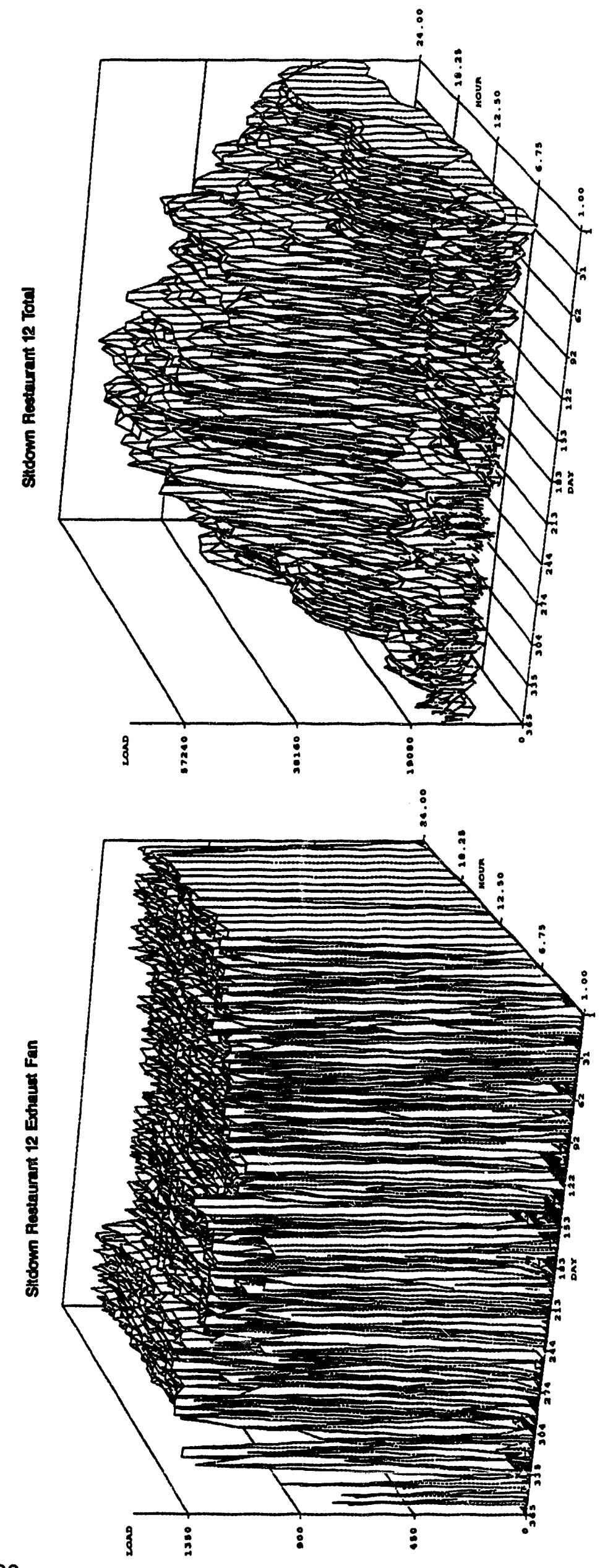



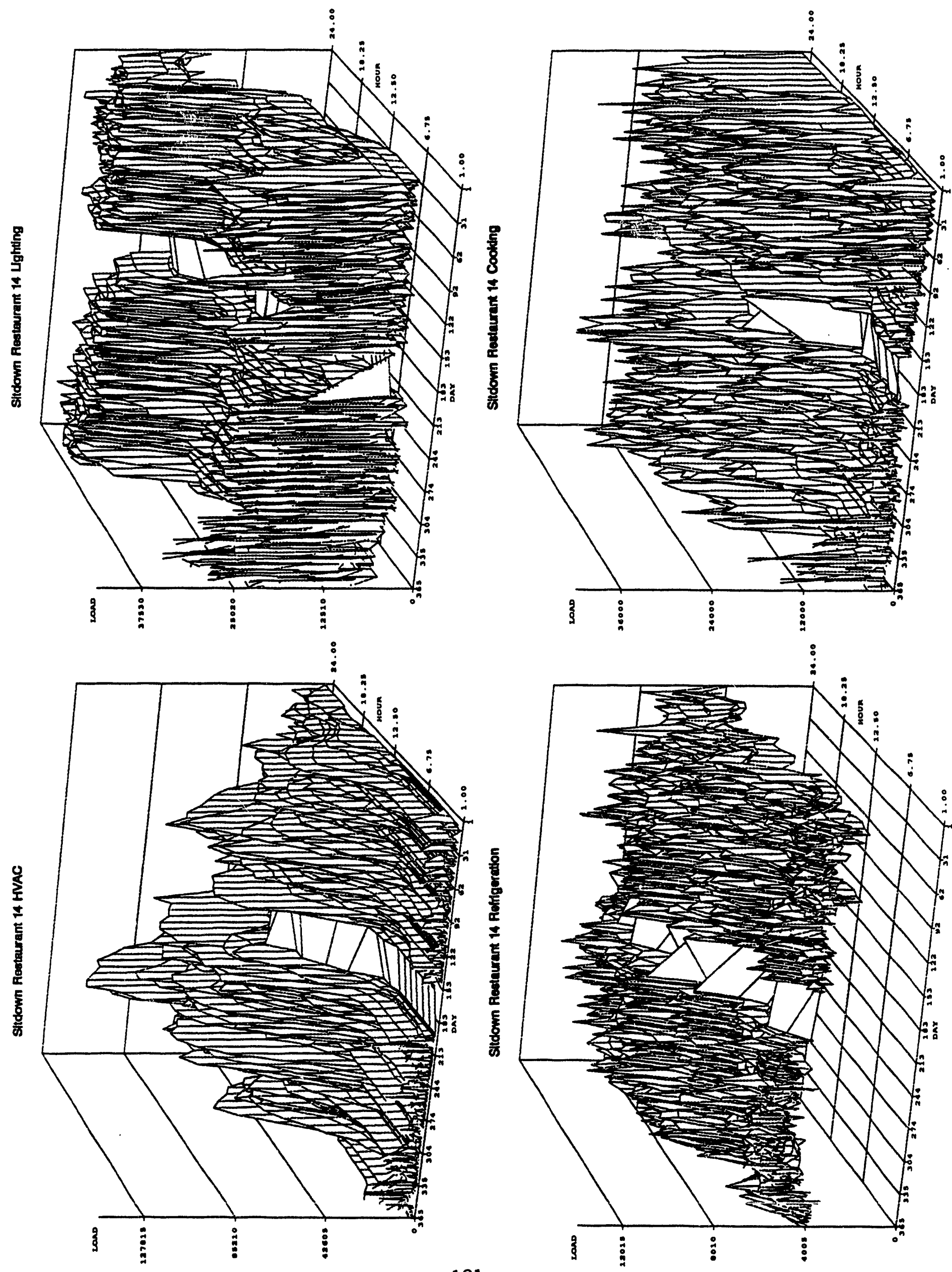

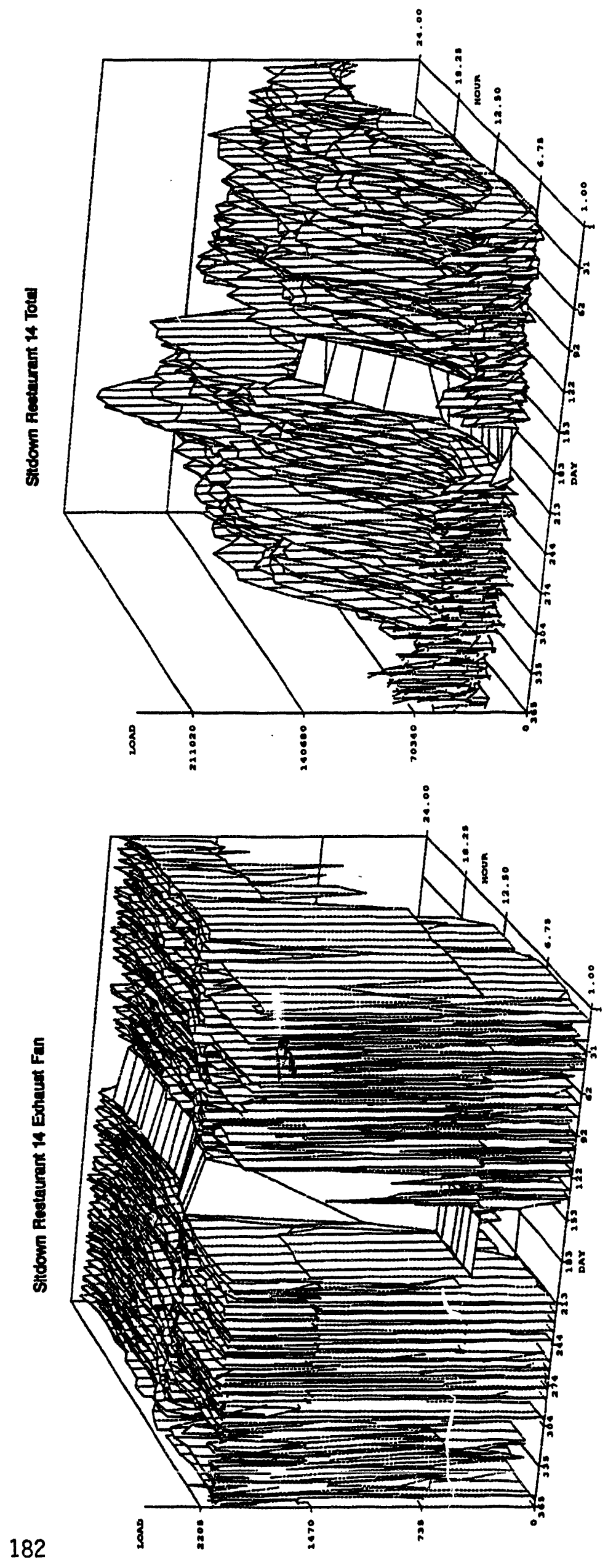

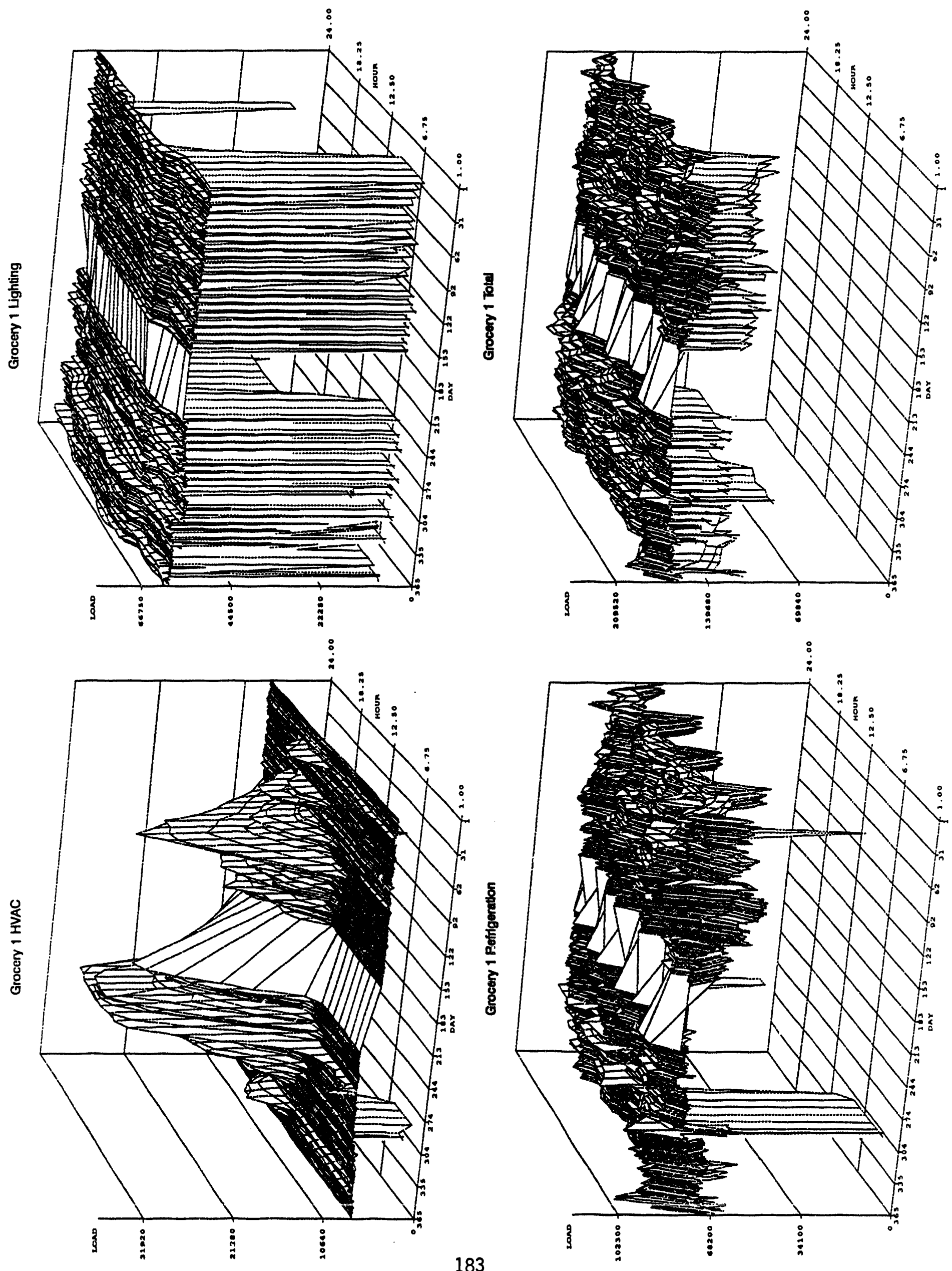

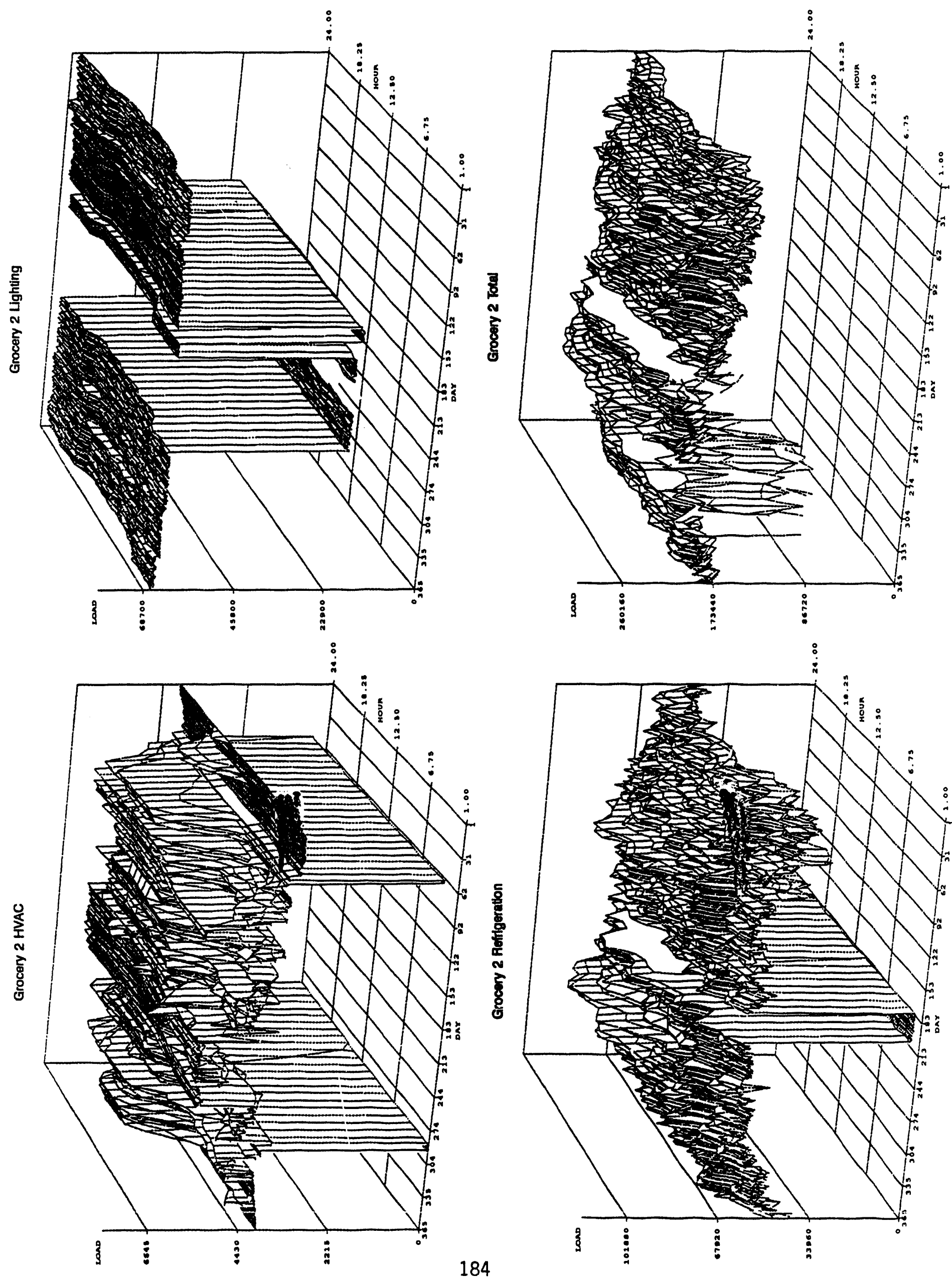

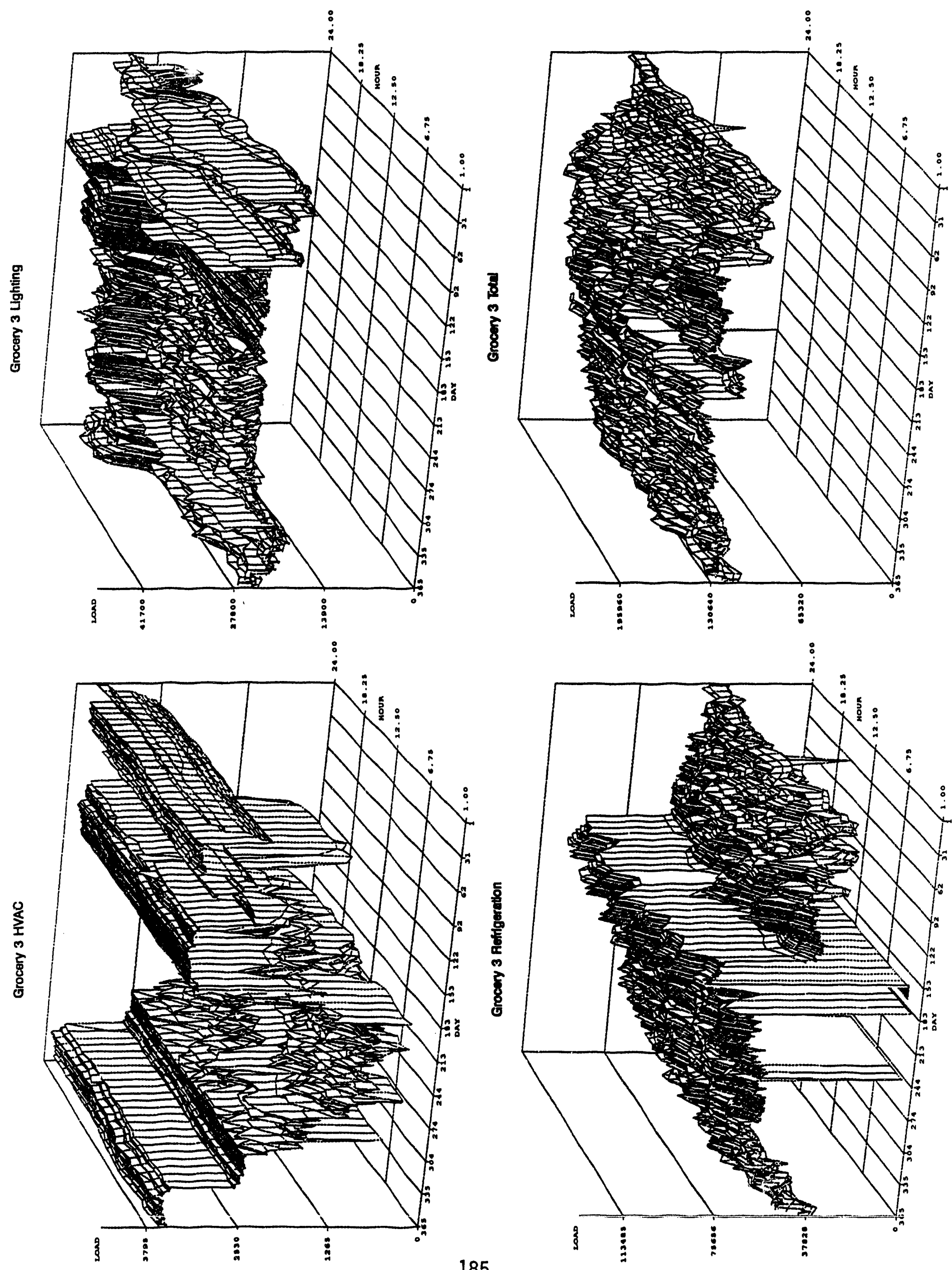

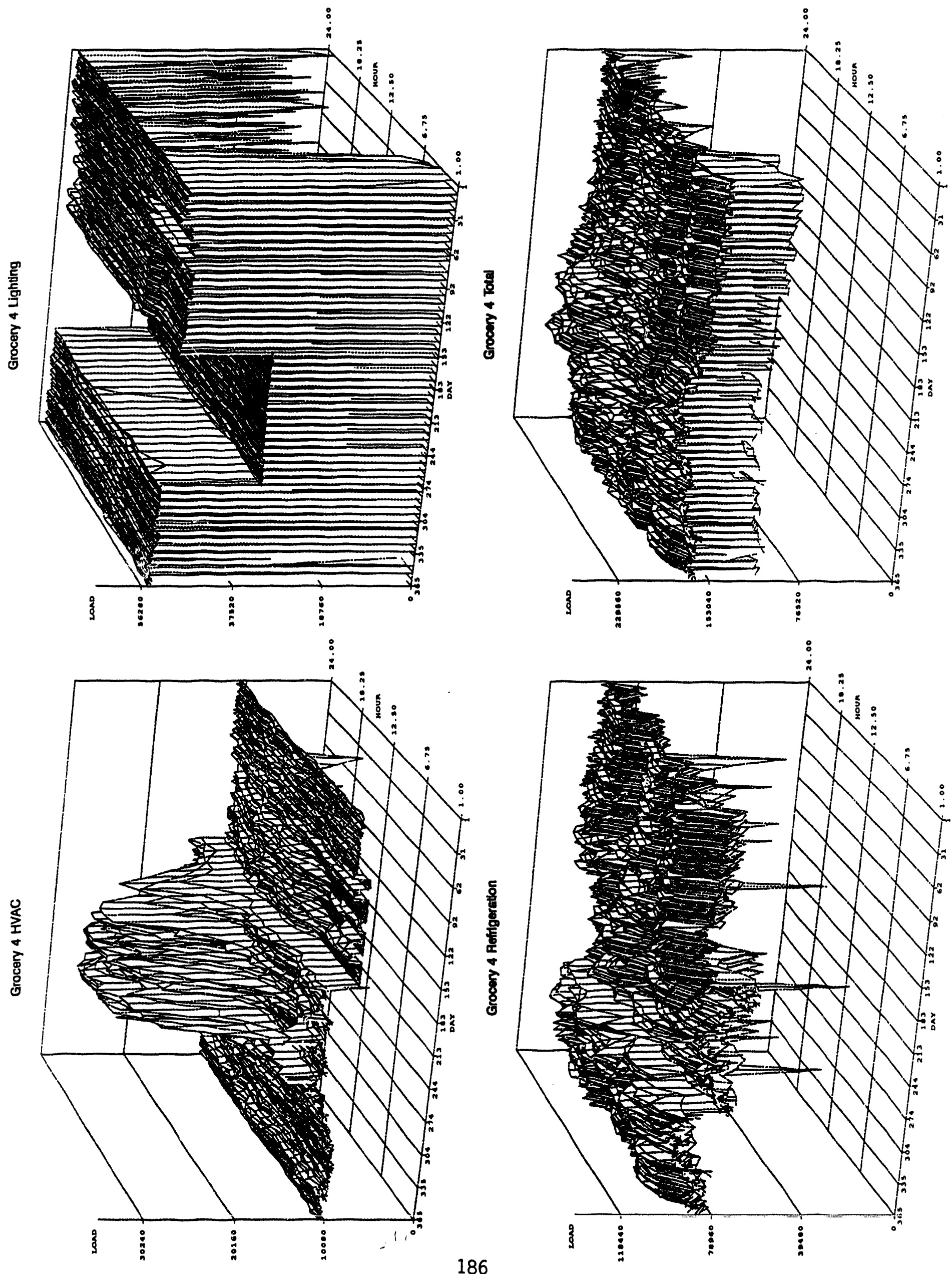

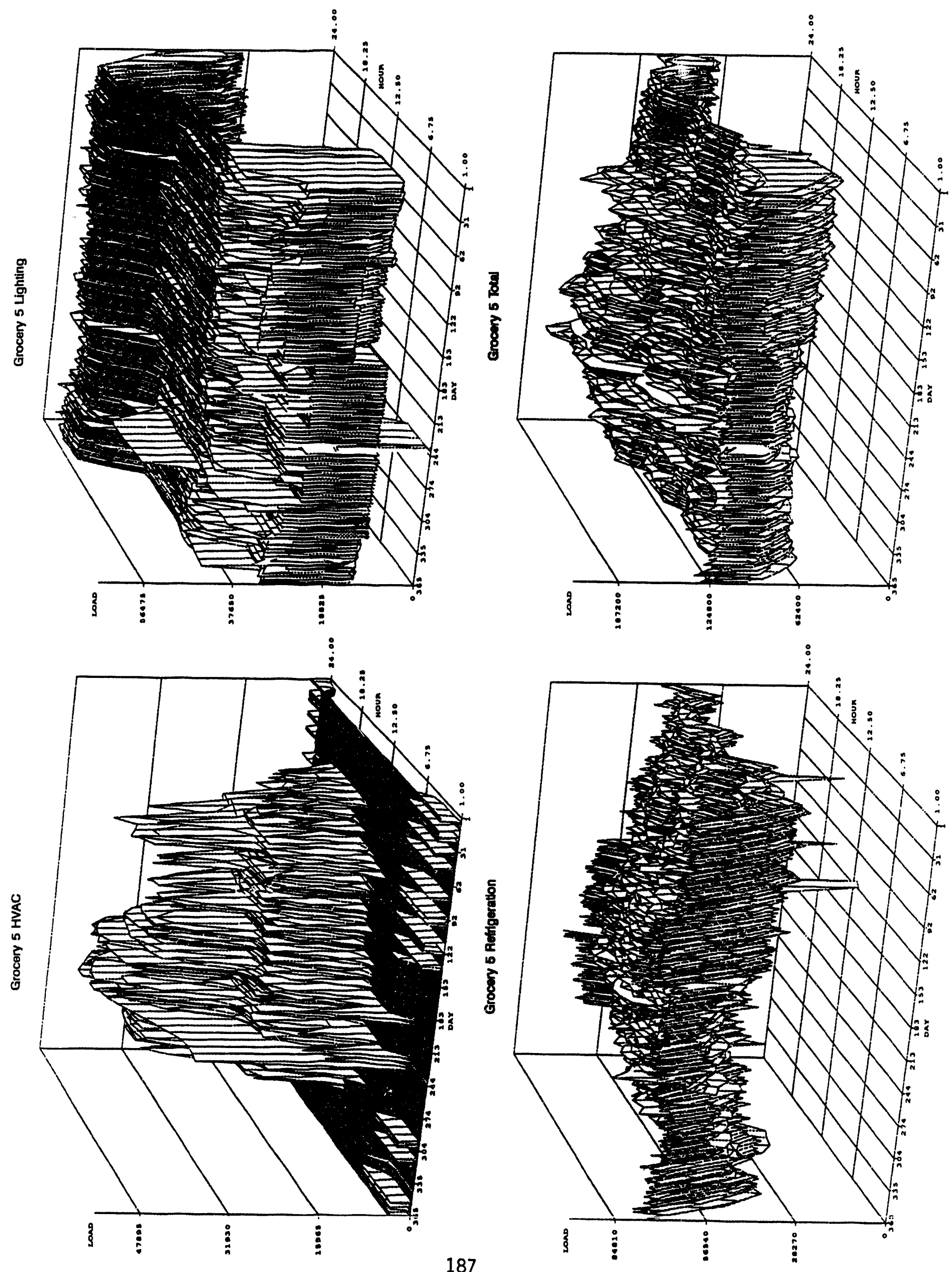

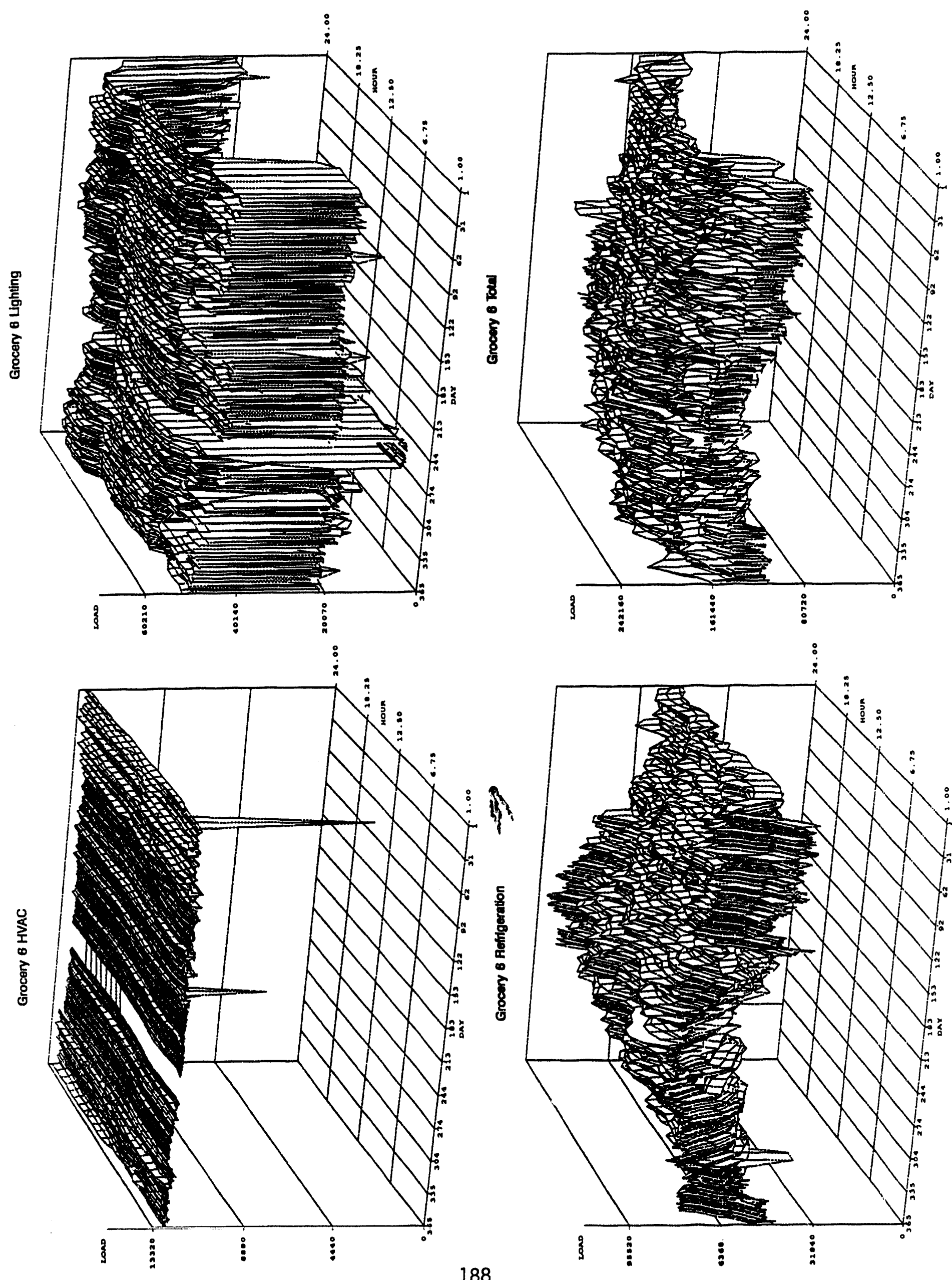

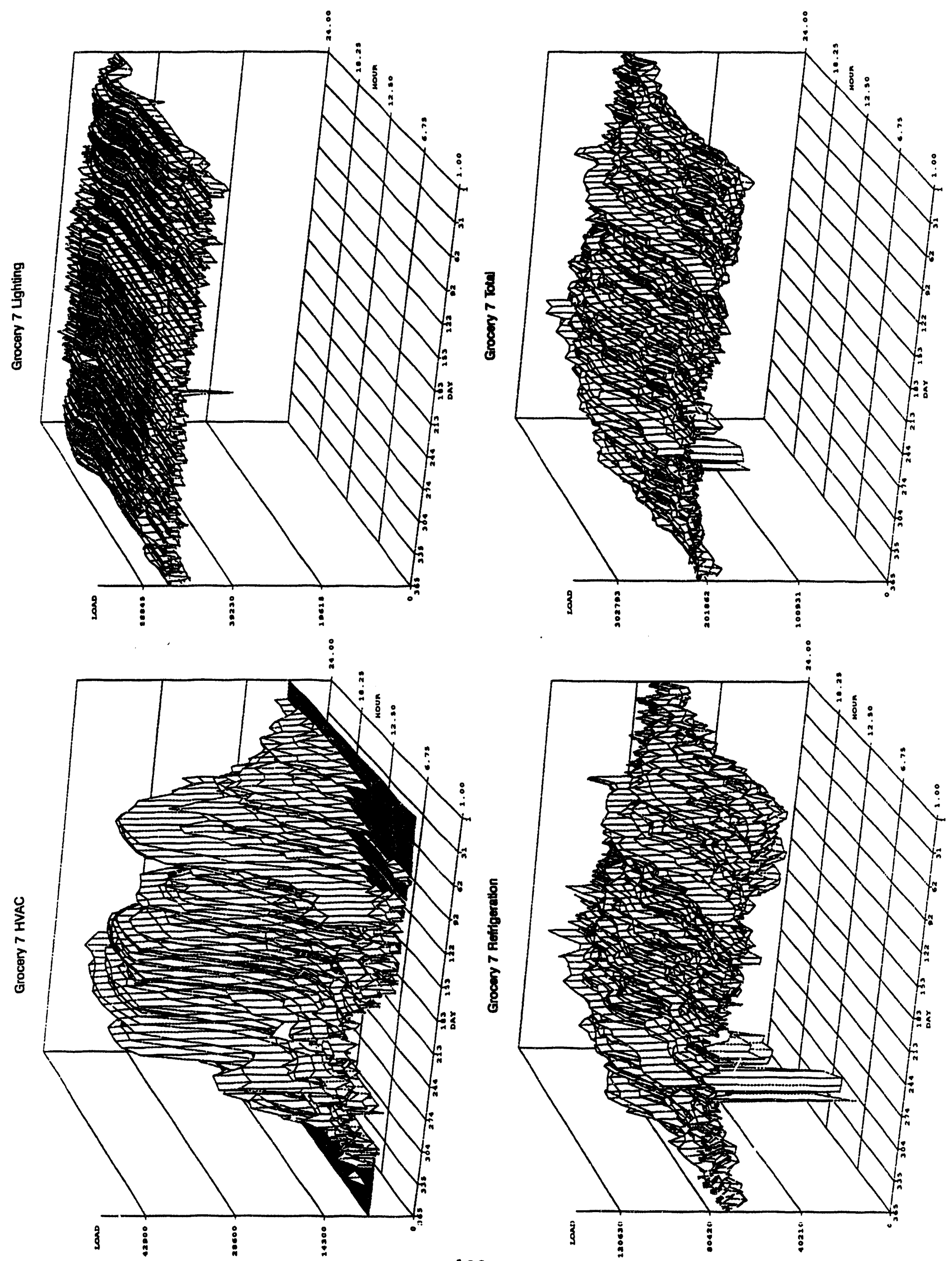

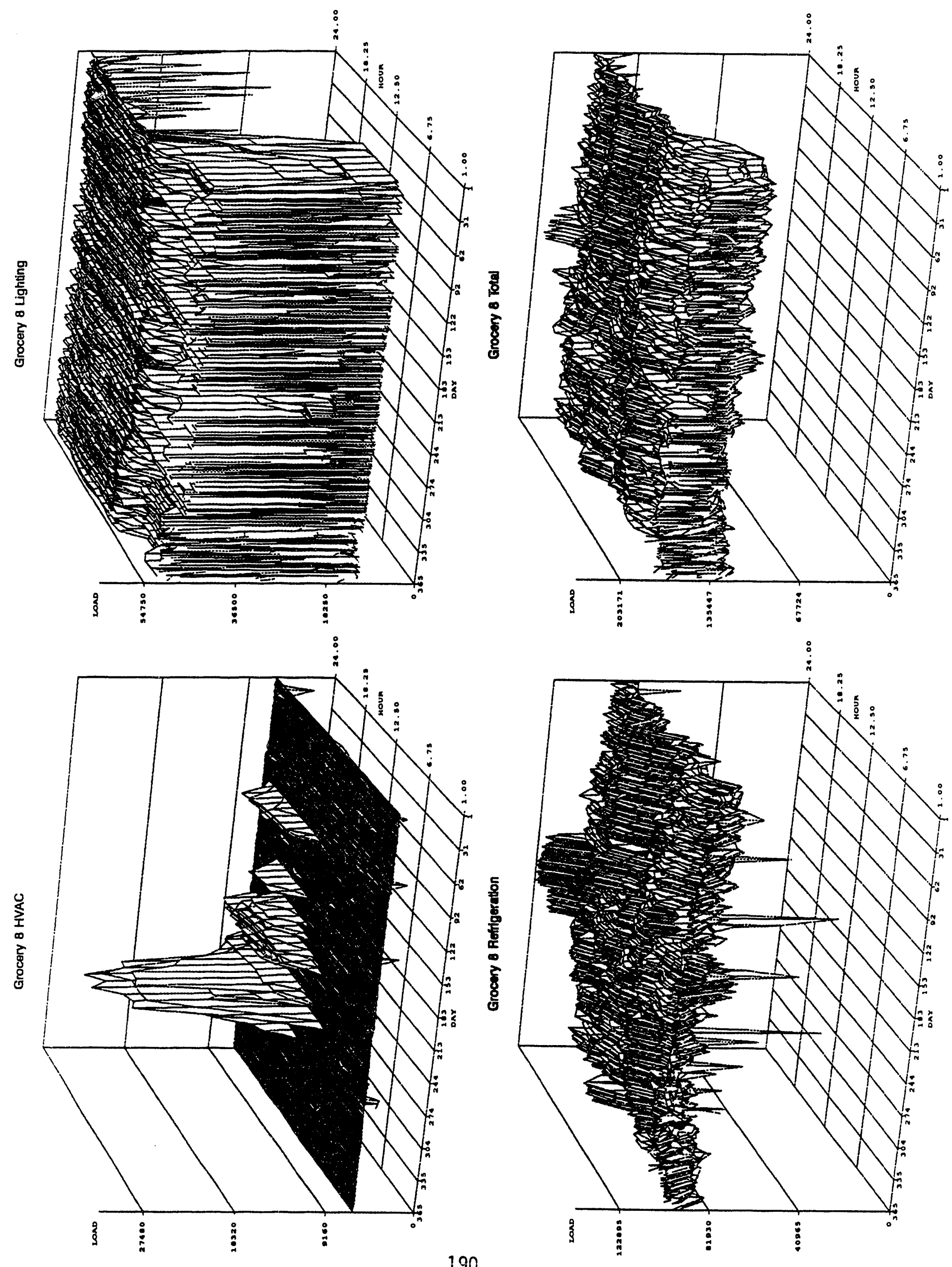

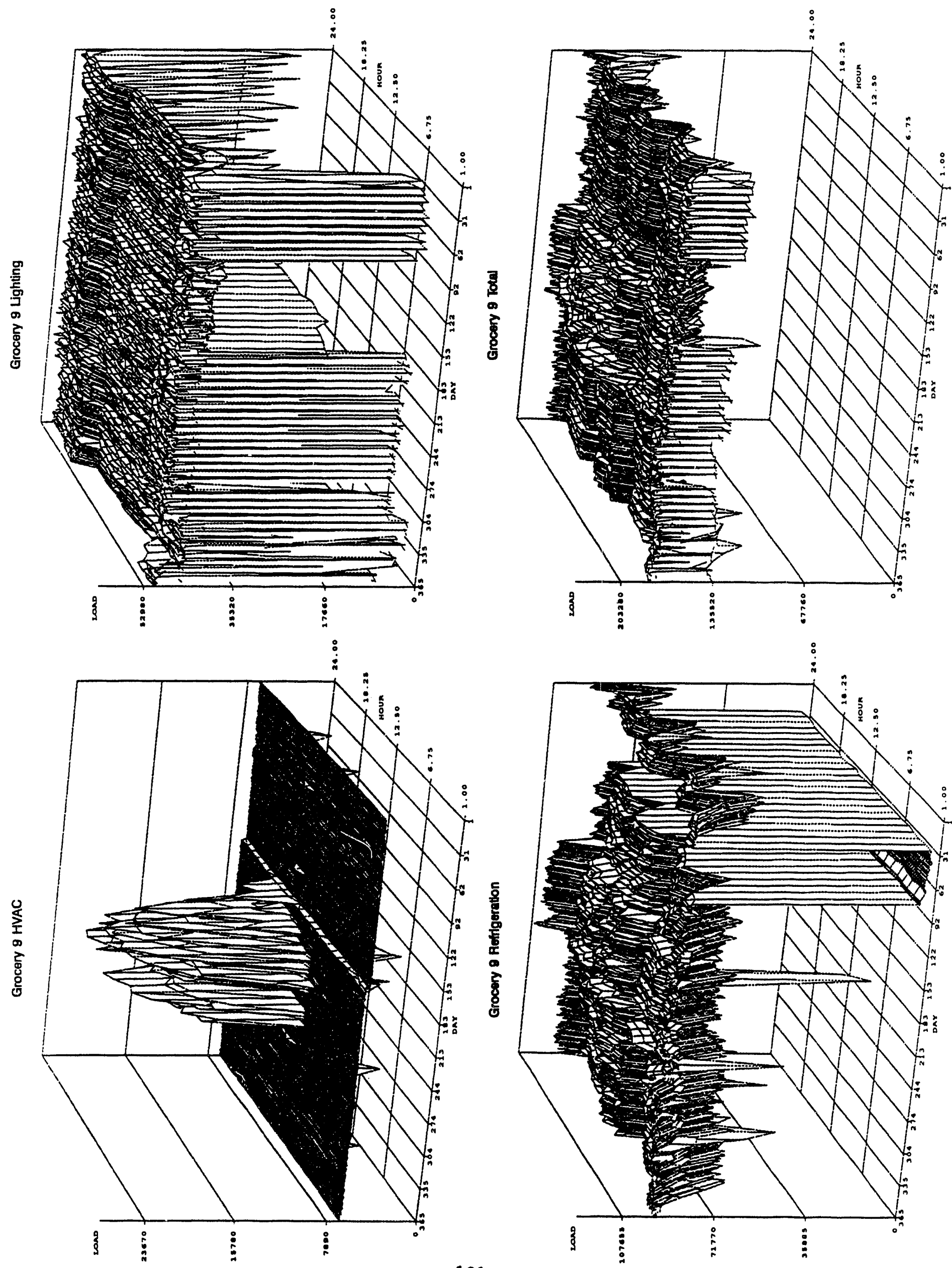

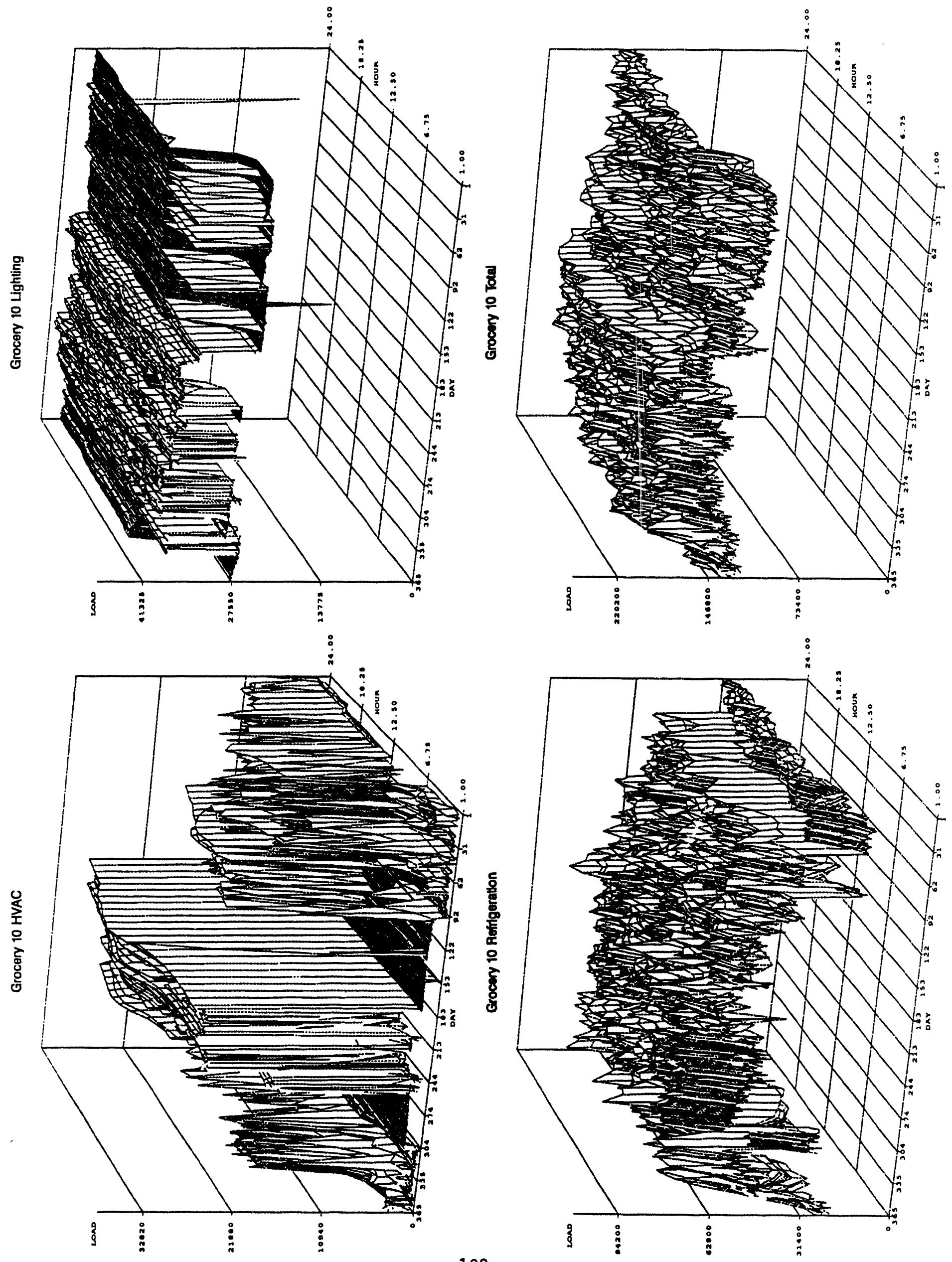

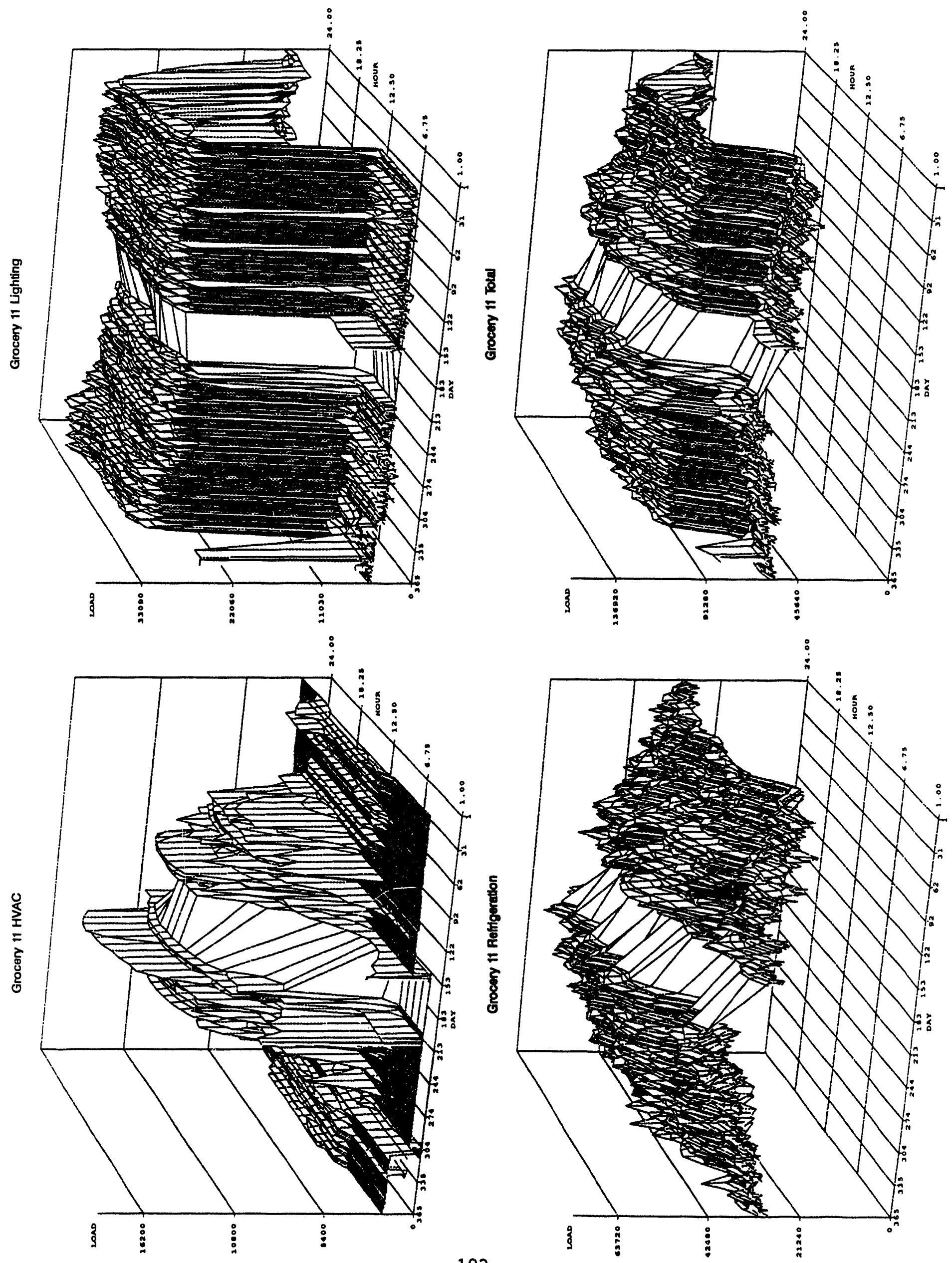

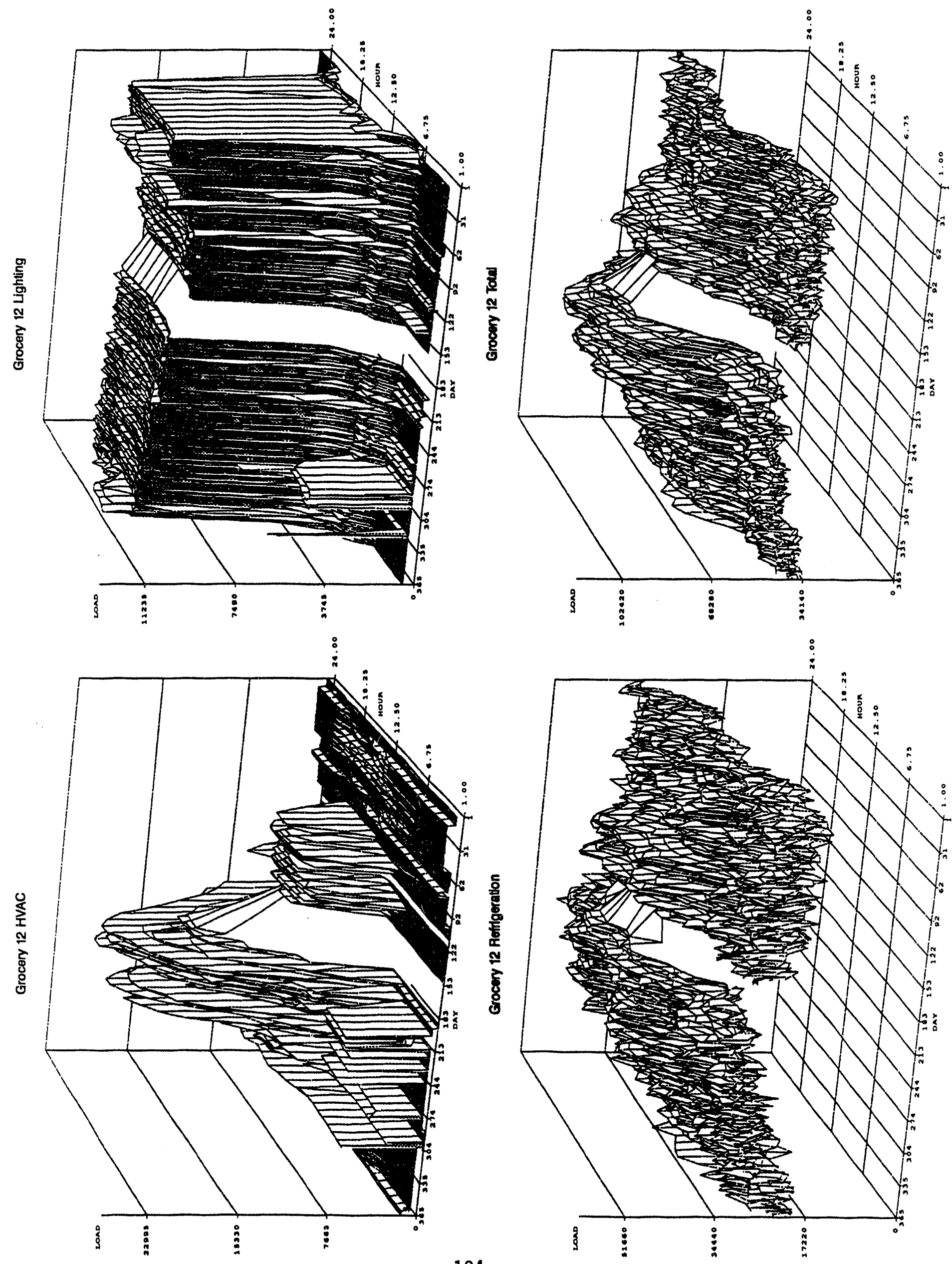

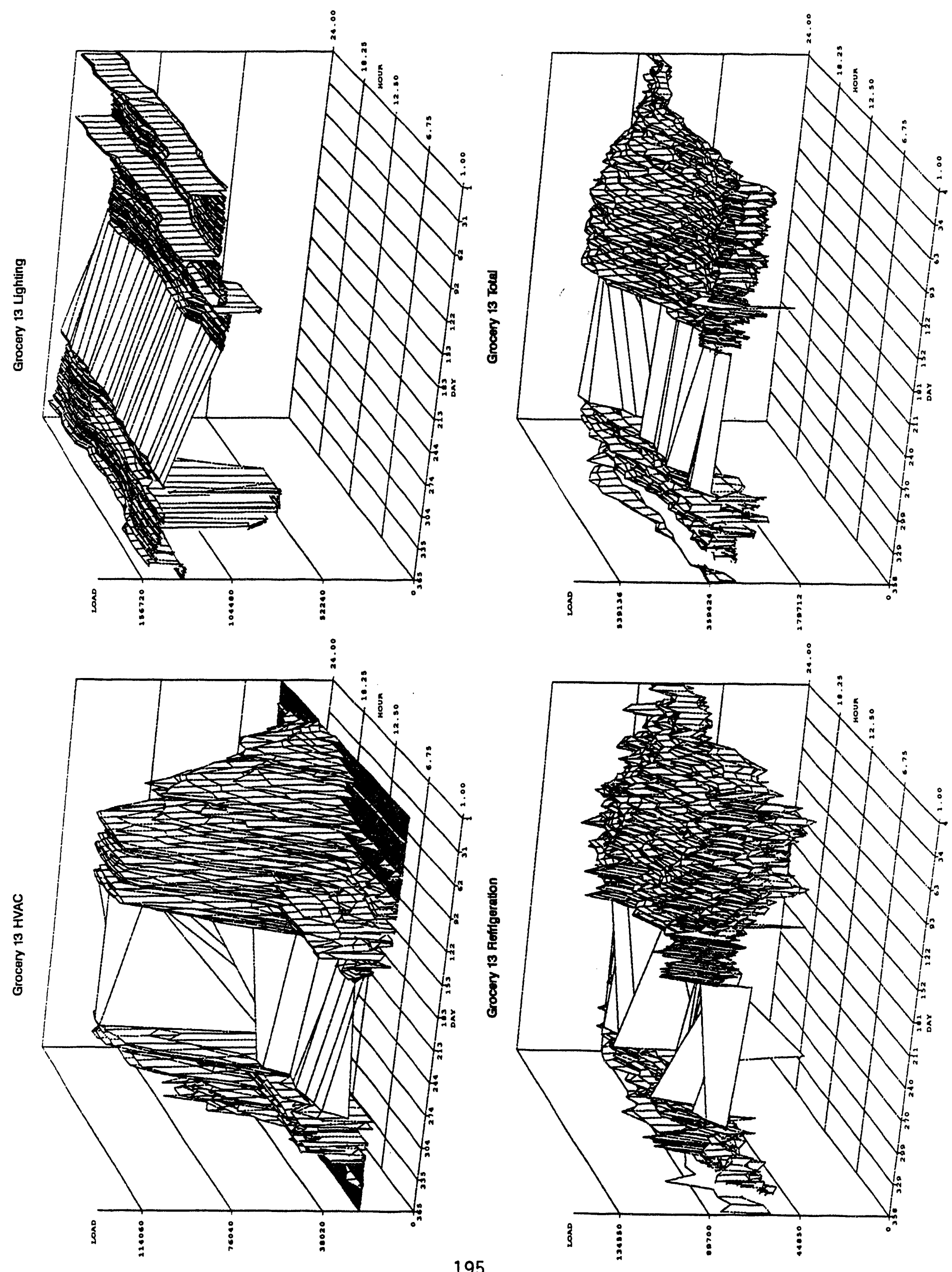

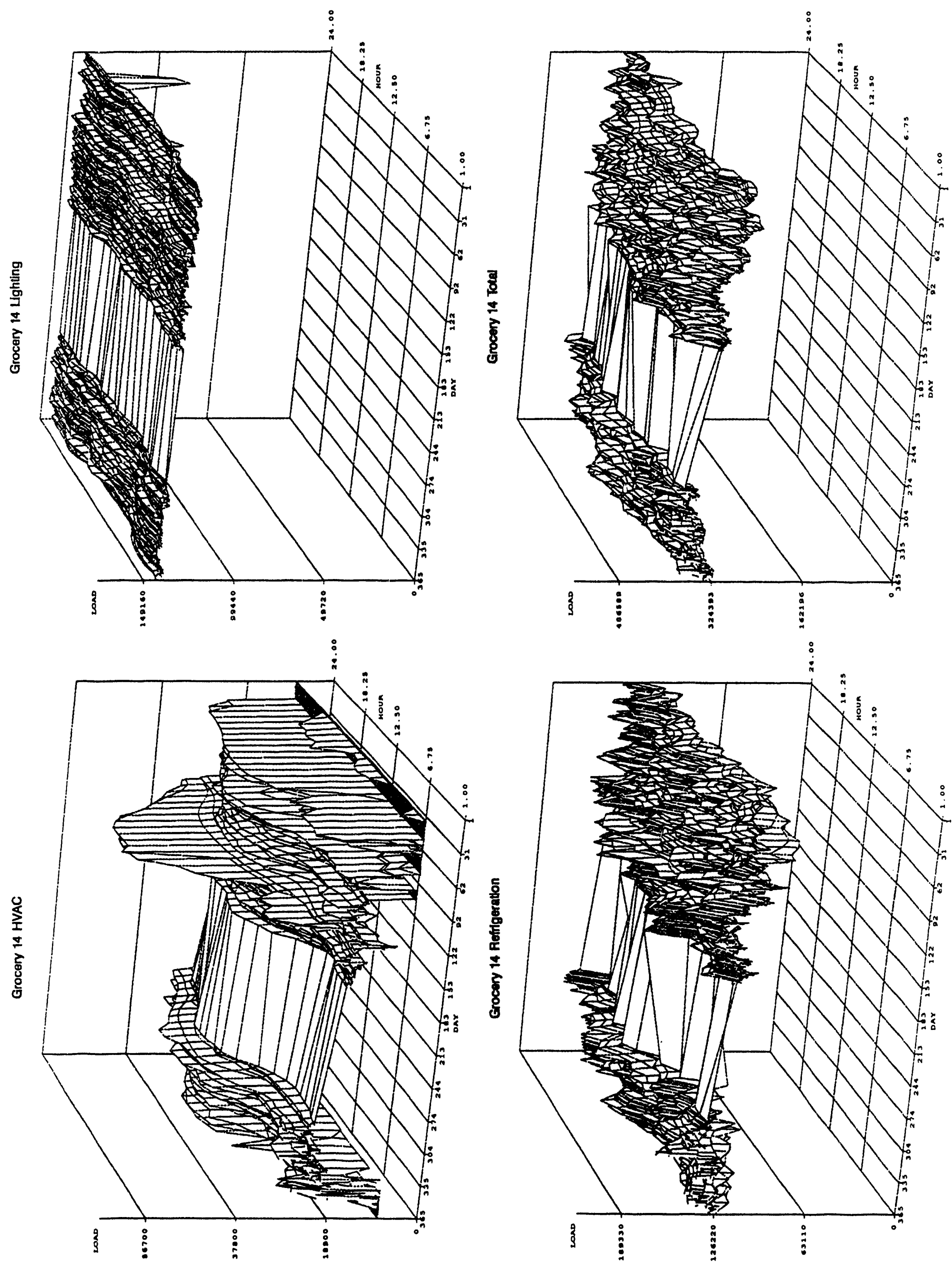

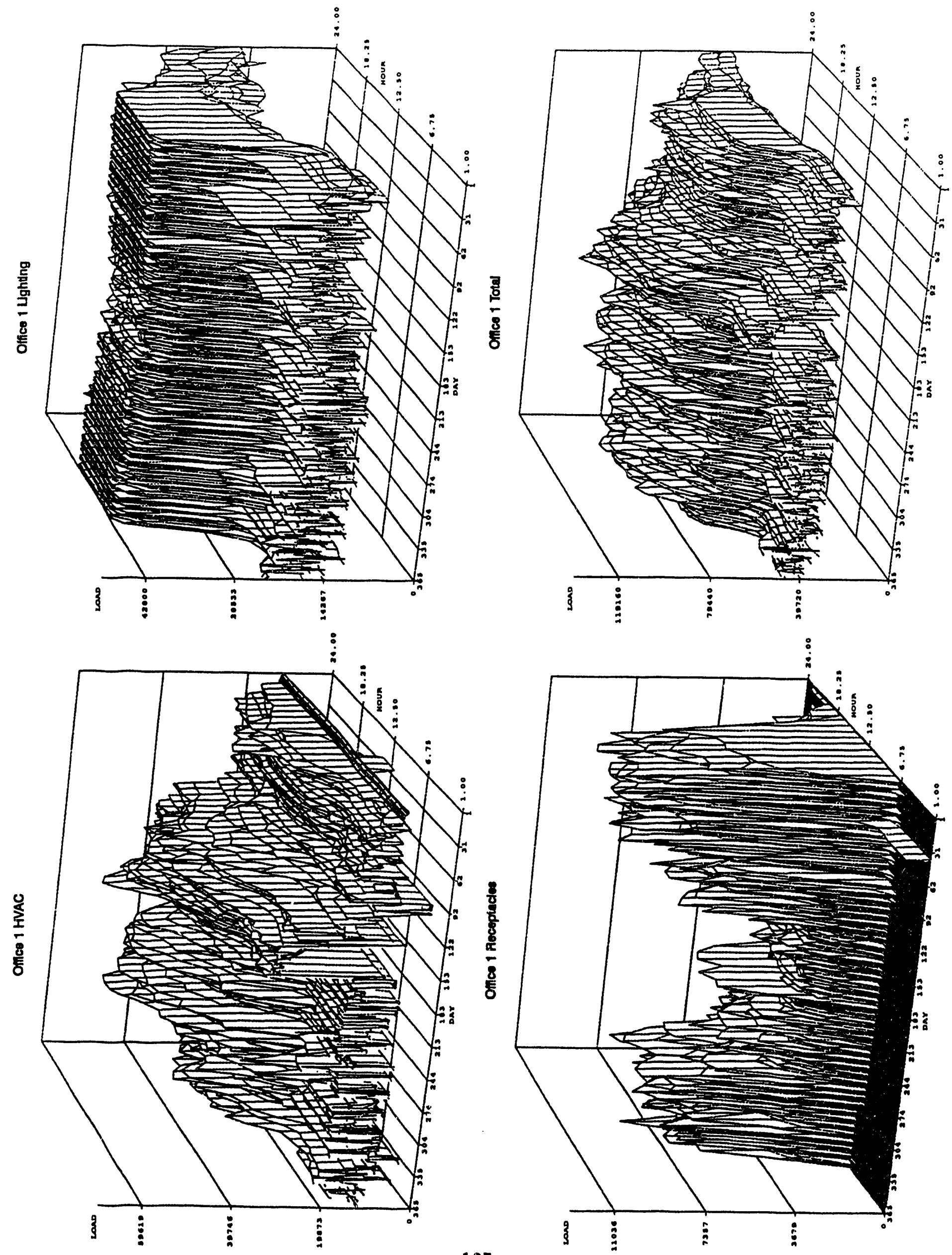

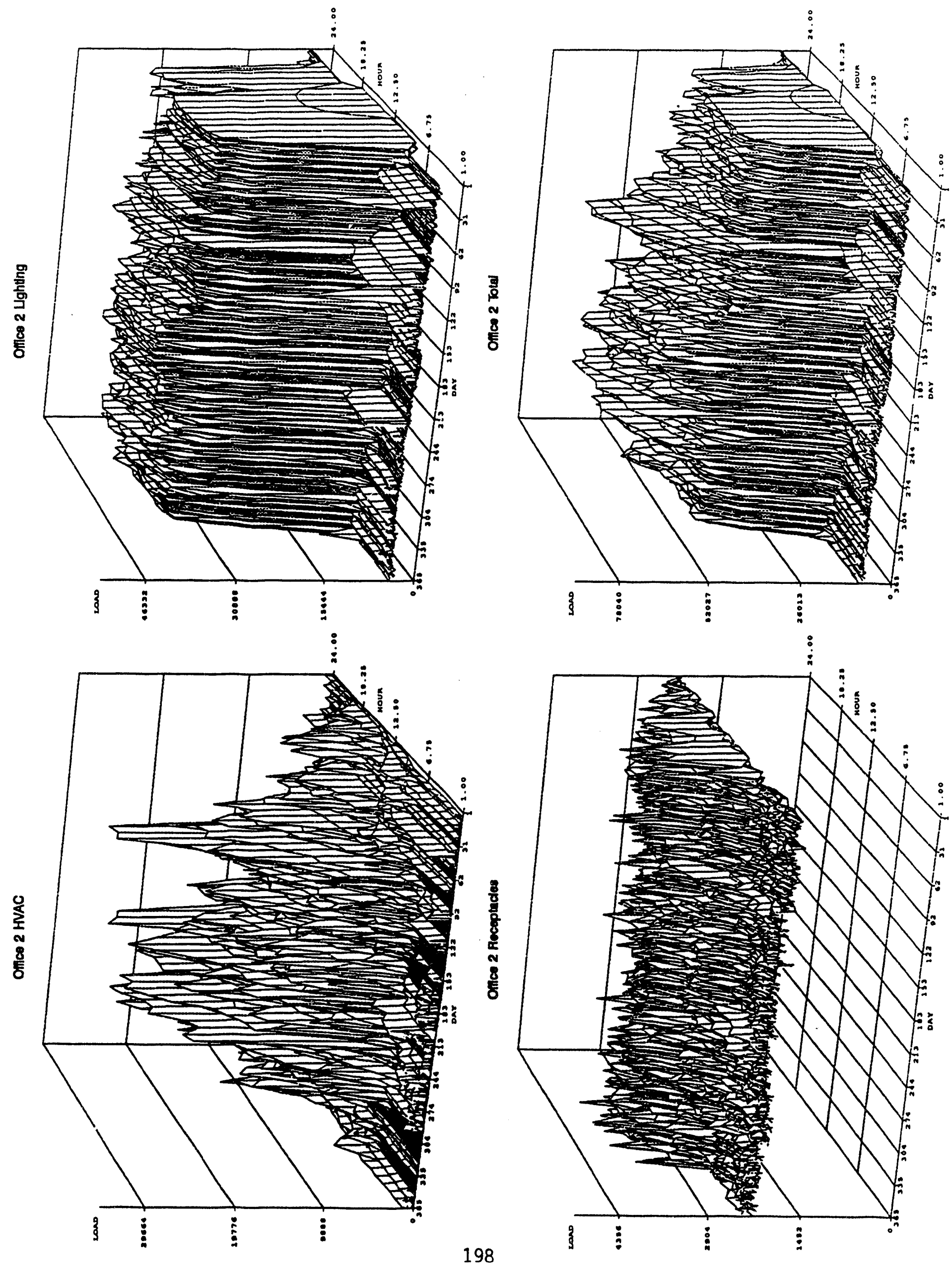

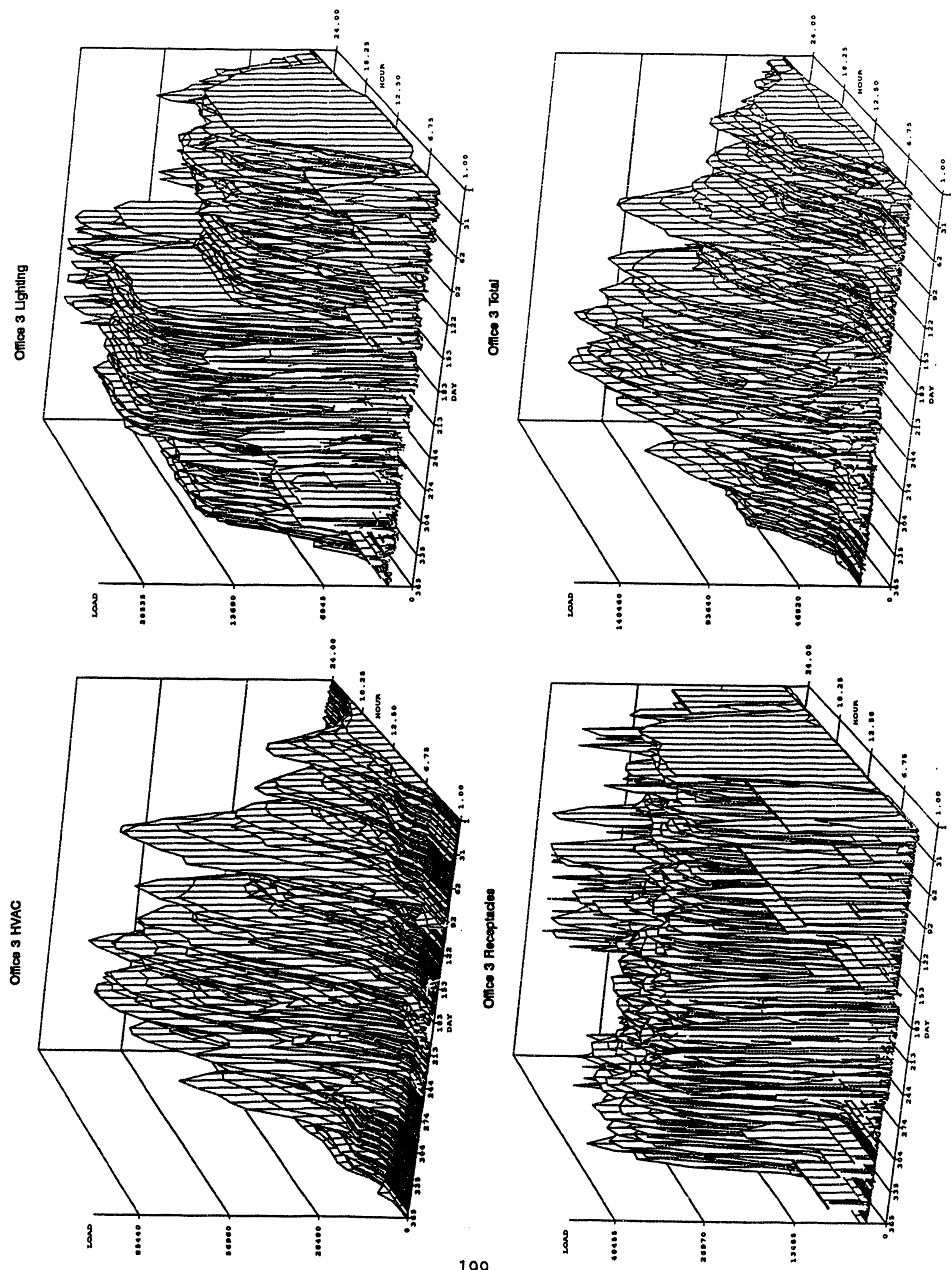

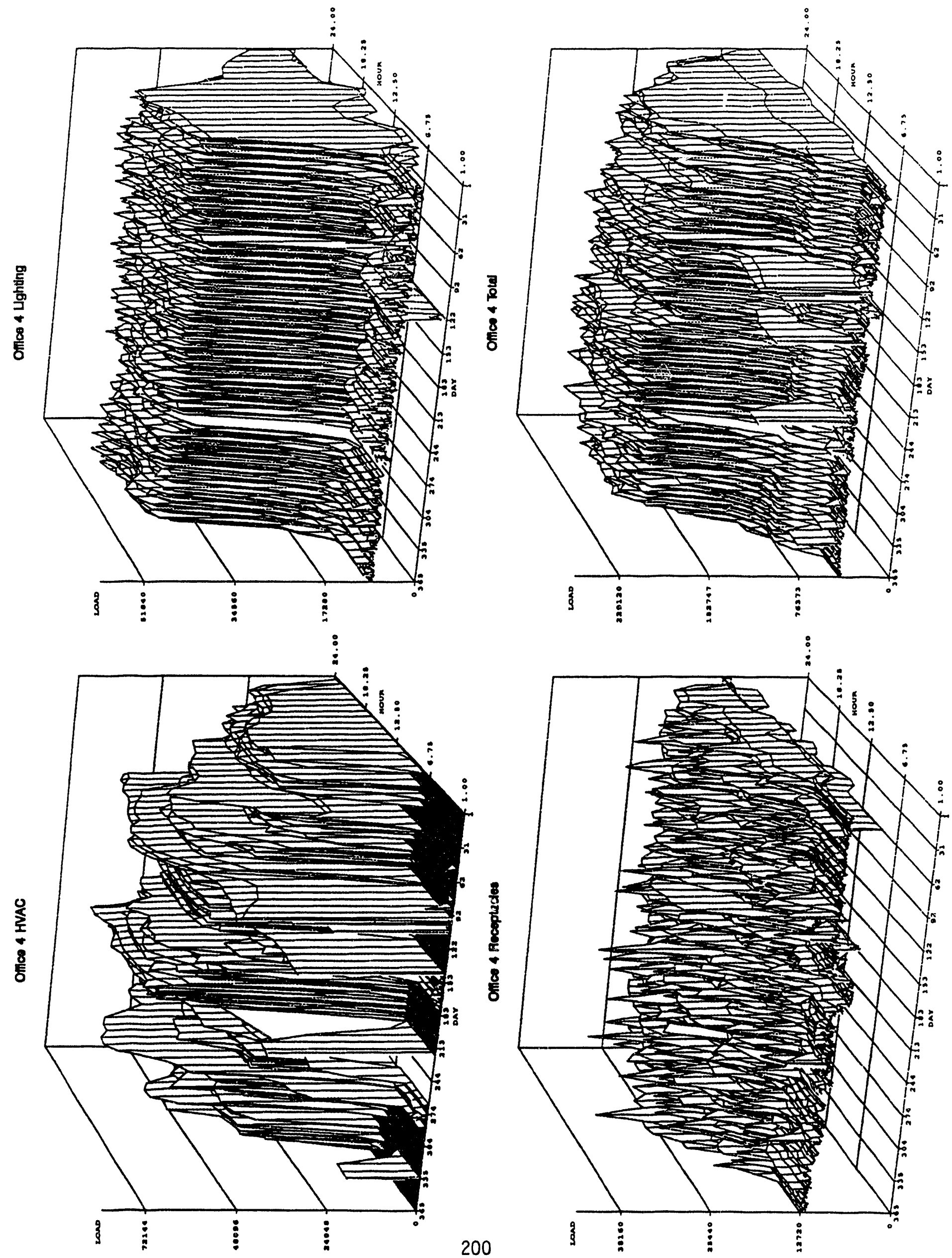

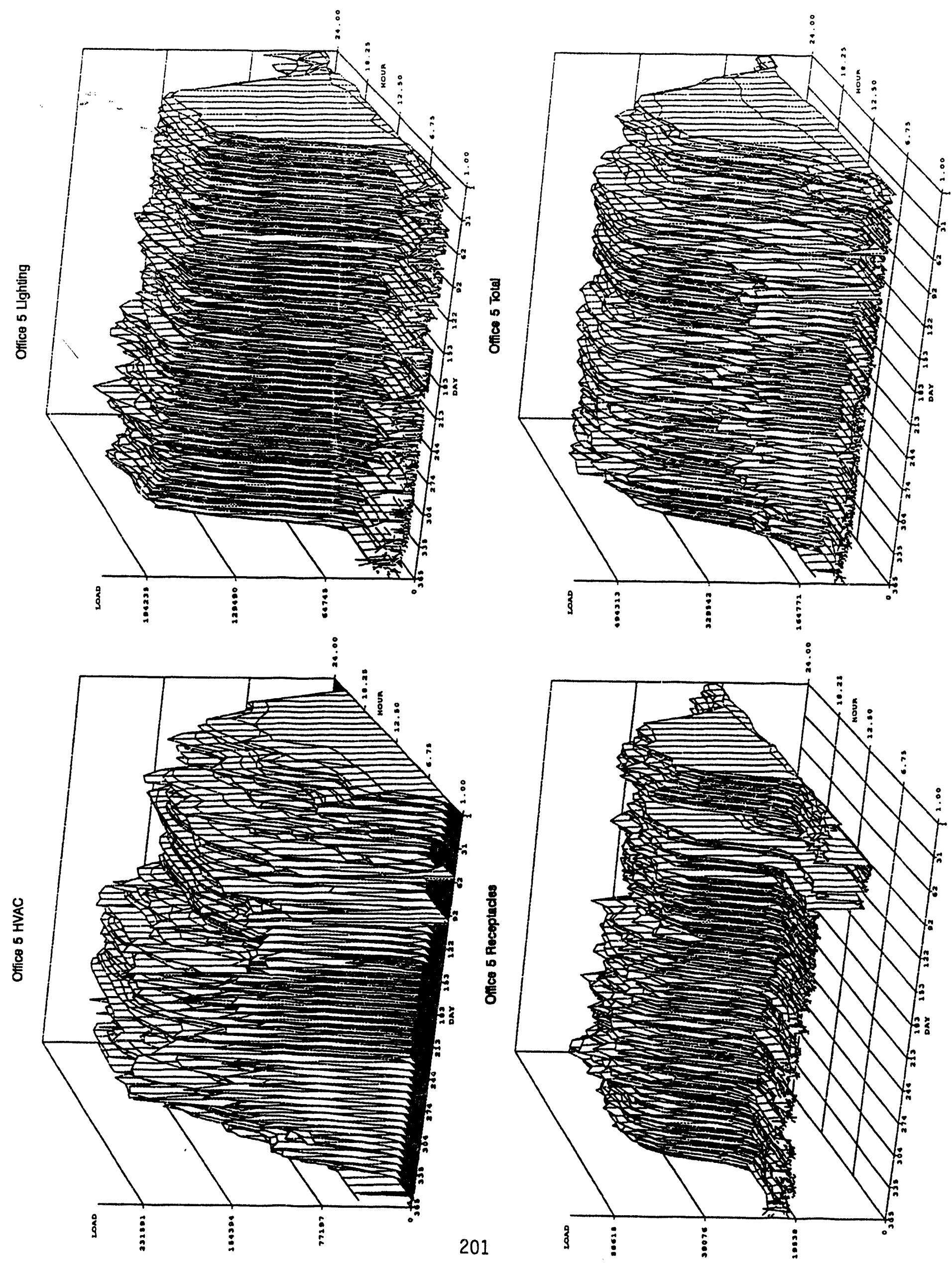


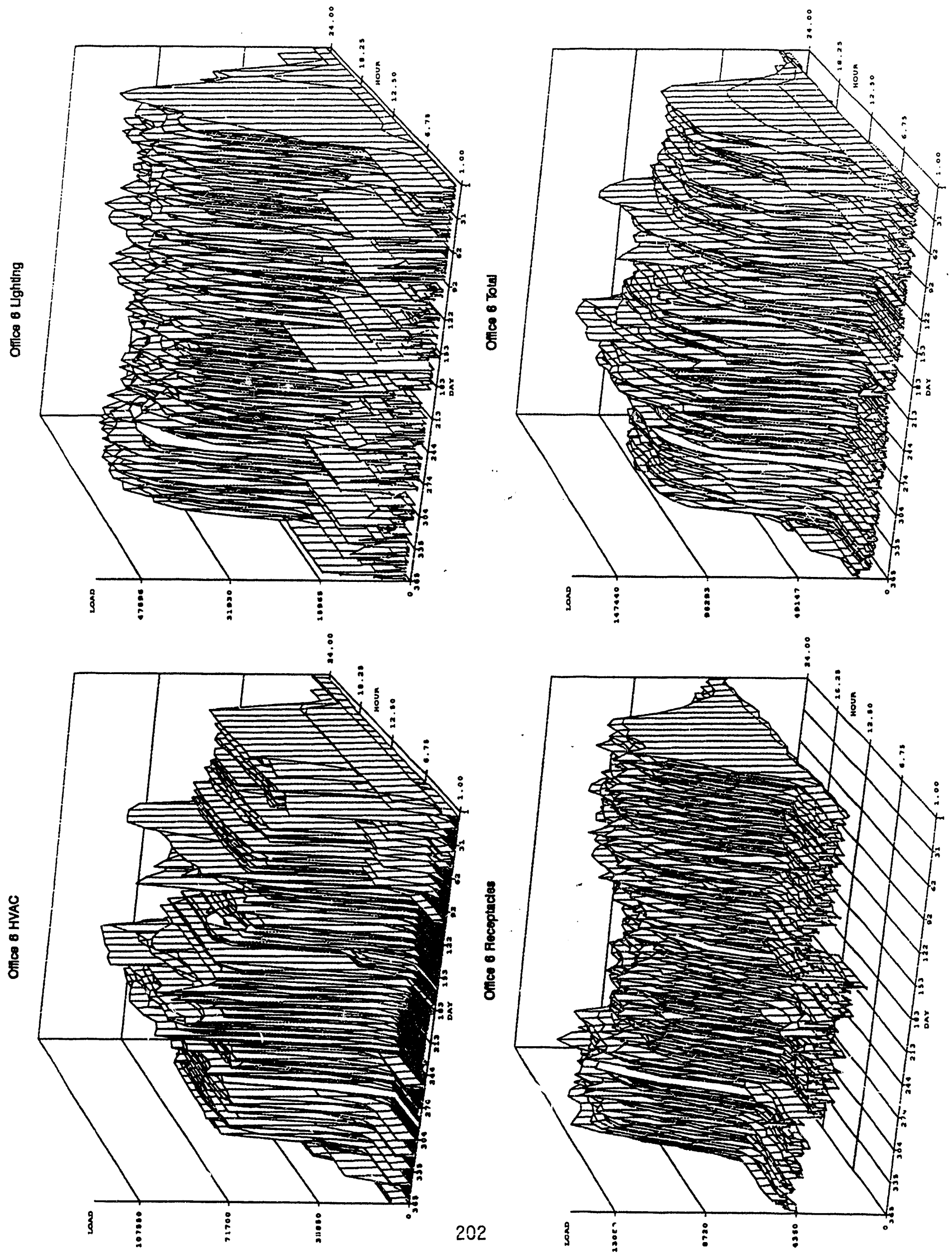




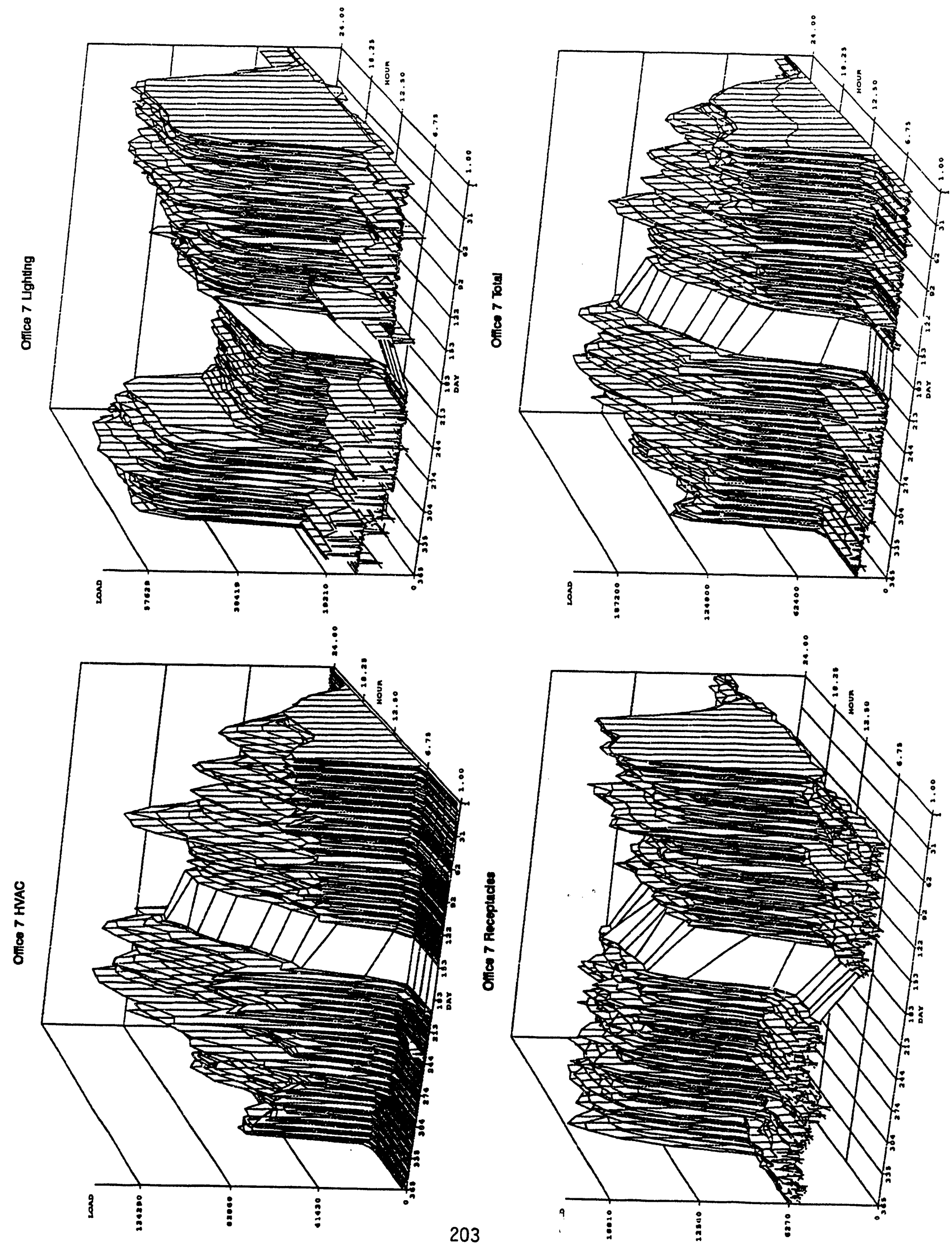



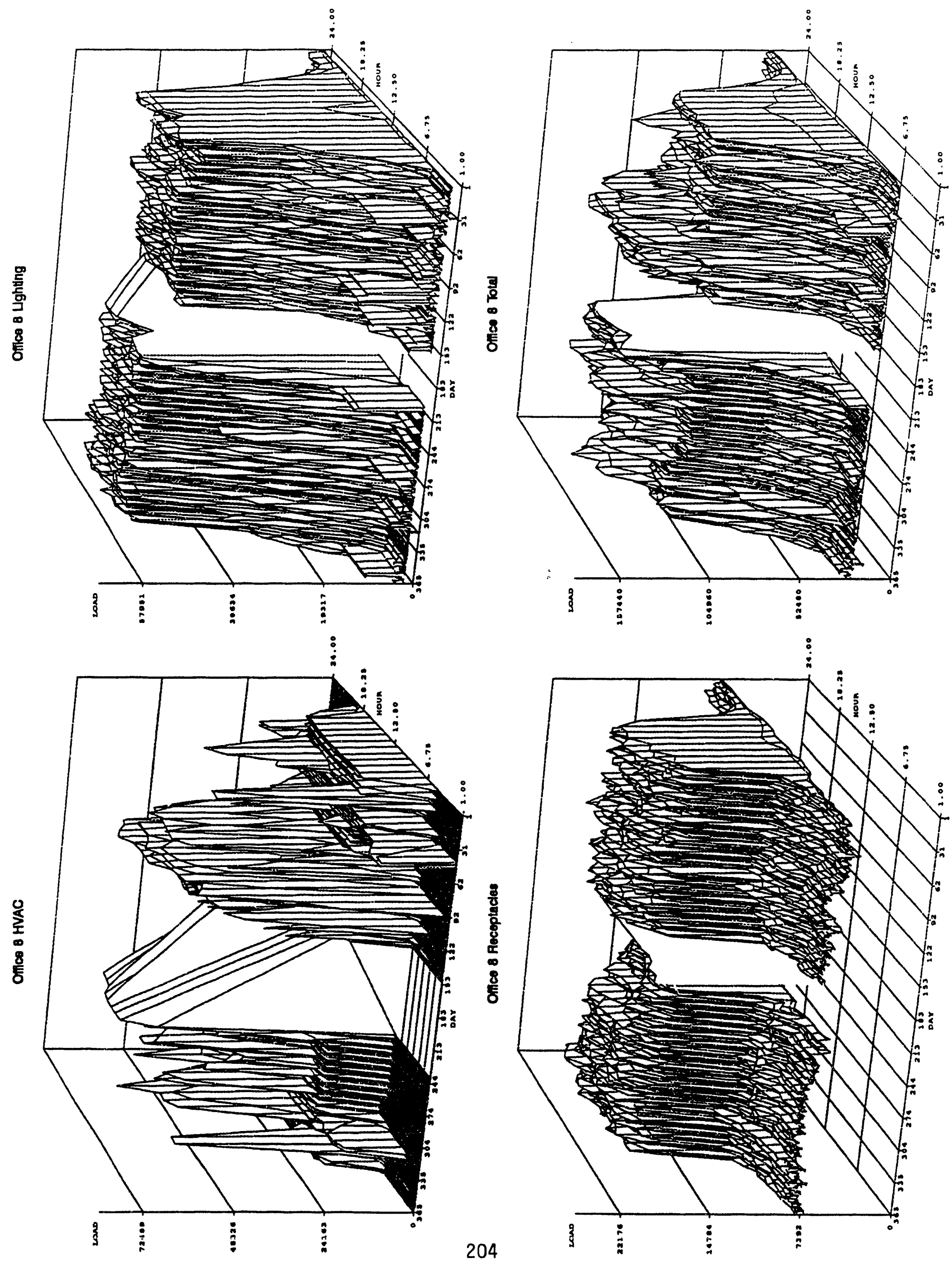


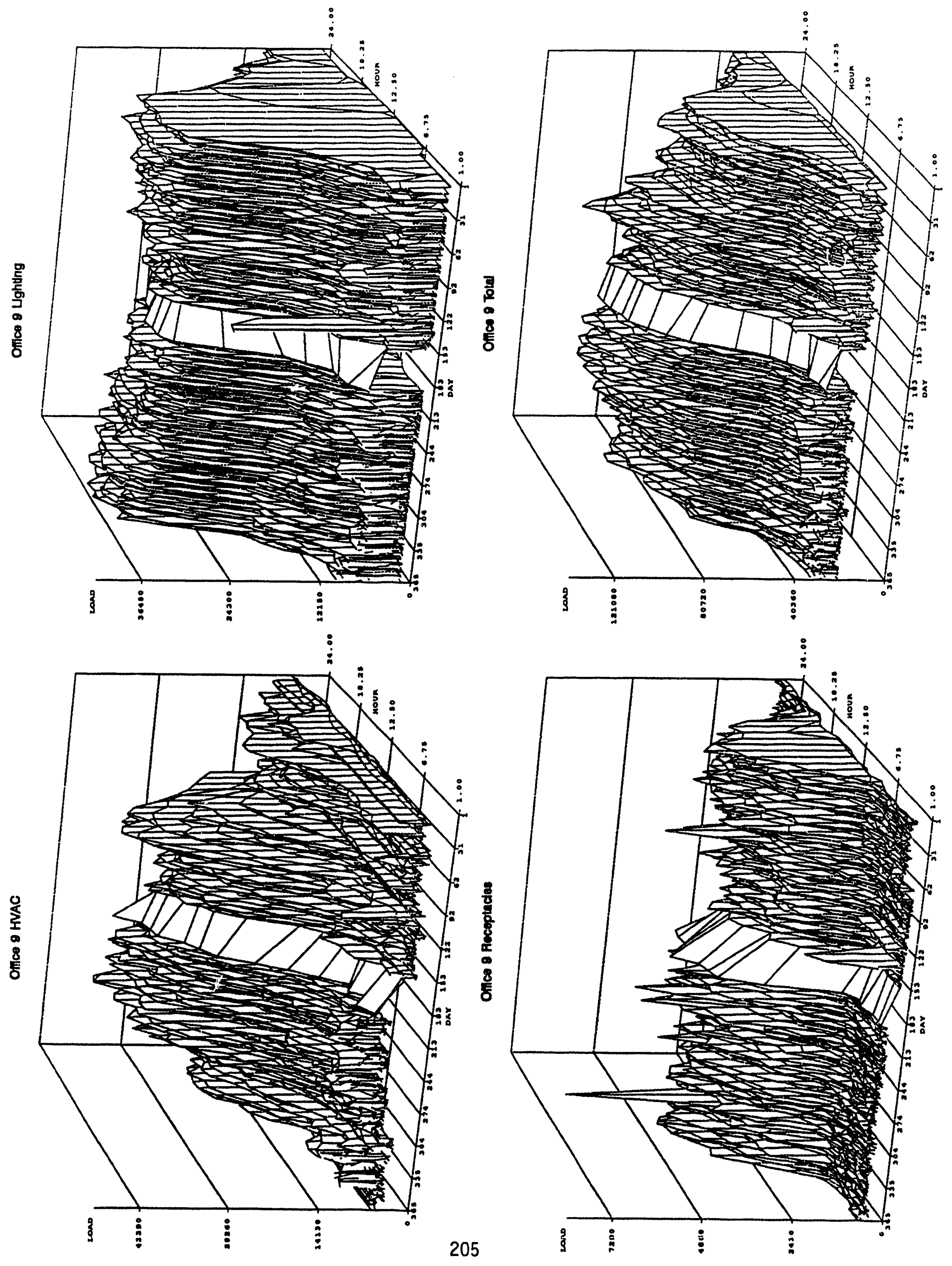



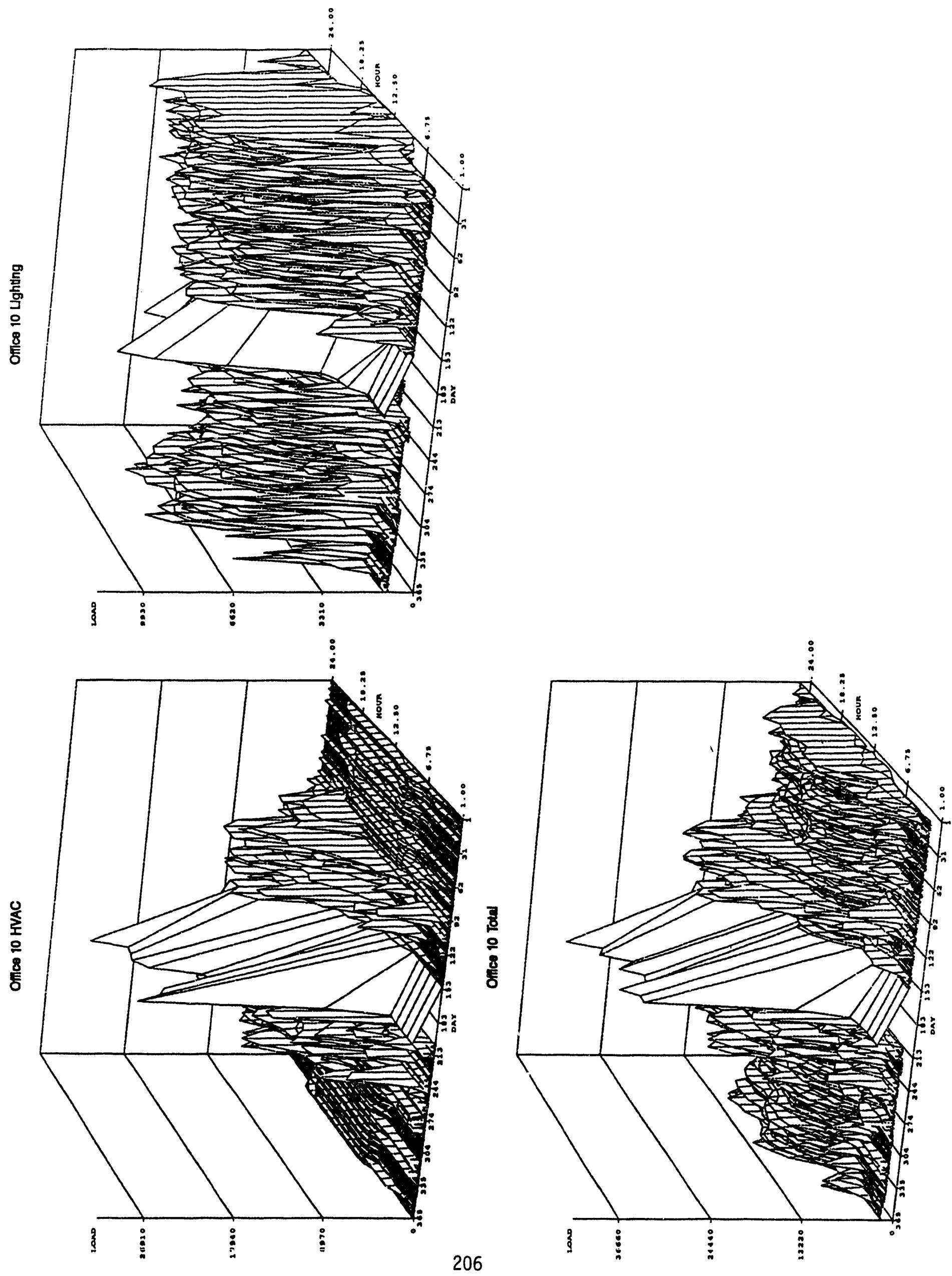


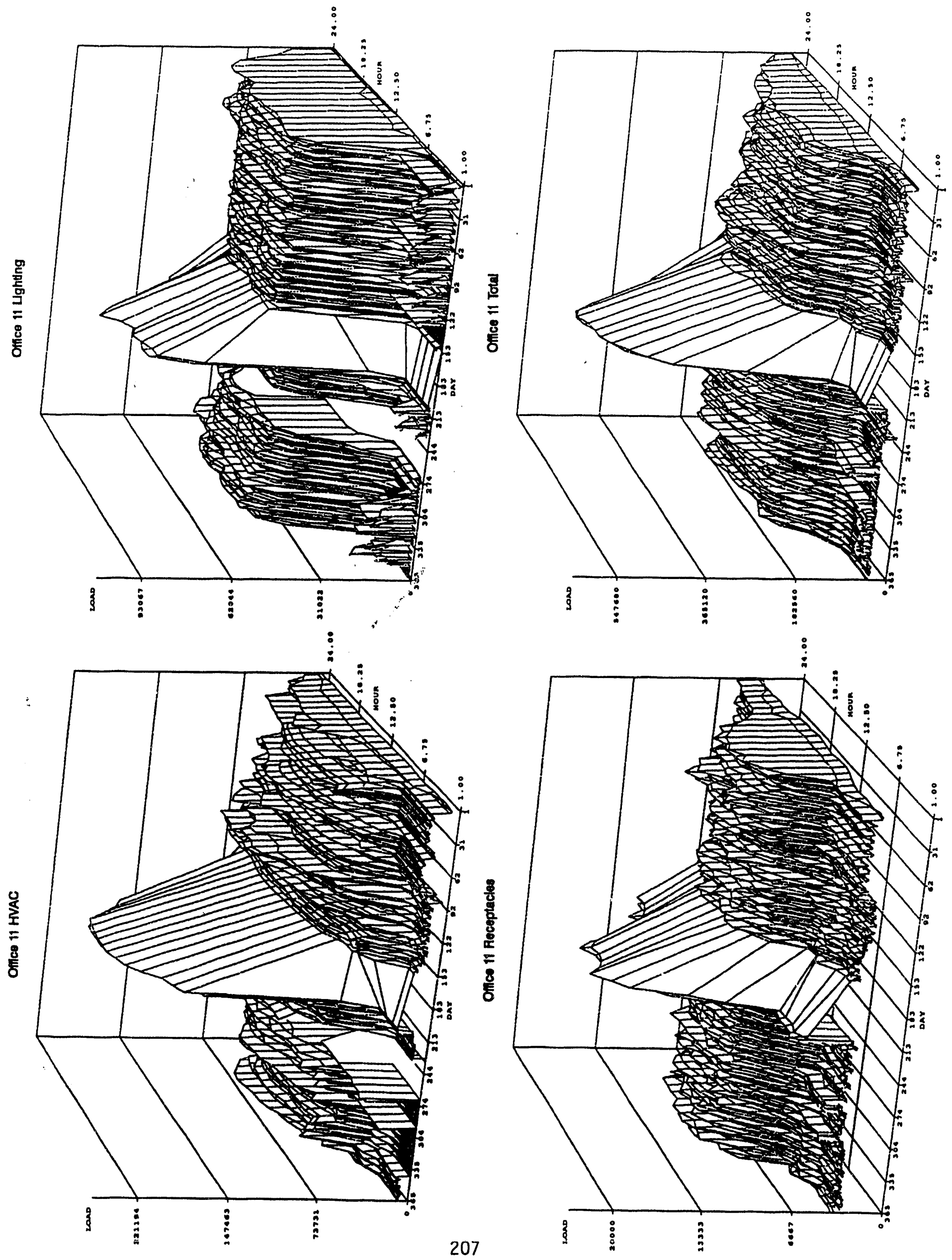



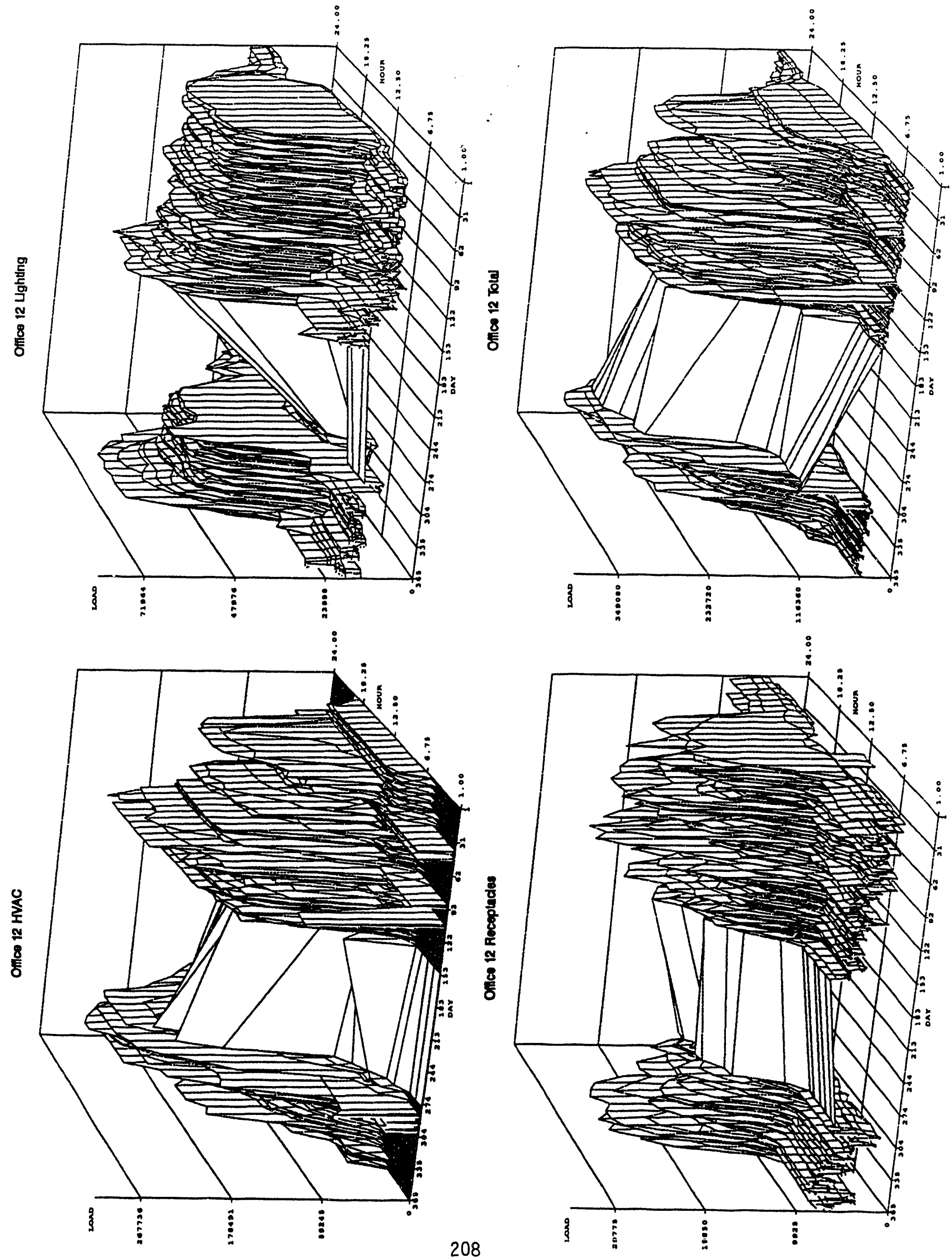

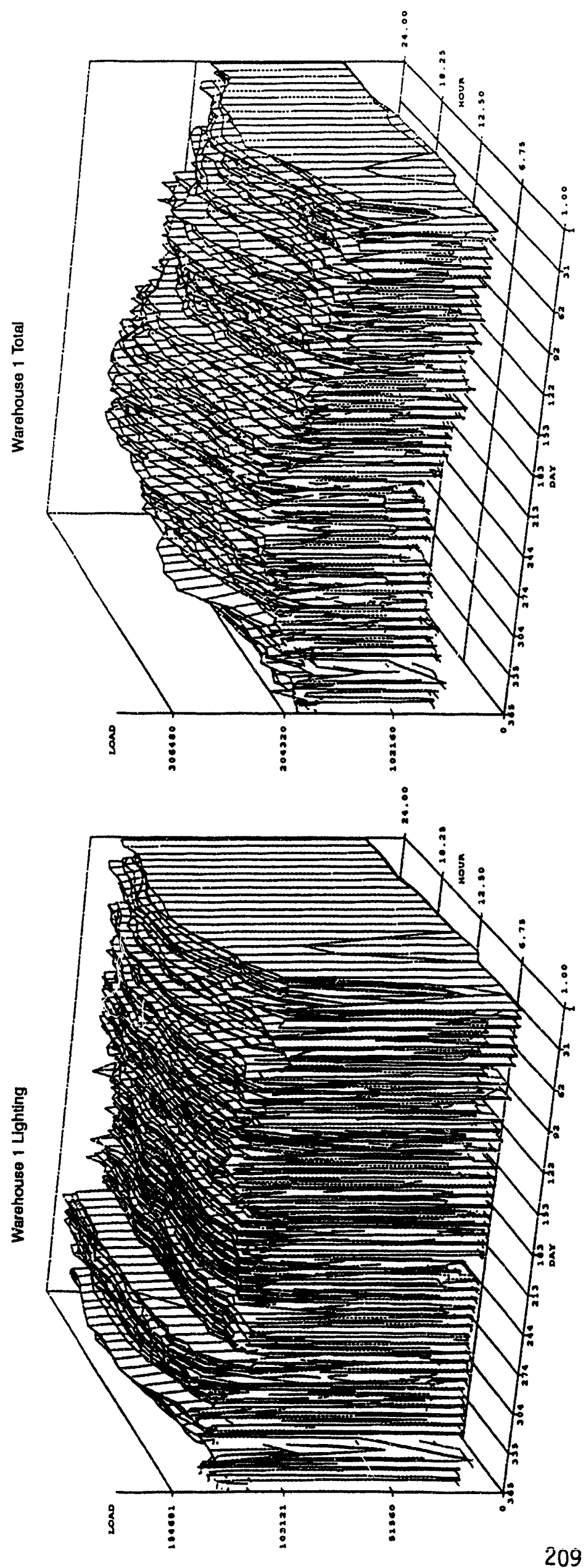

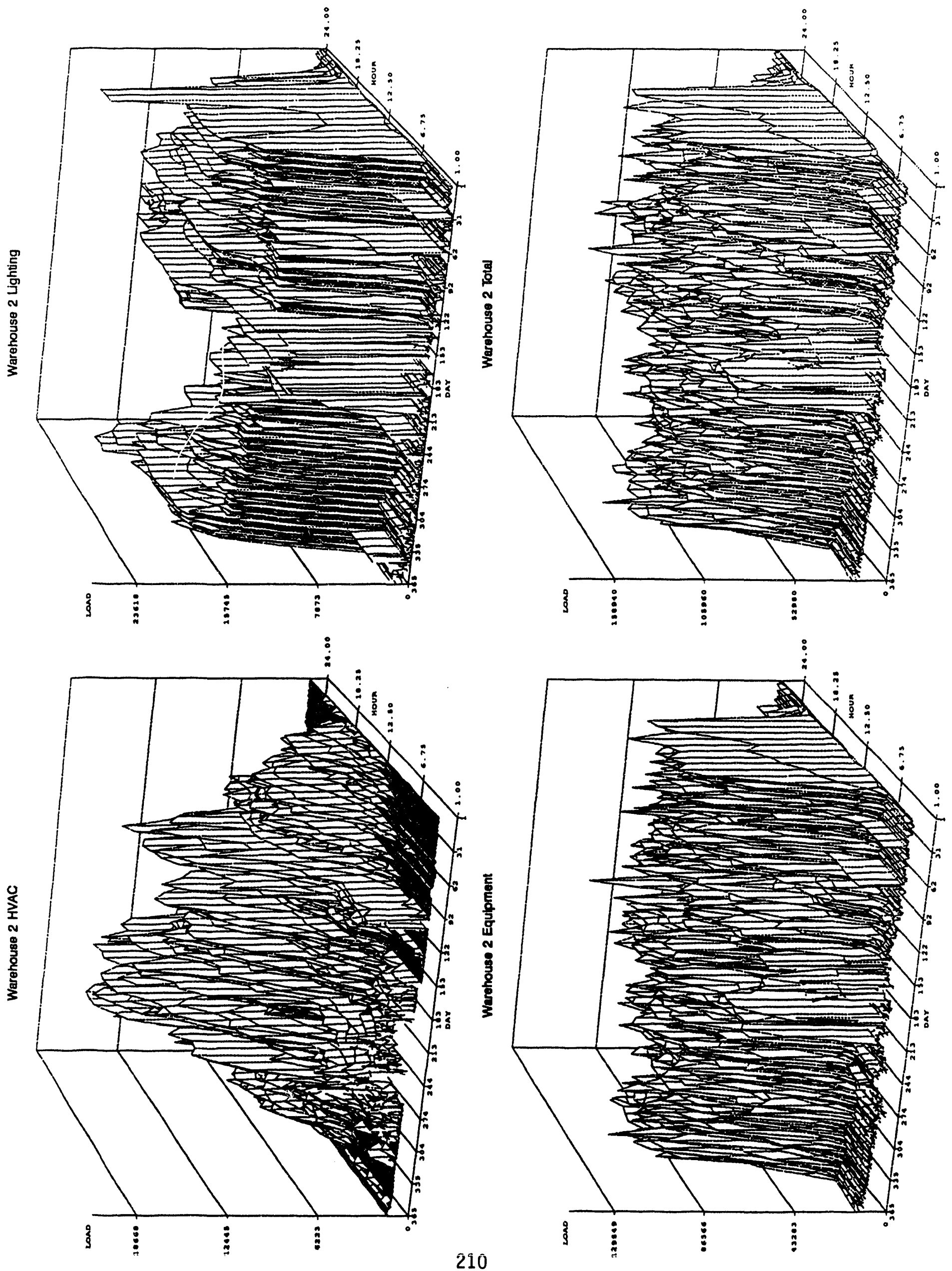

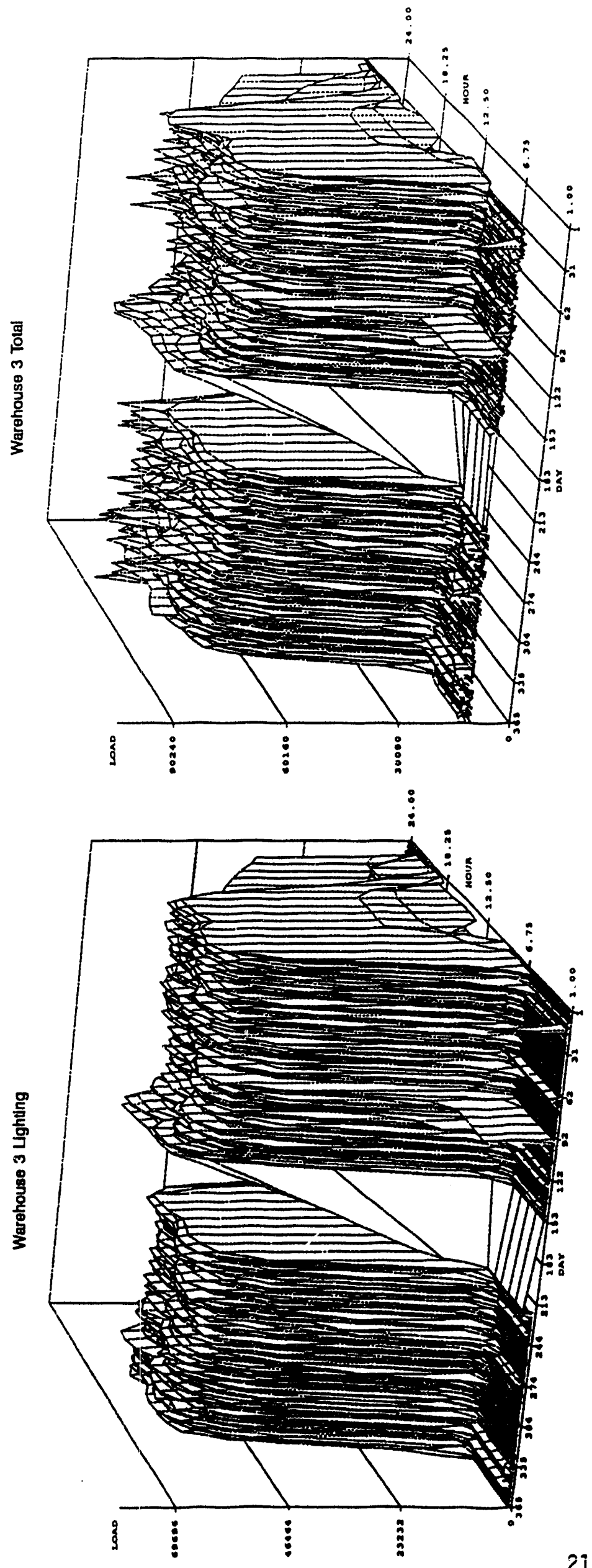

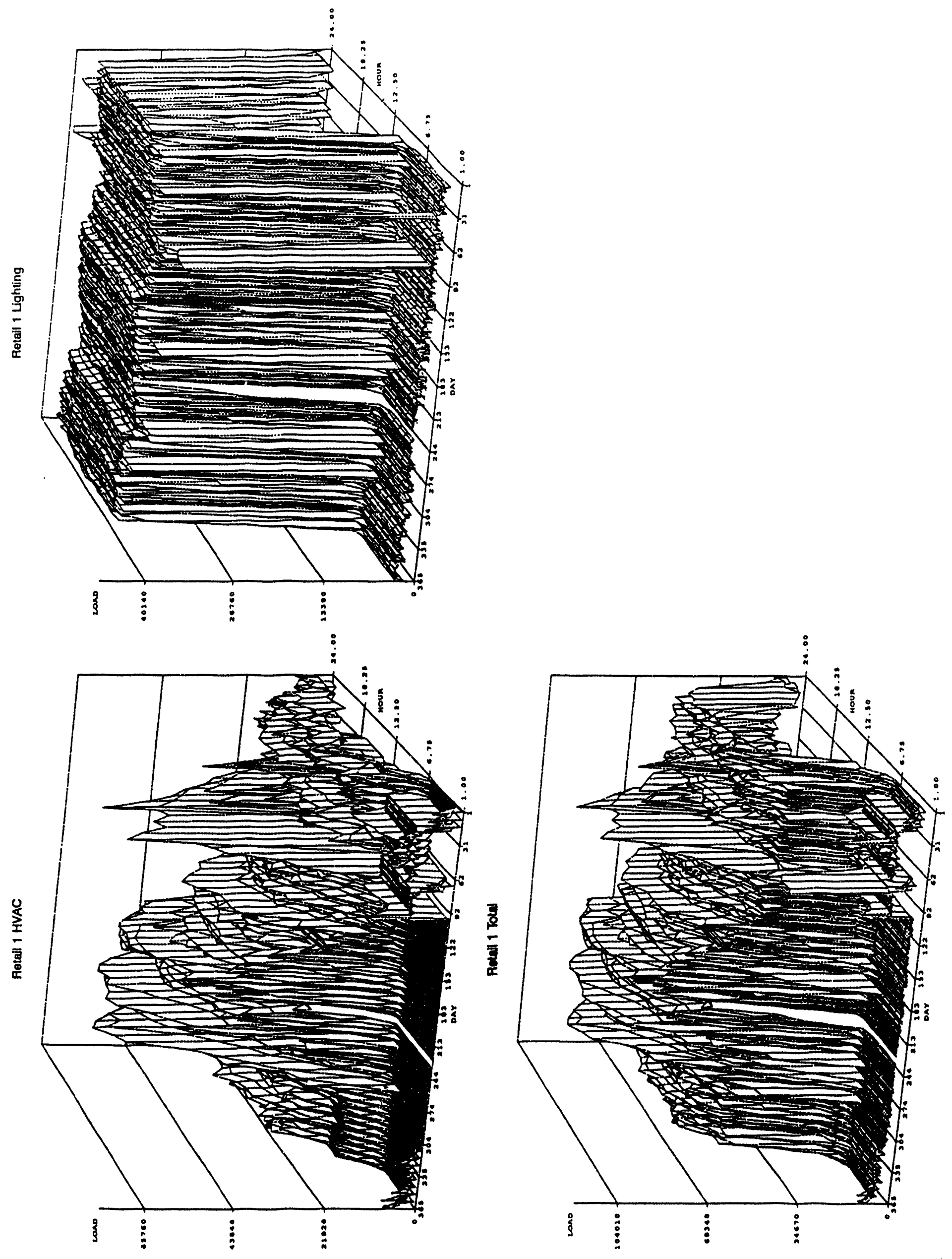

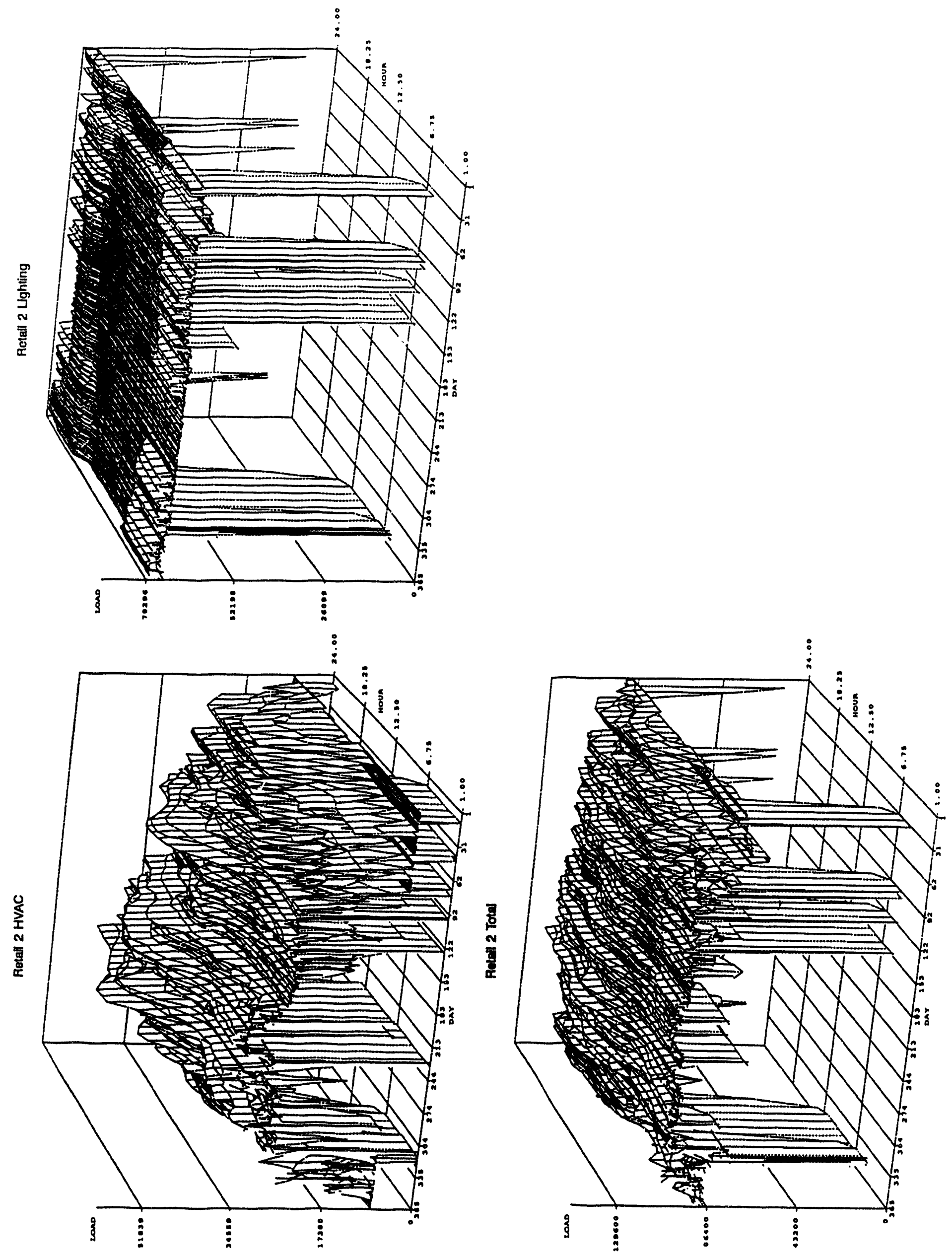

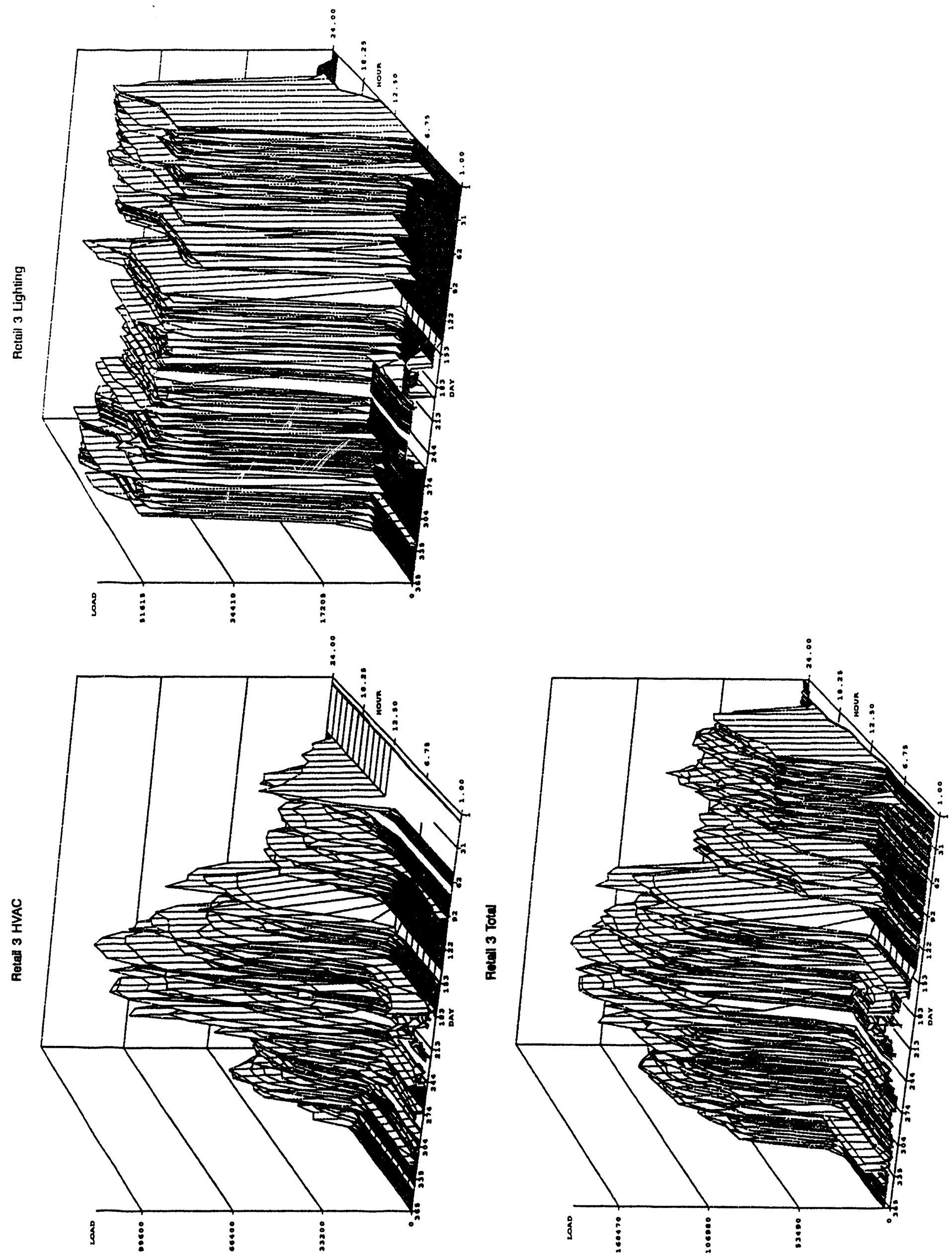

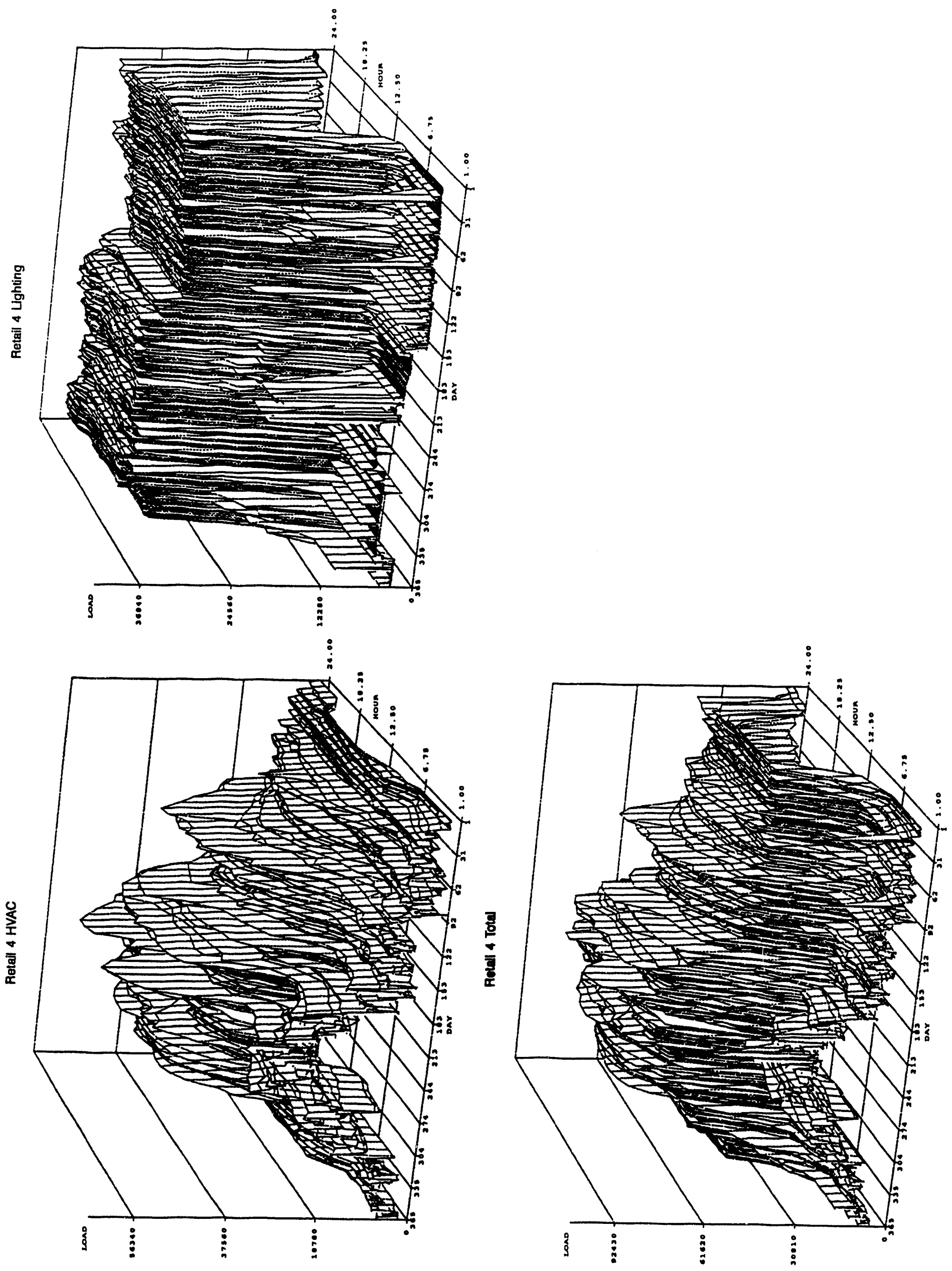

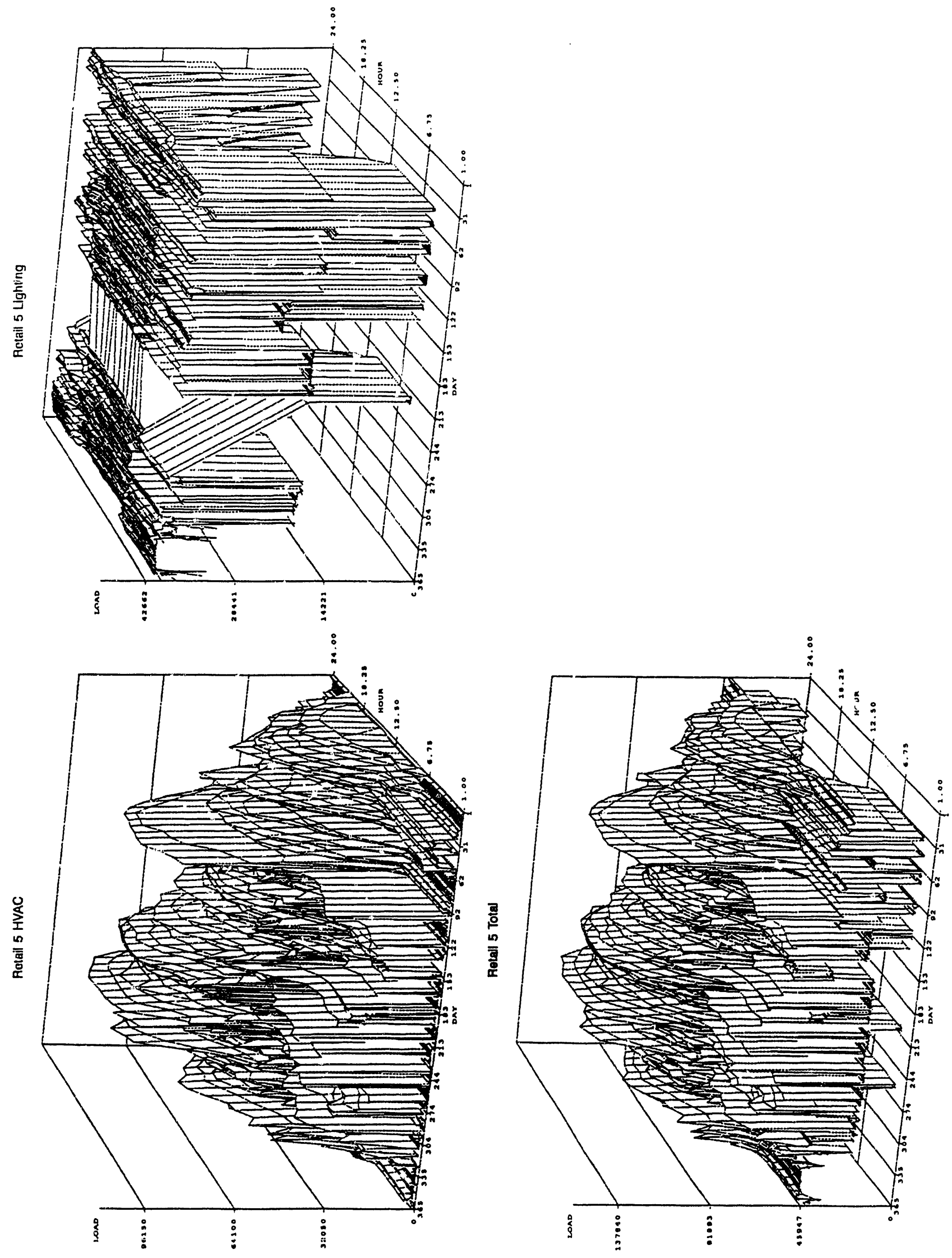

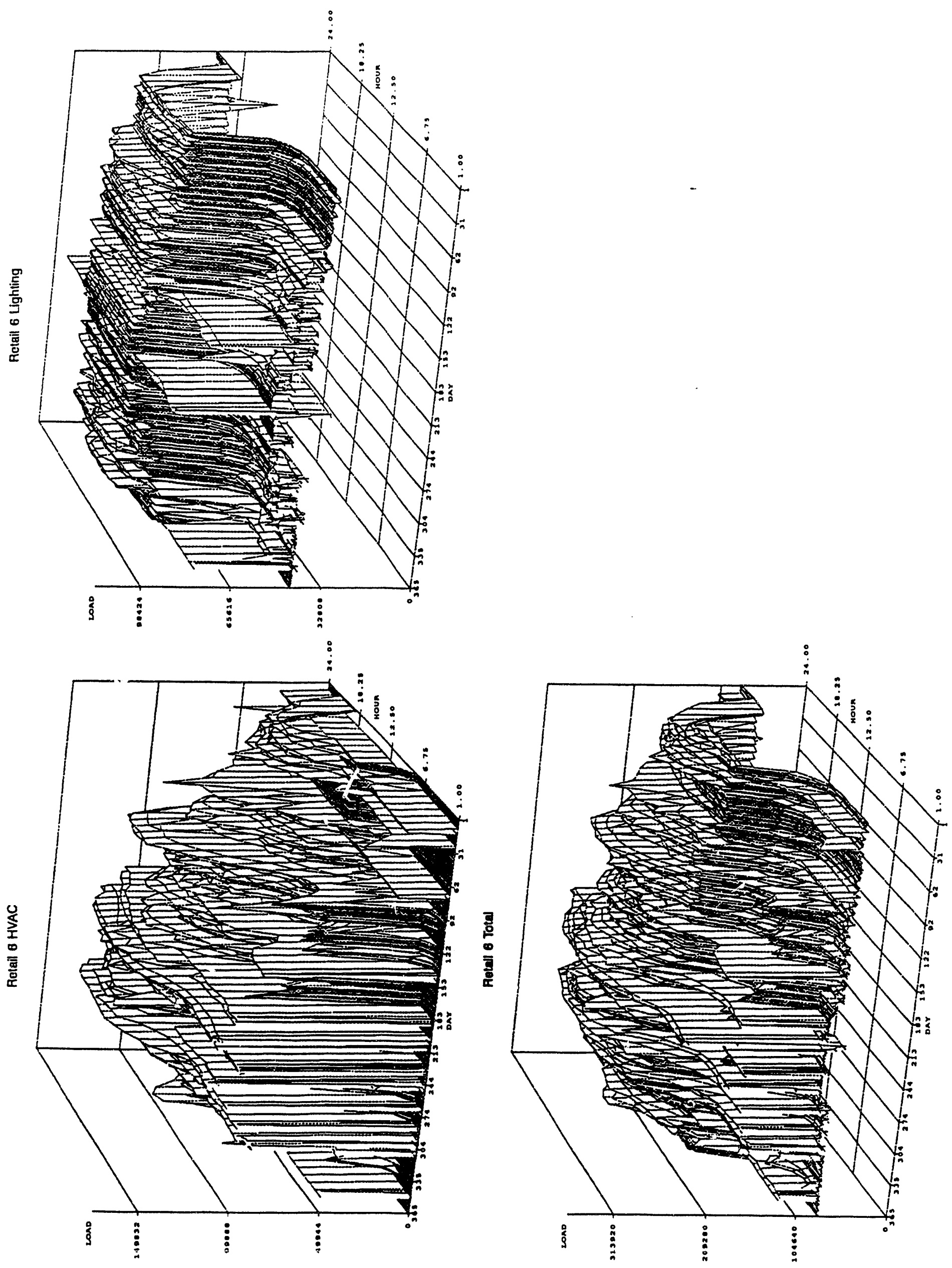

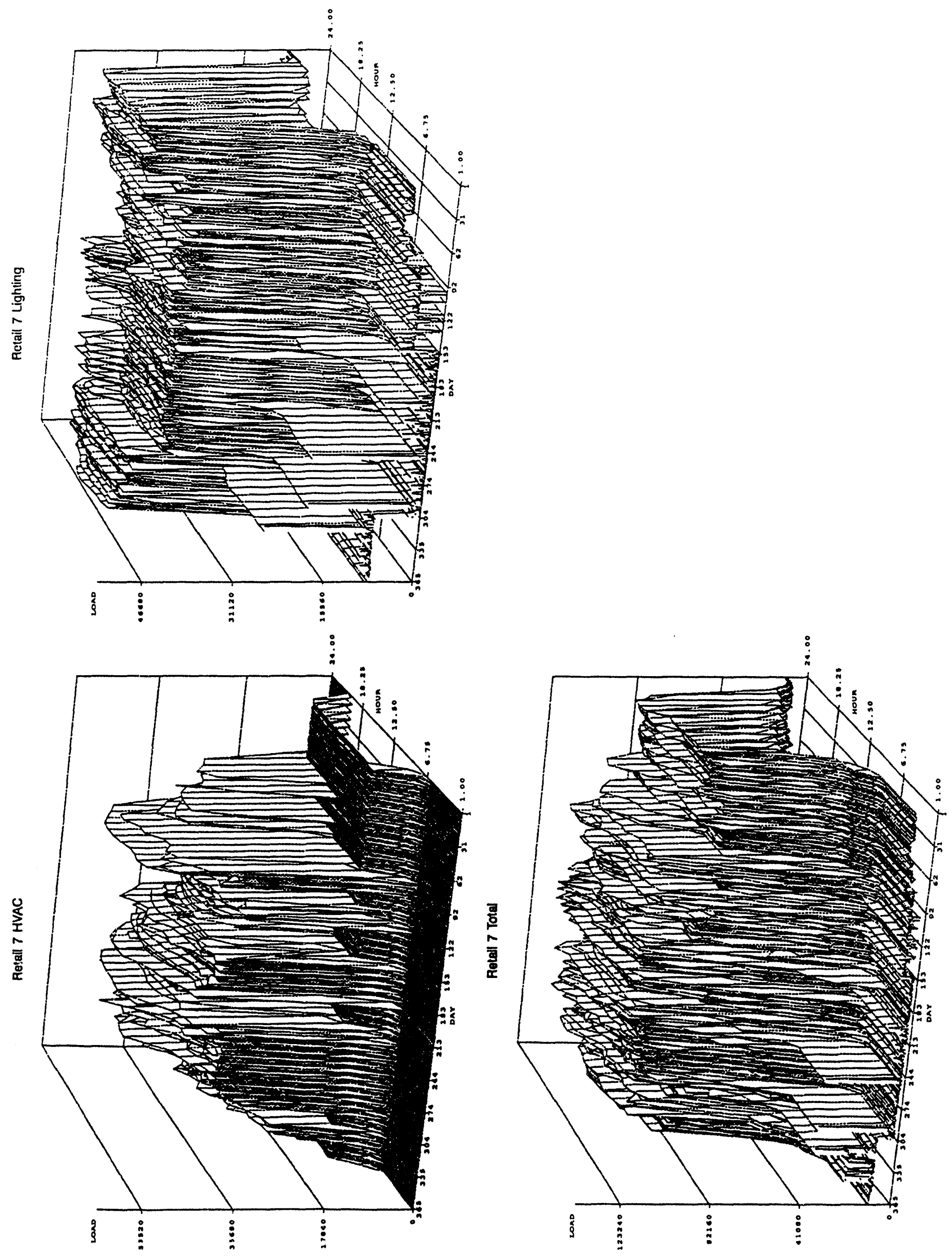


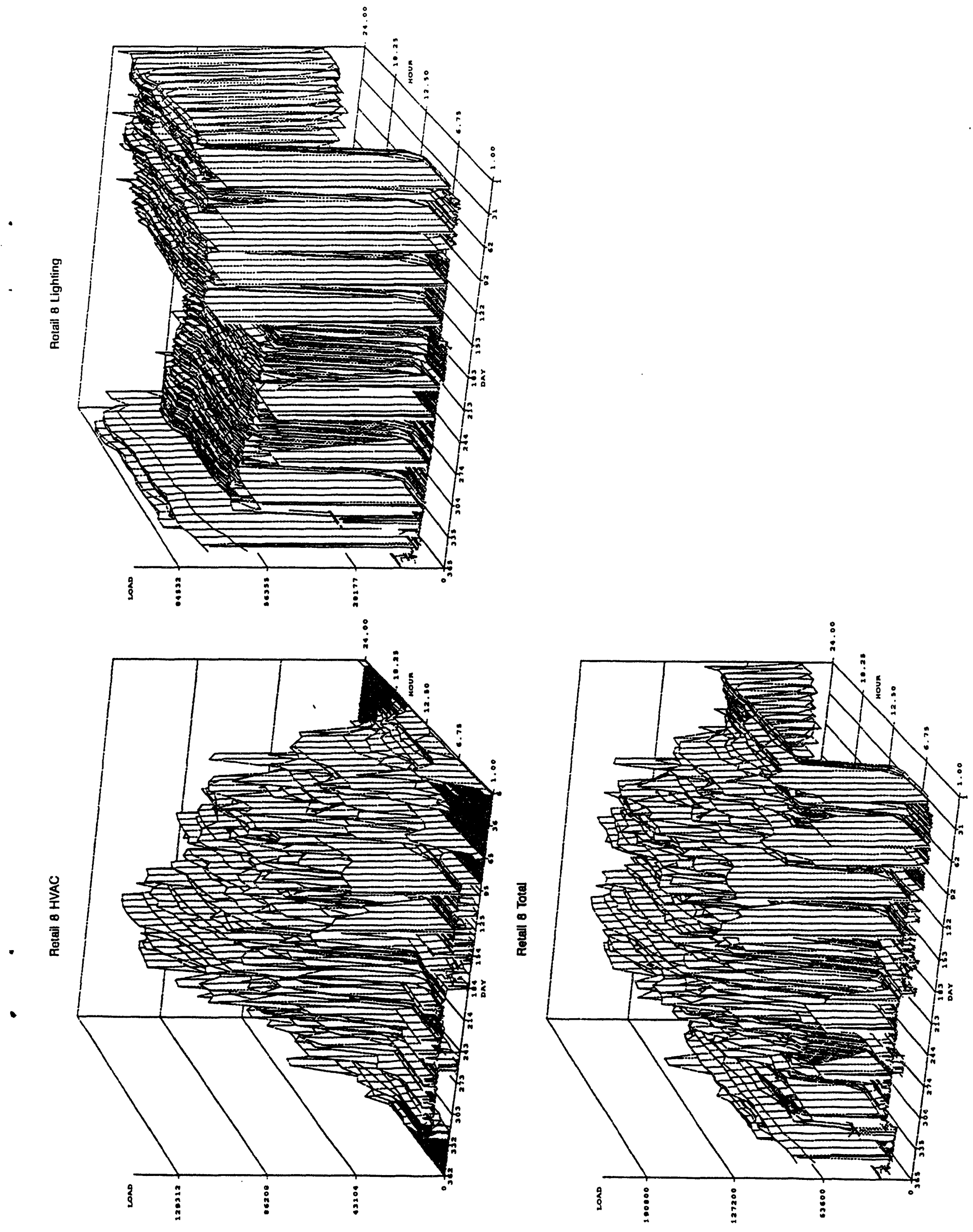



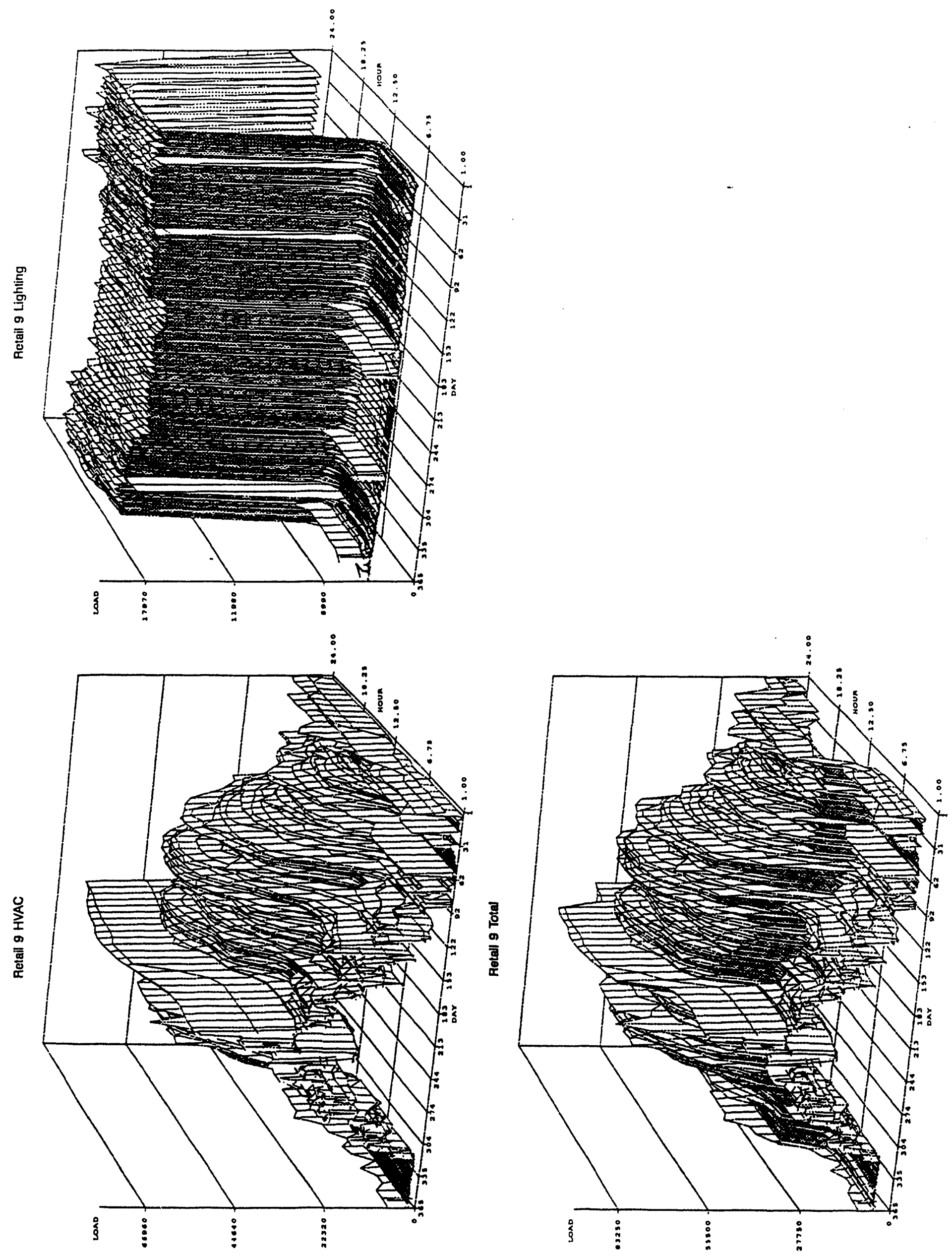

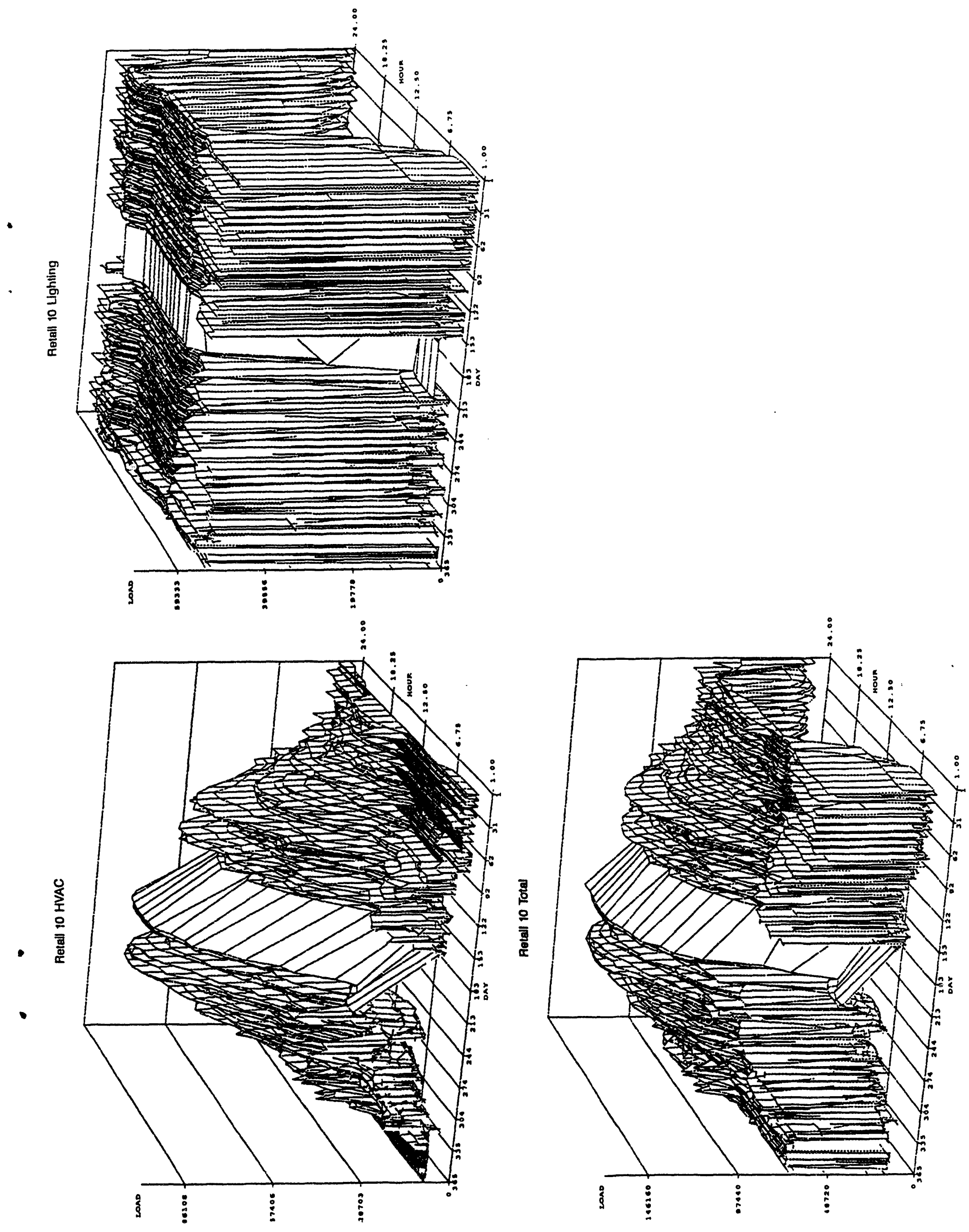

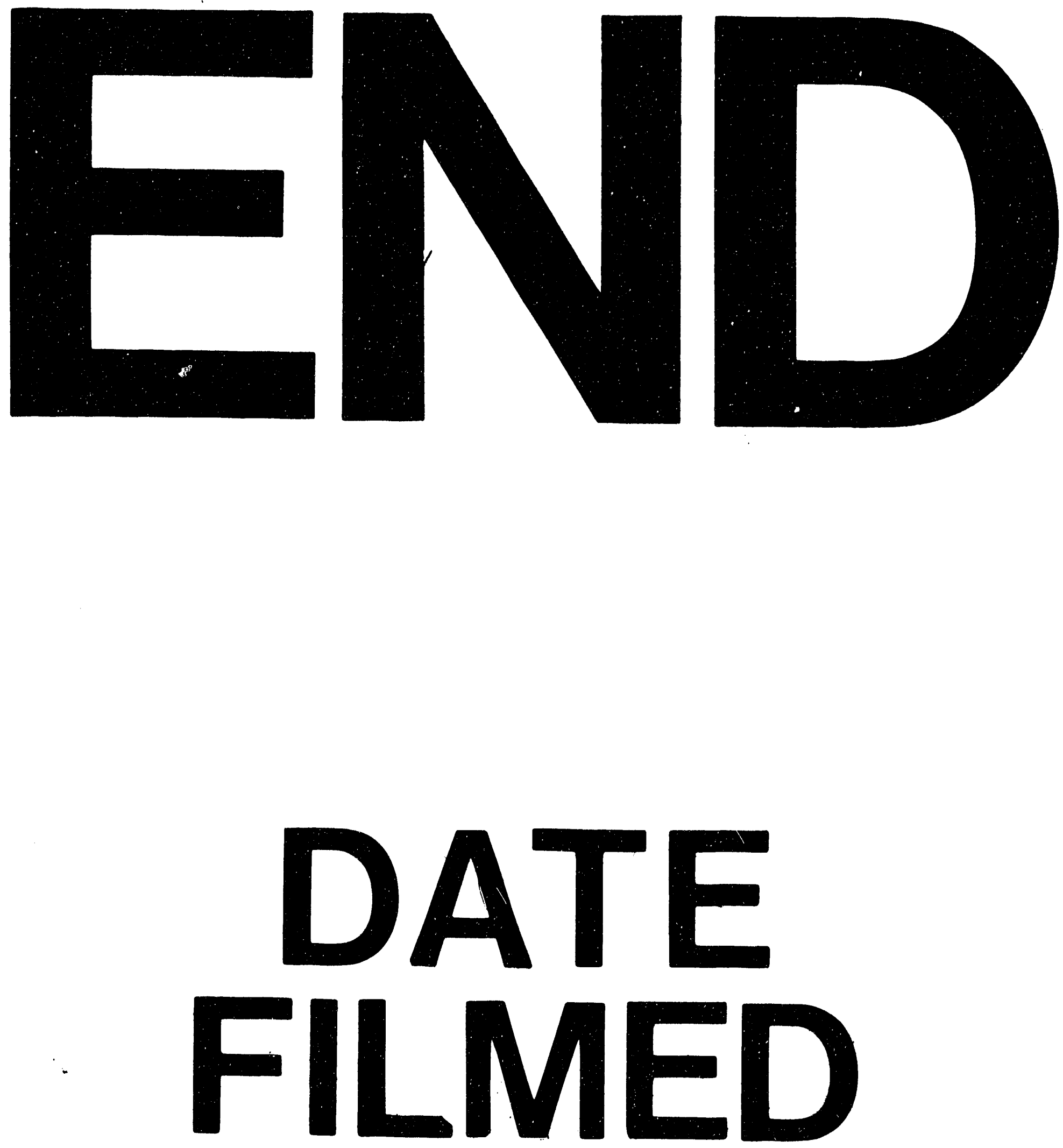

1

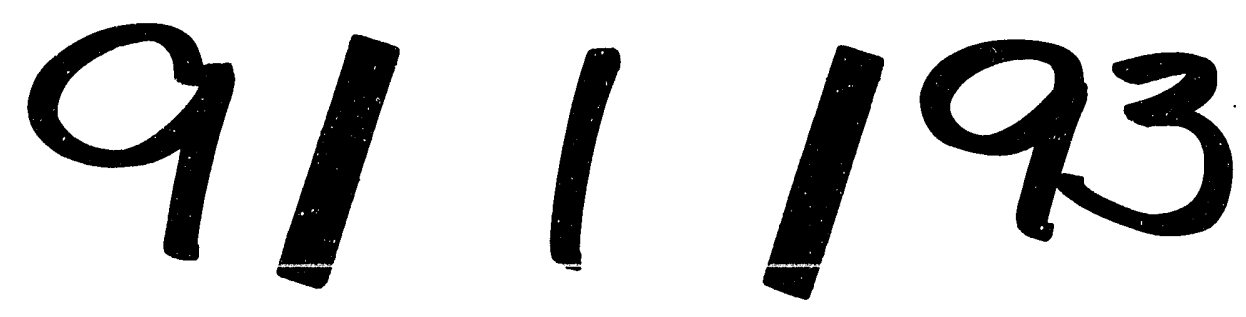


